\title{
Evidence Against Single-Electron Transfer in the Additions of Most Organomagnesium Reagents to Carbonyl Compounds
}

\author{
Nicole D. Bartolo and K. A. Woerpel* \\ Department of Chemistry, New York University \\ 100 Washington Square East, New York, New York 10003 \\ *kwoerpel@nyu.edu
}

Table of Contents

\section{Supporting Information}

I. Tentative Assignment of Relative Stereochemistry for Additions to Radical Clocks ....... S2

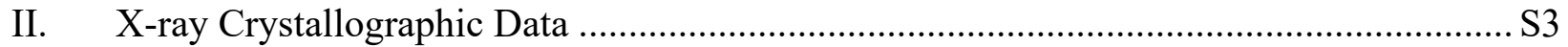

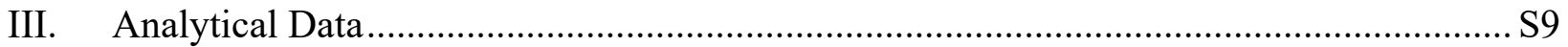




\section{Tentative Assignment of Relative Stereochemistry for Additions to Radical Clocks}

The tentative stereochemical assignment for the products formed in the additions of organometallic reagents to aldehyde $\mathbf{3}$ was made by analogy to the minor diastereomer $\mathbf{8 d}$ ' formed in the addition of phenylmagnesium chloride to aldehyde 3. The relative stereochemistry of alcohol 8d' was assigned by X-ray crystallography. Additionally, the ${ }^{1} \mathrm{H}$ NMR spectra of the two diastereomers formed in the additions to aldehyde $\mathbf{3}$ were consistently distinguished. In all cases, the three peaks corresponding to the cyclopropyl protons were resolved in the ${ }^{1} \mathrm{H}$ NMR spectra of the major diastereomer (Figure S1, a). Conversely, in the ${ }^{1} \mathrm{H}$ NMR spectra of the minor diastereomer, two of the three peaks corresponding to the cyclopropyl protons are overlapping (Figure S1, b).

(a)

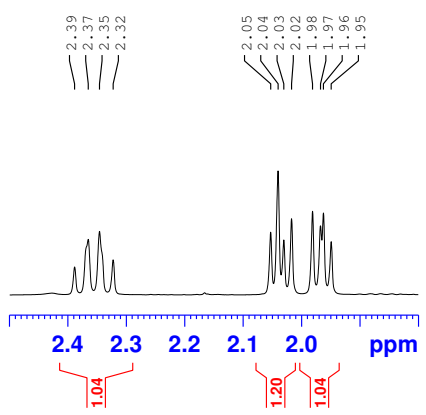

(b)

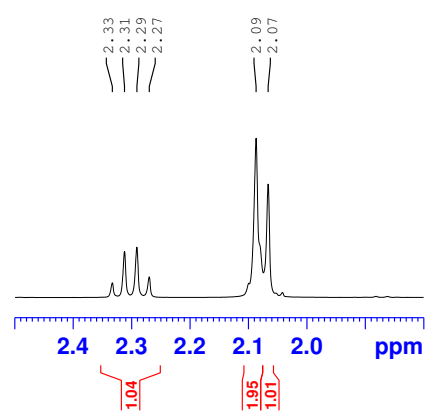

Figure S1. ${ }^{1} \mathrm{H}$ NMR spectra in $\mathrm{CDCl}_{3}$ of the peaks corresponding to the cyclopropyl protons for major diastereomer 8d (a) and minor diastereomer 8d' (b).

The tentative stereochemical assignment for the products formed in the additions of organometallic reagents to ketones 5, 6, and 7 were made by analogy to the alcohol 9d formed in the addition of phenylmagnesium chloride to ketone $\mathbf{5}$. The relative stereochemistry of alcohol $\mathbf{9 d}$ was assigned by X-ray crystallography. 


\section{X-ray Crystallographic Data}

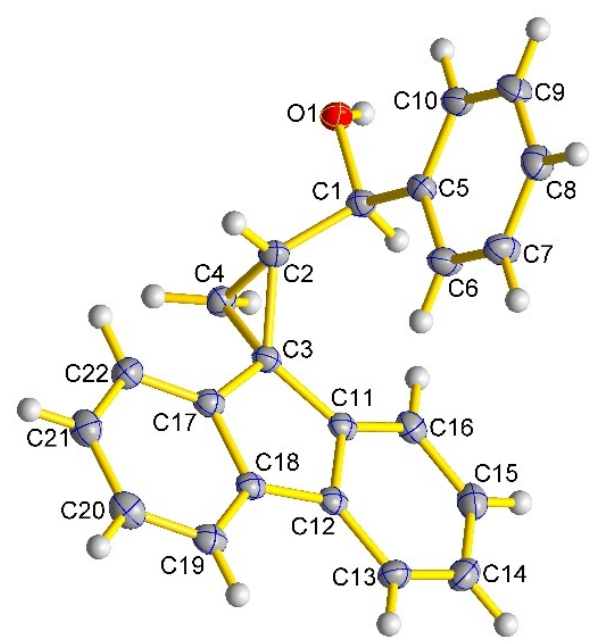

Figure S2. The molecular structure of alcohol 8d' (ellipsoids set at 50\% probability).

A colorless block-like specimen of $\mathrm{C}_{22} \mathrm{H}_{18} \mathrm{O}$ (grown by slow evaporation from ethyl acetate and hexanes), approximate dimensions $0.220 \mathrm{~mm} 0.360 \mathrm{~mm} \times 0.560 \mathrm{~mm}$, was used for the X-ray crystallographic analysis. The X-ray intensity data were measured on a Bruker APEX-II CCD system equipped with a graphite monochromator and a Mo sealed tube $(\lambda=0.71073 \AA)$.

A total of 1184 frames were collected. The total exposure time was 1.64 hours. The frames were integrated with the Bruker SAINT software package using a narrow-frame algorithm. The integration of the data using a tetragonal unit cell yielded a total of 35844 reflections to a maximum $\theta$ angle of $28.30^{\circ}(0.75 \AA$ resolution), of which 4003 were independent (average redundancy 8.954, completeness $\left.=100.0 \%, \mathrm{R}_{\text {int }}=3.72 \%, \mathrm{R}_{\text {sig }}=2.25 \%\right)$ and $3819(95.40 \%)$ were greater than $2 \sigma\left(\mathrm{F}^{2}\right)$. The final cell constants of $\underline{\mathrm{a}}=28.371(2) \AA, \underline{\mathrm{b}}=28.371(2) \AA, \underline{\mathrm{c}}=7.9856(6) \AA$, volume $=6427.7(11) \AA^{3}$, are based upon the refinement of the XYZ-centroids of 9897 reflections above $20 \sigma(\mathrm{I})$ with $4.54^{\circ}<2 \theta<55.54^{\circ}$. Data were corrected for absorption effects using the multi-scan method (SADABS). The ratio of minimum to maximum apparent transmission was 0.895 . The calculated minimum and maximum transmission coefficients (based on crystal size) are 0.6677 and 0.7457 .

The structure was solved and refined using the Bruker SHELXTL Software Package, using the space group I $41 \mathrm{c} \mathrm{d}$, with $\mathrm{Z}=16$ for the formula unit, $\mathrm{C}_{22} \mathrm{H}_{18} \mathrm{O}$. The final anisotropic full-matrix least-squares refinement on $\mathrm{F}^{2}$ with 212 variables converged at $\mathrm{R} 1=4.49 \%$, for the observed data and $\mathrm{wR} 2=11.48 \%$ for all data. The goodness-of-fit was 1.040 . The largest peak in the final difference electron density synthesis was $0.368 \mathrm{e}^{-} / \AA^{3}$ and the largest hole was $-0.172 \mathrm{e}^{-} / \AA^{3}$ with an RMS deviation of $0.047 \mathrm{e}^{-} / \AA^{3}$. On the basis of the final model, the calculated density was $1.233 \mathrm{~g} / \mathrm{cm}^{3}$ and $\mathrm{F}(000), 2528 \mathrm{e}^{-}$. 
Table S1. Sample and crystal data for $\mathbf{8 d}$ '.

\begin{tabular}{|c|c|c|}
\hline Identification code & \multicolumn{2}{|l|}{ 19kaw7h } \\
\hline Chemical formula & \multicolumn{2}{|l|}{$\mathrm{C}_{22} \mathrm{H}_{18} \mathrm{O}$} \\
\hline Formula weight & \multicolumn{2}{|l|}{$298.38 \mathrm{~g} / \mathrm{mol}$} \\
\hline Temperature & \multicolumn{2}{|l|}{$100(2) \mathrm{K}$} \\
\hline Wavelength & \multicolumn{2}{|l|}{$0.71073 \AA$} \\
\hline Crystal size & \multicolumn{2}{|c|}{$0.220 \times 0.360 \times 0.560 \mathrm{~mm}$} \\
\hline Crystal habit & \multicolumn{2}{|c|}{ colorless block } \\
\hline Crystal system & \multicolumn{2}{|l|}{ tetragonal } \\
\hline Space group & \multicolumn{2}{|l|}{ I $41 \mathrm{c} \mathrm{d}$} \\
\hline \multirow[t]{3}{*}{ Unit cell dimensions } & $\mathrm{a}=28.371(2) \AA$ & $\alpha=90^{\circ}$ \\
\hline & $\mathrm{b}=28.371(2) \AA$ & $\beta=90^{\circ}$ \\
\hline & $c=7.9856(6) \AA$ & $\gamma=90^{\circ}$ \\
\hline Volume & \multicolumn{2}{|l|}{$6427.7(11) \AA^{3}$} \\
\hline $\mathbf{Z}$ & \multicolumn{2}{|l|}{16} \\
\hline Density (calculated) & \multicolumn{2}{|l|}{$1.233 \mathrm{~g} / \mathrm{cm}^{3}$} \\
\hline Absorption coefficient & \multicolumn{2}{|l|}{$0.074 \mathrm{~mm}^{-1}$} \\
\hline$F(000)$ & \multicolumn{2}{|l|}{2528} \\
\hline
\end{tabular}


Table S2. Data collection and structure refinement for alcohol 8d'.

\begin{tabular}{|c|c|c|}
\hline Diffractometer & \multirow{2}{*}{\multicolumn{2}{|c|}{ Bruker APEX-II CCD }} \\
\hline Radiation source & & sealed tube, Mo \\
\hline Theta range for data collection & \multicolumn{2}{|c|}{1.44 to $28.30^{\circ}$} \\
\hline Index ranges & \multicolumn{2}{|c|}{$-37<=\mathrm{h}<=37,-36<=\mathrm{k}<=37,-10<=1<=10$} \\
\hline Reflections collected & \multicolumn{2}{|l|}{35844} \\
\hline Independent reflections & \multicolumn{2}{|c|}{$4003[\mathrm{R}(\mathrm{int})=0.0372]$} \\
\hline Coverage of independent reflections & \multicolumn{2}{|l|}{$100.0 \%$} \\
\hline Absorption correction & \multicolumn{2}{|l|}{ multi-scan } \\
\hline Max. and min. transmission & \multicolumn{2}{|l|}{0.7457 and 0.6677} \\
\hline Structure solution technique & \multicolumn{2}{|l|}{ direct methods } \\
\hline Structure solution program & \multicolumn{2}{|c|}{ SHELXT (Sheldrick 2015) } \\
\hline Refinement method & \multicolumn{2}{|c|}{ Full-matrix least-squares on $\mathrm{F}^{2}$} \\
\hline Refinement program & \multicolumn{2}{|c|}{ SHELXL-2018/3 (Sheldrick, 2018) } \\
\hline Function minimized & \multicolumn{2}{|c|}{$\Sigma \mathrm{w}\left(\mathrm{F}_{\mathrm{o}}^{2}-\mathrm{F}_{\mathrm{c}}^{2}\right)^{2}$} \\
\hline Data / restraints / parameters & \multicolumn{2}{|l|}{$4003 / 2 / 212$} \\
\hline Goodness-of-fit on $\mathbf{F}^{2}$ & \multicolumn{2}{|l|}{1.040} \\
\hline \multirow[t]{2}{*}{ Final $\mathbf{R}$ indices } & 3819 data; $\mathrm{I}>2 \sigma(\mathrm{I})$ & $\begin{array}{l}\mathrm{R} 1=0.0449 \\
\mathrm{wR} 2=0.1130\end{array}$ \\
\hline & all data & $\begin{array}{l}\mathrm{R} 1=0.0472 \\
\mathrm{wR} 2=0.1148\end{array}$ \\
\hline Weighting scheme & \multicolumn{2}{|c|}{$\begin{array}{l}\mathrm{w}=1 /\left[\sigma^{2}\left(\mathrm{~F}_{\mathrm{o}}^{2}\right)+(0.0677 \mathrm{P})^{2}+4.0750 \mathrm{P}\right] \\
\text { where } \mathrm{P}=\left(\mathrm{F}_{\mathrm{o}}^{2}+2 \mathrm{~F}_{\mathrm{c}}^{2}\right) / 3\end{array}$} \\
\hline Absolute structure parameter & \multicolumn{2}{|l|}{$0.3(5)$} \\
\hline Largest diff. peak and hole & \multicolumn{2}{|c|}{0.368 and $-0.172 \mathrm{e}^{-3}$} \\
\hline R.M.S. deviation from mean & \multicolumn{2}{|l|}{$0.047 \mathrm{e}^{-3}$} \\
\hline
\end{tabular}




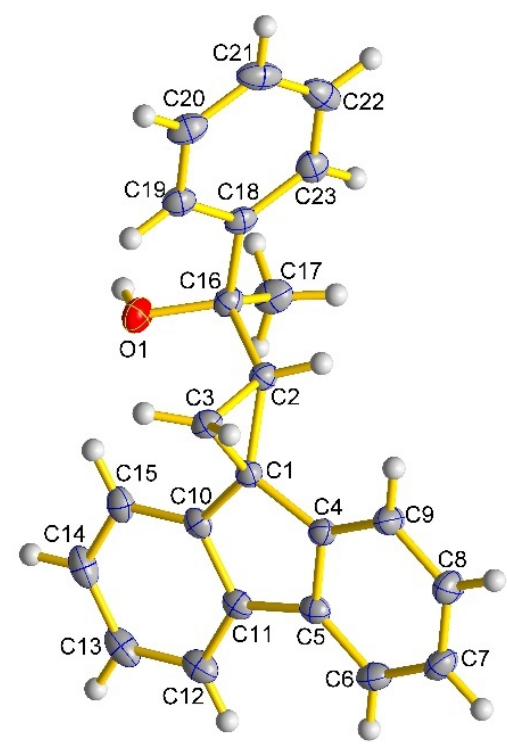

Figure S3. The molecular structure of alcohol 9d (ellipsoids set at 50\% probability).

A colorless block-like specimen of $\mathrm{C}_{23} \mathrm{H}_{20} \mathrm{O}$ (grown by slow evaporation from ethyl acetate and hexanes), approximate dimensions $0.230 \mathrm{~mm} \times 0.320 \mathrm{~mm} \times 0.540 \mathrm{~mm}$, was used for the X-ray crystallographic analysis. The X-ray intensity data were measured on a Bruker APEX-II CCD system equipped with a graphite monochromator and a Mo sealed tube $(\lambda=0.71073 \AA)$.

A total of 1490 frames were collected. The total exposure time was 2.07 hours. The frames were integrated with the Bruker SAINT software package using a narrow-frame algorithm. The integration of the data using an orthorhombic unit cell yielded a total of 44773 reflections to a maximum $\theta$ angle of $28.29^{\circ}(0.75 \AA$ resolution), of which 4059 were independent (average redundancy 11.031 , completeness $\left.=100.0 \%, \mathrm{R}_{\text {int }}=4.13 \%, \mathrm{R}_{\text {sig }}=2.14 \%\right)$ and $3507(86.40 \%)$ were greater than $2 \sigma\left(\mathrm{F}^{2}\right)$. The final cell constants of $\underline{\mathrm{a}}=19.1861(10) \AA, \underline{\mathrm{b}}=8.0835(4) \AA$, $\underline{\mathrm{c}}=21.0439(11) \AA$, volume $=3263.7(3) \AA^{3}$, are based upon the refinement of the XYZ-centroids of 9944 reflections above $20 \sigma(\mathrm{I})$ with $4.415^{\circ}<2 \theta<55.80^{\circ}$. Data were corrected for absorption effects using the multi-scan method (SADABS). The ratio of minimum to maximum apparent transmission was 0.924 . The calculated minimum and maximum transmission coefficients (based on crystal size) are 0.6891 and 0.7457 . 
Table S3. Sample and crystal data for alcohol 9d.

\begin{tabular}{|c|c|c|}
\hline Identification code & \multicolumn{2}{|l|}{$19 \mathrm{kaw} 2 \mathrm{~h}$} \\
\hline Chemical formula & \multicolumn{2}{|l|}{$\mathrm{C}_{23} \mathrm{H}_{20} \mathrm{O}$} \\
\hline Formula weight & \multicolumn{2}{|l|}{$312.40 \mathrm{~g} / \mathrm{mol}$} \\
\hline Temperature & \multicolumn{2}{|l|}{$100(2) \mathrm{K}$} \\
\hline Wavelength & \multicolumn{2}{|l|}{$0.71073 \AA$} \\
\hline Crystal size & \multicolumn{2}{|c|}{$0.230 \times 0.320 \times 0.540 \mathrm{~mm}$} \\
\hline Crystal habit & \multicolumn{2}{|c|}{ colorless block } \\
\hline Crystal system & \multicolumn{2}{|l|}{ orthorhombic } \\
\hline Space group & \multicolumn{2}{|l|}{$\mathrm{Pb} \mathrm{c} \mathrm{a}$} \\
\hline \multirow[t]{3}{*}{ Unit cell dimensions } & $\mathrm{a}=19.1861(10) \AA$ & $\alpha=90^{\circ}$ \\
\hline & $\mathrm{b}=8.0835(4) \AA$ & $\beta=90^{\circ}$ \\
\hline & $\mathrm{c}=21.0439(11) \AA$ & $\gamma=90^{\circ}$ \\
\hline Volume & $3263.7(3) \AA^{3}$ & \\
\hline $\mathbf{Z}$ & 8 & \\
\hline Density (calculated) & $1.272 \mathrm{~g} / \mathrm{cm}^{3}$ & \\
\hline Absorption coefficient & $0.076 \mathrm{~mm}^{-1}$ & \\
\hline $\mathbf{F}(000)$ & 1328 & \\
\hline
\end{tabular}


Table S4. Data collection and structure refinement for alcohol 9d.

\begin{tabular}{|c|c|c|}
\hline Diffractometer & \multirow{2}{*}{\multicolumn{2}{|c|}{$\begin{array}{l}\text { Bruker APEX-II CCD } \\
\text { sealed tube, Mo }\end{array}$}} \\
\hline Radiation source & & \\
\hline Theta range for data collection & \multicolumn{2}{|c|}{1.94 to $28.29^{\circ}$} \\
\hline Index ranges & \multicolumn{2}{|c|}{$-25<=\mathrm{h}<=25,-10<=\mathrm{k}<=10,-28<=1<=28$} \\
\hline Reflections collected & \multicolumn{2}{|c|}{44773} \\
\hline Independent reflections & \multicolumn{2}{|c|}{$4059[\mathrm{R}(\mathrm{int})=0.0413]$} \\
\hline $\begin{array}{l}\text { Coverage of independent } \\
\text { reflections }\end{array}$ & \multicolumn{2}{|l|}{$100.0 \%$} \\
\hline Absorption correction & \multicolumn{2}{|l|}{ multi-scan } \\
\hline Max. and min. transmission & \multicolumn{2}{|l|}{0.7457 and 0.6891} \\
\hline Structure solution technique & \multicolumn{2}{|l|}{ direct methods } \\
\hline Structure solution program & \multicolumn{2}{|c|}{ SHELXT (Sheldrick 2015) } \\
\hline Refinement method & \multicolumn{2}{|c|}{ Full-matrix least-squares on $\mathrm{F}^{2}$} \\
\hline Refinement program & \multicolumn{2}{|c|}{ SHELXL-2018/3 (Sheldrick, 2018) } \\
\hline Function minimized & \multicolumn{2}{|c|}{$\Sigma \mathrm{w}\left(\mathrm{F}_{\mathrm{o}}^{2}-\mathrm{F}_{\mathrm{c}}^{2}\right)^{2}$} \\
\hline Data / restraints / parameters & \multicolumn{2}{|l|}{4059 / 0 / 222} \\
\hline Goodness-of-fit on $F^{2}$ & \multicolumn{2}{|l|}{1.063} \\
\hline \multirow[t]{2}{*}{ Final $\mathbf{R}$ indices } & 3507 data; $\mathrm{I}>2 \sigma(\mathrm{I})$ & $\begin{array}{l}\mathrm{R} 1=0.0472 \\
\mathrm{wR} 2=0.1191\end{array}$ \\
\hline & all data & $\begin{array}{l}\mathrm{R} 1=0.0550 \\
\mathrm{wR} 2=0.1244\end{array}$ \\
\hline Weighting scheme & \multicolumn{2}{|c|}{$\begin{array}{l}\mathrm{w}=1 /\left[\sigma^{2}\left(\mathrm{~F}_{\mathrm{o}}^{2}\right)+(0.0600 \mathrm{P})^{2}+1.3976 \mathrm{P}\right] \\
\text { where } \mathrm{P}=\left(\mathrm{F}_{\mathrm{o}}^{2}+2 \mathrm{~F}_{\mathrm{c}}^{2}\right) / 3\end{array}$} \\
\hline Largest diff. peak and hole & \multicolumn{2}{|c|}{0.359 and $-0.229 \mathrm{e}^{-3}$} \\
\hline R.M.S. deviation from mean & \multicolumn{2}{|c|}{$0.047 \mathrm{e}^{-3}$} \\
\hline
\end{tabular}




\section{Analytical Data}

\section{A. Selected HPLC Traces}

The following pages contain HPLC traces used to analyze the unpurified reaction mixtures for the presence of ring-opened products indicating single-electron transfer.

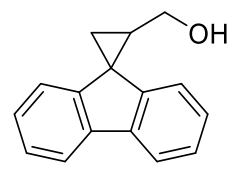

s1
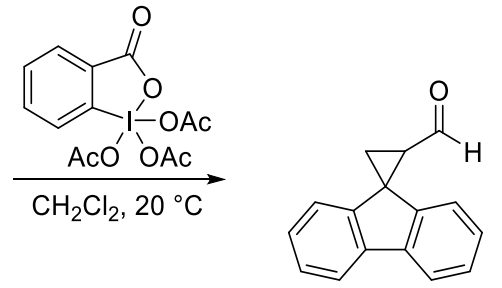

3

eluent $=0.5: 99.5 i-\mathrm{PrOH}:$ hexanes; flow rate $=1.0 \mathrm{~mL} / \mathrm{min}$
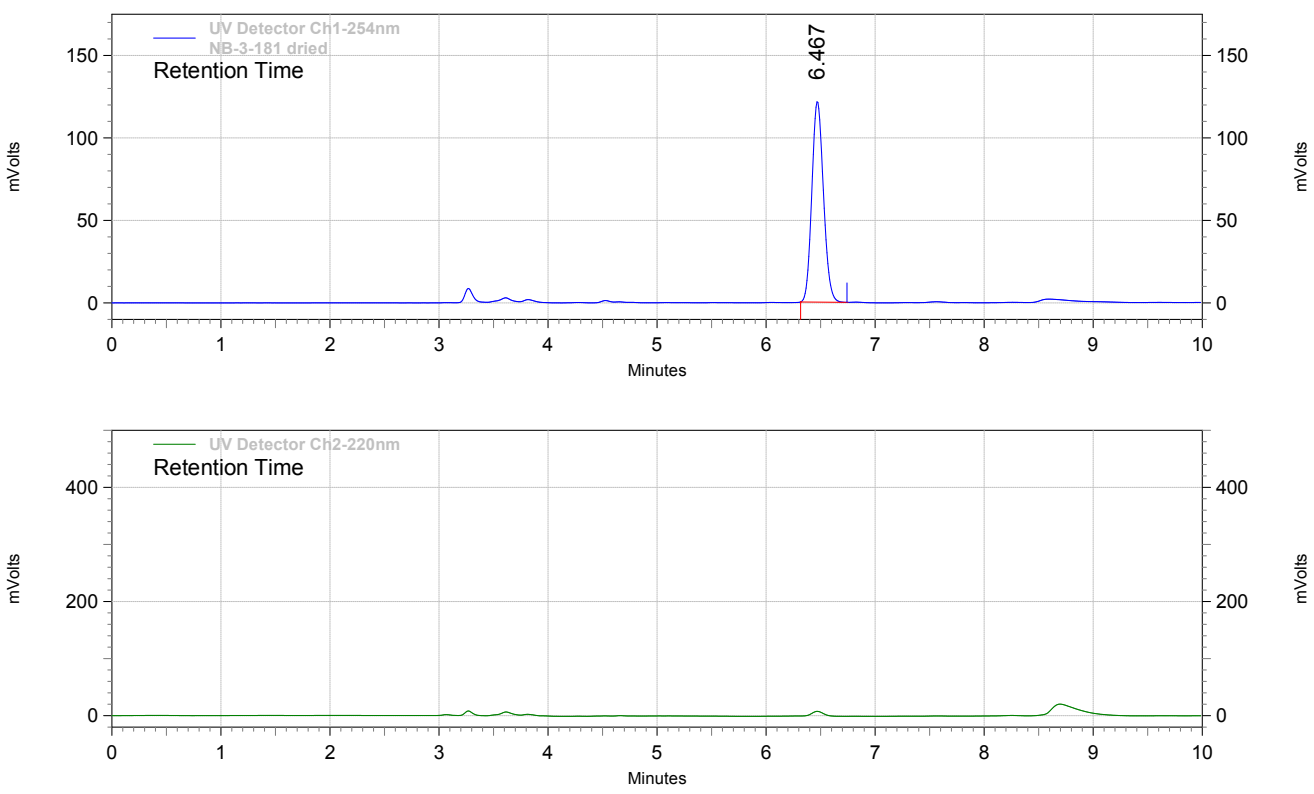

UV Detector Ch1-254nm Results Retention Time 6.467 Area Area \% 100.00 Height 121438 Height \% 100.00

\begin{tabular}{|r|r|r|r|r|}
\hline Totals & 900569 & 100.00 & 121438 & 100.00 \\
\hline
\end{tabular}




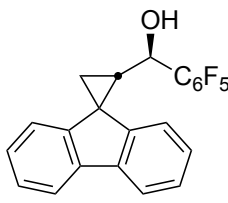

20
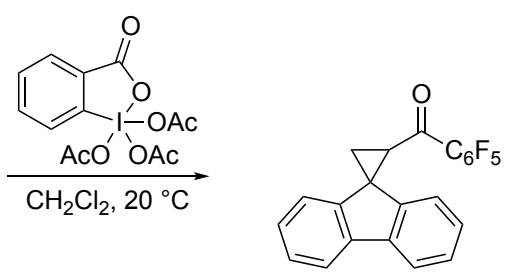

5

eluent $=0.5: 99.5 i-\mathrm{PrOH}:$ hexanes; flow rate $=1.0 \mathrm{~mL} / \mathrm{min}$
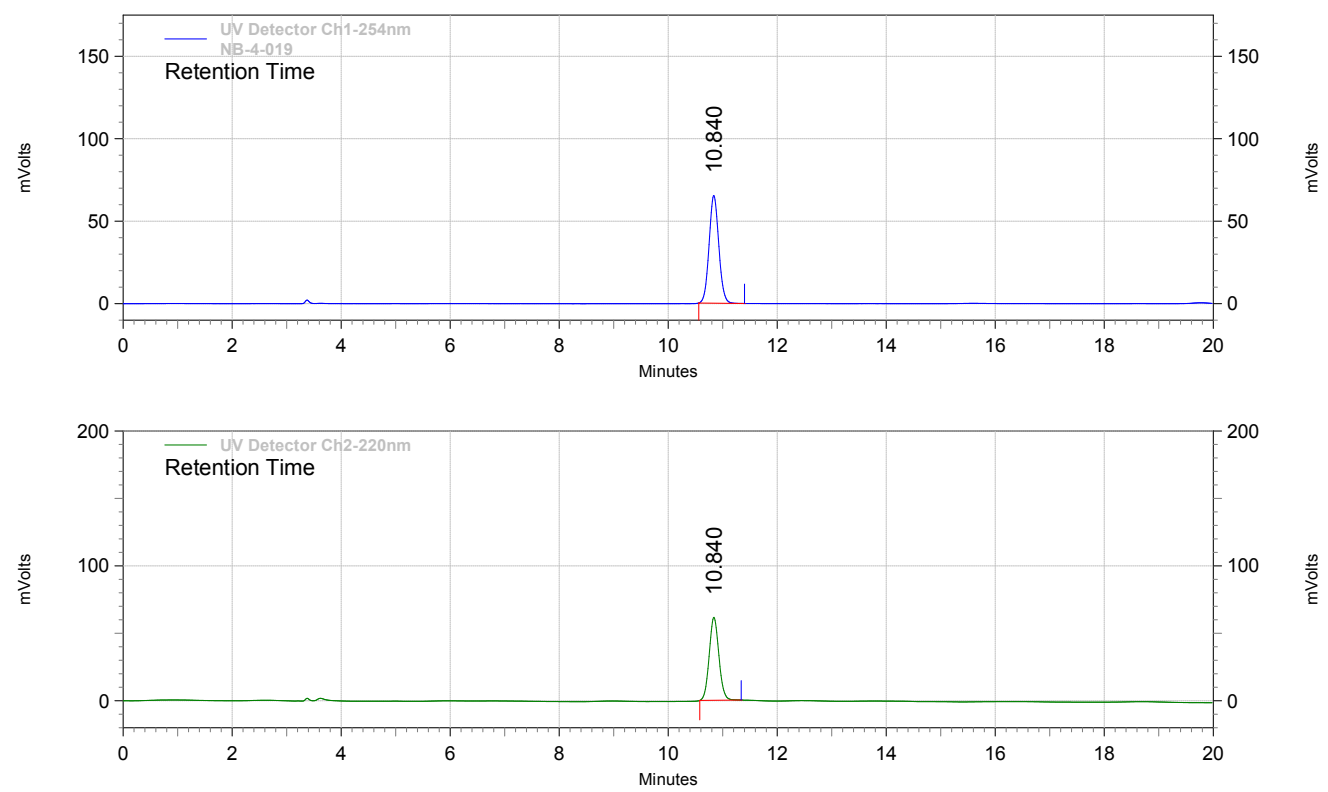

UV Detector

Ch1-254nm Results Retention Time 10.840 Area Area \% Height Height \%

\begin{tabular}{rrrr|r|r|}
\multicolumn{2}{r}{ Retention Time } & Area & Area \% & Height & Height \% \\
\hline 10.840 & 805774 & 100.00 & 65243 & 100.00 \\
\hline Totals & 805774 & 100.00 & 65243 & 100.00 \\
\hline
\end{tabular}

UV Detector Ch2-220nm Results Retention Time 10.840 Area Area \% Height Height \%$$
\text { Totals }
$$

\begin{tabular}{|r|r|r|r|r|}
\hline Totals & 759267 & 100.00 & 61503 & 100.00 \\
\hline
\end{tabular}




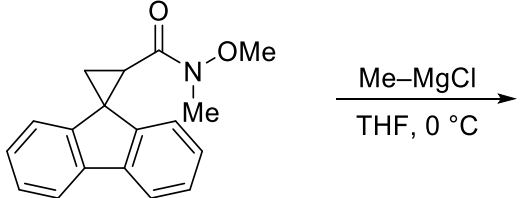

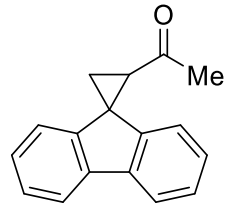

eluent $=0.5: 99.5 i-\mathrm{PrOH}:$ hexanes; flow rate $=1.0 \mathrm{~mL} / \mathrm{min}$
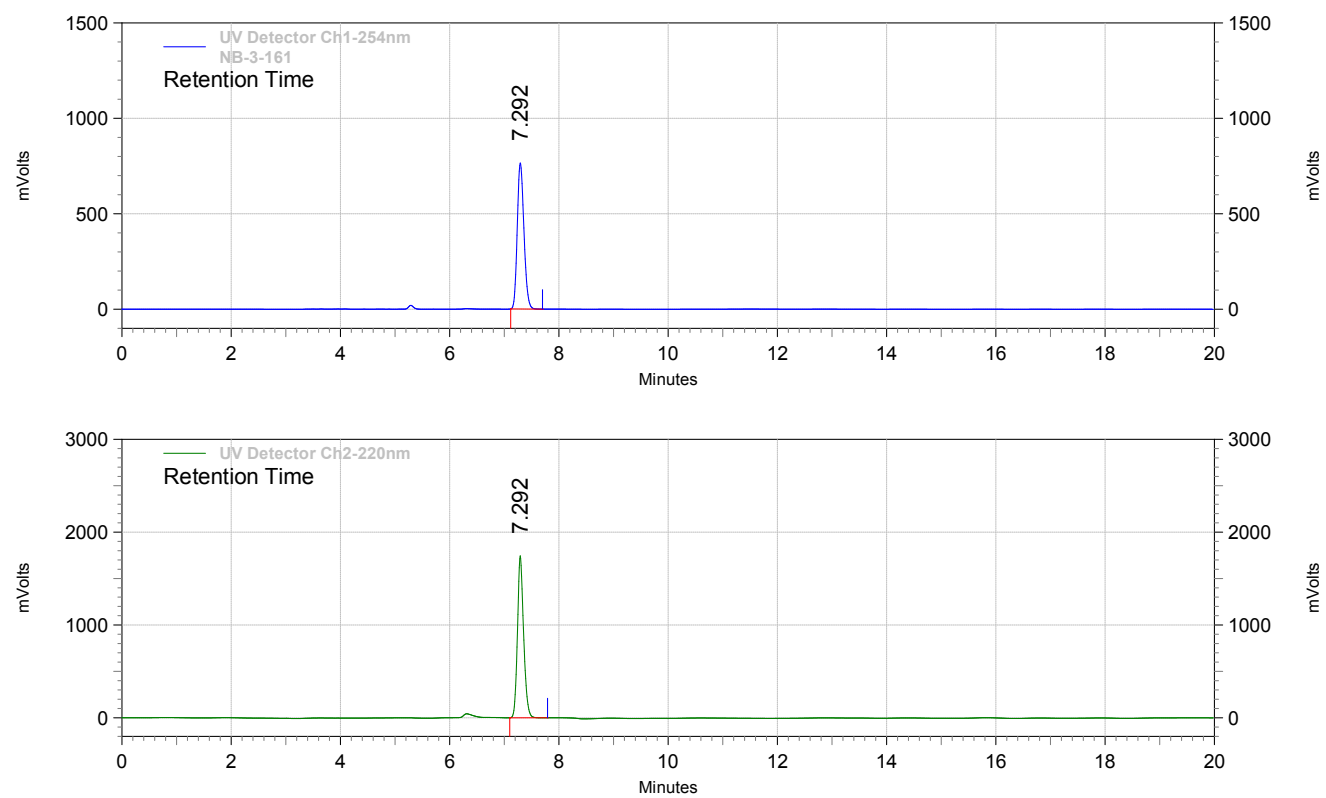

UV Detector

Ch1-254nm Results

Retention Time

7.292

Area

Area \%

Height

Height \%

100.00

764688

100.00

\begin{tabular}{|r|r|r|r|r|}
\hline Totals & 6488627 & 100.00 & 764688 & 100.00 \\
\hline
\end{tabular}

UV Detector Ch2-220nm Results Retention Time 7.292

Area

Area \%

Height

Height \%

7.292

13589597

100.00

1744702 100.00

\begin{tabular}{|r|r|r|r|r|}
\hline Totals & 13589597 & 100.00 & 1744702 & 100.00 \\
\hline
\end{tabular}


<smiles>CN(C)C(=O)C1CC12c1ccccc1-c1ccccc12</smiles>

4

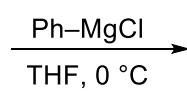

THF, $0{ }^{\circ} \mathrm{C}$

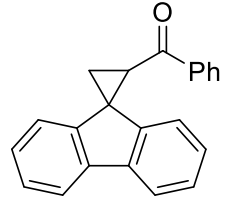

eluent $=0.5: 99.5 i$-PrOH:hexanes; flow rate $=1.0 \mathrm{~mL} / \mathrm{min}$
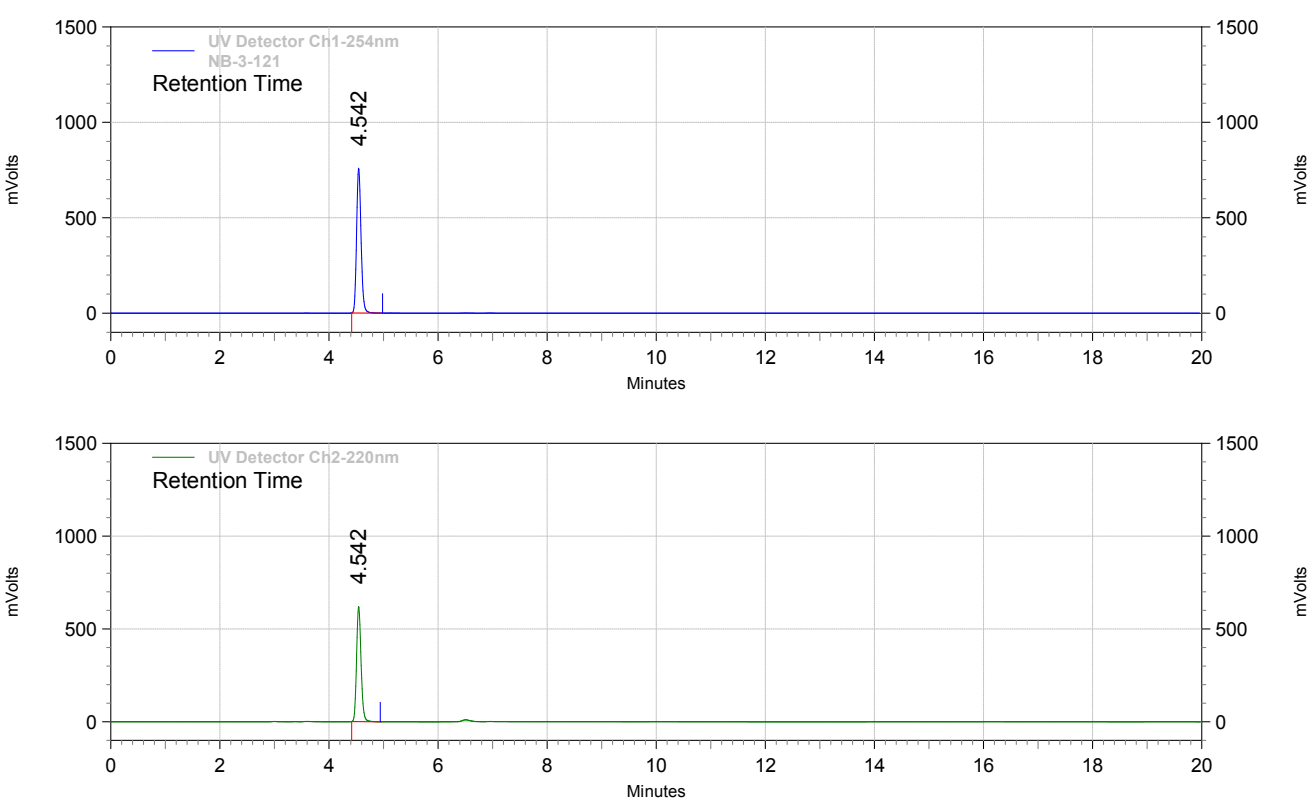

UV Detector

Ch1-254nm Results

Retention Time

4.542

Area

Area $\%$

Height

Height \%

100.00

758195

100.00

\begin{tabular}{|r|r|r|r|r|}
\hline Totals & 4315863 & 100.00 & 758195 & 100.00 \\
\hline
\end{tabular}

UV Detector

Ch2-220nm Results

Retention Time

Area

Area \%

Height

Height \%

\begin{tabular}{|r|r|r|r|r|}
\hline \multicolumn{1}{|c}{3542} & 3538163 & 100.00 & 619446 & 100.00 \\
\hline Totals & 3538163 & 100.00 & 619446 & 100.00 \\
\hline
\end{tabular}




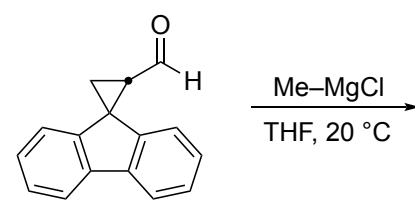

3

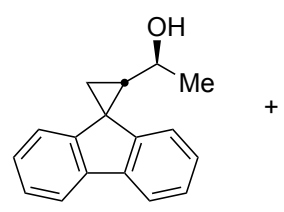

$8 a$

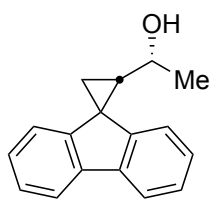

$8 a^{\prime}$

eluent $=1.0: 99.0 i$-PrOH:hexanes; flow rate $=1.0 \mathrm{~mL} / \mathrm{min}$
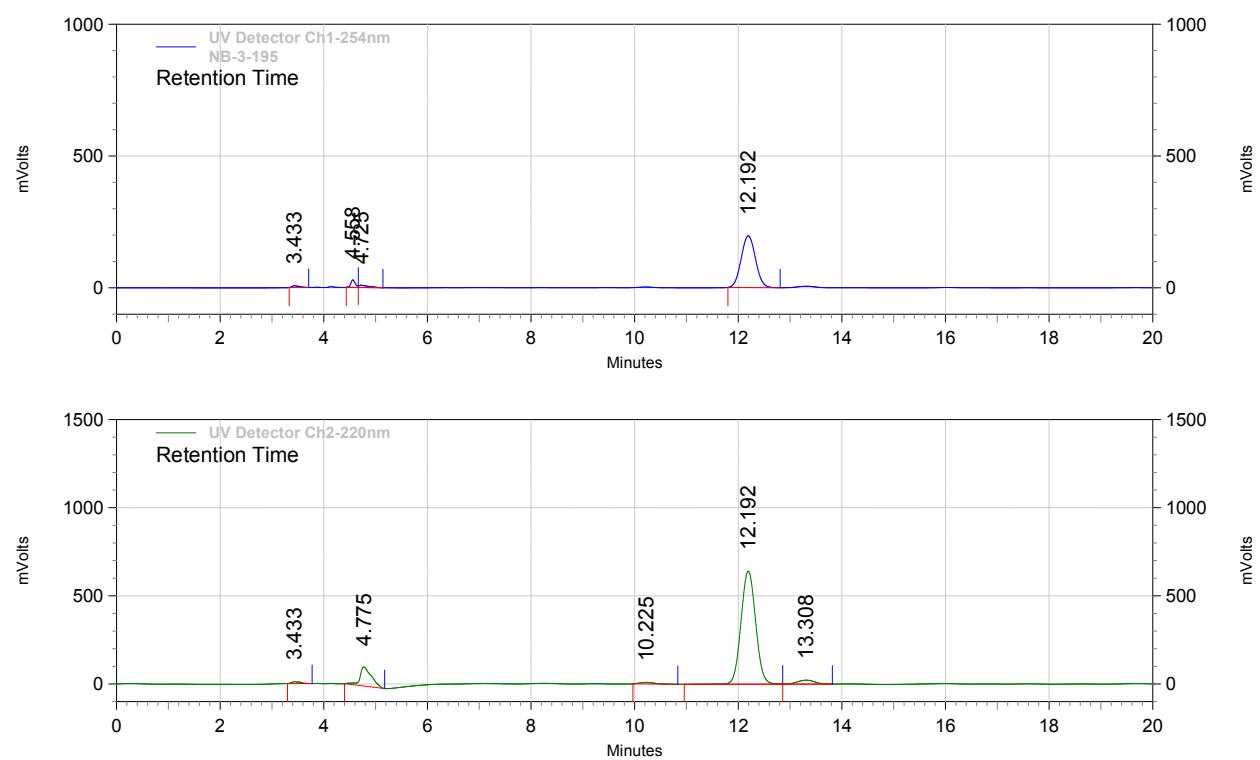

UV Detector

Ch1-254nm Results

Retention Time

3.433

4.558
4.725

12.192

Area

61767
166843

111407

3759353

Area \%

$4.07 \quad 28376$

8744

2.82

$91.71 \quad 195940$

3.65

81.70

\begin{tabular}{|r|r|r|r|r|}
\hline Totals & 4099370 & 100.00 & 239829 & 100.00 \\
\hline
\end{tabular}

UV Detector

Ch2-220nm Results

Retention Time

3.433
4.775

4.775
10.225

12.192

13.308

Area

125100

1674053

184830

12589402

521329

Totals

15094714

\begin{tabular}{|r|r|r|r|r|}
\hline Totals & 15094714 & 100.00 & 791667 & 100.00 \\
\hline
\end{tabular}

\begin{tabular}{|r|r|r|r|r|}
\hline Totals & 15094714 & 100.00 & 791667 & 100.00 \\
\hline
\end{tabular}

\begin{tabular}{|r|r|r|r|r|}
\hline Totals & 15094714 & 100.00 & 791667 & 100.00 \\
\hline
\end{tabular}

Area \%

Height Height \%

$0.83 \quad 10743 \quad 1.36$

$11.09 \quad 108232$

13.67

$1.22 \quad 8980$

$\begin{array}{rr}83.40 & 641354 \\ 3.45 & 22358\end{array}$

1.13

2.82 


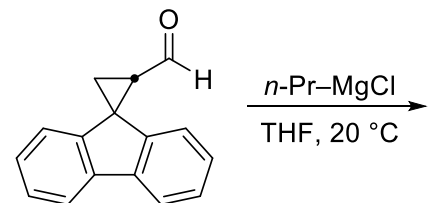

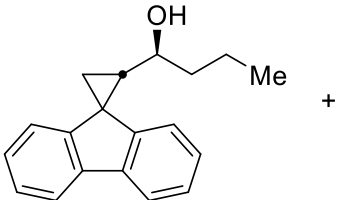

$8 b$

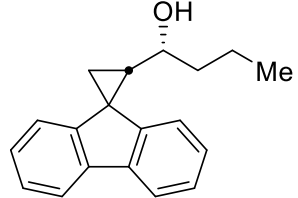

8b'

eluent $=1.0: 99.0 i-\mathrm{PrOH}:$ hexanes; flow rate $=1.0 \mathrm{~mL} / \mathrm{min}$
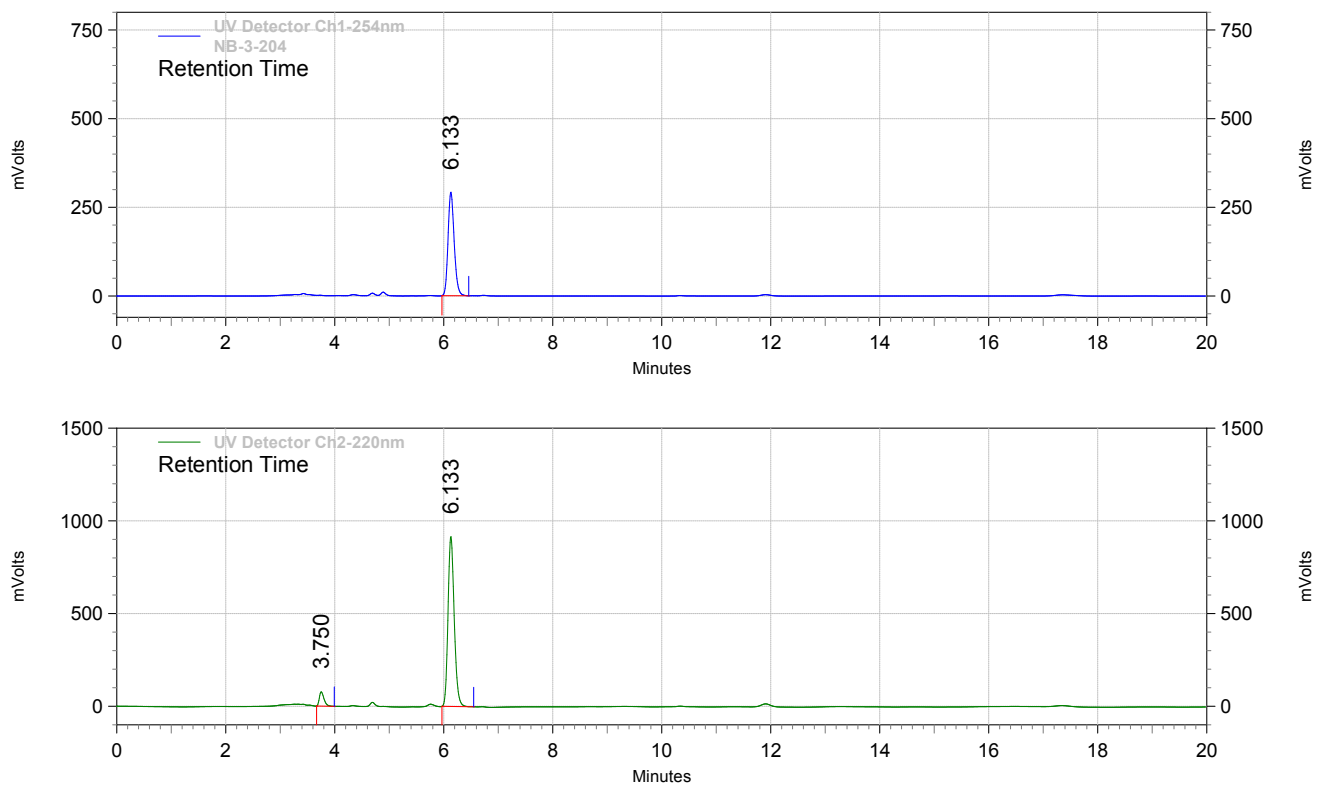

UV Detector

Ch1-254nm Results

Retention Time

6.133

Area

2284290

Area \%

Height

Height \%

100.00

291745 100.00

\begin{tabular}{|r|r|r|r|r|}
\hline Totals & 2284290 & 100.00 & 291745 & 100.00 \\
\hline
\end{tabular}

UV Detector

Ch2-220nm Results

Retention Time

3.750

Area

455904

7239721

Area \%

5.92

Height

76817

Height \%

6.133

94.08

915760

92.26

\begin{tabular}{|r|r|r|r|r|}
\hline Totals & 7695625 & 100.00 & 992577 & 100.00 \\
\hline
\end{tabular}




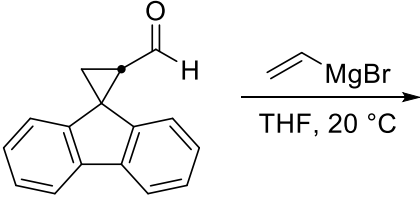

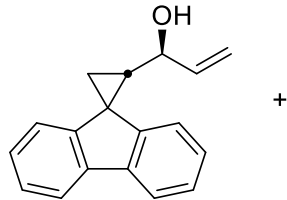

$8 \mathrm{c}$

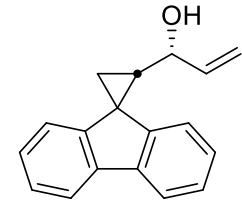

$8 c^{\prime}$

eluent $=1.0: 99.0 i-\mathrm{PrOH}:$ hexanes; flow rate $=1.0 \mathrm{~mL} / \mathrm{min}$
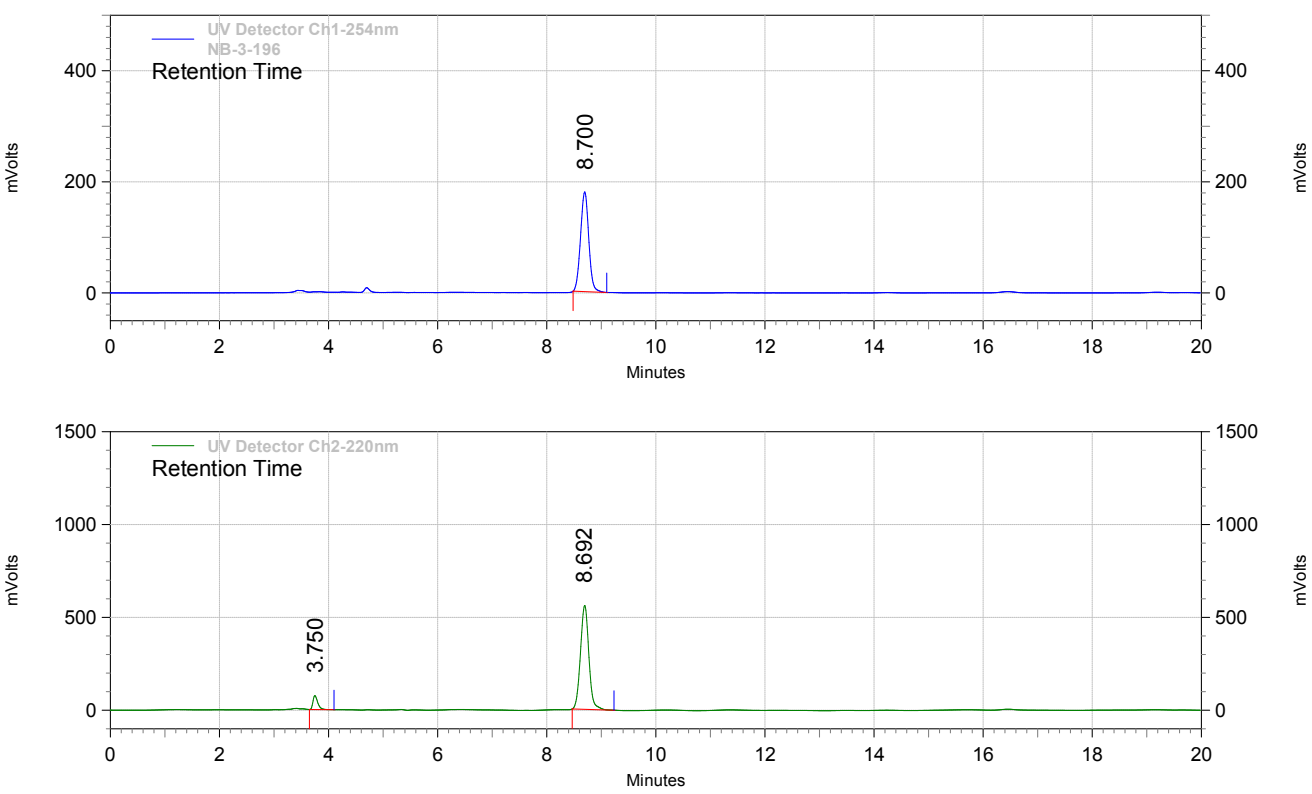

UV Detector

Ch1-254nm Results

Retention Time

8.700

Area

Area \%

Height

Height \%

Totals

877074

100.00

179326

100.00

\begin{tabular}{|r|r|r|r|r|}
\hline Totals & 1877074 & 100.00 & 179326 & 100.00 \\
\hline
\end{tabular}

UV Detector

Ch2-220nm Results

Retention Time

3.750

Area

454210

5980266

Area \%

7.06

Height

74840

Height \%

8.692

92.94

559268

88.20

Totals 


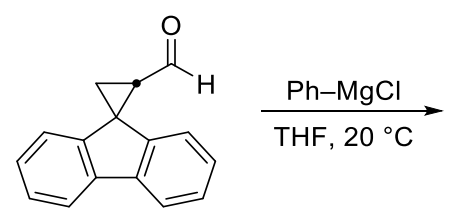

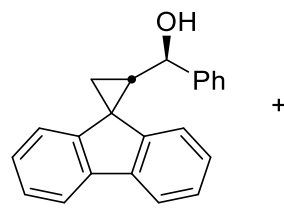

8d<smiles>OC(c1ccccc1)C12CC1(C1=C(c3ccccc3)c3ccccc31)C2</smiles>

8d'

eluent $=1.0: 99.0 i-\mathrm{PrOH}:$ hexanes; flow rate $=1.0 \mathrm{~mL} / \mathrm{min}$
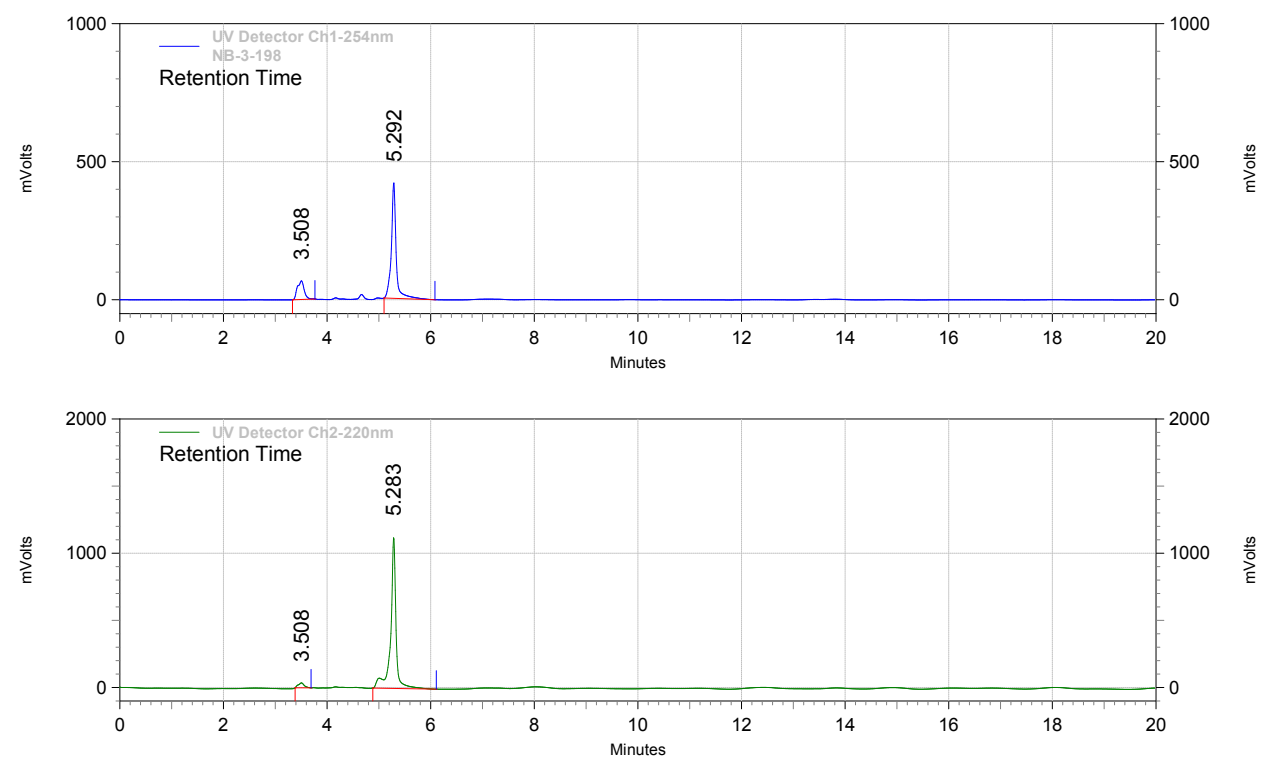

UV Detector Ch1-254nm Results Retention Time 3.508
5.292 Area 609957 2709200 Area \% 18.38 81.62

\begin{tabular}{rr} 
Height & Height \% \\
\hline 67496 & 13.92 \\
417414 & 86.08
\end{tabular}

Totals 3319157 100.00

484910 100.00

UV Detector Ch2-220nm Results Retention Time 3.508 5.283 Area 291823 8105200 Area \% 3.48 96.52

\begin{tabular}{|r|r|r|r|r|}
\hline Totals & 8397023 & 100.00 & 1158249 & 100.00 \\
\hline
\end{tabular}




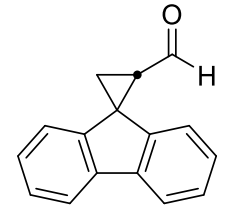

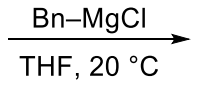<smiles>OC(Br)C1CC12c1ccccc1-c1ccccc12</smiles>

$8 \mathrm{e}$

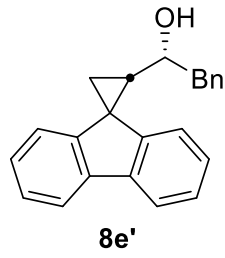

$8 e^{\prime}$

eluent $=0.5: 99.5 i-\mathrm{PrOH}:$ hexanes; flow rate $=1.0 \mathrm{~mL} / \mathrm{min}$
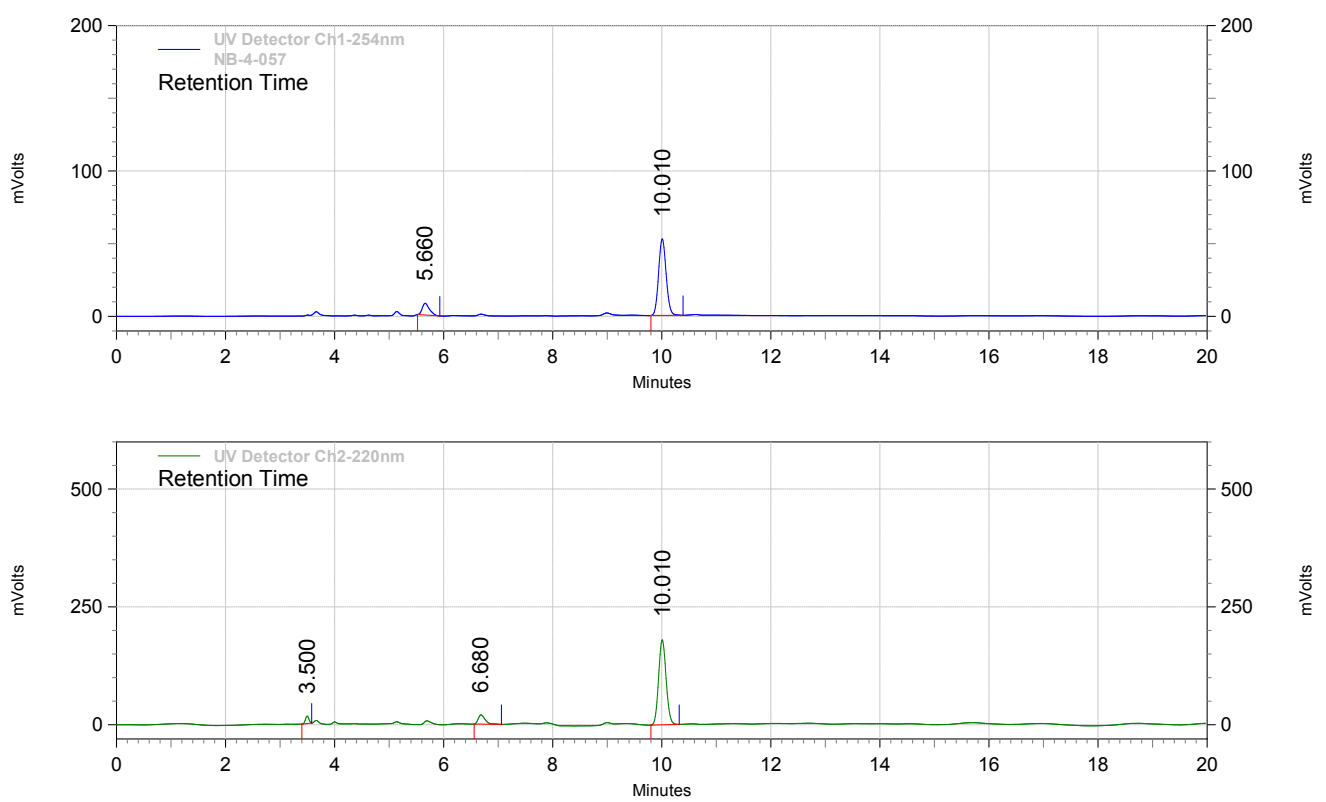

UV Detector

Ch1-254nm Results

Retention Time

5.660

Area

74270

Area \%

12.80

87.20

Height

8072

Height \%

10.010

505759

52680

86.71

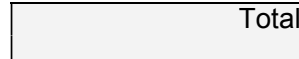

580029

100.00

60752

100.00

UV Detector

Ch2-220nm Results

Retention Time

3.500
6.680
10.010

Area

Area \%

Height

Height \%

170512

3.61
8.69

87.71

15600

7.24
9.02

1721660

180324

83.73

\begin{tabular}{|l|r|r|r|r|}
\hline Totals & 1962937 & 100.00 & 215354 & 100.00 \\
\hline
\end{tabular}



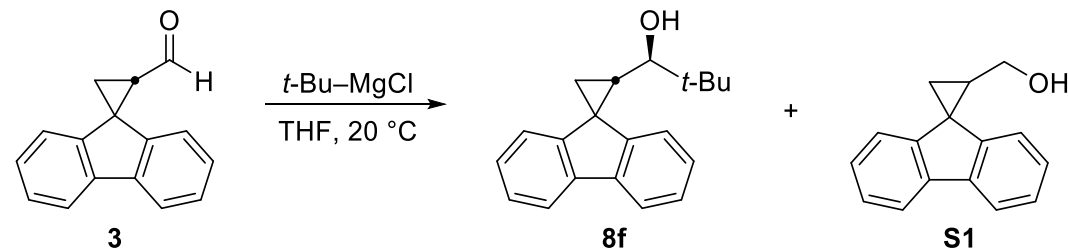

eluent $=0.5: 99.5 i-\mathrm{PrOH}:$ hexanes; flow rate $=1.0 \mathrm{~mL} / \mathrm{min}$
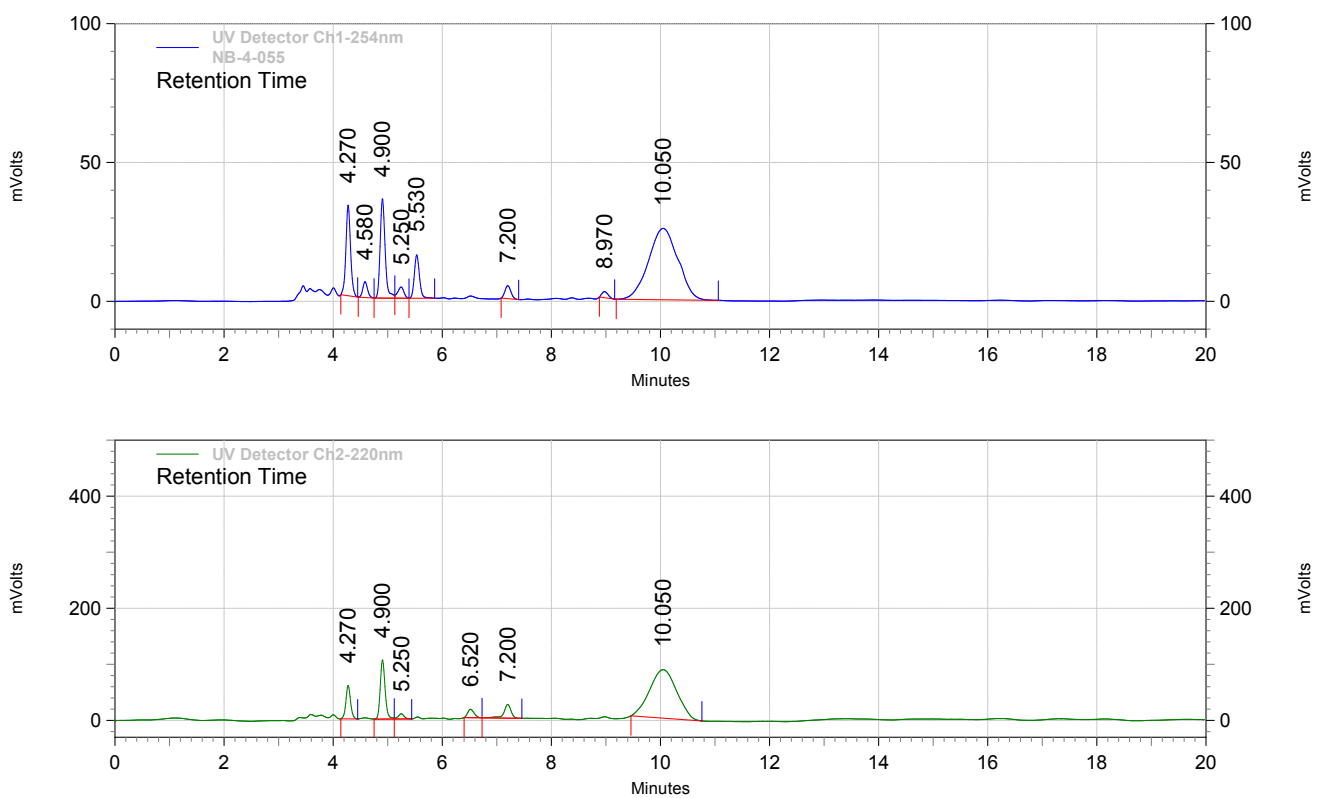

UV Detector Ch1-254nm Results Retention Time 4.270
4.580 4.580
4.900 5.250

5.530

7.200

8.970

10.050

Area

Area \%

Height Height \% 194391

34552

227499

12.09

32575

31341

101803

14.15

35714

28.32

1.95

35315

6.33

3998

3.17

2.20

15616

12.39

17339

1.08

4666

3.70

965341

25697

20.38

\begin{tabular}{|r|r|r|r|r|}
\hline Totals & 1607581 & 100.00 & 126087 & 100.00 \\
\hline
\end{tabular}

UV Detector

Ch2-220nm Results

Retention Time

4.270
4.900

4.900

5.250

6.520

7.200

10.050

Area

356154

656696

69253

124060

225288

2989162

Totals

4420613

\begin{tabular}{|r|r|r|r|r|}
\hline Totals & 4420613 & 100.00 & 300272 & 100.00 \\
\hline
\end{tabular}

\begin{tabular}{|r|r|r|r|r|}
\hline Totals & 4420613 & 100.00 & 300272 & 100.00 \\
\hline
\end{tabular}

\begin{tabular}{l|r|r|}
100.00 & 126087 & 100.00 \\
\hline
\end{tabular}


<smiles>O=CC1CC12c1ccccc1-c1ccccc12</smiles>

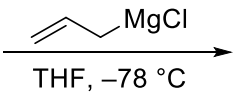

eluent $=1.0: 99.0 i-\mathrm{PrOH}:$ hexanes; flow rate $=1.0 \mathrm{~mL} / \mathrm{min}$

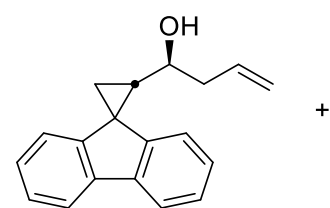

$8 \mathrm{~g}$

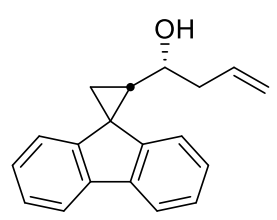

$8 g^{\prime}$
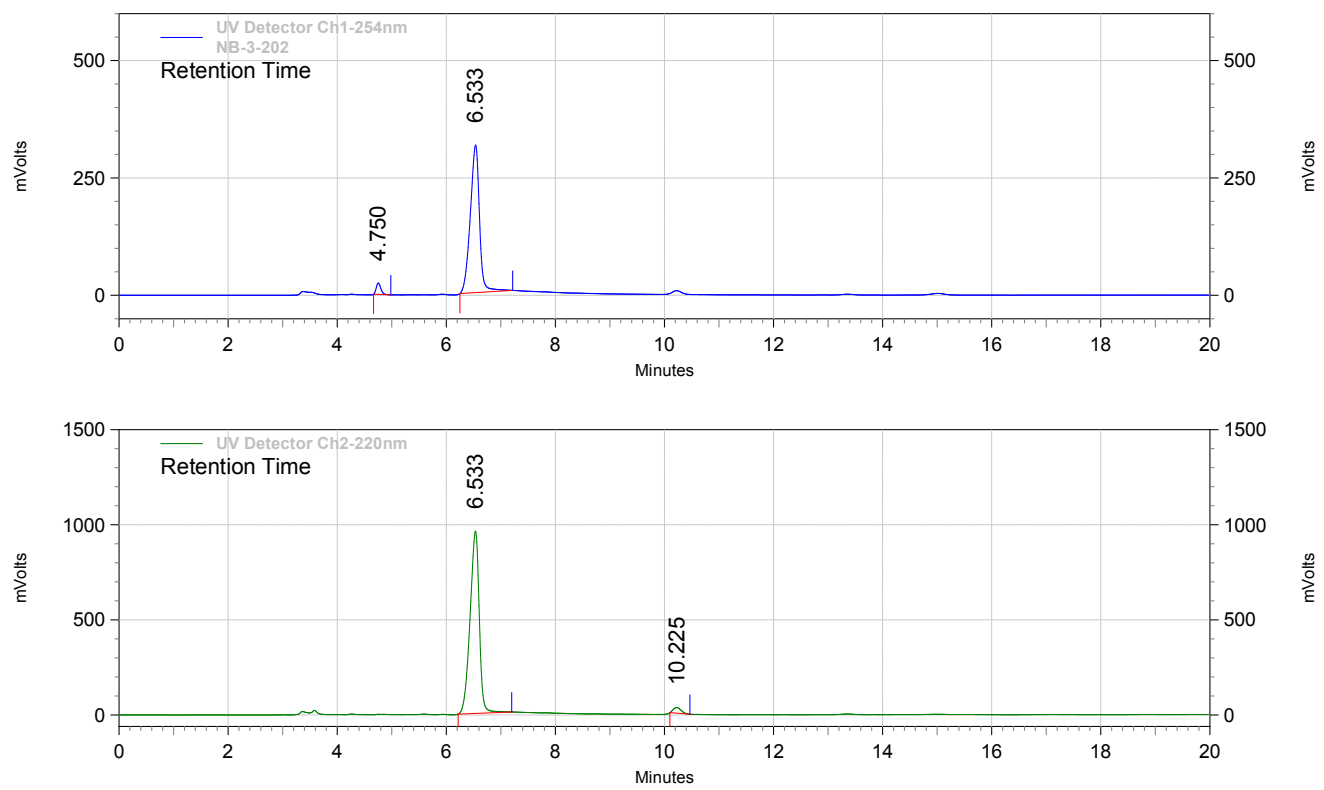

UV Detector Ch1-254nm Results Retention Time 4.750 6.533 $\begin{array}{rr}\text { Area } & \text { Area \% } \\ 140624 & 3.68\end{array}$ 96.32 Height Height \%

Totals 3680555 92.87

\begin{tabular}{|r|r|r|r|r|}
\hline Totals & 3821179 & 100.00 & 338151 & 100.00 \\
\hline
\end{tabular}

UV Detector Ch2-220nm Results Retention Time 6.533 10.225 Area Area \% 97.44 295469 2.56 Height Height \% 958061 96.98

Totals 11528699 100.00 987892 


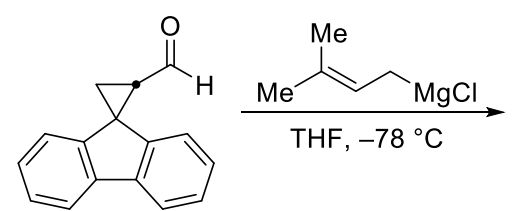

3

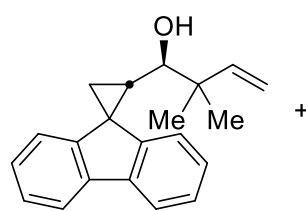

$8 \mathrm{~h}$

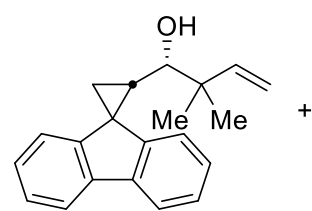

$8 h^{\prime}$

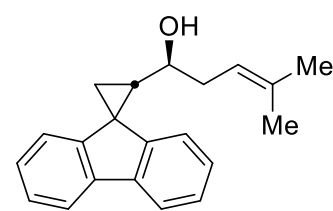

8h"

eluent $=1.0: 99.0 i$-PrOH:hexanes; flow rate $=1.0 \mathrm{~mL} / \mathrm{min}$
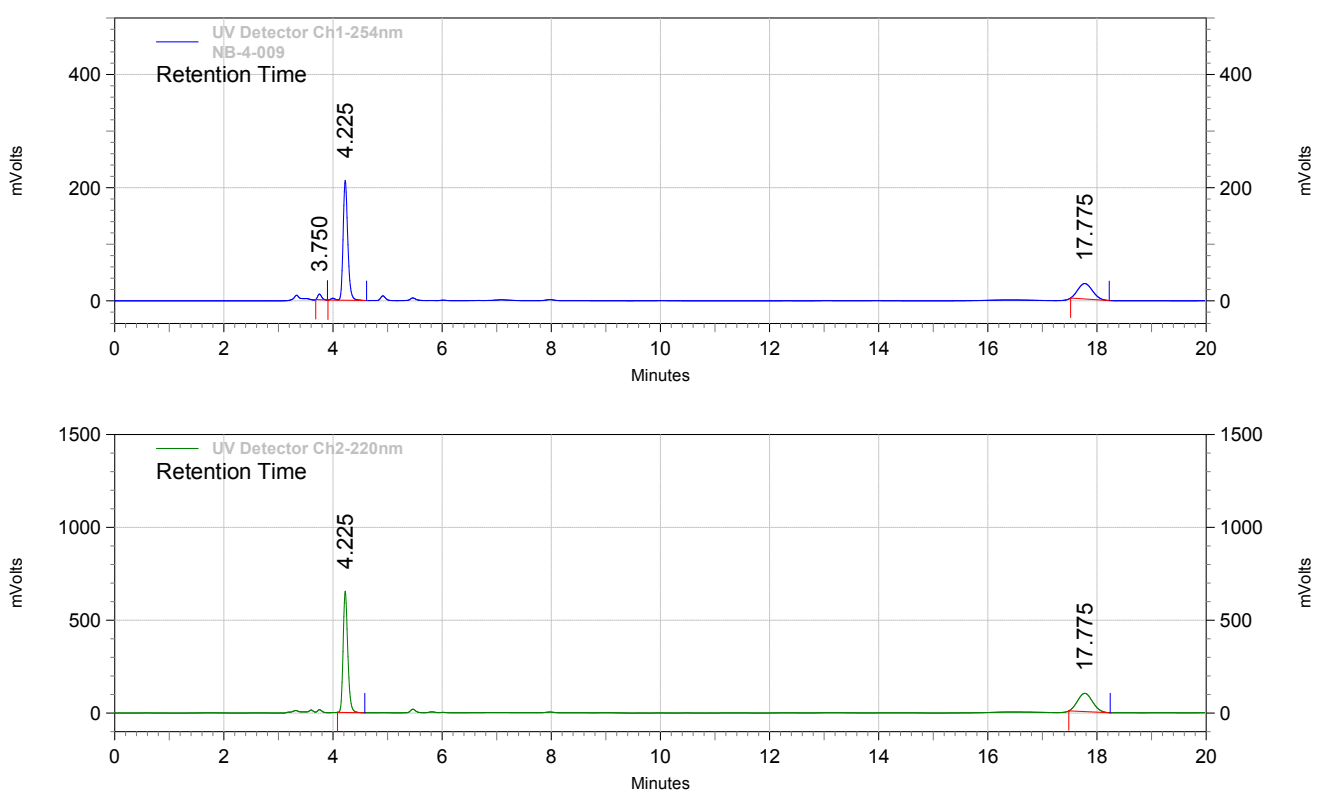

UV Detector

Ch1-254nm Results Retention Time

3.750
4.225

17.775

Area

Area \%

Height

Height \%

2.73

69.85

9973

27.41

27242

85.06

498513

100.00

249039

100.00

UV Detector

Ch2-220nm Results

Retention Time

4.225

Area

3849539

1866011

Area \%

67.35

Height

652982

32.65

98443

86.90

Totals 


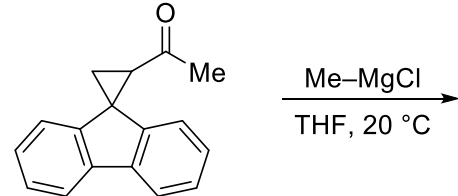

6

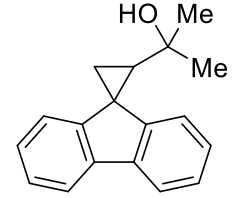

9a

eluent $=0.5: 99.5 i-\mathrm{PrOH}:$ hexanes; flow rate $=1.0 \mathrm{~mL} / \mathrm{min}$
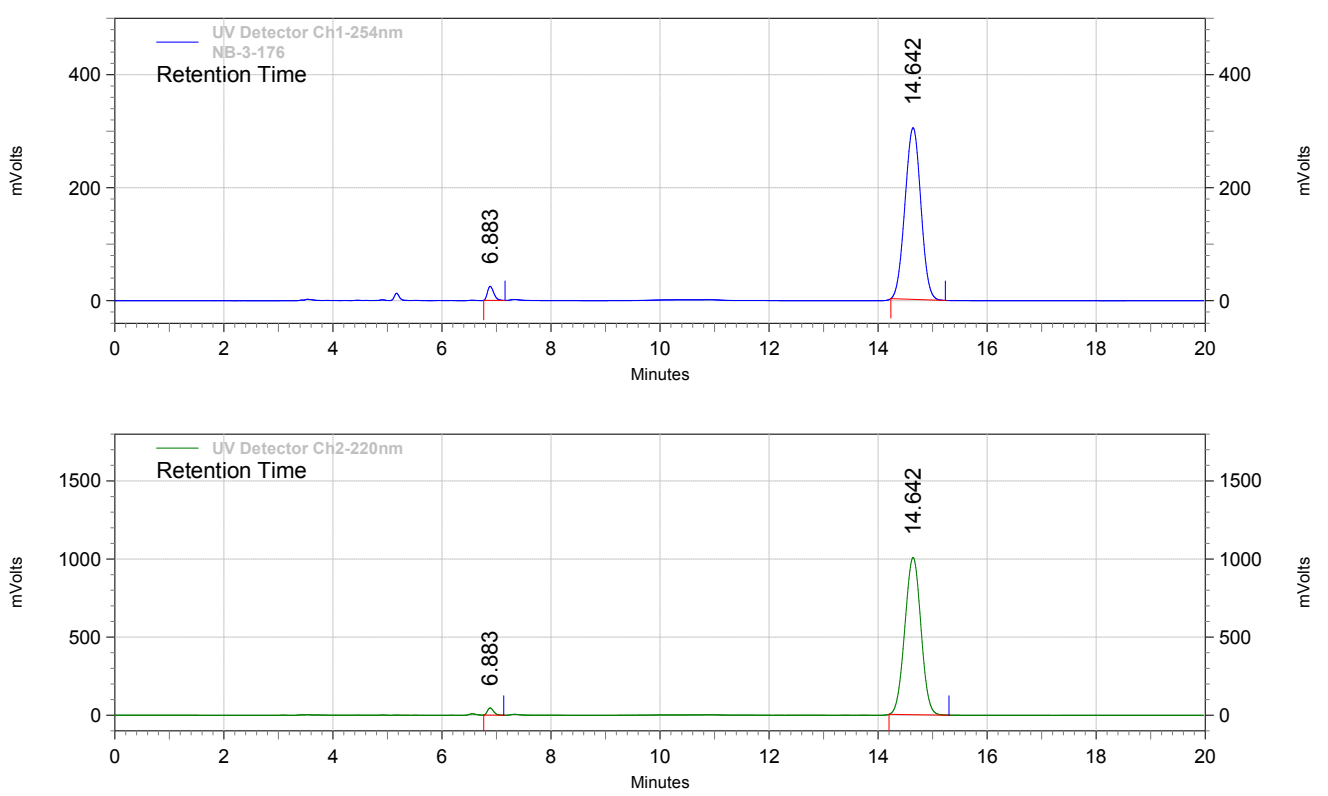

UV Detector

Ch1-254nm Results Retention Time 6.883

14.642 Area $\quad$ Area \%

Height Height \% 199622 3.14 $\begin{array}{rr}24871 & 7.57 \\ 303875 & 92.43\end{array}$

\begin{tabular}{|r|r|r|r|r|}
\hline Totals & 6349593 & 100.00 & 328746 & 100.00 \\
\hline
\end{tabular}

UV Detector Ch2-220nm Results Retention Time 6.883 Area 360203 20621539 Area \% 


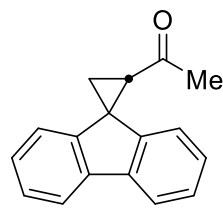

6
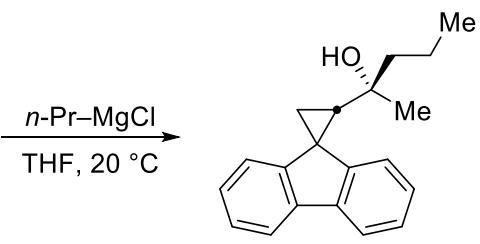

9b

eluent $=0.5: 99.5 i-\mathrm{PrOH}:$ hexanes; flow rate $=1.0 \mathrm{~mL} / \mathrm{min}$
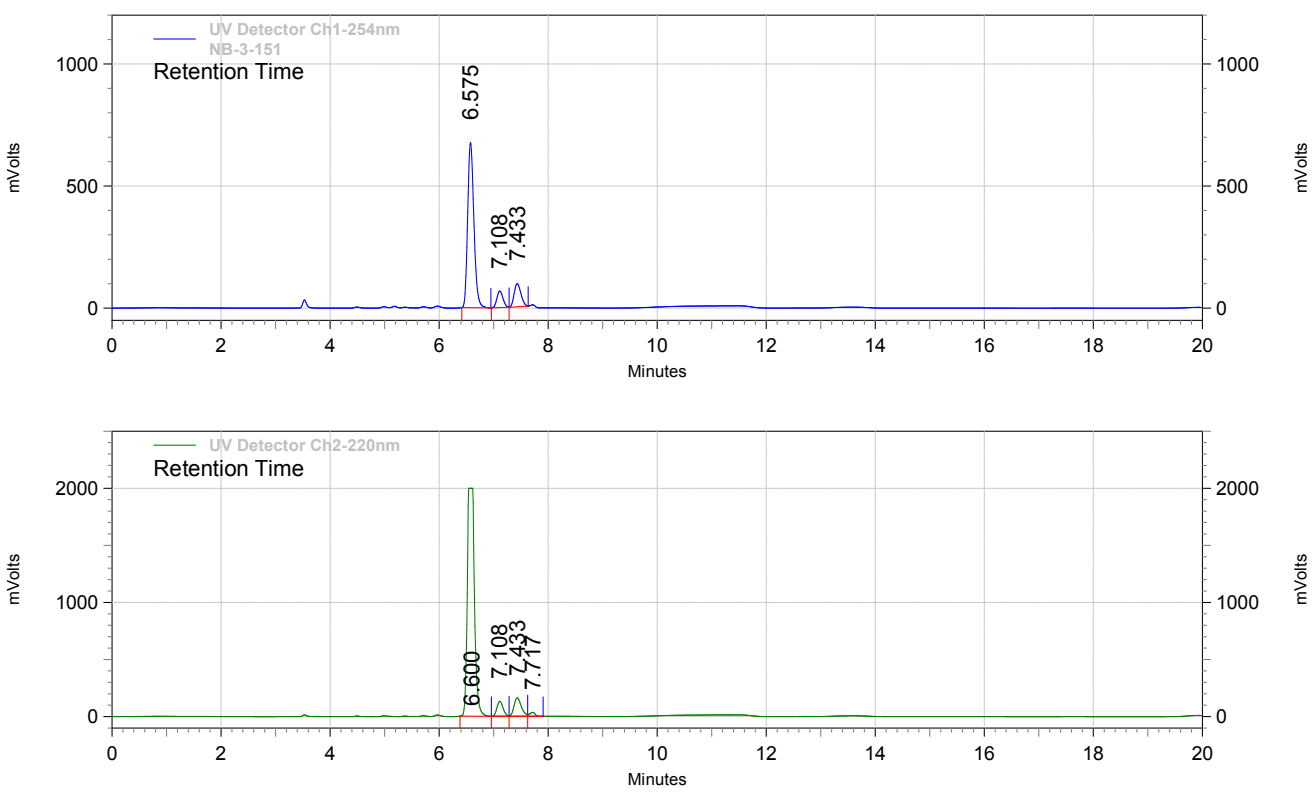

UV Detector

Ch1-254nm Results

Retention Time

6.575

7.433

Area

Area \%

Height

Height \%

$\begin{array}{lll}79.98 & 676957 & 80.69\end{array}$

$\begin{array}{lrr}7.78 & 67541 & 8.05\end{array}$

12.23

94486

11.26

\begin{tabular}{|r|r|r|r|r|}
\hline Totals & 6694632 & 100.00 & 838984 & 100.00 \\
\hline
\end{tabular}

UV Detector

Ch2-220nm Results

Retention Time

6.600

7.108

7.433

Area

18226095

Area \%

86.70

Height

1996713

131565

$7.20 \quad 161488$

35033

85.89

7.717

241464

1.15

6.95

Totals 


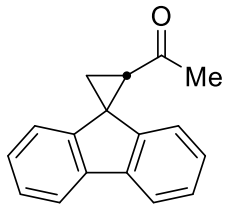

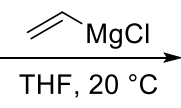

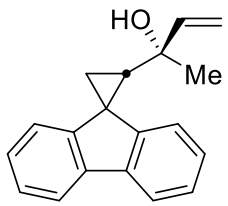

9c

eluent $=0.5: 99.5 i-\mathrm{PrOH}$ :hexanes; flow rate $=1.0 \mathrm{~mL} / \mathrm{min}$
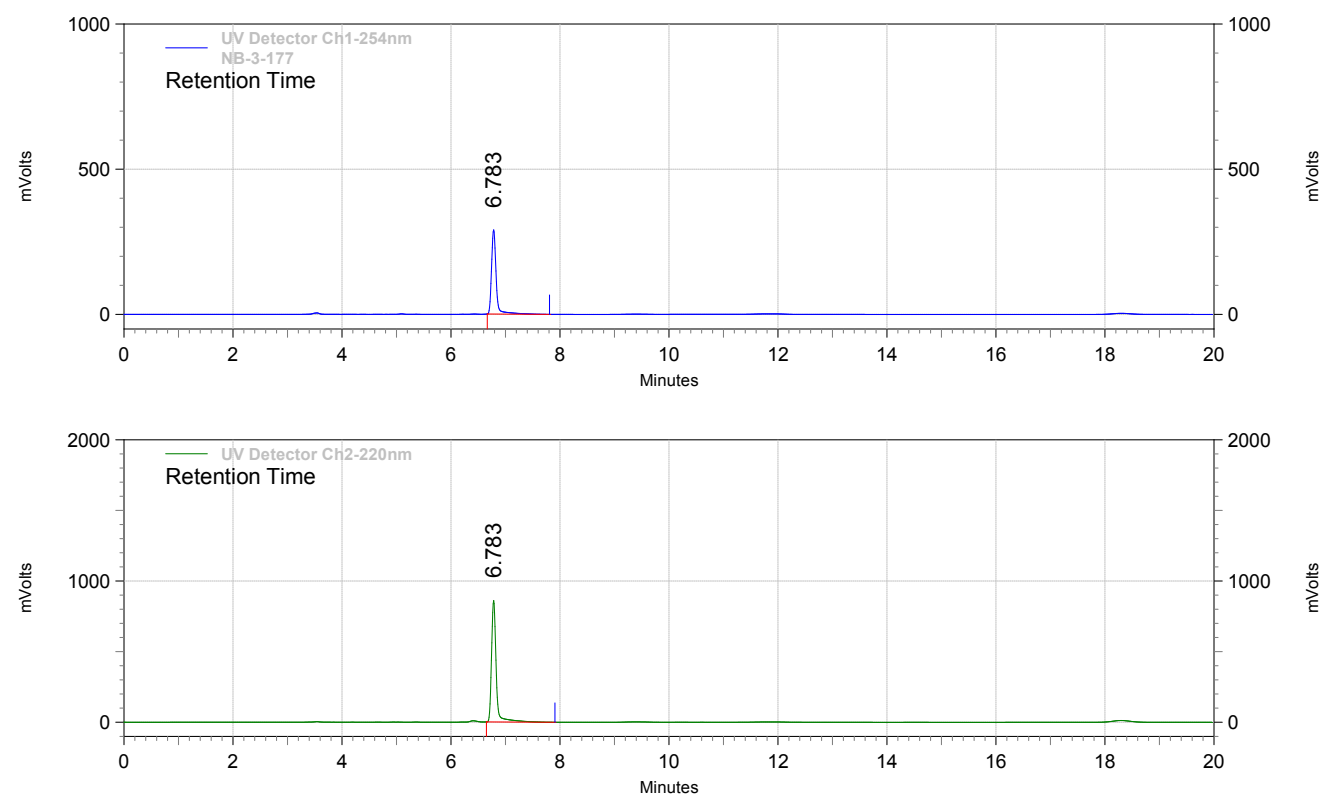

UV Detector

Ch1-254nm Results

Retention Time 6.783

Area

Area \%

Height

Height \%

100.00

289708

100.00

\begin{tabular}{|r|r|r|r|r|}
\hline Totals & 1712220 & 100.00 & 289708 & 100.00 \\
\hline
\end{tabular}

UV Detector

Ch2-220nm Results

Retention Time

6.783

Area

Area \%

Height

Height \%

Totals

5129130

100.00

859909

100.00

\begin{tabular}{|r|r|r|r|r|}
\hline Totals & 5129130 & 100.00 & 859909 & 100.00 \\
\hline
\end{tabular}



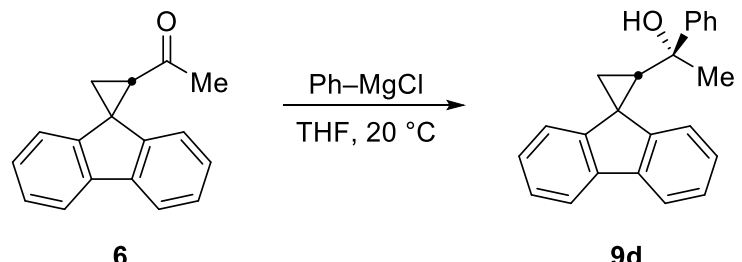

eluent $=0.5: 99.5 i-\mathrm{PrOH}:$ hexanes; flow rate $=1.0 \mathrm{~mL} / \mathrm{min}$
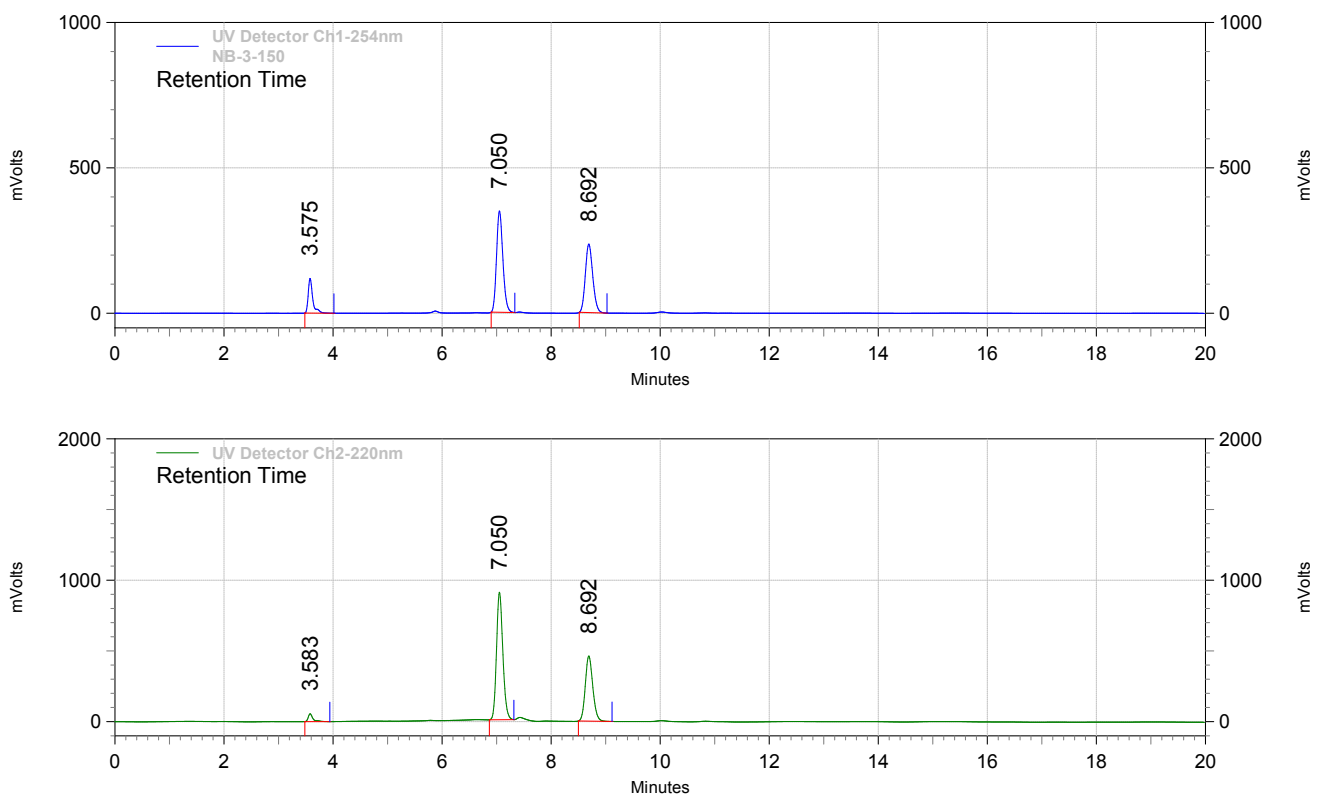

UV Detector

Ch1-254nm Results Retention Time

\begin{tabular}{|c|c|c|c|c|}
\hline TIIIE & Mirea & Aled $\%$ & Hergmil & пелgm \% \\
\hline 3.575 & 690298 & 12.04 & 119025 & 16.94 \\
\hline 7.050 & 2815391 & 49.10 & 348197 & 49.54 \\
\hline 8.692 & 2228142 & 38.86 & 235589 & 33.52 \\
\hline
\end{tabular}

UV Detector

Ch2-220nm Results

Retention Time

3.583
7.050

Area

Area \%

Height

Height \%

8.692

327577
7270619

2.73

54905

3.88

4390246

36.62

460404

32.50

Totals

11988442

100.00

1416419

100.00 


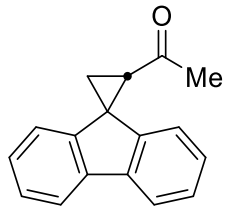

6
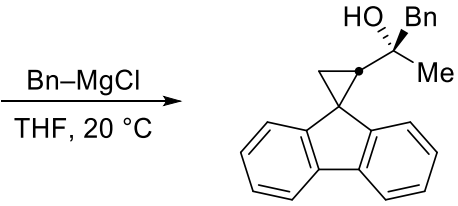

$9 e$

eluent $=0.5: 99.5 i-\mathrm{PrOH}:$ hexanes; flow rate $=1.0 \mathrm{~mL} / \mathrm{min}$
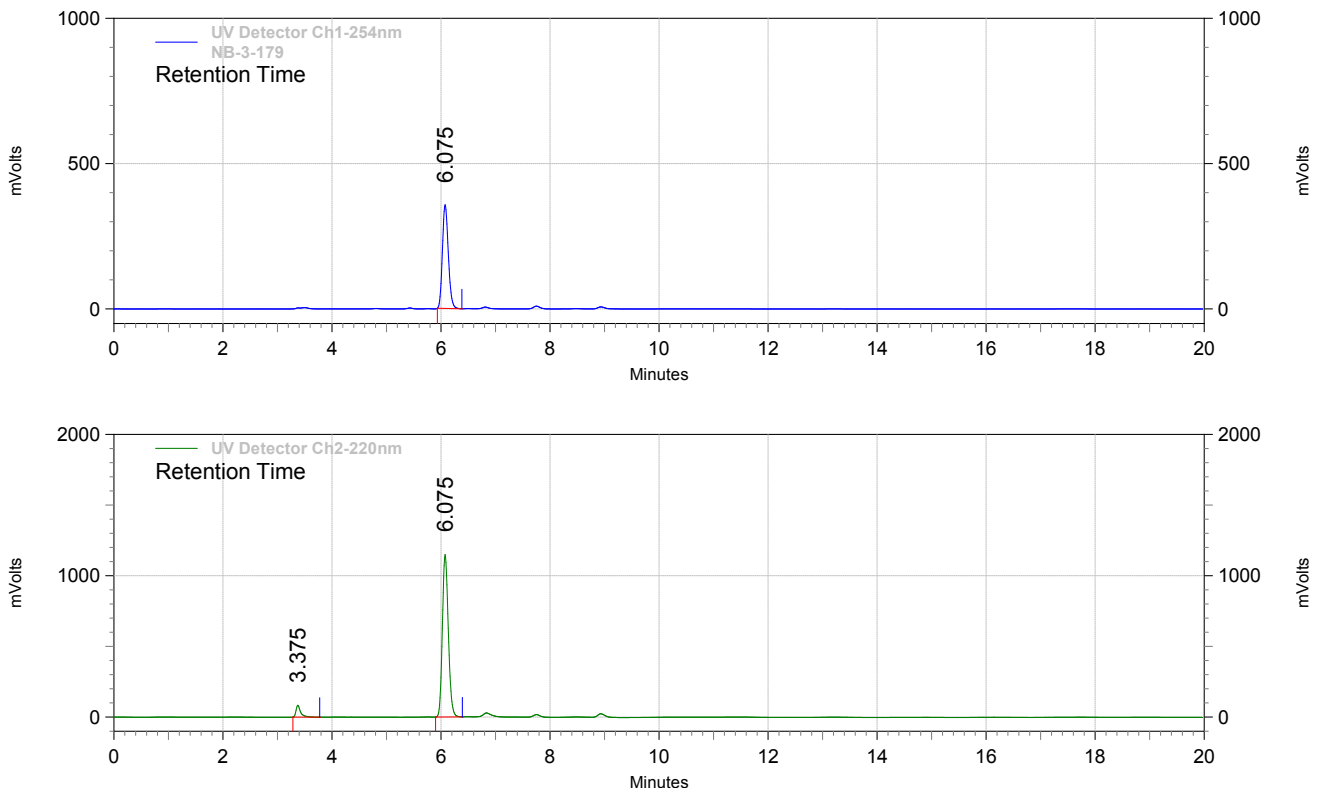

UV Detector

Ch1-254nm Results Retention Time 6.075 Area Area $\%$ Height Height \%

\begin{tabular}{|r|r|r|r|r|}
\hline Retention Time & Area & Area \% & Height & Height \% \\
\hline 6.075 & 2676429 & 100.00 & 356794 & 100.00 \\
\hline Totals & 2676429 & 100.00 & 356794 & 100.00 \\
\hline
\end{tabular}

UV Detector

Ch2-220nm Results Retention Time 3.375

6.075 Area 493868 Area \% Height

5.39 84004 Height \% 8675325

94.61

1149065

93.19

\begin{tabular}{|r|r|r|r|r|}
\hline Totals & 9169193 & 100.00 & 1233069 & 100.00 \\
\hline
\end{tabular}




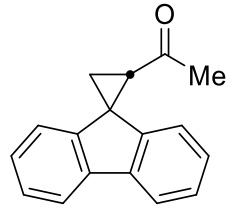

6
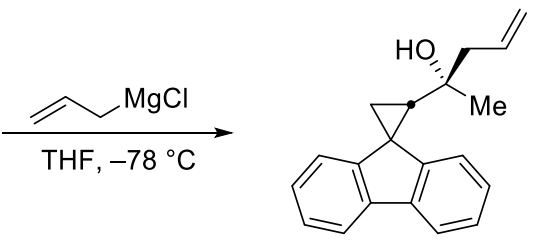

9f

eluent $=0.5: 99.5 i-\mathrm{PrOH}:$ hexanes; flow rate $=1.0 \mathrm{~mL} / \mathrm{min}$
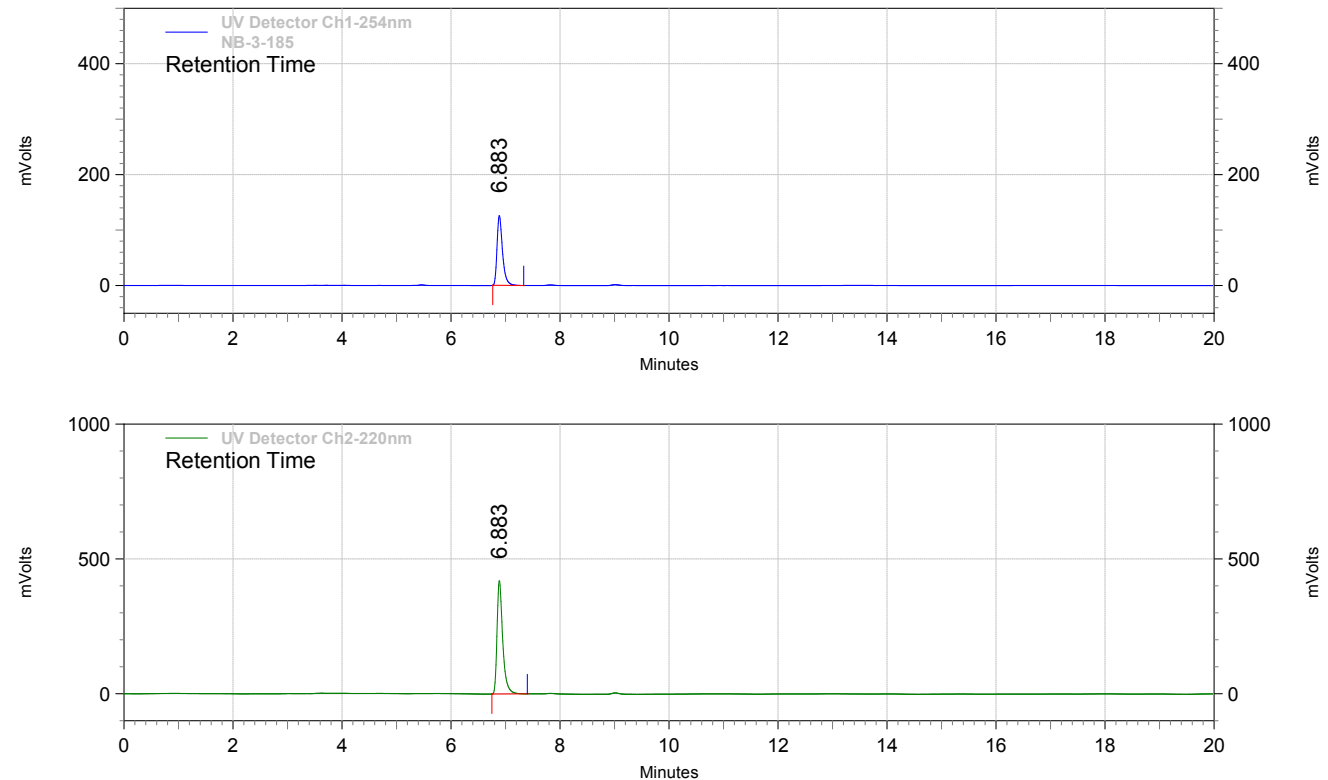

UV Detector

Ch1-254nm Results

Retention Time

Area

Area \%

Height

Height \%

6.883

879060

100.00

125664

100.00

\begin{tabular}{|r|r|r|r|r|}
\hline Totals & 879060 & 100.00 & 125664 & 100.00 \\
\hline
\end{tabular}

UV Detector

Ch2-220nm Results

Retention Time

6.883

Area

Area \%

100.00

Height

419269

Height \%

Totals

3044286

|

100.00

419269

100.00 


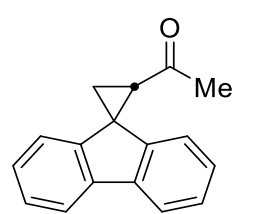

6

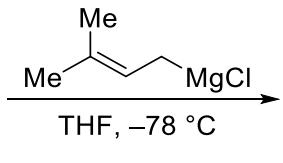

eluent $=1.0: 99.0 i$-PrOH:hexanes; flow rate $=1.0 \mathrm{~mL} / \mathrm{min}$
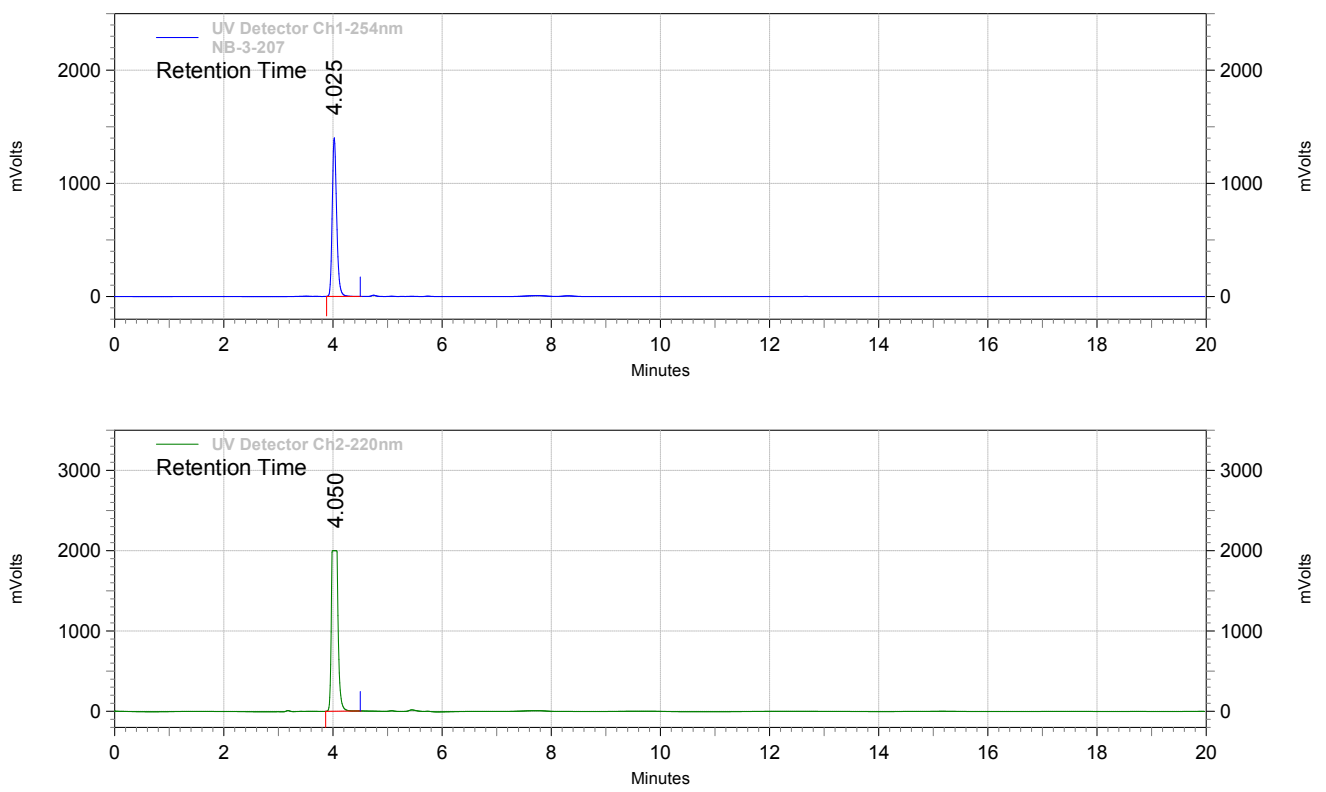

UV Detector

Ch1-254nm Results Retention Time 4.025 Area Area \% Height Height \%

\begin{tabular}{|r|r|r|r|r|}
\hline Retention Time & Area & Area \% & Height & Height \% \\
\hline 4.025 & 7899805 & 100.00 & 1400949 & 100.00 \\
\hline Totals & 7899805 & 100.00 & 1400949 & 100.00 \\
& &
\end{tabular}

UV Detector Ch2-220nm Results Retention Time 4.050

\begin{tabular}{|r|r|r|r|r|}
\hline Totals & 17084473 & 100.00 & 1999722 & 100.00 \\
\hline
\end{tabular}



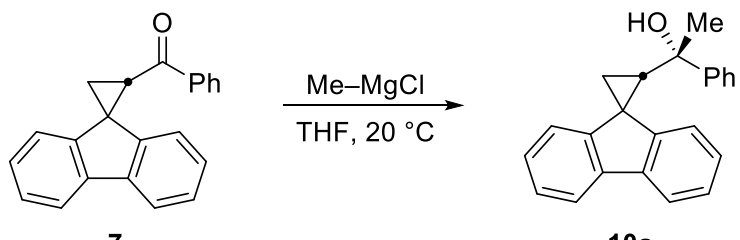

10a

eluent $=$ 0.5:99.5 $i$-PrOH:hexanes; flow rate $=1.0 \mathrm{~mL} / \mathrm{min}$
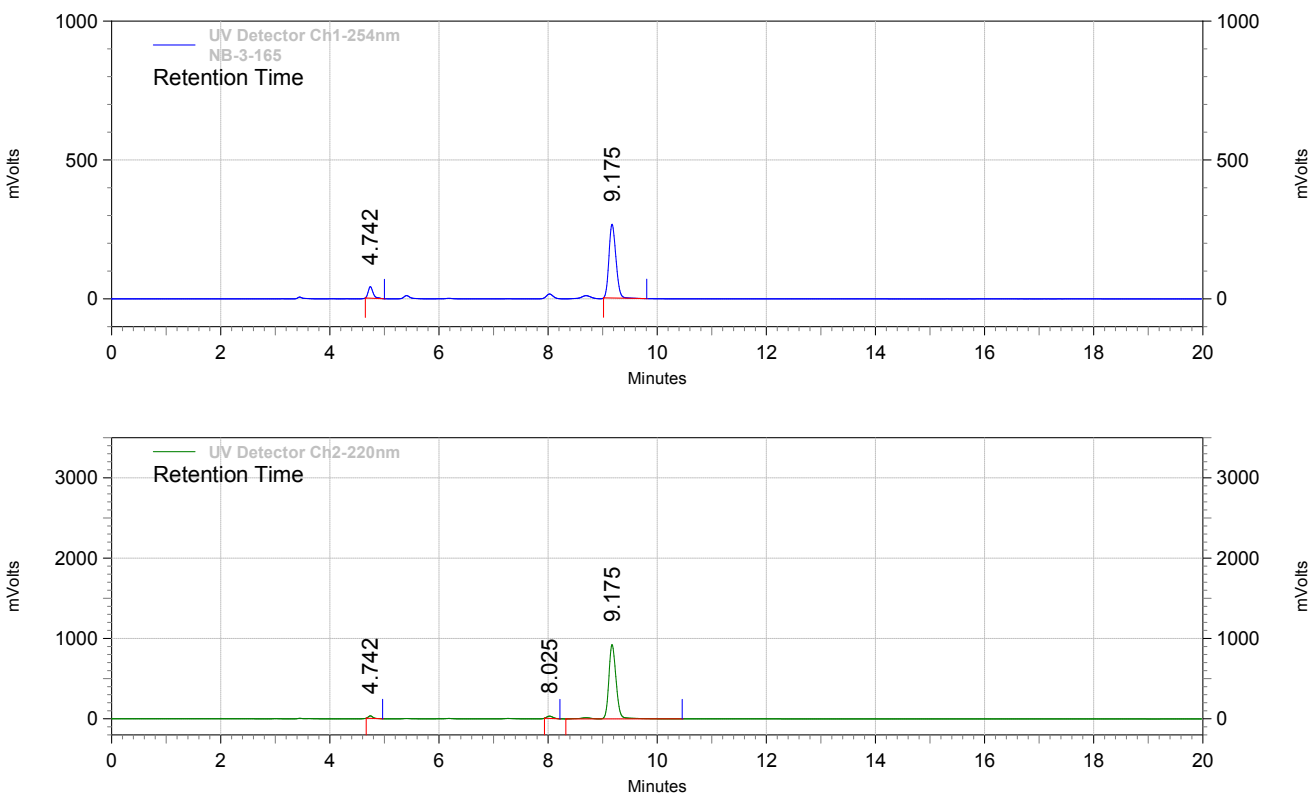

UV Detector

Ch1-254nm Results

Retention Time

4.742

9.175

Area

Area \%

9.70

90.30

Height

$41280-13.46$

2359017

86.54

\begin{tabular}{|r|r|r|r|r|}
\hline Totals & 2612355 & 100.00 & 306668 & 100.00 \\
\hline
\end{tabular}

UV Detector

Ch2-220nm Results

Retention Time

4.742

8.025

9.175

Area

Area \%

1.89

Height

Height \%

171109

2.32

30769

3.13

8684574

95.79

923066

2.86

Totals 
<smiles>O=C(c1ccccc1)C1CC12c1ccccc1-c1ccccc12</smiles>

7

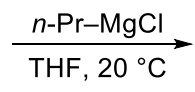

(1)

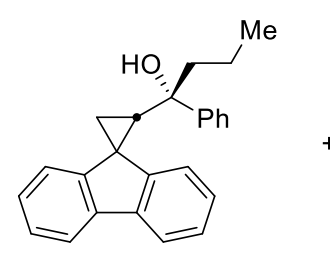

$10 \mathrm{~b}$

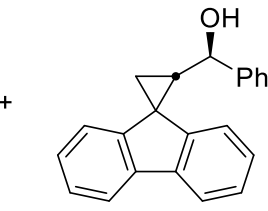

8d
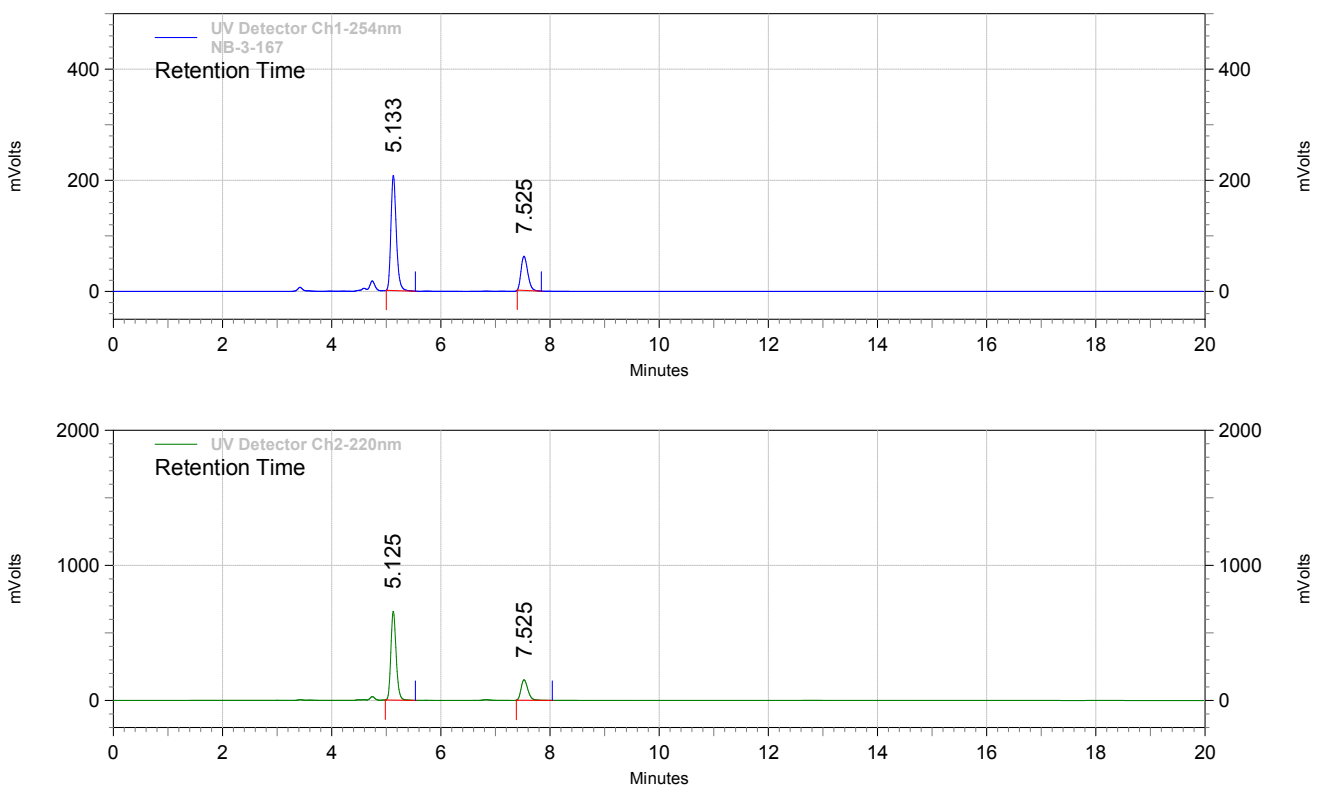

UV Detector

Ch1-254nm Results

Retention Time

5.133

Area

Area \%

Height

$206837 \quad 77.09$

521210

26.88

61472

22.91

Totals

1938763

100.00

268309 100.00

UV Detector Ch2-220nm Results Retention Time

5.125

7.525

Area

1307356

Area \%

77.08

Height

656870

151412

Height \%

22.92

81.27
18.73

\begin{tabular}{|r|r|r|r|r|}
\hline Totals & 5703537 & 100.00 & 808282 & 100.00 \\
\hline
\end{tabular}



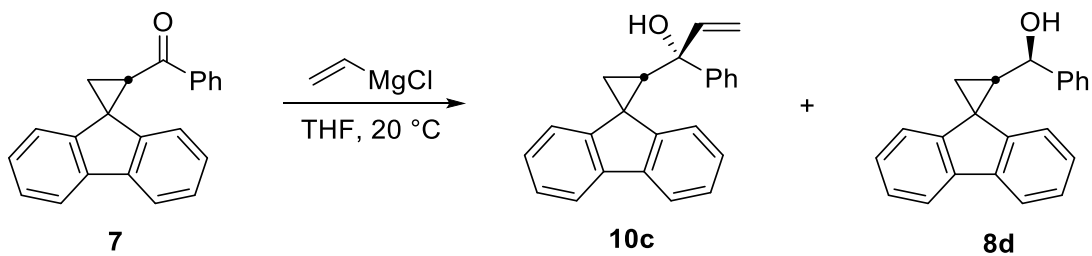

eluent $=0.5: 99.5 i$-PrOH:hexanes; flow rate $=1.0 \mathrm{~mL} / \mathrm{min}$
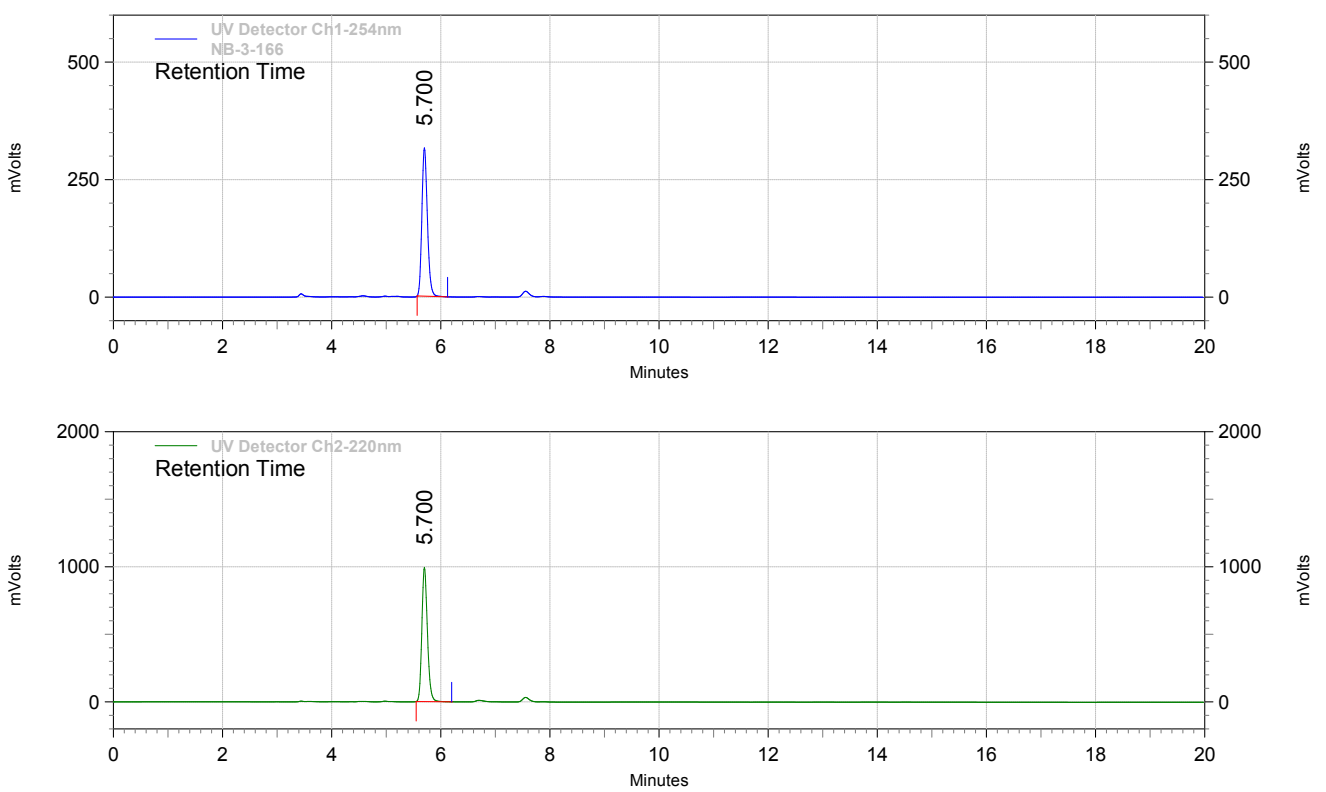

UV Detector

Ch1-254nm Results

Retention Time

5.700

Area

Area \%

Height

Height \%

Totals

2213589

100.00

315530 100.00

\begin{tabular}{|r|r|r|r|r|}
\hline Totals & 2213589 & 100.00 & 315530 & 100.00 \\
\hline
\end{tabular}

UV Detector

Ch2-220nm Results

Retention Time

5.700

Area

Area \%

Height

992990

Height \%

7020252

100.00

\begin{tabular}{|r|r|r|r|r|}
\hline Totals & 7020252 & 100.00 & 992990 & 100.00 \\
\hline
\end{tabular}




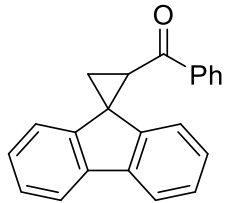

7
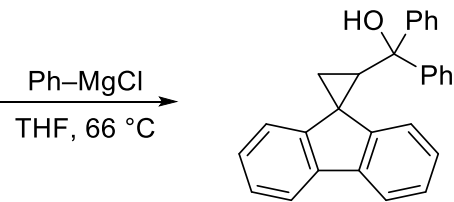

$10 d$

eluent $=0.1: 99.9 i-\mathrm{PrOH}:$ hexanes; flow rate $=1.0 \mathrm{~mL} / \mathrm{min}$
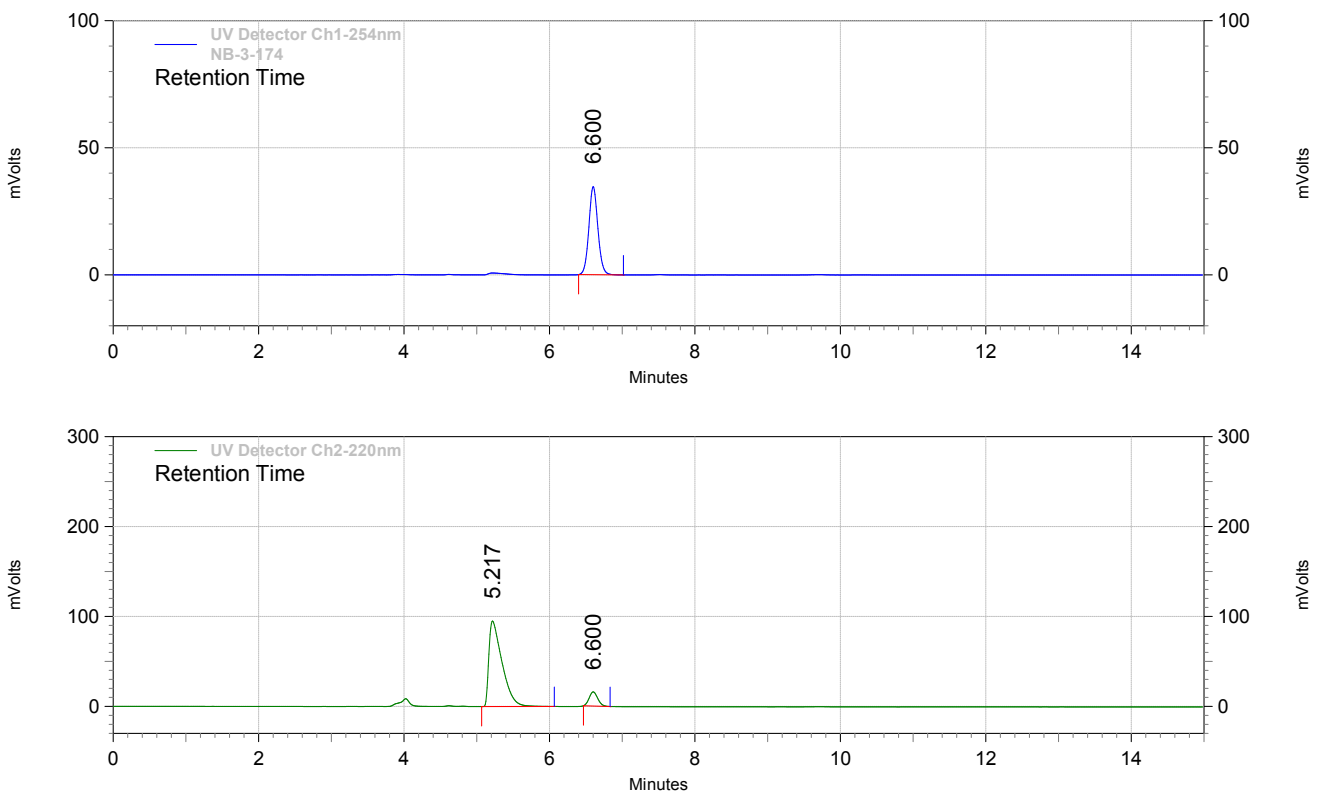

UV Detector

Ch1-254nm Results

Retention Time

6.600

Area

Area \%

Height

Height \%

298288

100.00

34675

100.00

\begin{tabular}{|r|r|r|r|r|}
\hline Totals & 298288 & 100.00 & 34675 & 100.00 \\
\hline
\end{tabular}

UV Detector

Ch2-220nm Results

Retention Time

5.217

Area

1195122

128560

Area \%

90.29

Height

95133

Height \%

9.71

15745

14.20

\begin{tabular}{|r|r|r|r|r|}
\hline Totals & 1323682 & 100.00 & 110878 & 100.00 \\
\hline
\end{tabular}




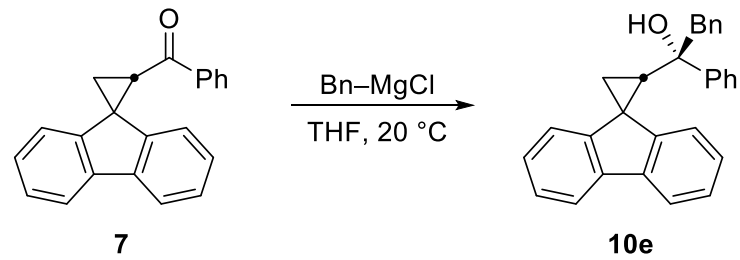

eluent $=1.0: 99.0 i-\mathrm{PrOH}:$ hexanes; flow rate $=1.0 \mathrm{~mL} / \mathrm{min}$
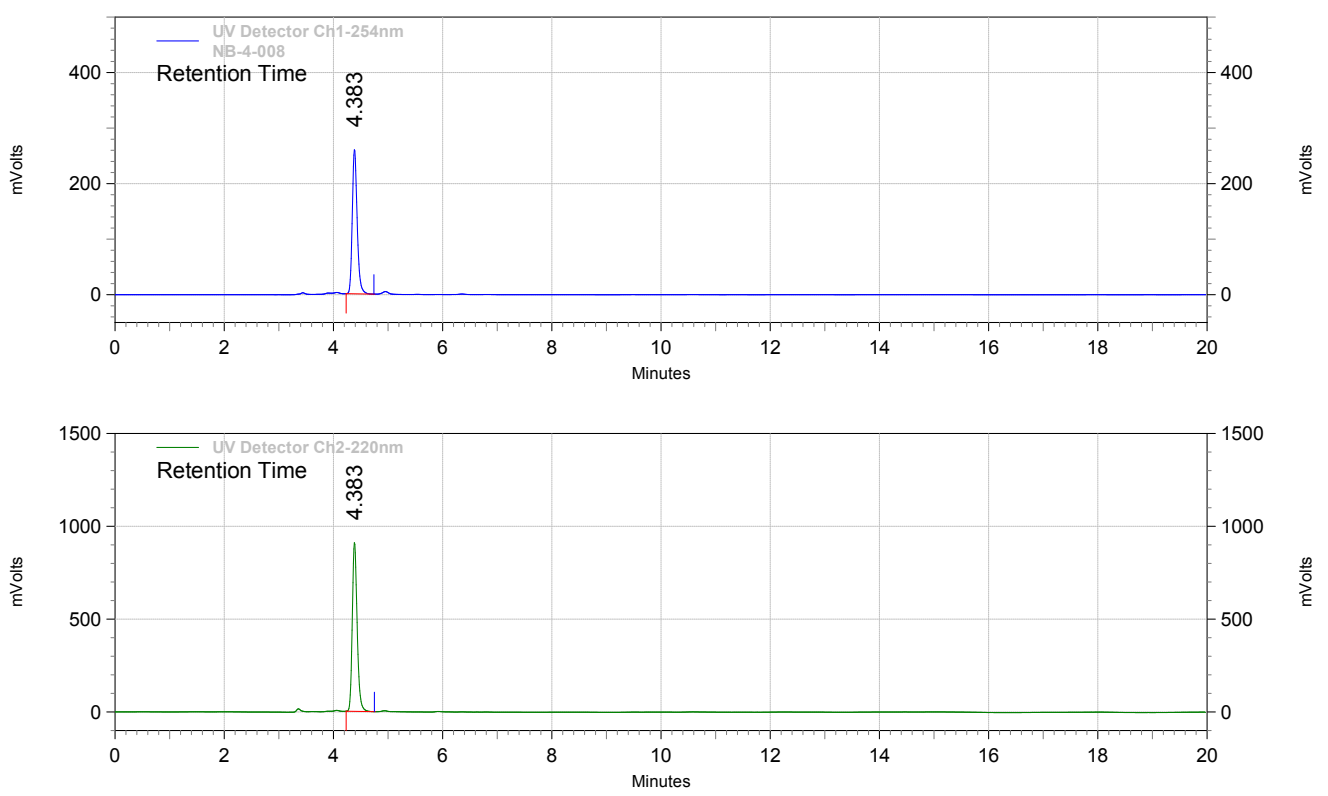

UV Detector

Ch1-254nm Results

Retention Time

4.383

Area

Area \%

Height

Height \%

1611807

100.00

259475 100.00

\begin{tabular}{|r|r|r|r|r|}
\hline Totals & 1611807 & 100.00 & 259475 & 100.00 \\
\hline
\end{tabular}

UV Detector

Ch2-220nm Results

Retention Time

4.383

Area

Area \%

Height

Height \%

Totals 5612985 100.00 908821 100.00

\begin{tabular}{|r|r|r|r|r|}
\hline Totals & 5612985 & 100.00 & 908821 & 100.00 \\
\hline
\end{tabular}




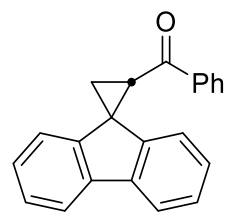

7
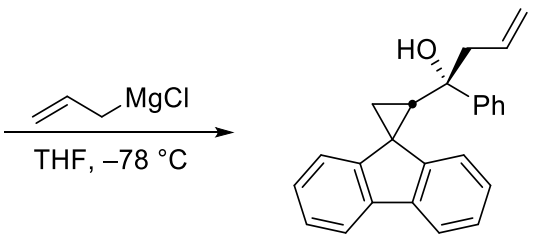

$10 f$

eluent $=0.5: 99.5 i-\mathrm{PrOH}:$ hexanes; flow rate $=1.0 \mathrm{~mL} / \mathrm{min}$
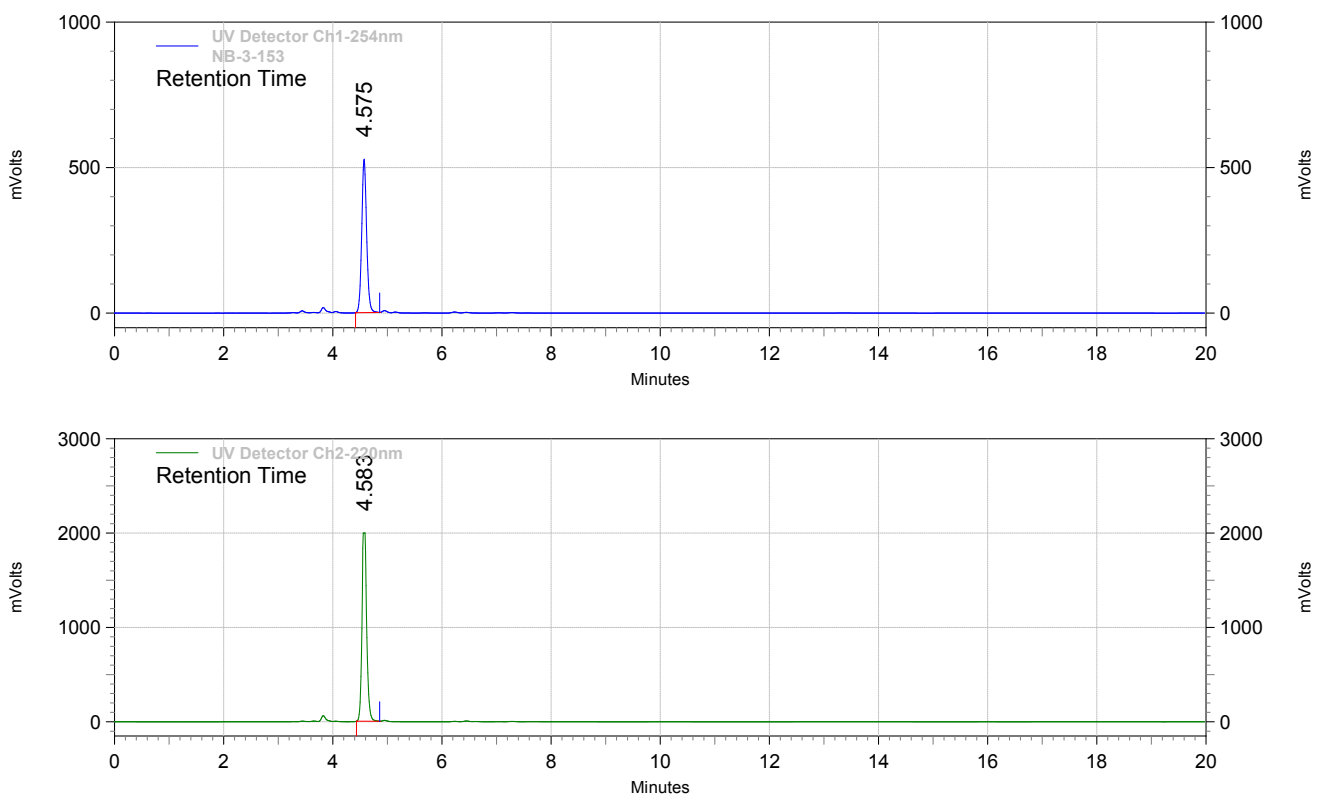

UV Detector

Ch1-254nm Results

Retention Time

4.575

Area

Area \%

Height

Height \%

$100.00 \quad 527028$

\begin{tabular}{|r|r|r|r|r|}
\hline Totals & 3287876 & 100.00 & 527028 & 100.00 \\
\hline
\end{tabular}

UV Detector

Ch2-220nm Results

Retention Time 4.583

Area \%

Height

Height \% 12022846

$100.00 \quad 1994222$
100.00

\begin{tabular}{|r|r|r|r|r|}
\hline Totals & 12022846 & 100.00 & 1994222 & 100.00 \\
\hline
\end{tabular}


<smiles>O=C(c1ccccc1)[C@@H]1CC12c1ccccc1-c1ccccc12</smiles>

7
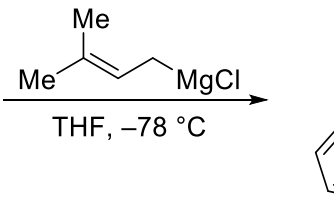

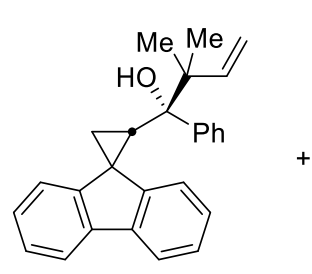

$10 \mathrm{~g}$

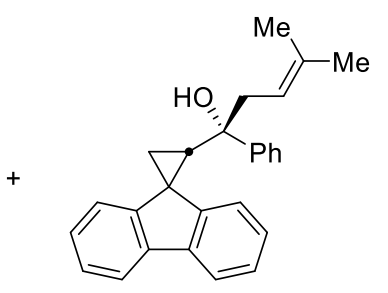

$10 g^{\prime}$

eluent $=1.0: 99.0 i$-PrOH:hexanes; flow rate $=1.0 \mathrm{~mL} / \mathrm{min}$
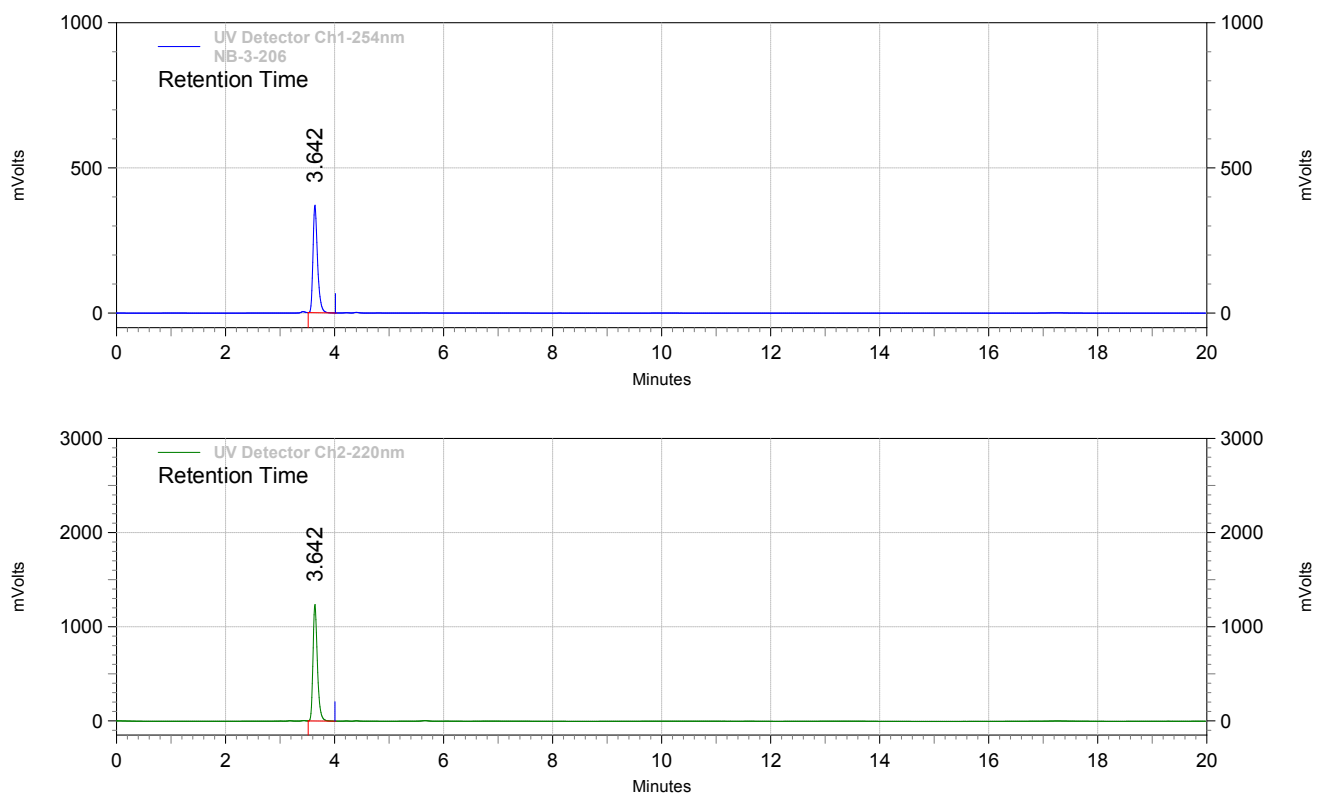

UV Detector

Ch1-254nm Results Retention Time 3.642 Area Area \% 100.00 Height 370088 Height \%

Totals 2138277 100.00 370088 100.00

UV Detector Ch2-220nm Results Retention Time 2138277

Area \%

Height Height \% 3.642 6928914 100.00 1237318 100.00

\begin{tabular}{|r|r|r|r|r|}
\hline Totals & 6928914 & 100.00 & 1237318 & 100.00 \\
\hline
\end{tabular}




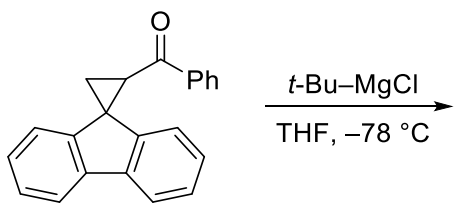

7

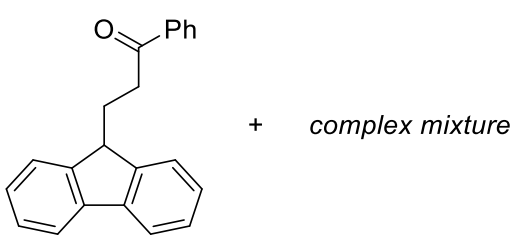

11

eluent $=0.1: 99.9 i-\mathrm{PrOH}:$ hexanes; flow rate $=1.0 \mathrm{~mL} / \mathrm{min}$
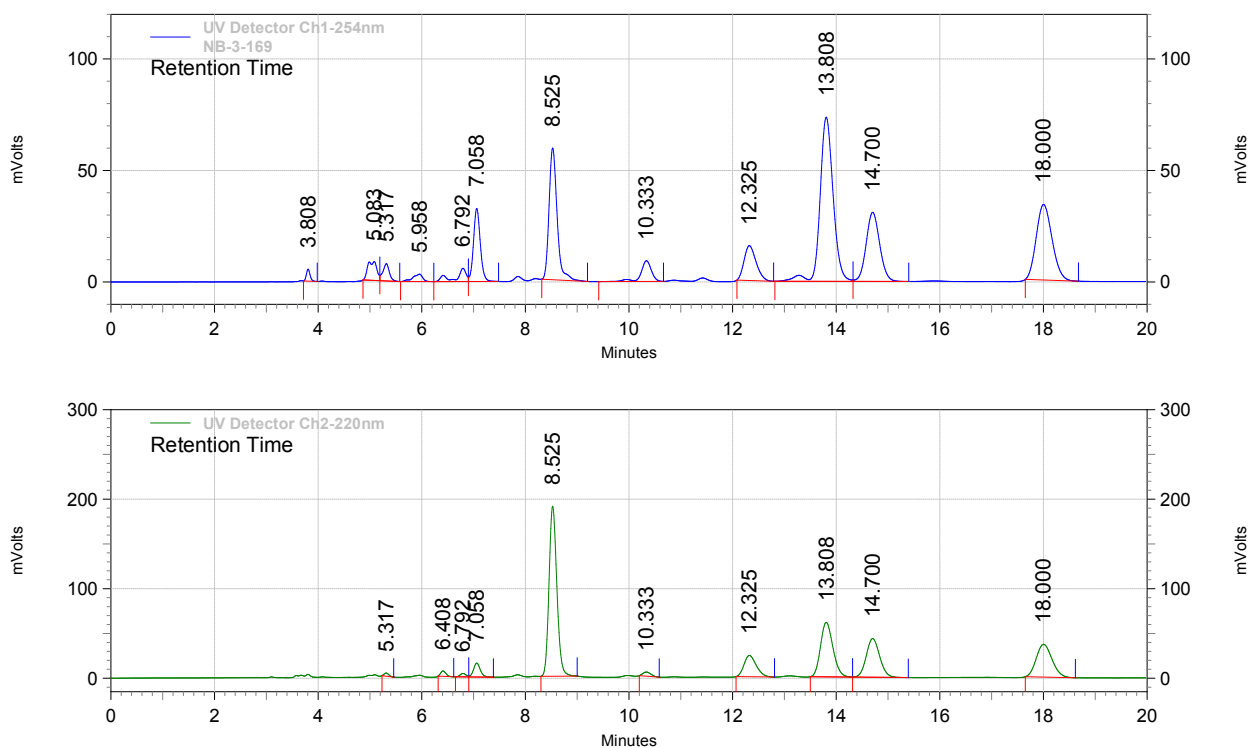

UV Detector

Ch1-254nm Results

Retention Time

3.808
5.083

5.083

5.958

6.792

7.058

8.525

10.333

12.325

13.808

14.700

18.000

Area

26147
103822

66868

43620

78841

299656

638898

129168

251514

1294852

563674

719552

Totals

4216612

\begin{tabular}{|r|r|r|r|r|}
\hline Totals & 4216612 & 100.00 & 285903 & 100.00 \\
\hline
\end{tabular}

Area \%

Height

$2.46-5346 \quad 1.87$

159

7743

3319

5931

32708

59037

9278

15614

73628

30988

33880

2.95

2.71

1.87

15.15

5.96

30.71

13.37
17.06

1.16
2.07

11.44

(20.65

3.25

5.46

25.75

10.84

UV Detector Ch2-220nm Results Retention Time

5.317

6.408

6.792

7.058

8.525

10.333

12.325

13.808

14.700

18.000

Area

42223

30131

140911

1990539

49194

392082

1015372

778453

774965

Totals

5235290

Area \%

Height

Height \%

$3502 \quad 0.90$

5967

3840

15521

189922

4627

23844

60864

43238

36645

1.54

0.99

4.00

38.02

7.49

19.39

14.87

14.80

1.19

6.15

15.69

11.14

9.45

\begin{tabular}{|r|r|r|r|r|}
\hline Totals & 5235290 & 100.00 & 387970 & 100.00 \\
\hline
\end{tabular}




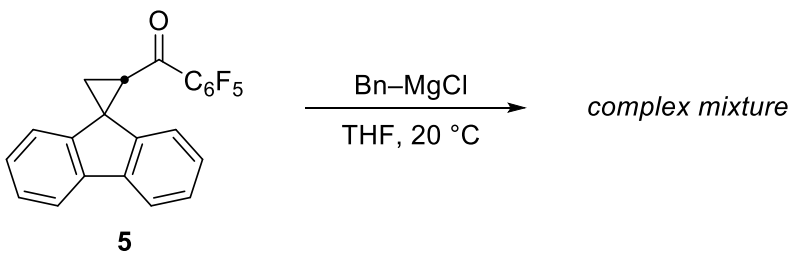

eluent $=$ 0.5:99.5 $i$-PrOH:hexanes; flow rate $=1.0 \mathrm{~mL} / \mathrm{min}$
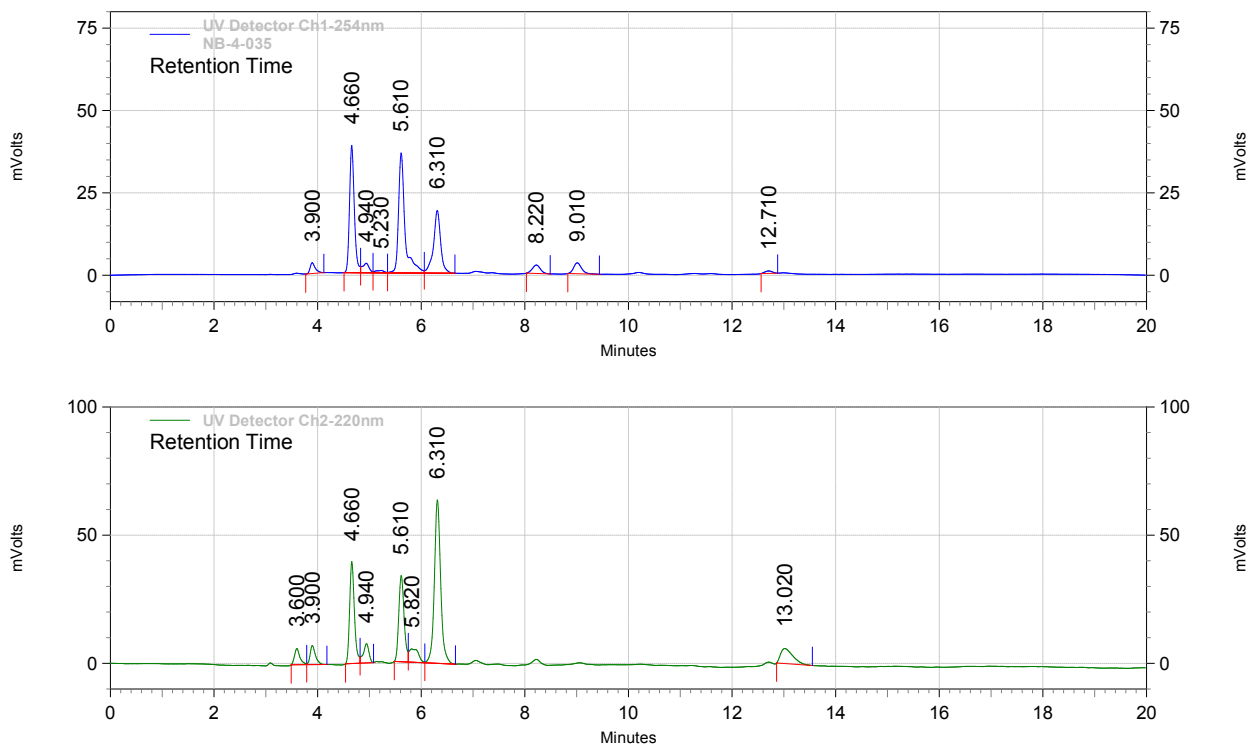

UV Detector

Ch1-254nm Results

Retention Time

3.900
4.660

4.660
4.940

5.230

5.610

6.310

8.220

9.010

12.710

Are
2286
249170

Area

Area \%

Height

Height \%

$2.613266 \quad 3.04$

$\begin{array}{lll}28.46 & 38639 & 35.92\end{array}$

25721

2.94

2889

2.69

8321

36408

183115

27472

35254

20.91

19055

33.85

3.14

2545

4.03

2.37

$0.84 \quad 737-0.69$

\begin{tabular}{|r|r|r|r|r|}
\hline Totals & 875609 & 100.00 & 107562 & 100.00 \\
\hline
\end{tabular}

UV Detector Ch2-220nm Results

Retention Time

3.600

3.900

4.660

4.940

5.610

5.820

6.310

13.020

Area

45641

53196

255114

56555

239385

65206

539999

96832

100.00

Totals

(2)




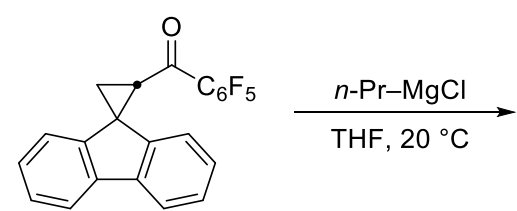

5<smiles>OC([C@H]1CC12c1ccccc1-c1ccccc12)C(F)(F)F</smiles>

20<smiles>O[C@@H](C1CC12c1ccccc1-c1ccccc12)C(F)(F)F</smiles>

20'

eluent $=$ 0.5:99.5 $i$-PrOH:hexanes; flow rate $=1.0 \mathrm{~mL} / \mathrm{min}$
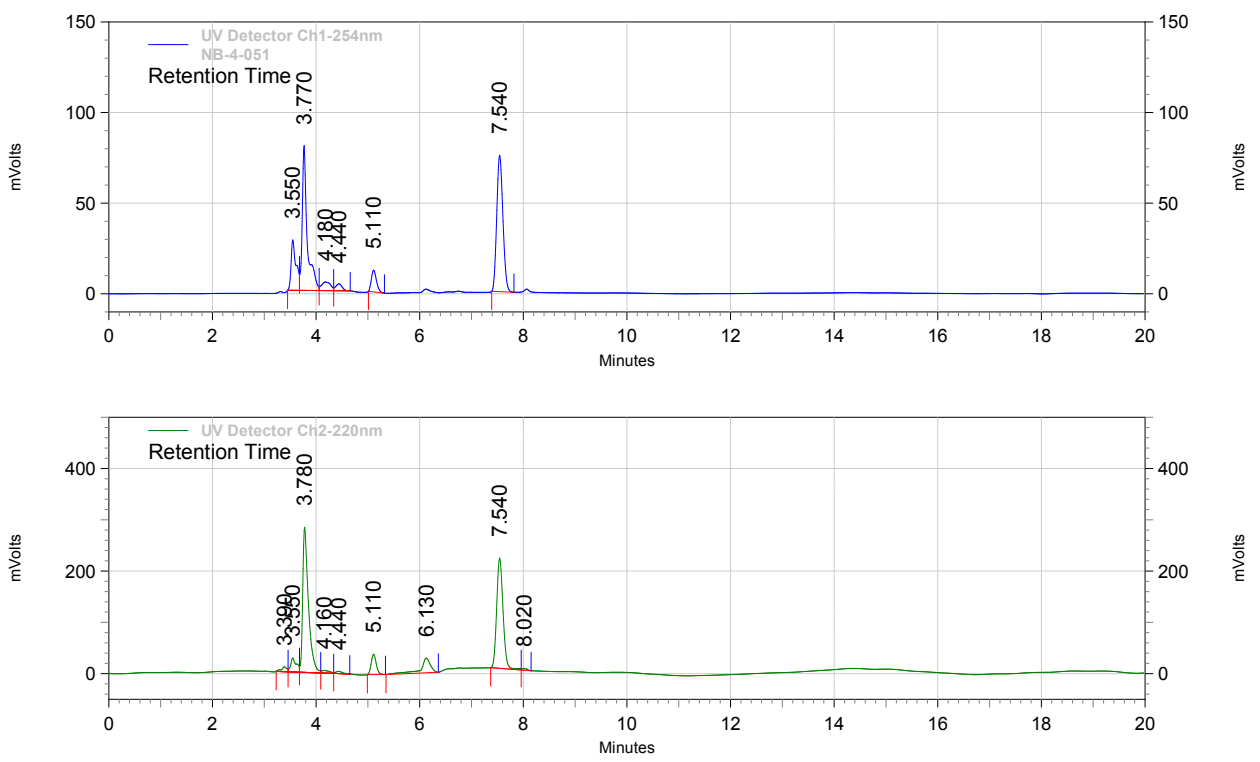

UV Detector

Ch1-254nm Results

Retention Time

3.550
3.770

3.770
4.180

4.440

5.110

7.540

Area

186390

540738

57955

32102

82234

623871

Area \%

Area \% Heigh

27844

13.69

35.50

3.80

2.11

5.40

40.96

4722

3719

11981

75247

39.28

2.32

1.83

Totals

1523290

100.00

203429

100.00

UV Detector

Ch2-220nm Results

Retention Time

3.390
3.550

3.550
3.780

4.160

4.440

5.110

6.130

7.540

8.020

Area

211738

2158892

55301

37663

266792

364250

1828704

24415

Totals

Area \%

Height

Height \%

1.40

9474

1.54

4.22
43.02

1.10

0.75

5.32

7.26

36.44

0.49

27238
282993

4960

4168

39603

28500

214847

3329

46.01

0.81

0.68

6.44

4.63

34.93

0.54

\begin{tabular}{|r|r|r|r|r|}
\hline Totals & 5018131 & 100.00 & 615112 & 100.00 \\
\hline
\end{tabular}


<smiles>O=C(C1CC12c1ccccc1-c1ccccc12)C(F)(F)F</smiles>

5
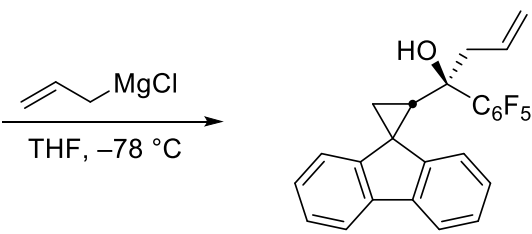

21
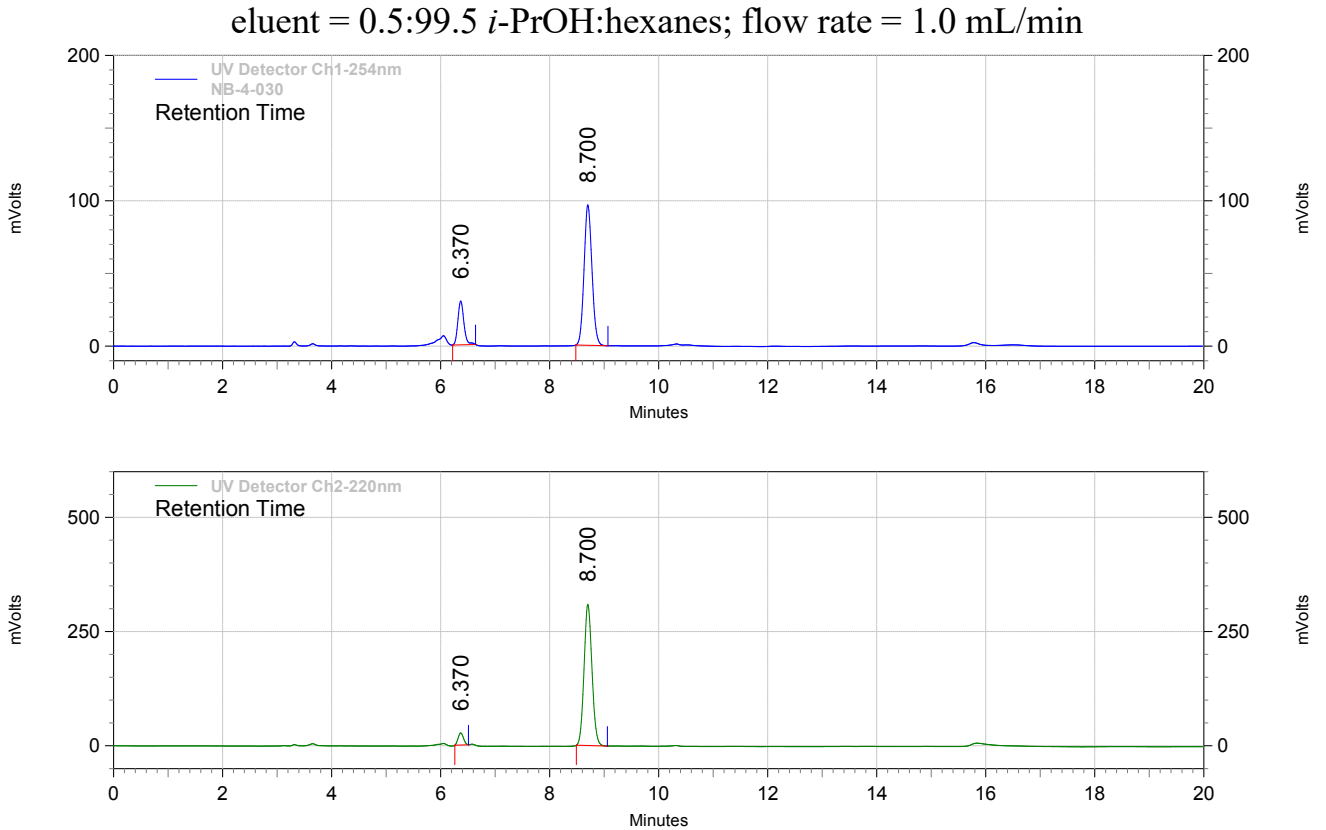

UV Detector

Ch1-254nm Results Retention Time 6.370

8.700 Area 230429 975812 Area \% 80.90

Height 30125 96600 Height \% 23.77 76.23

\begin{tabular}{|r|r|r|r|r|}
\hline Totals & 1206241 & 100.00 & 126725 & 100.00 \\
\hline
\end{tabular}

UV Detector Ch2-220nm Results Retention Time 6.370 Area Area \% Height

26388
Height \%

8.700 3116865

94.55 309182

Totals 


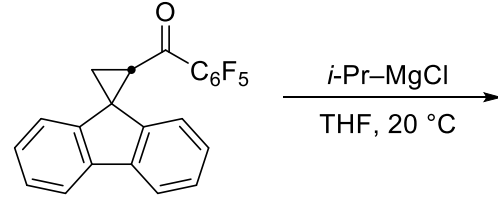

5<smiles>O[C@@H]([C@H]1CC12c1ccccc1-c1ccccc12)C(F)(F)F</smiles>

20

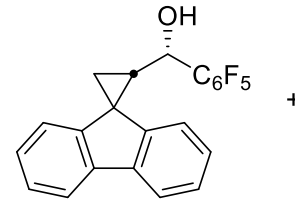

20'

eluent $=$ 0.5:99.5 $i-\mathrm{PrOH}:$ hexanes
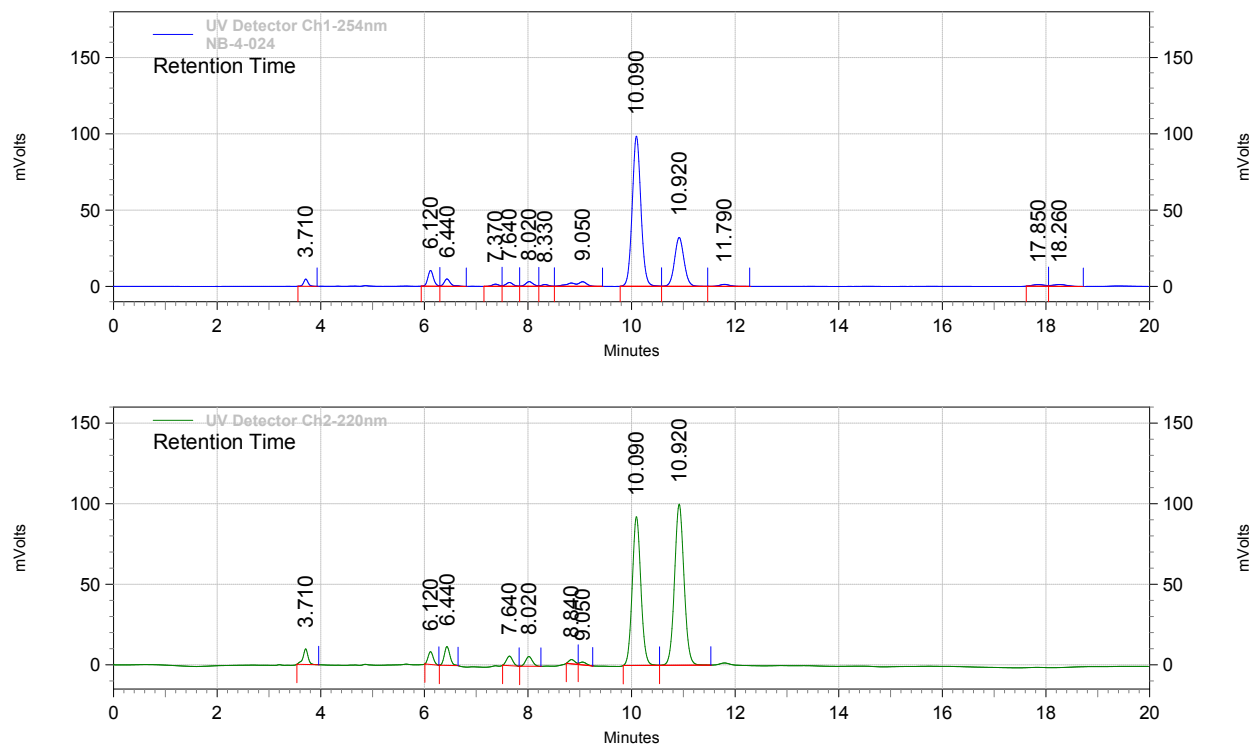

UV Detector

Ch1-254nm Results

Retention Time

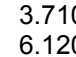

6.120
6.440

7.370

7.640

8.020

8.330

9.050

10.090

10.920

11.790

17.850

18.260

Area

27833

77767

40998

13740

24991

32411

12050

63301

1147294

407825

17730

17456

21763

Area $\%$
1.46
4.08
2.15
0.72
1.31
1.70
0.63
3.32
60.22
21.41
0.93
0.92
1.14

\begin{tabular}{rrr} 
ea $\%$ & Height & Height \% \\
\hline 1.46 & 4723 & 2.86 \\
4.08 & 10344 & 6.26 \\
2.15 & 4816 & 2.91 \\
0.72 & 1483 & 0.90 \\
1.31 & 2573 & 1.56 \\
1.70 & 3133 & 1.90 \\
0.63 & 1236 & 0.75 \\
3.32 & 3053 & 1.85 \\
0.22 & 98338 & 59.50 \\
21.41 & 32033 & 19.38 \\
0.93 & 1303 & 0.79 \\
0.92 & 1065 & 0.64 \\
1.14 & 1176 & 0.71
\end{tabular}

\begin{tabular}{|r|r|r|r|r|}
\hline Totals & 1905159 & 100.00 & 165276 & 100.00 \\
\hline $\begin{array}{r}\text { UV Detector } \\
\text { Ch2-220nm Results }\end{array}$ & Area & Area \% & Height & Height \% \\
Retention Time & 64804 & 2.41 & 9693 & 4.07 \\
\hline 3.710 & 54867 & 2.04 & 7931 & 3.33 \\
6.120 & 88176 & 3.28 & 11667 & 4.90 \\
6.440 & 49358 & 1.84 & 5936 & 2.50 \\
7.640 & 55793 & 2.08 & 6060 & 1.12 \\
8.020 & 21670 & 0.81 & 2658 & 0.73 \\
8.840 & 15335 & 0.57 & 1746 & 42.77 \\
9.050 & 1065990 & 39.68 & 92226 & \\
10.090 & 1270522 & 47.29 & 99984 & 100.00 \\
10.920 & \multicolumn{5}{|c|}{} \\
\hline
\end{tabular}




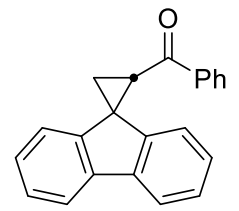

7

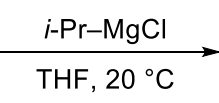

$\mathrm{THF}, 20^{\circ} \mathrm{C}$

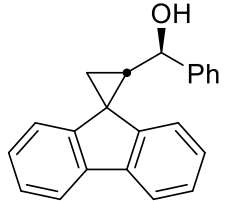

$8 d$

eluent $=0.5: 99.5 i-\mathrm{PrOH}:$ hexanes; flow rate $=1.0 \mathrm{~mL} / \mathrm{min}$
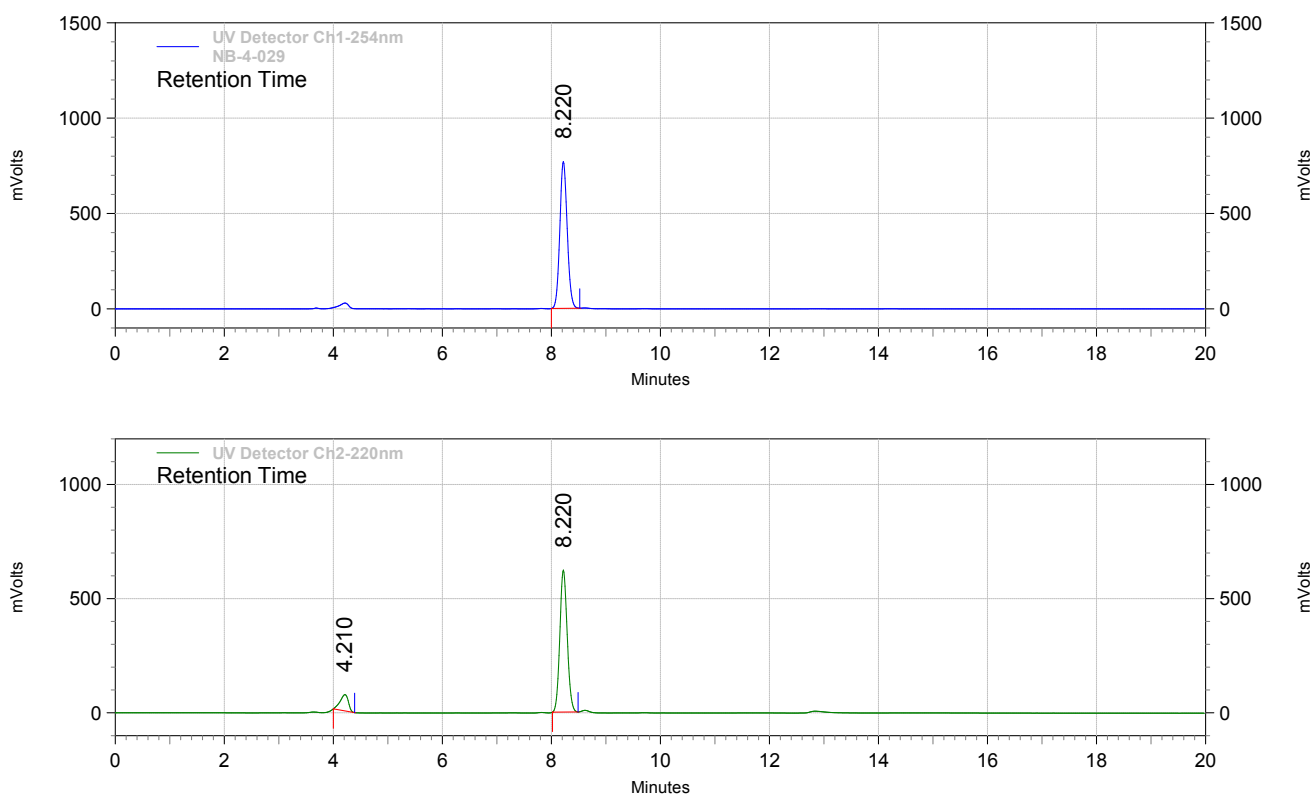

UV Detector

Ch1-254nm Results

Retention Time

8.220

Area

Area $\%$

$\begin{array}{llll}226963 & 100.00 & 769938 & 100.00\end{array}$

Height \%

\begin{tabular}{|r|r|r|r|r|}
\hline Totals & 7226963 & 100.00 & 769938 & 100.00 \\
\hline
\end{tabular}

UV Detector

Ch2-220nm Results

Retention Time

4.210

8.220

Area

Area \%

11.37

Height

Height \%

Totals

5824645

88.63

71301

10.28 

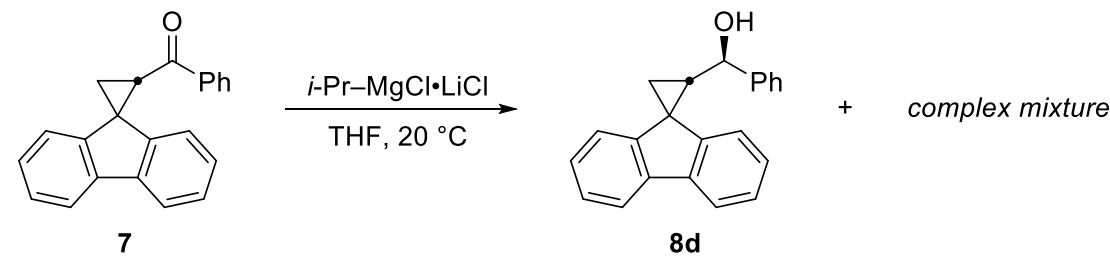

eluent $=0.5: 99.5 i$-PrOH:hexanes; flow rate $=1.0 \mathrm{~mL} / \mathrm{min}$
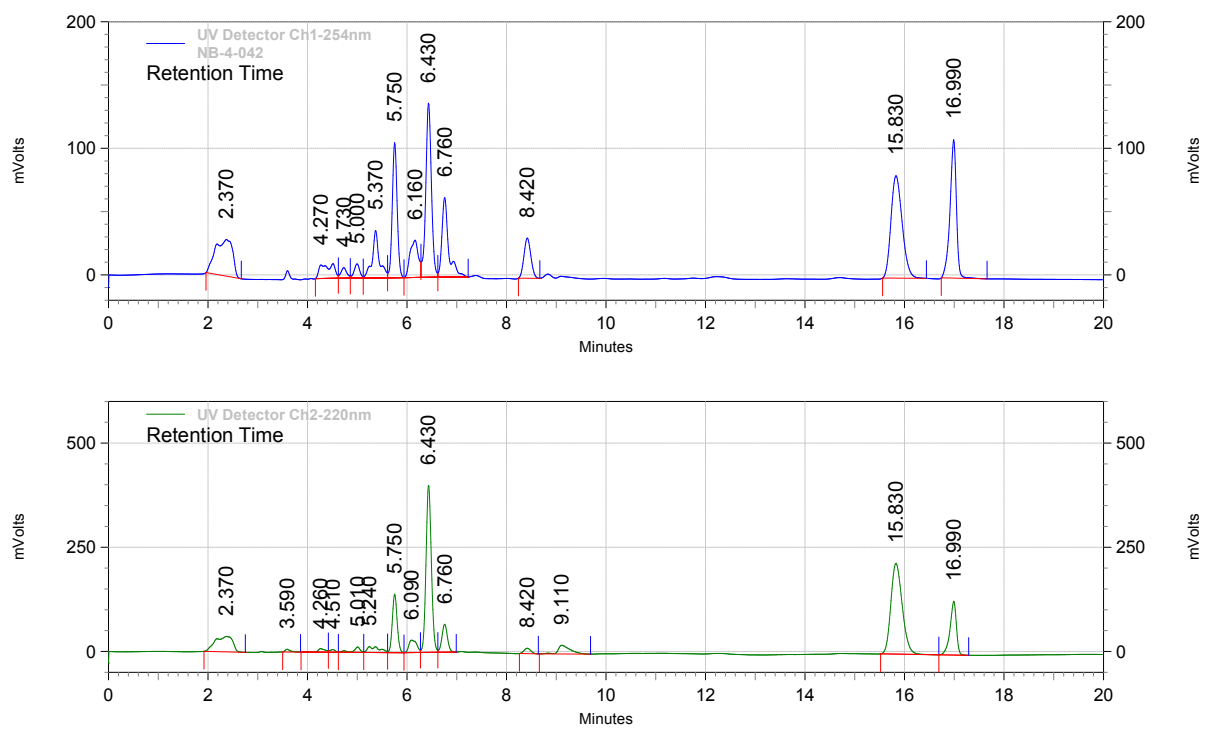

\begin{tabular}{|c|c|c|c|c|}
\hline $\begin{array}{r}\text { UV Detector } \\
\text { Ch1-254nm Results } \\
\text { Retention Time }\end{array}$ & Area & Area \% & Height & Height $\%$ \\
\hline 2.370 & 697748 & 10.37 & 28987 & 4.42 \\
\hline 4.270 & 211845 & 3.15 & 10682 & 1.63 \\
\hline 4.730 & 68234 & 1.01 & 8390 & 1.28 \\
\hline 5.000 & 86844 & 1.29 & 11206 & 1.71 \\
\hline 5.370 & 368084 & 5.47 & 37439 & 5.71 \\
\hline 5.750 & 727086 & 10.80 & 106697 & 16.28 \\
\hline 6.160 & 343180 & 5.10 & 29515 & 4.50 \\
\hline 6.430 & 1087441 & 16.16 & 137543 & 20.99 \\
\hline 6.760 & 585406 & 8.70 & 62836 & 9.59 \\
\hline 8.420 & 307096 & 4.56 & 31771 & 4.85 \\
\hline 15.830 & 1267929 & 18.84 & 80914 & 12.35 \\
\hline 16.990 & 980273 & 14.56 & 109369 & 16.69 \\
\hline \multirow[t]{2}{*}{ Totals } & & & & \\
\hline & 6731166 & 100.00 & 655349 & 100.00 \\
\hline \multicolumn{5}{|l|}{$\begin{array}{c}\text { UV Detector } \\
\text { Ch2-220nm Results }\end{array}$} \\
\hline Retention Time & Area & Area \% & Height & Height \% \\
\hline 2.370 & 913312 & 8.06 & 37203 & 3.37 \\
\hline 3.590 & 48149 & 0.42 & 6548 & 0.59 \\
\hline 4.260 & 91066 & 0.80 & 8603 & 0.78 \\
\hline 4.510 & 50108 & 0.44 & 6601 & 0.60 \\
\hline 5.010 & 127390 & 1.12 & 13013 & 1.18 \\
\hline 5.240 & 236144 & 2.08 & 13956 & 1.26 \\
\hline 5.750 & 947309 & 8.36 & 140288 & 12.70 \\
\hline 6.090 & 329368 & 2.91 & 29909 & 2.71 \\
\hline 6.430 & 3014204 & 26.59 & 400599 & 36.27 \\
\hline 6.760 & 515485 & 4.55 & 67022 & 6.07 \\
\hline 8.420 & 122291 & 1.08 & 13029 & 1.18 \\
\hline 9.110 & 352881 & 3.11 & 20941 & 1.90 \\
\hline 15.830 & 3446976 & 30.41 & 217652 & 19.71 \\
\hline 16.990 & 1139731 & 10.06 & 128993 & 11.68 \\
\hline Totals & 11334414 & 100.00 & 1104357 & 100.00 \\
\hline
\end{tabular}


<smiles>O=C(c1ccccc1)C1CC12c1ccccc1-c1ccccc12</smiles>

7
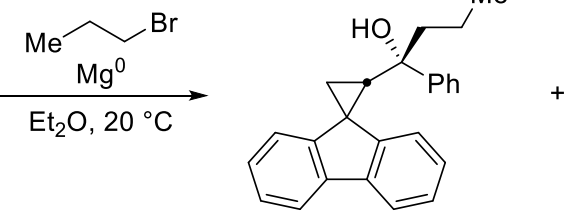

10b<smiles>OC(c1ccccc1)[C@H]1CC12c1ccccc1-c1ccccc12</smiles>

$8 d$

eluent $=0.5: 99.5 i-\mathrm{PrOH}:$ hexanes; flow rate $=1.0 \mathrm{~mL} / \mathrm{min}$
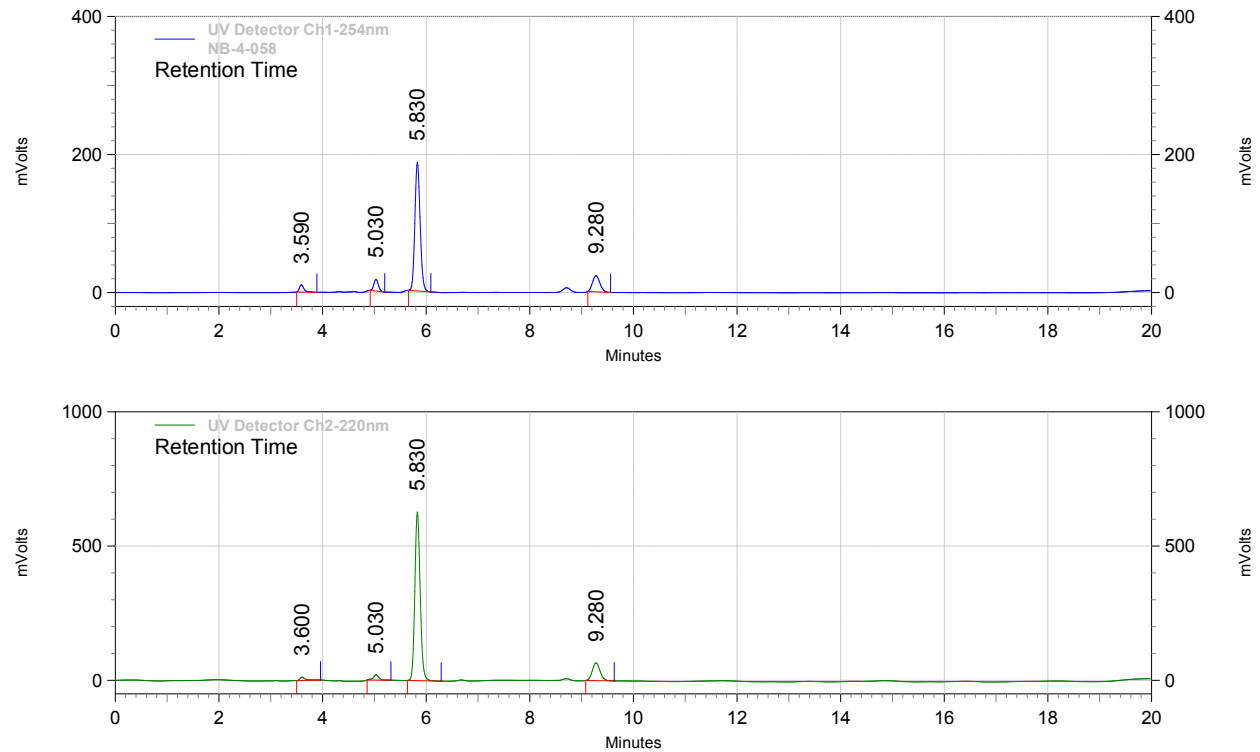

UV Detector Ch1-254nm Results Retention Time 3.590

5.030

9.280

Area

102837

1311753

232752

Totals

1711372

UV Detector

Ch2-220nm Results

Retention Time

3.600

5.830

9.280

Area \%

Height

10657

16889

186406

23422

76.65

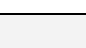

237374

100.00

\begin{tabular}{rrrrrr|} 
Retention Time & Area & Area \% & Height & Height \% \\
\hline 3.600 & 91714 & 1.69 & 12274 & 1.69 \\
5.030 & 144311 & 2.66 & 19276 & 2.66 \\
5.830 & 4495408 & 82.97 & 627723 & 86.55 \\
9.280 & 686458 & 12.67 & 66040 & 9.11 \\
\hline Totals & 5417891 & 100.00 & 725313 & 100.00 \\
\hline
\end{tabular}


<smiles>O=C(c1ccccc1)C1CC12c1ccccc1-c1ccccc12</smiles>

7

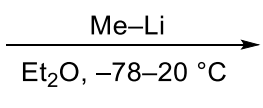

eluent $=0.5: 99.5 i$-PrOH:hexanes; flow rate $=1.0 \mathrm{~mL} / \mathrm{min}$
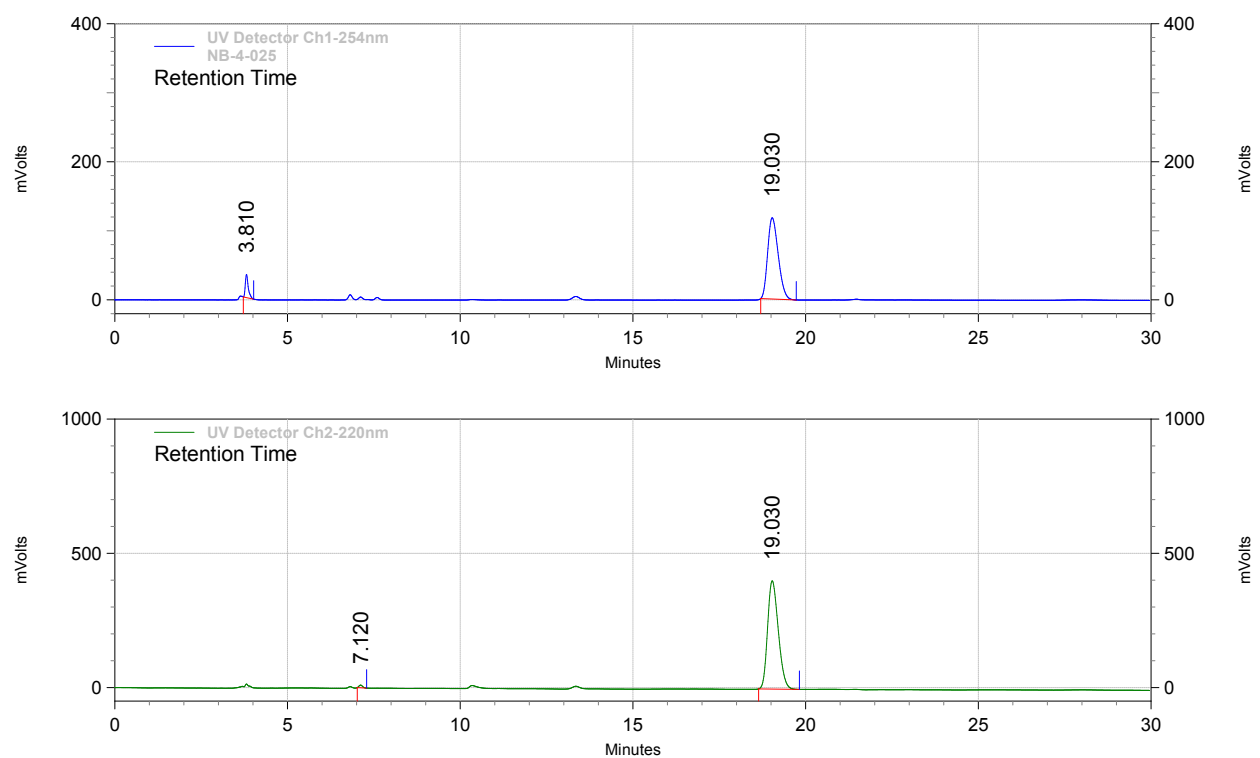

UV Detector

Ch1-254nm Results

Retention Time

3.810

19.030

Area

197805

2502064

Area \%

Height \%

$\begin{array}{rrr}92.67 & 33542 & 22.19 \\ & 117639 & 77.81\end{array}$

\begin{tabular}{|r|r|r|r|r|}
\hline Totals & 2699869 & 100.00 & 151181 & 100.00 \\
\hline
\end{tabular}

UV Detector

Ch2-220nm Results

Retention Time

7.120
19.030

Area

Area \%

0.82
99.18

Height

9993

Height \%

71554
8675747

99.1

402846

97.58

\begin{tabular}{|r|r|r|r|r|}
\hline Totals & 8747301 & 100.00 & 412839 & 100.00 \\
\hline
\end{tabular}


<smiles>O=C(c1ccccc1)C1CC12c1ccccc1-c1ccccc12</smiles>

7

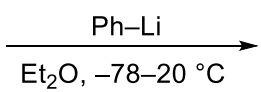

eluent $=0.5: 99.5 i-\mathrm{PrOH}:$ hexanes; flow rate $=1.0 \mathrm{~mL} / \mathrm{min}$
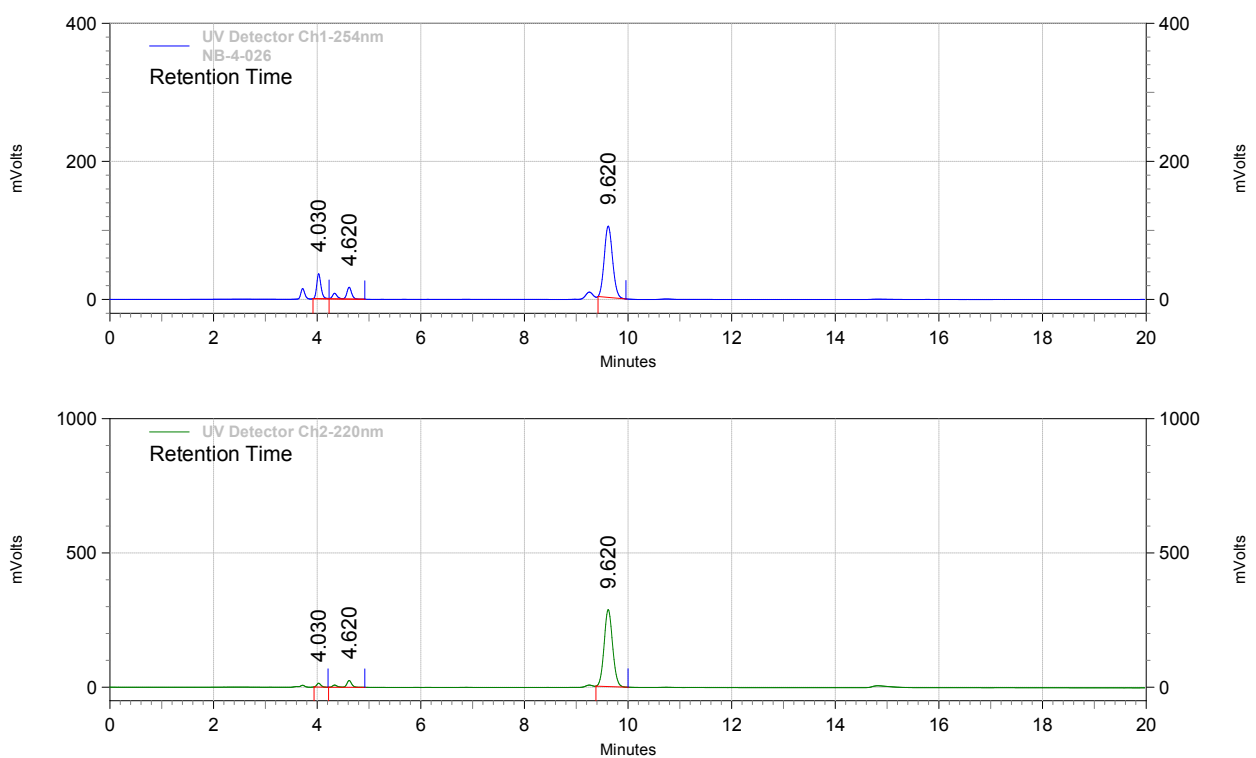

UV Detector Ch1-254nm Results Retention Time 4.030
4.620 9.620 Area 208583 160385 1149107

Area \%

13.74

10.57

75.70

Height

36295

17159

103294

Height \%

10.95

65.90

\begin{tabular}{|r|r|r|r|r|}
\hline Totals & 1518075 & 100.00 & 156748 & 100.00 \\
\hline
\end{tabular}

UV Detector Ch2-220nm Results Retention Time 4.030

9.620

Area Area \%

2.22

6.02

91.76

Height

14399

Height \%

\begin{tabular}{l|l} 
Totals & 3542577
\end{tabular} 3250787

25218 286349

7.74

100.00

325966

100.00 


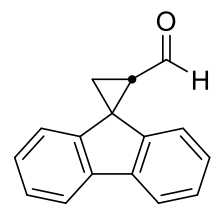

3

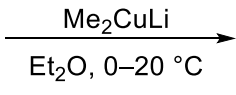

$\mathrm{Et}_{2} \mathrm{O}, 0-20{ }^{\circ} \mathrm{C}$

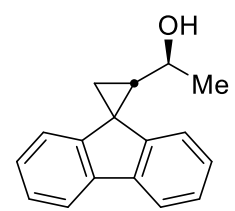

$8 a$

eluent $=0.5: 99.5 i$-PrOH:hexanes; flow rate $=1.0 \mathrm{~mL} / \mathrm{min}$
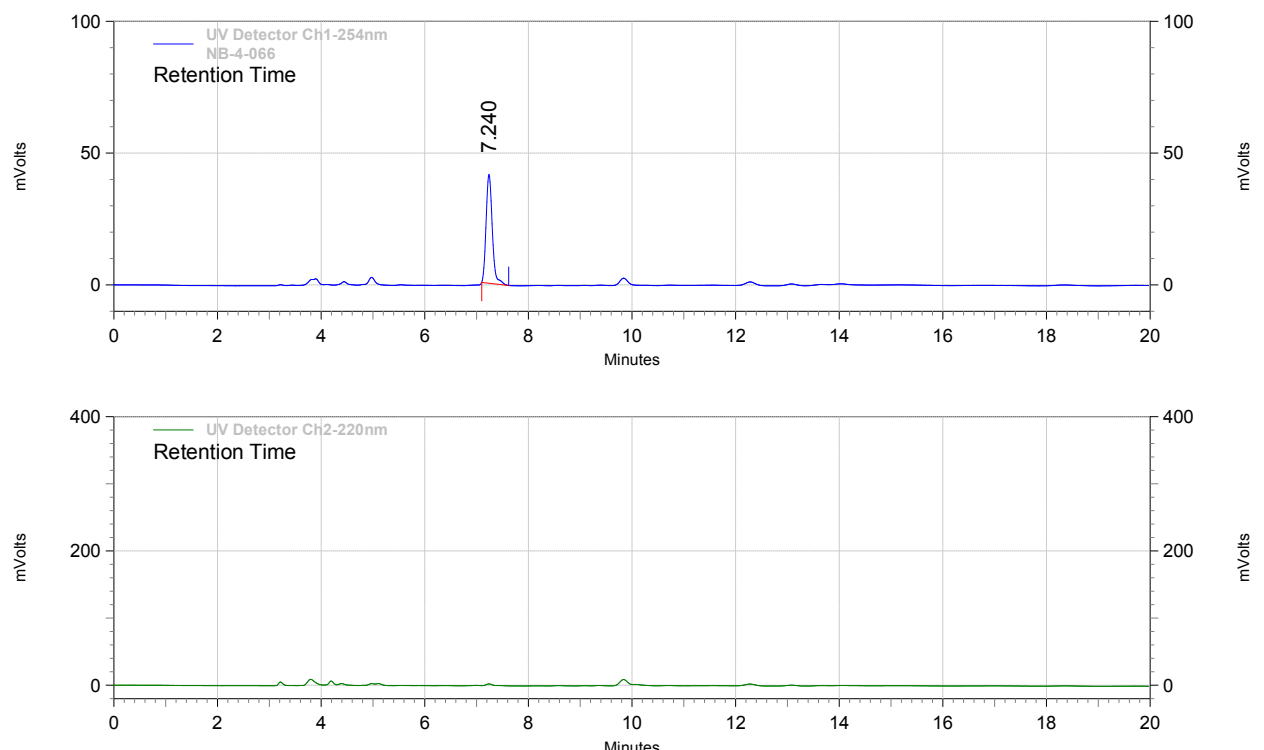

UV Detector

Ch1-254nm Results

Retention Time

7.240

Area

341802

Area \%

100.00

Height

Height \%

Totals 


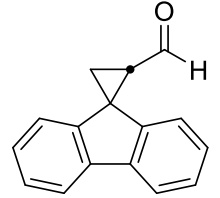

3

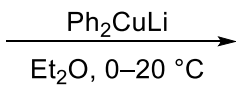

$\mathrm{Et}_{2} \mathrm{O}, 0-20{ }^{\circ} \mathrm{C}$

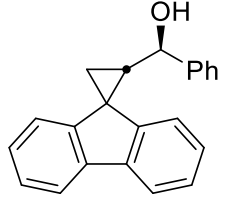

$8 d$

eluent $=0.5: 99.5 i-\mathrm{PrOH}:$ hexanes; flow rate $=1.0 \mathrm{~mL} / \mathrm{min}$
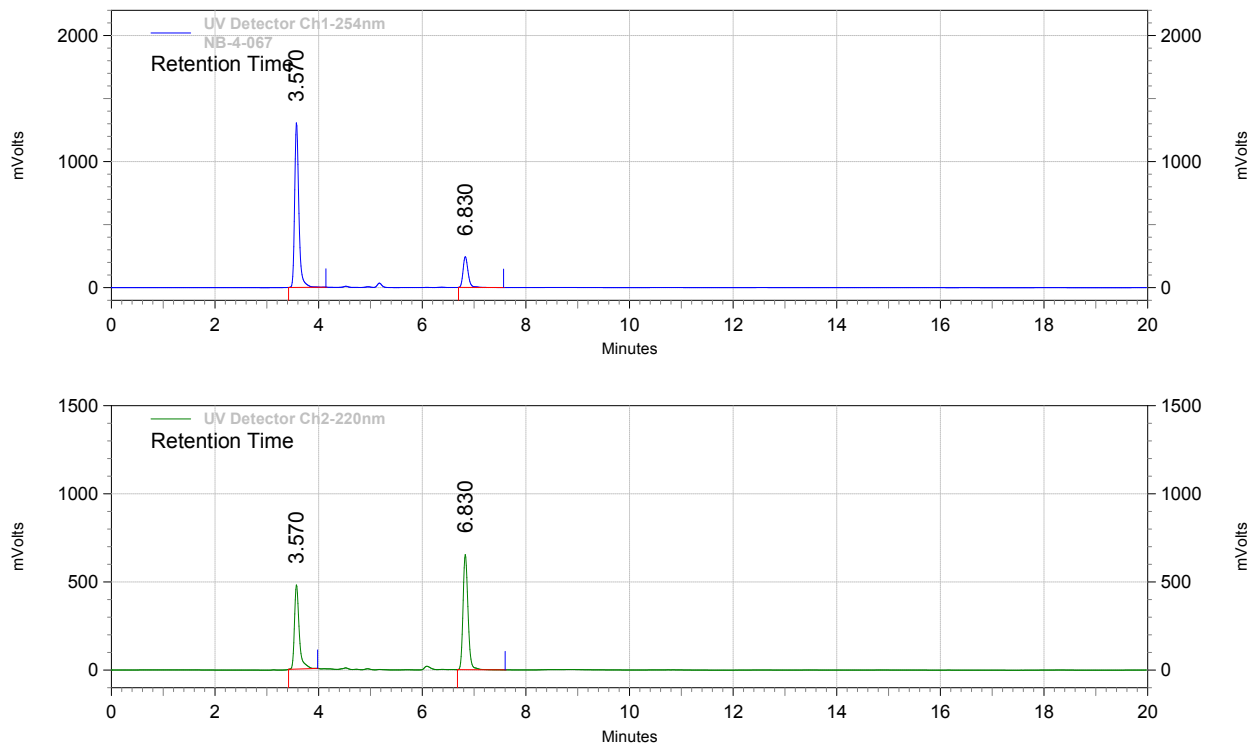

UV Detector Ch1-254nm Results
Retention Time 3.570 6.830 Area 7306885 1642643 Area \% Height 81.65

18.35 1305194 1305194
244563 Height \% 8949528 100.00 1549757

100.00

UV Detector Ch2-220nm Results
Retention Time 3.570 6.830 Area 4342168 Area \% 41.07

58.93

Height

477358 653618

Height \%

Totals 7367957 100.00 

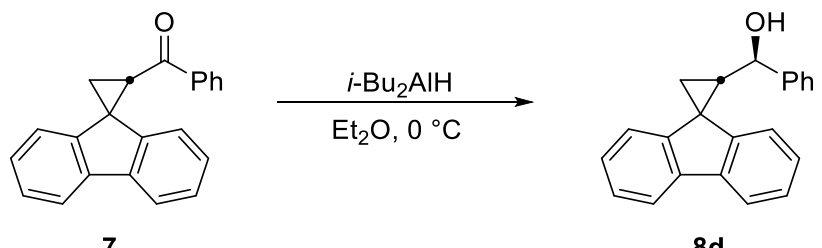

8d

eluent $=0.5: 99.5 i-\mathrm{PrOH}:$ hexanes; flow rate $=1.0 \mathrm{~mL} / \mathrm{min}$
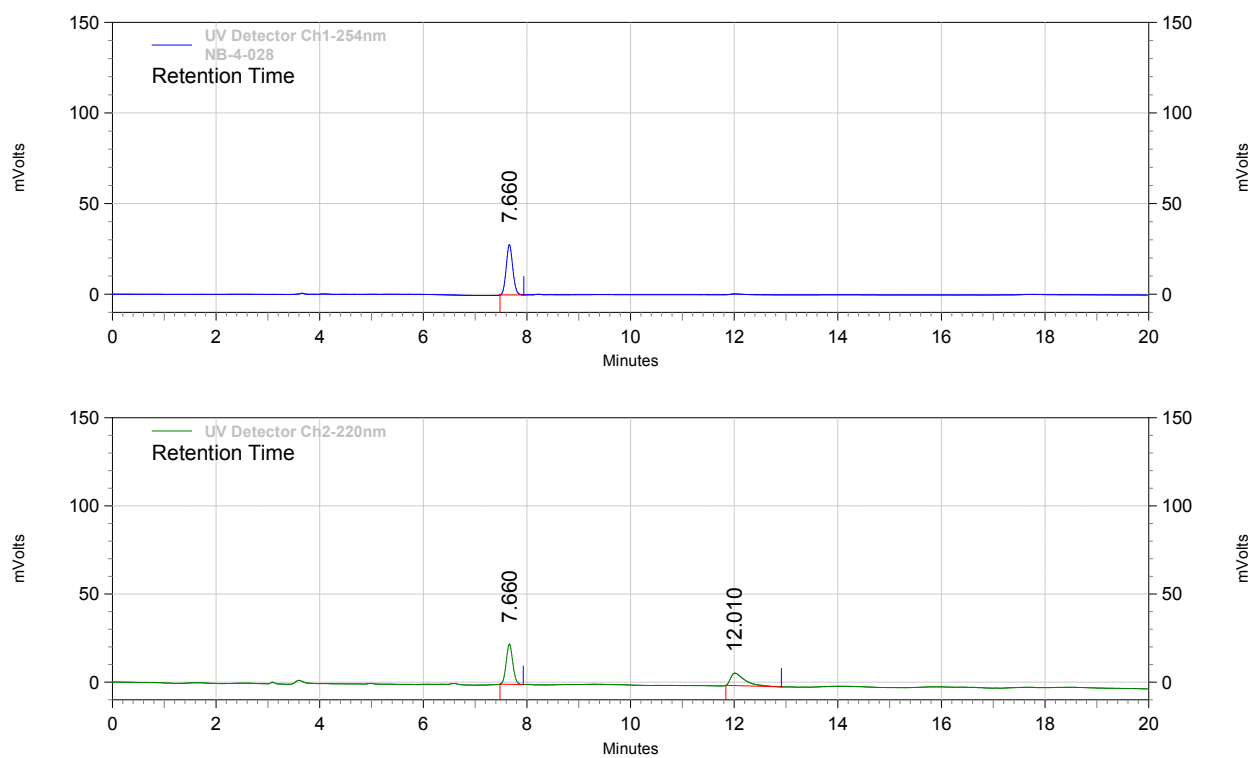

UV Detector

Ch1-254nm Results

Retention Time

7.660

Area

Area $\%$

244042

Height

Height \%

27789

100.00

\begin{tabular}{|r|r|r|r|r|}
\hline Totals & 244042 & 100.00 & 27789 & 100.00 \\
\hline
\end{tabular}

UV Detector

Ch2-220nm Results

Retention Time

7.660

Area

Area \%

Height

22942

7088

Height \%

12.010

134647

40.03

30030

23.60

Totals 

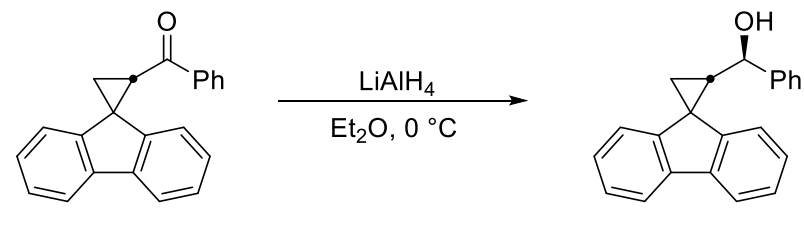

7

8d

eluent $=$ 0.5:99.5 $i$-PrOH:hexanes; flow rate $=1.0 \mathrm{~mL} / \mathrm{min}$
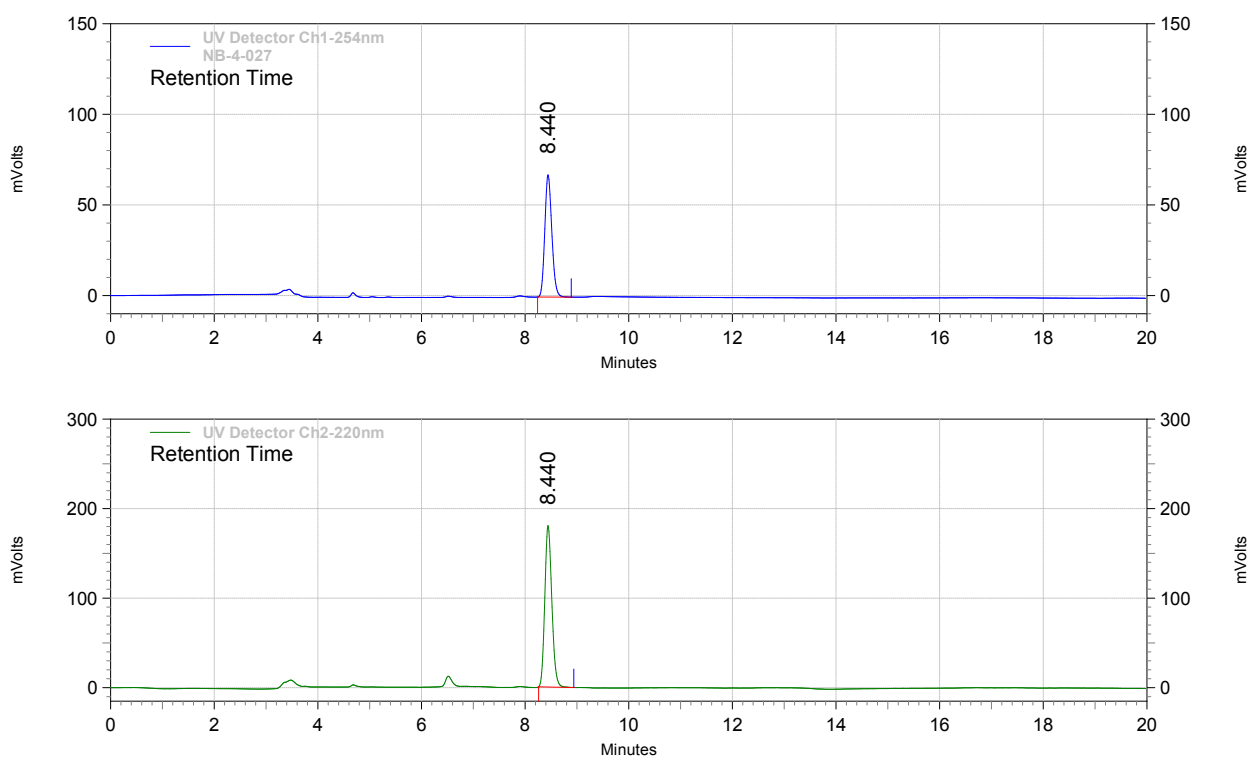

UV Detector

Ch1-254nm Results

Retention Time

8.440

Area

Area \%

Height

Height \%

100.00

67435

100.00

\begin{tabular}{|r|r|r|r|r|}
\hline Totals & 635194 & 100.00 & 67435 & 100.00 \\
\hline
\end{tabular}

UV Detector

Ch2-220nm Results

Retention Time

Area

Area \%

Heigh

Height \%

8.440

100.00

180275

100.00

\begin{tabular}{|r|r|r|r|r|}
\hline Totals & 1696182 & 100.00 & 180275 & 100.00 \\
\hline
\end{tabular}




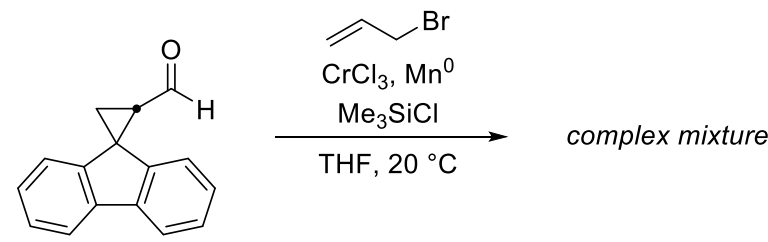

3

eluent $=0.1: 99.9 i-\mathrm{PrOH}:$ hexanes; flow rate $=1.0 \mathrm{~mL} / \mathrm{min}$
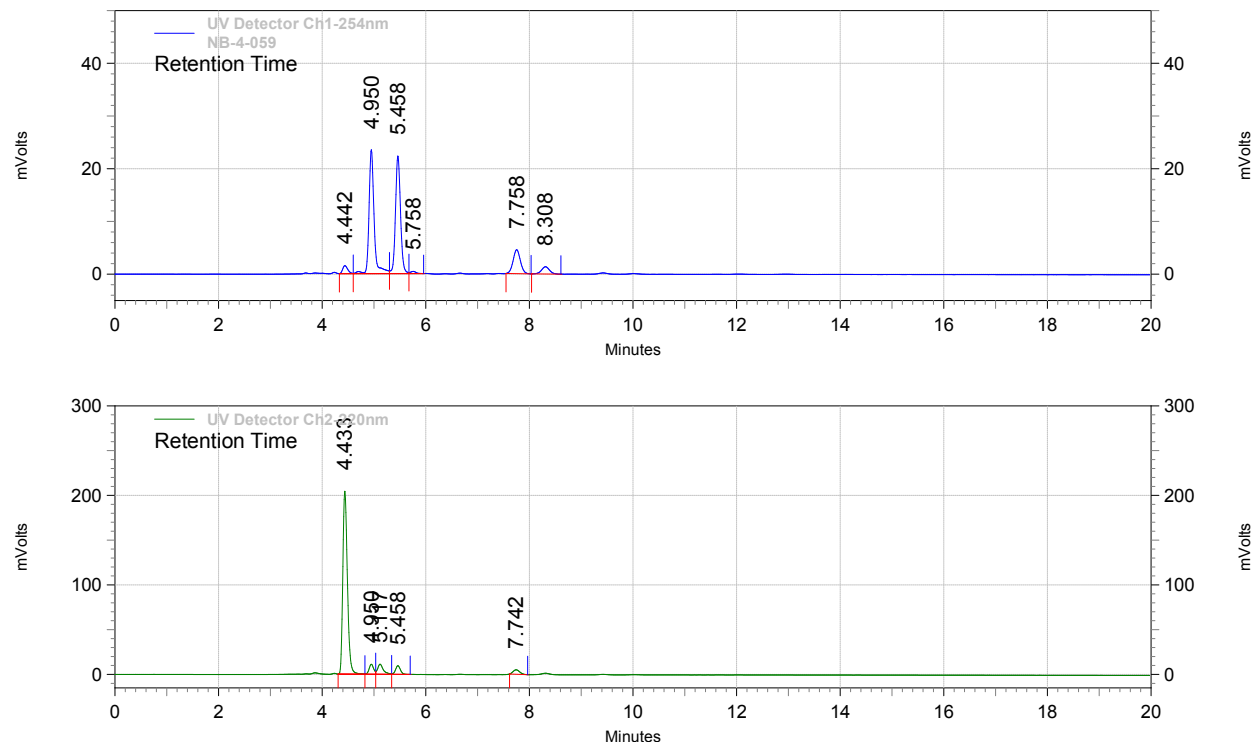

UV Detector Ch1-254nm Results Retention Time $4.442 \quad 10139$ $4.950 \quad 160054$ $5.458 \quad 150349$

$5.758 \quad 2995$

$7.758 \quad 44187$

$8.308 \quad 14805$

$14805-11.55$

Totals

\begin{tabular}{|r|r|r|r|r|}
\hline Totals & 382529 & 100.00 & 53621 & 100.00 \\
\hline
\end{tabular}

Area \%

Height Height \%

$2.65 \quad 1506 \quad 2.81$

$\begin{array}{lll}41.84 & 23508 & 43.84\end{array}$

$39.30 \quad 22290$

41.57

$0.78 \quad 413$

4528

0.77

$3.87 \quad 1376$

2.57

UV Detector

Ch2-220nm Results

Retention Time

4.433

4.950

5.117

5.458

7.742

Area

Area

68121

86849

63560

43496

100.00

Totals

1523805

100.00

241316

100.00 


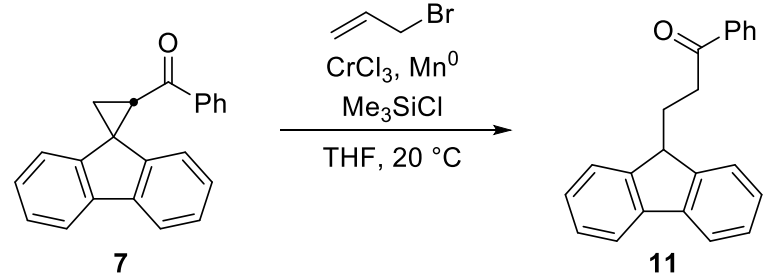

eluent $=0.5: 99.5 i-\mathrm{PrOH}:$ hexanes; flow rate $=1.0 \mathrm{~mL} / \mathrm{min}$
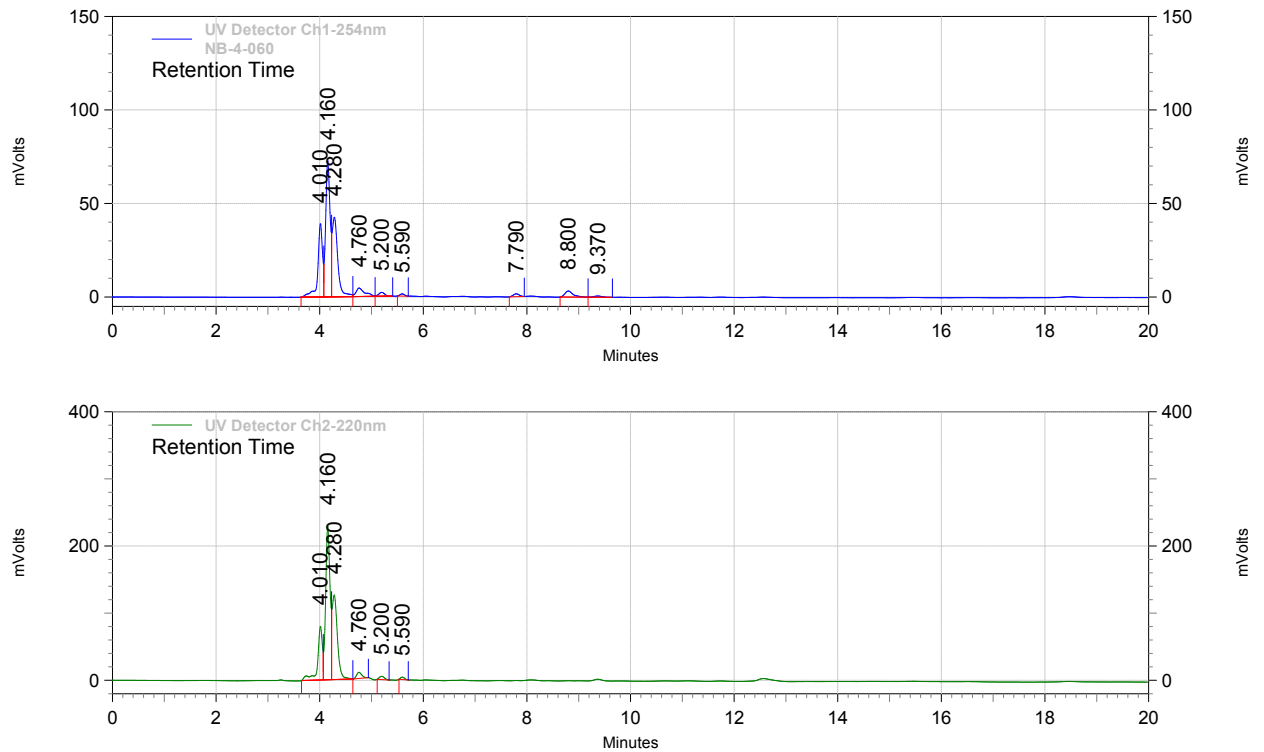

UV Detector

Ch1-254nm Results

Retention Time

4.010
4.160

4.160

4.760

5.200

5.590

7.790

8.800

9.370

Are
247901
43452
32107
53561
16335
687
11993
35271
6768

Area \%

Height

39095

72666

42513

4532

2075

1164

1504

3264

645

Height \%

43.39

25.39

4.72

0.61

1.06

3.11

16748

100.00

167458

100.00

\begin{tabular}{|r|r|r|r|r|}
\hline Totals & 1134302 & 100.00 & 167458 & 100.00 \\
\hline
\end{tabular}

UV Detector

Ch2-220nm Results

Retention Time

4.010

4.160

4.280

4.760

5.200

5.590

Are
50936
1370769
89619
63608
3132
1905

\begin{tabular}{rrrr} 
Area & Area \% & Height & Height \% \\
\hline 360 & 17.62 & 79706 & 17.64 \\
769 & 47.43 & 228753 & 50.62 \\
191 & 31.01 & 126024 & 27.89 \\
608 & 2.20 & 9161 & 2.03 \\
325 & 1.08 & 4752 & 1.05 \\
055 & 0.66 & 3523 & 0.78
\end{tabular}

Totals 

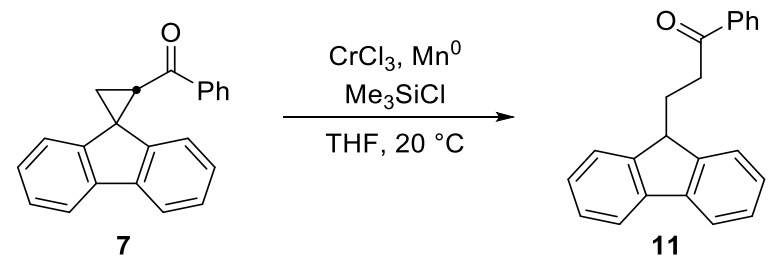

eluent $=0.5: 99.5 i-\mathrm{PrOH}:$ hexanes; flow rate $=1.0 \mathrm{~mL} / \mathrm{min}$
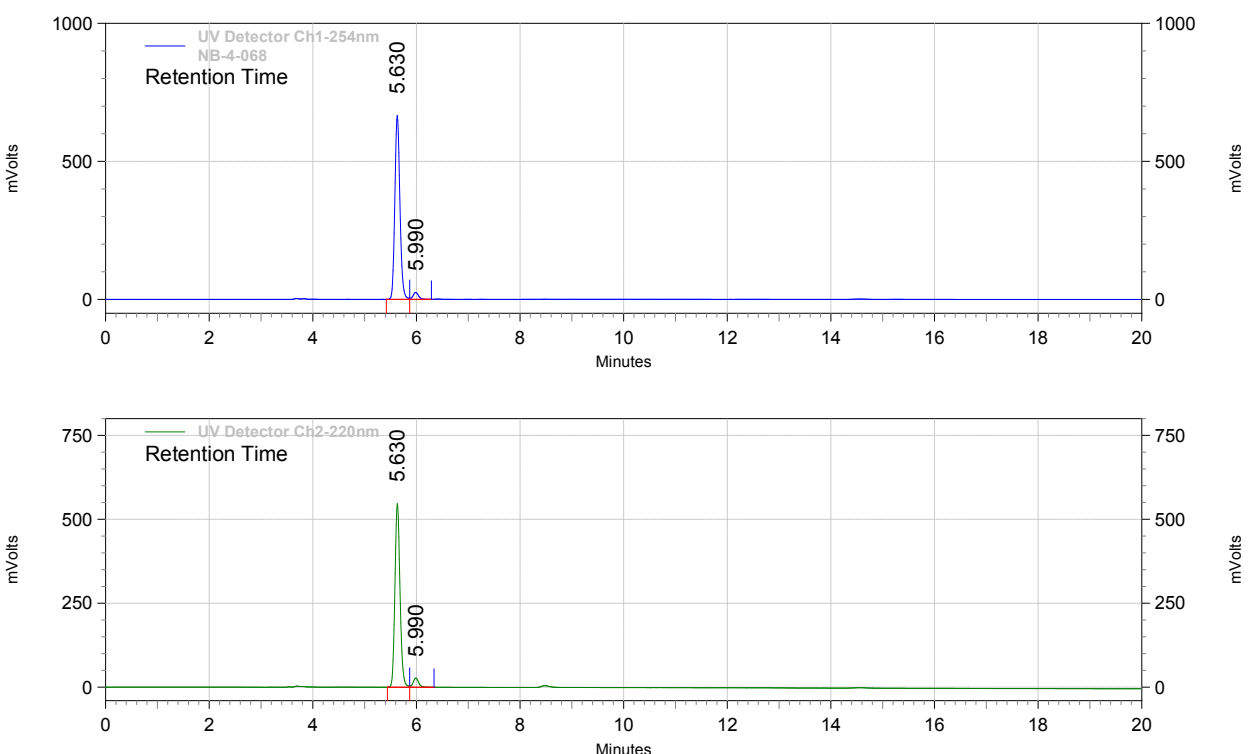

UV Detector

Ch1-254nm Results Retention Time 5.630 5.990 Area 4530714 184837

Area \%

3.92

666385 Height \%

Totals 4715551

100.00

691514

100.00

UV Detector Ch2-220nm Results Retention Time 5.630 5.990 Area 3740656 Area \% 94.90

5.10

Height Height \% 201065

27449

95.22
4.78

\begin{tabular}{|r|r|r|r|r|}
\hline Totals & 3941721 & 100.00 & 574772 & 100.00 \\
\hline
\end{tabular}




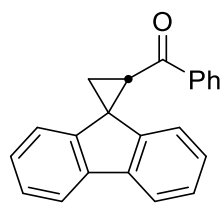

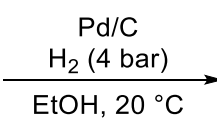

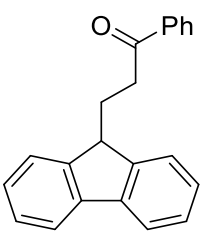

11

eluent $=0.1: 99.9 i-\mathrm{PrOH}:$ hexanes; flow rate $=1.0 \mathrm{~mL} / \mathrm{min}$
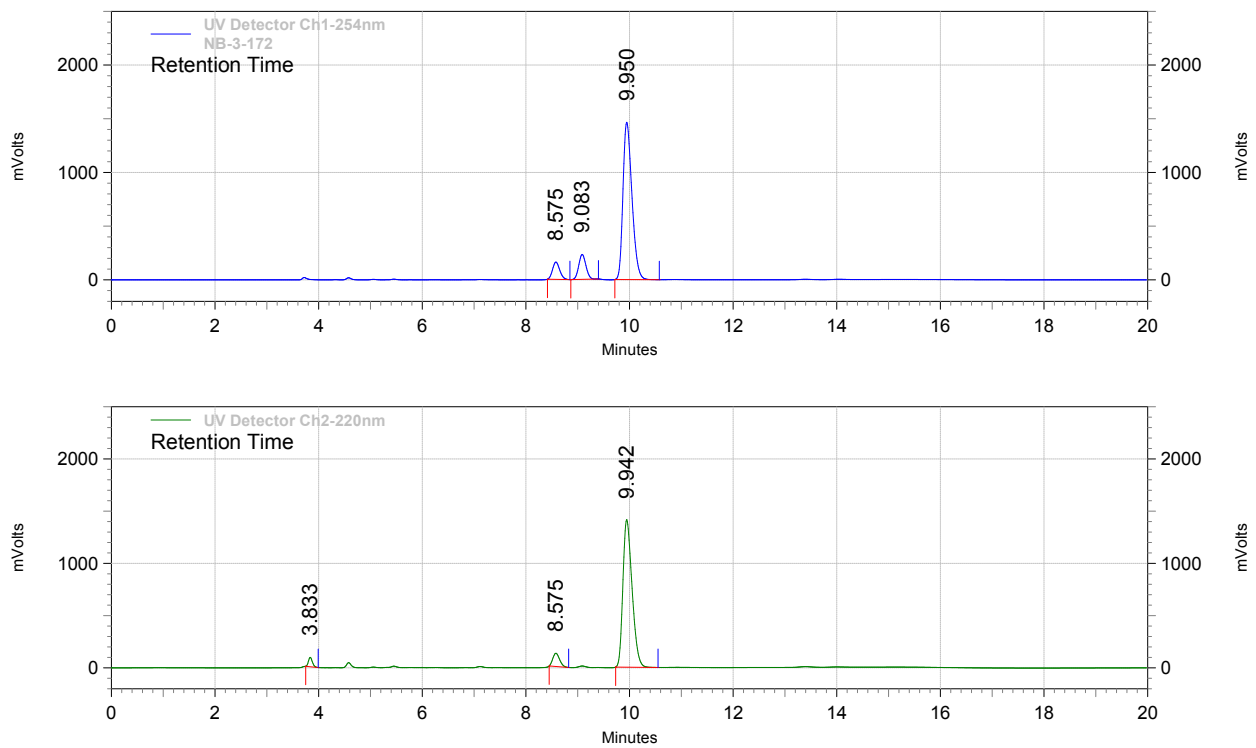

UV Detector Ch1-254nm Results Retention Time 8.575

9.950 Area 1552706 2246717 17599308 Area \% 160643 $\begin{array}{rr}10.50 & 231850 \\ 82.24 & 1464084\end{array}$ Height \%

\begin{tabular}{|r|r|r|r|r|}
\hline Totals & 21398731 & 100.00 & 1856577 & 100.00 \\
\hline
\end{tabular}

12.49

UV Detector Ch2-220nm Results

Retention Time 3.833 8.575 9.942 Area 474436 1154613 17330356 Area \%

$\begin{array}{rrr}2.50 & 88999 & 5.47 \\ 6.09 & 126102 & 7.75\end{array}$

126102
1411220 86.77

\begin{tabular}{|r|r|r|r|r|}
\hline Totals & 18959405 & 100.00 & 1626321 & 100.00 \\
\hline
\end{tabular}




\section{B. Selected LC-MS Traces}

The following pages contain HPLC traces used to analyze the unpurified reaction mixtures for the presence of ring-opened products indicating single-electron transfer.

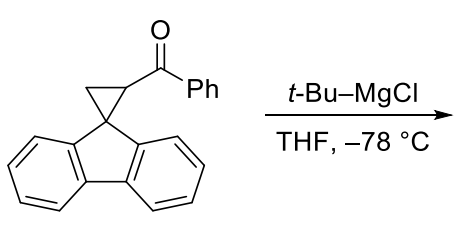

7<smiles>O=C(CCC1c2ccccc2-c2ccccc21)c1ccccc1</smiles>

11

$+\quad$ complex mixture

eluent $=50: 50$ to $0: 100 \mathrm{H}_{2} \mathrm{O}: \mathrm{MeCN}$; flow rate $=1.0 \mathrm{~mL} / \mathrm{min}$

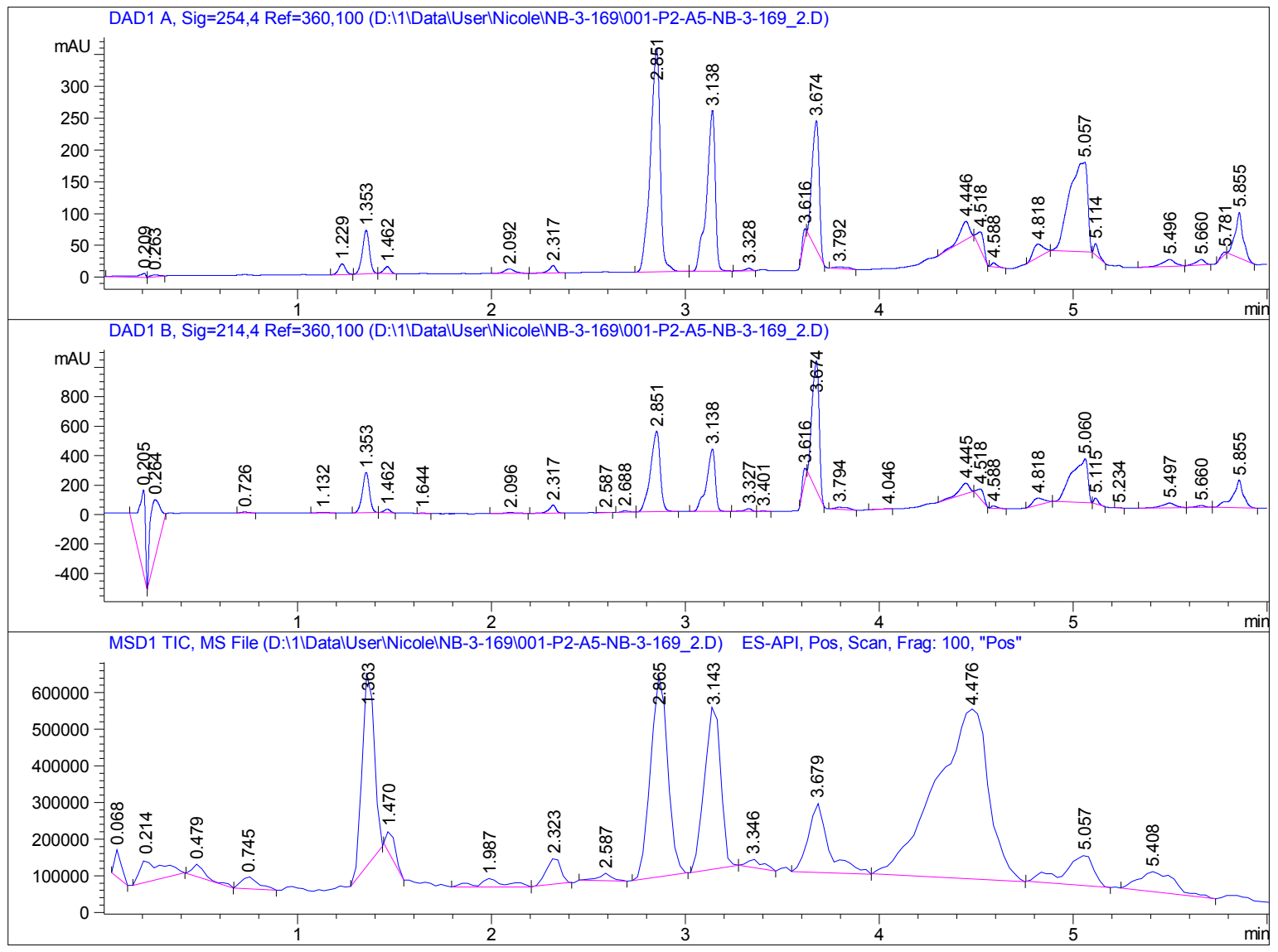


Si gnal 1: DAD1 A, Si $g=254,4$ Ref $=360,100$

\begin{tabular}{|c|c|c|c|c|c|c|}
\hline $\begin{array}{l}\text { eak } \\
\#\end{array}$ & $\begin{array}{l}\text { Ret Ti me } \\
{[\text { m n }]}\end{array}$ & Type & $\begin{array}{l}W d t h \\
{[m n]}\end{array}$ & $\begin{array}{c}\text { Ar ea } \\
{\left[\mathrm{mAU}^{*} \mathrm{~s}\right]}\end{array}$ & $\begin{array}{l}\text { Hei ght } \\
\text { [ } \mathrm{mAU}]\end{array}$ & $\begin{array}{c}\text { Ar ea } \\
\%\end{array}$ \\
\hline & & & & & & \\
\hline 1 & 0.209 & BB & 0.0415 & 21. 39212 & 6. 83146 & 0.4881 \\
\hline 2 & 0.263 & BB & 0.0416 & 9. 49946 & 3. 07244 & 0.2168 \\
\hline 3 & 1. 229 & BB & 0.0372 & 40.34 & 16. 69099 & 0.9206 \\
\hline 4 & 1. 353 & BB & 0.0382 & 171.3 & 68.5 & 3. 9087 \\
\hline 5 & & & & 24.5 & 10. & .5591 \\
\hline 6 & 2. 092 & BB & & 27. 0 & 7. & 0.6162 \\
\hline 7 & 317 & BB & & 30.3 & 11.9 & 0.6929 \\
\hline 8 & 2. 851 & BB & 0547 & 1225. 58081 & 351.01 & 27. 9643 \\
\hline 9 & 3. 138 & & 477 & 783. 80096 & 252.5 & 17. 8841 \\
\hline 10 & 328 & B & & 8. 6 & 3. & 0.1980 \\
\hline 11 & 3. 616 & BB & & 24.5 & 15. 0 & 0.5592 \\
\hline 12 & 3. 674 & 3B & & 507.0 & 202. 4 & 11.5693 \\
\hline 13 & 3. 792 & BB & 0.0669 & 13. 43054 & 2. 68 & 0.3064 \\
\hline 12 & 4. 446 & BB & 545 & 103. 5 & 28. 7 & 2. 3632 \\
\hline 15 & & 3B & 397 & 65.2 & 26. 0 & 1. 4892 \\
\hline 16 & & 3B & & 13. 26 & 6.5 & 0.3026 \\
\hline 17 & 4. 818 & BB & 590 & 77. 29041 & 20.7 & 1. 7635 \\
\hline 18 & & BB & 330 & 916.9 & 140.8 & 20. 9229 \\
\hline 19 & & $B$ & 48 & 28. 84156 & 17. 86 & 0.6581 \\
\hline 20 & & B & & 49. 85591 & 10. 91074 & 1. 1376 \\
\hline 21 & 5. 660 & BB & 0.0475 & 27. 10433 & 8. 43671 & 0.6184 \\
\hline 22 & 5. 781 & BB & 408 & 9. 14785 & 3. 47005 & 0.2087 \\
\hline 23 & 5. 855 & BB & 0.0389 & 203. 88135 & 71. 24210 & 4. 6520 \\
\hline
\end{tabular}

Tot al s :

4382. 660701287.37598

Si gnal 2: DAD1 B, Si g=214, 4 Ref $=360,100$

\begin{tabular}{|c|c|c|c|c|c|c|}
\hline $\begin{array}{c}\text { eak } \\
\#\end{array}$ & $\begin{array}{c}\text { Ret Ti me } \\
{[\mathrm{min}]}\end{array}$ & Type & $\begin{array}{l}\text { Wdth } \\
{[\mathrm{m} \mathrm{n}]}\end{array}$ & $\begin{array}{c}\text { Ar ea } \\
\left.\text { [ } \mathrm{mAU}^{*} \mathrm{~s}\right]\end{array}$ & $\begin{array}{l}\text { Hei ght } \\
\text { [ } \mathrm{mAU}]\end{array}$ & $\begin{array}{c}\text { Area } \\
\%\end{array}$ \\
\hline & & & & & & \\
\hline 1 & 0.205 & $\mathrm{BB}$ & 0.0345 & 1413. 58179 & 568. 34723 & 11.0413 \\
\hline 2 & 0.264 & BB & & 1415. 52 & 394. & 11. 0565 \\
\hline 3 & 0.726 & BB & 0310 & 12. 57 & $5 . \varsigma$ & 0.0982 \\
\hline 4 & & B & 0486 & 13. 29 & 3. 51 & 0.1039 \\
\hline 5 & & & 85 & $692.7 C$ & 274 & 5. 4106 \\
\hline 6 & 1. 462 & $B$ & 0.0 & 54.2 & 24. & 0.4236 \\
\hline 7 & 1. 644 & $B$ & 0.0 & 5.7 & & 0.0447 \\
\hline 8 & 2. 096 & & $0 . \mathrm{C}$ & 29.5 & & 312 \\
\hline 9 & & & 0.0 & 132. 3 & 54. & .0341 \\
\hline 10 & & & 38 & & & 0.0 \\
\hline 11 & 2. 688 & & 0.0406 & 25.7 & 52 & 0.2010 \\
\hline 12 & 2. 851 & & 0.0546 & 1898. 36 & 545.6 & 14. 8279 \\
\hline 13 & 3. 138 & & 0.0478 & 1317. 73 & 423. 1 & 10. 2926 \\
\hline 14 & 3. 327 & & 0.0 & 45.2 & 18. & 0.3 \\
\hline 15 & & & & & & 0.0437 \\
\hline 16 & 3. 616 & & 0.0235 & 118. 70556 & 79. 28957 & 0.9272 \\
\hline 17 & 3. 674 & $3 B$ & 0.0421 & 2183. 88281 & 876. 15161 & 17. 0580 \\
\hline 18 & 3. 794 & BB & 0.0646 & 66. 44868 & 13. 70462 & 0.5190 \\
\hline
\end{tabular}




\begin{tabular}{|c|c|c|c|c|c|}
\hline $\begin{array}{c}\text { Peak } \\
\text { \# }\end{array}$ & $\begin{array}{l}\text { Ret Ti me Type } \\
{[\mathrm{m} \mathrm{n}]}\end{array}$ & $\begin{array}{l}W d t h \\
{[m n]}\end{array}$ & $\begin{array}{c}\text { Area } \\
{\left[\mathrm{mAU}^{*} \mathrm{~s}\right]}\end{array}$ & $\begin{array}{l}\text { Hei ght } \\
\text { [ } \mathrm{mAU}]\end{array}$ & $\begin{array}{c}\text { Ar ea } \\
\%\end{array}$ \\
\hline---1 & - & | - - - - - - & - - - - - - - - - - - & | - - - - - - - - - - & |- - - - - - - \\
\hline 19 & 4. $046 \mathrm{BB}$ & 0.1097 & 13. 08859 & 1. 41120 & 0.1022 \\
\hline 20 & 4. $445 \mathrm{BB}$ & 0.0550 & 255. 51256 & 69. 36913 & 1. 9958 \\
\hline 21 & 4. $518 \mathrm{BB}$ & 0.0422 & 158. 00124 & 62. 07660 & 1. 2341 \\
\hline 22 & 4. $588 \mathrm{BB}$ & 0.0313 & 31.60922 & 15. 48234 & 0.2469 \\
\hline 23 & 4. $818 \mathrm{BB}$ & 0.0615 & 180. 84517 & 45. 81736 & 1. 4126 \\
\hline 24 & 5. $060 \mathrm{BB}$ & 0.0758 & 1748. 13123 & 298. 60910 & 13. 6544 \\
\hline 25 & 5. $115 \mathrm{BB}$ & 0.0236 & 60. 63888 & 40. 12679 & 0.4736 \\
\hline 26 & 5. $234 \mathrm{BB}$ & 0.0248 & 5. 93648 & 3. 90021 & 0.0464 \\
\hline 27 & 5. $497 \mathrm{BB}$ & 0.0660 & 151. 78252 & 31. 92945 & 1. 1856 \\
\hline 28 & 5. $660 \mathrm{BB}$ & 0.0474 & 38. 11666 & 11.90031 & 0. 2977 \\
\hline 29 & 5. $855 \mathrm{BB}$ & 0.0506 & 721.06189 & 188. 71233 & 5. 6321 \\
\hline Tot & & & 1. $28027 \mathrm{e} 4$ & 4075. 08 & \\
\hline
\end{tabular}

Si gnal 3: NSD1 TIC, MS File

\begin{tabular}{|c|c|c|c|c|c|}
\hline $\begin{array}{c}\text { Peak } \\
\text { \# }\end{array}$ & $\begin{array}{l}\text { Ret Ti me Type } \\
\text { [ } \mathrm{m} \text { n] }\end{array}$ & $\begin{array}{l}W d t h \\
{[m n]}\end{array}$ & Ar ea & Hei ght & $\begin{array}{c}\text { Ar ea } \\
\%\end{array}$ \\
\hline & & & & & \\
\hline 1 & $0.068 \mathrm{BB}$ & 0.0401 & 1. $79584 \mathrm{e} 5$ & 7. $46737 \mathrm{e} 4$ & 0.8152 \\
\hline 2 & $0.214 \mathrm{BB}$ & 0.1324 & 4. $94692 \mathrm{e} 5$ & 6. $22778 \mathrm{e} 4$ & 2. 2455 \\
\hline 3 & $0.479 \mathrm{BB}$ & 0.0772 & 1. $58345 \mathrm{e} 5$ & 3. $41965 \mathrm{e} 4$ & 0.7188 \\
\hline 4 & $0.745 \mathrm{BB}$ & 0.0975 & 2. $04072 \mathrm{e} 5$ & 3. $27023 \mathrm{e} 4$ & 0.9263 \\
\hline 5 & 1. $363 \mathrm{BB}$ & 0.0703 & 2. $27602 \mathrm{e} 6$ & 5. $39371 \mathrm{e} 5$ & 10. 3312 \\
\hline 6 & 1. $470 \mathrm{BB}$ & 0.0567 & 1. $97104 \mathrm{e} 5$ & 5. $79790 \mathrm{e} 4$ & 0.8947 \\
\hline 7 & 1. $987 \mathrm{BB}$ & 0.1384 & 2. $20735 \mathrm{e} 5$ & 2. $26344 \mathrm{e} 4$ & 1. 0020 \\
\hline 8 & 2. $323 \mathrm{BB}$ & 0.0847 & 3. $70257 \mathrm{e} 5$ & 7. $19895 \mathrm{e} 4$ & 1. 6807 \\
\hline 9 & 2. $587 \mathrm{BB}$ & 0.0769 & 9. $27494 \mathrm{e} 4$ & 2. $00914 \mathrm{e} 4$ & 0.4210 \\
\hline 10 & 2. $865 \mathrm{BB}$ & 0.0962 & 3. $37781 \mathrm{e} 6$ & 5. $50518 \mathrm{e} 5$ & 15. 3324 \\
\hline 11 & 3. $143 \mathrm{BB}$ & 0.0920 & 2. $59137 \mathrm{e} 6$ & 4. $49155 \mathrm{e} 5$ & 11. 7626 \\
\hline 12 & 3. $346 \mathrm{BB}$ & 0.0834 & 1. $31896 e 5$ & 2. $29748 \mathrm{e} 4$ & 0.5987 \\
\hline 13 & 3. $679 \mathrm{BB}$ & 0.1098 & 1. $37680 \mathrm{e} 6$ & 1. $88711 \mathrm{e} 5$ & 6. 2495 \\
\hline 14 & 4. $476 \mathrm{BB}$ & 0.2616 & 8. $75507 \mathrm{e} 6$ & 4. $63276 \mathrm{e} 5$ & 39. 7405 \\
\hline 15 & 5. $057 \mathrm{BB}$ & 0.1578 & 8. $90946 \mathrm{e} 5$ & 8. $32049 \mathrm{e} 4$ & 4. 0441 \\
\hline 16 & 5. $408 \mathrm{BB}$ & 0.1814 & 7. $13113 \mathrm{e} 5$ & 5. $38192 \mathrm{e} 4$ & 3. 2369 \\
\hline \multicolumn{2}{|c|}{ Tot al s } & & 2. $20306 \mathrm{e} 7$ & 2. $72757 \mathrm{e} 6$ & \\
\hline
\end{tabular}




\section{Selected ${ }^{1} \mathrm{H}$ NMR, ${ }^{13} \mathrm{C}$ NMR, and ${ }^{19}$ F NMR Spectra}

The following pages contain ${ }^{1} \mathrm{H}$ NMR, ${ }^{13} \mathrm{C}$ NMR, and ${ }^{19} \mathrm{~F}$ NMR spectra for all new compounds. Unpurified ${ }^{1} \mathrm{H}$ NMR or ${ }^{13} \mathrm{C}$ NMR are also included if they were used to calculate diastereomeric ratios or if they were used to determine if ring-opening had occurred. There are no resonances with chemical shifts beyond the shown area of the spectra. 

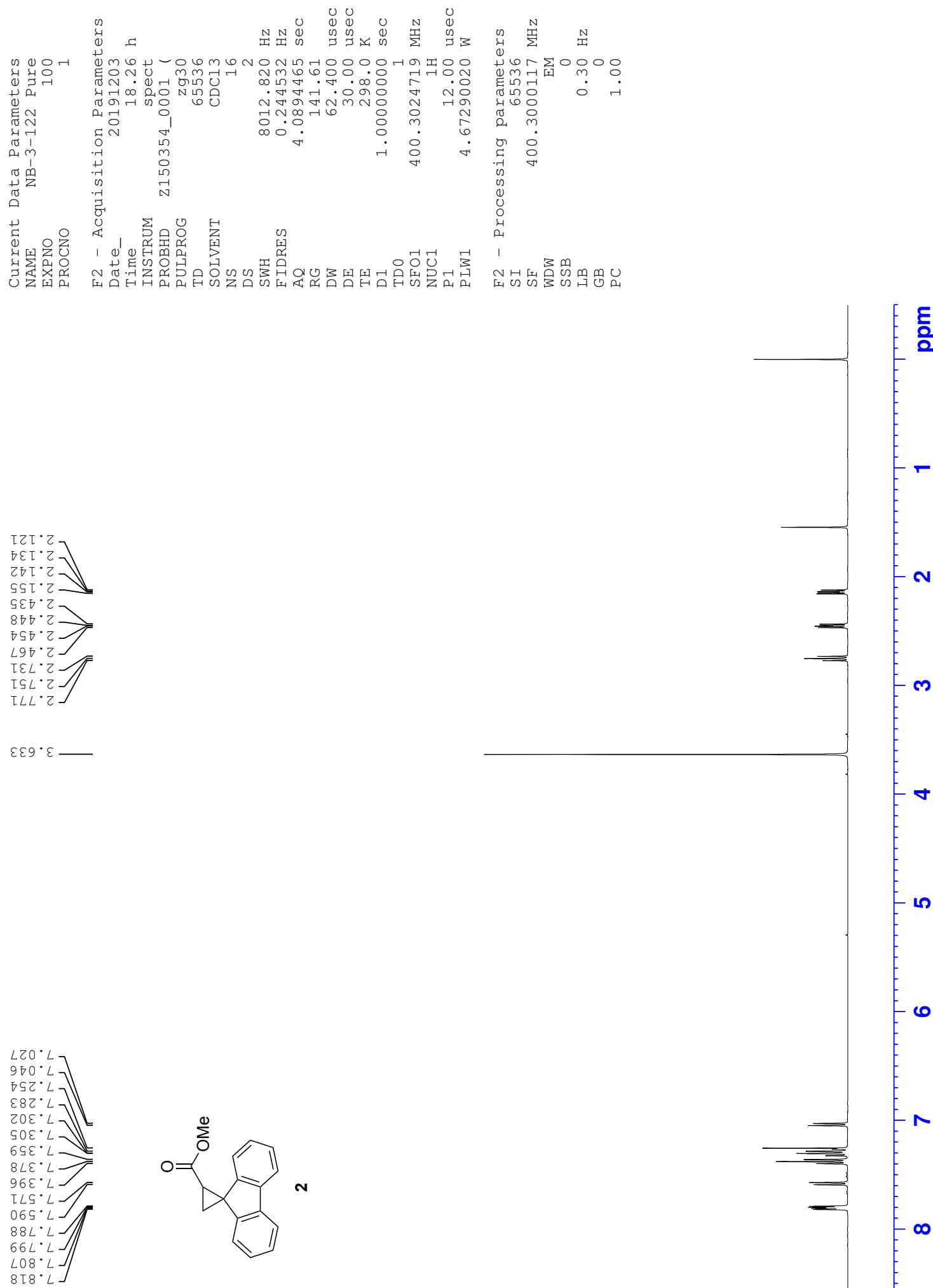

틍
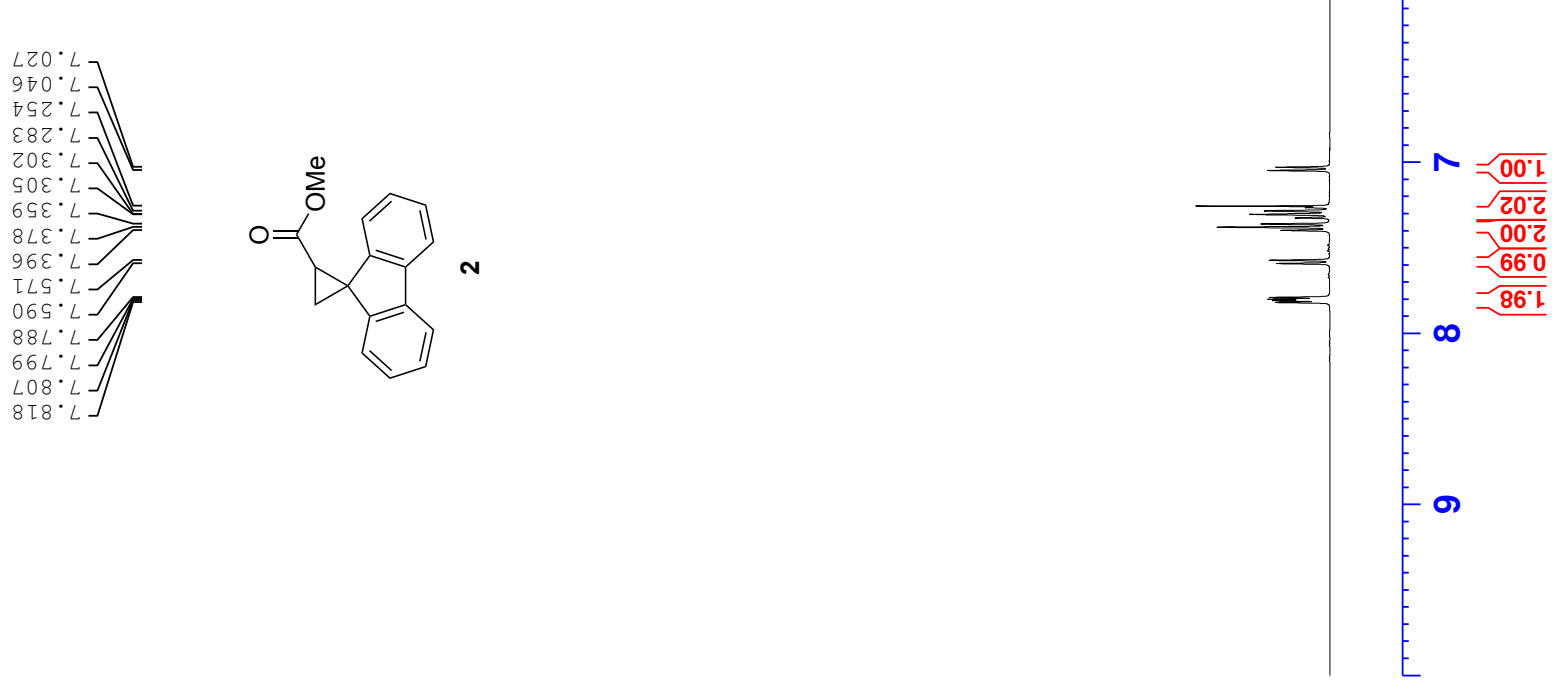

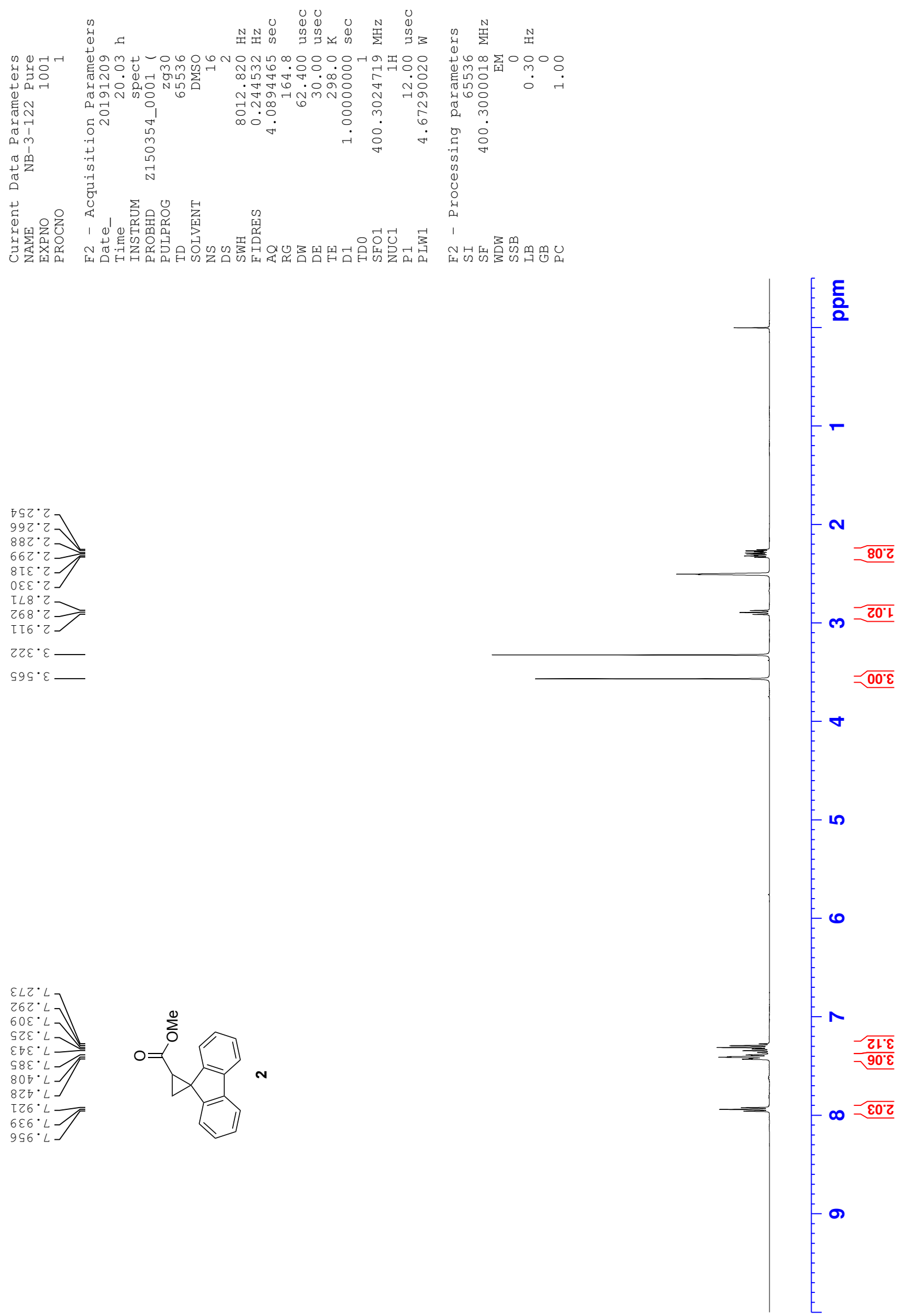

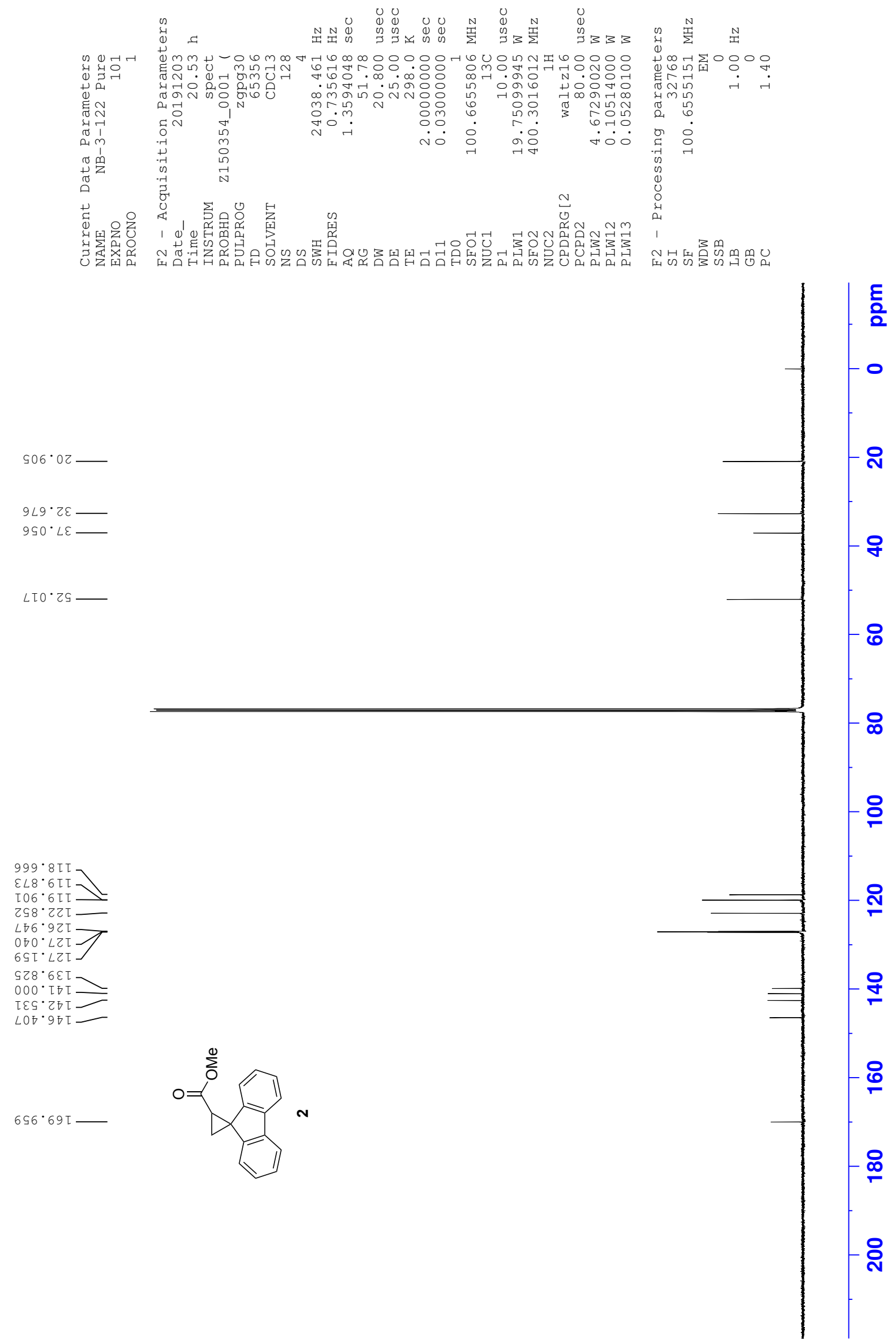

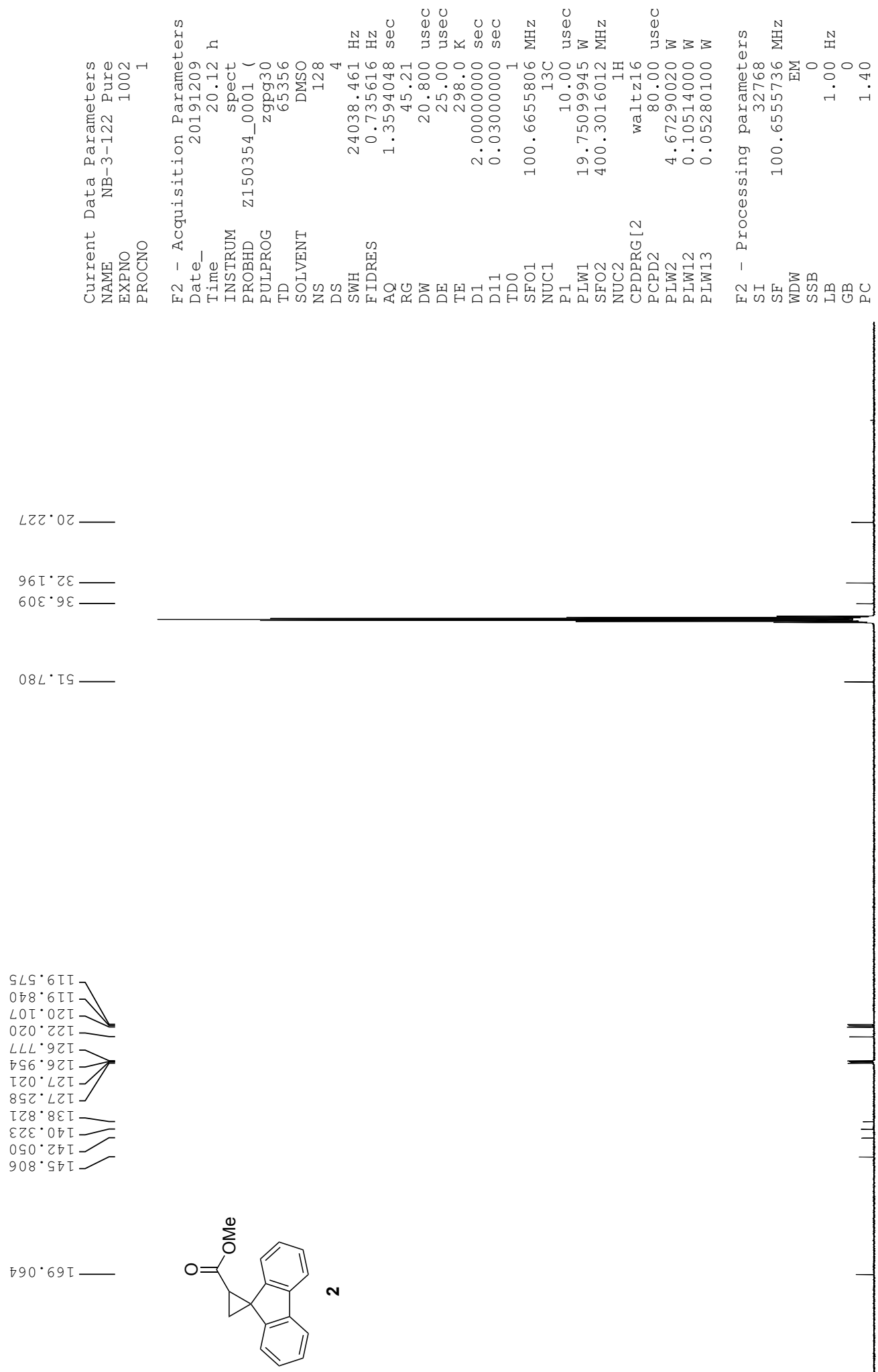

ลิ

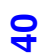

8

$\infty$

움

ิㅗำ

g

$\stackrel{\circ}{\circ}$

$\stackrel{\infty}{\stackrel{\infty}{0}}$

ㅇํ 

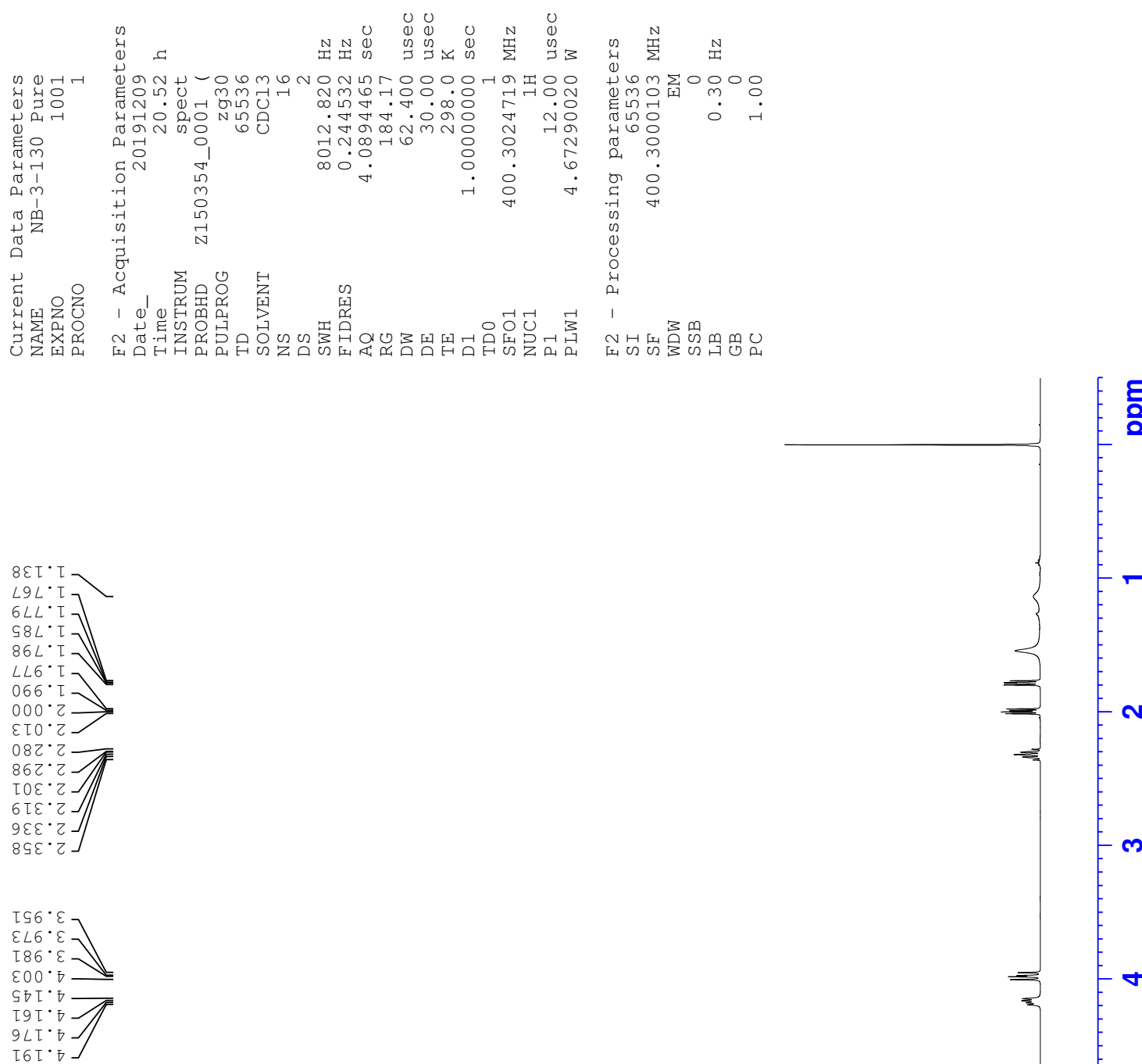

틍

$m$

$\frac{207}{90.1}$
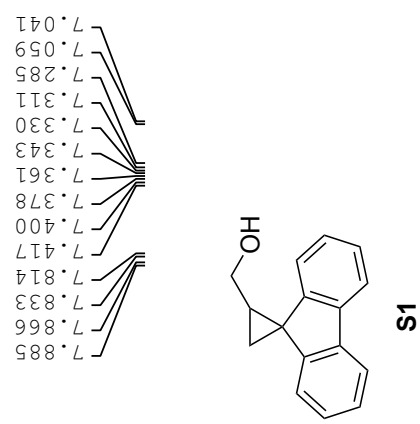

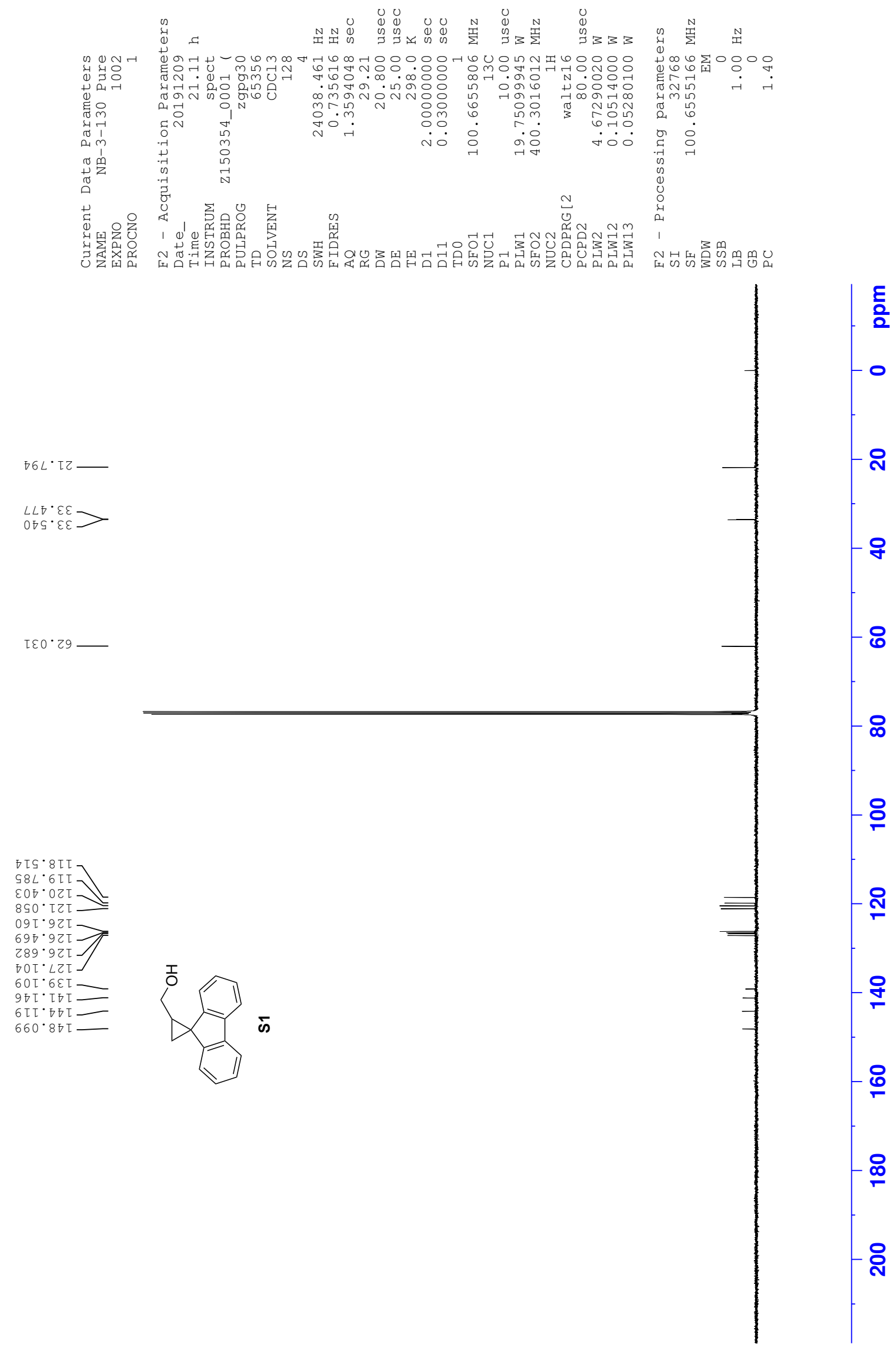

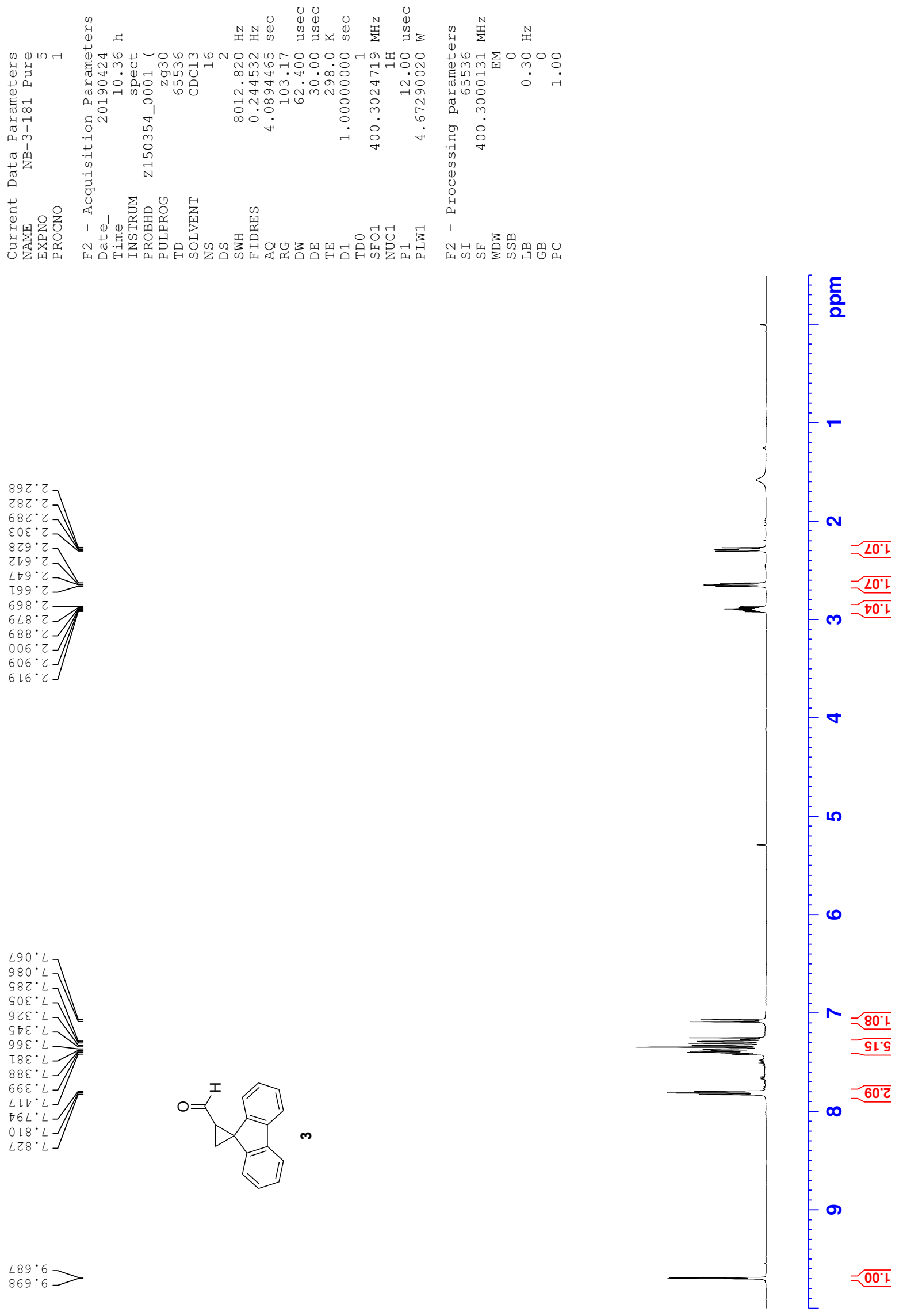

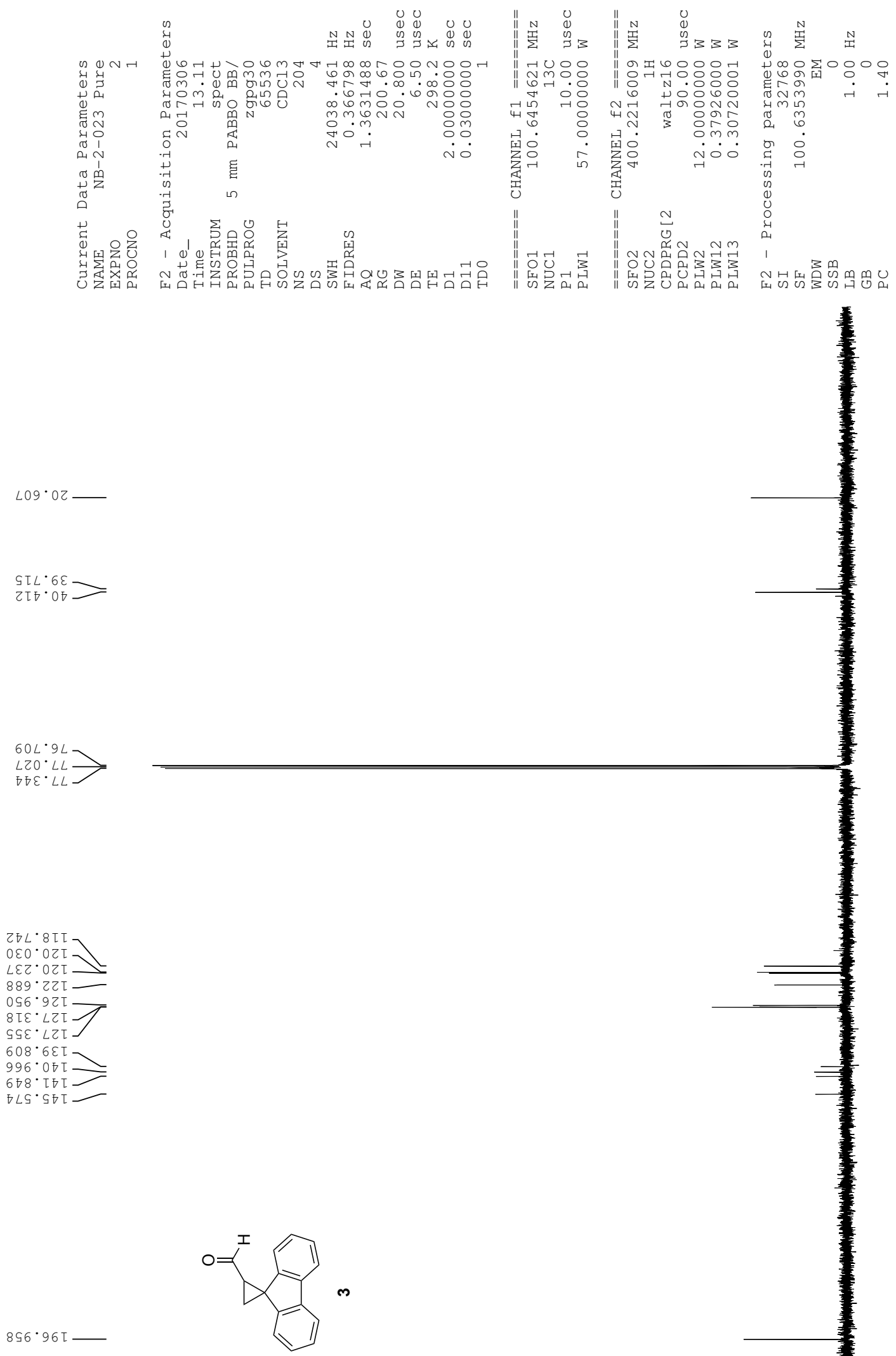

튼 $-0$

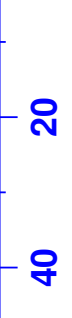



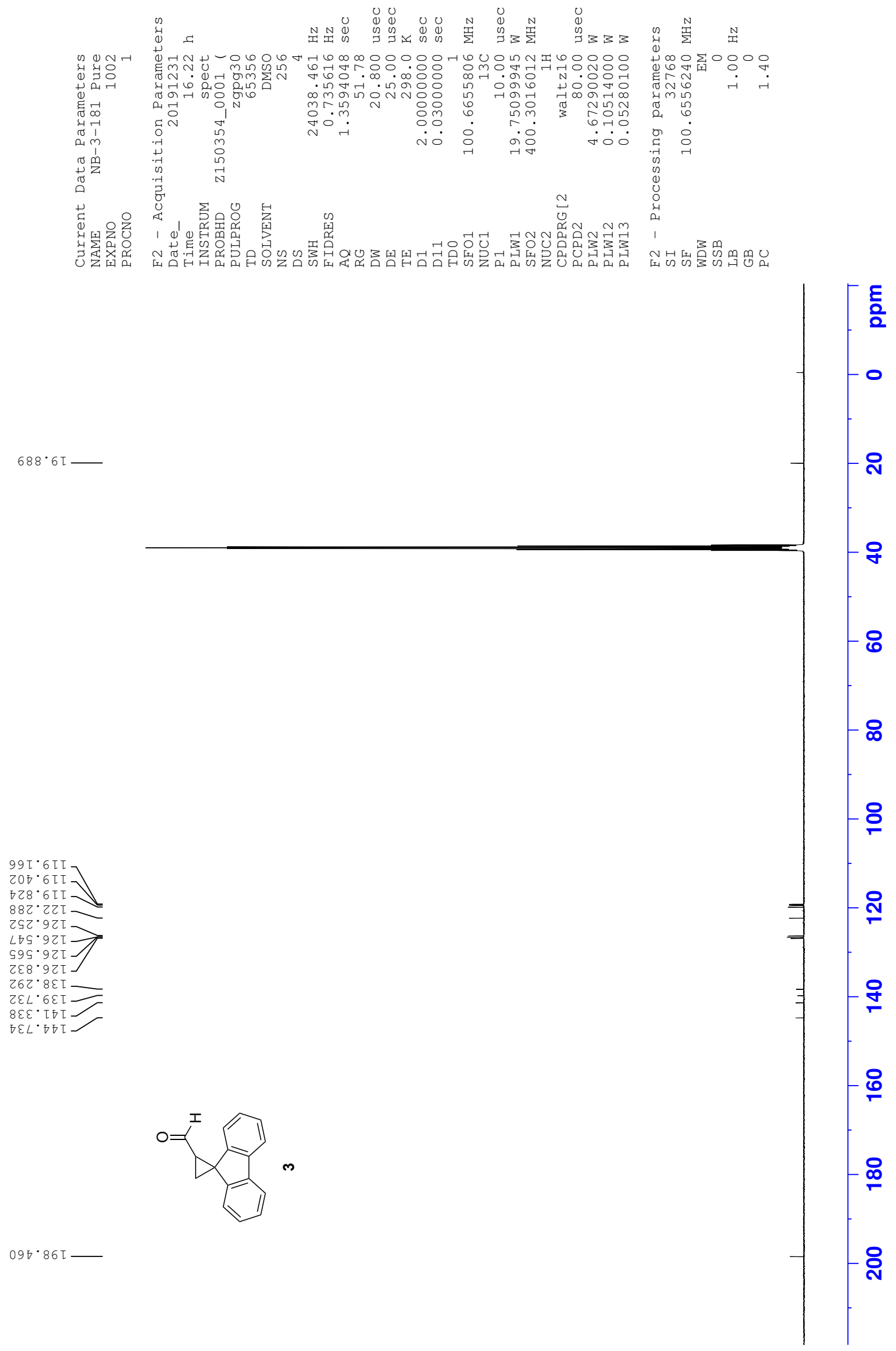

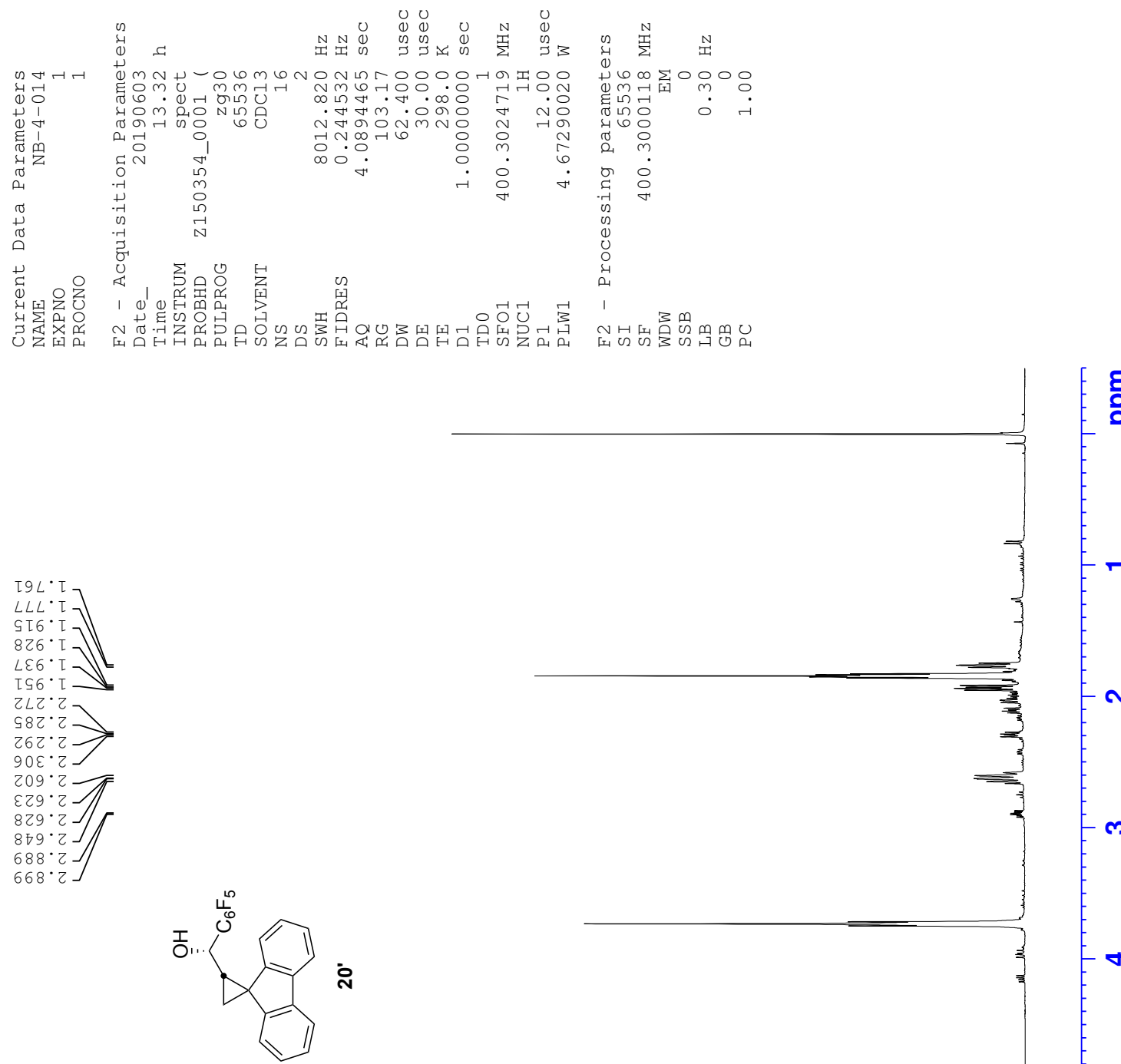

틍

2
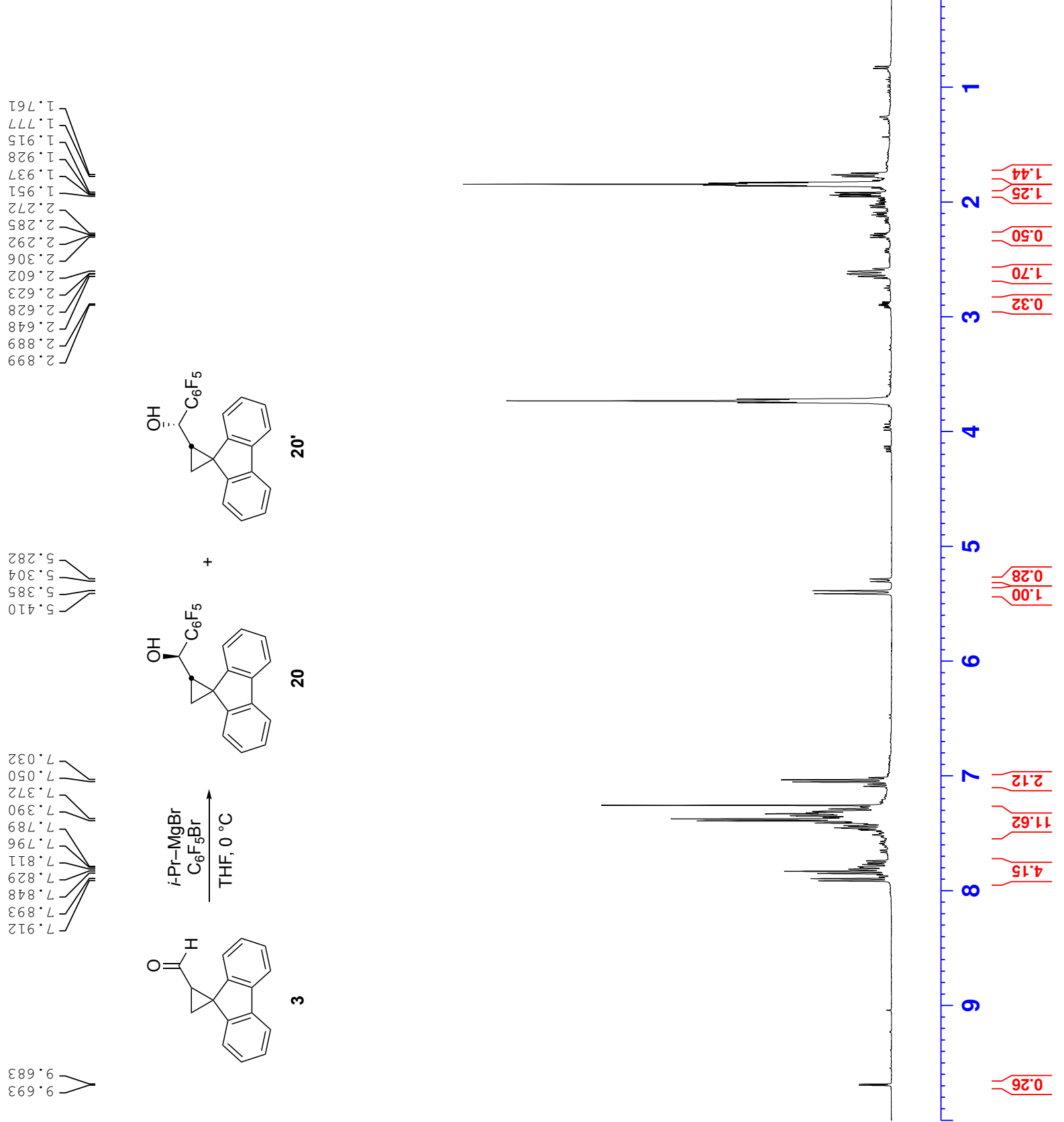

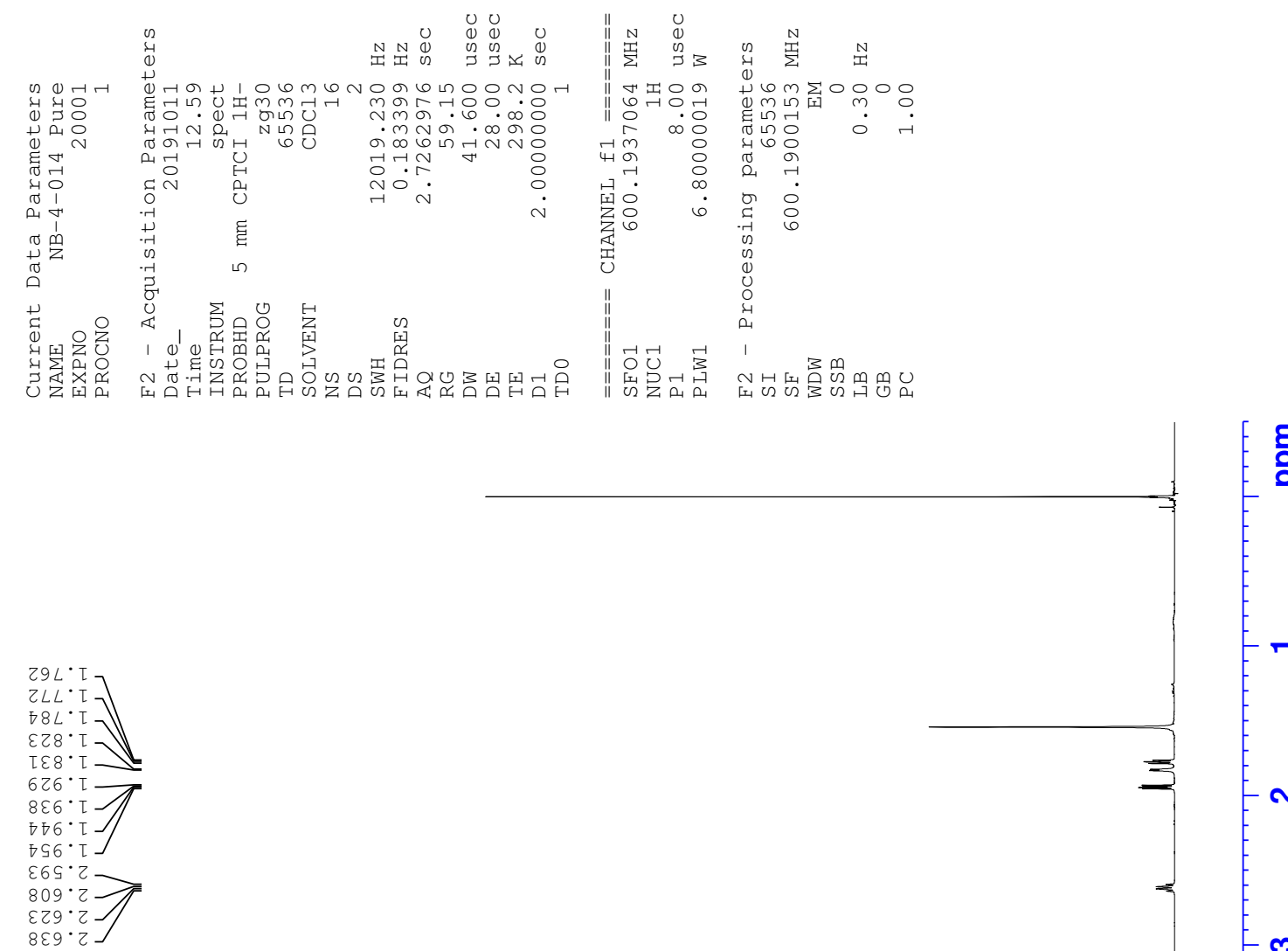

틍

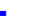



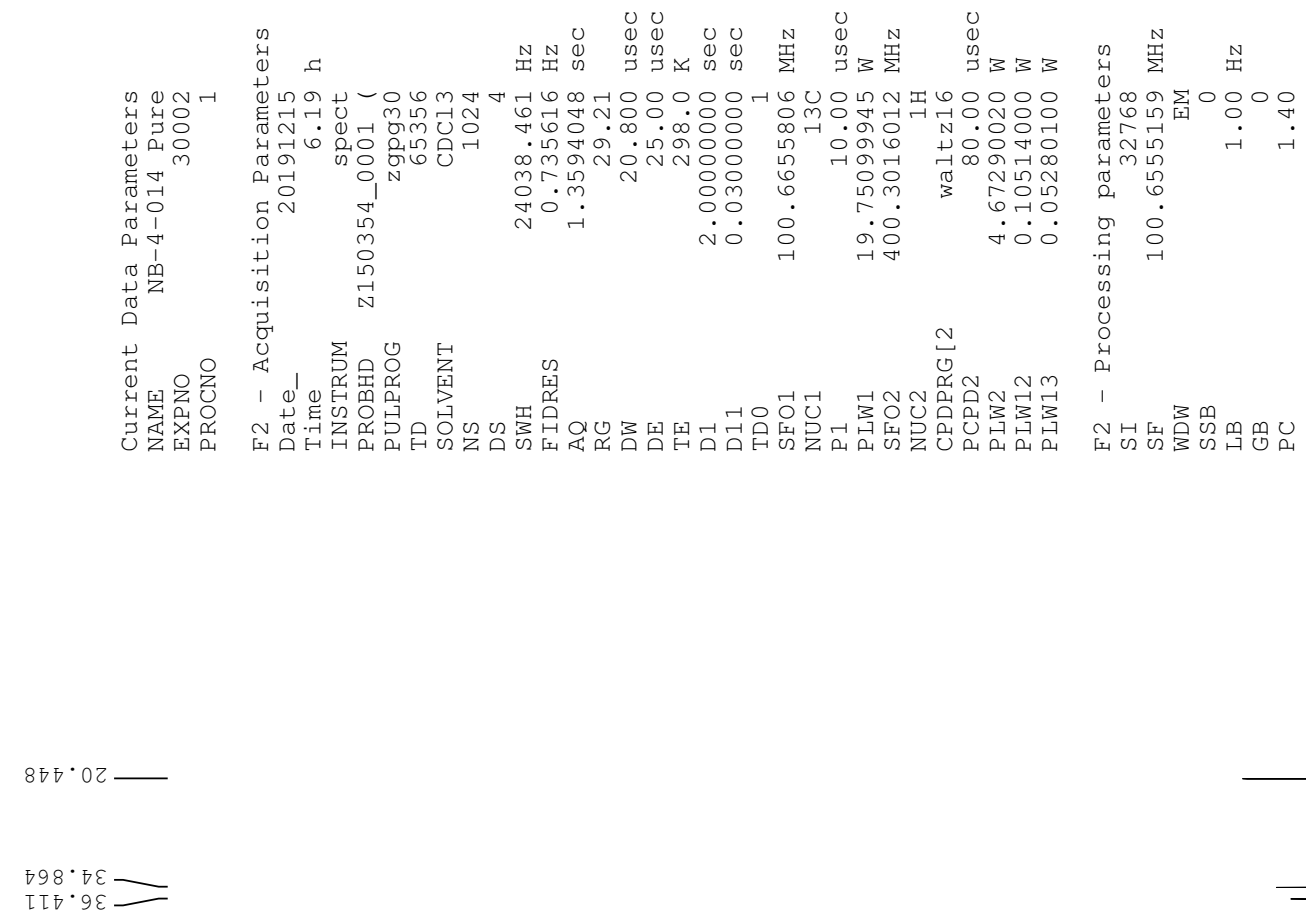

$009 \cdot 59$
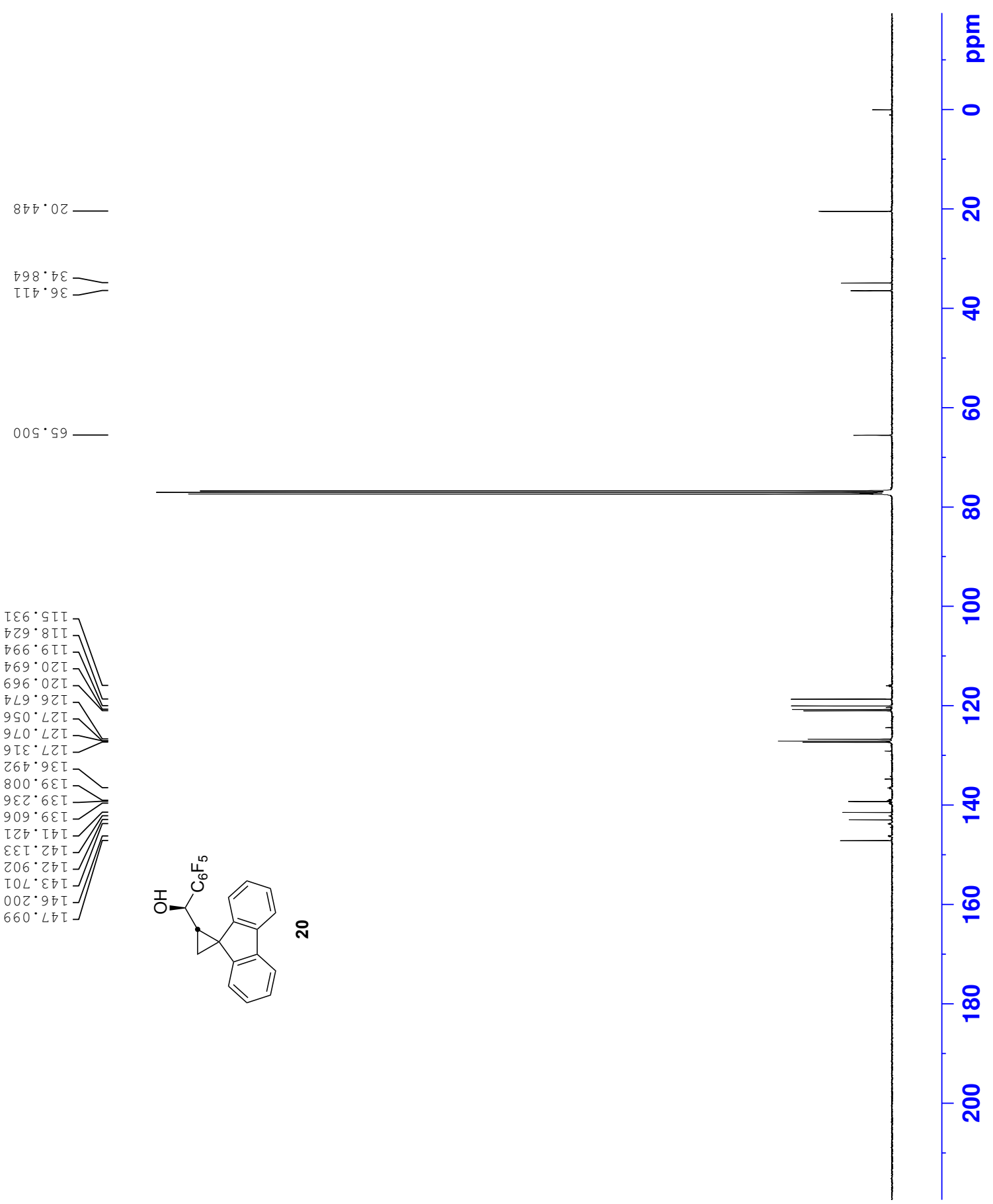

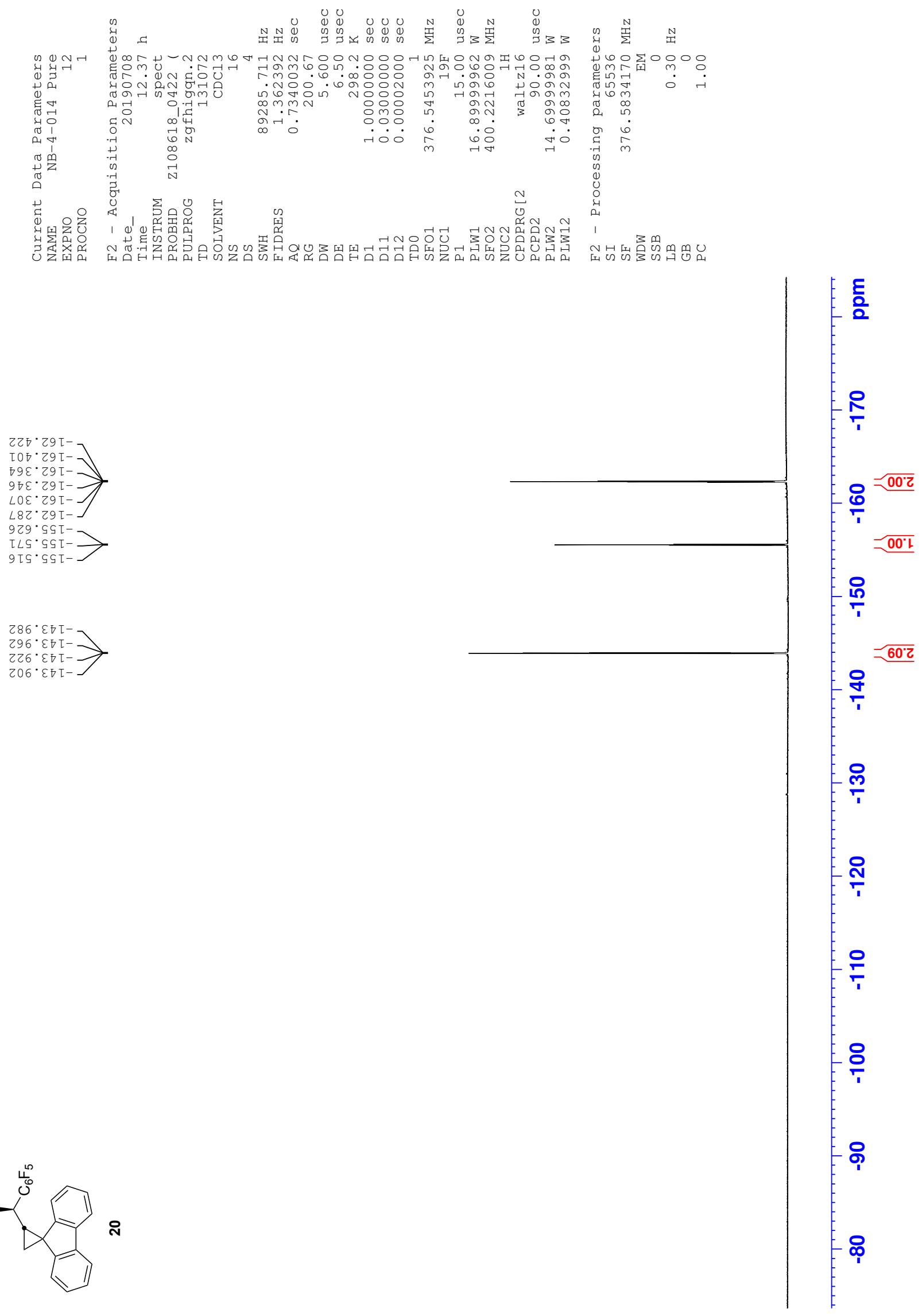

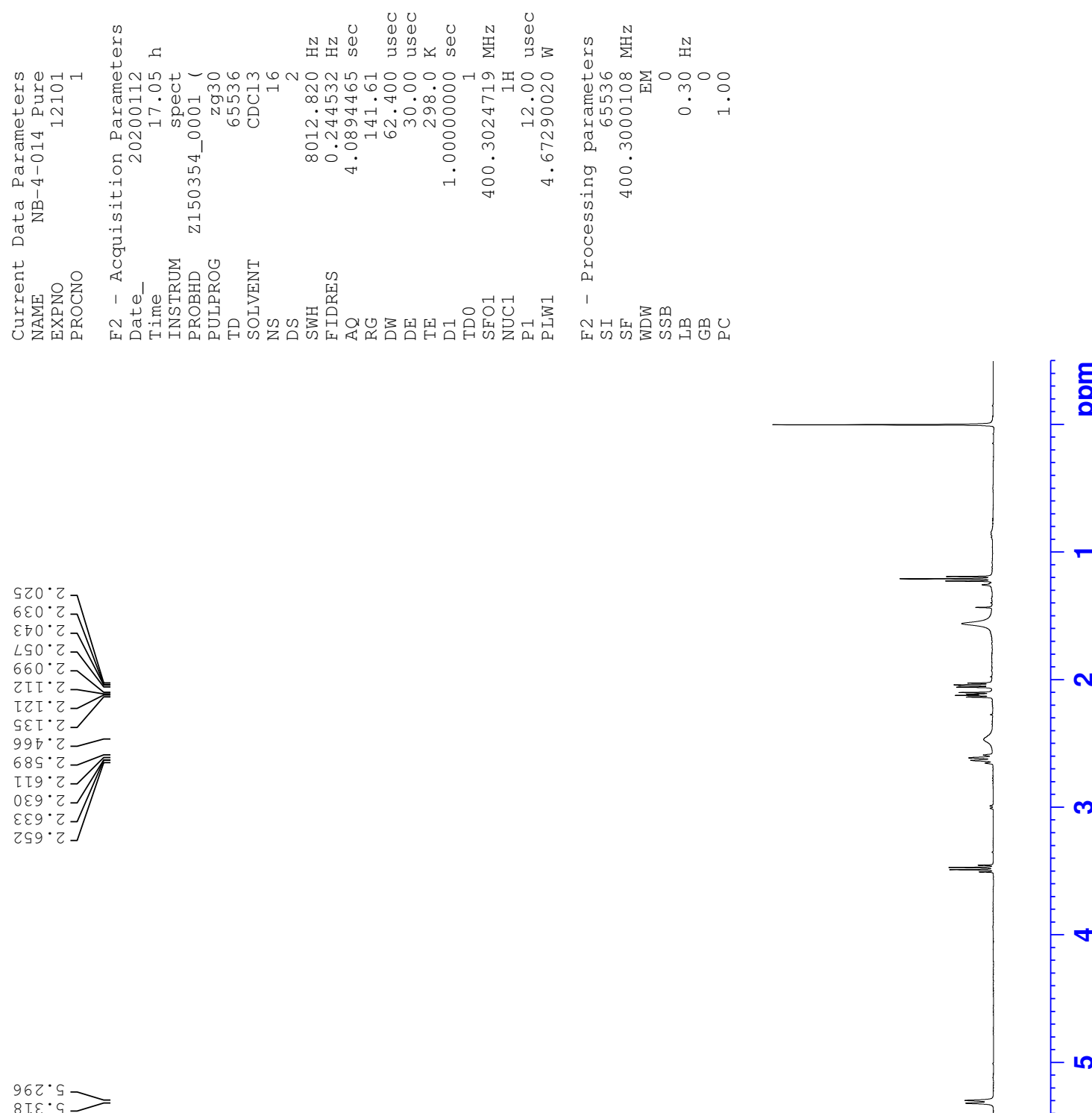

틍

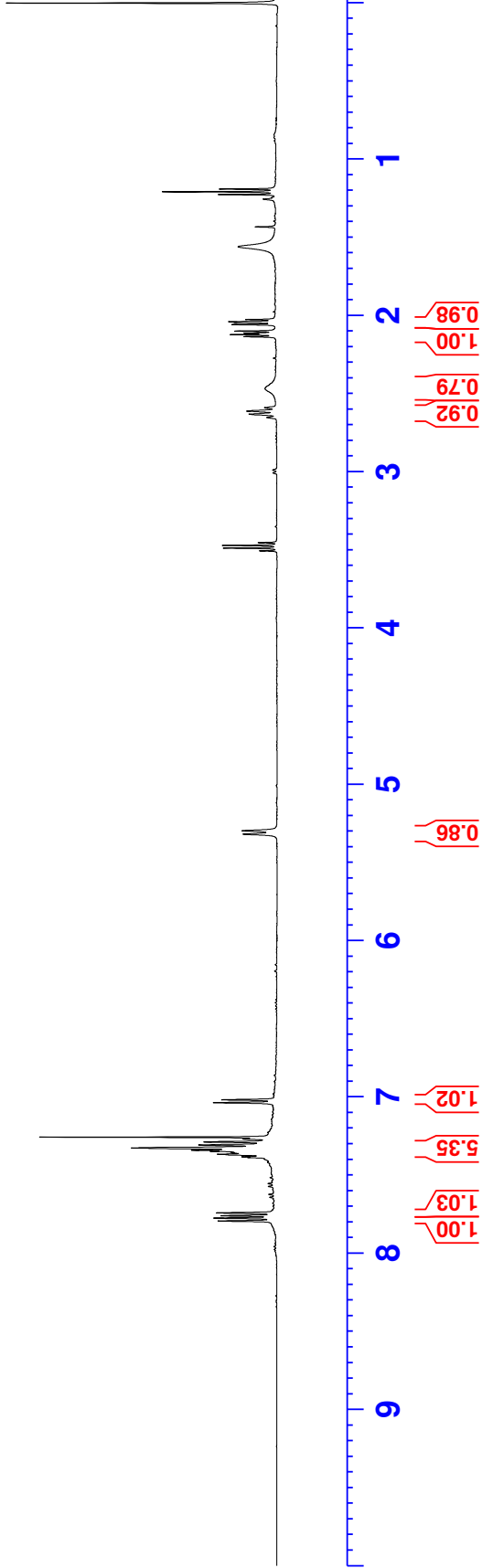



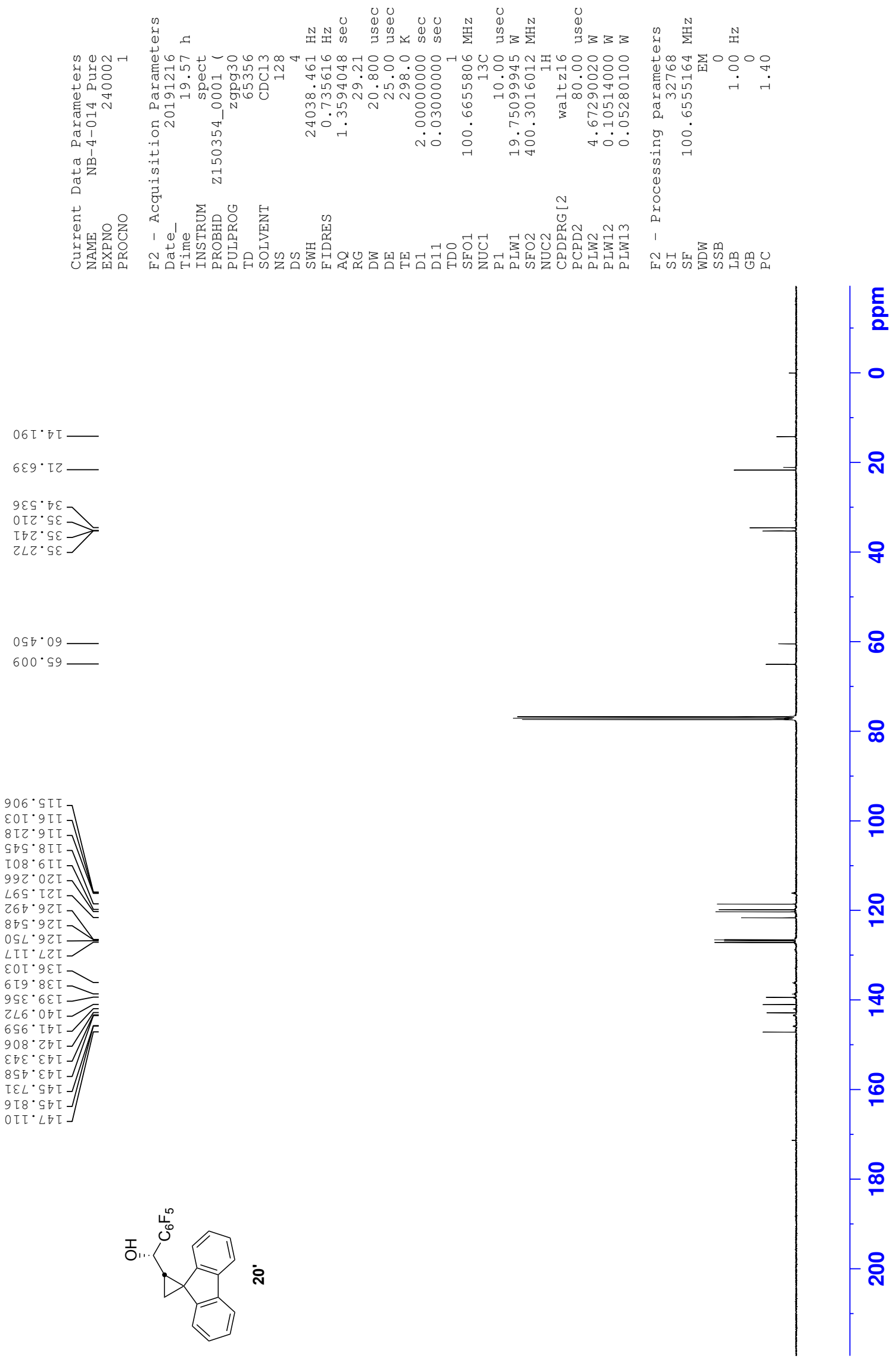

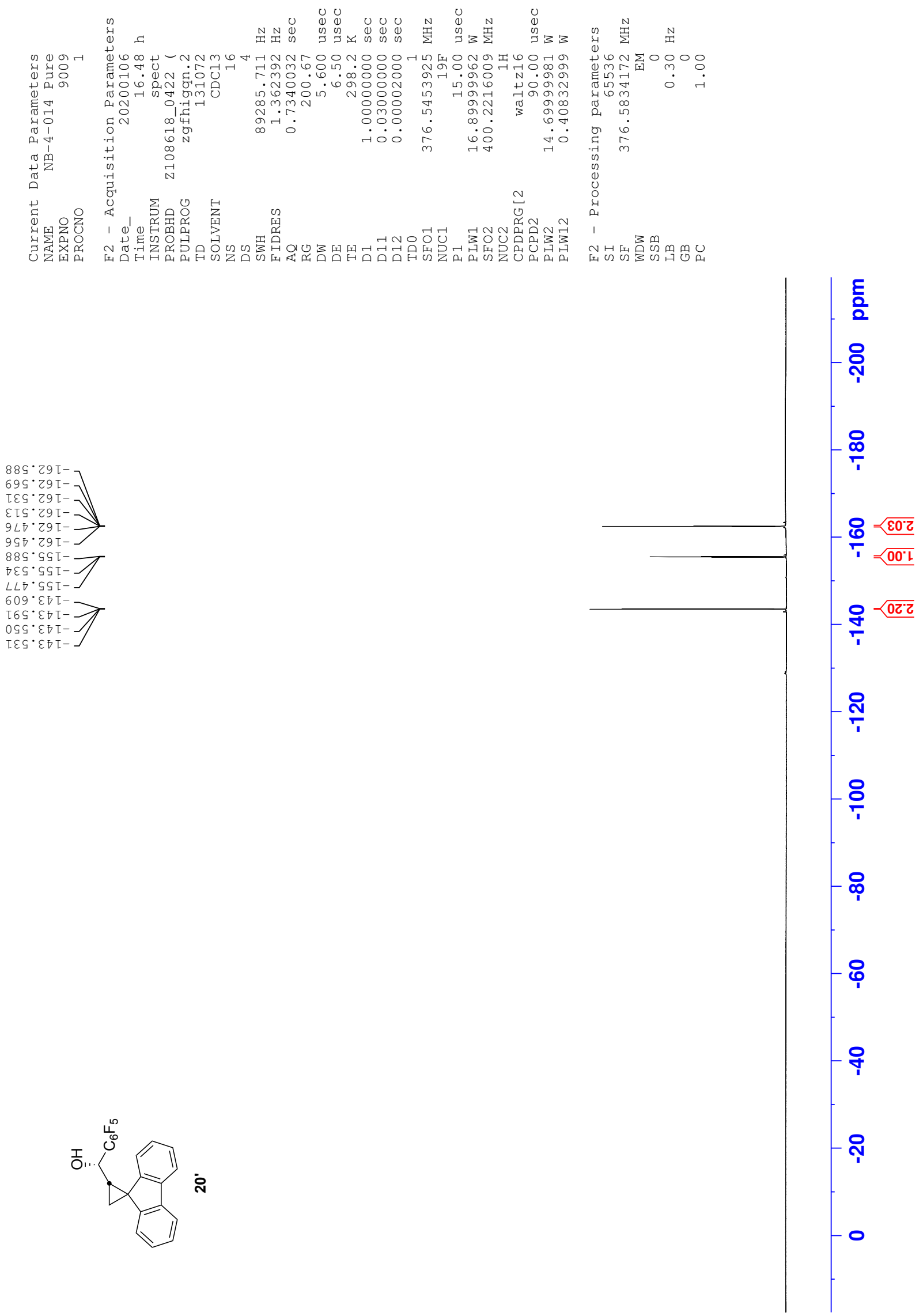

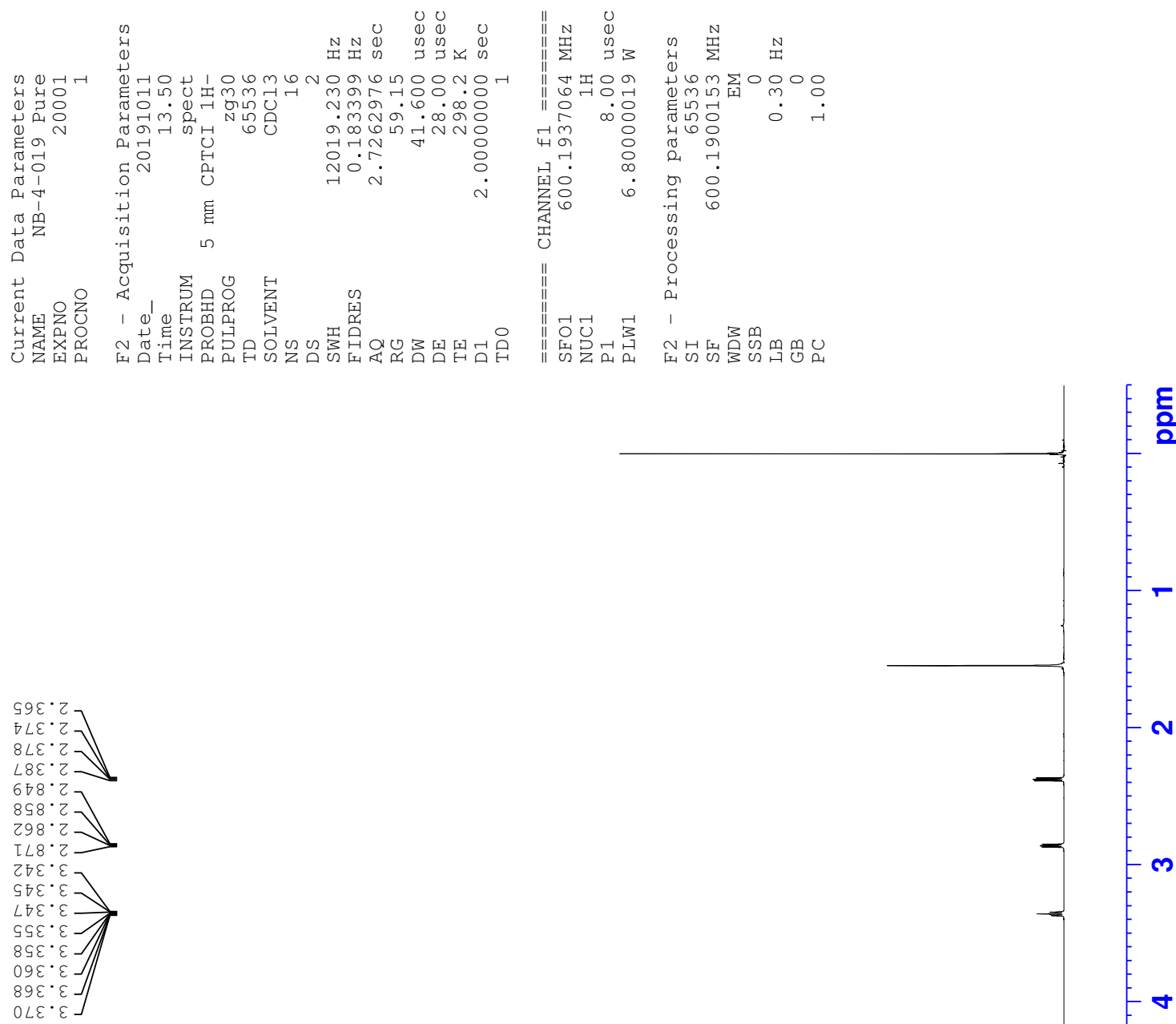

$\frac{\varepsilon}{\circ}$
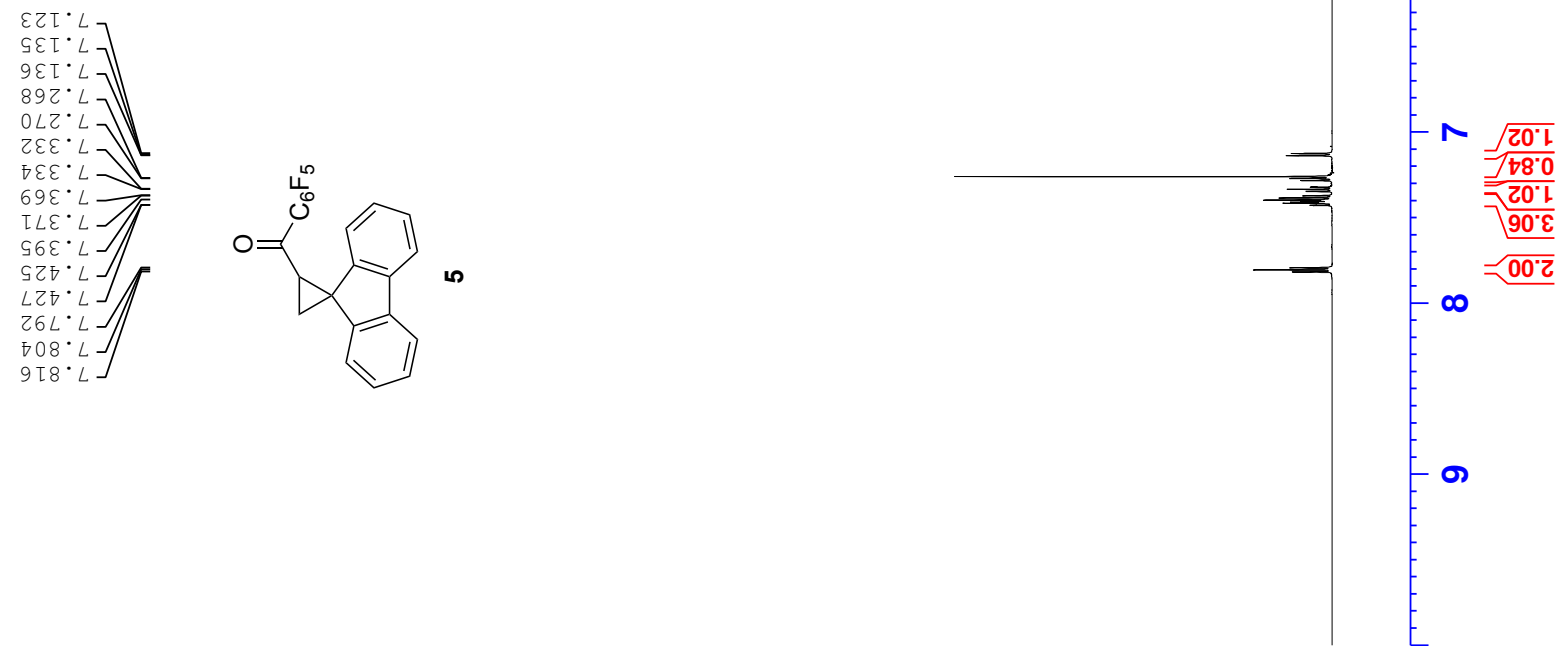

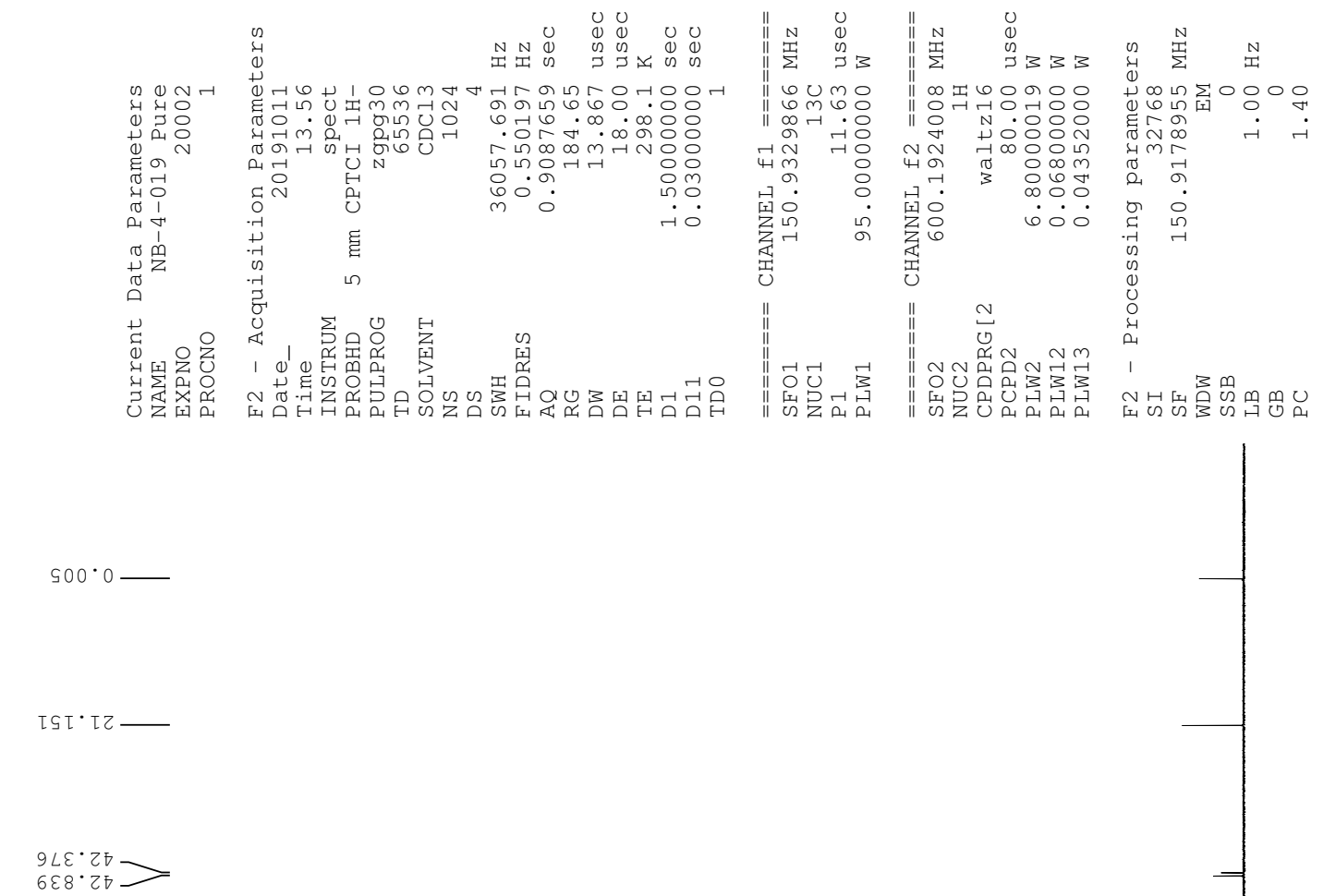

흥

- 0
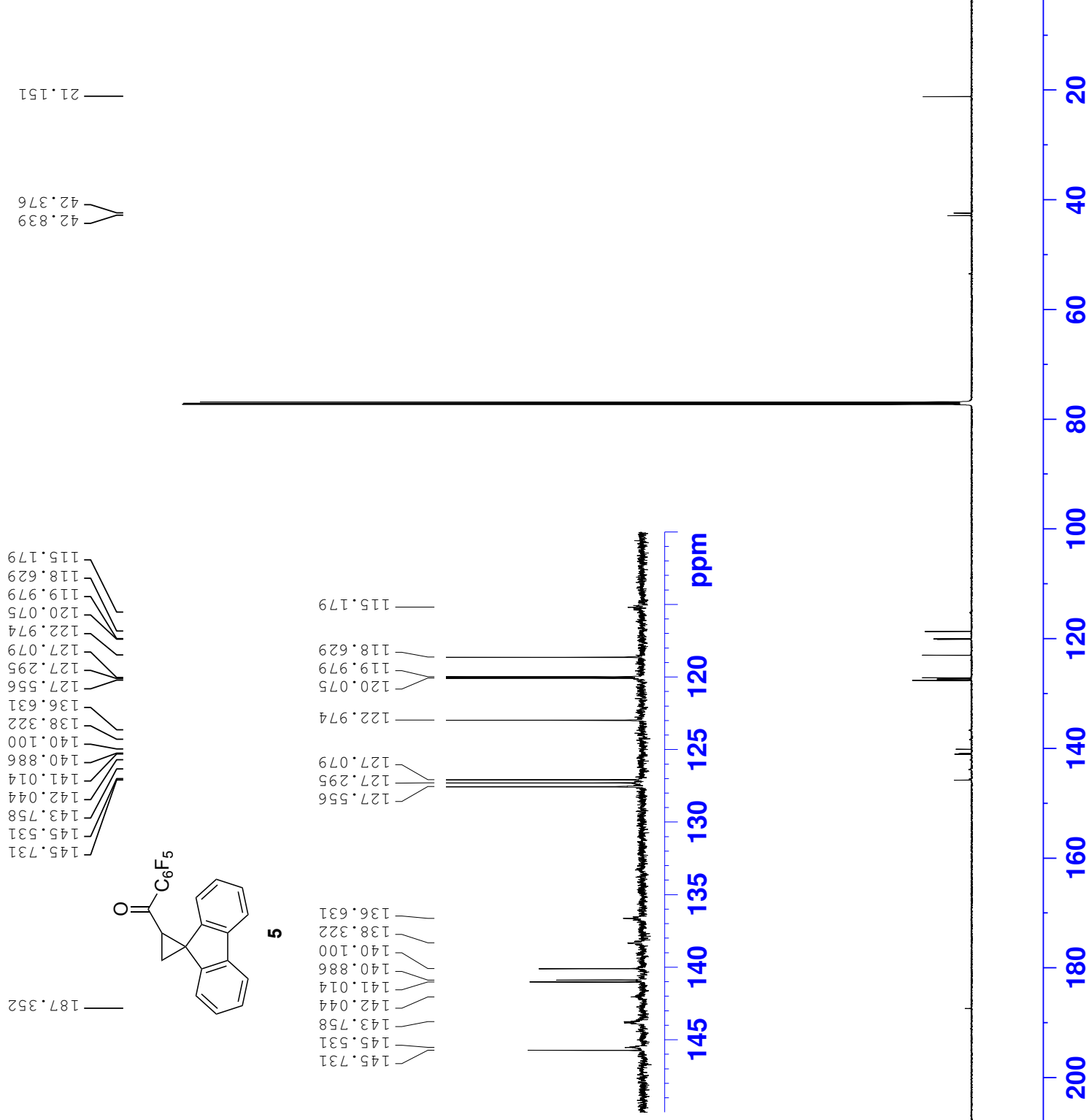

을

웅

ำ

守

$\stackrel{\circ}{\circ}$

$\stackrel{\infty}{\circ}$

우 

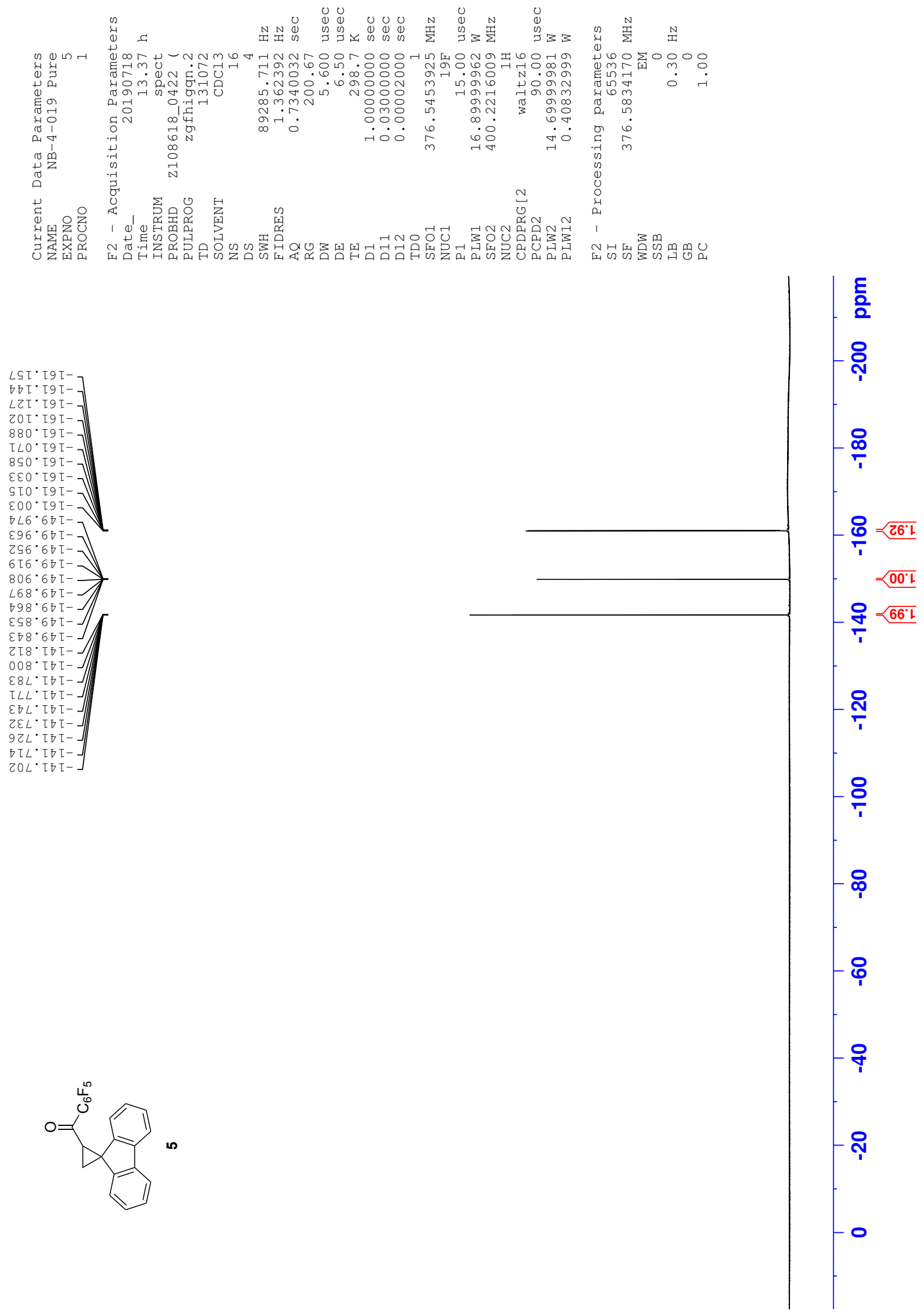

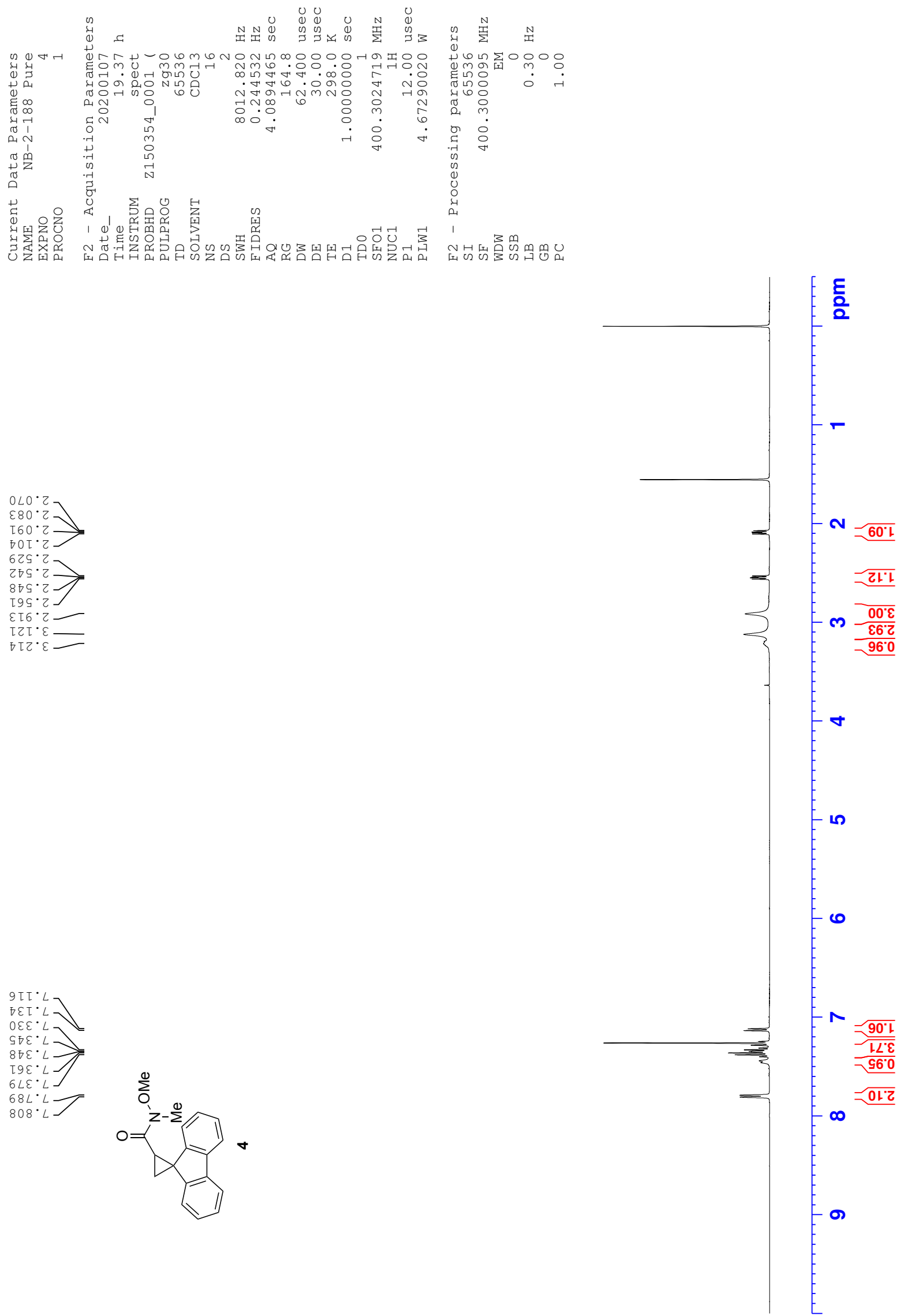

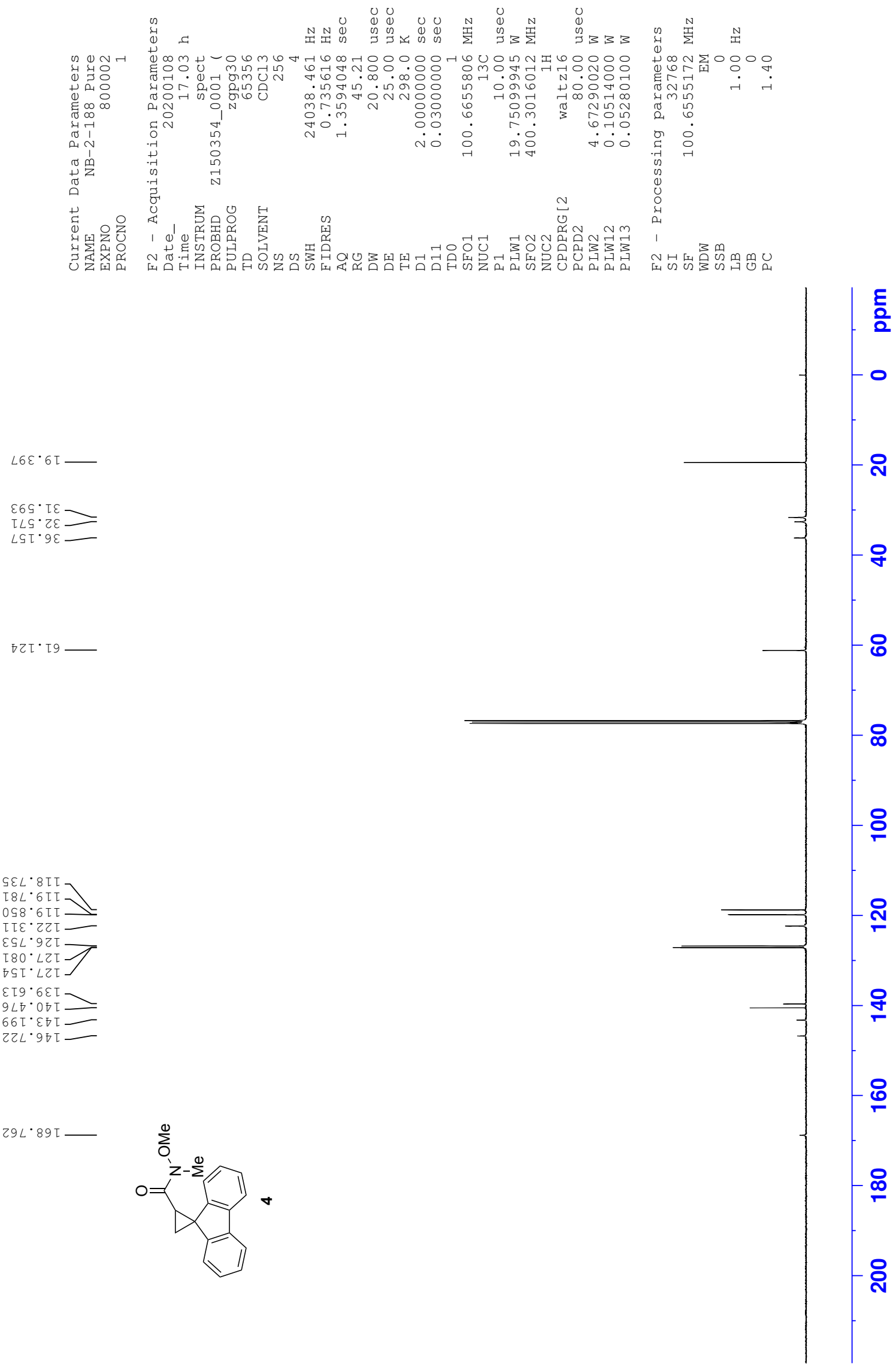

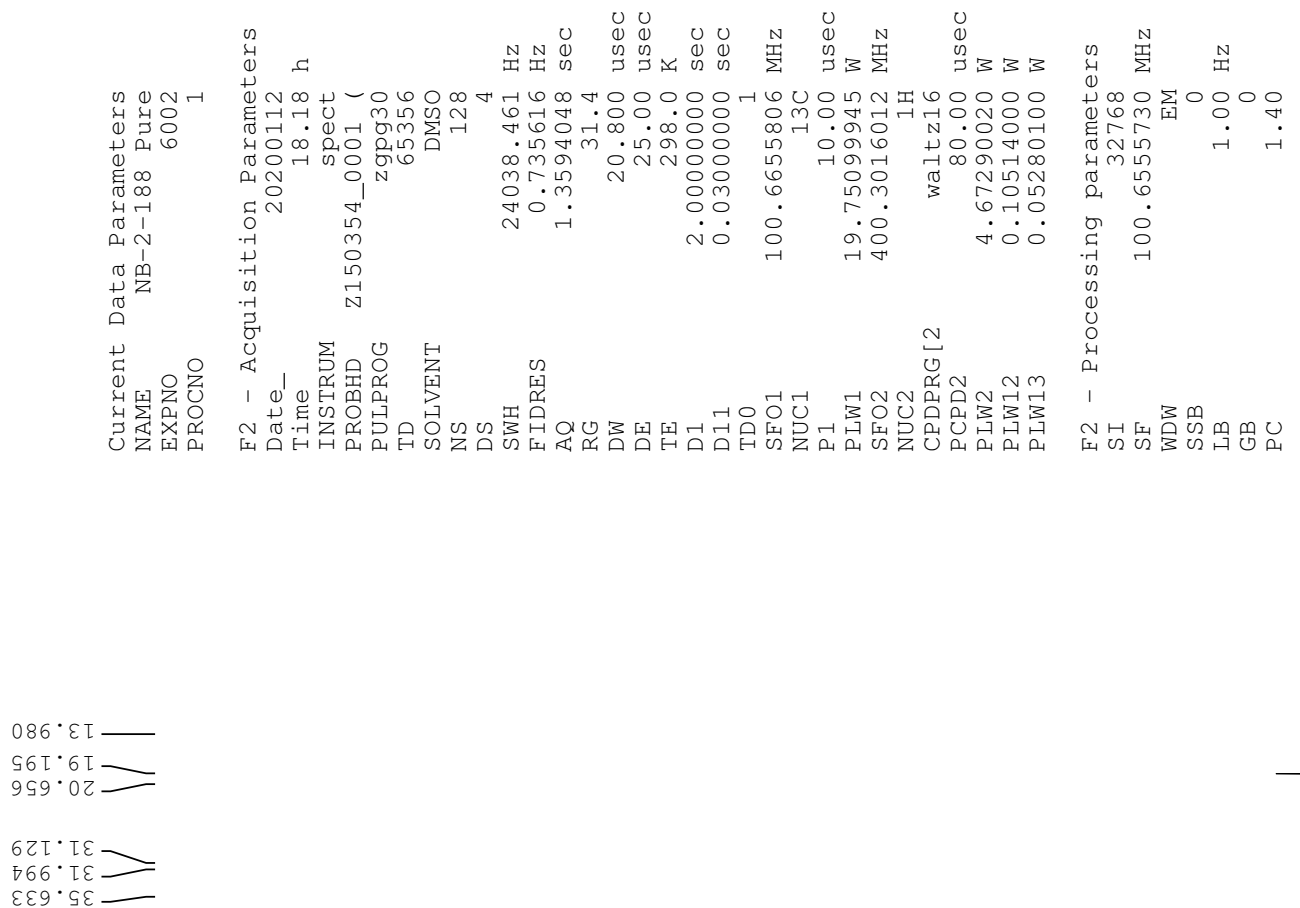

$579 \cdot 69=$

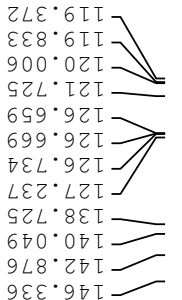

음

오

운

8

I9L. L9T

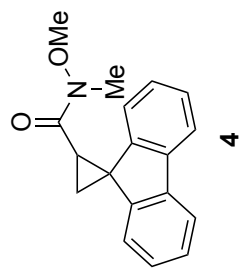



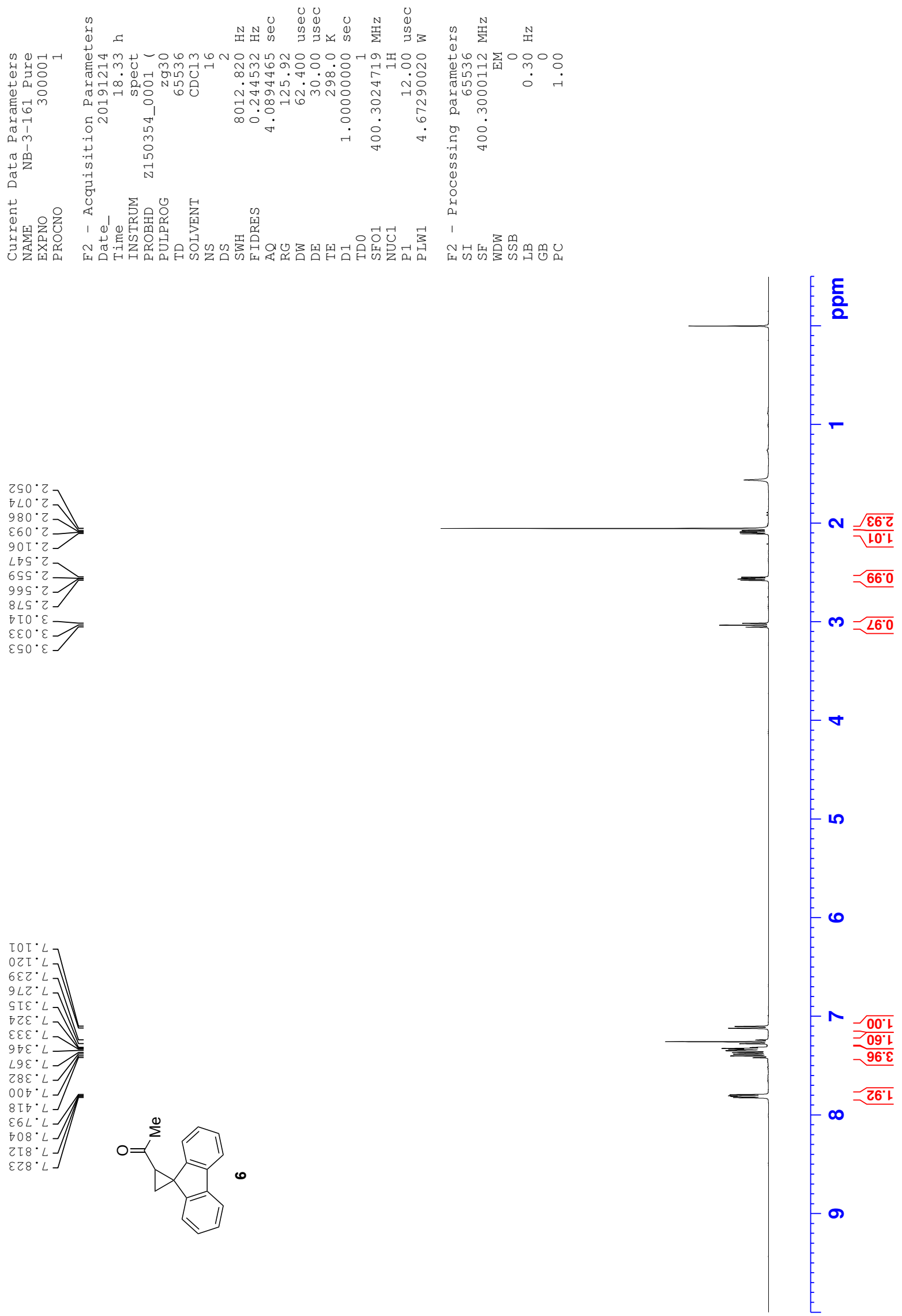

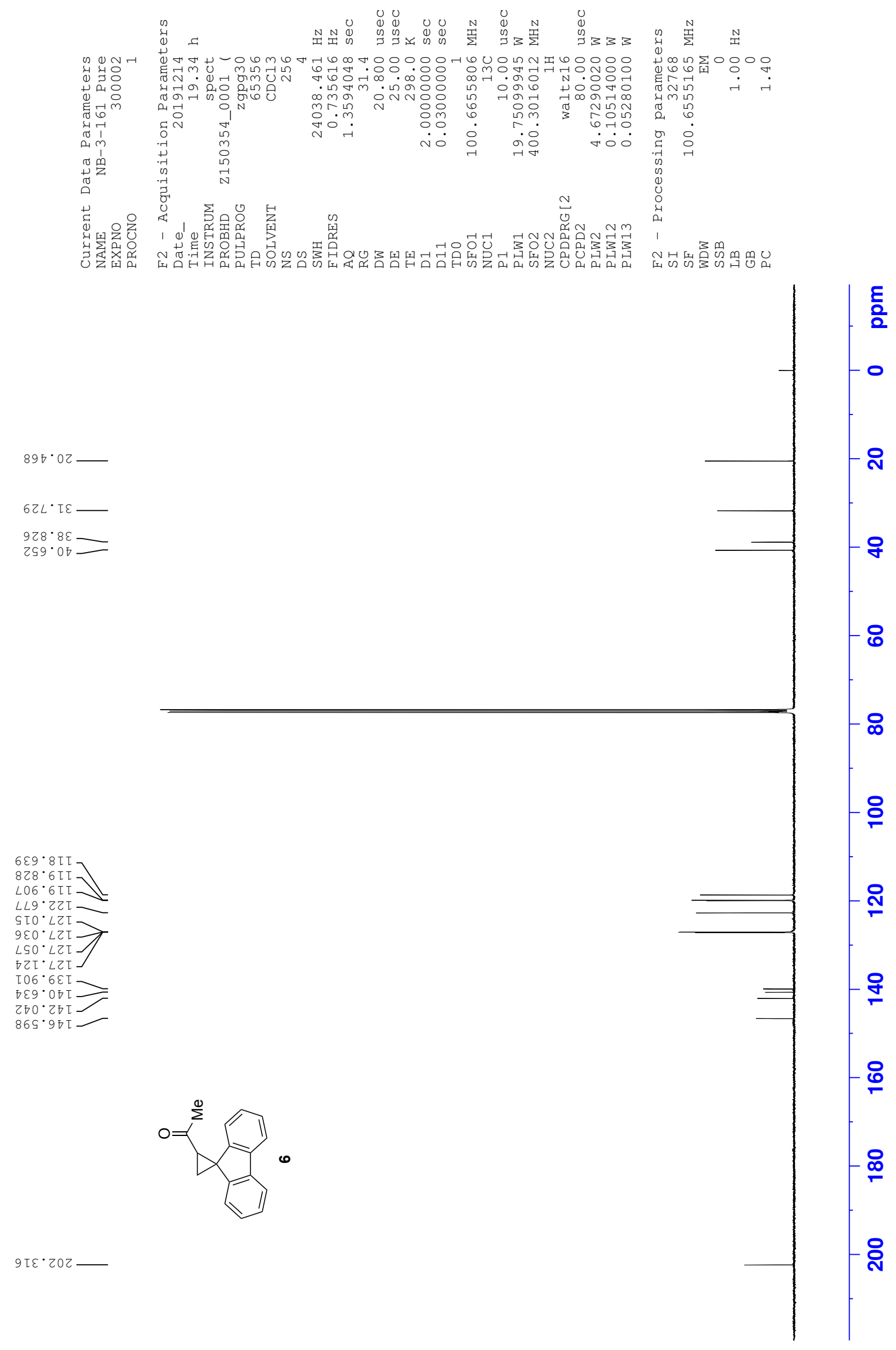

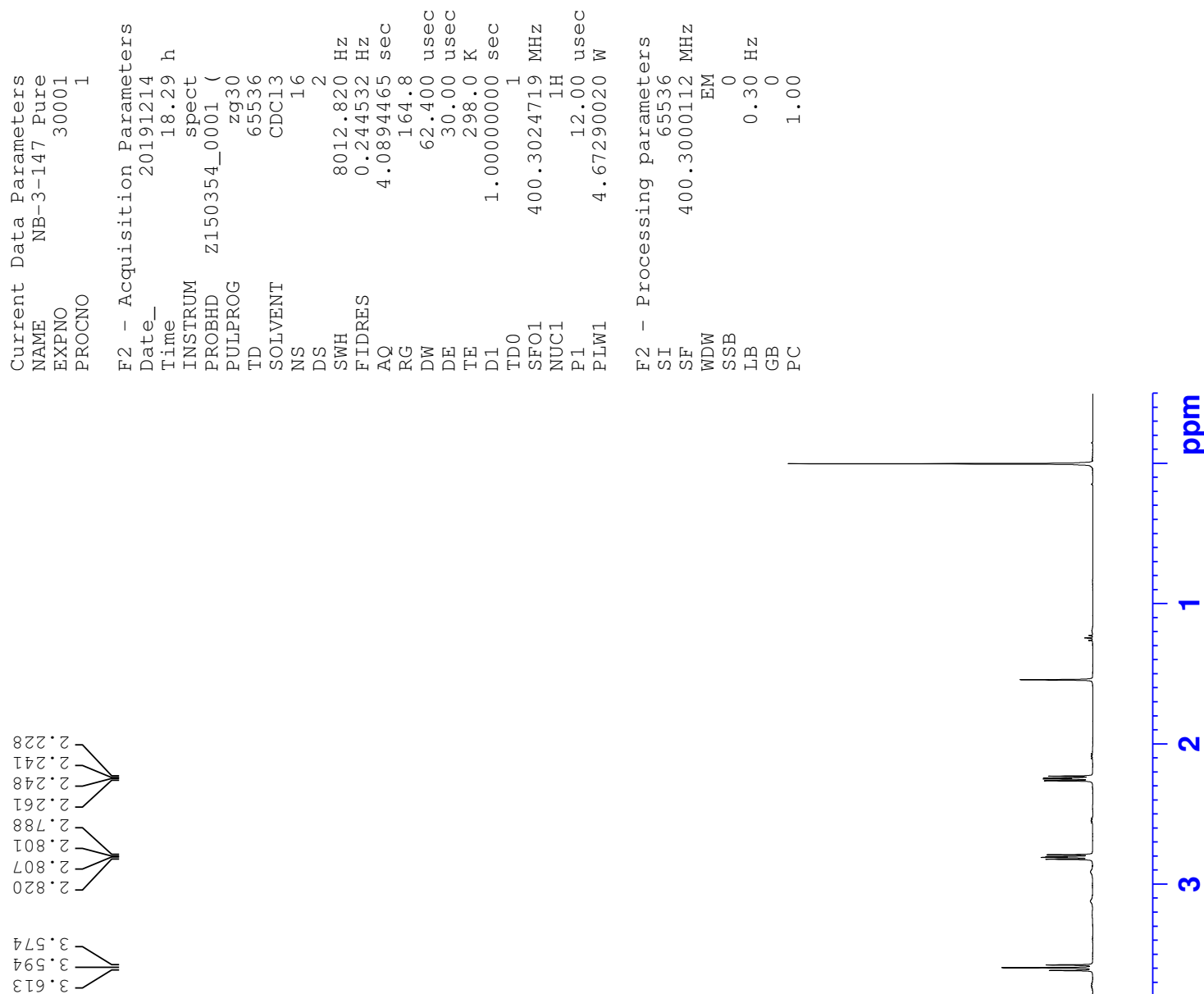

틍

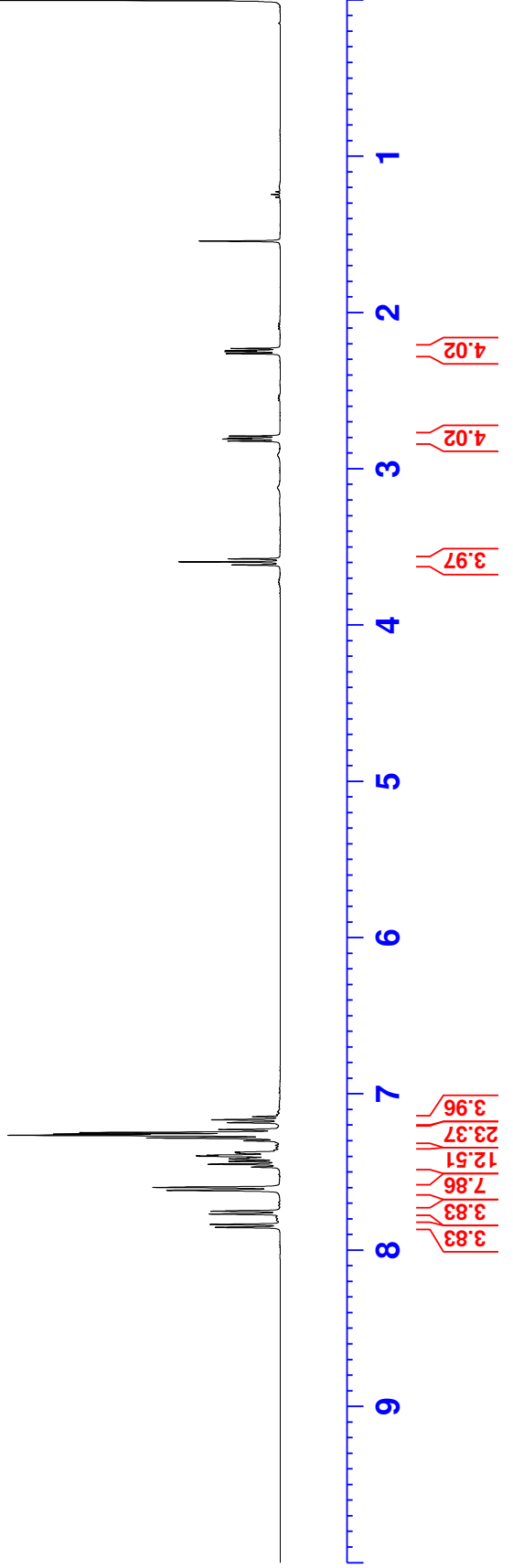



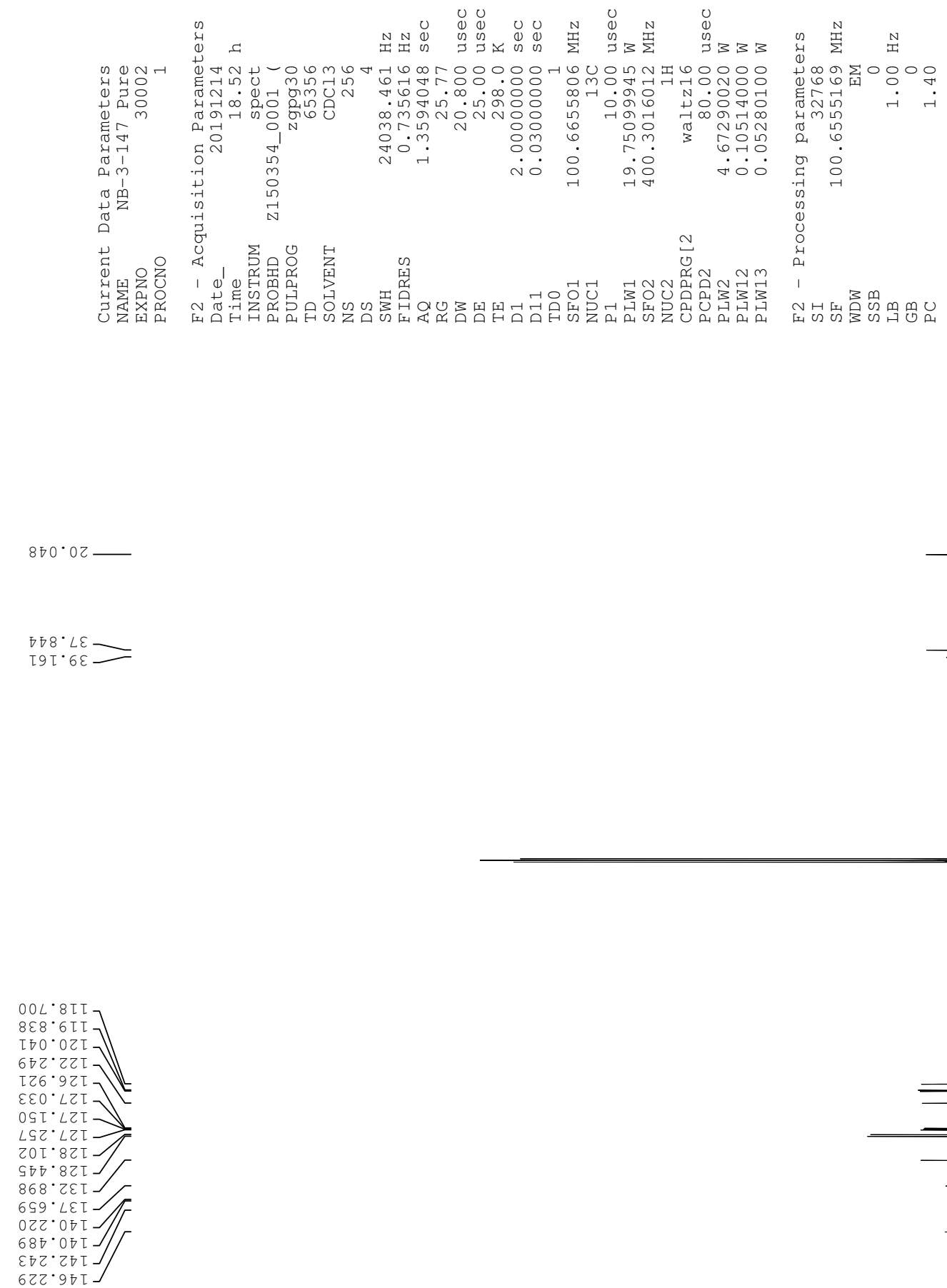

$967 \cdot 76 \mathrm{~T}$
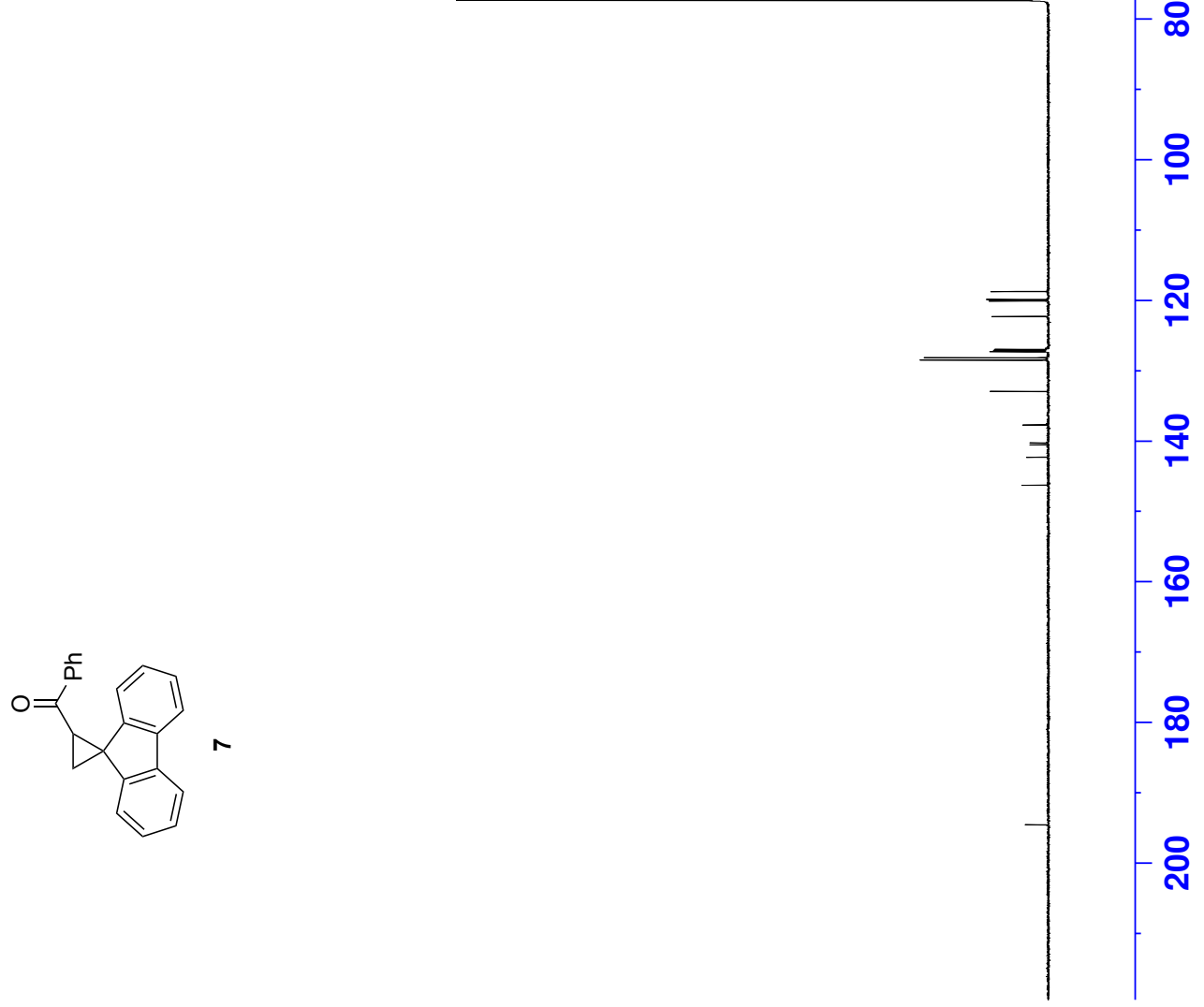

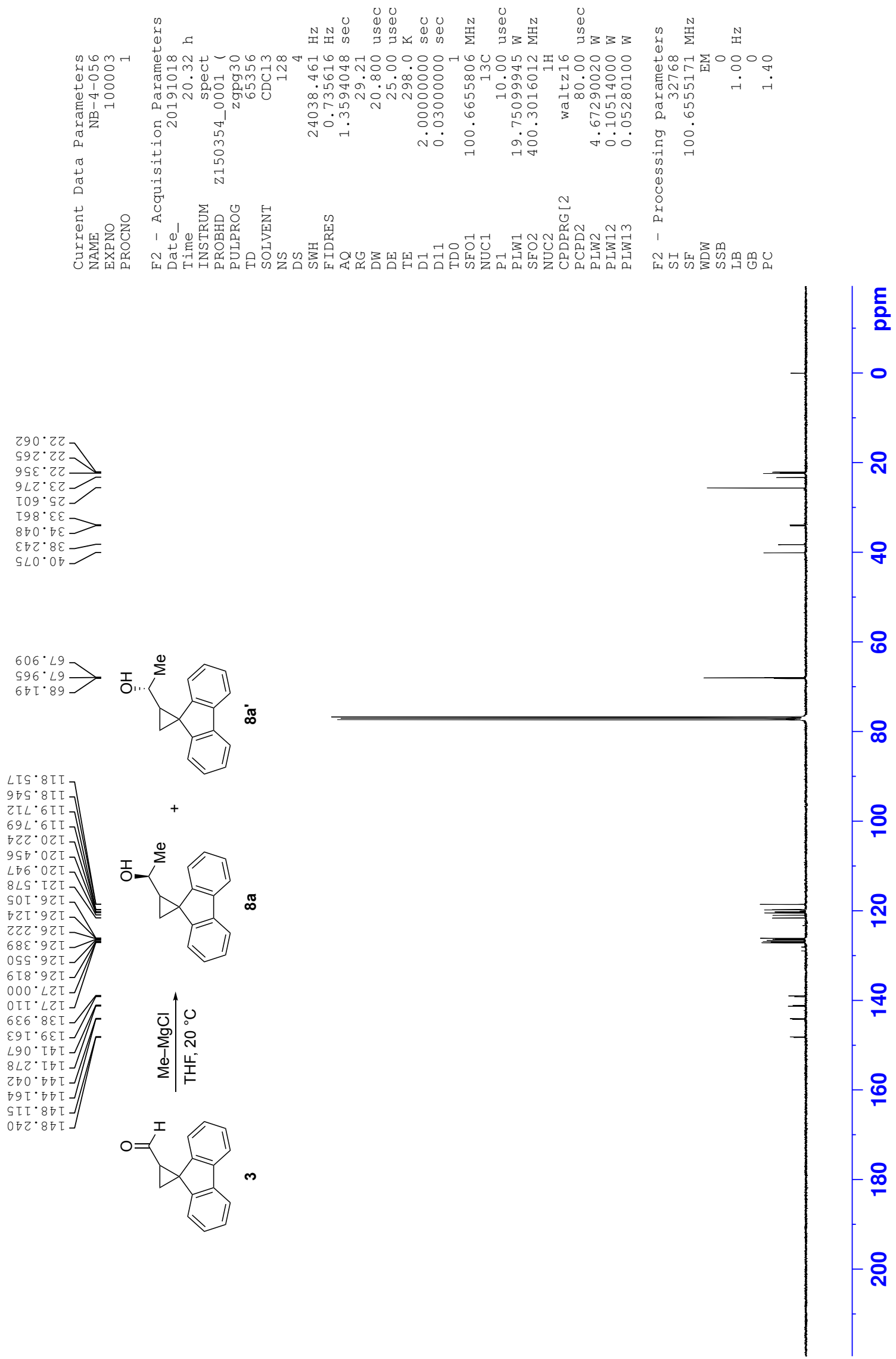

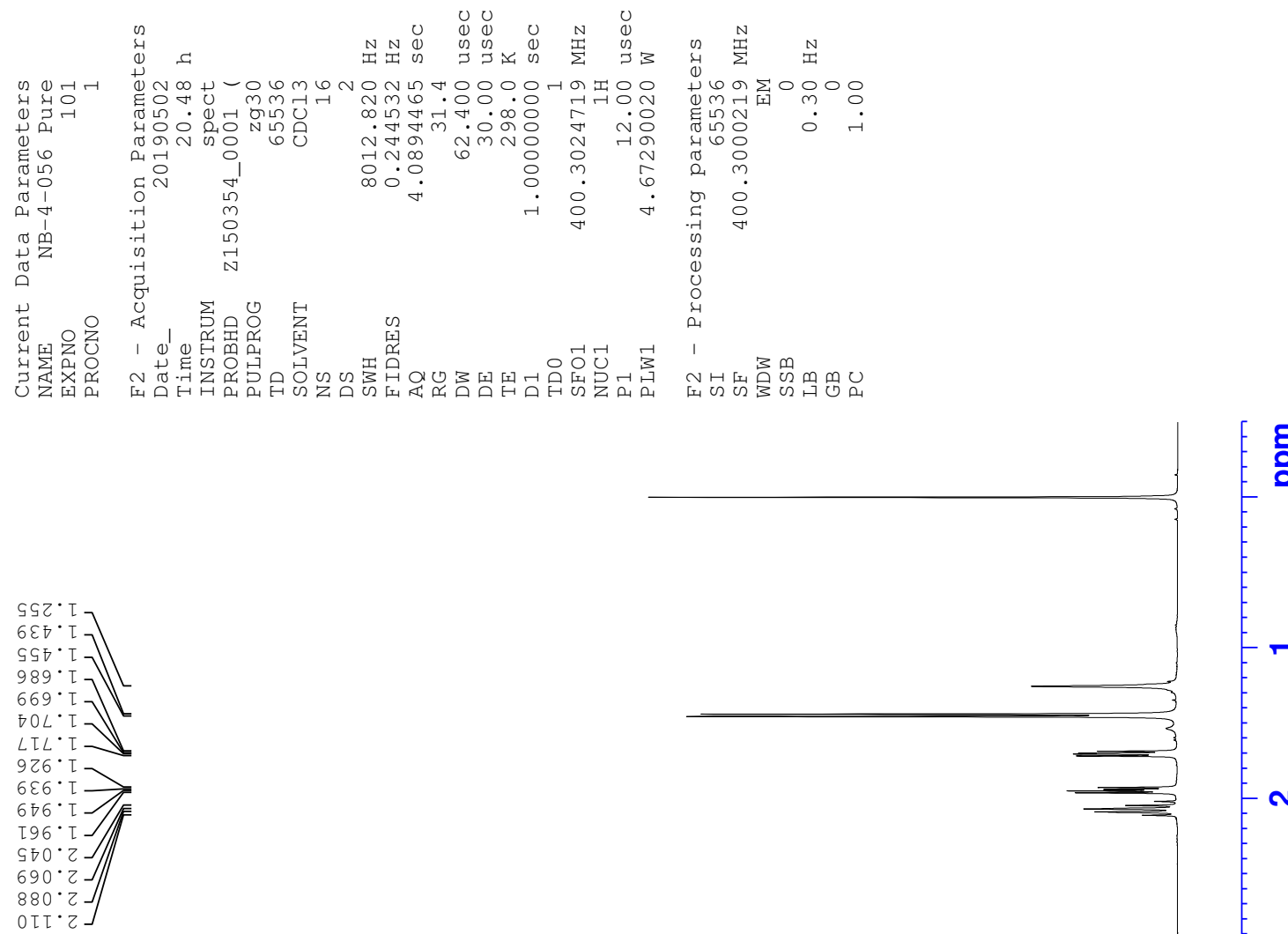

틍
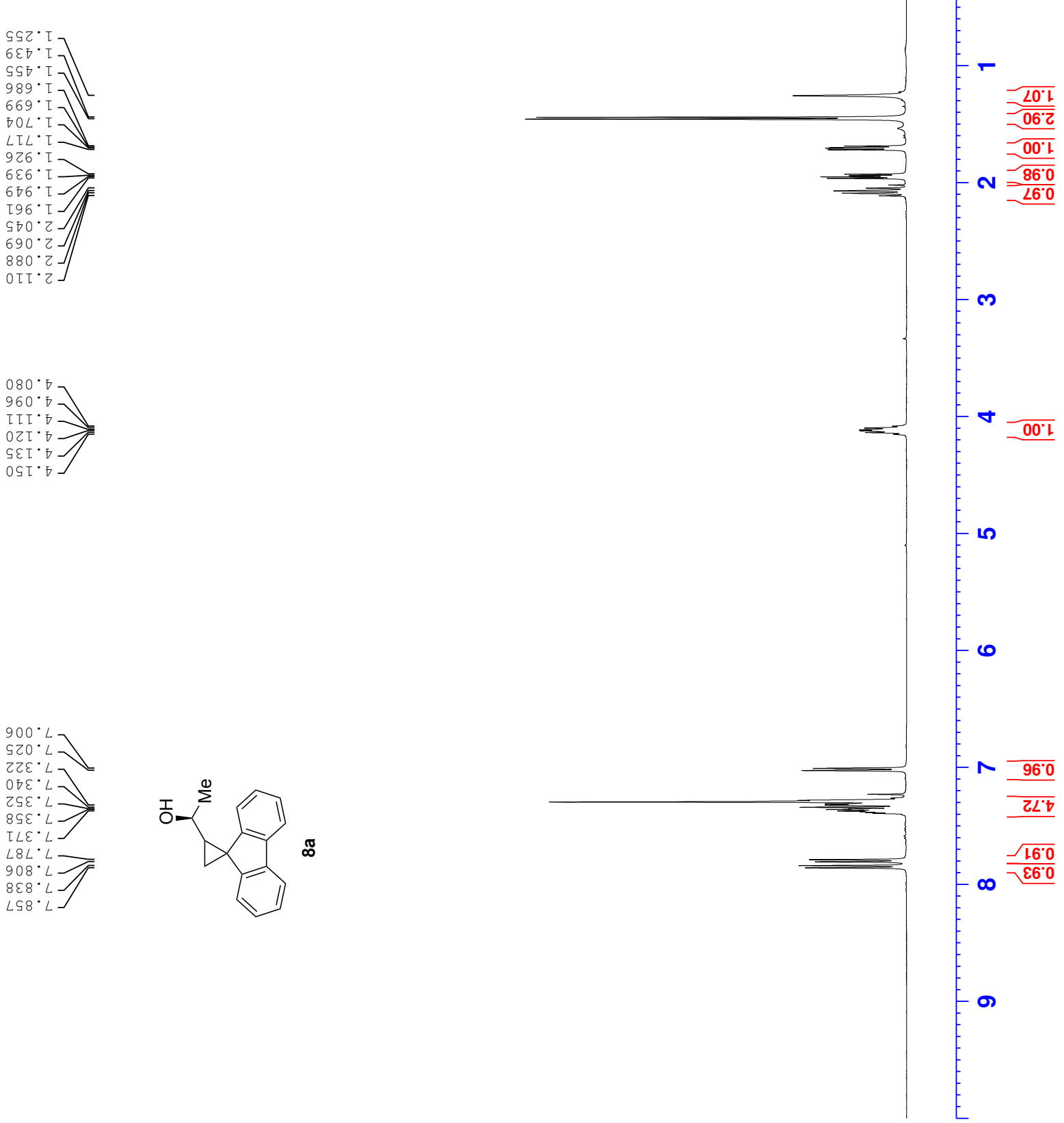

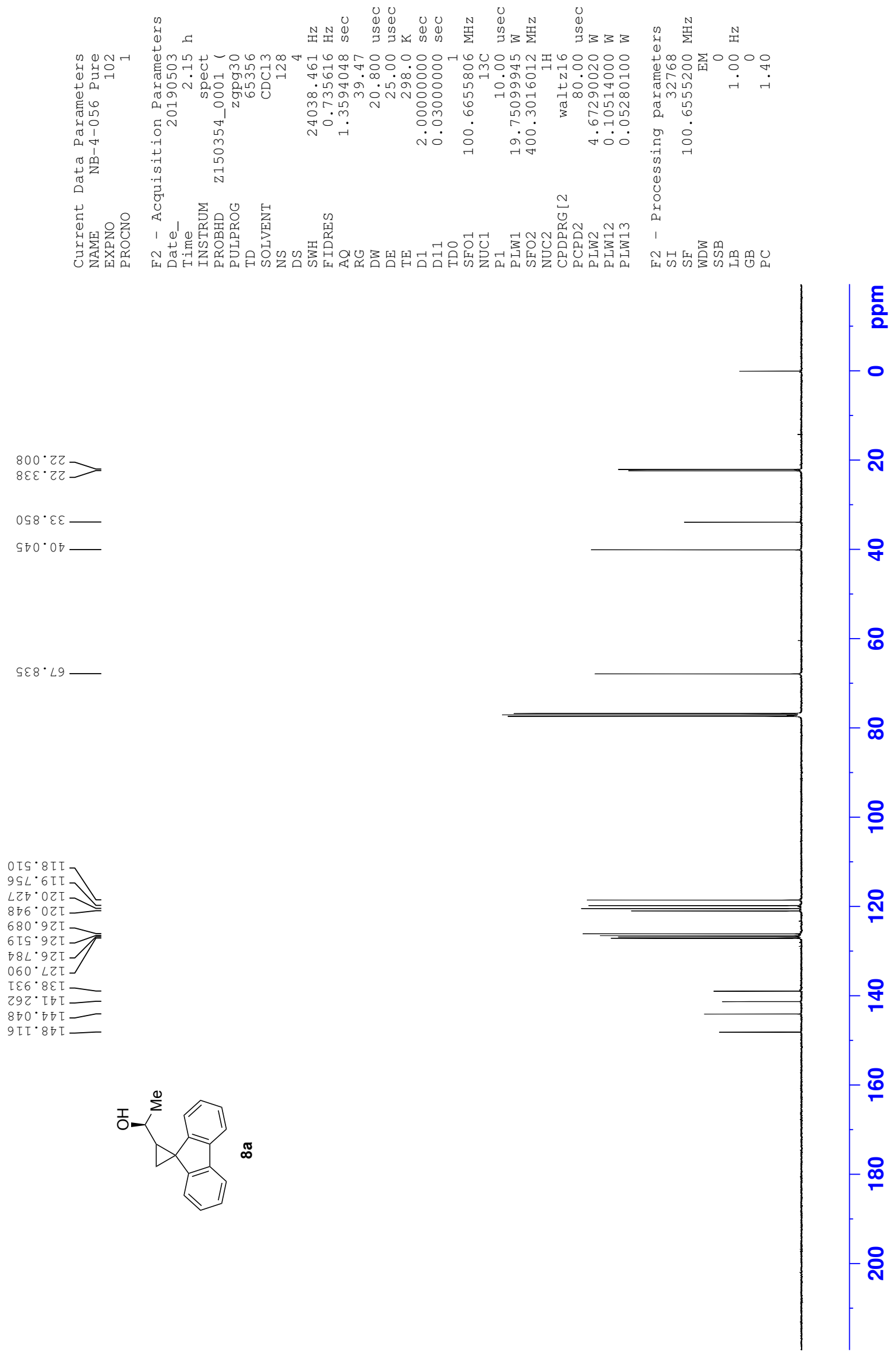

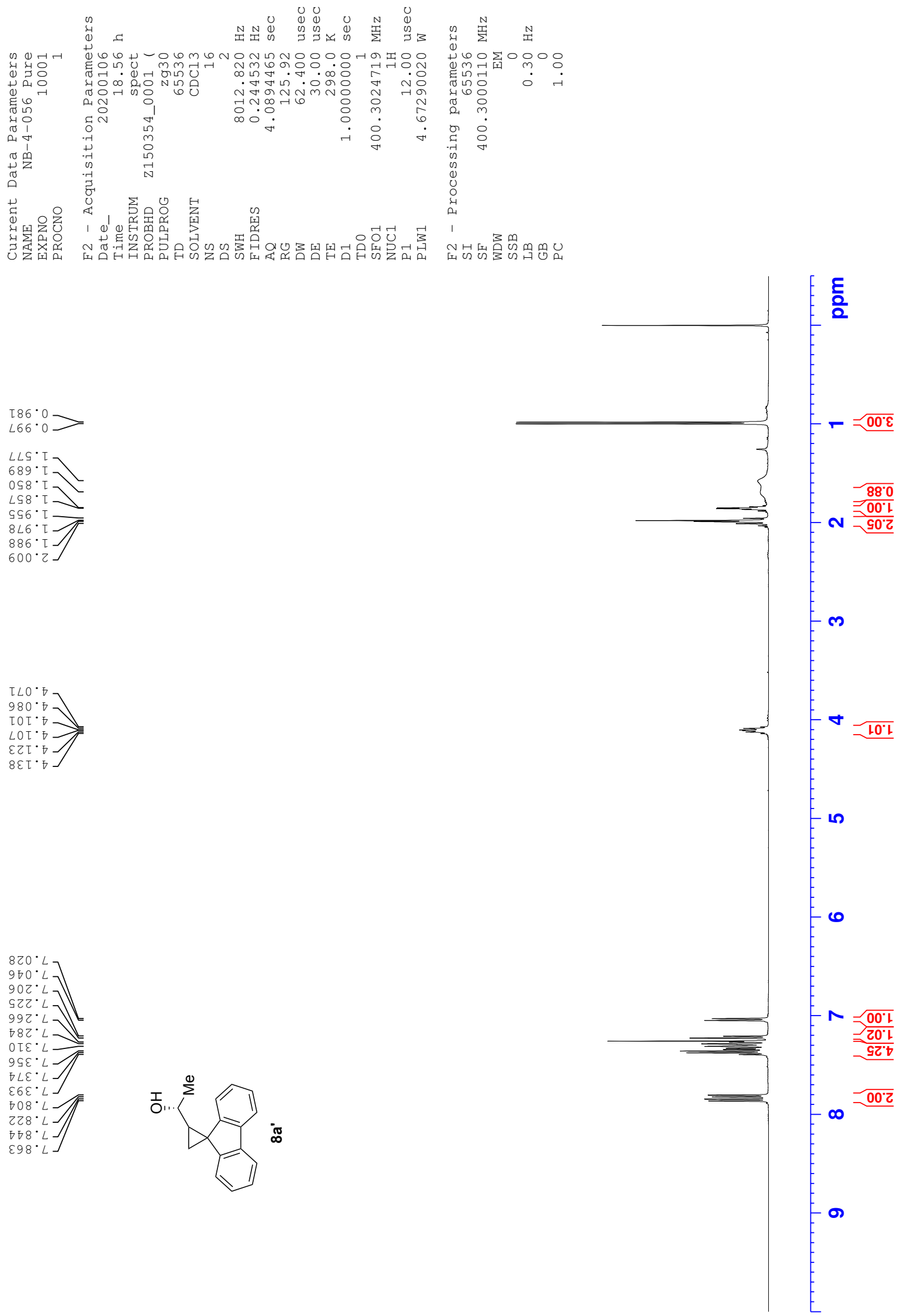

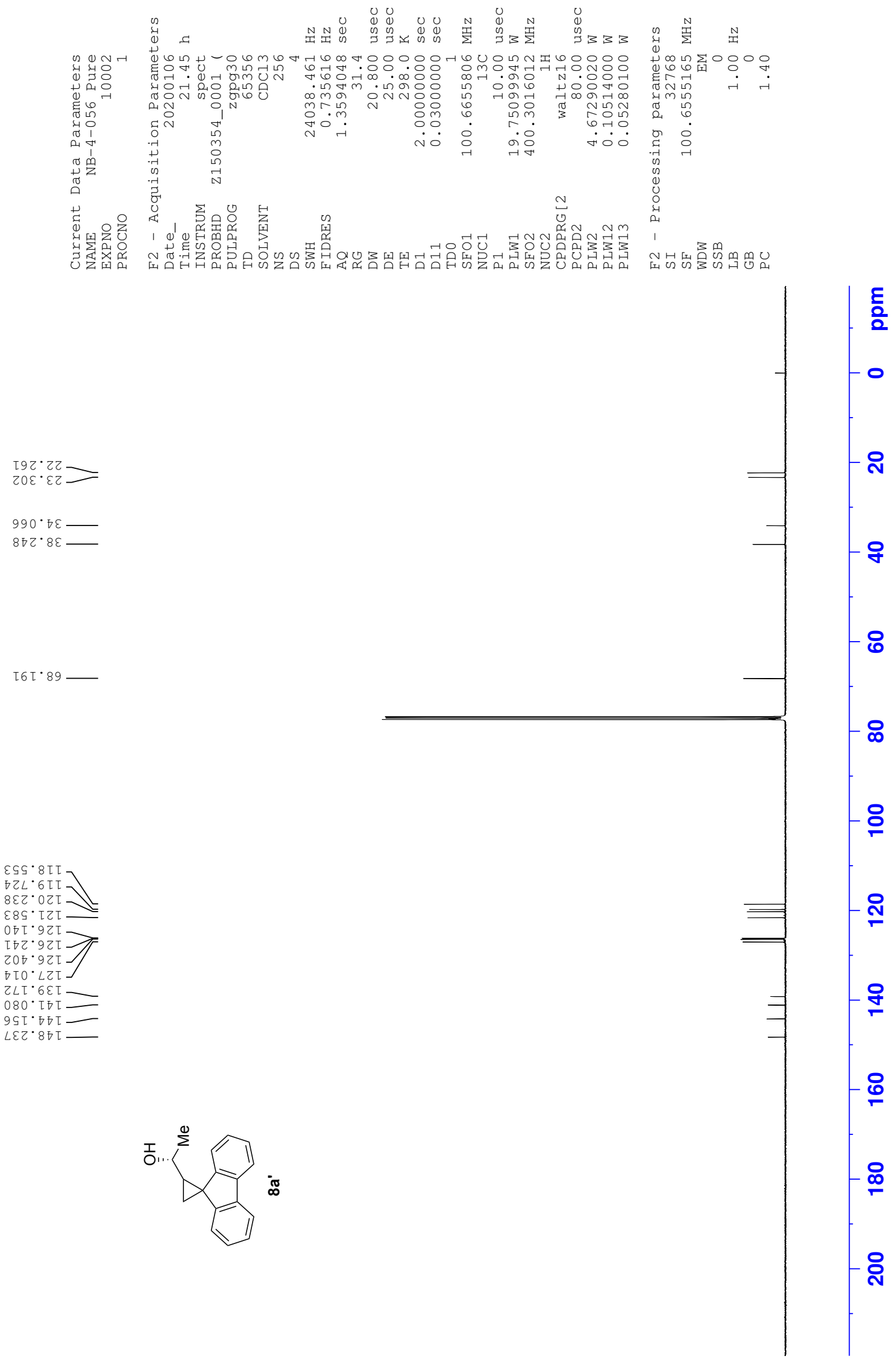

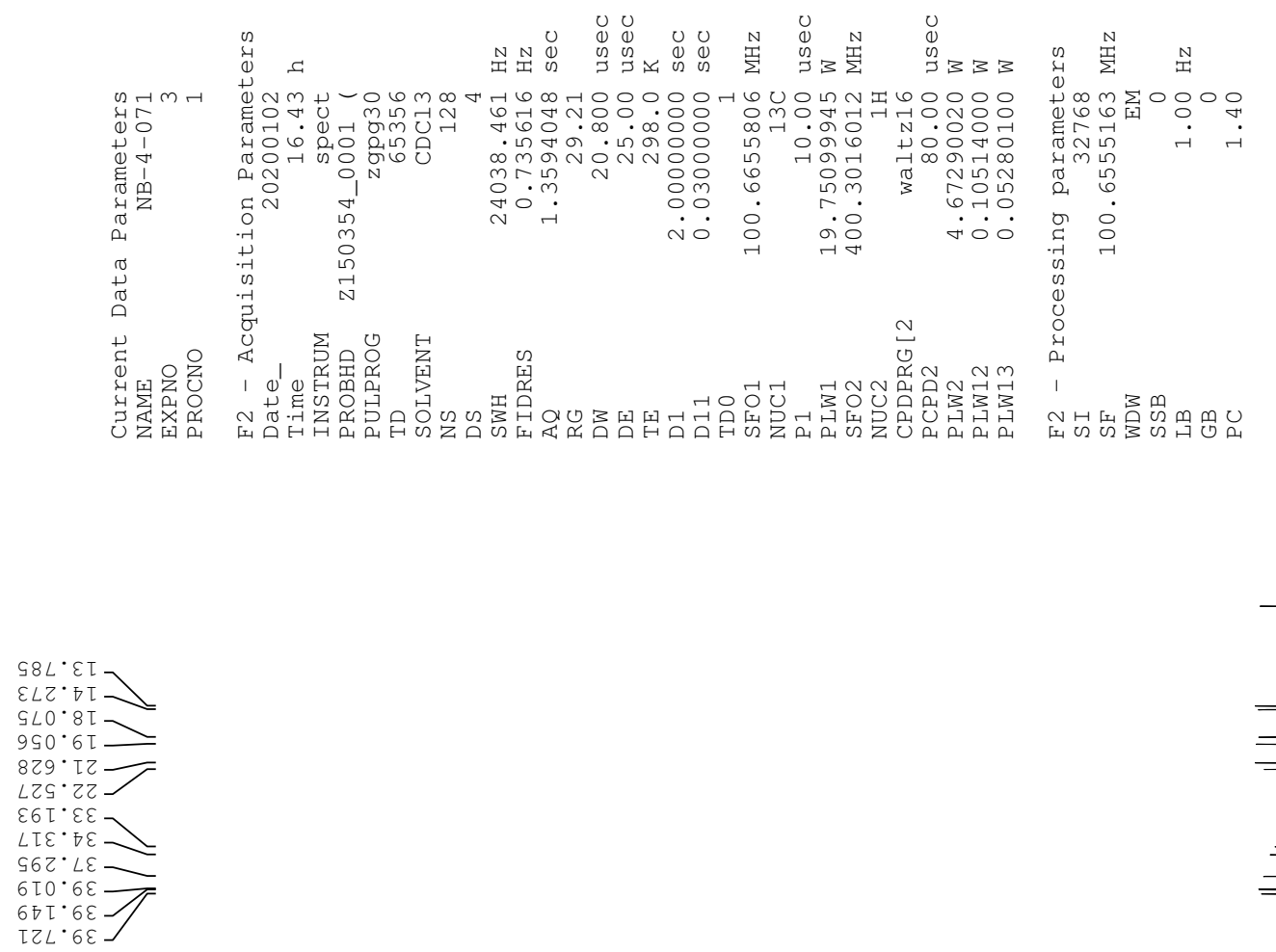

$\mid$

등
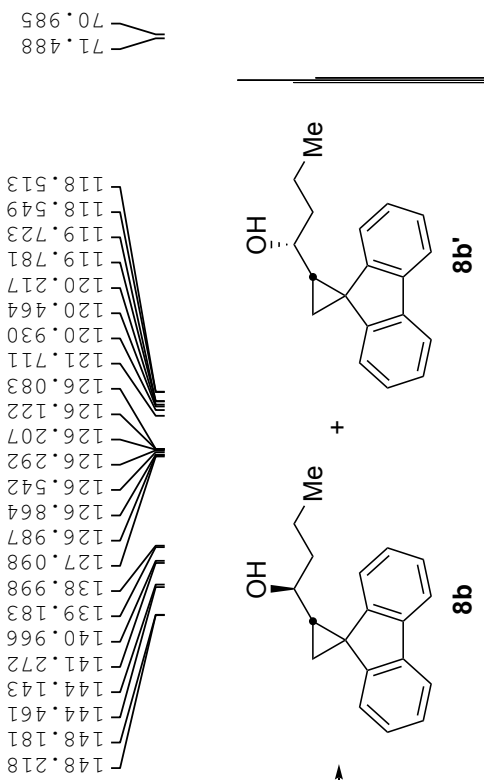

$\infty$

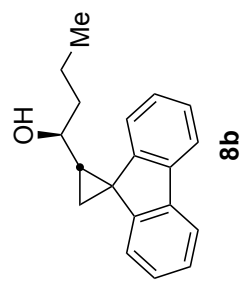

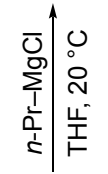

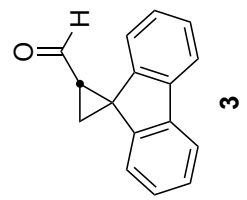



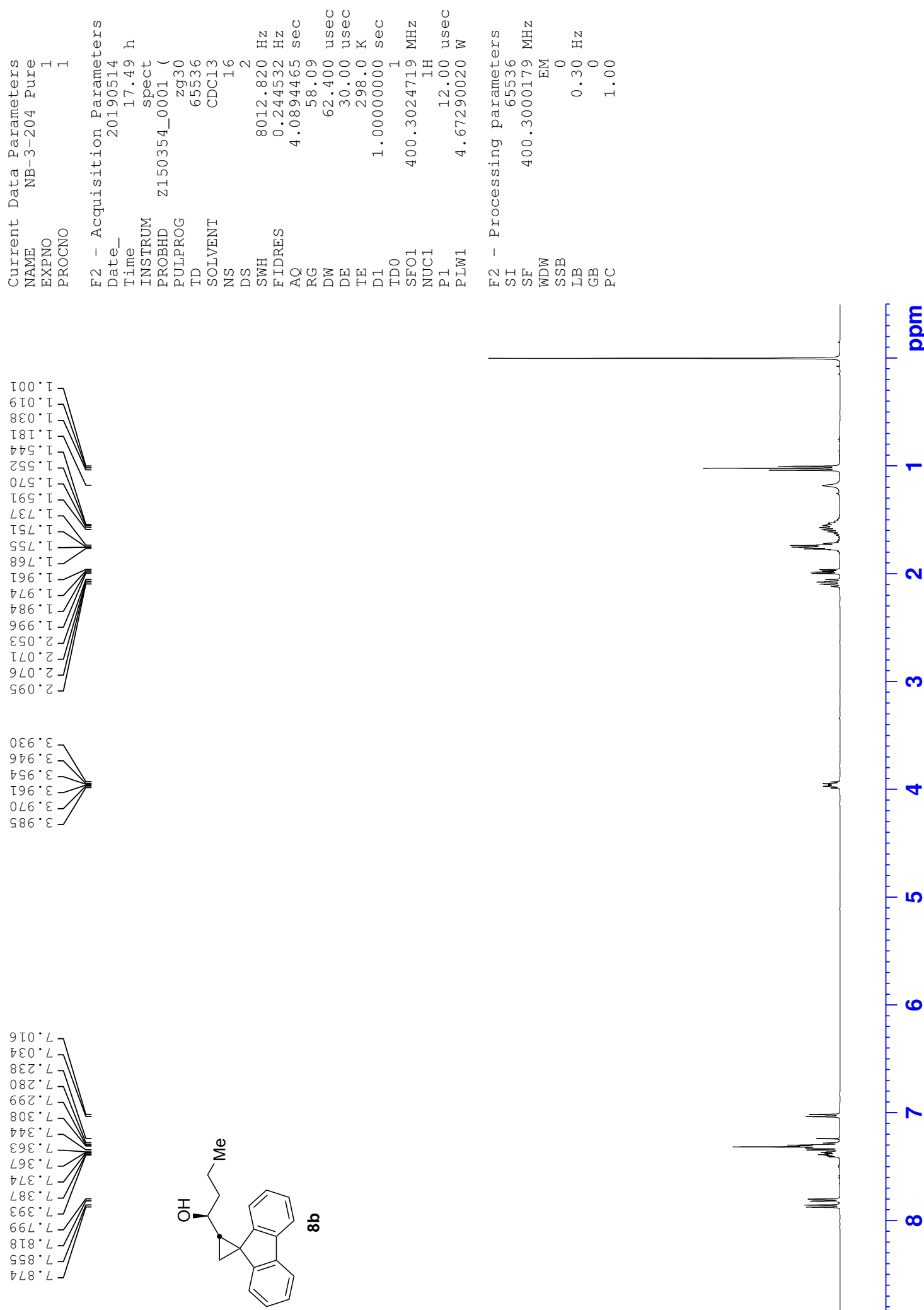

틍
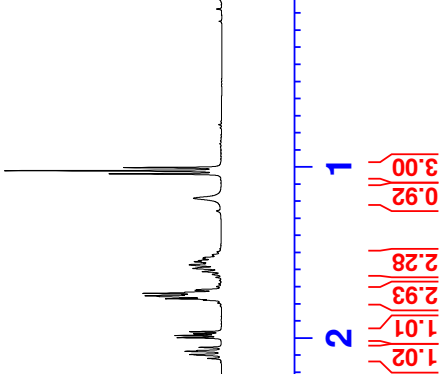

m

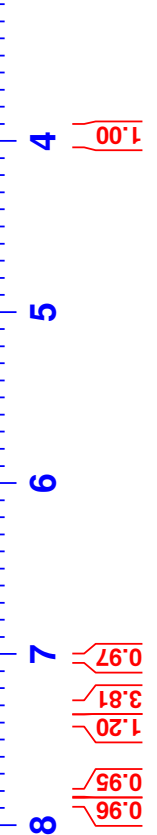



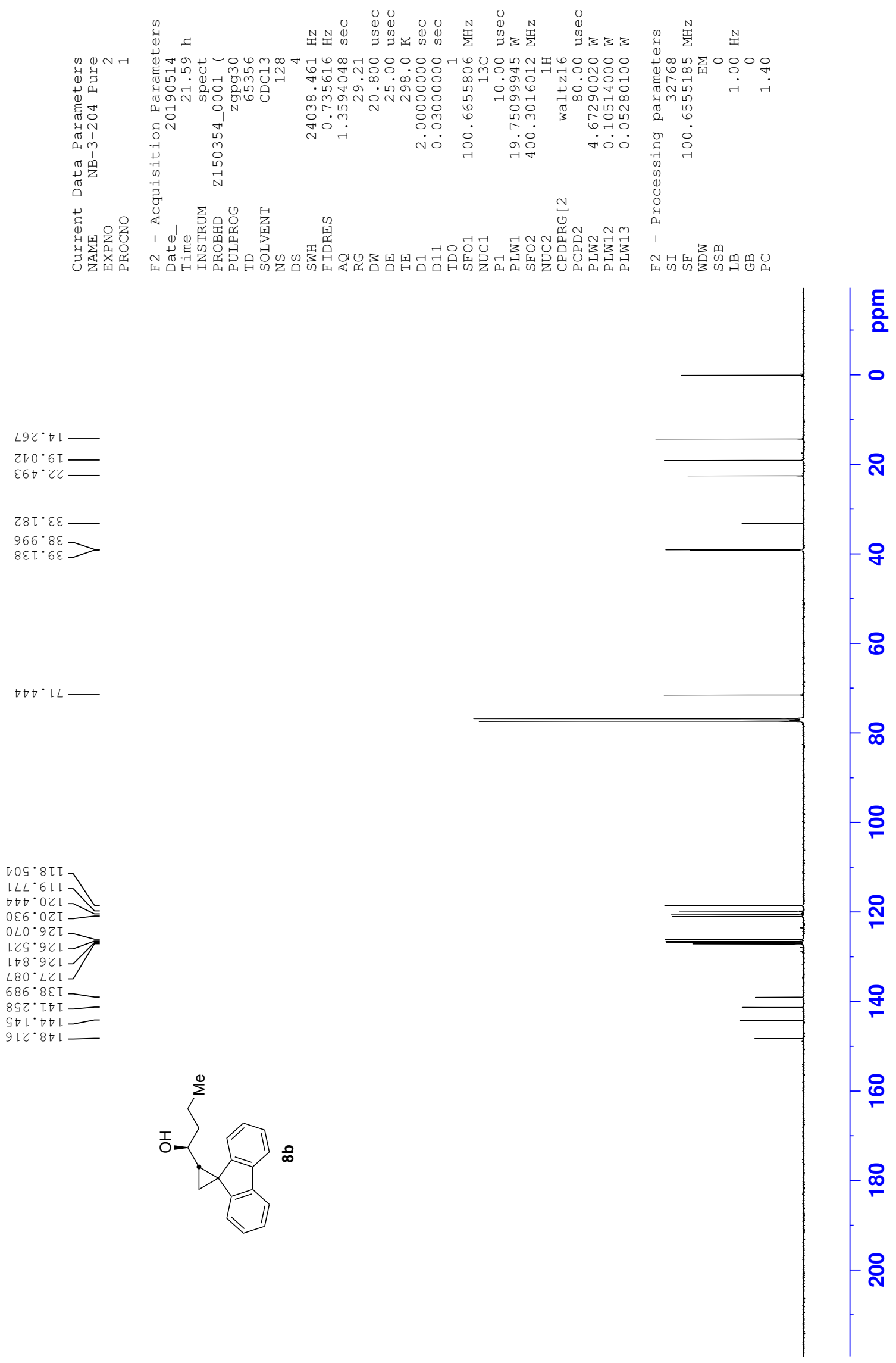

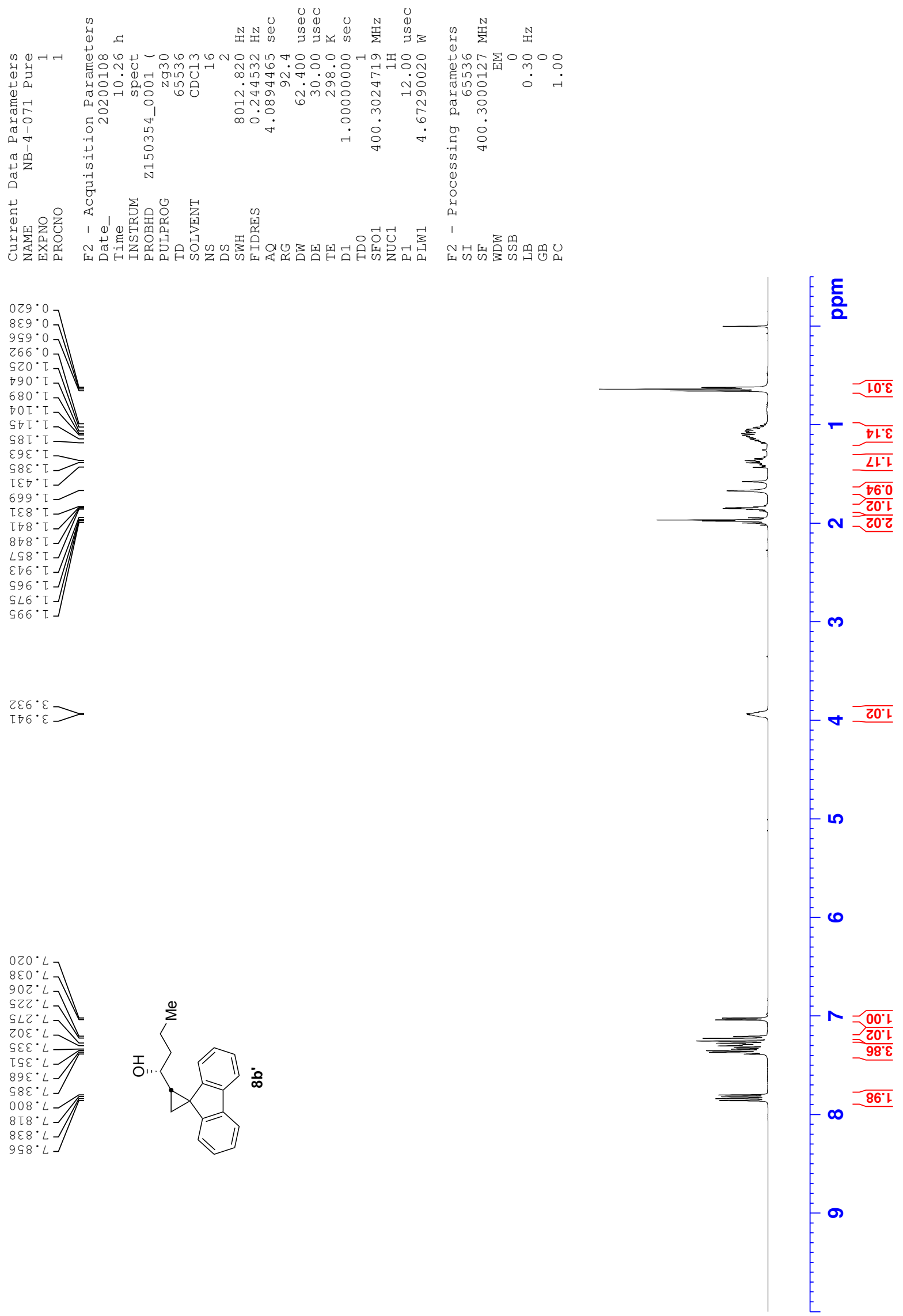

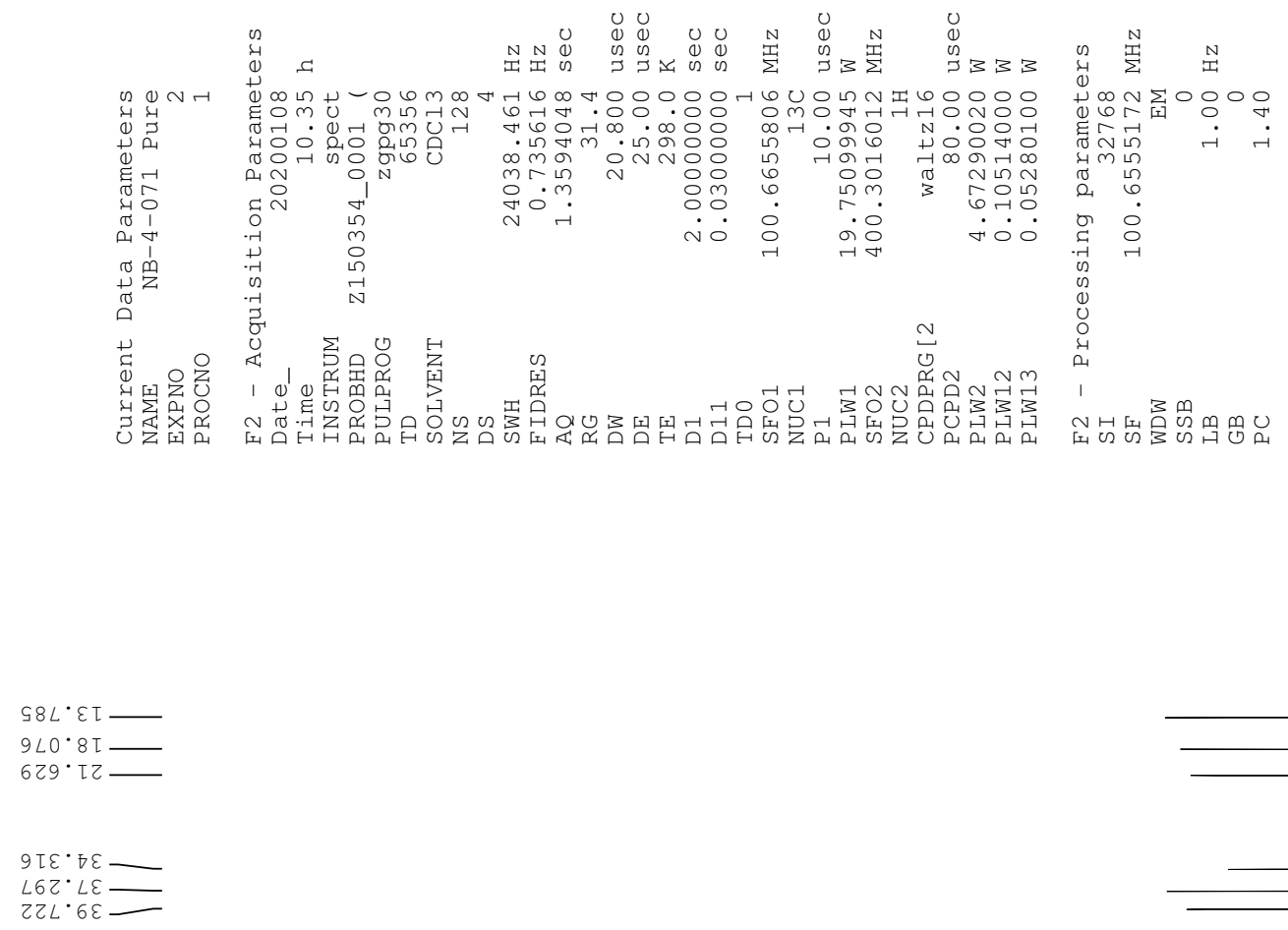

$6 \angle 6 \cdot O L-$
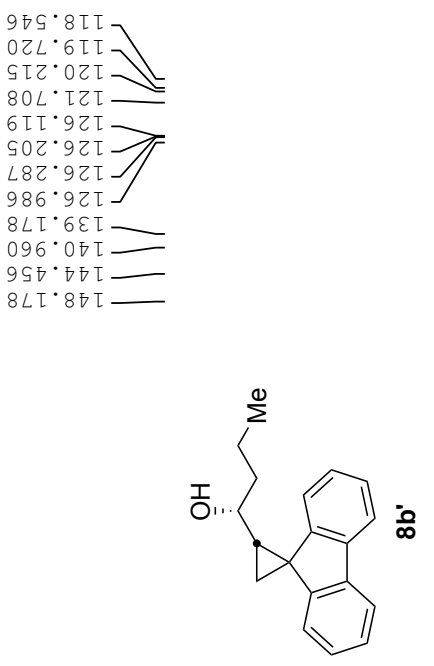

틍

- 

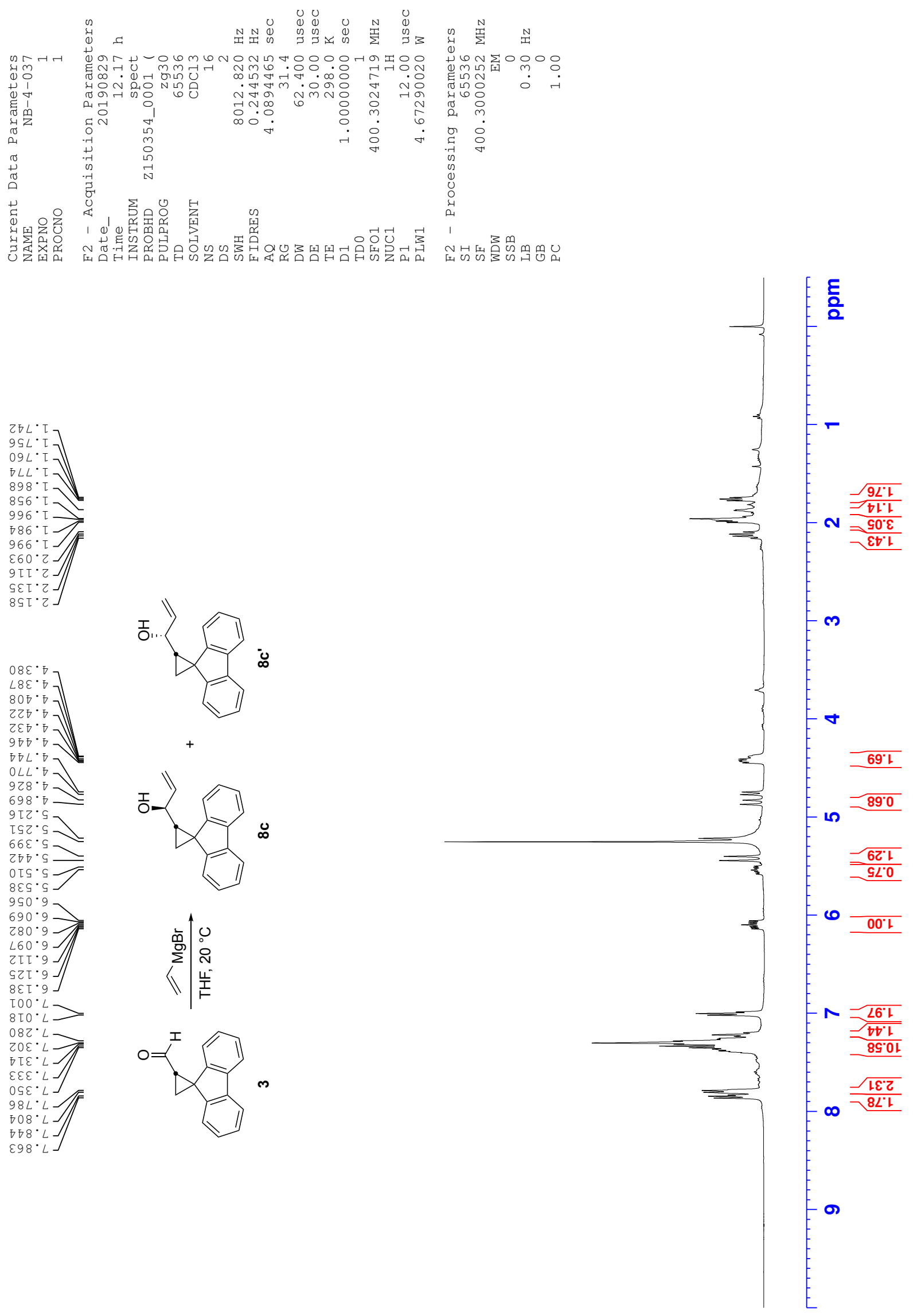

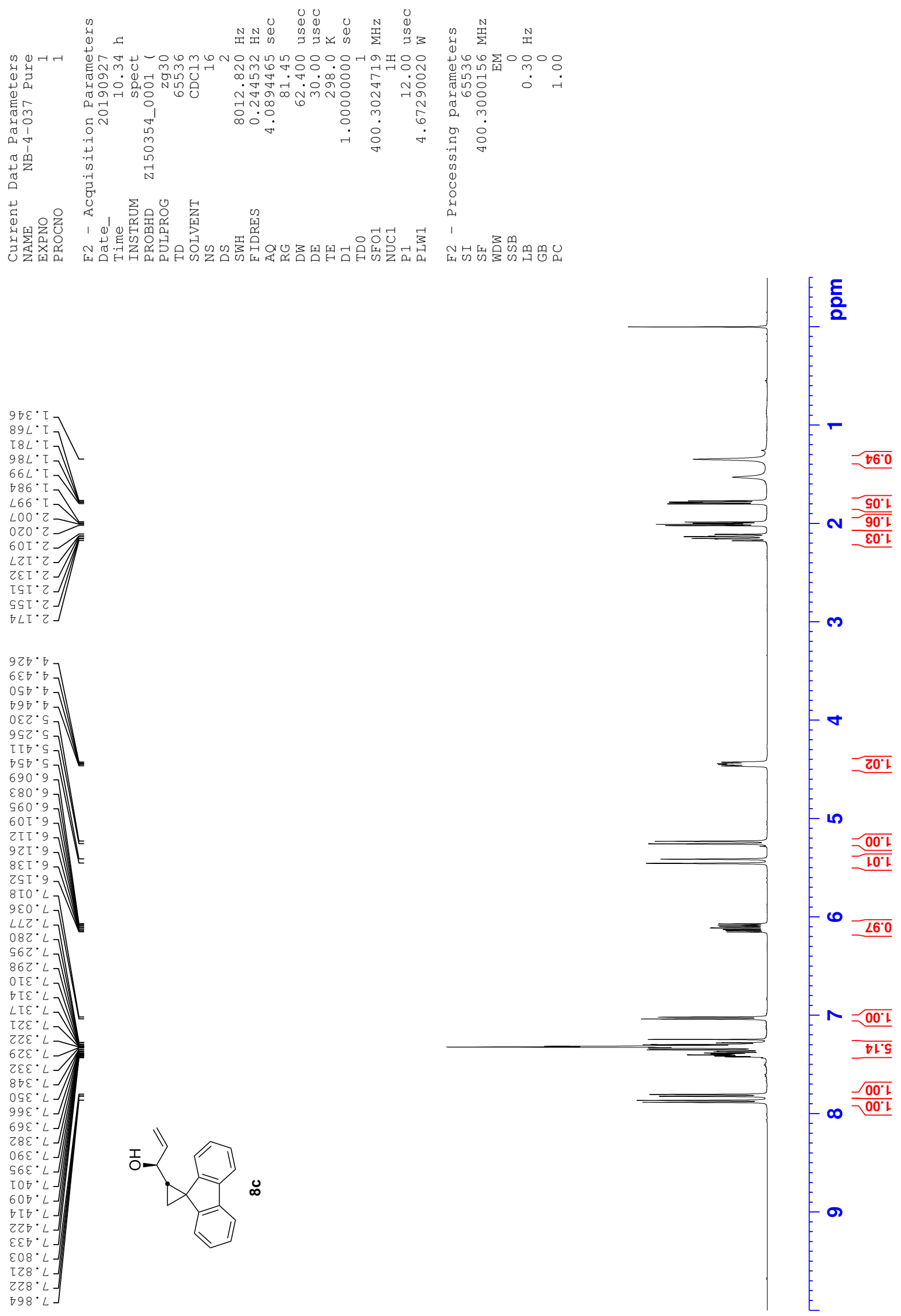

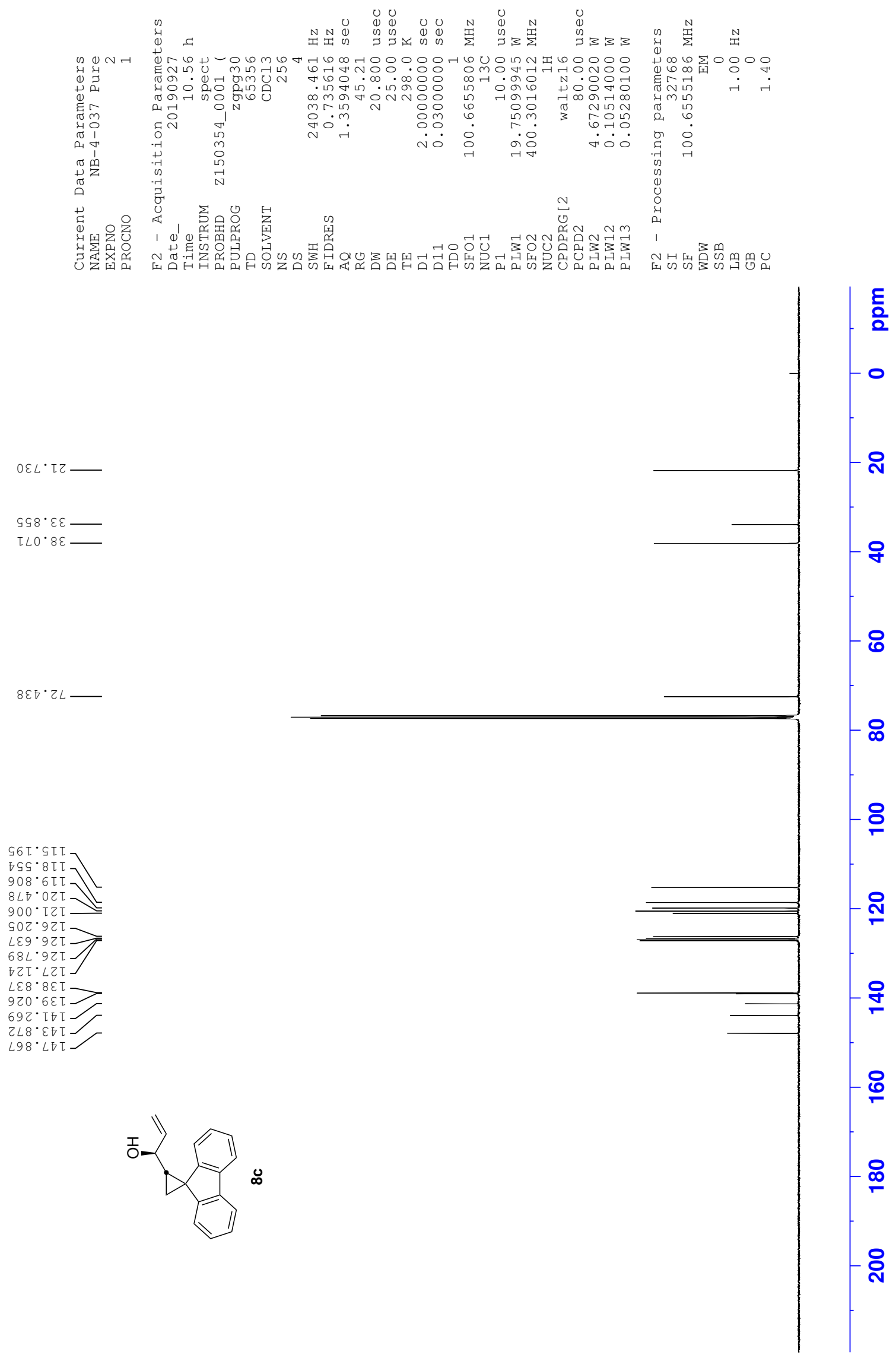

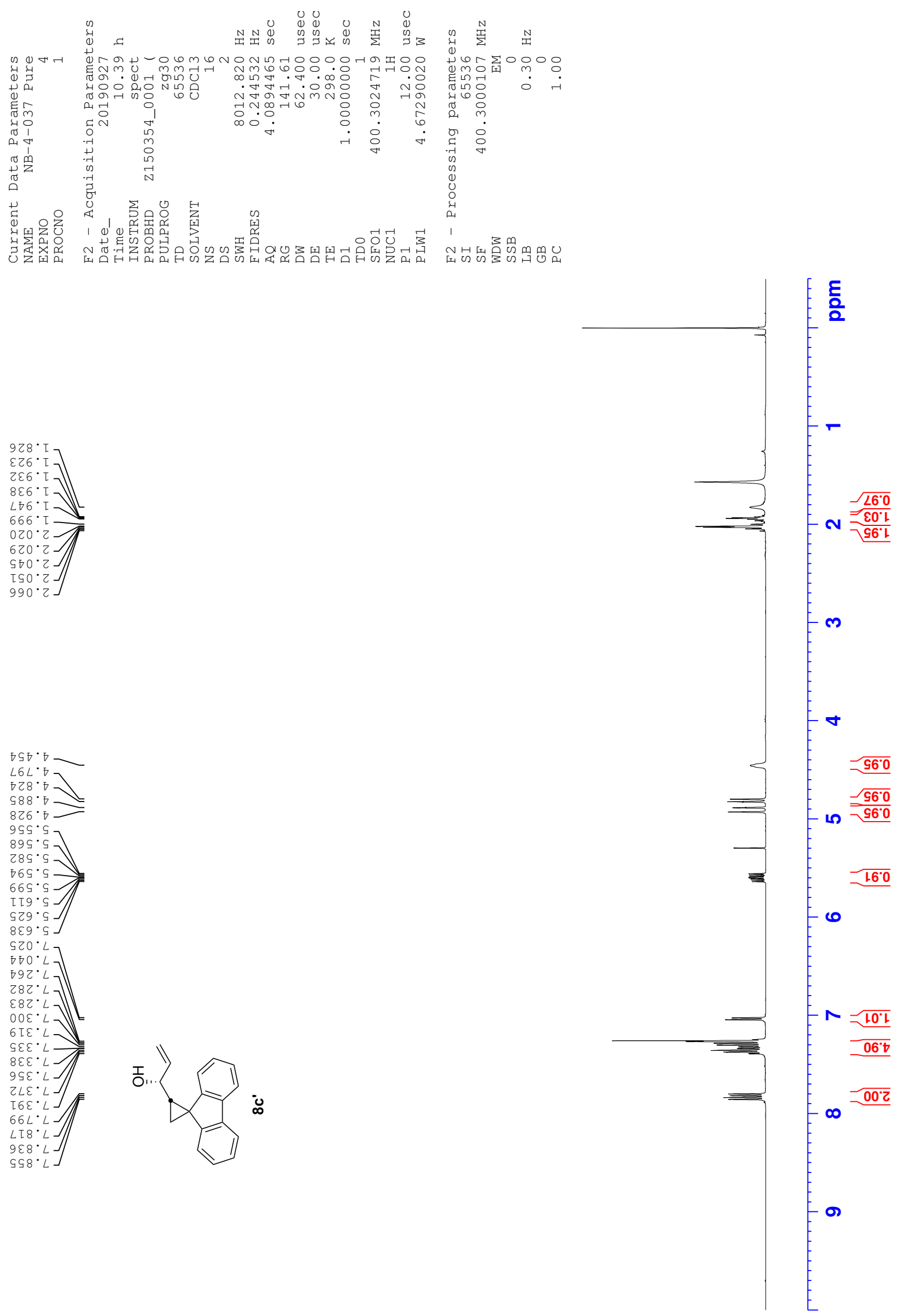

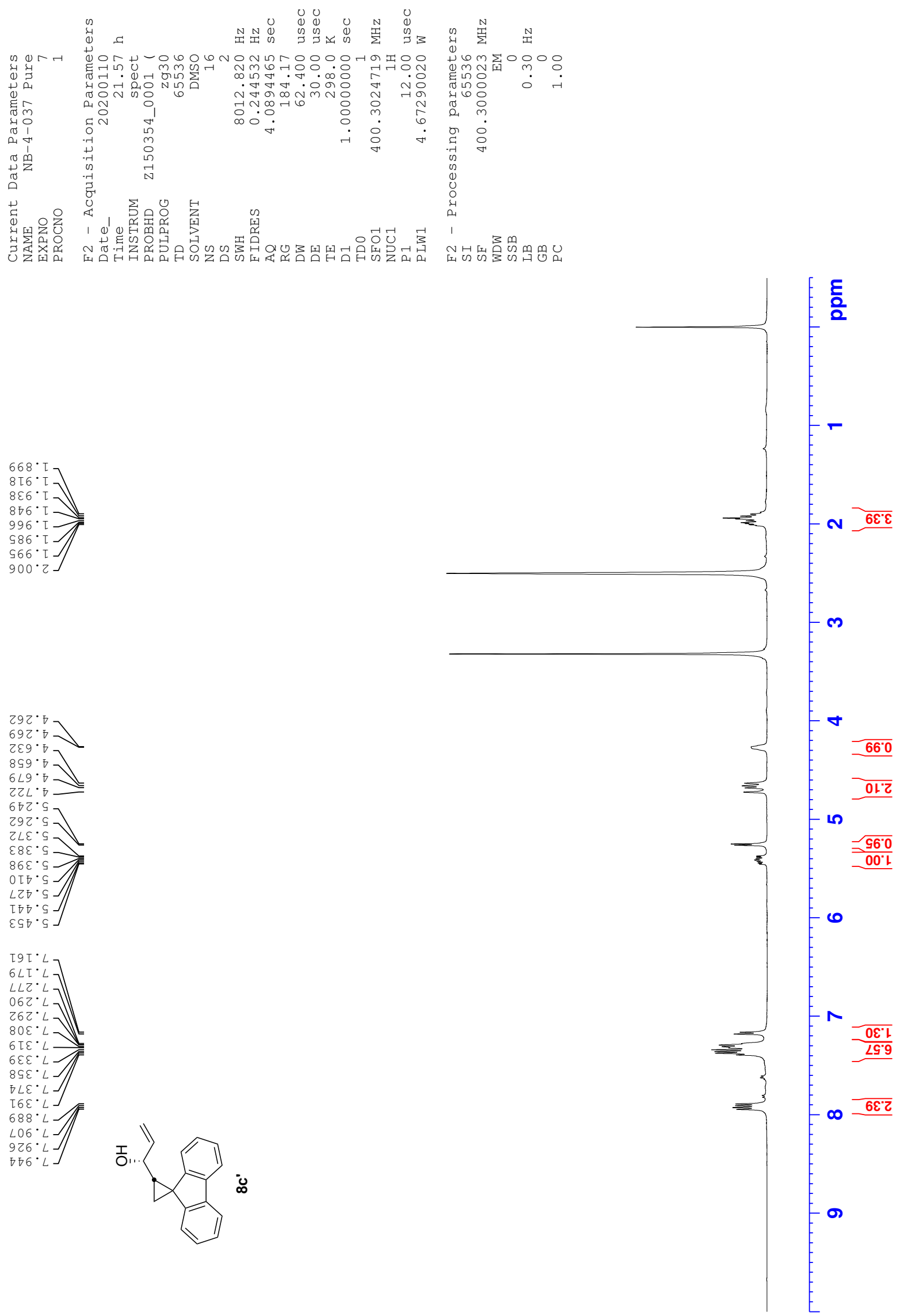

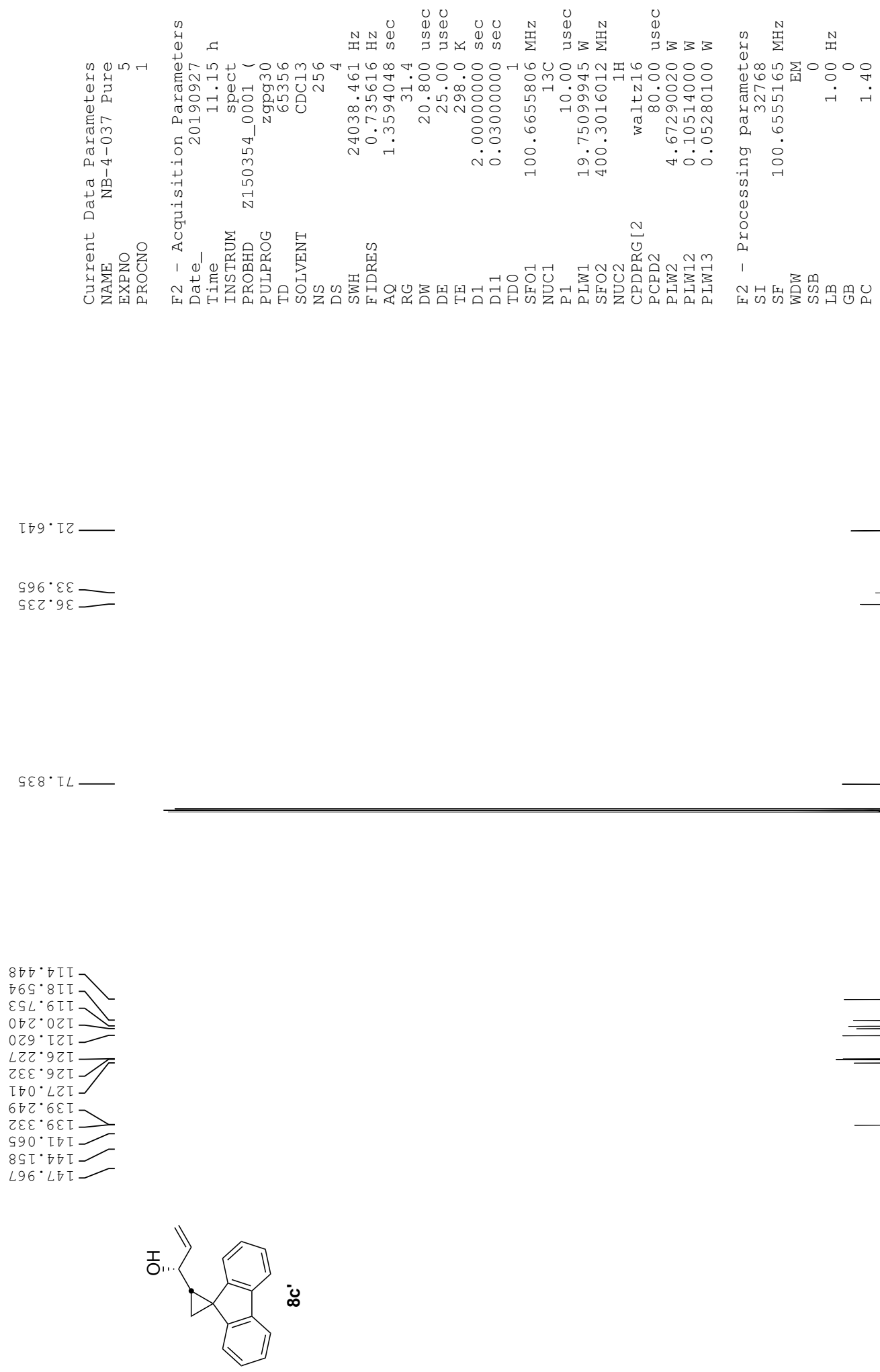

통 

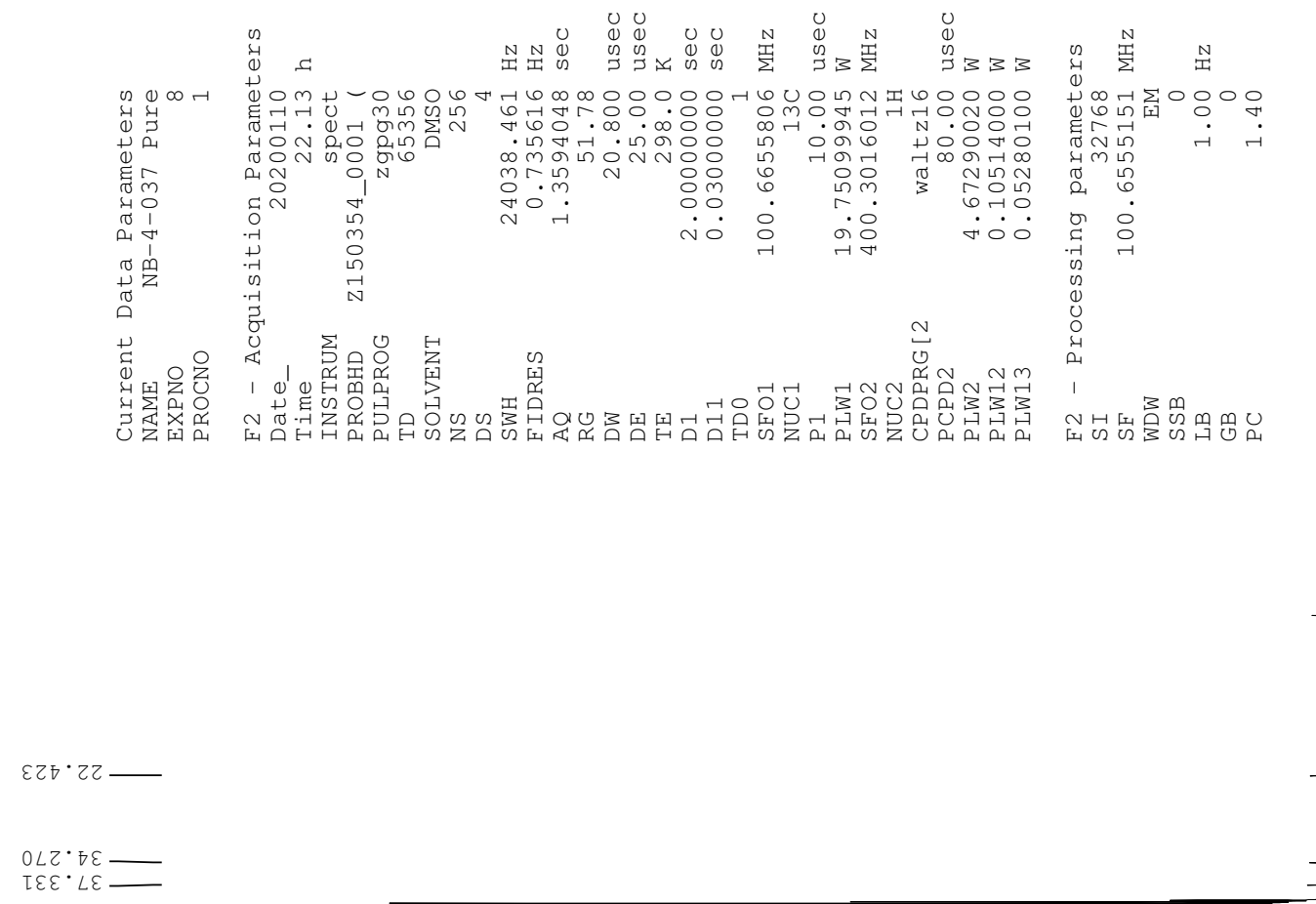

IOZ.OL
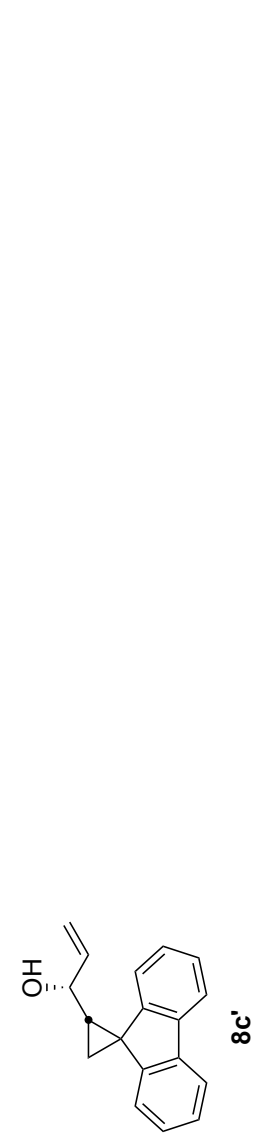

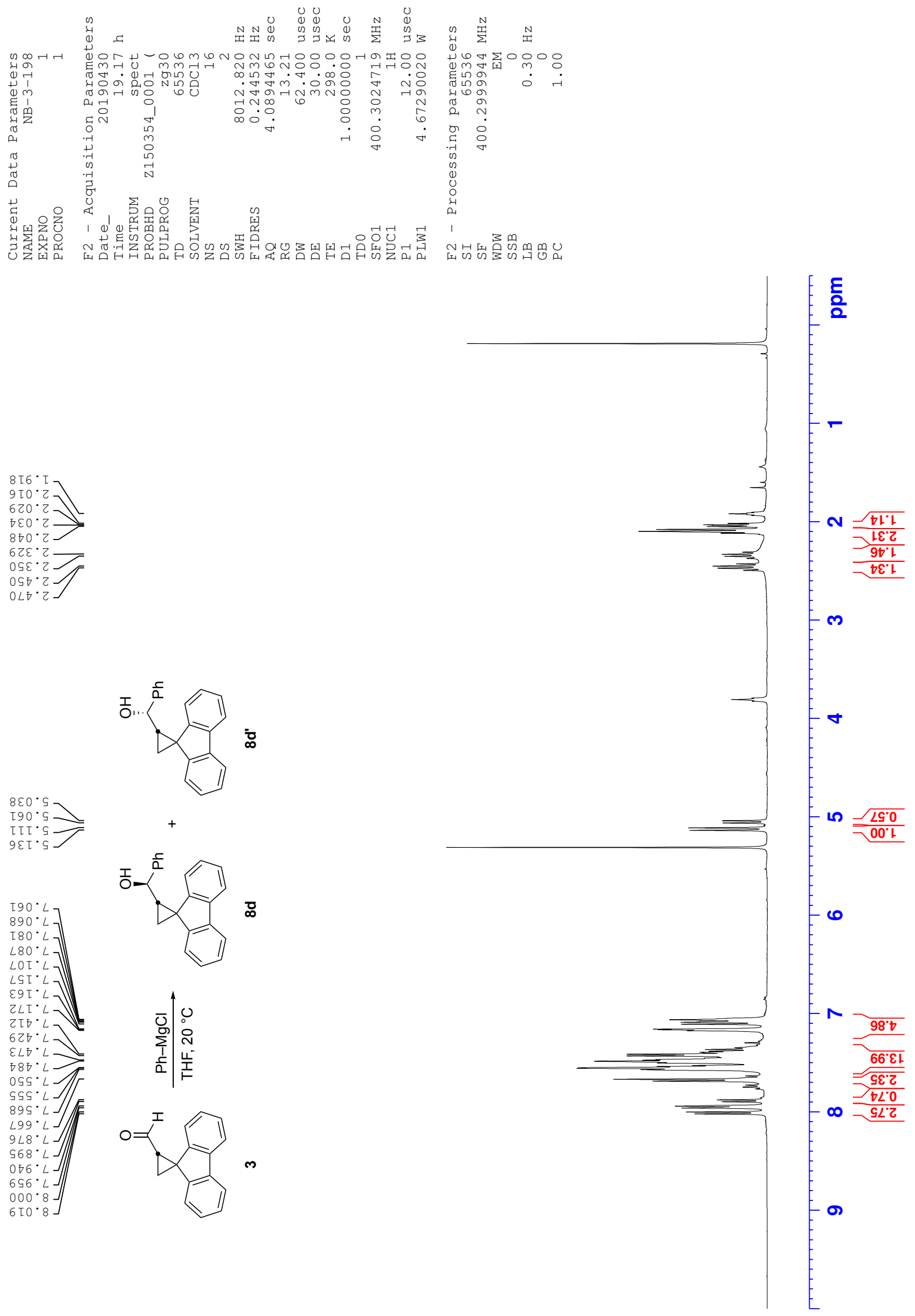

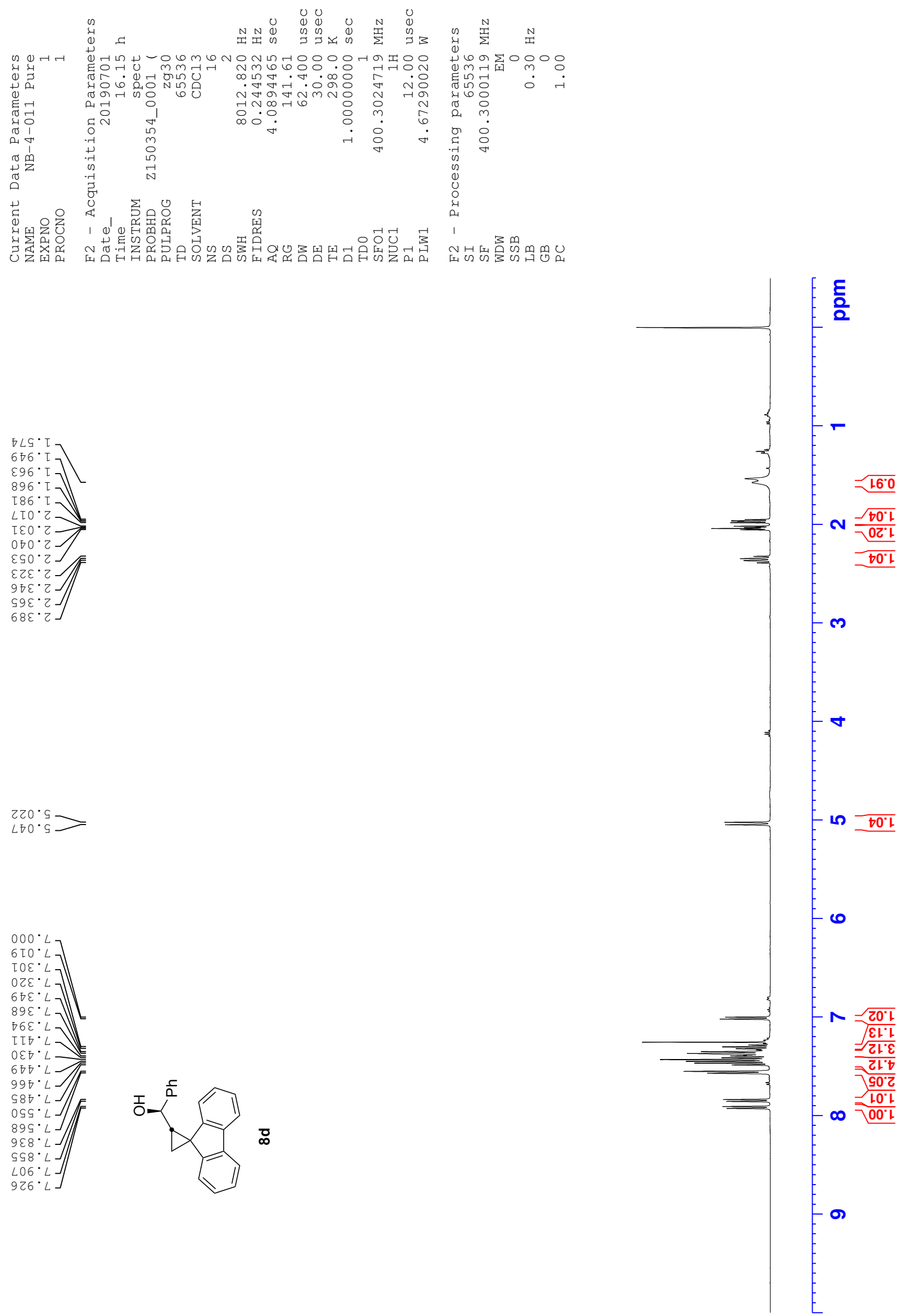

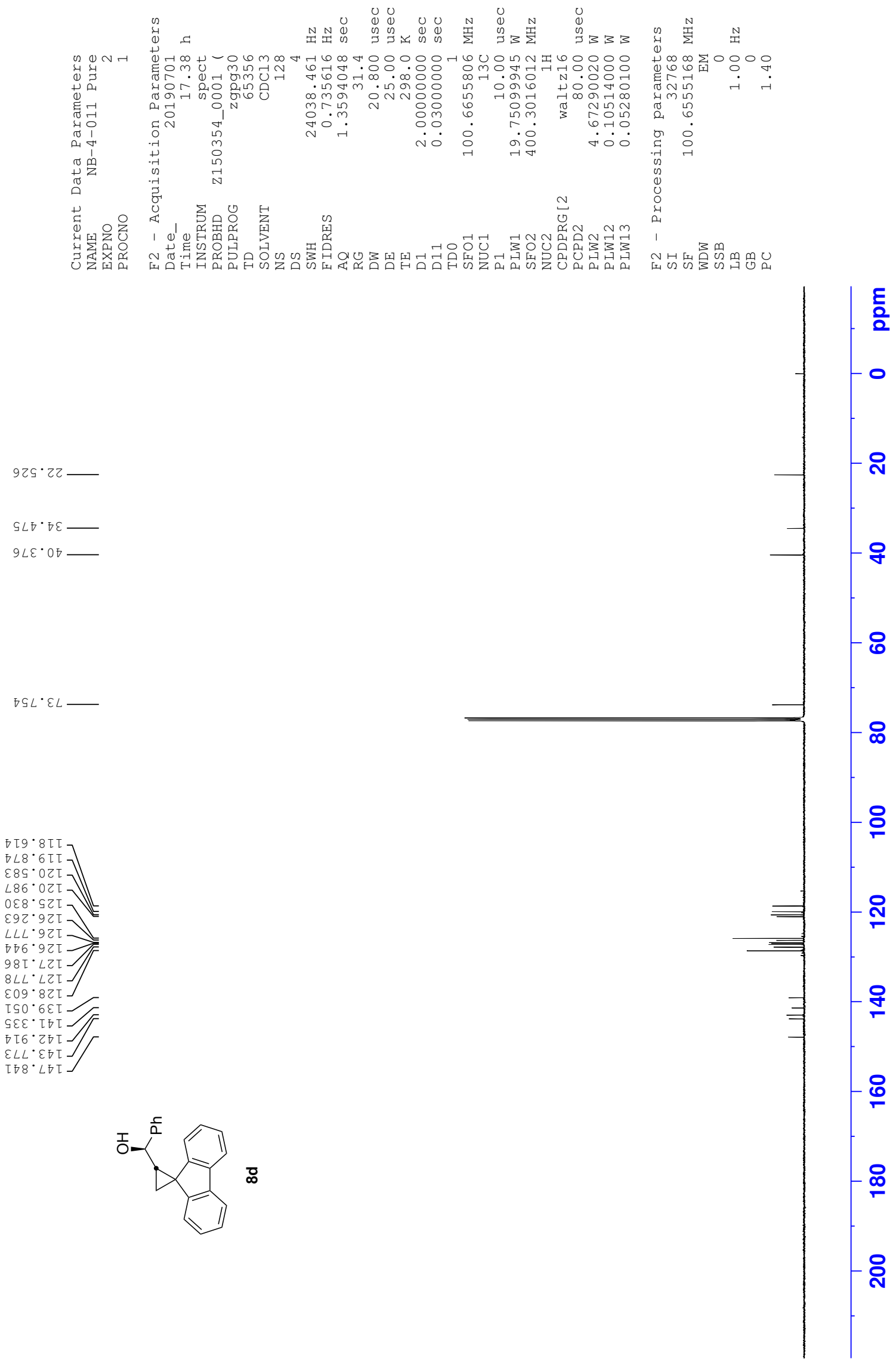

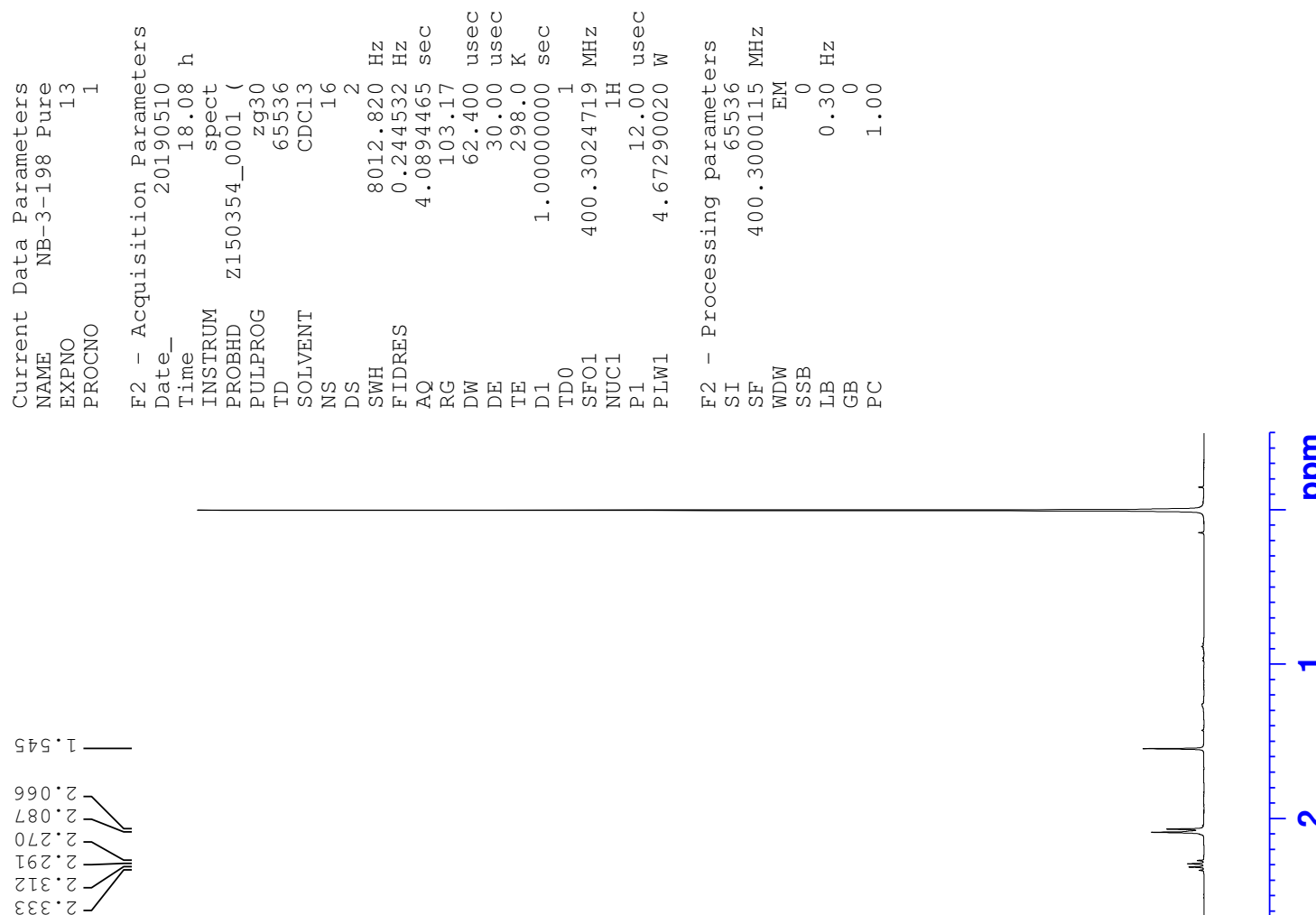

틍
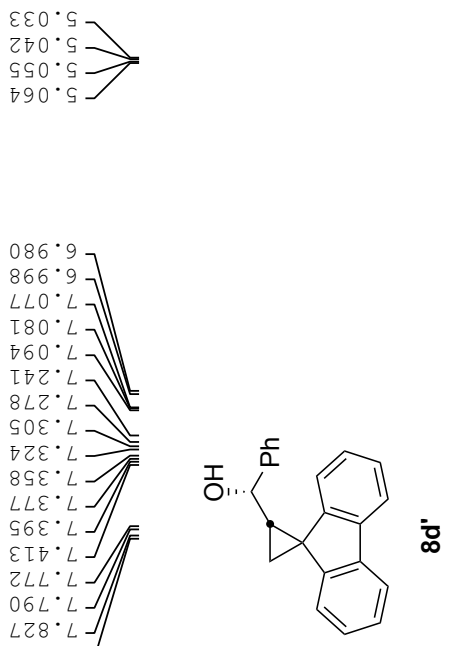

(n)

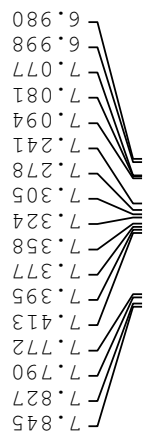

i

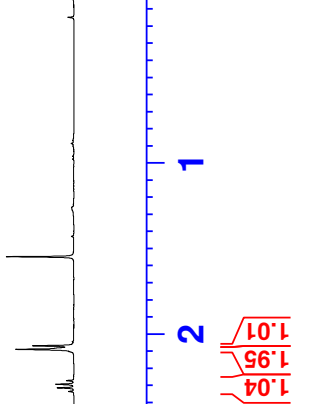

m

6

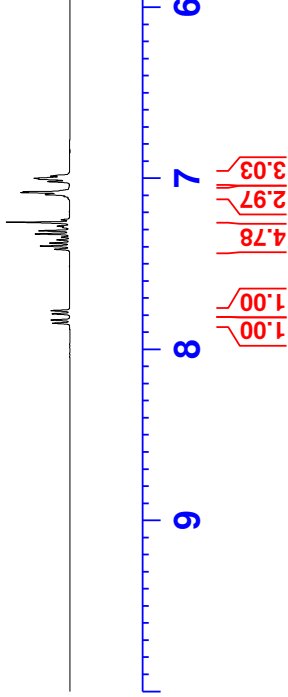



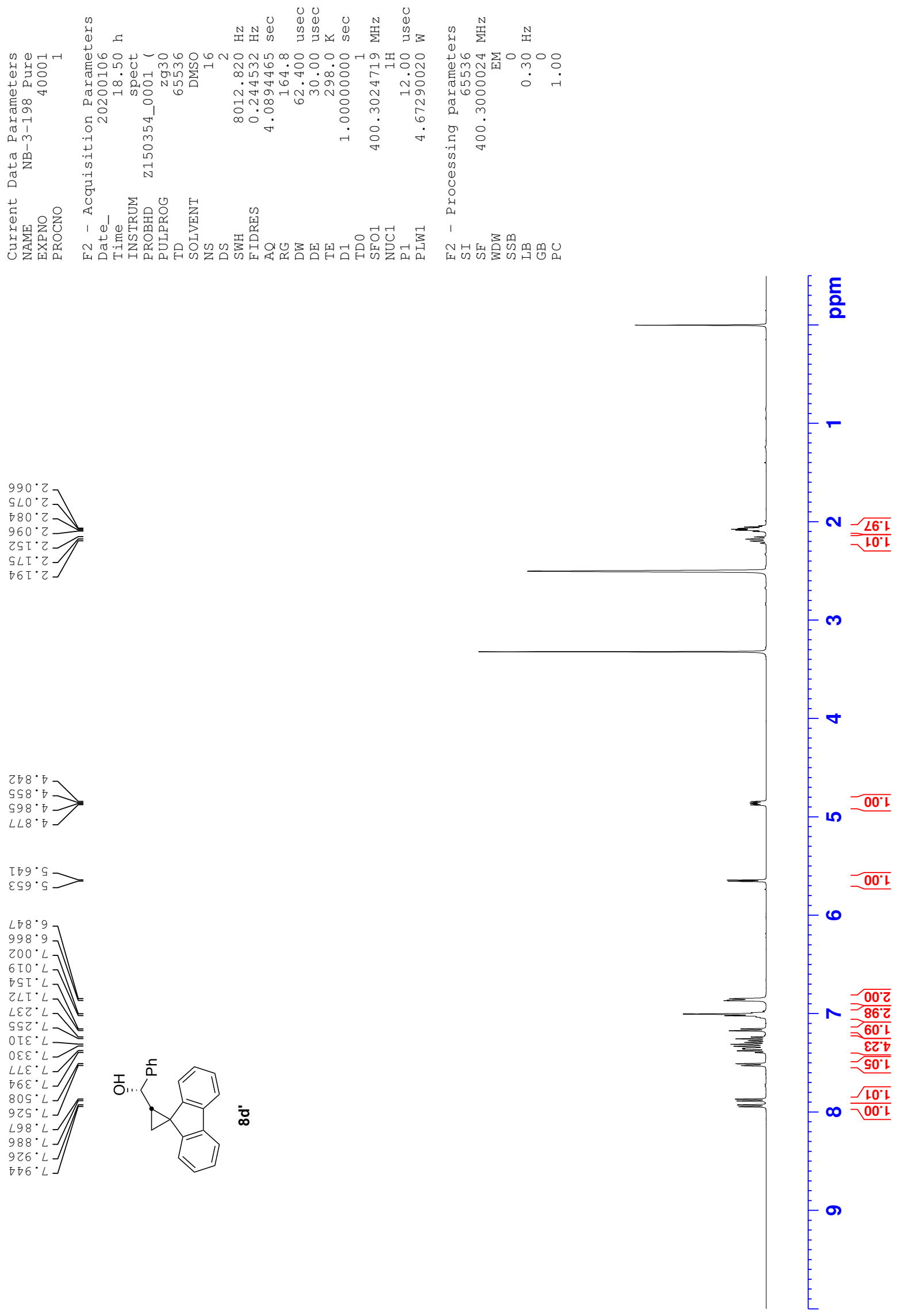

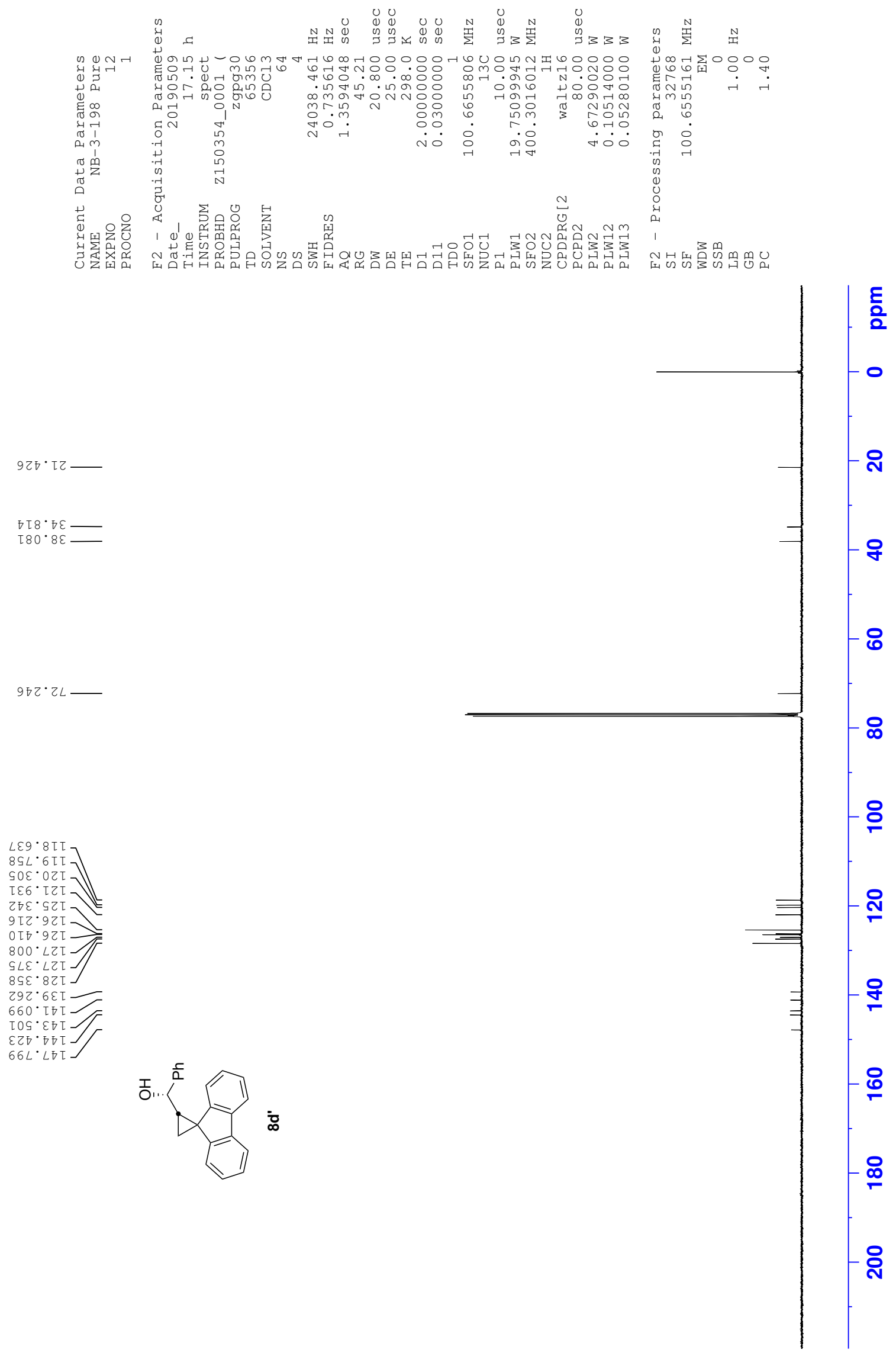

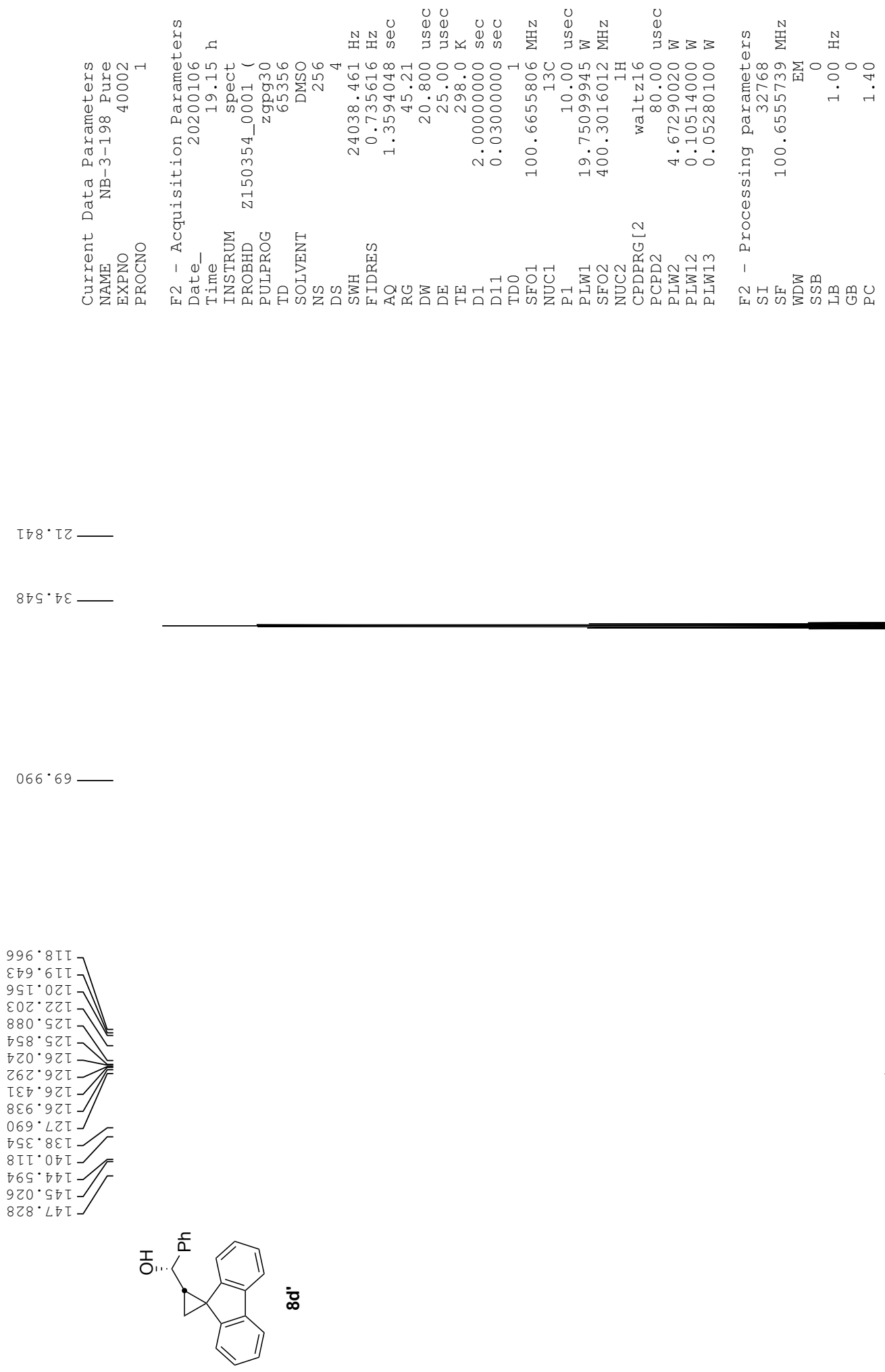

틍

ㅇํ

우

8

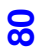

움

ลิ

$\stackrel{q}{\square}$

음

$\stackrel{\infty}{\circ}$

ఫั่ 

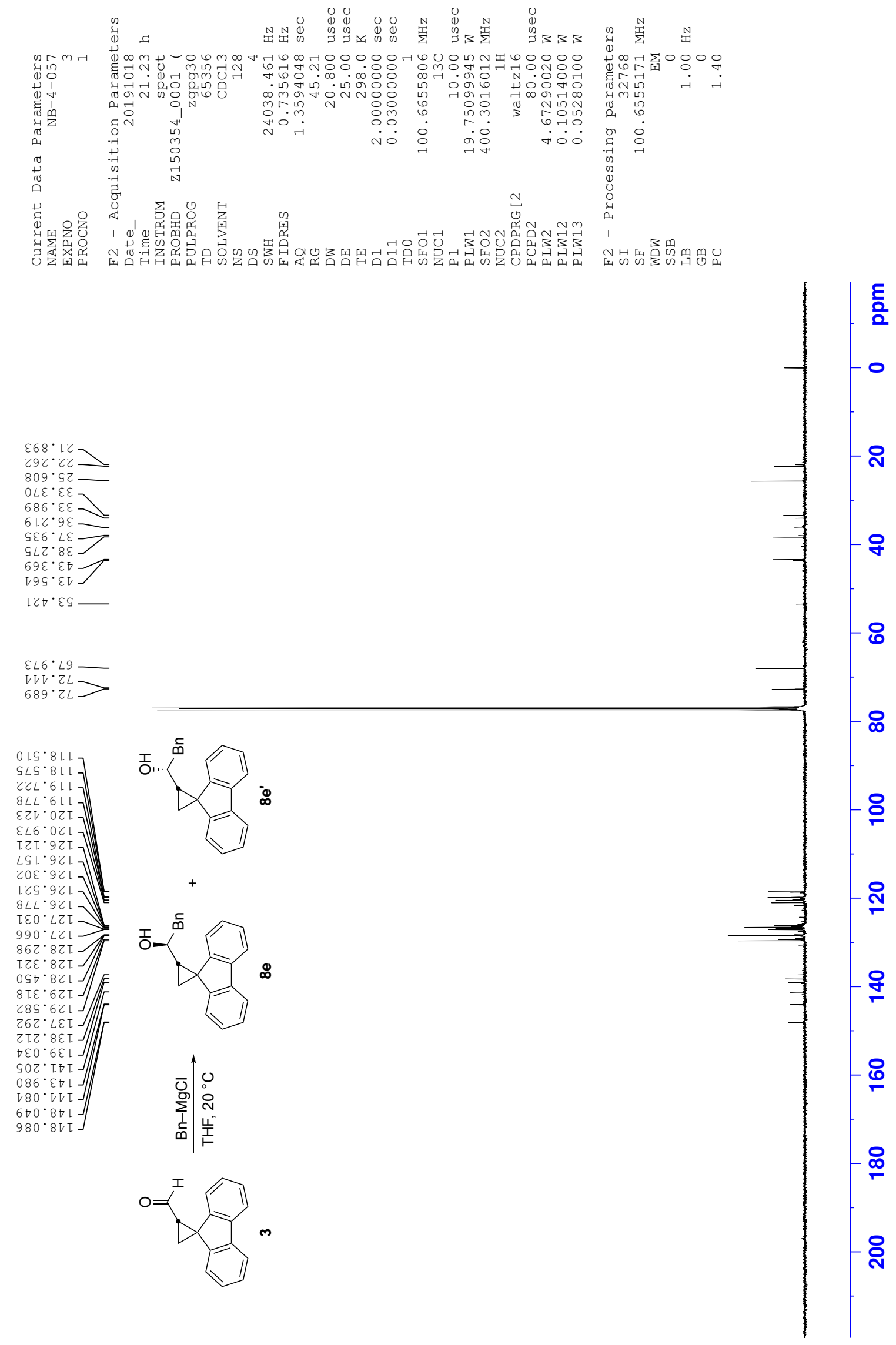

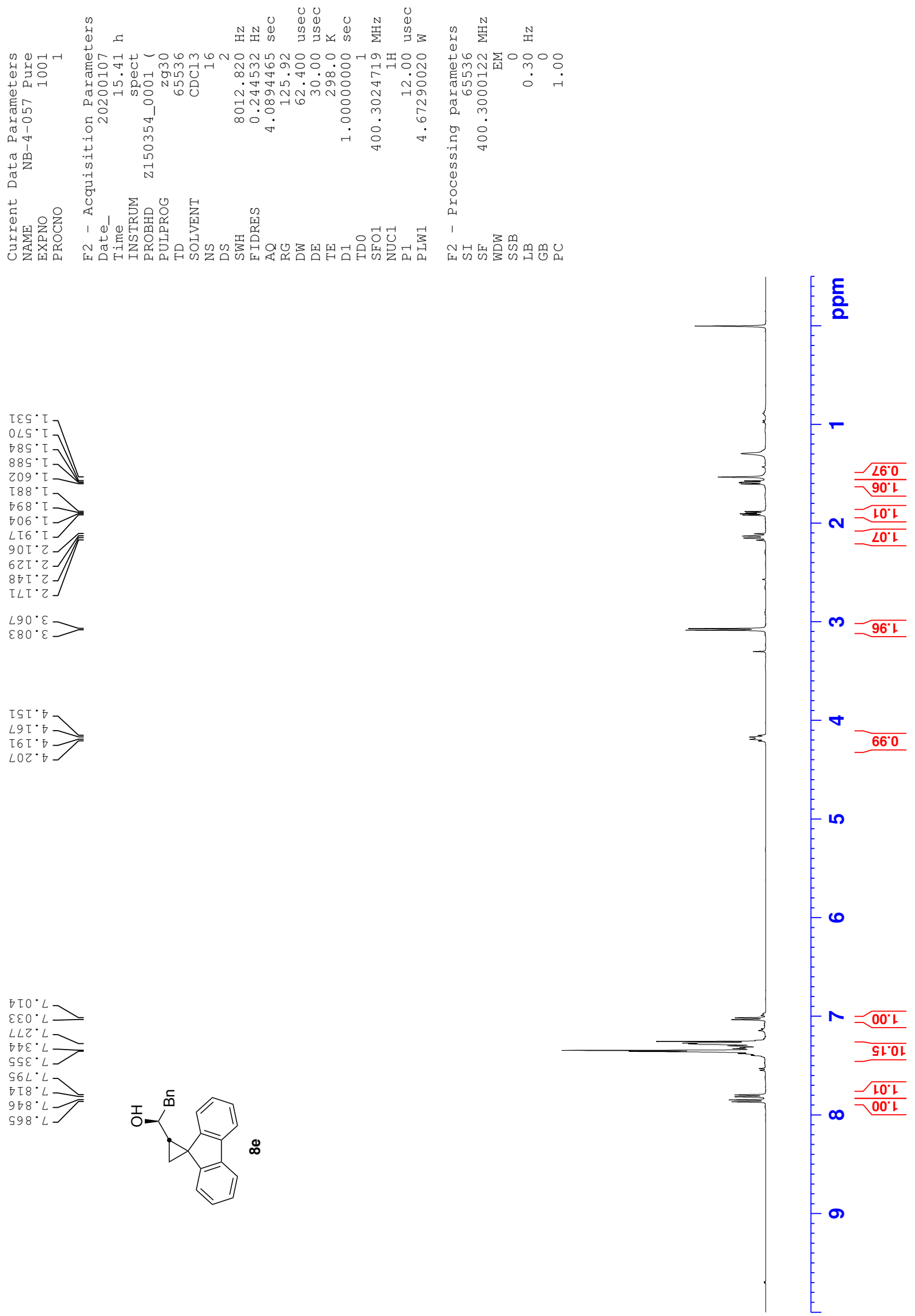

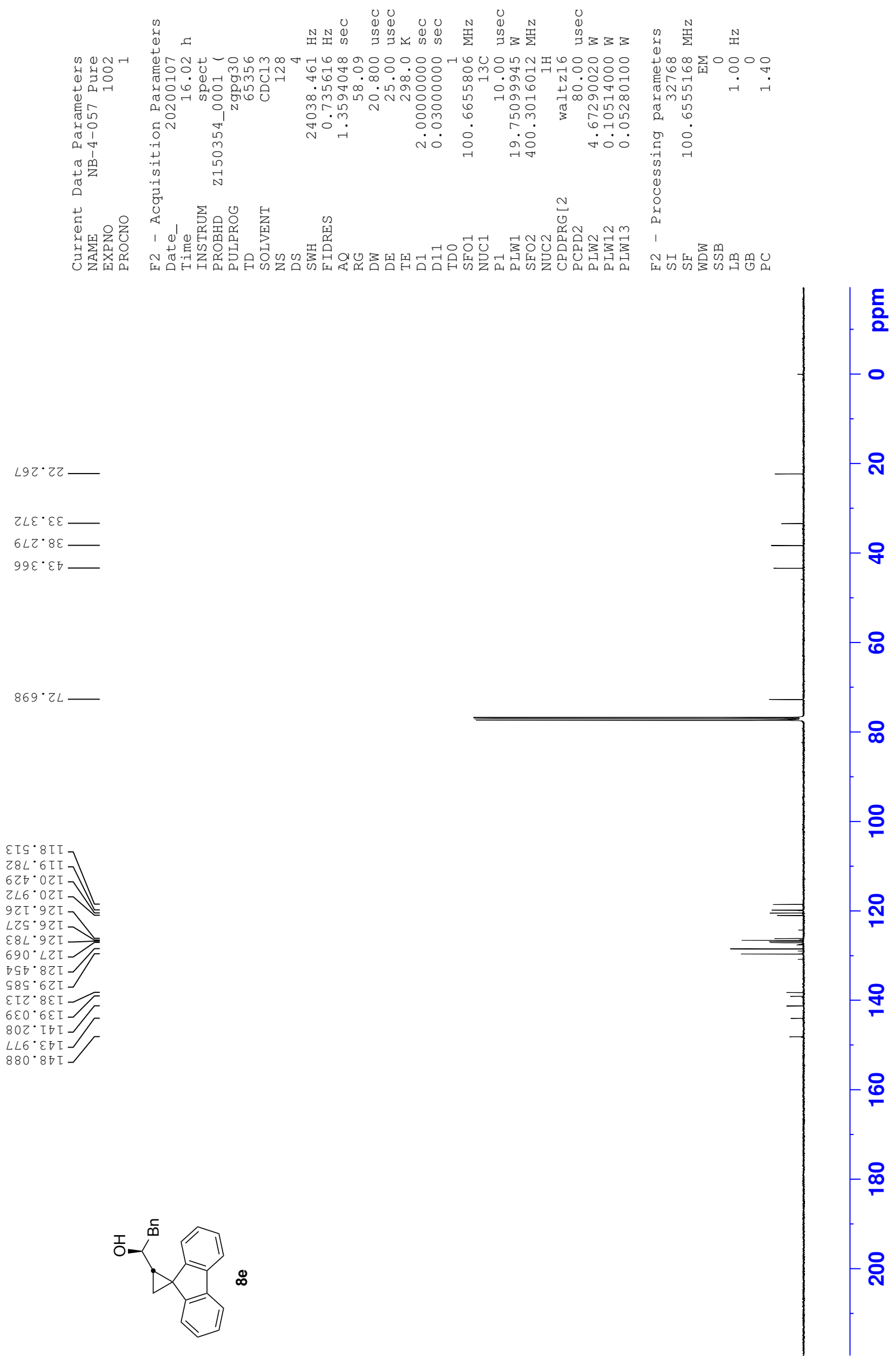

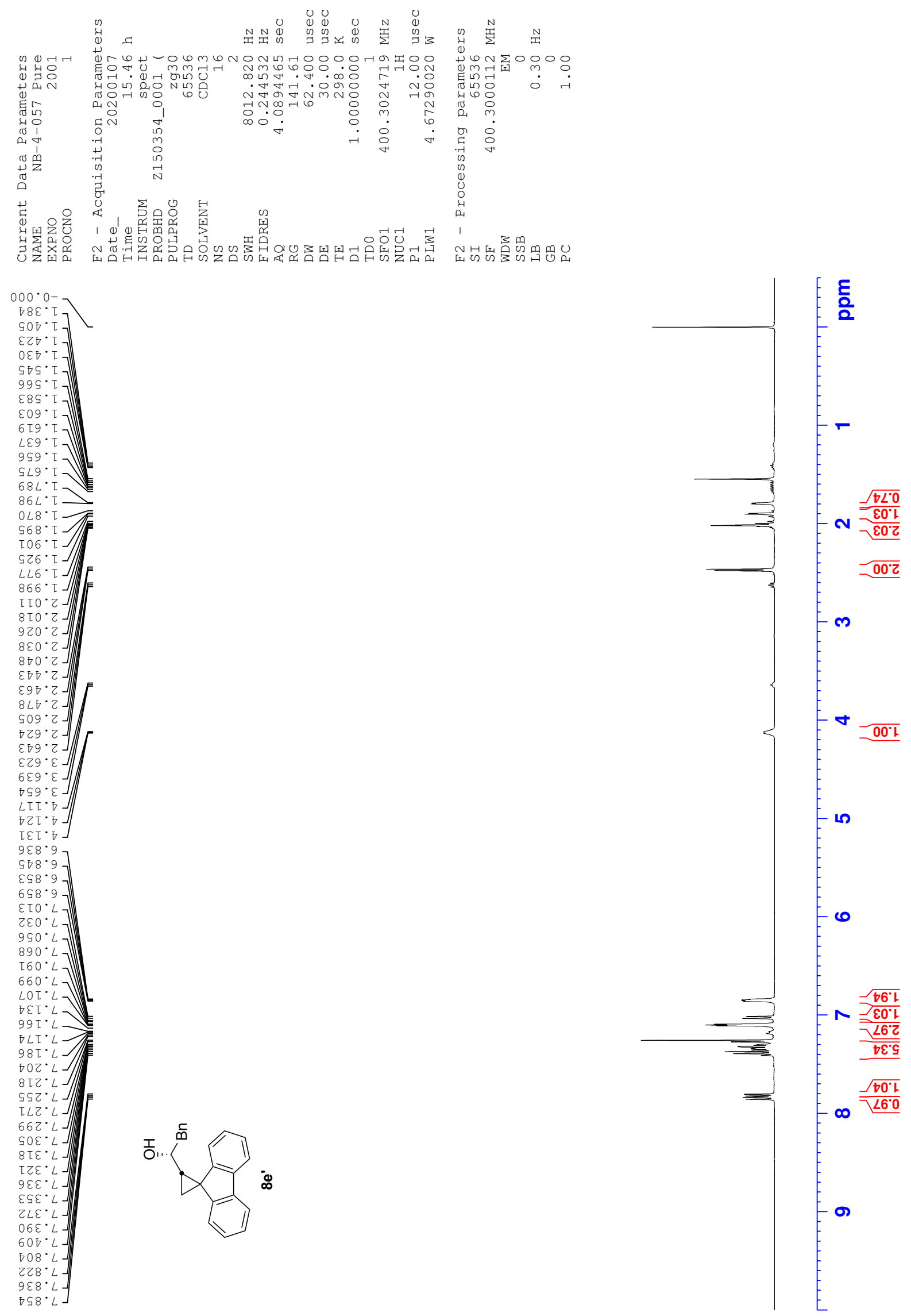

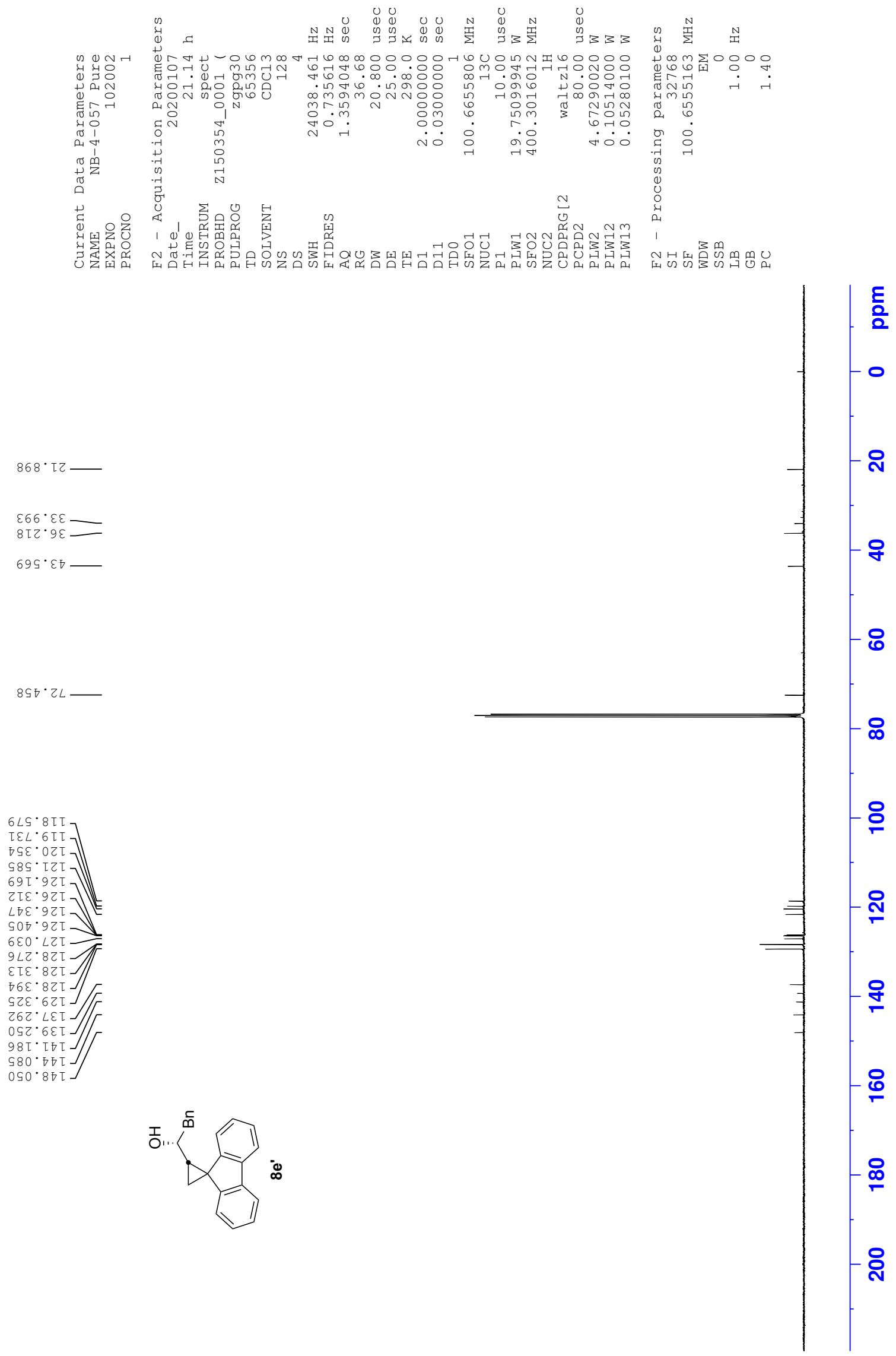

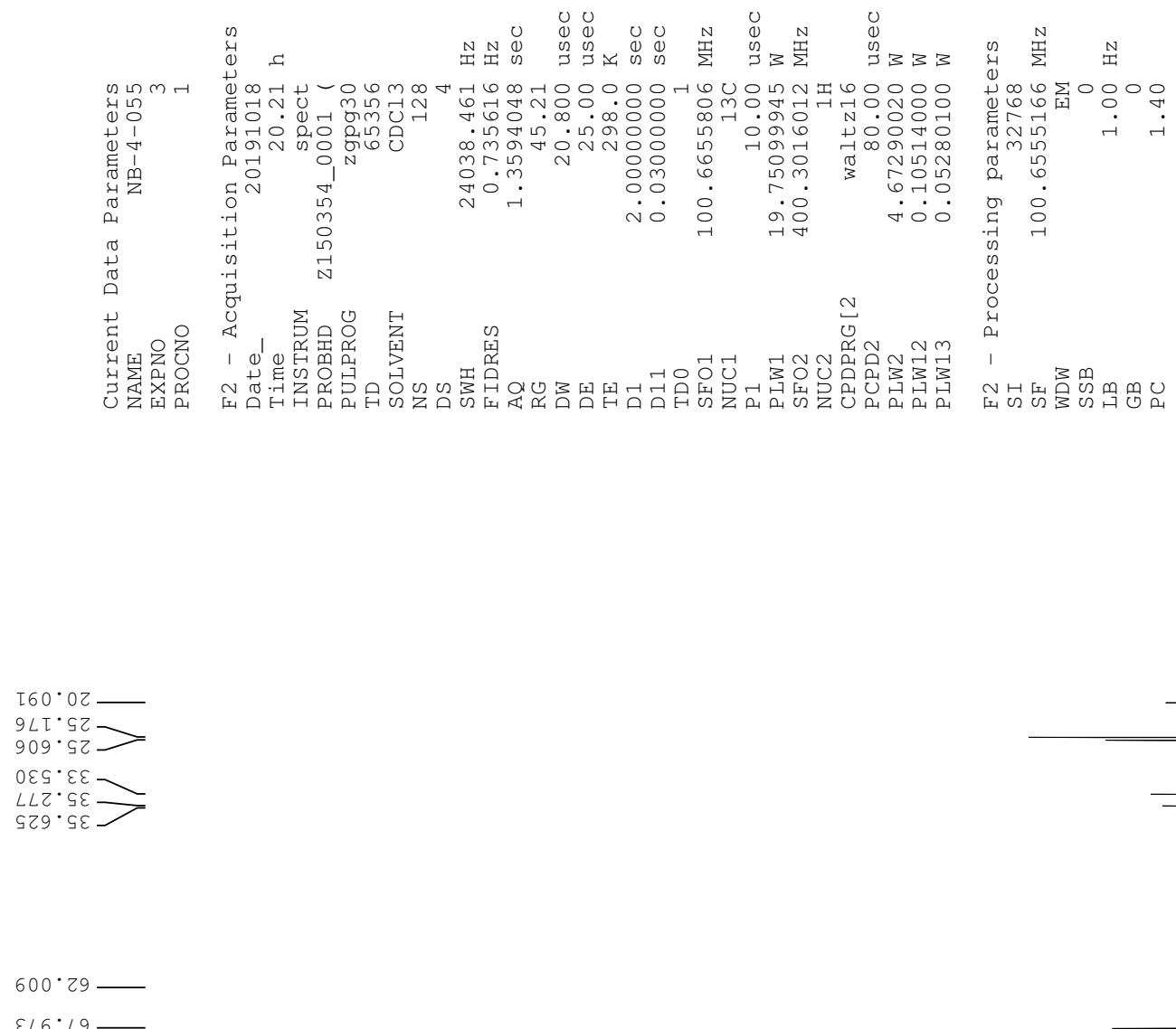

I
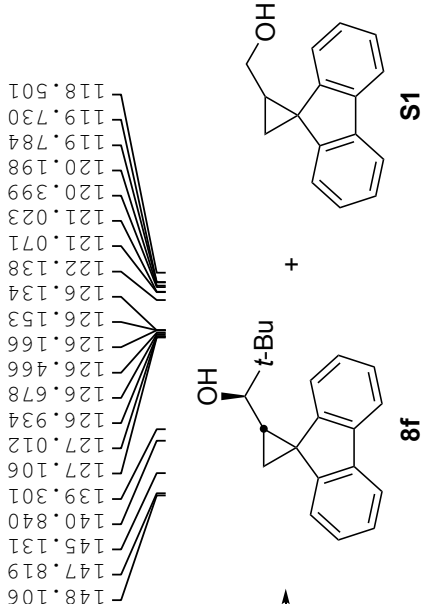

$+$
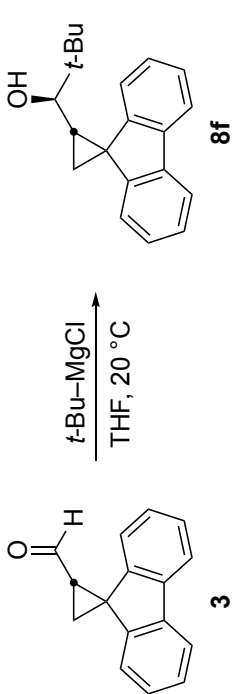

틍

ำ

q

$\infty$

움

กิ

움

음

$\stackrel{\square}{\square}$

ัิ 

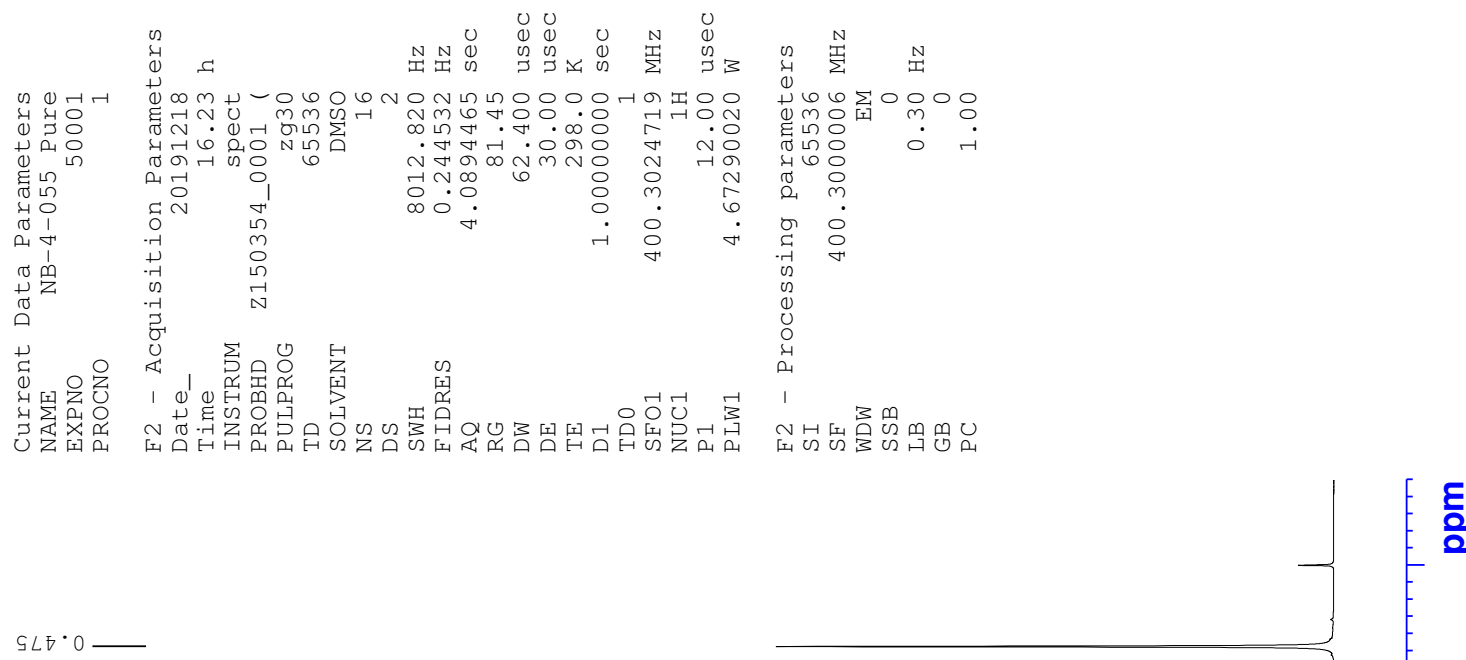

$S \angle D^{\circ} 0$
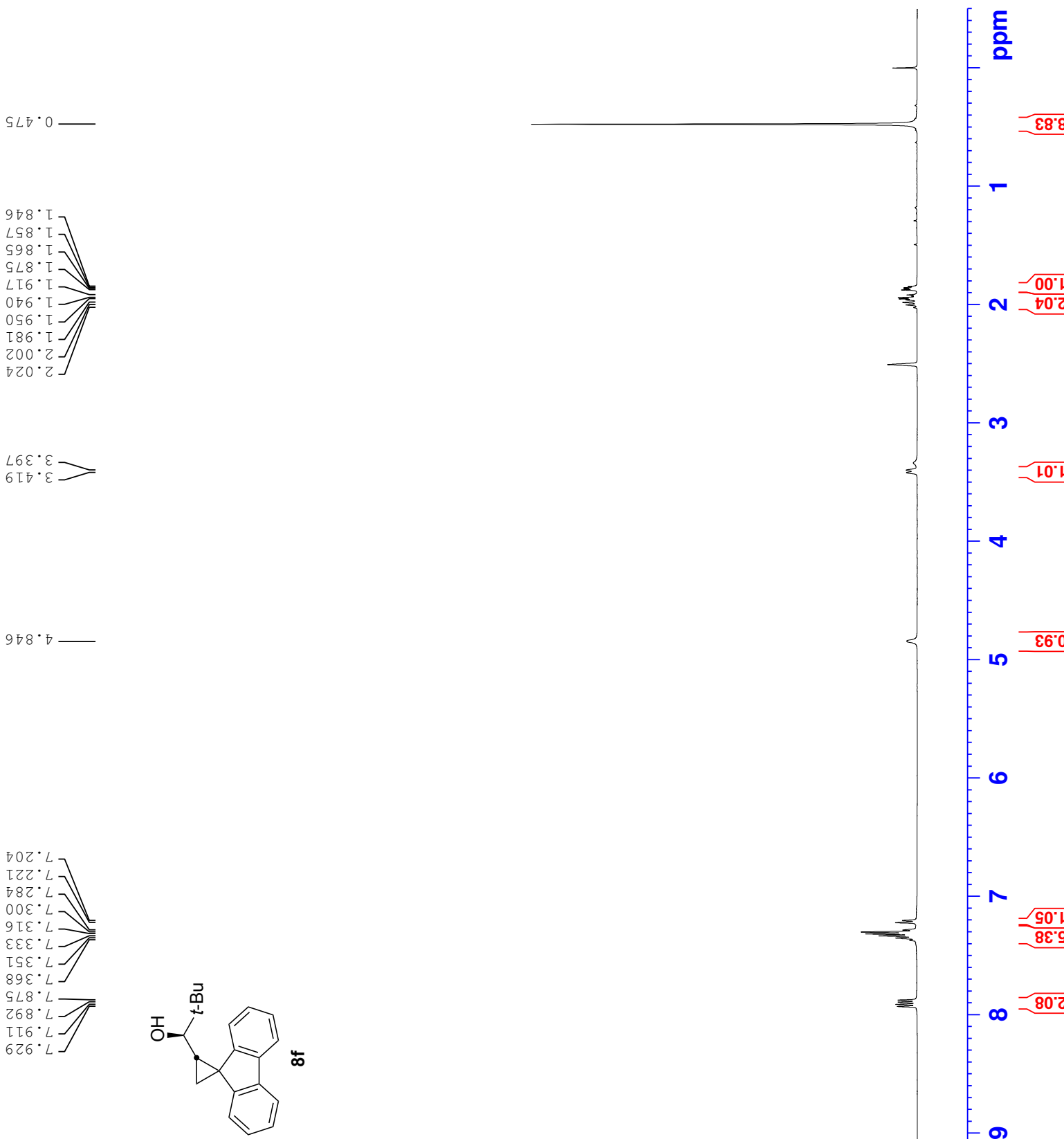

$\frac{1}{2}$
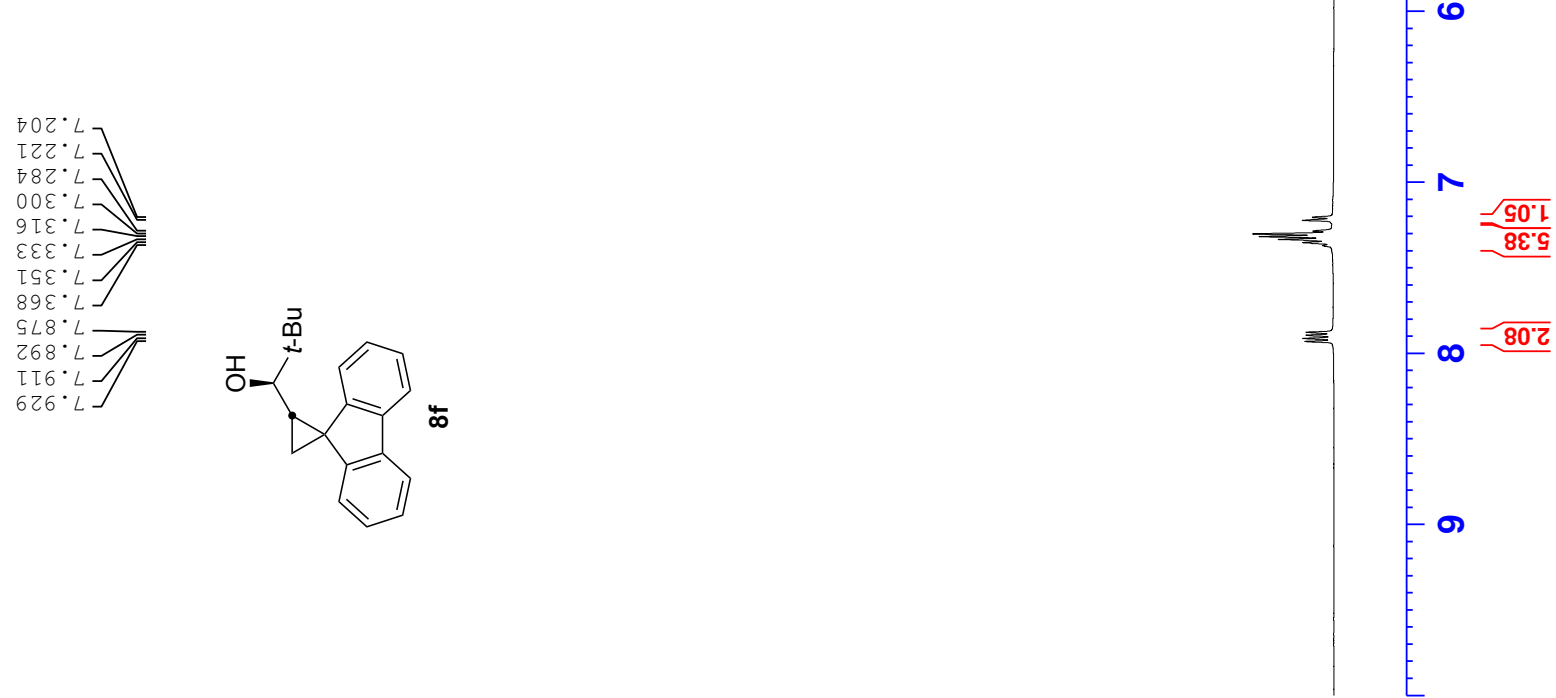

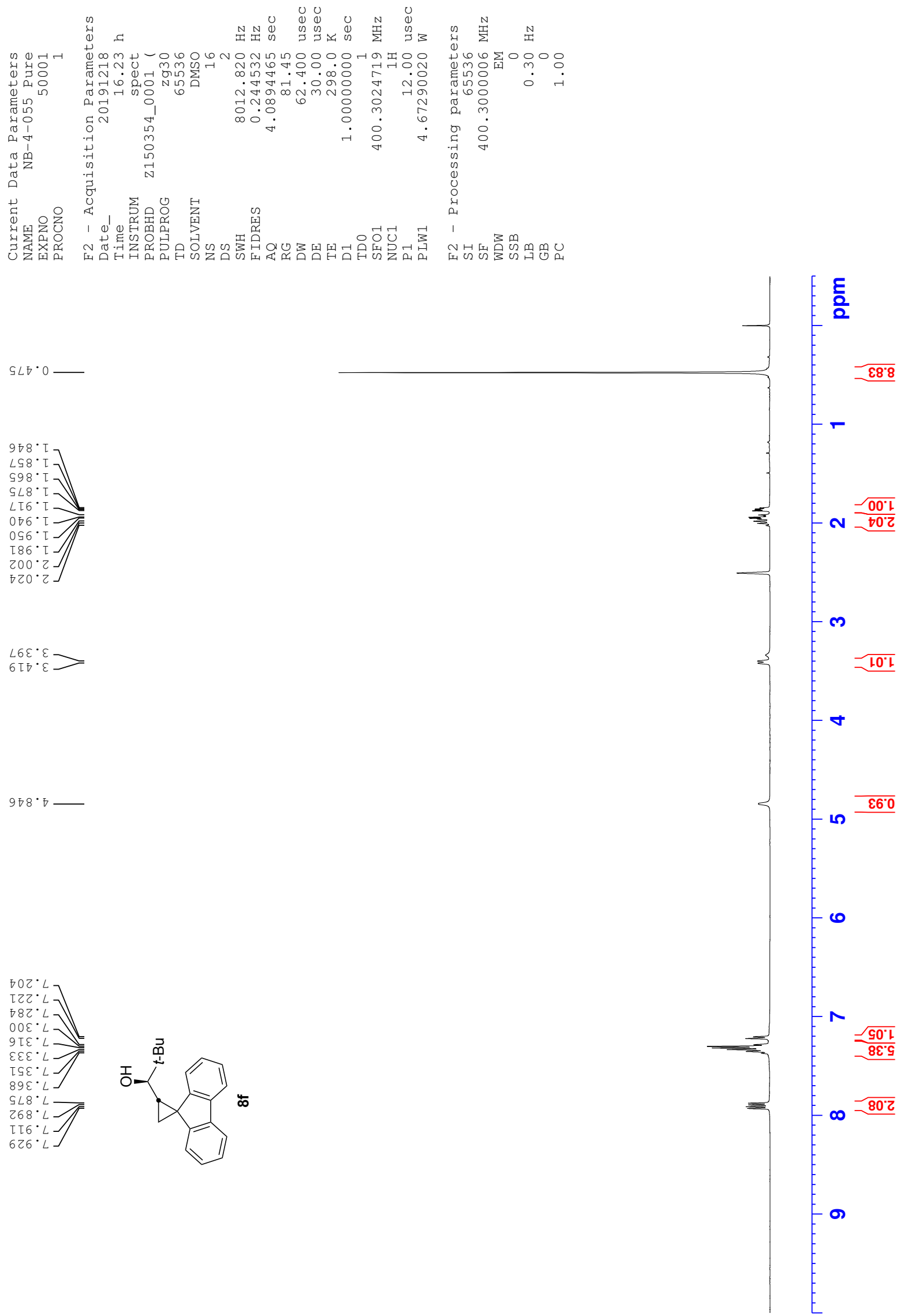

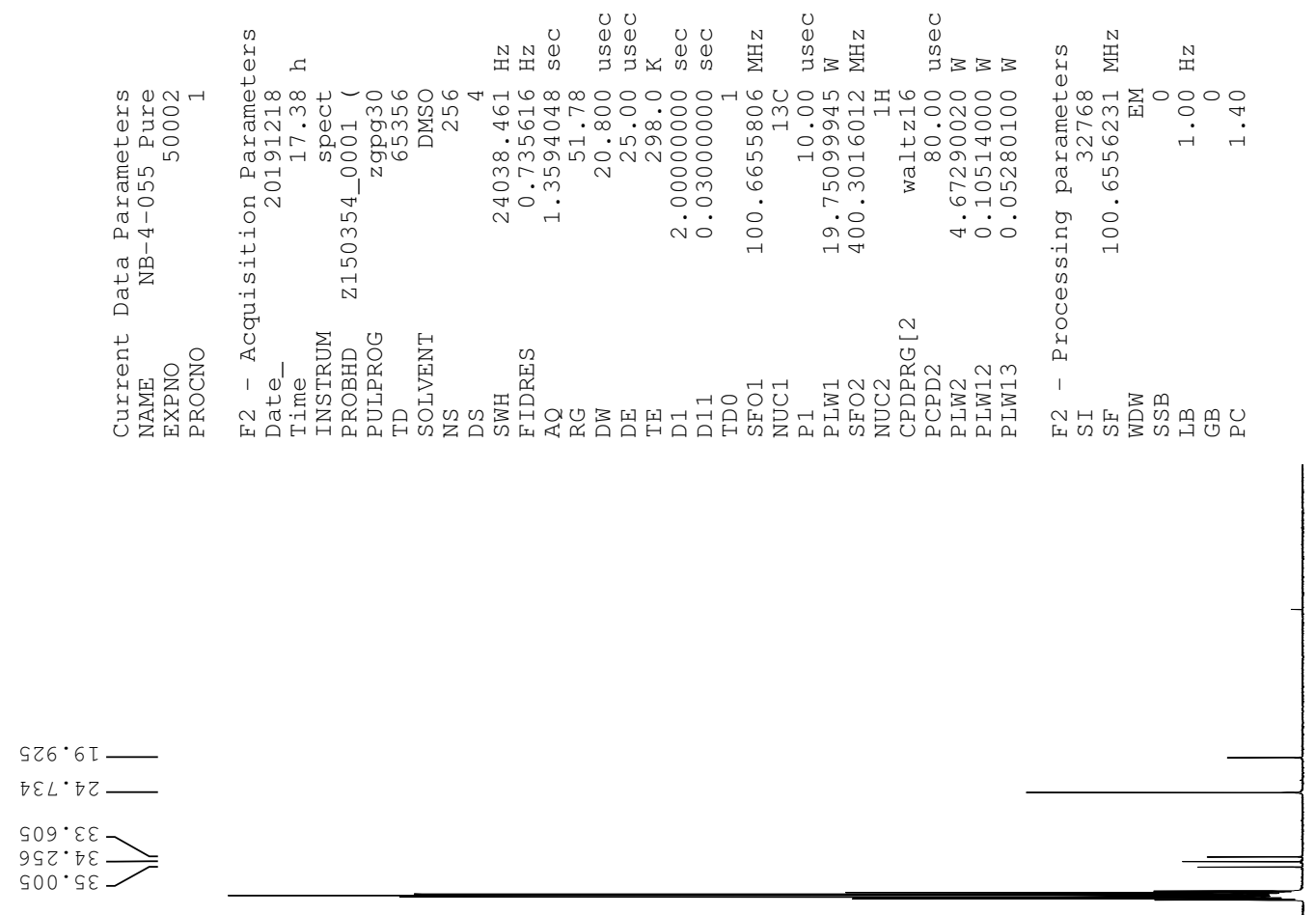

틍

$\varepsilon L 8 \cdot \varepsilon L$
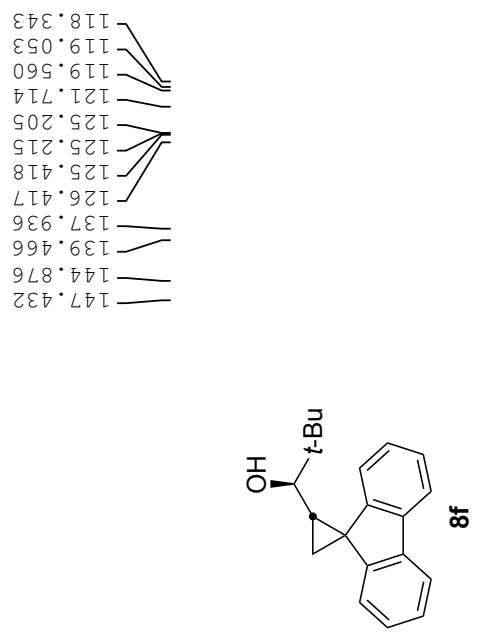

กิ

q

$-8$

ळి

음

ำ

웅

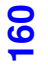

$\stackrel{\infty}{\check{\infty}}$

: 

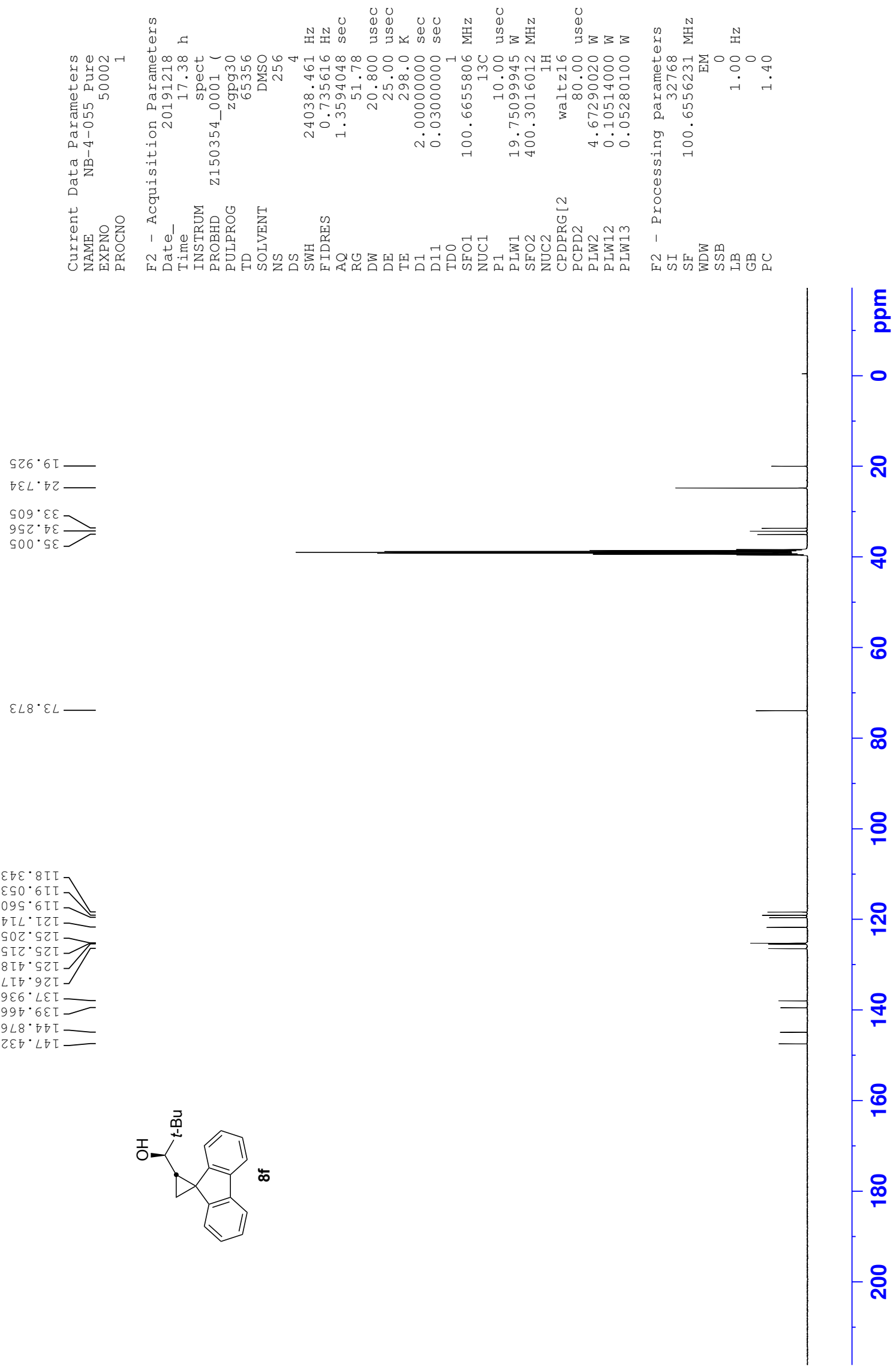

ㅇํ 

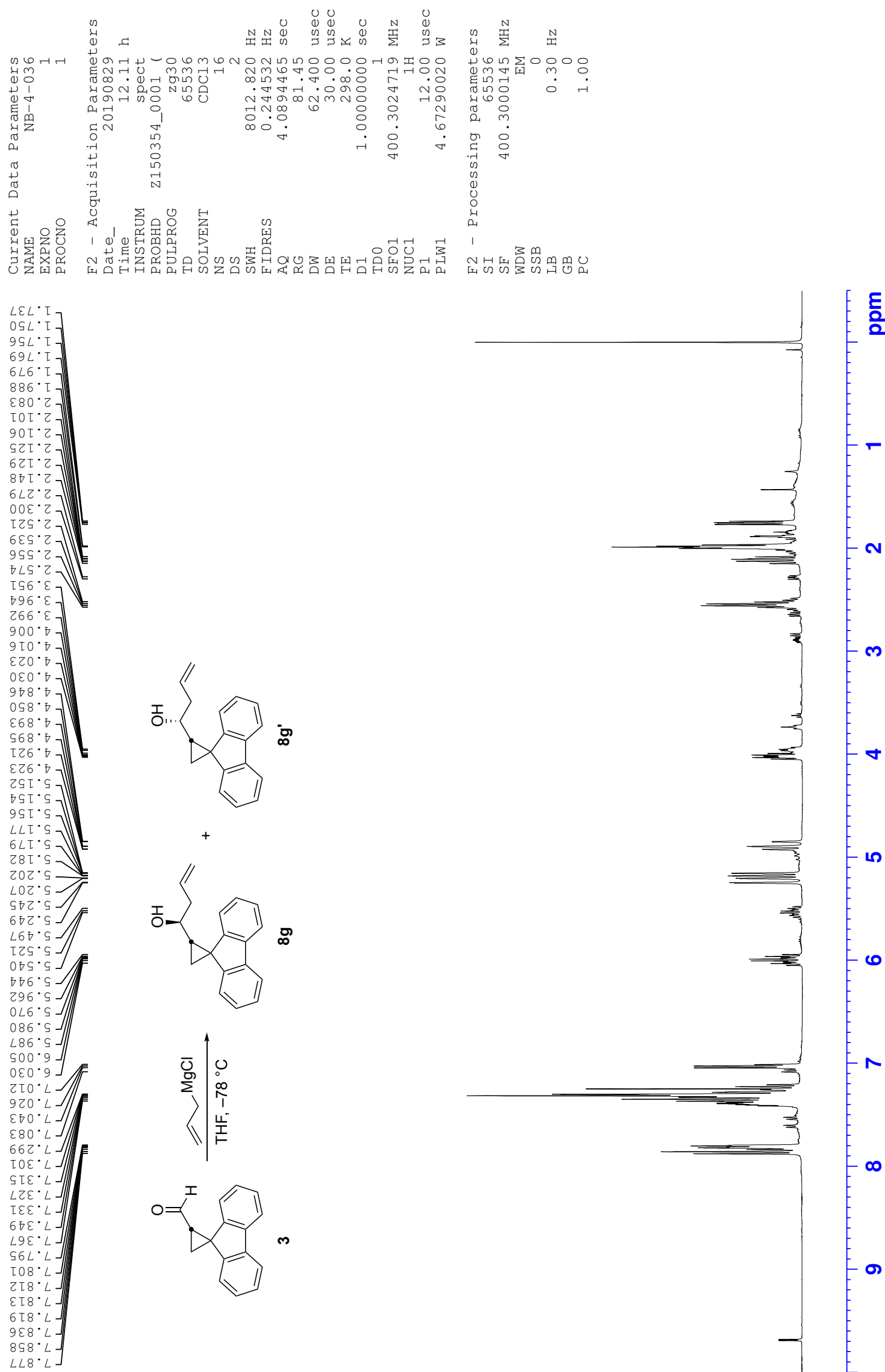

틍
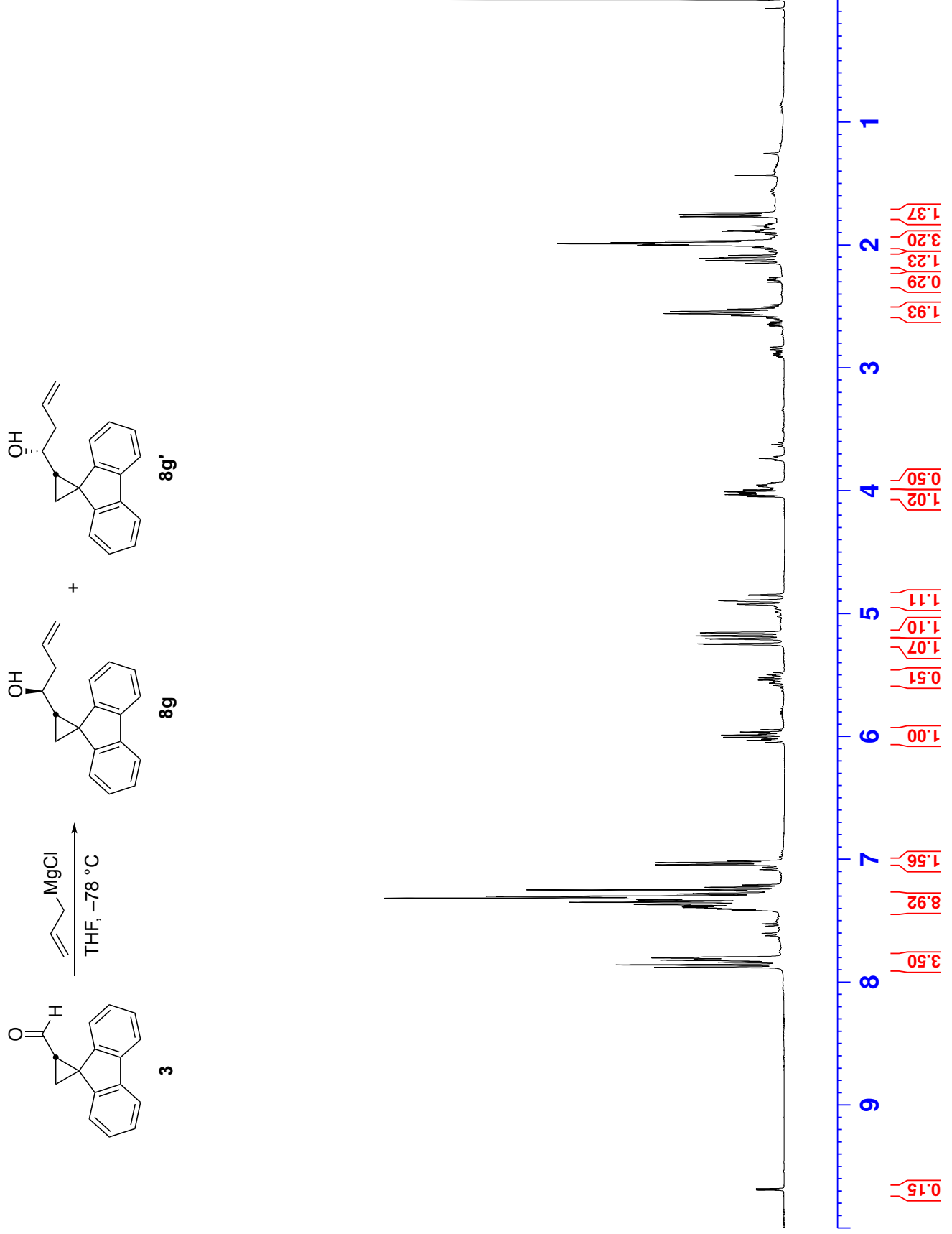

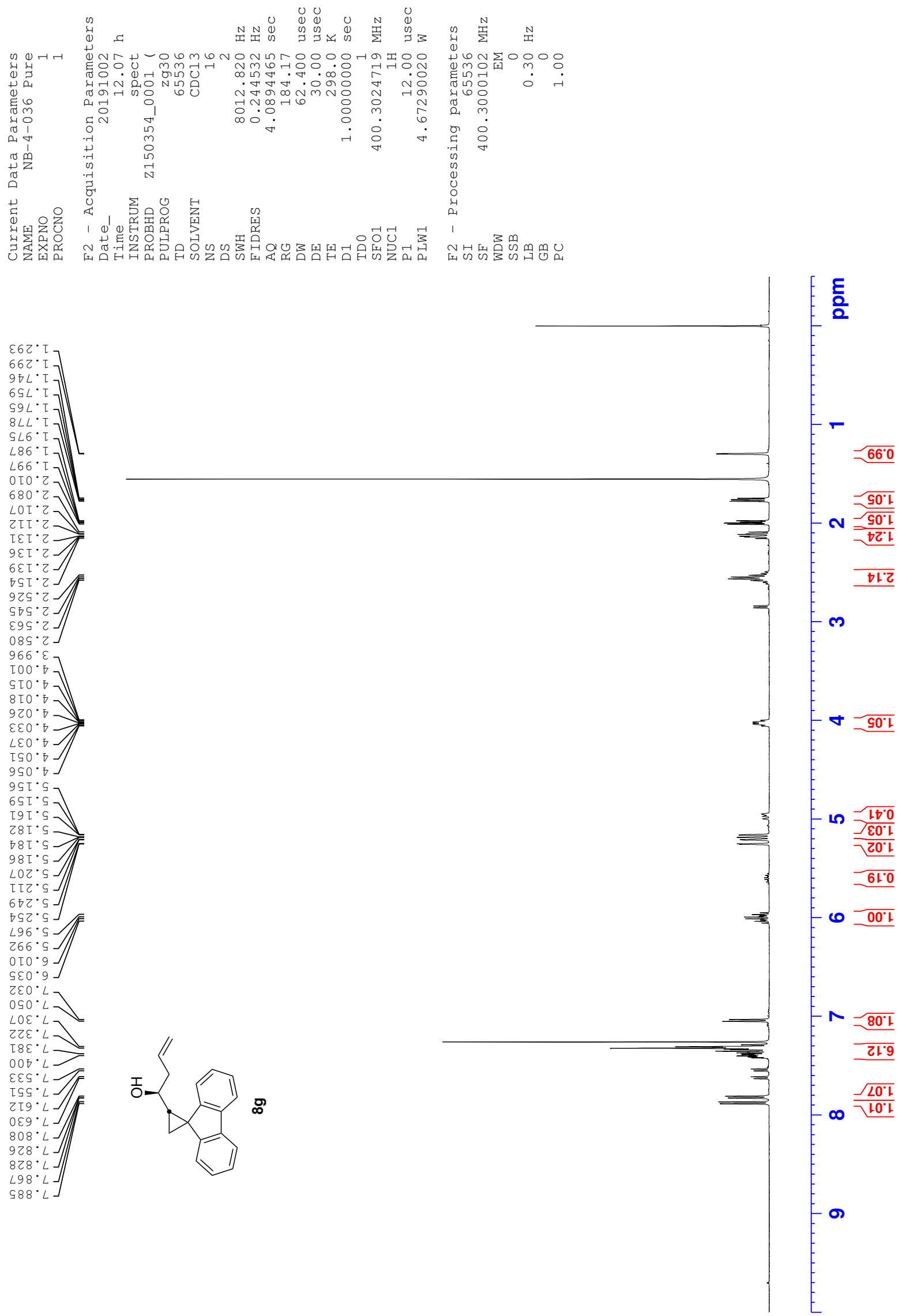

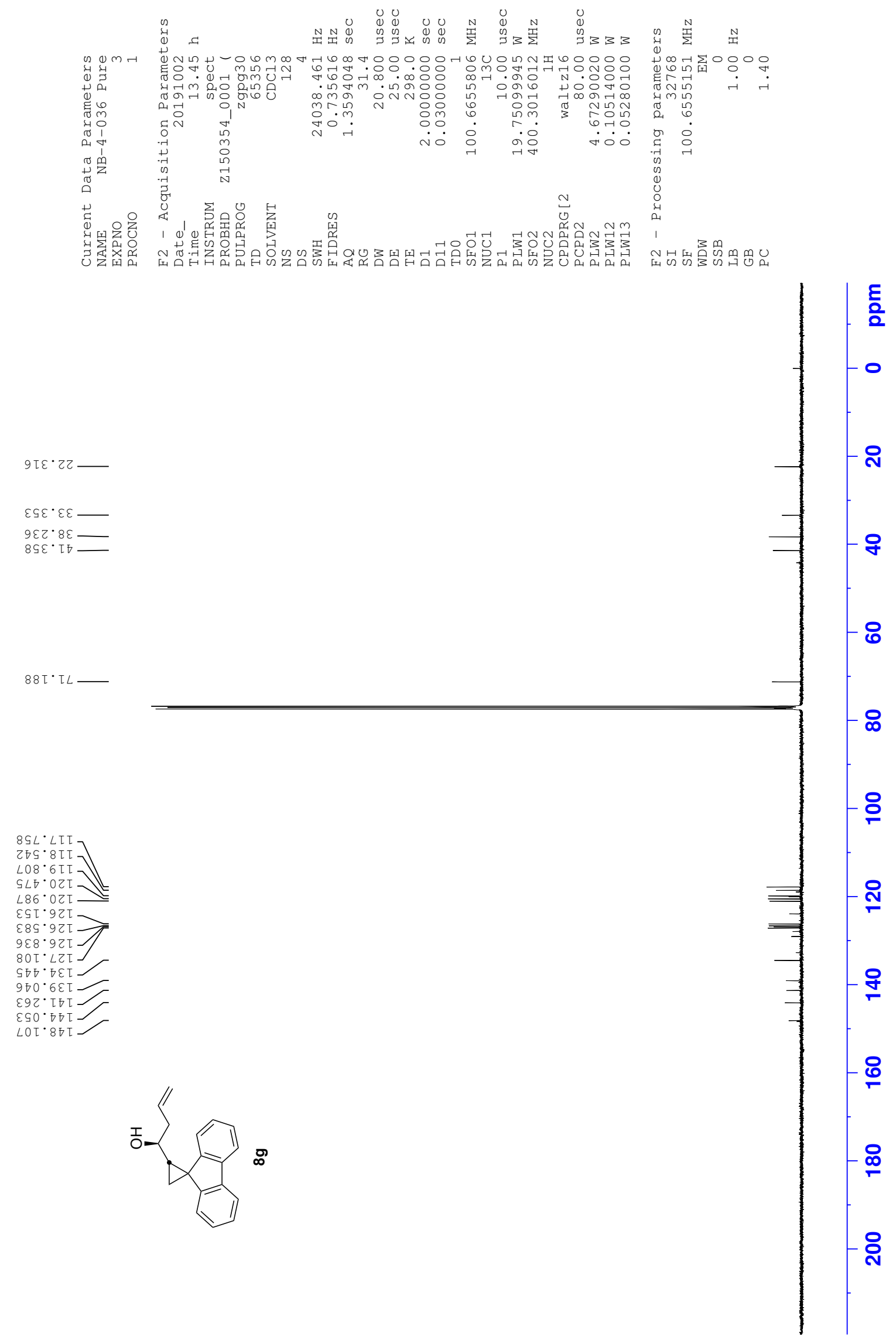

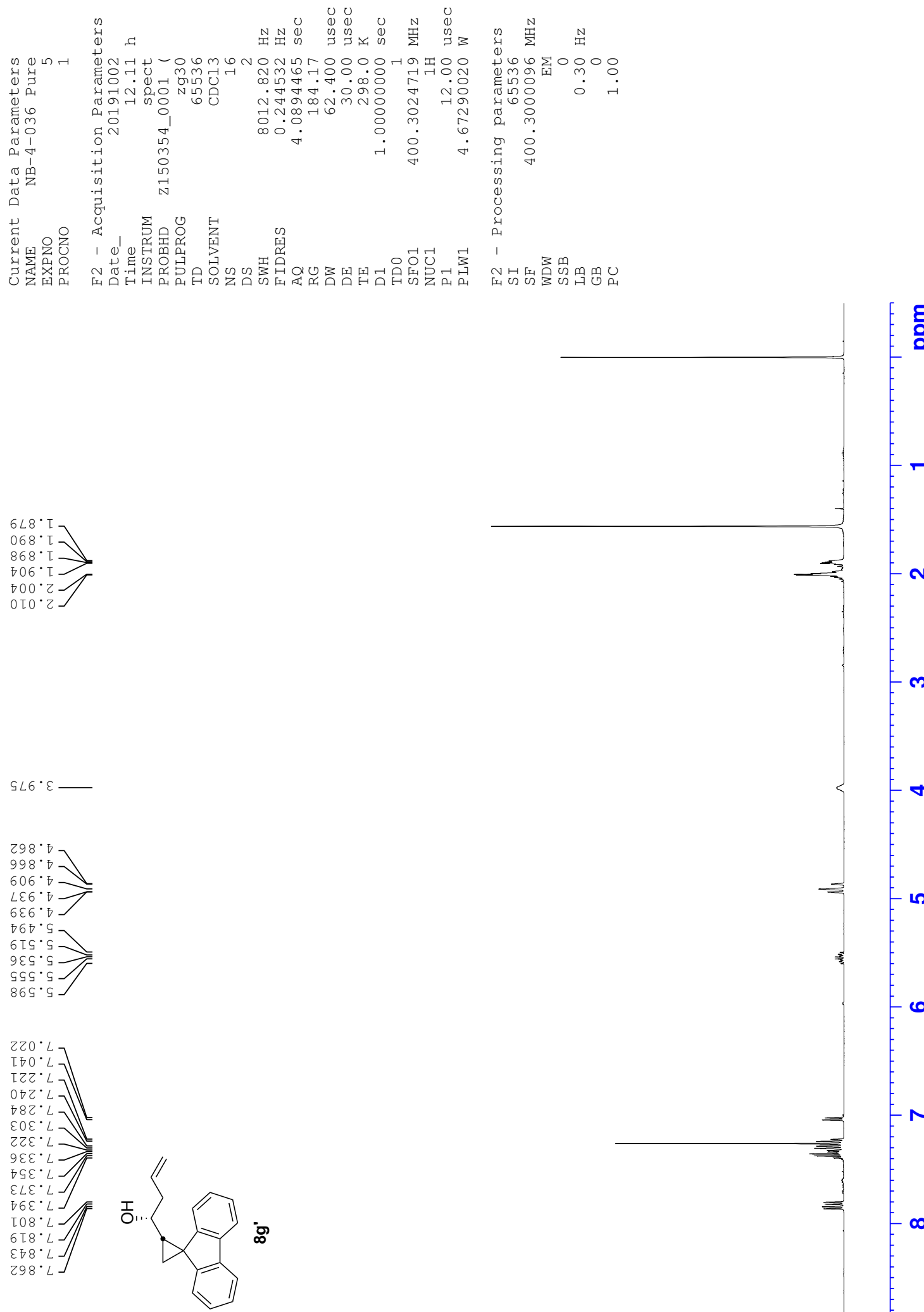

틍

2

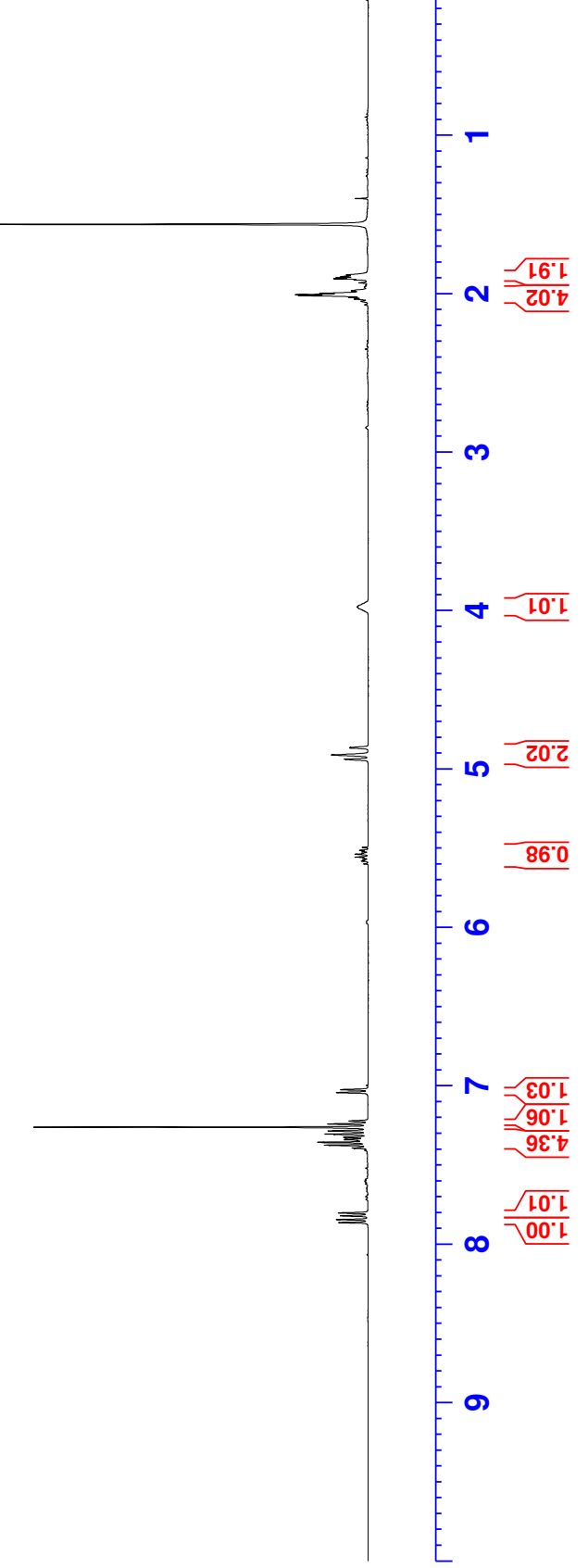



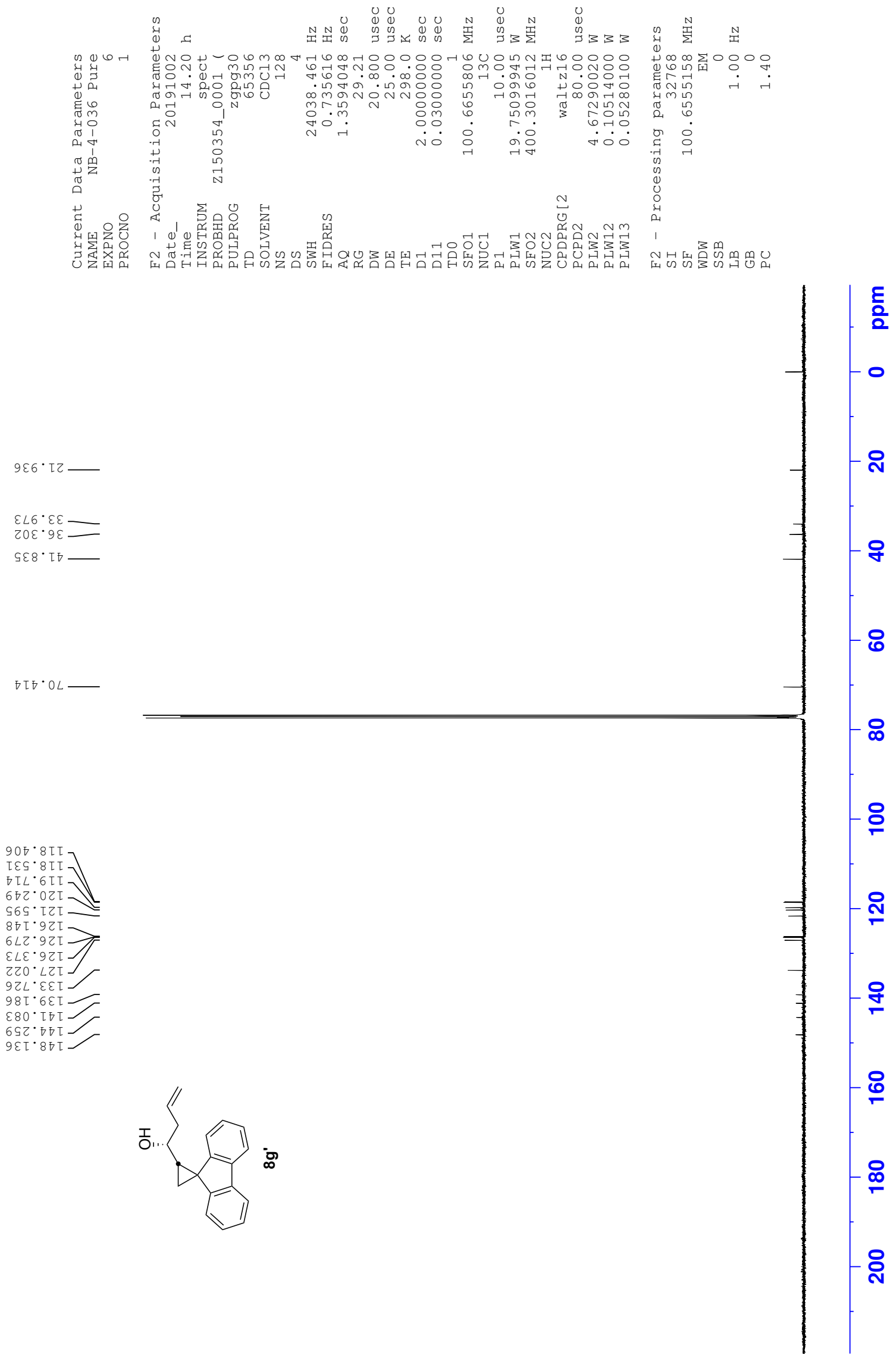

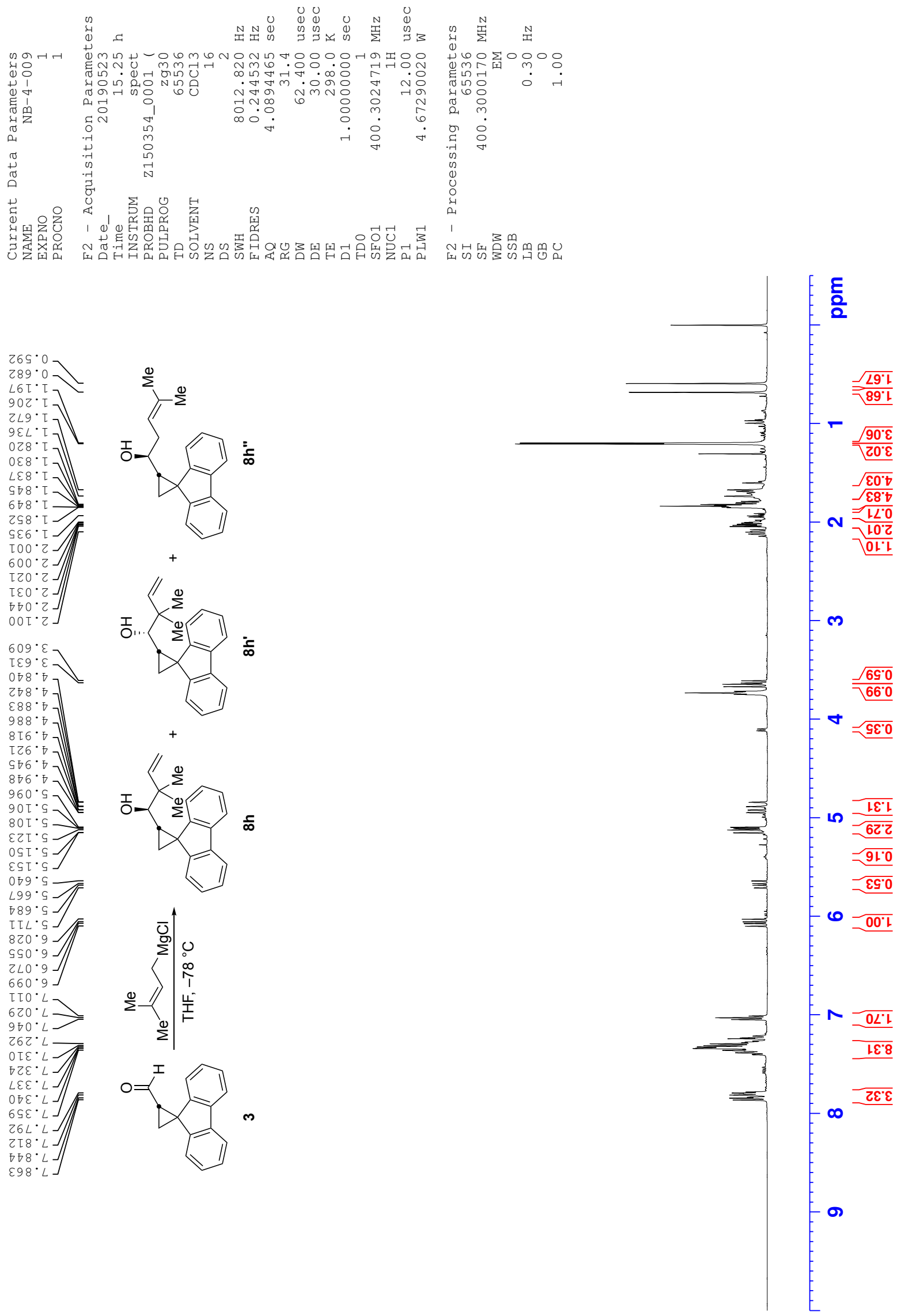

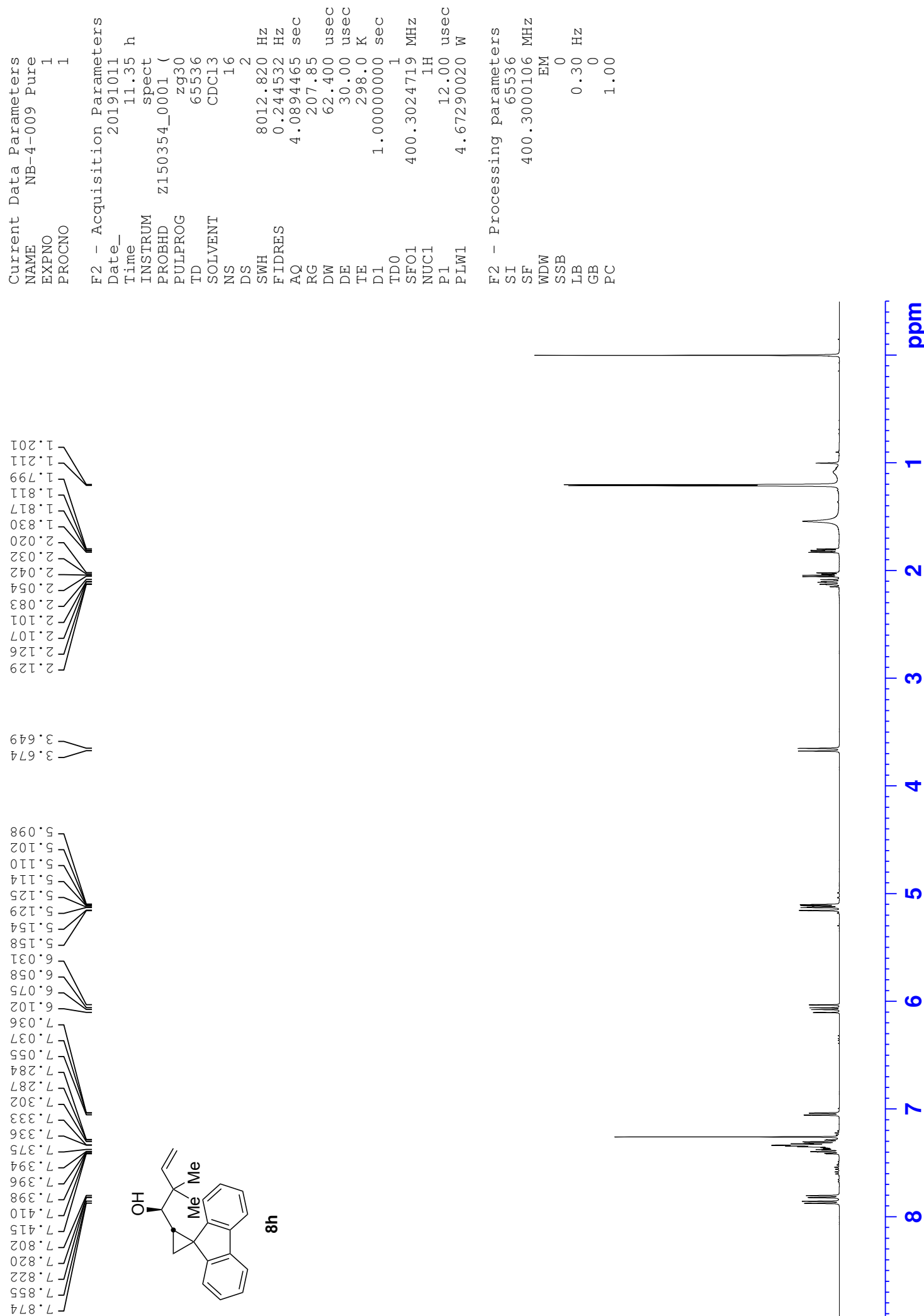

$\frac{5}{2}$

m

in $\longdiv { \angle 6 0 }$ 

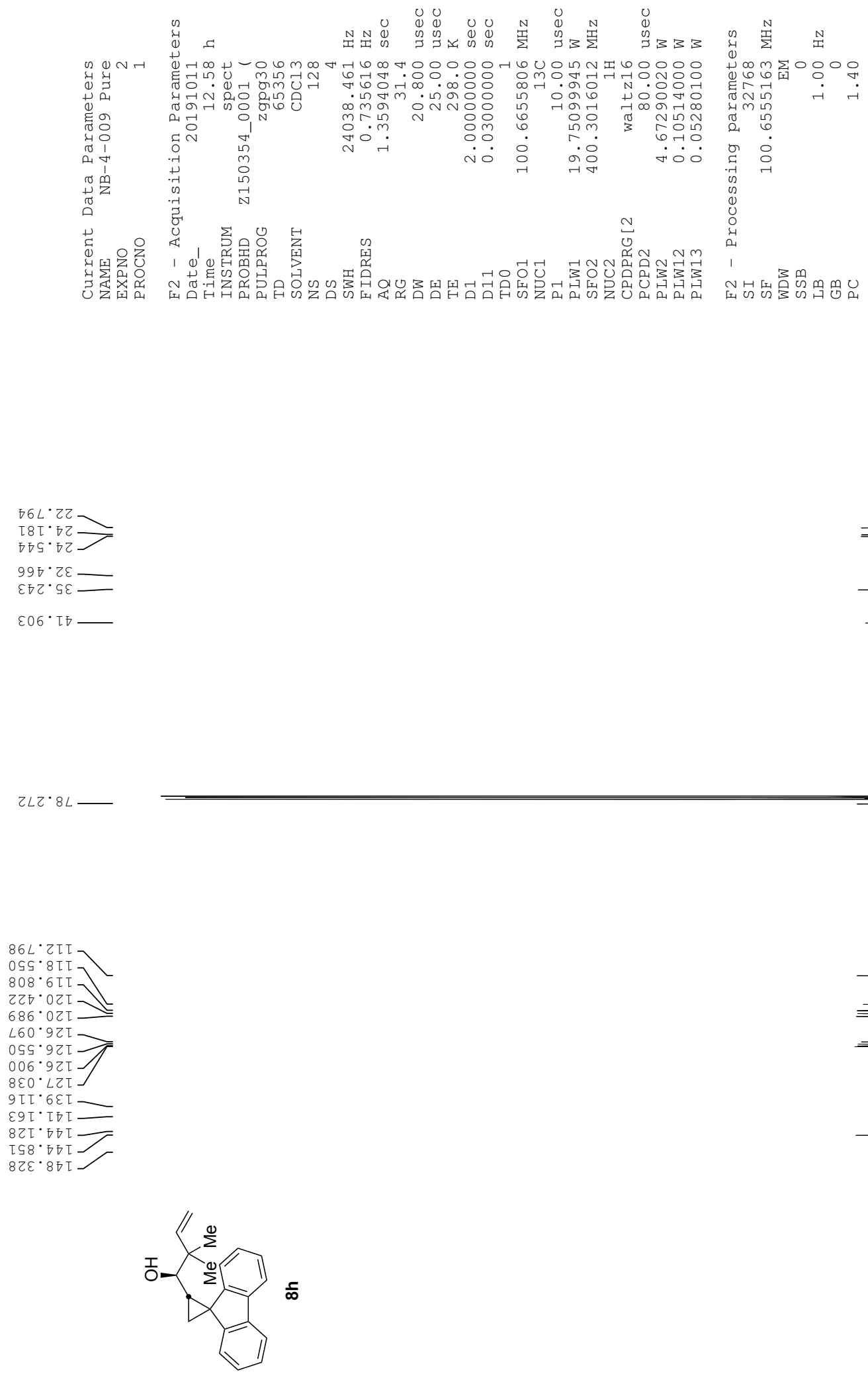

틍 

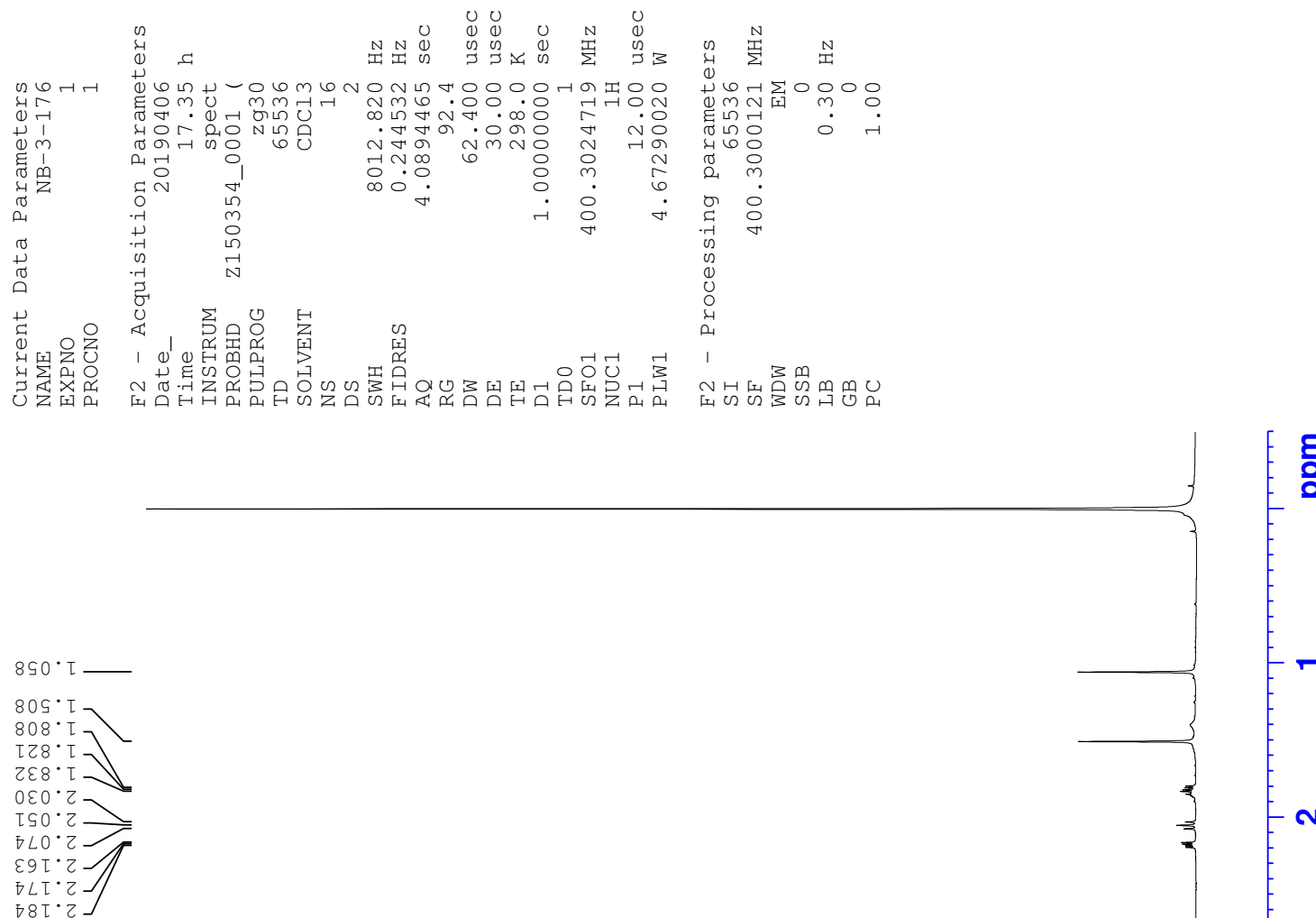

틍

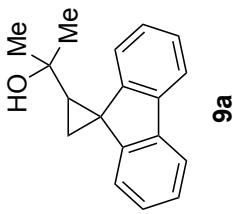

10
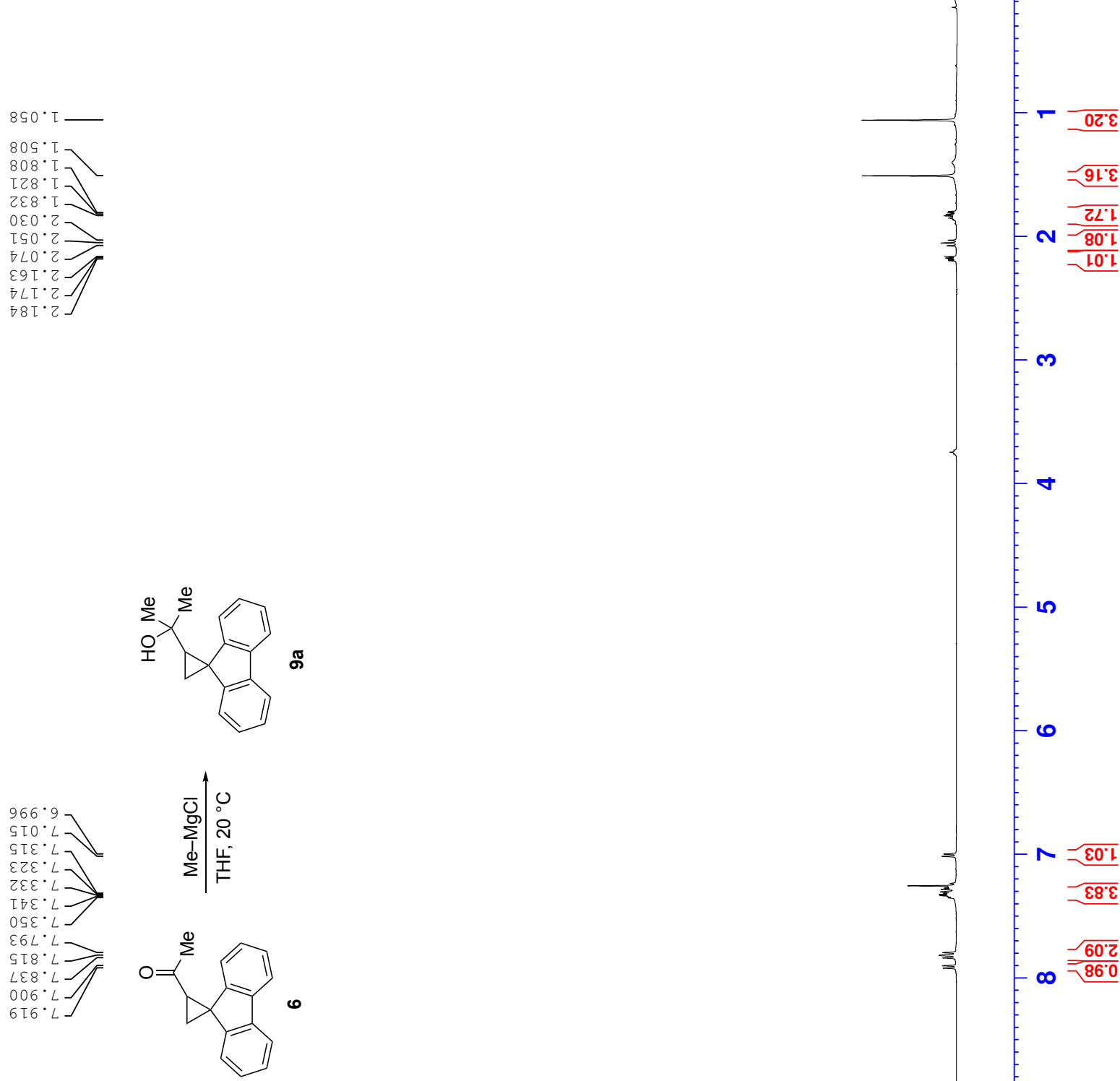

$-\infty$

$\nabla$

$-6$

$\Lambda \widehat{\varepsilon 0^{\circ}}$

$\varepsilon 8^{\prime} \varepsilon$

$\sqrt{60 \mathrm{z}}$

$\infty \longdiv { 8 6 ^ { \circ } 0 }$ 


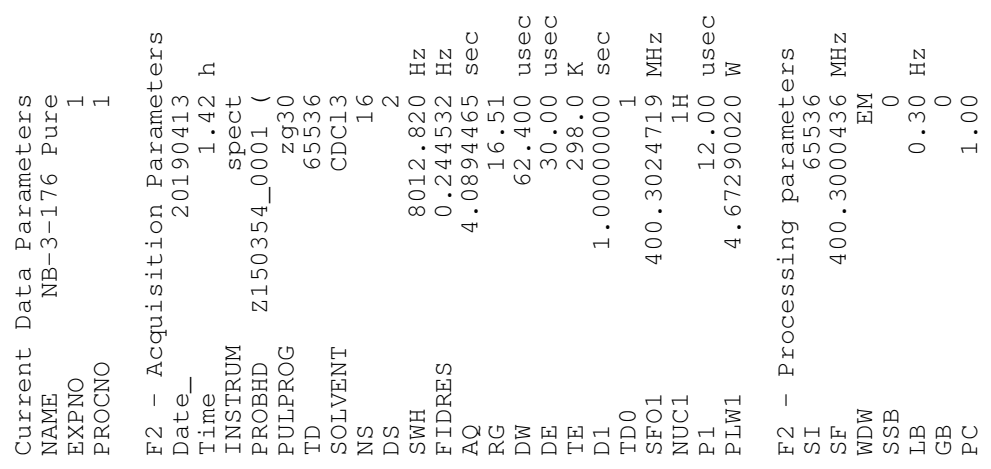

$200 \cdot 0-$

$000 \cdot 0->$

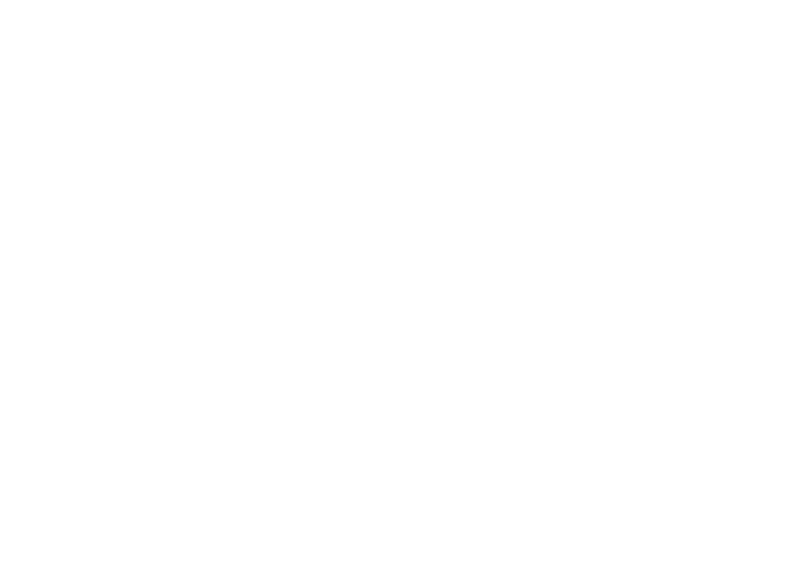

_ $=\frac{E}{2}$
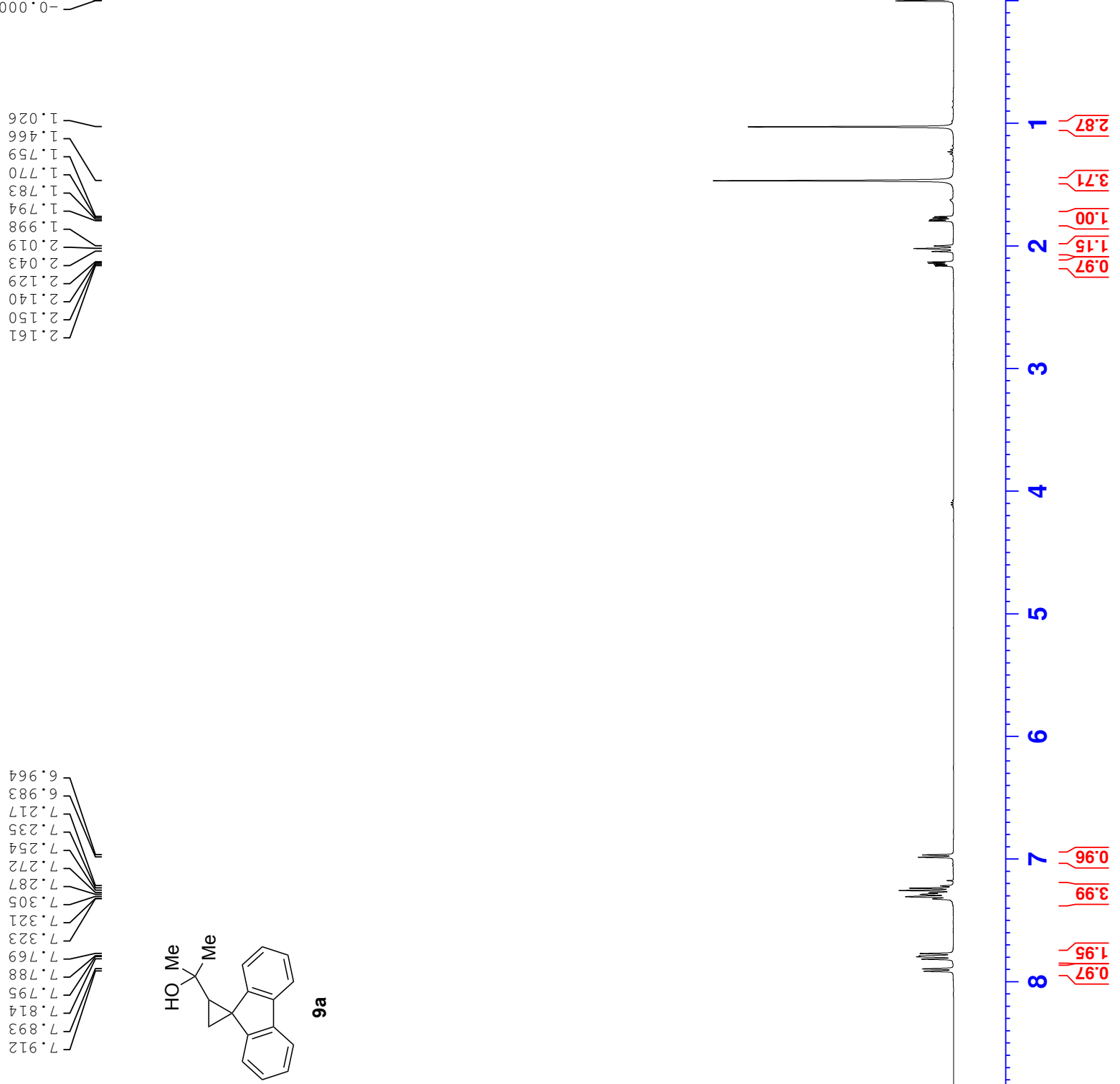

言 

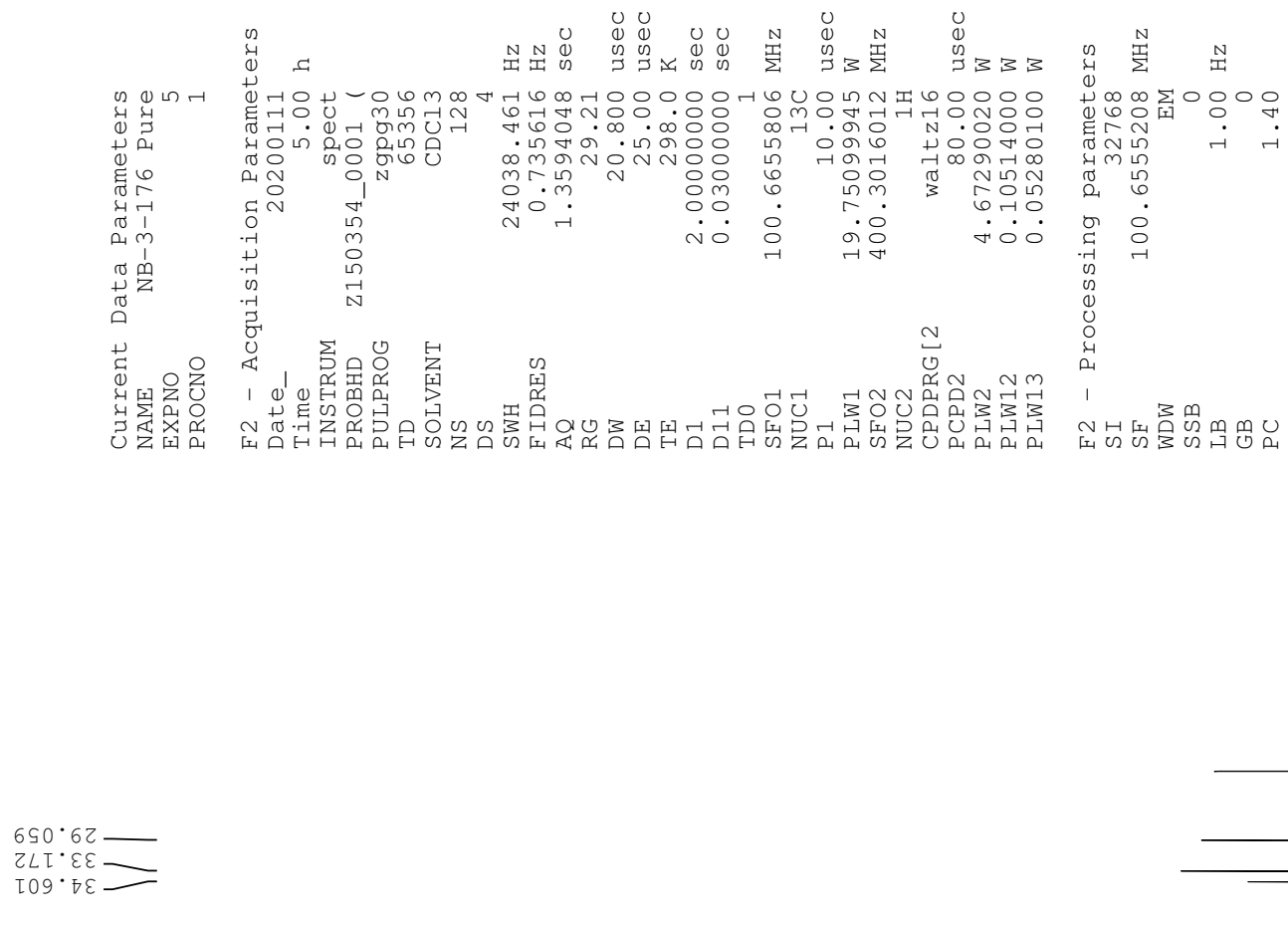

$8 \tau \mp \cdot \varepsilon \mp$
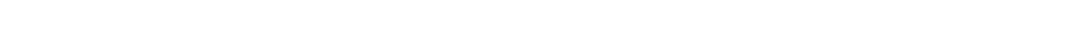

TOO.OL
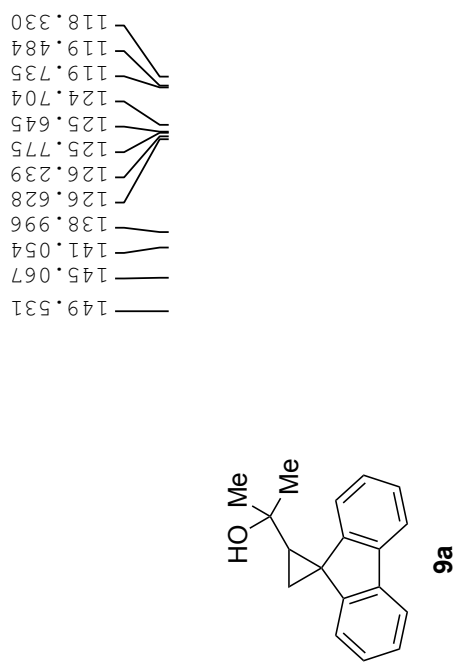

틀

요

q

ㅇ

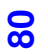

8

ิㅗำ

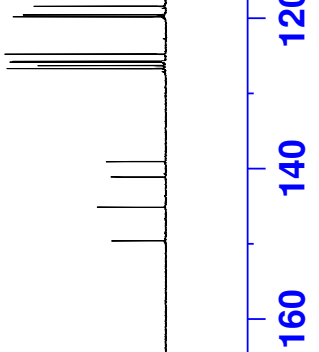

$\stackrel{\circ}{\circ}$

$\stackrel{\infty}{\infty}$

ఫ్ 

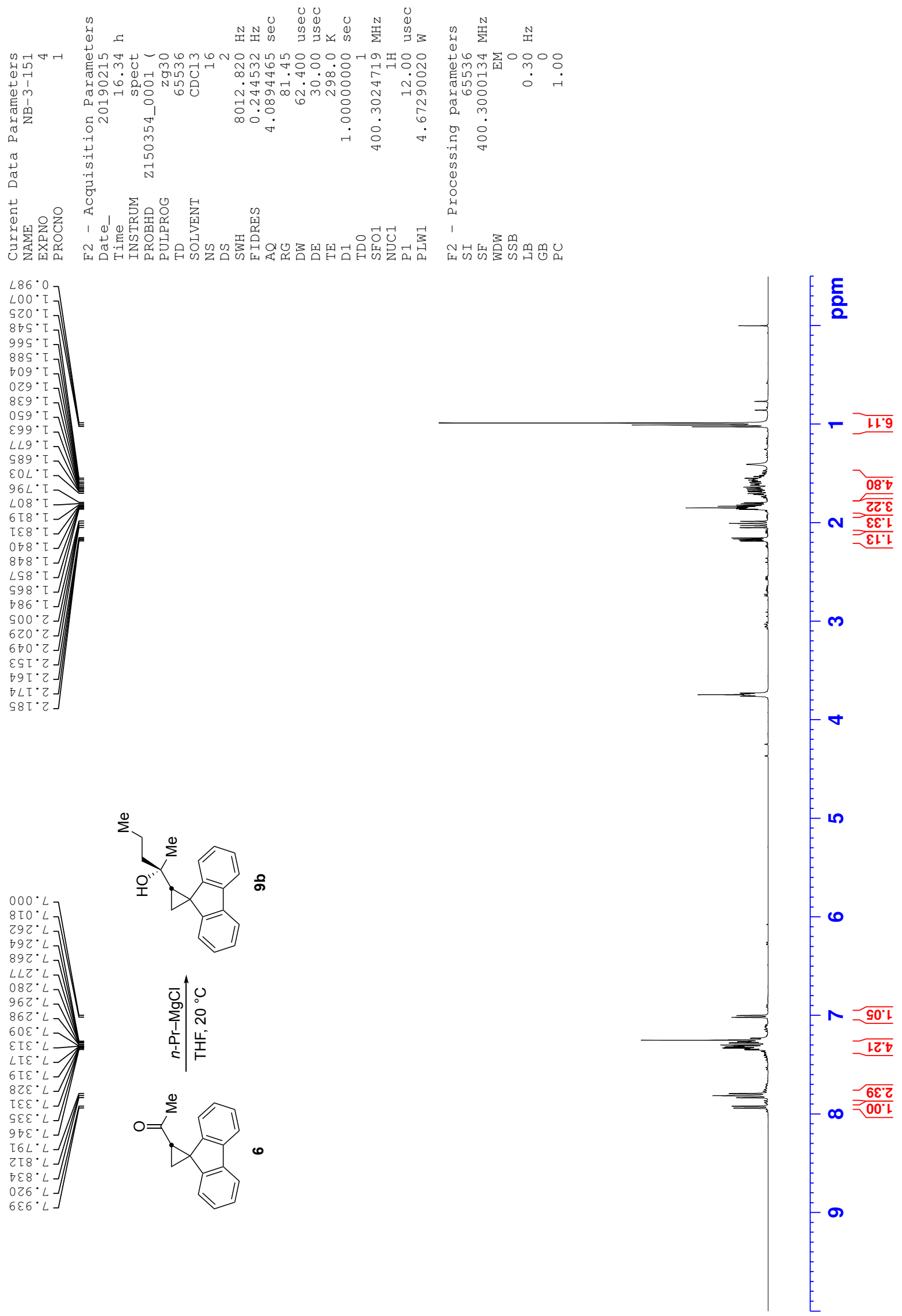

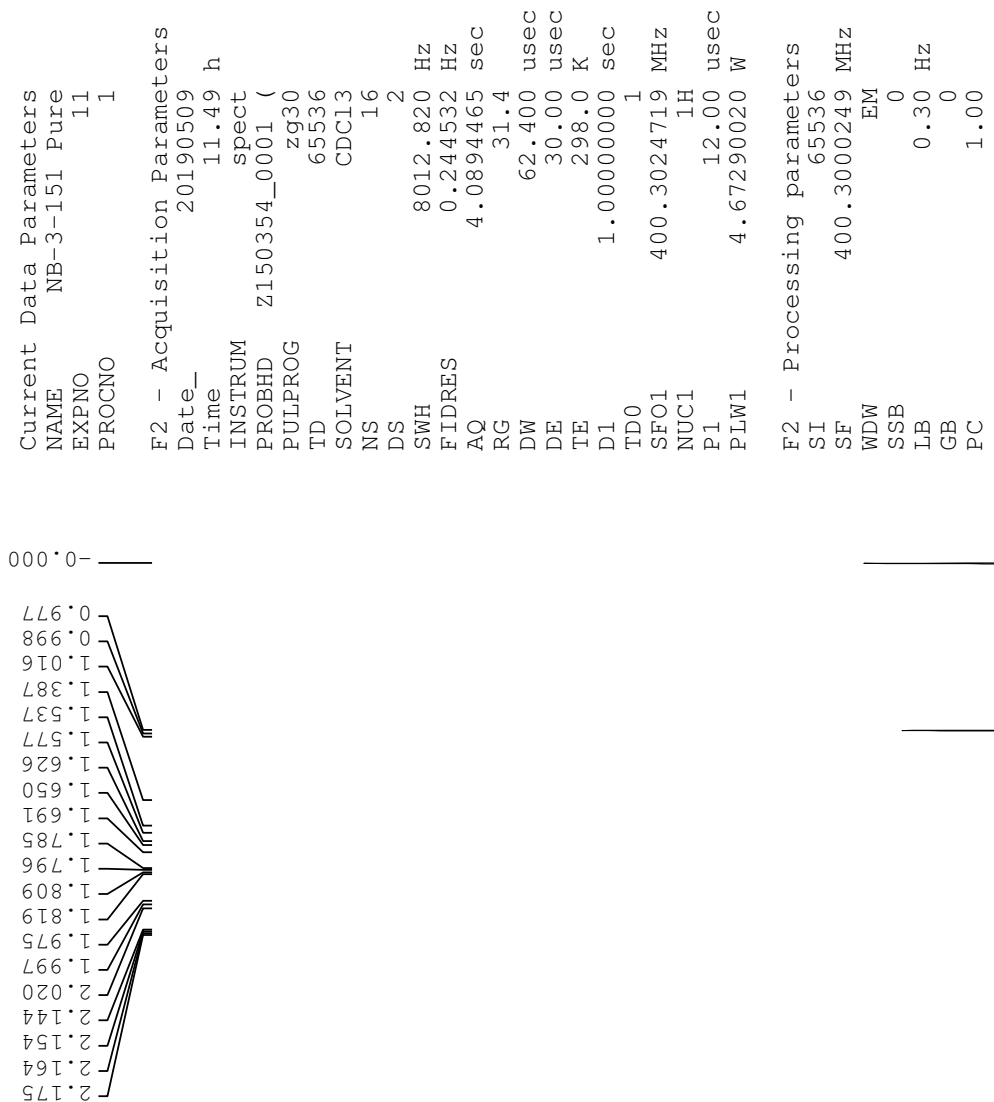

F 틍
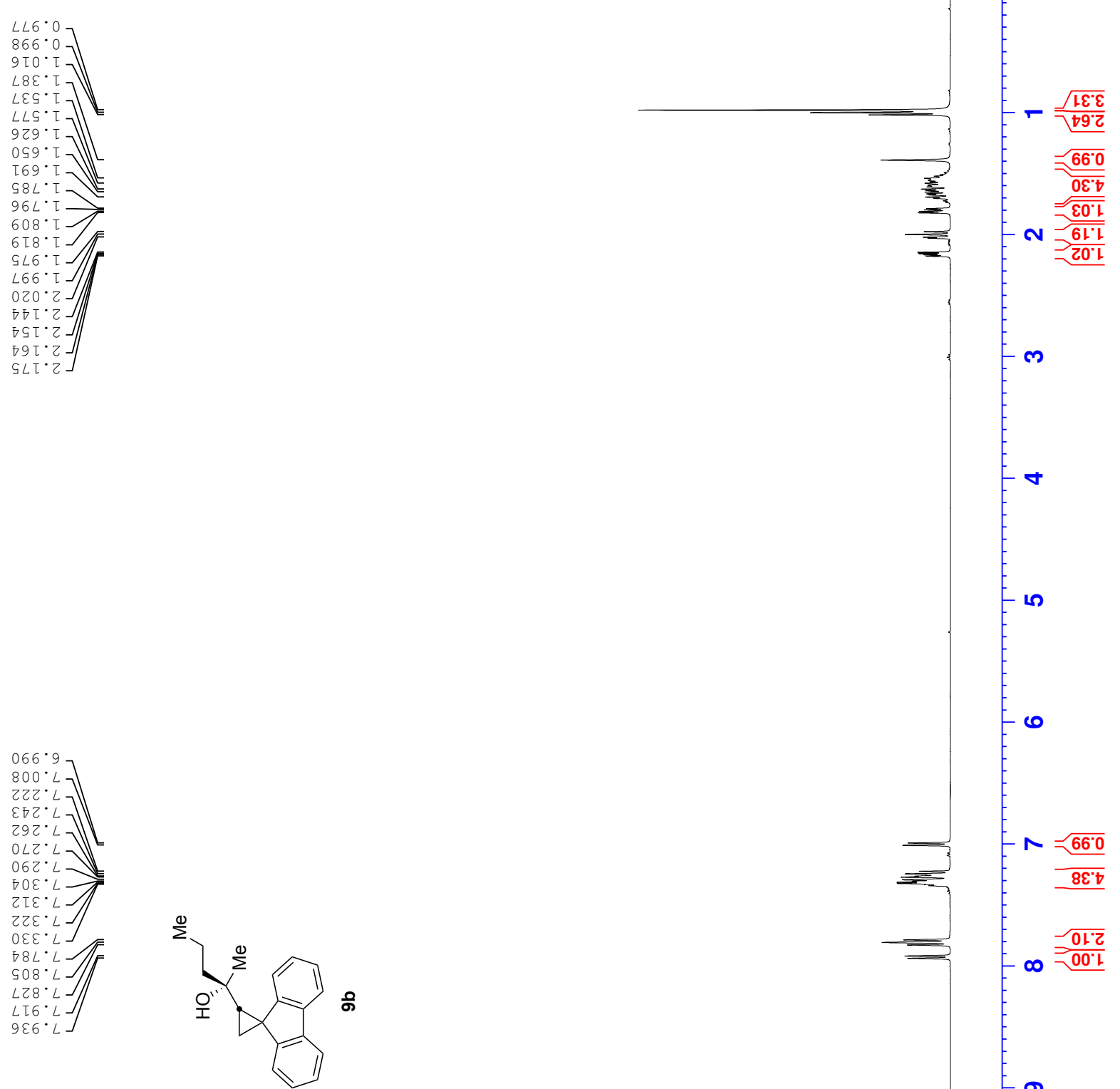

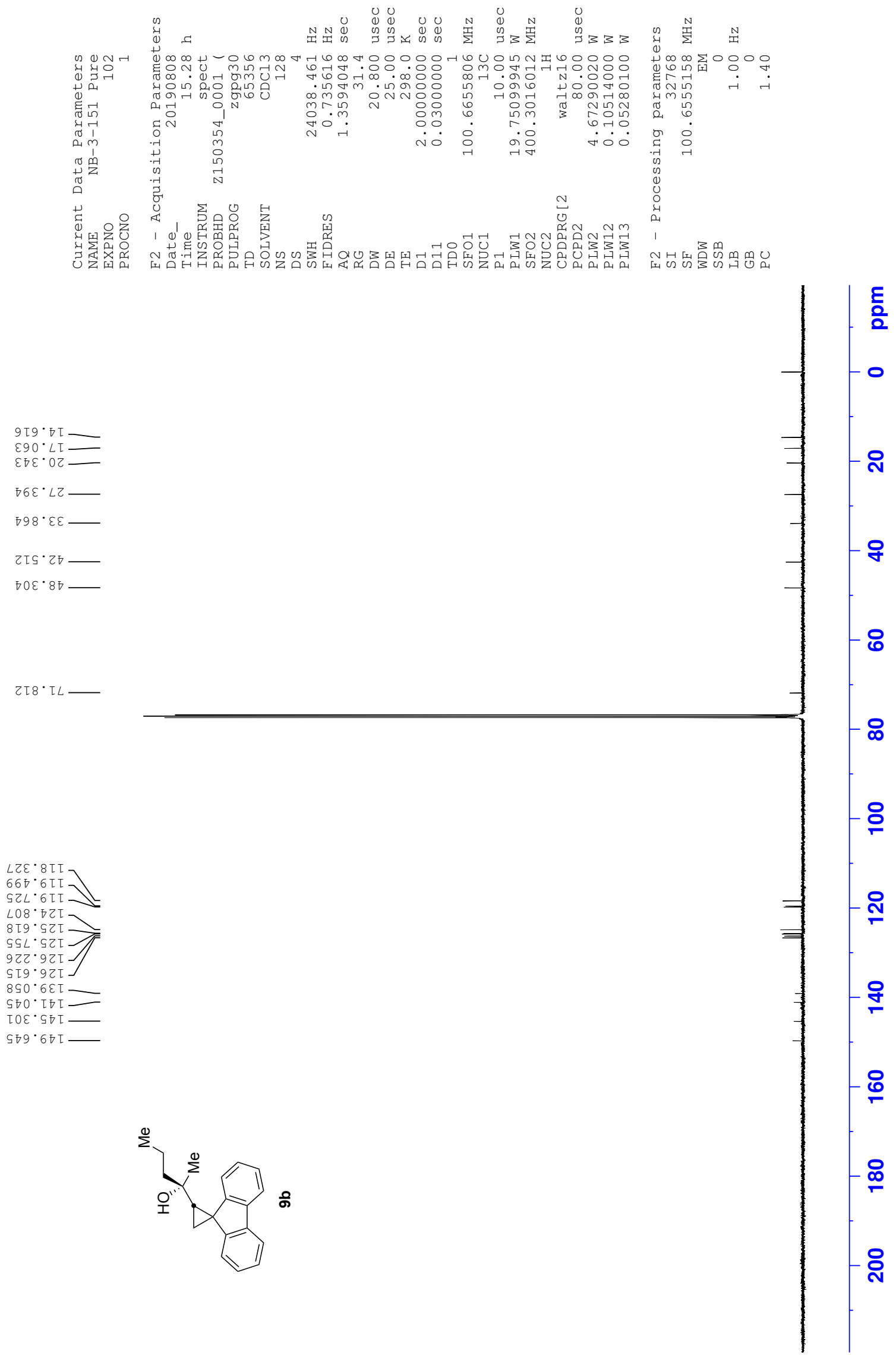

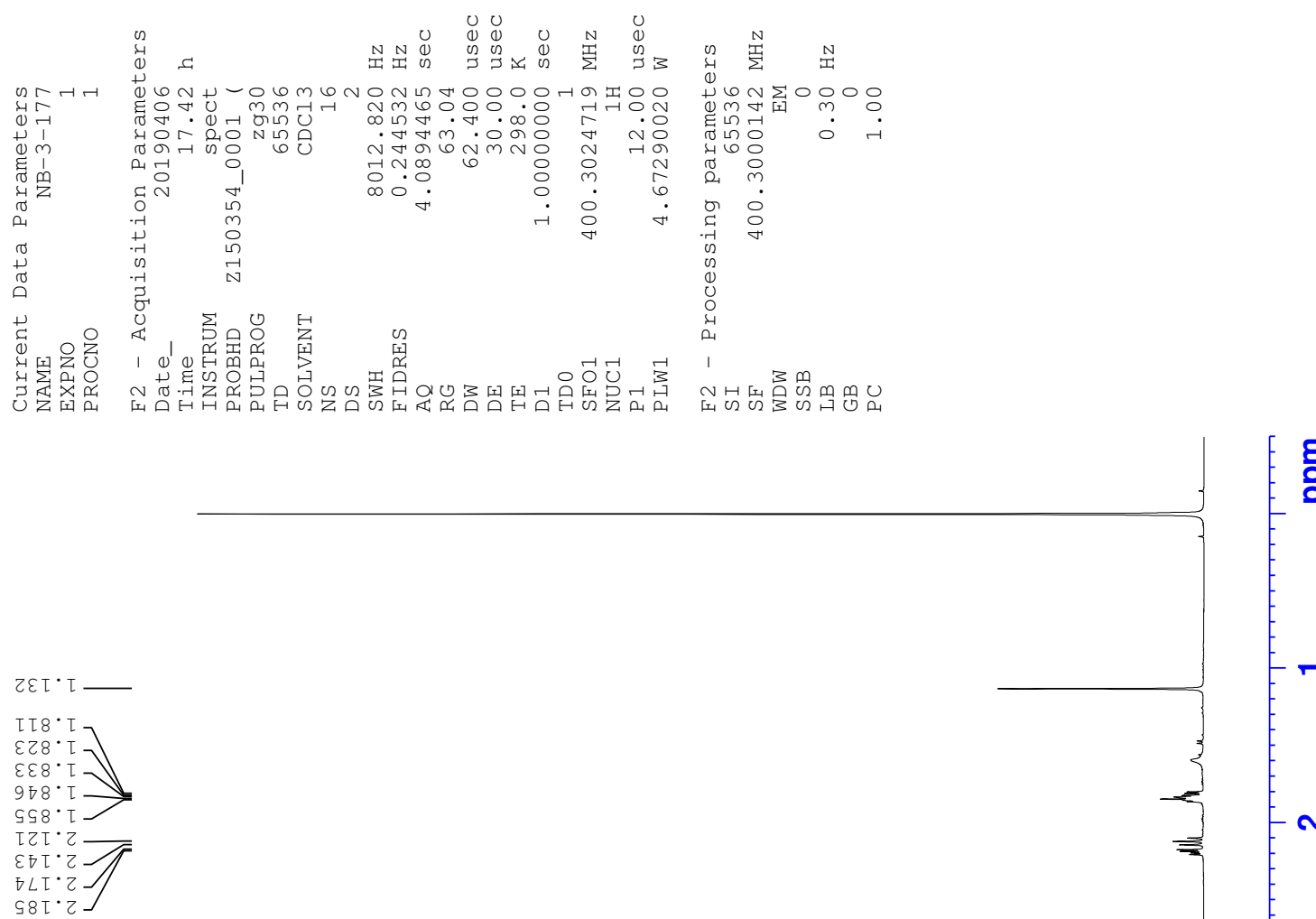

틍
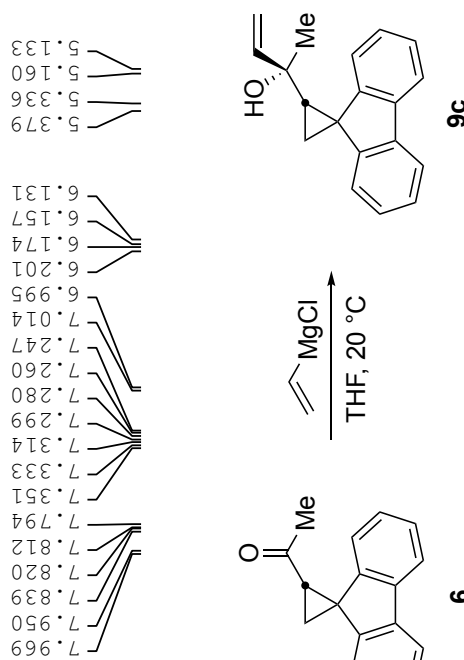

- 10

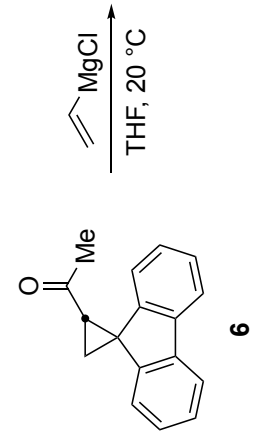

(26.0

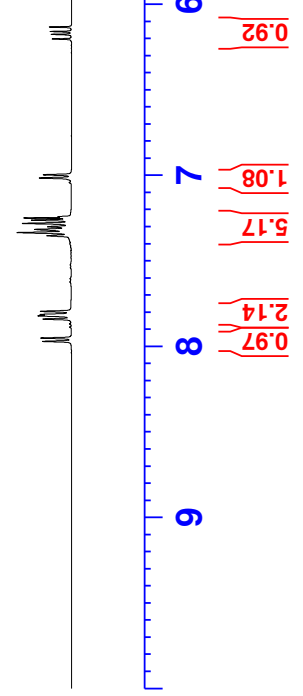



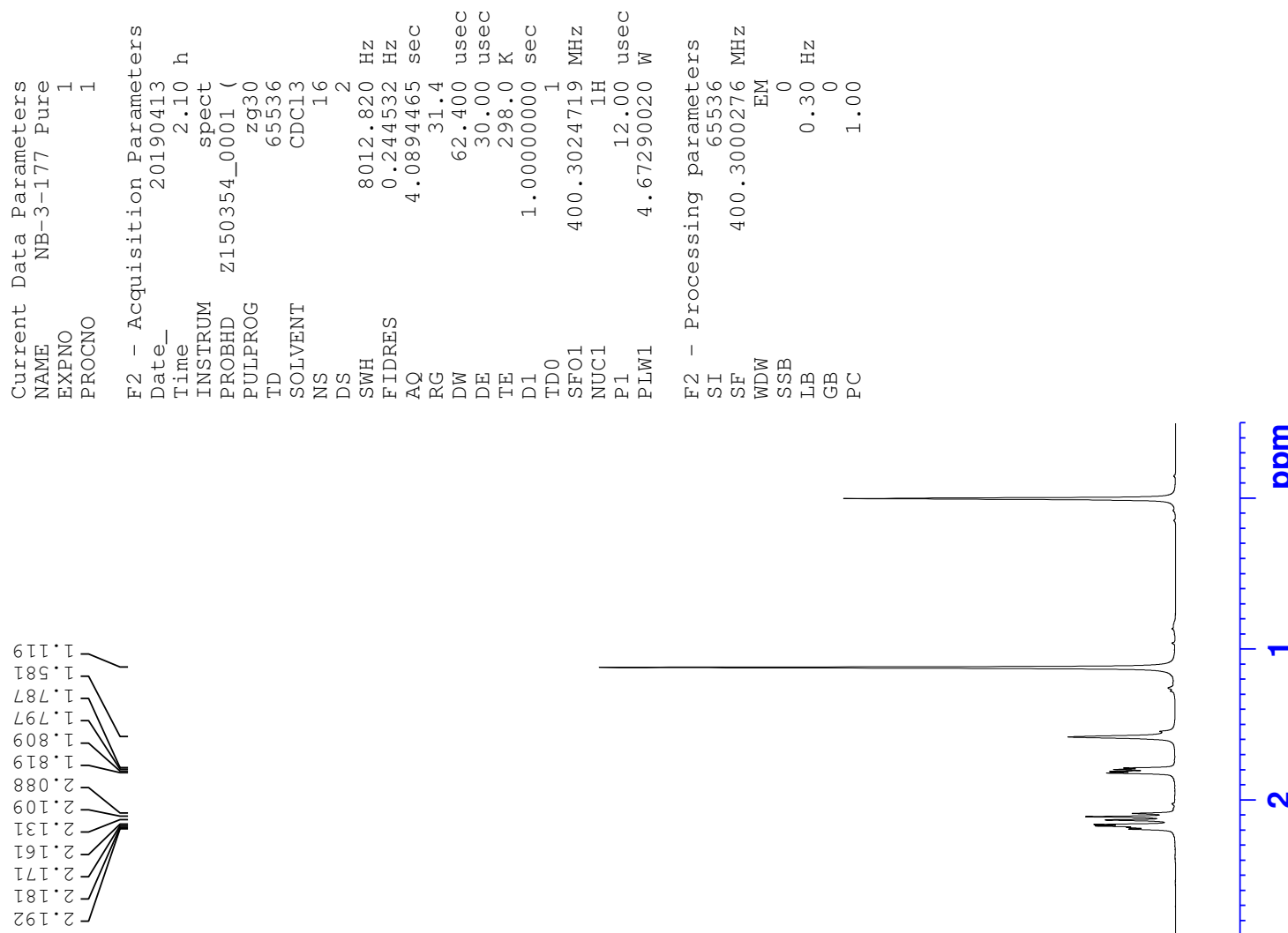

틍

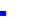

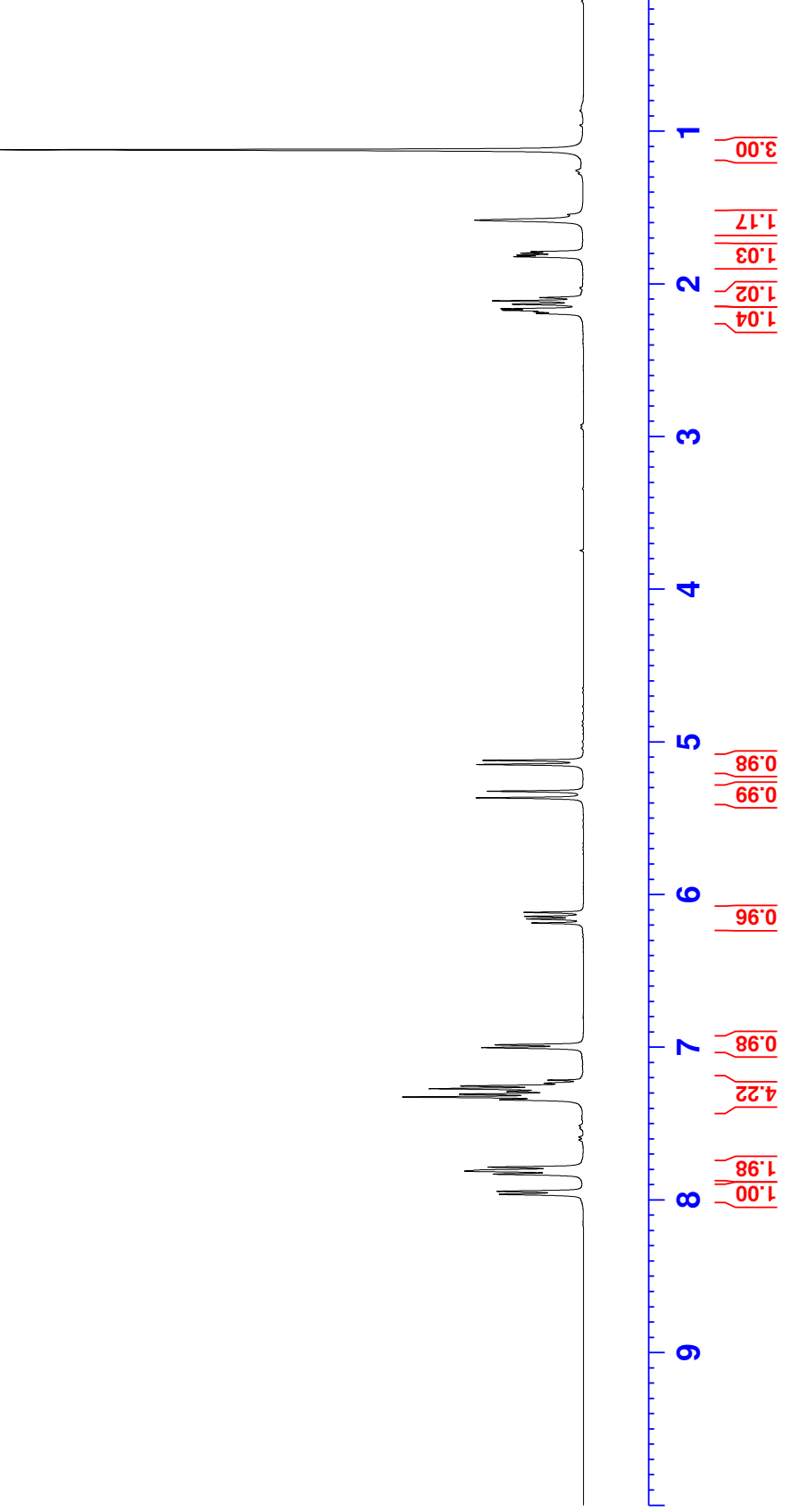



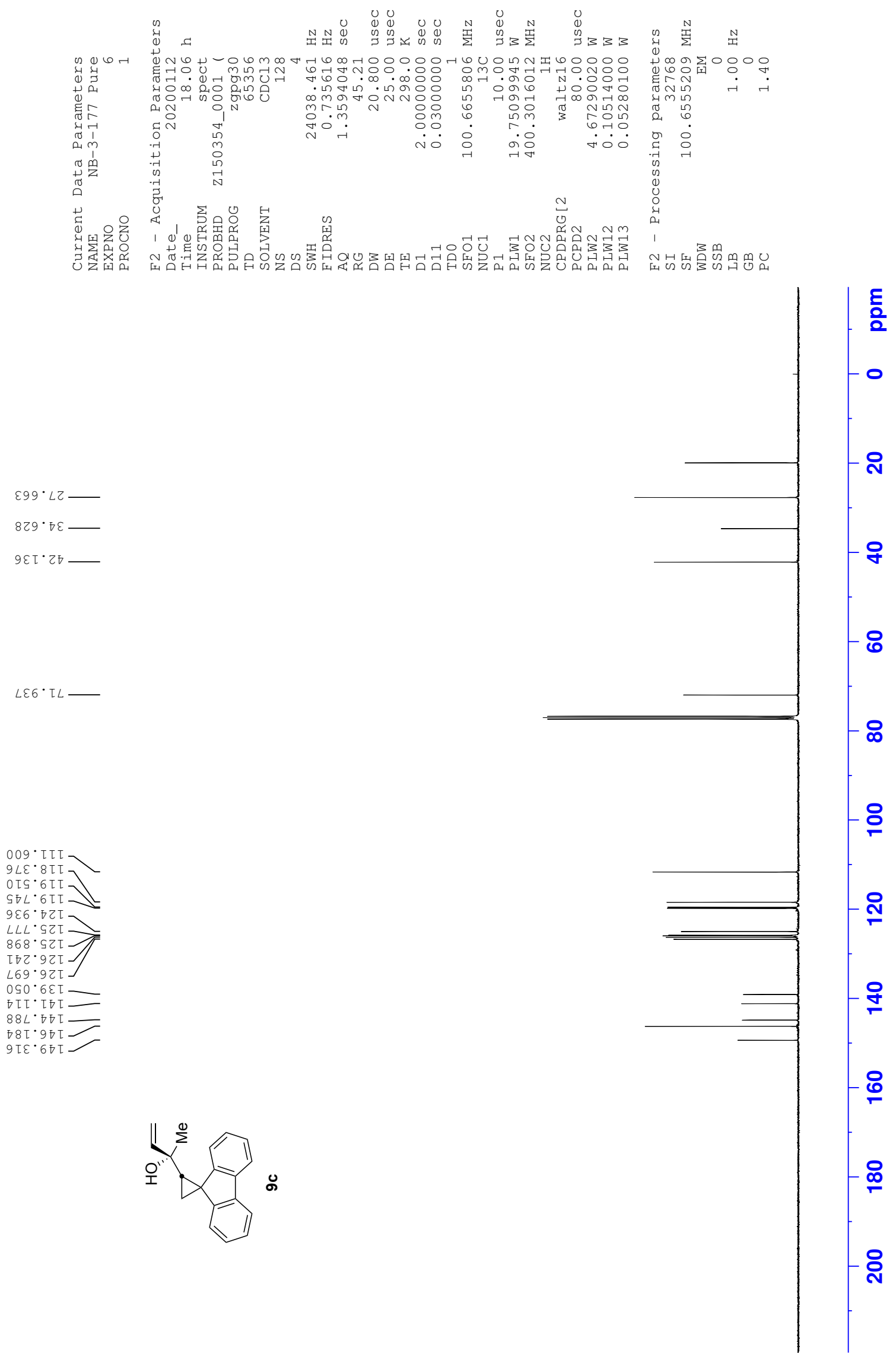

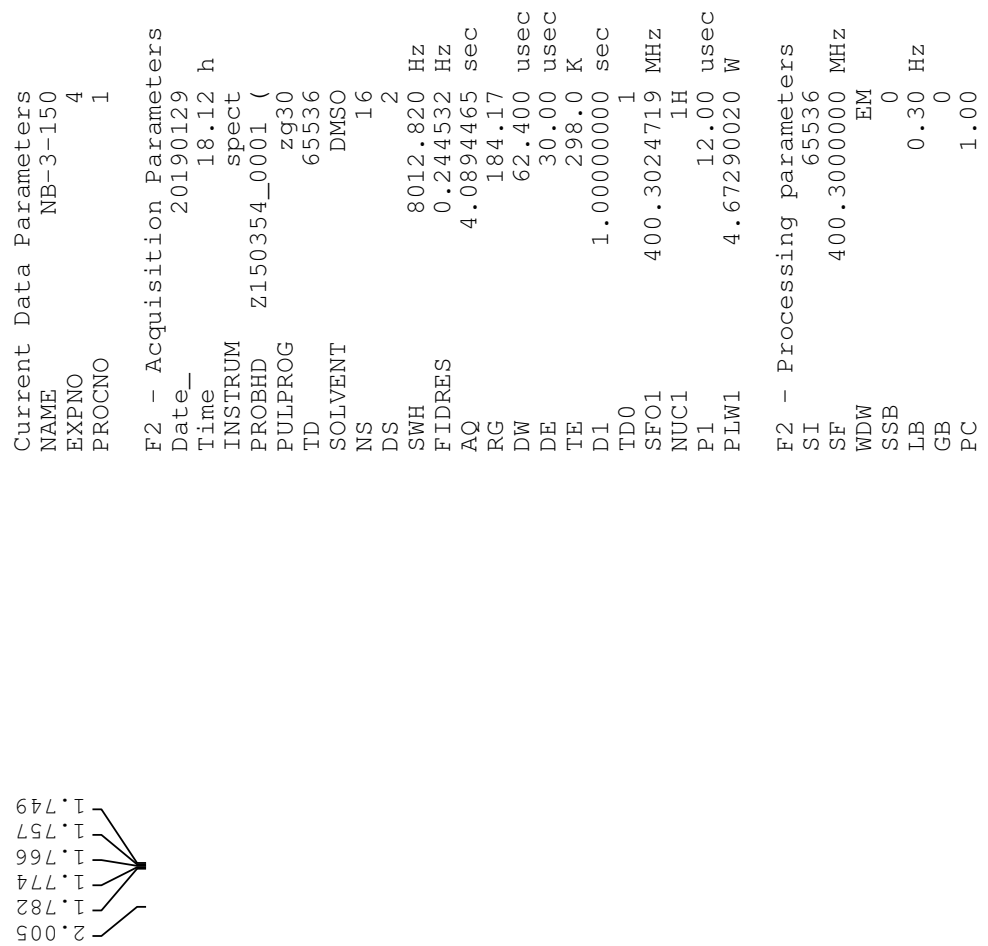

$\tau 乙 \varepsilon \cdot \varepsilon$
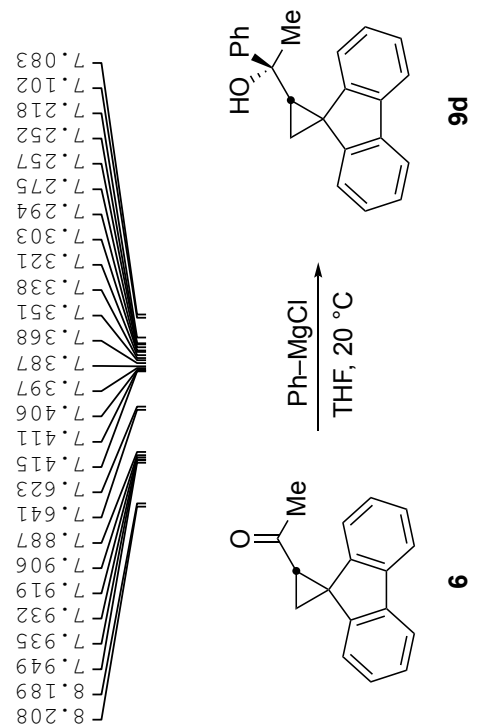

틍

( $)$

$-\infty$

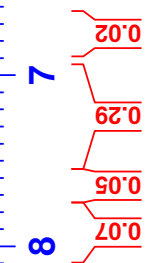



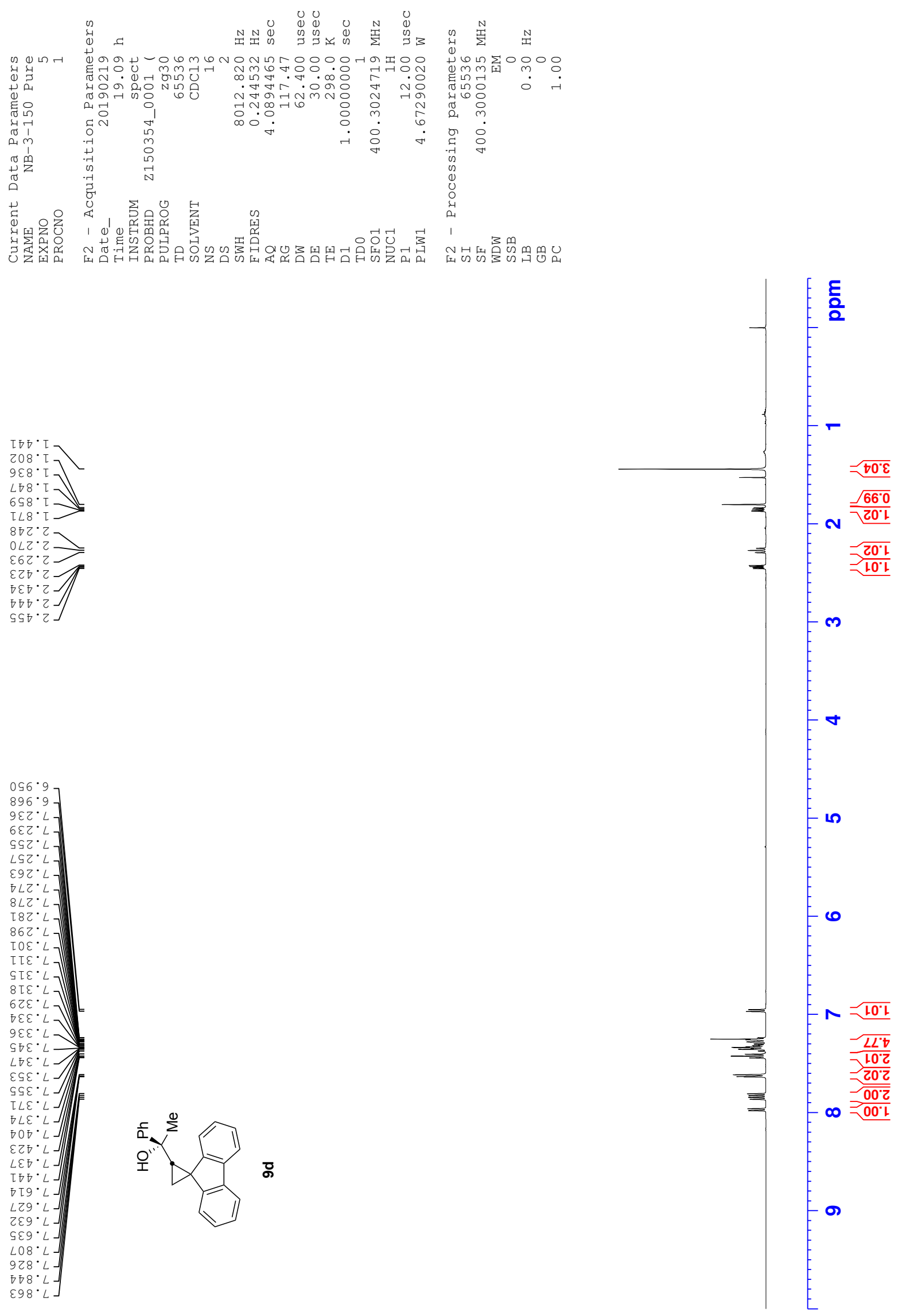

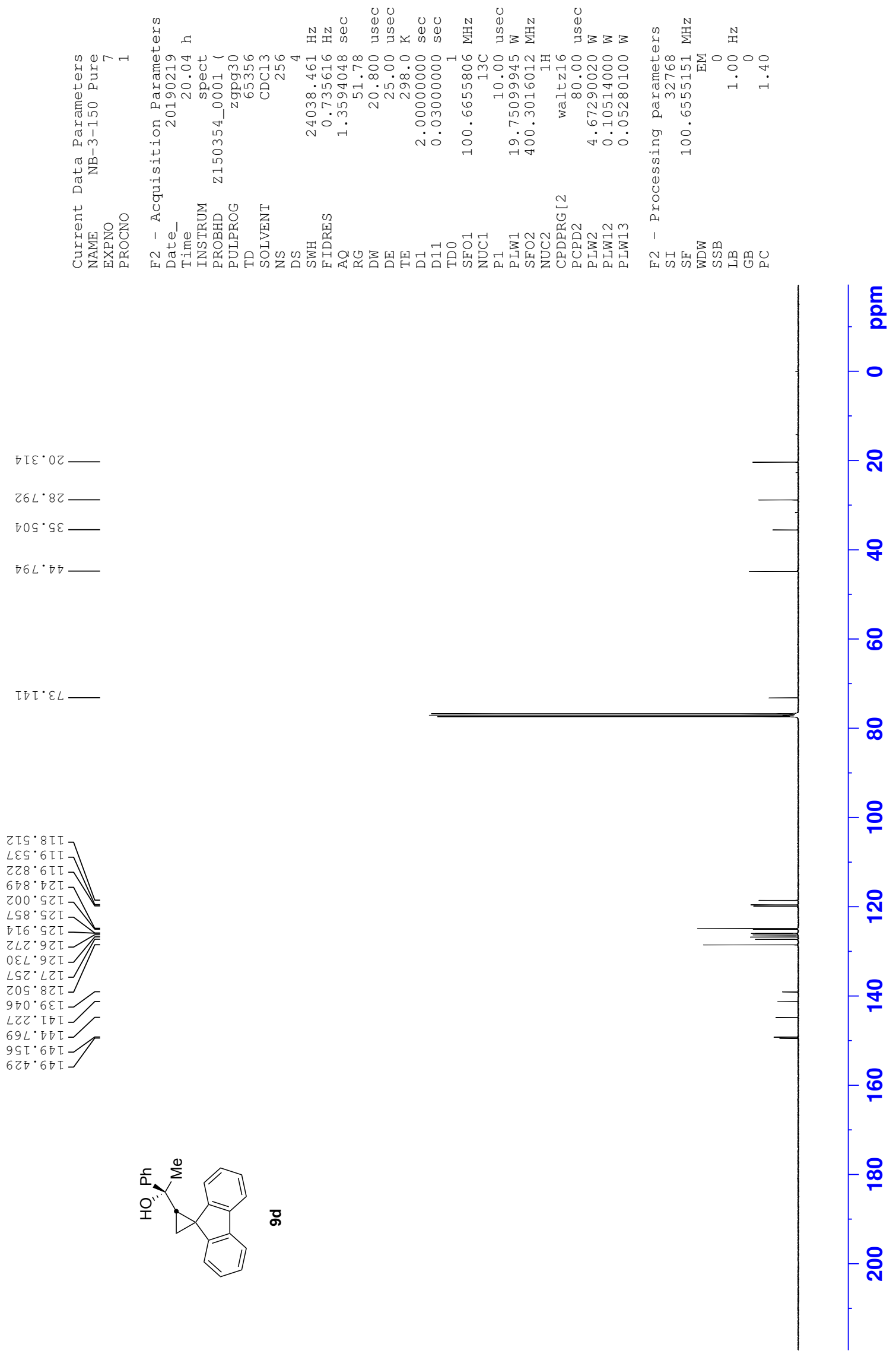

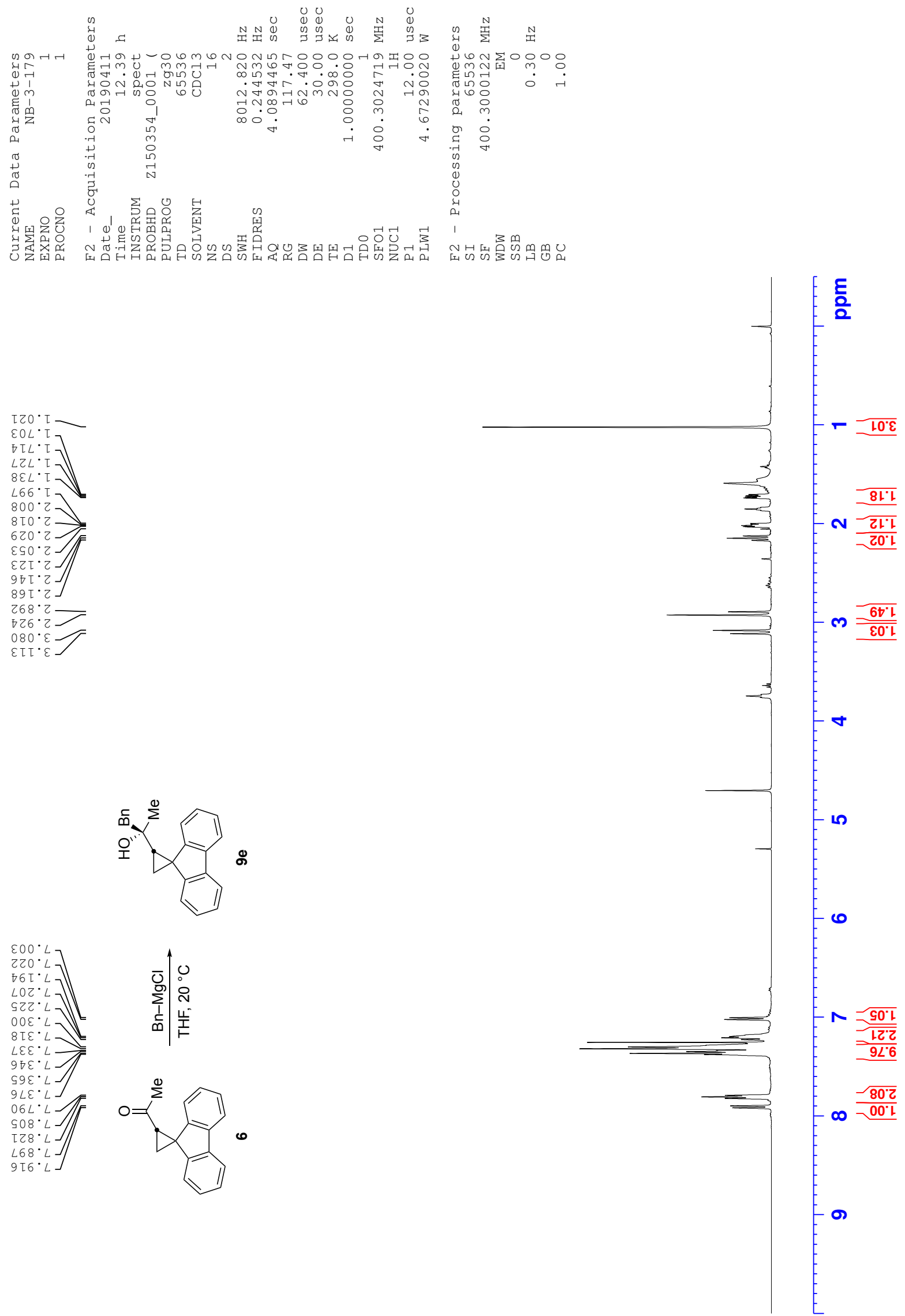

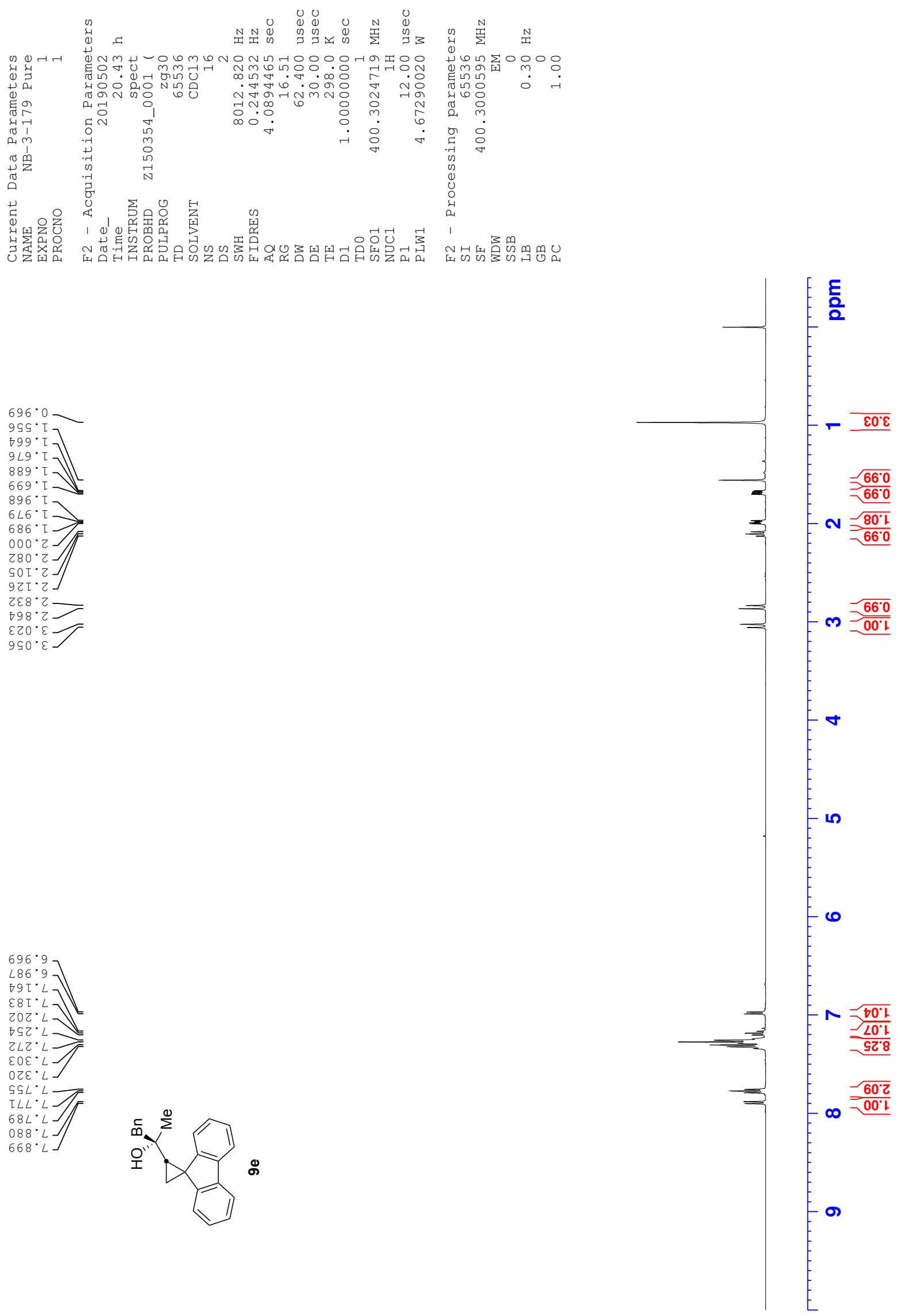

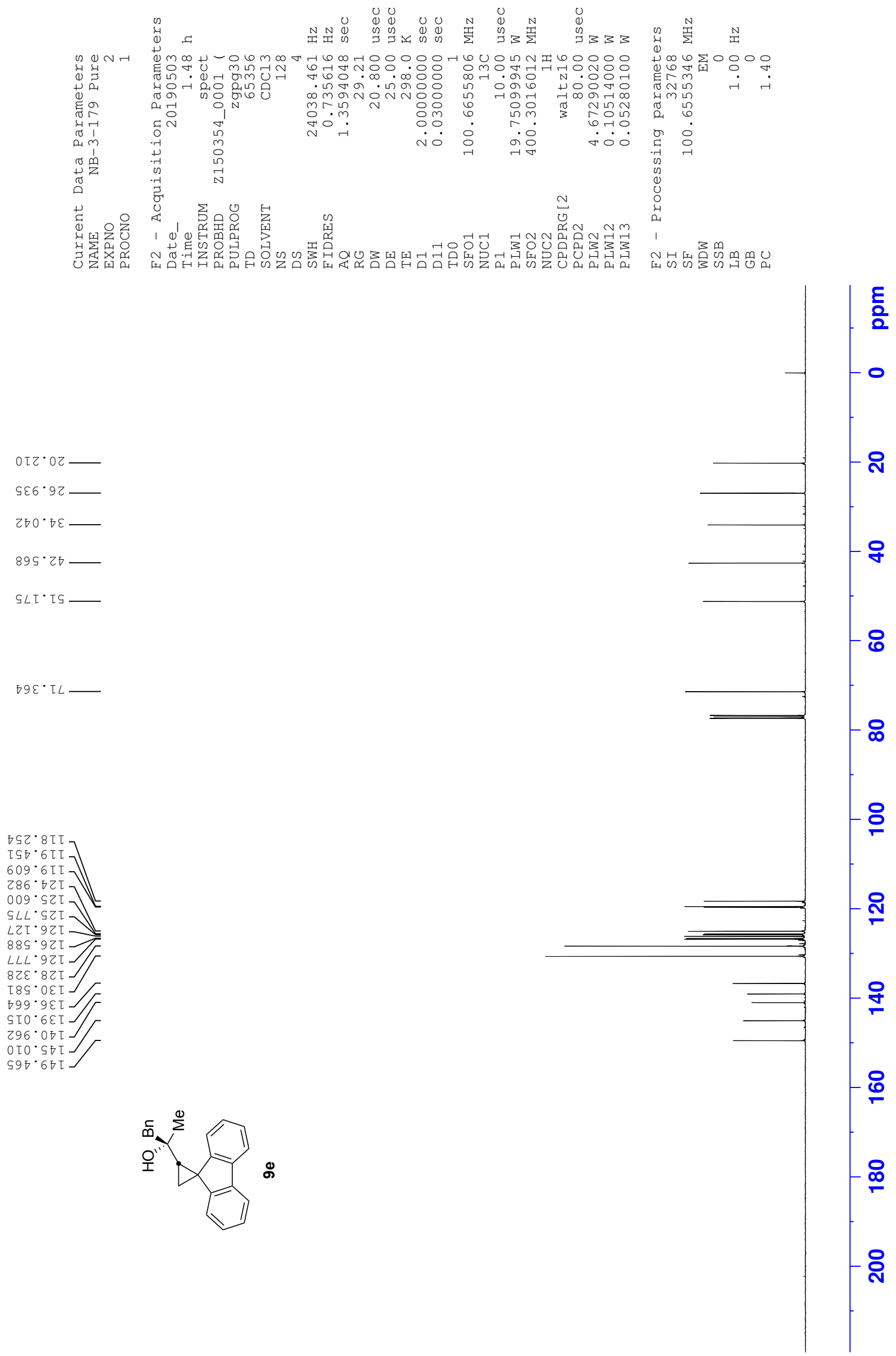

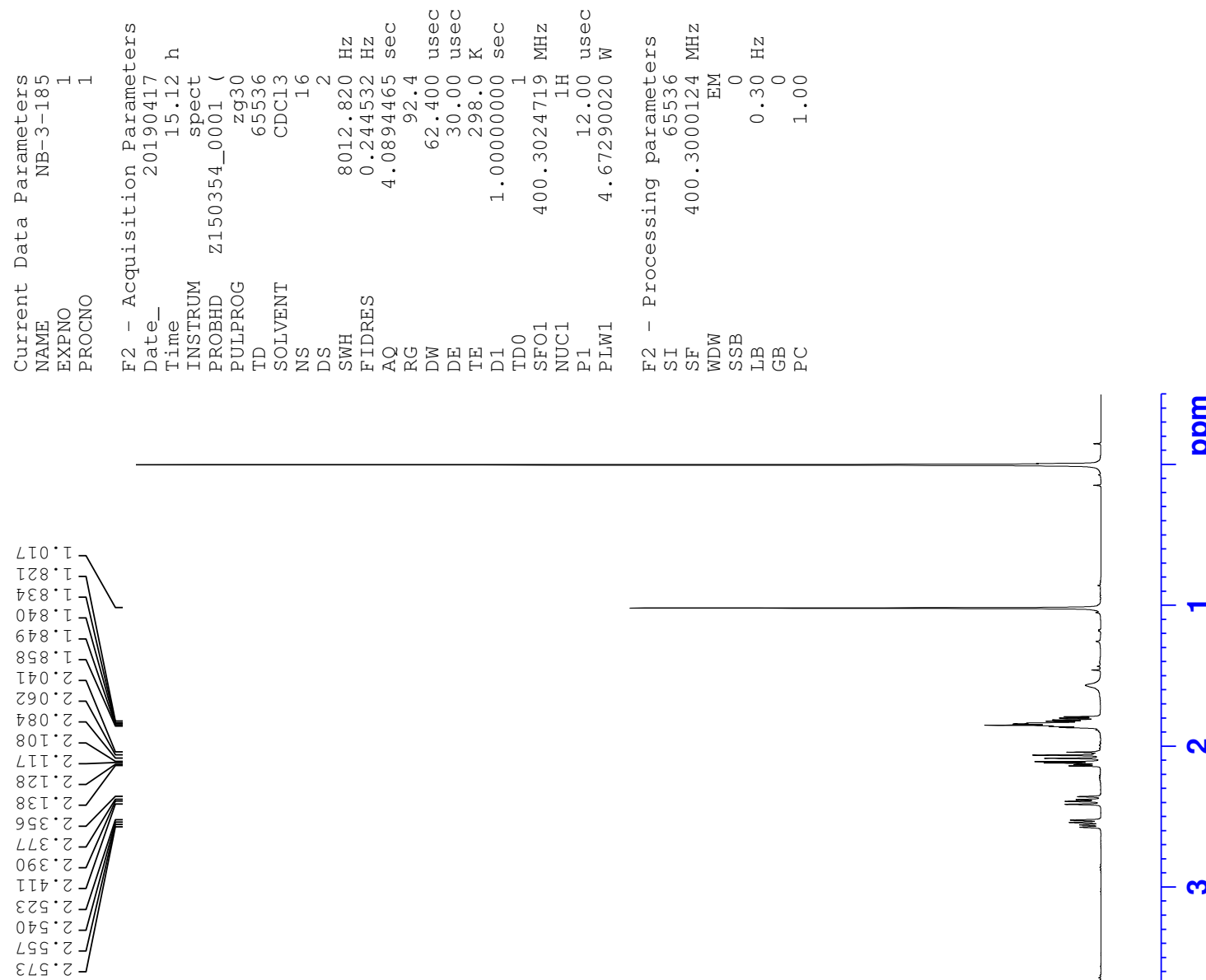

틍
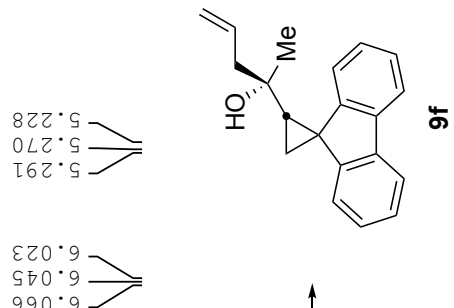

990.9
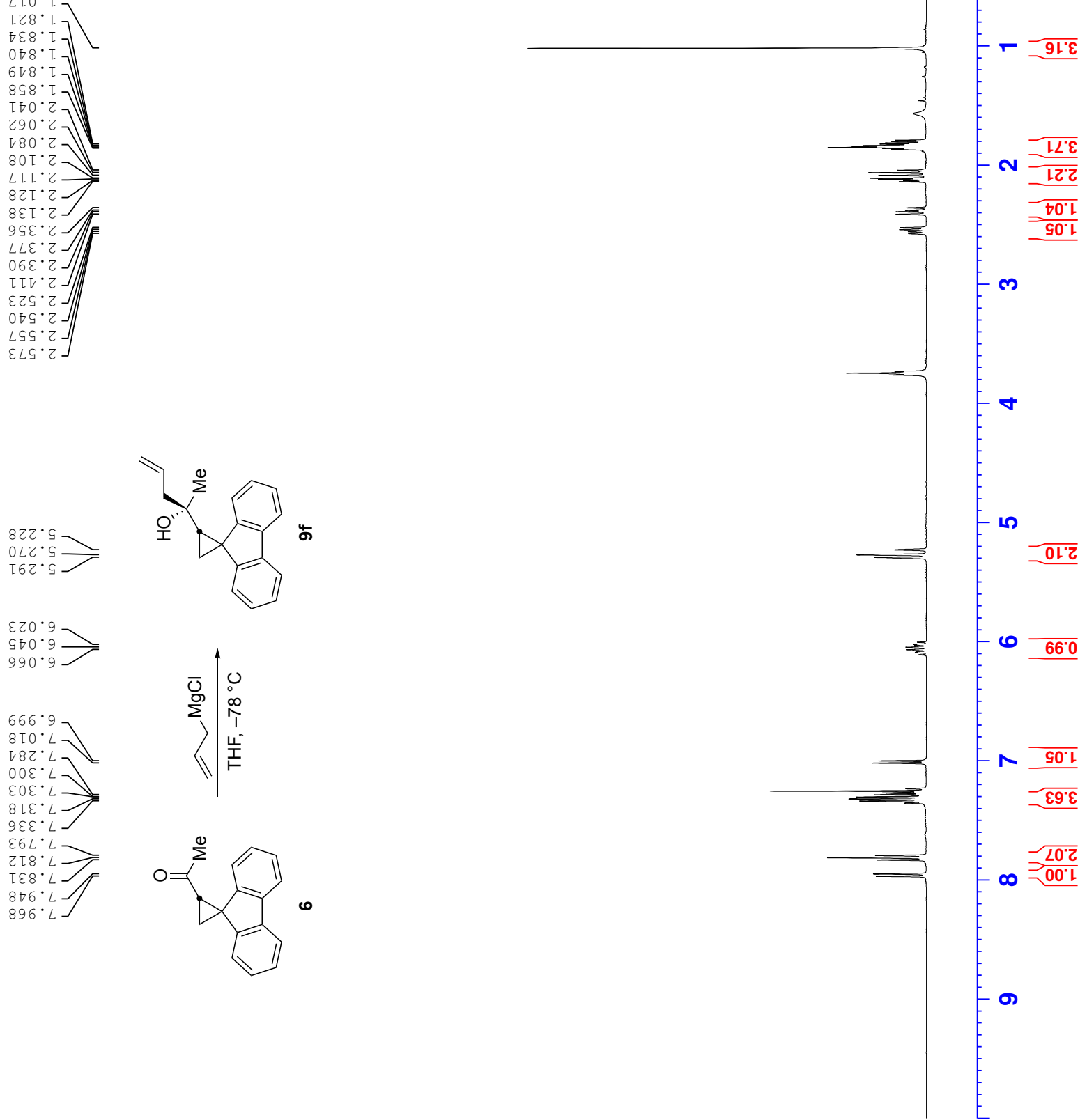

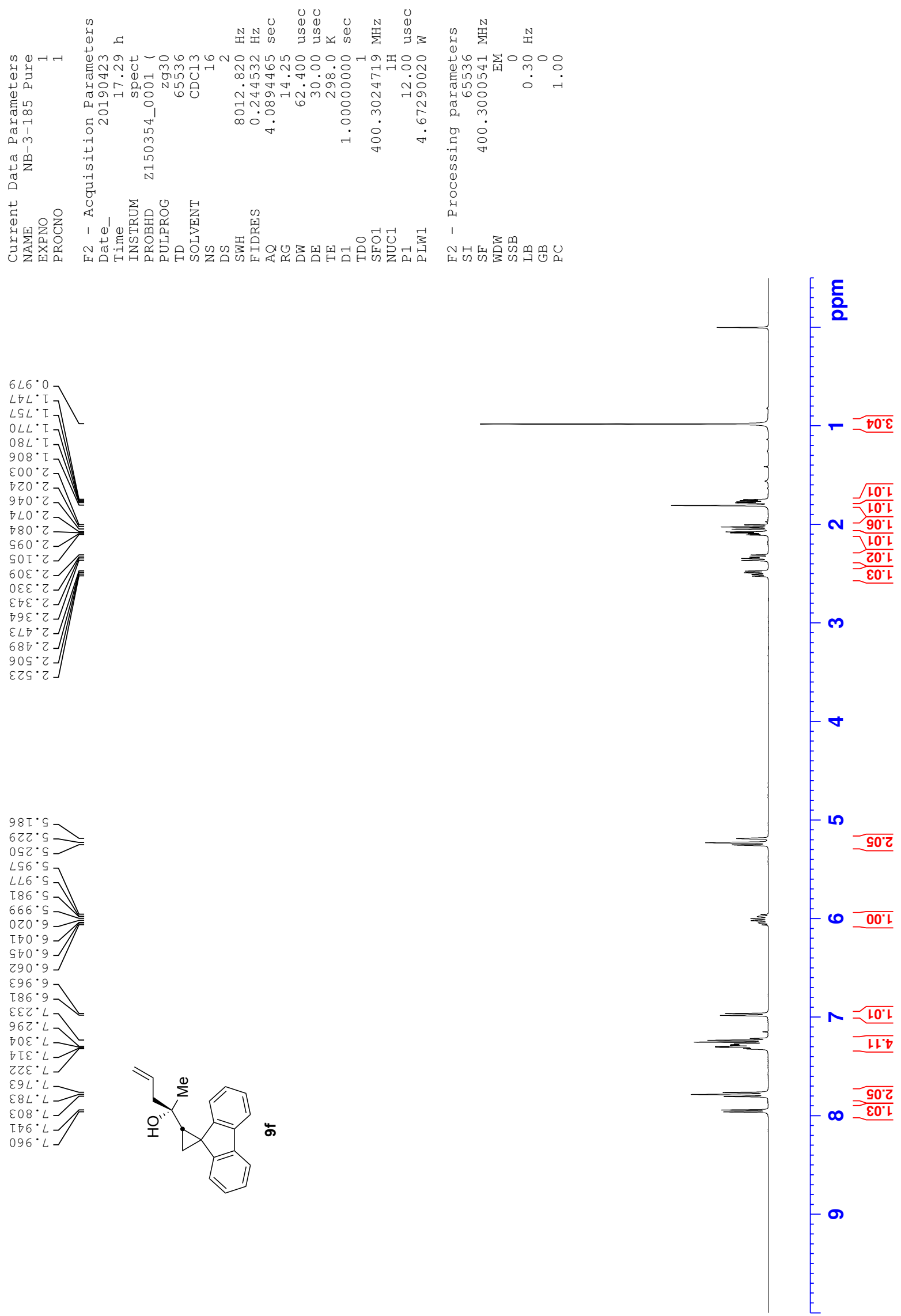

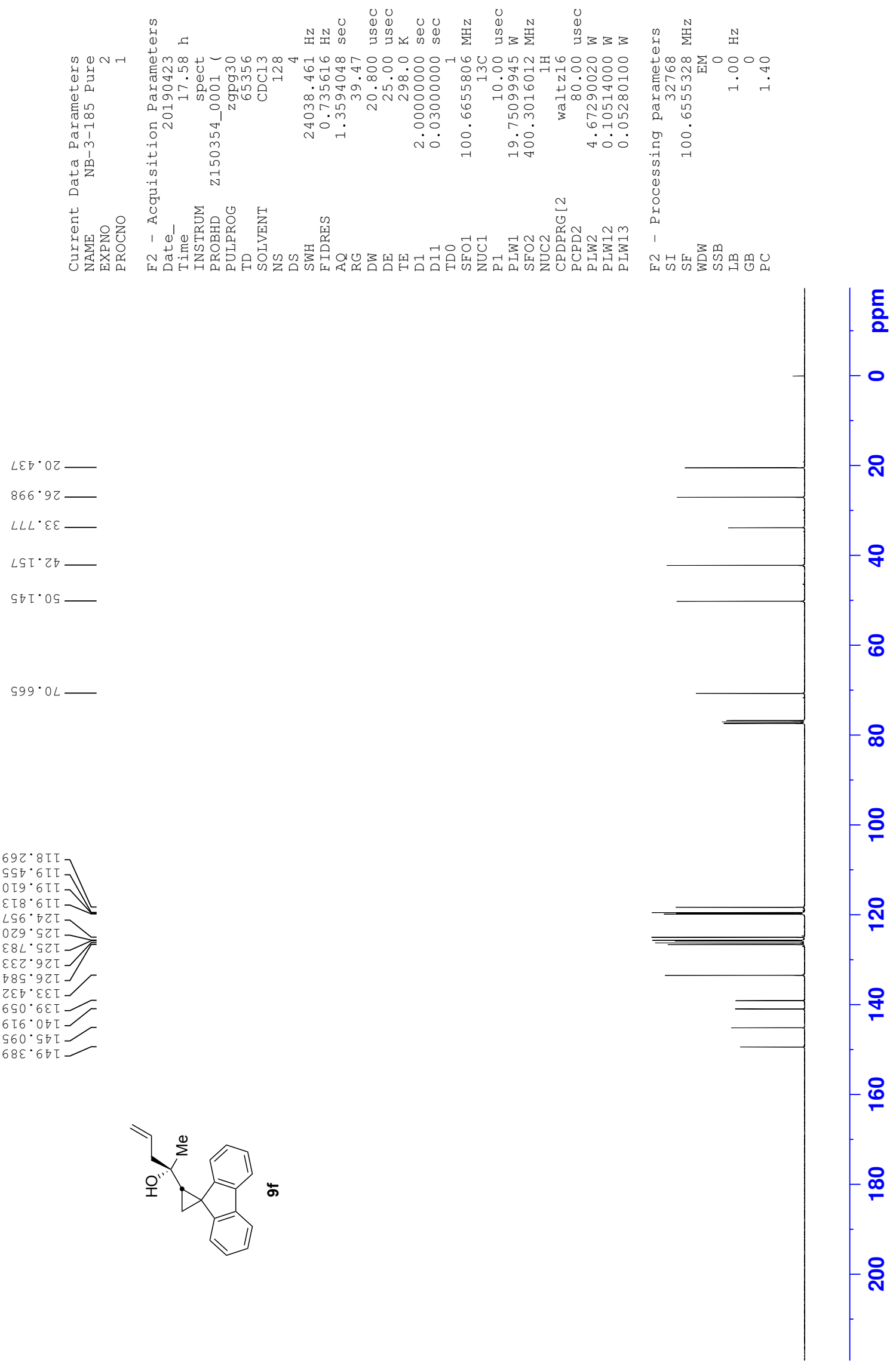

틍

$599^{\circ} 0 L$

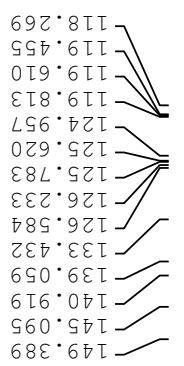



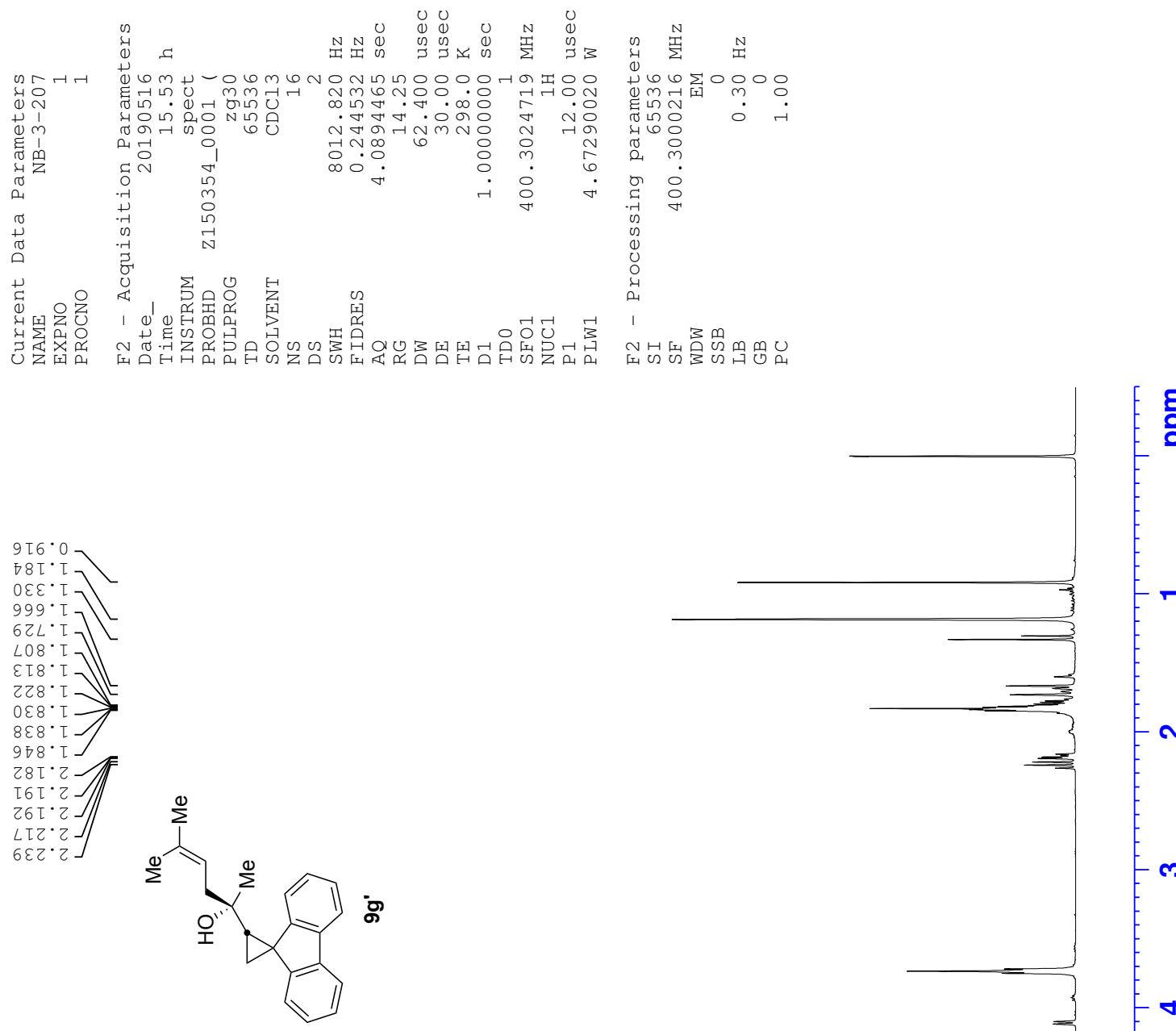

등
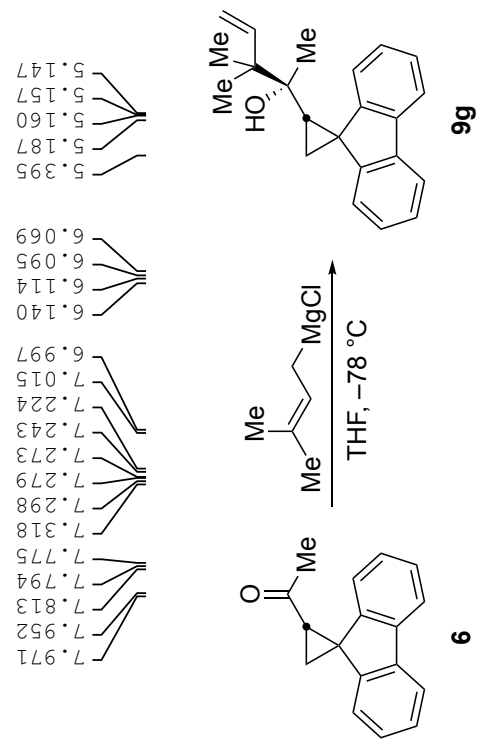

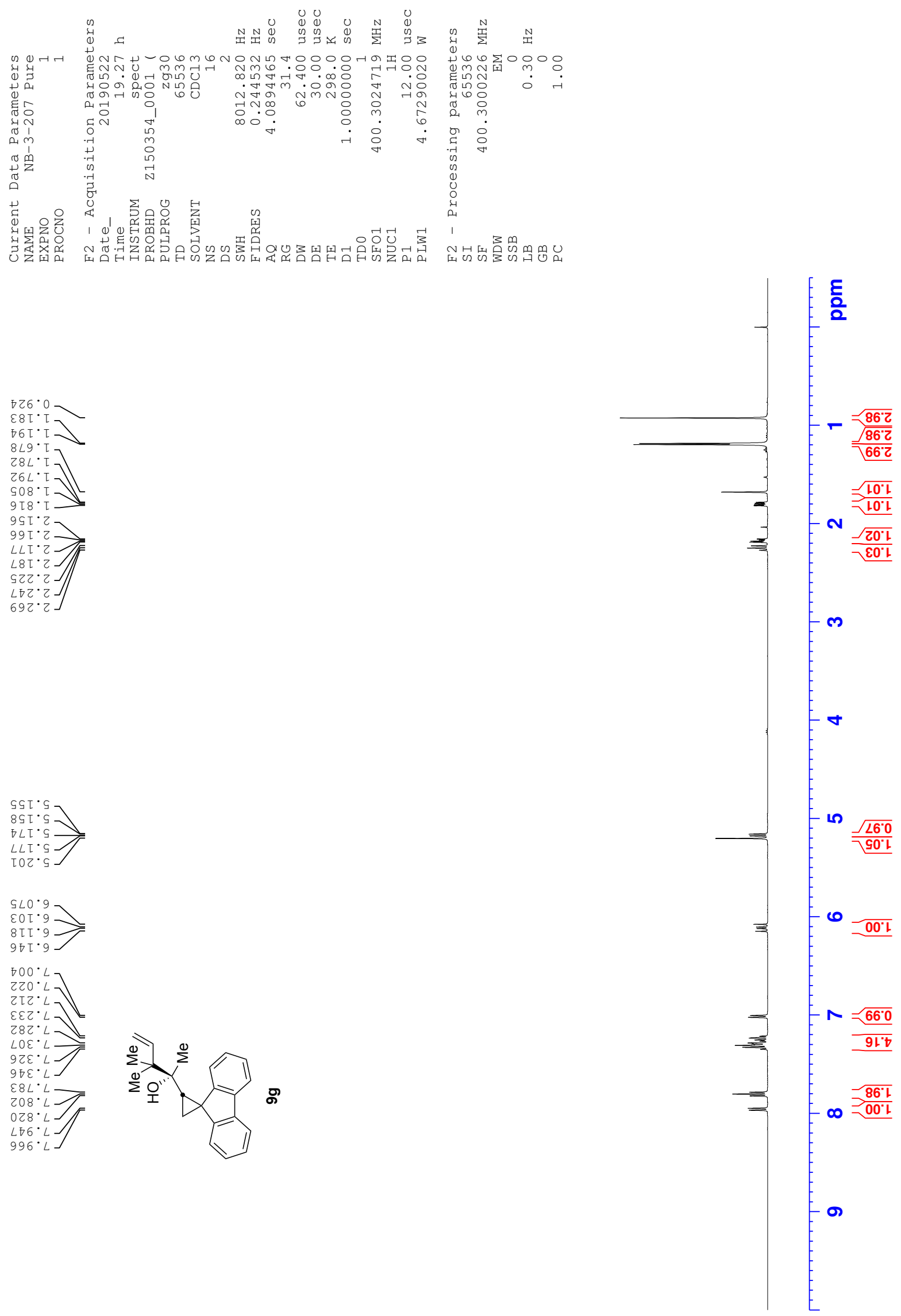

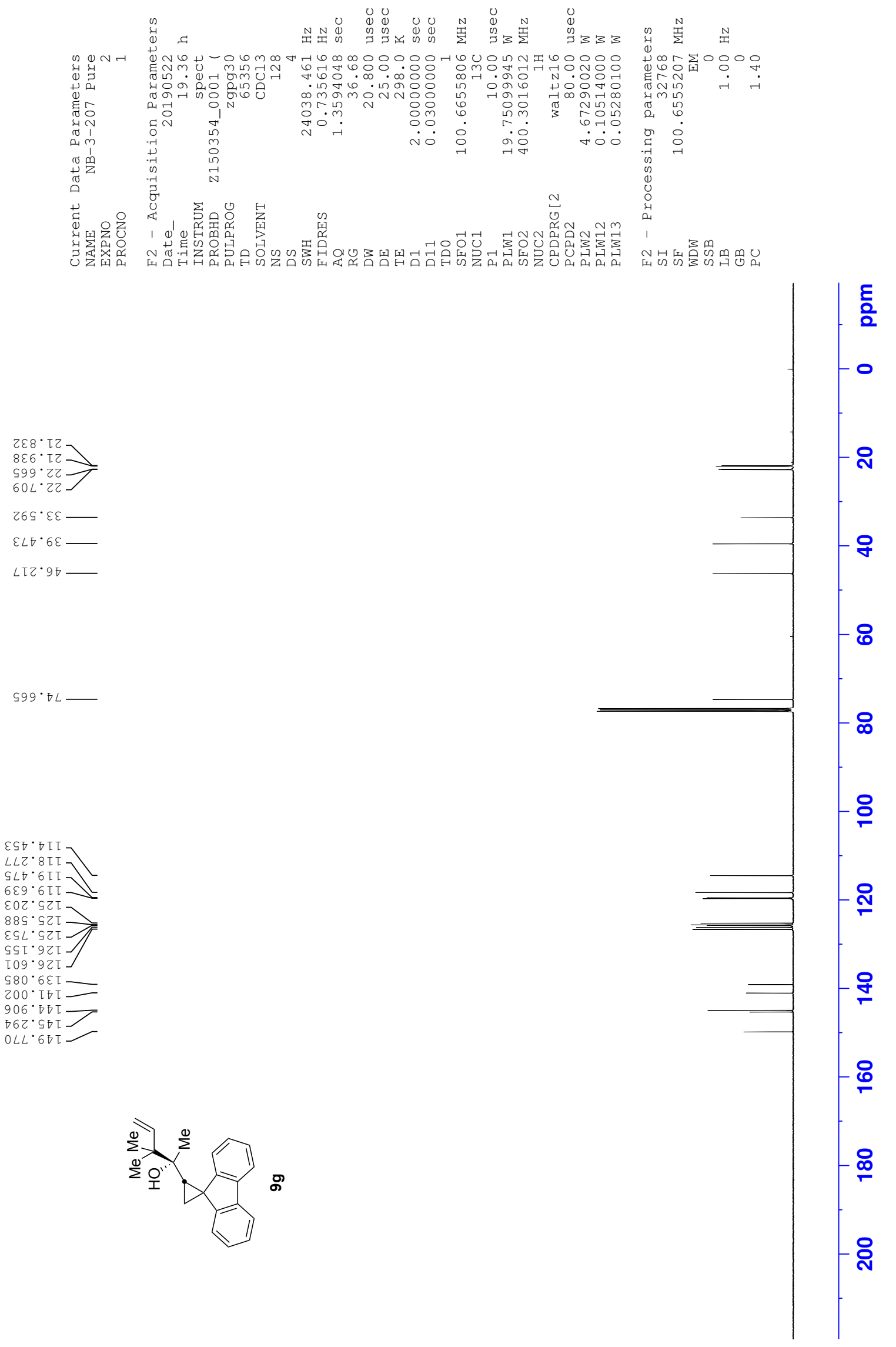

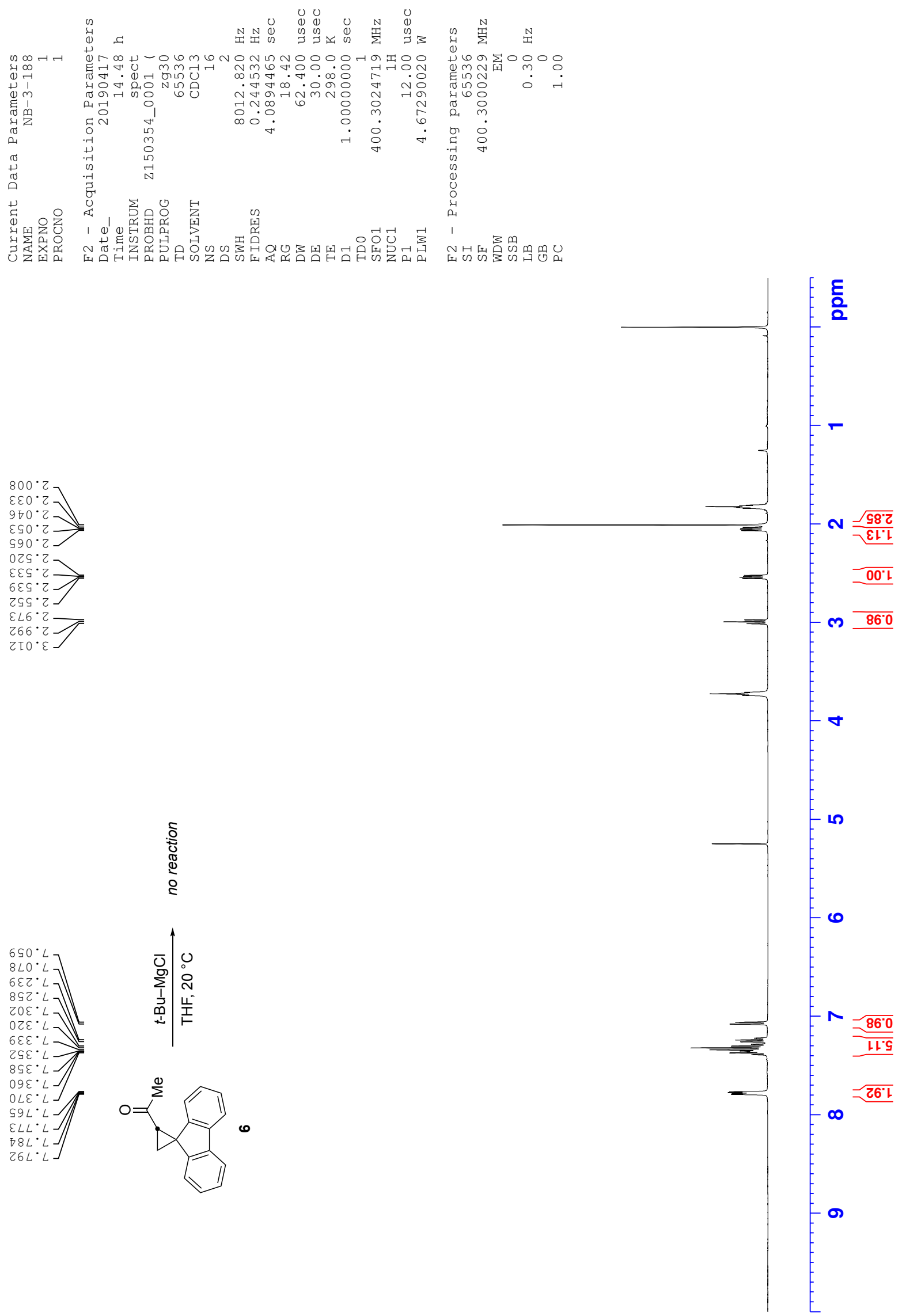

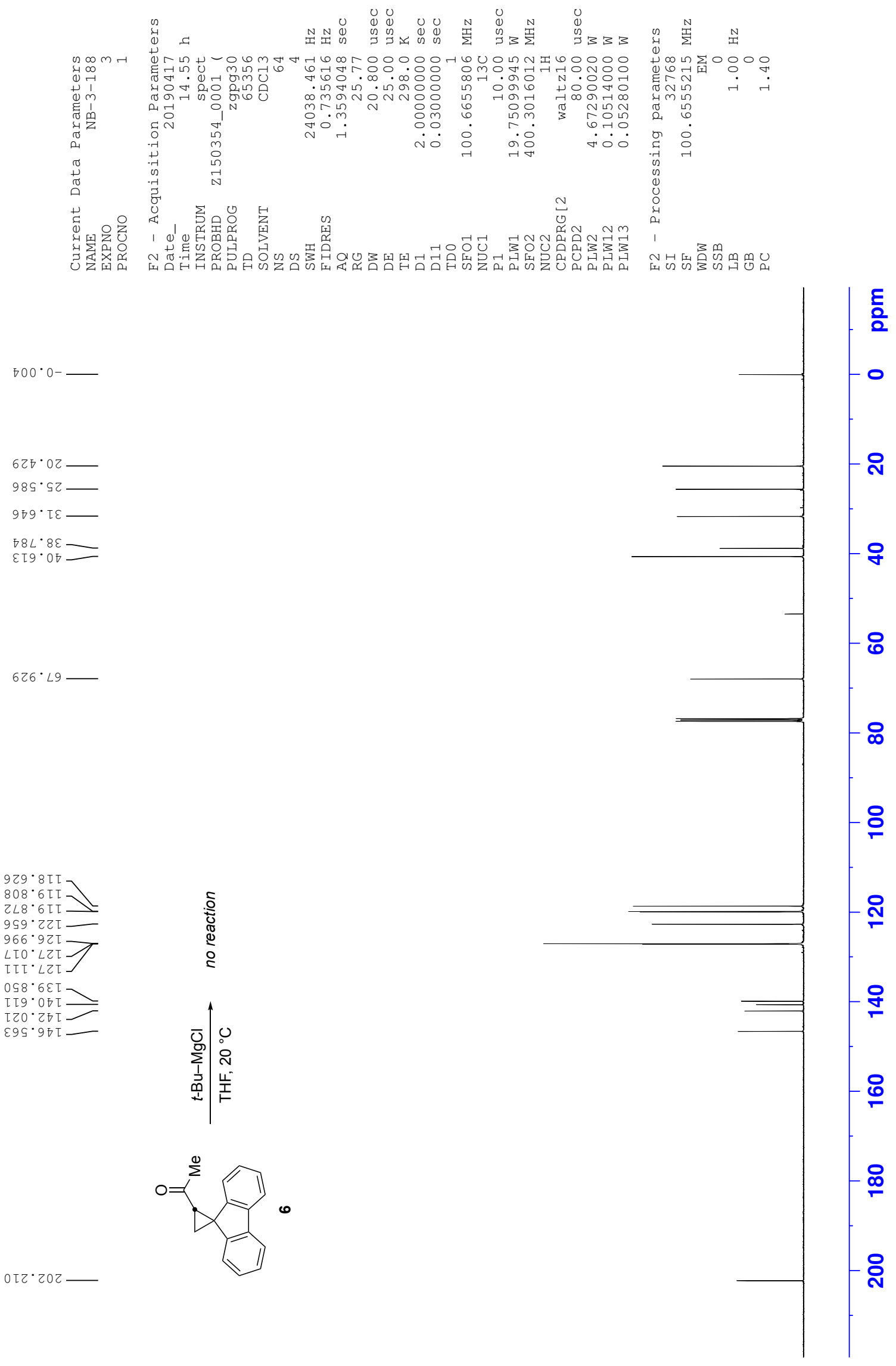

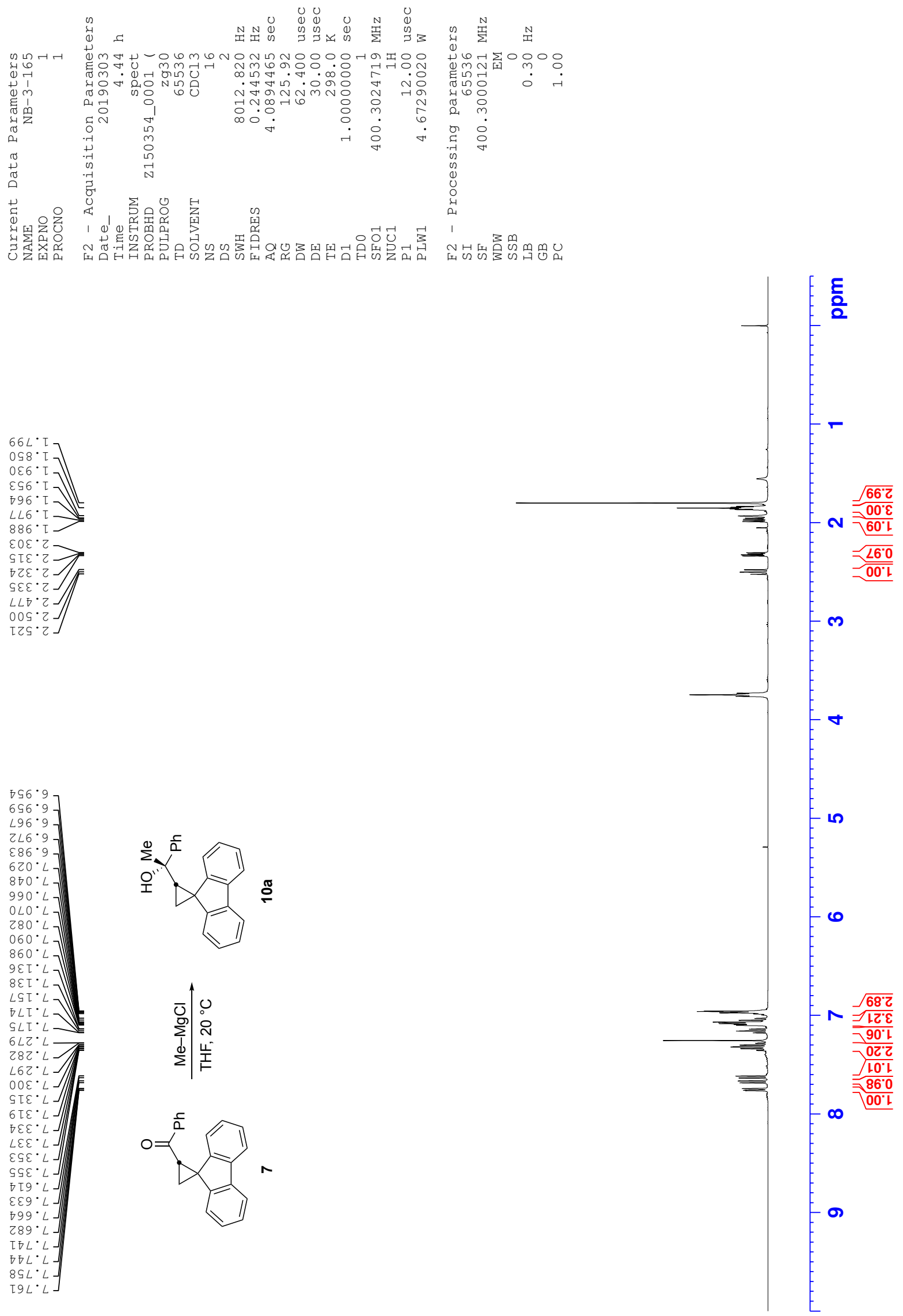

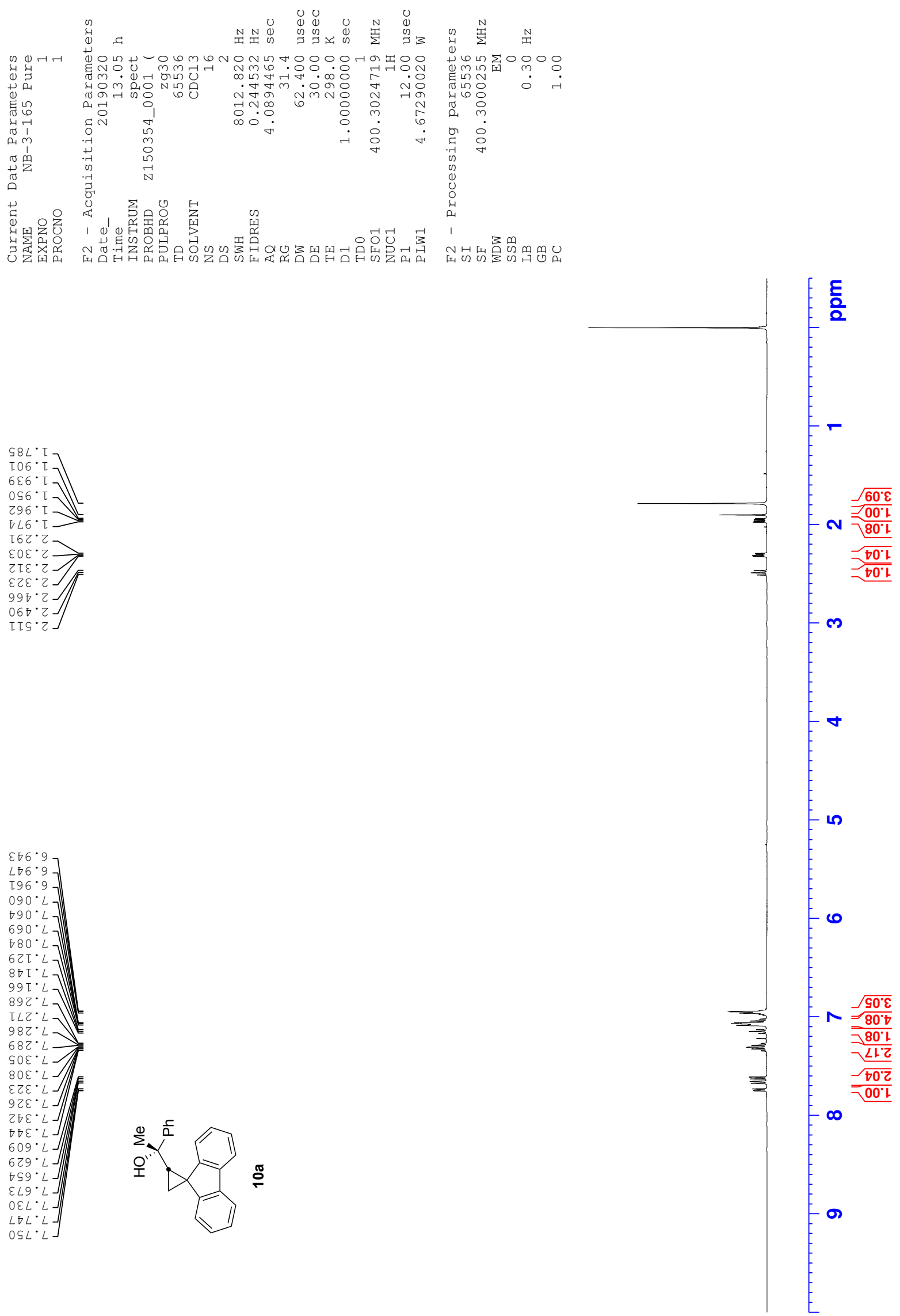

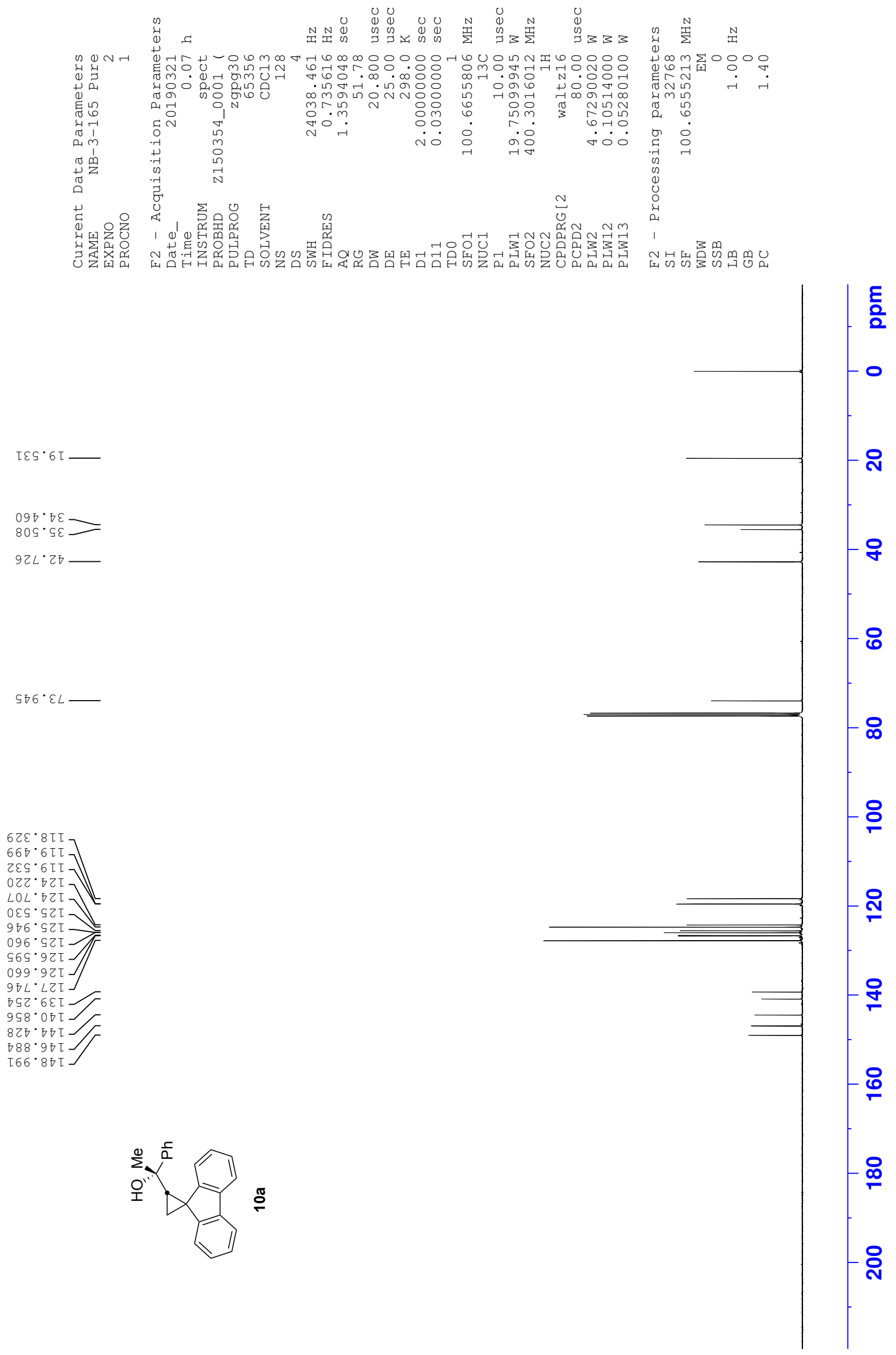

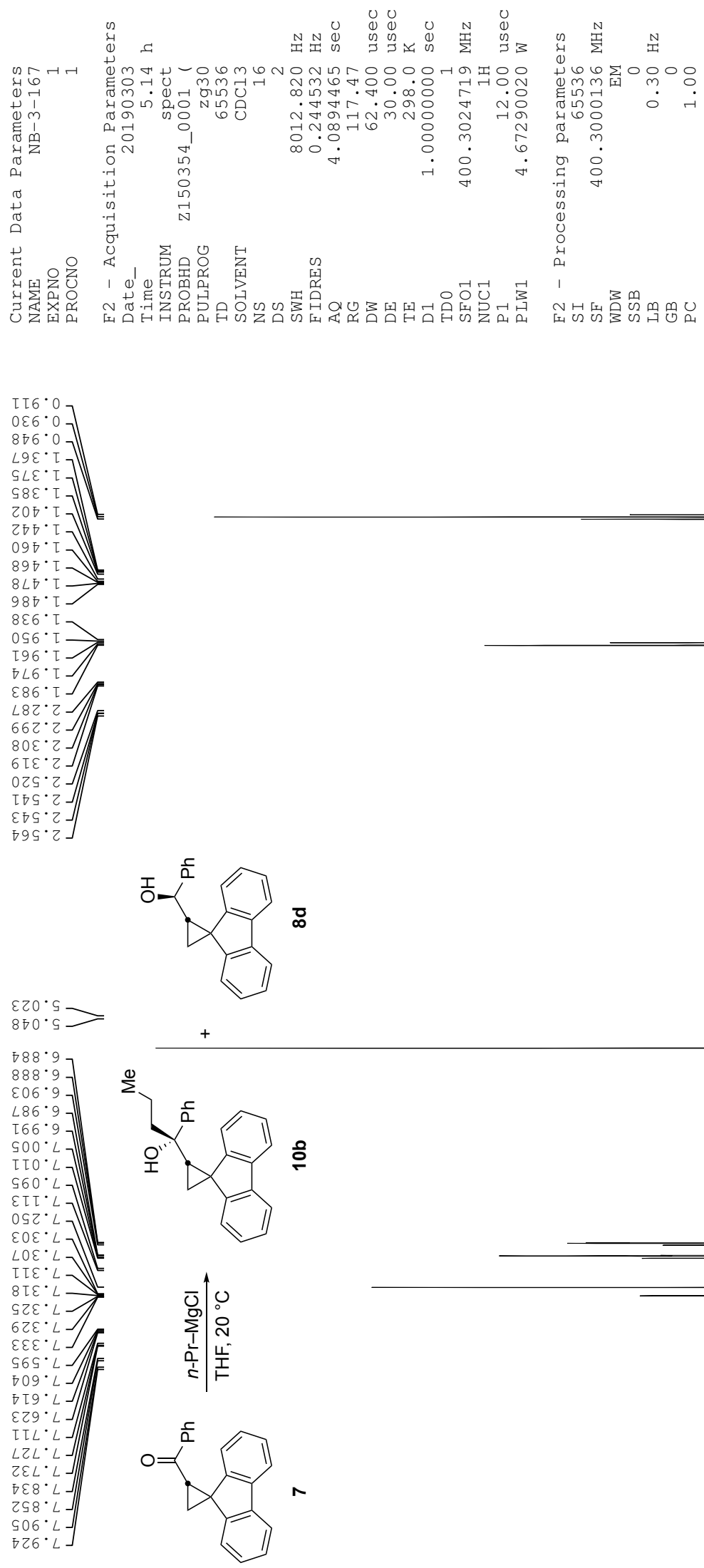

흘

$-\overline{\mathrm{G} 0^{\circ} \varepsilon}$ $\frac{78^{2} \mathrm{z}}{}$

$N=20^{\circ}$

$=90^{\circ} \mathrm{t}$

$-00^{\circ}$

-

$-\sqrt{92^{\circ} 0}$

$-6$

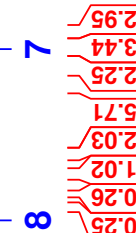

a 

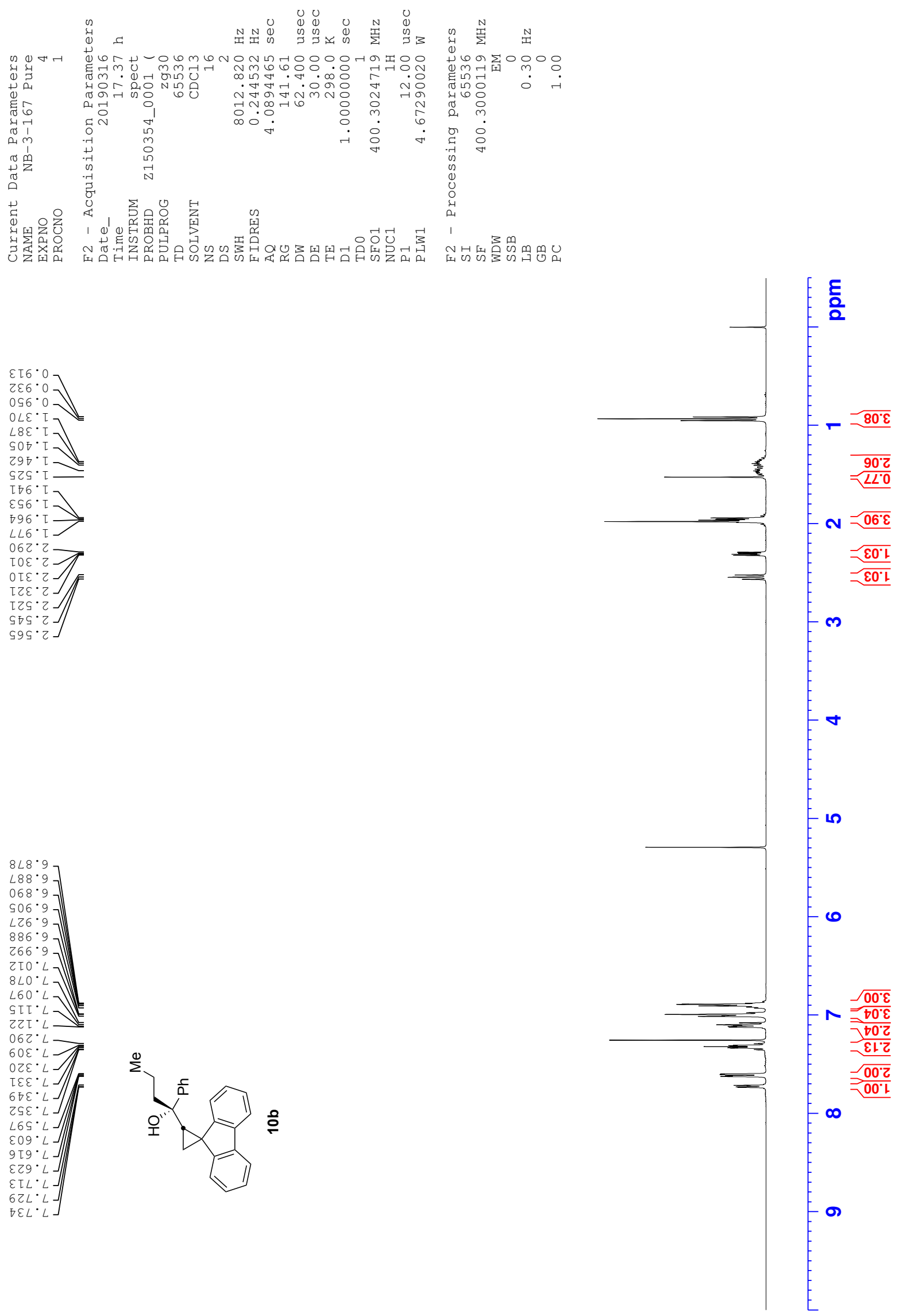

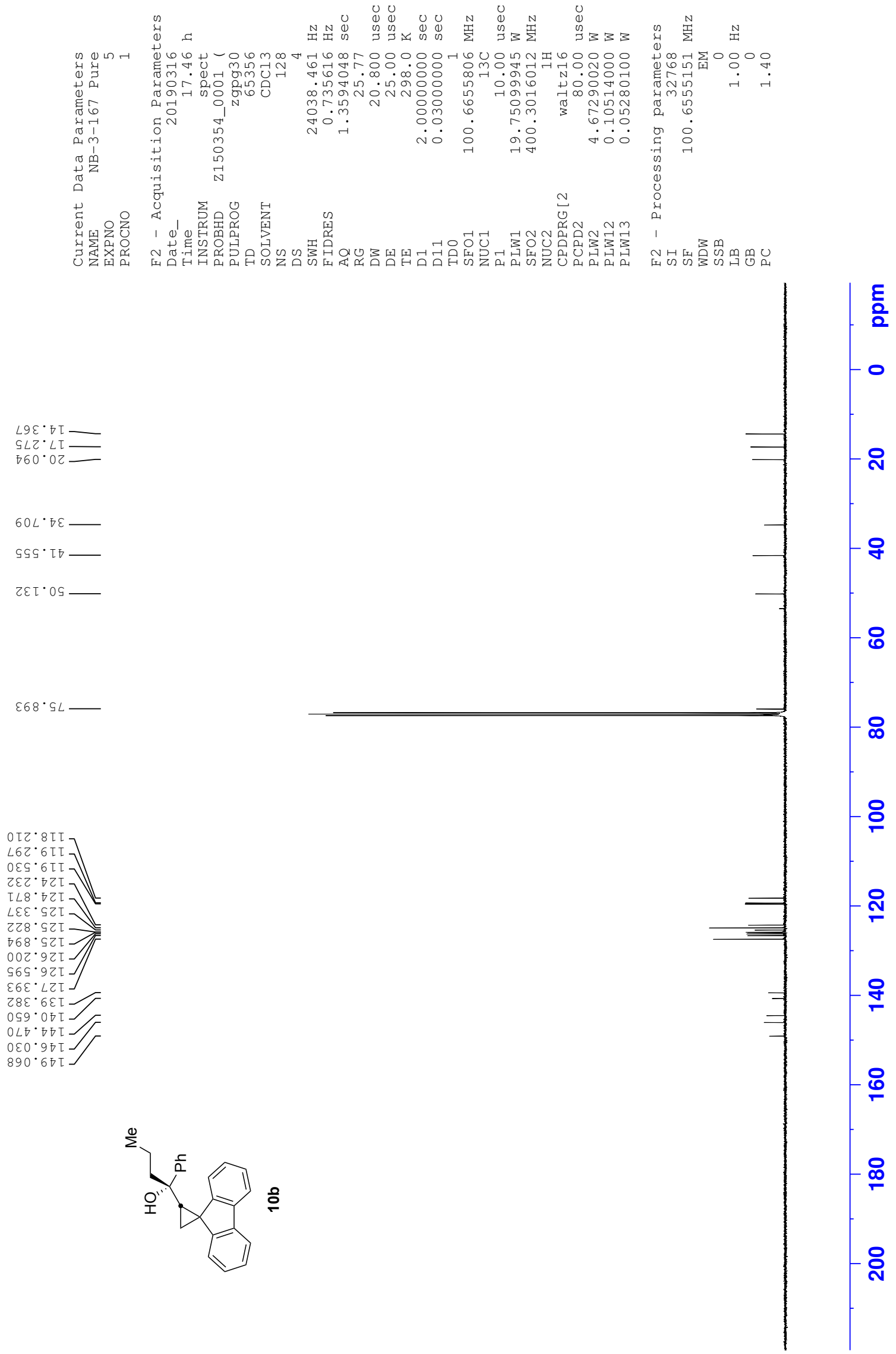

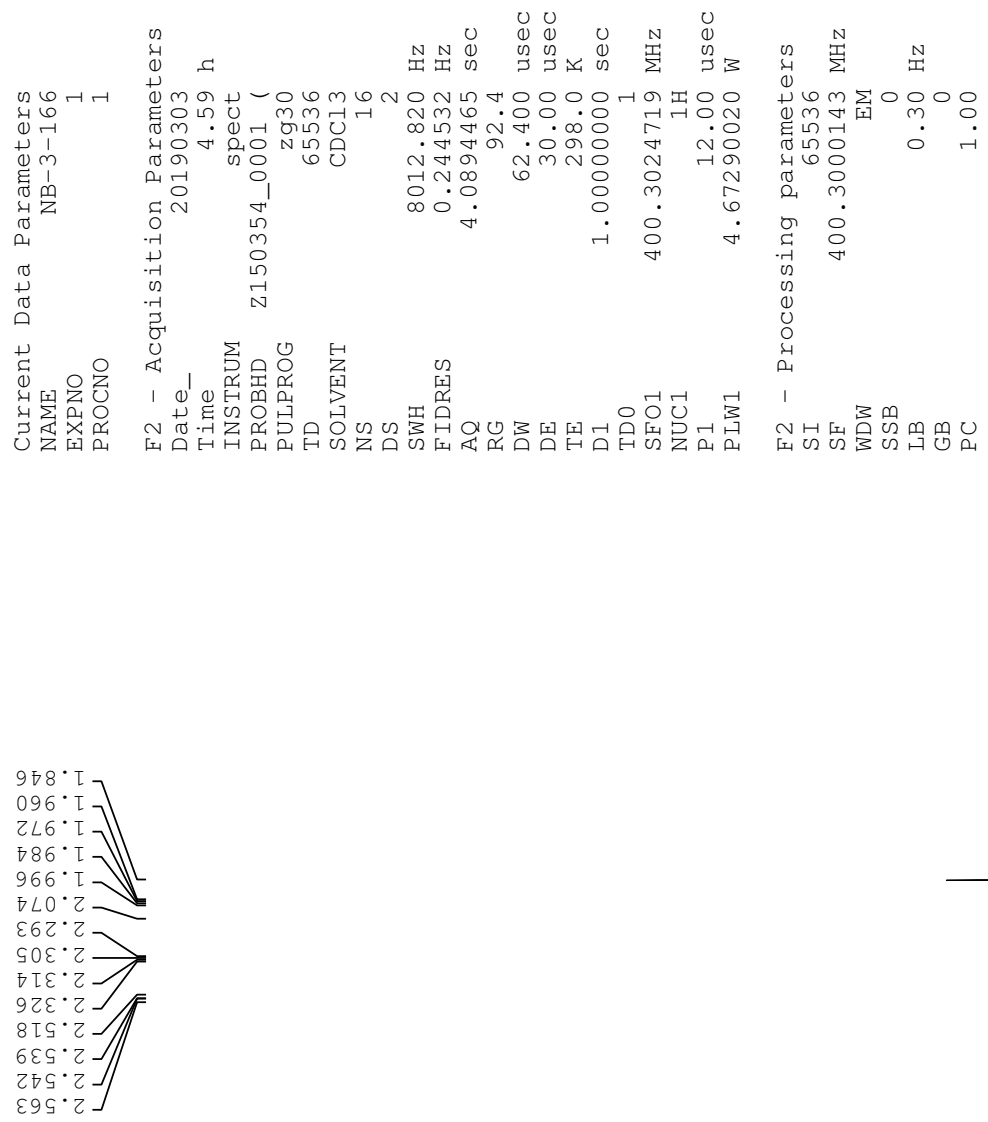

$\operatorname{IFL} L \cdot$

$\varepsilon[\tau \cdot \varsigma]$

$0 \oplus z \cdot c$

乙๘乙·与]

$\varepsilon 8 z^{\circ} \varsigma$

$8 \angle \varepsilon \cdot s]$

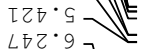

$\left.\begin{array}{ll}L Z Z \cdot 9 \\ \varepsilon L Z \cdot 9 \\ 06 z \cdot 9]\end{array}\right]$

$062 \cdot 9]$

9[ย.9]

$\left.\begin{array}{l}986.9 \\ 266.9\end{array}\right]$

$266 \cdot 9$
$[00 \cdot L]$

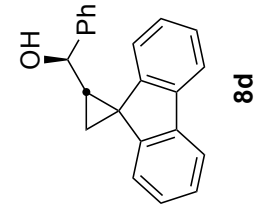

ర్ర
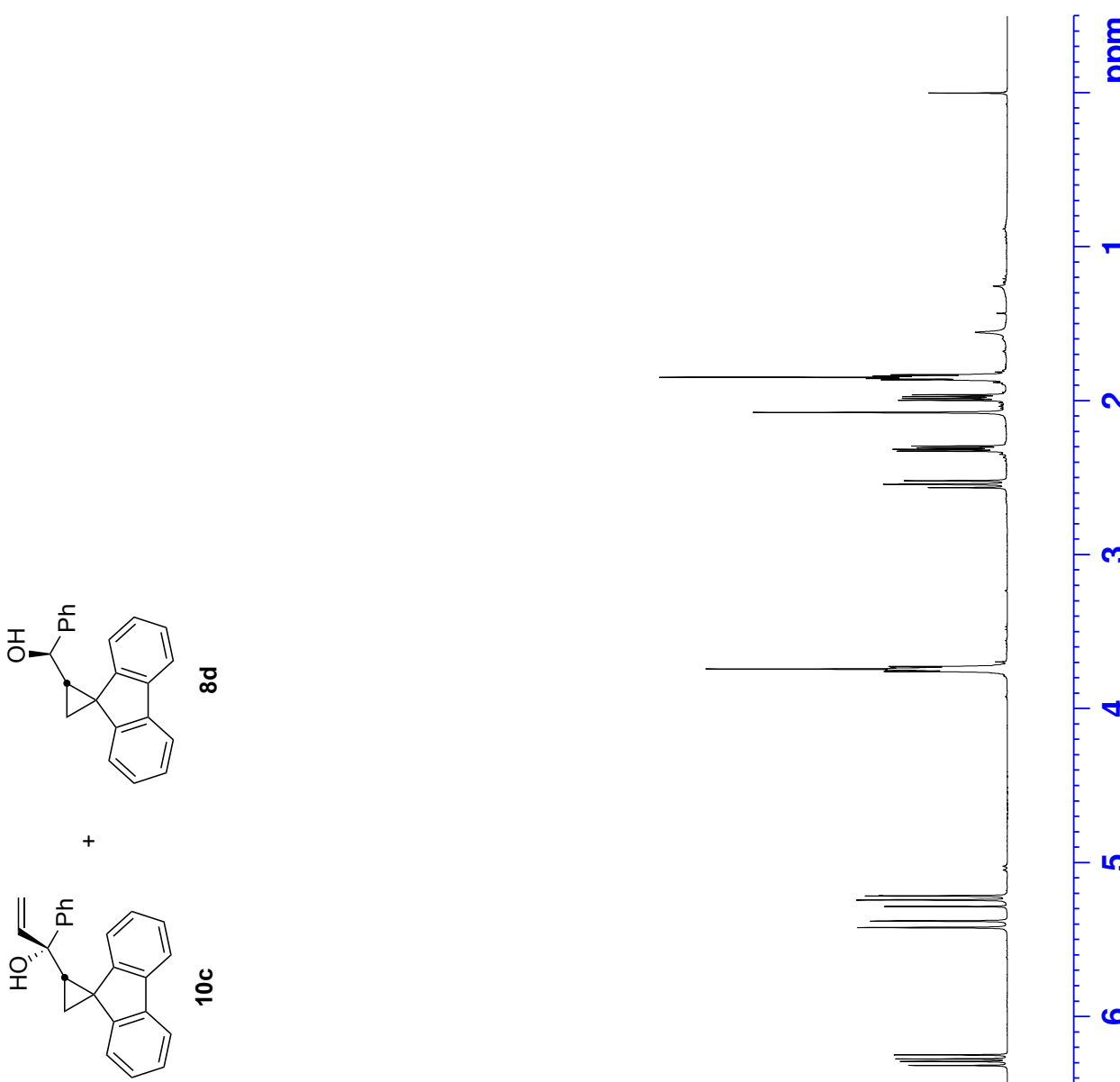

$\frac{\varepsilon}{2}$

$\left.\begin{array}{lll}\varepsilon & 00^{\circ} \\ \varepsilon S 0^{\circ} & L\end{array}\right]$

$\left.890^{\circ} \mathrm{L}\right]$

$\angle 90^{\circ} \mathrm{L}$

$\varepsilon \angle O^{\circ} L$

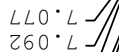

${ }^{\circ} 60^{\circ} \mathrm{L} J$

$L 6 Z \cdot L J$
$00 \varepsilon \cdot L]$

$90 \varepsilon \cdot L]$

$9 \nabla \varepsilon \cdot L J$

$\left.\angle 89^{\circ} L\right]$

$00 L \cdot L]$

$20 L: L$

SOL $\angle L$

$6 \tau L \cdot L J$

$99 L \cdot L J$
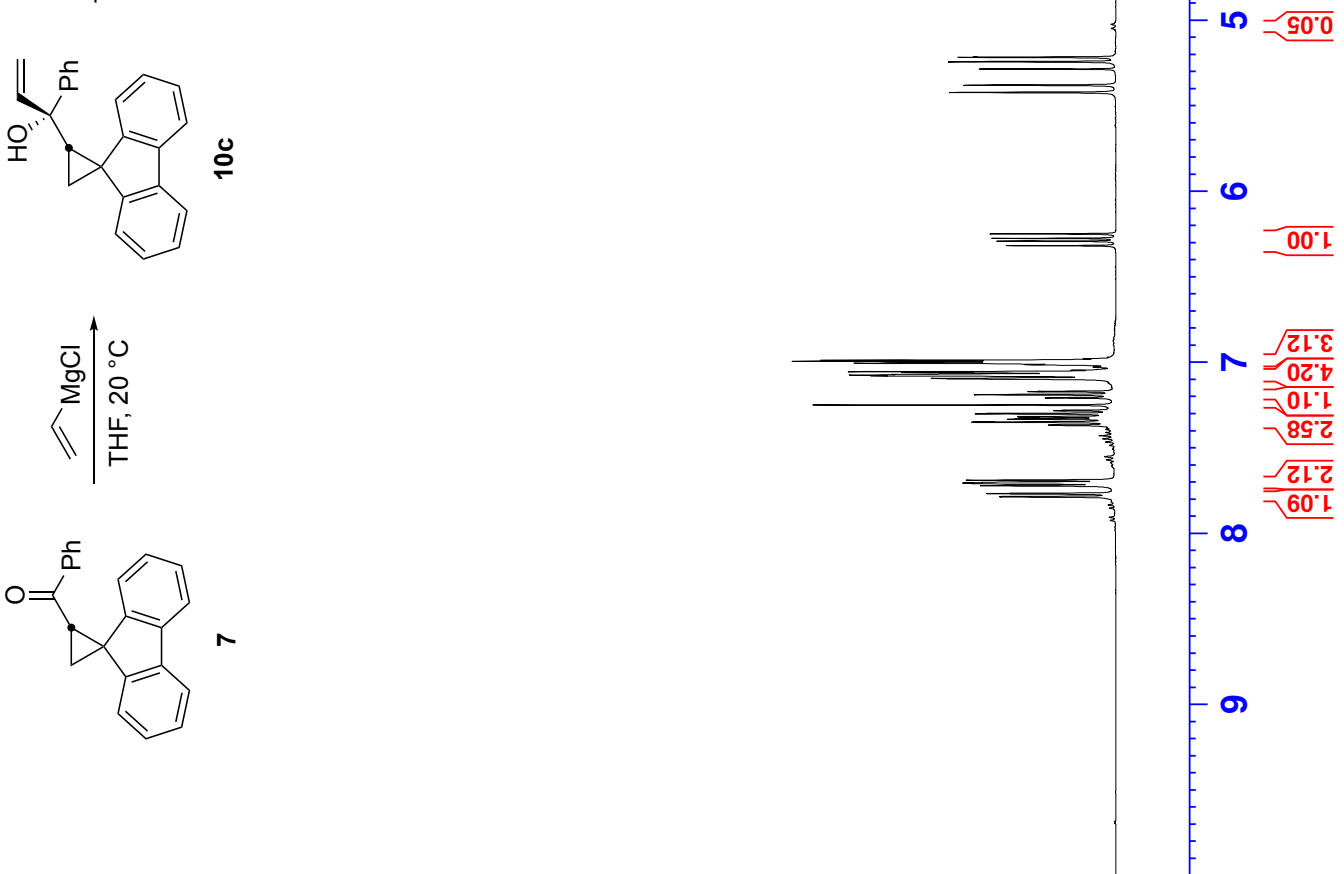

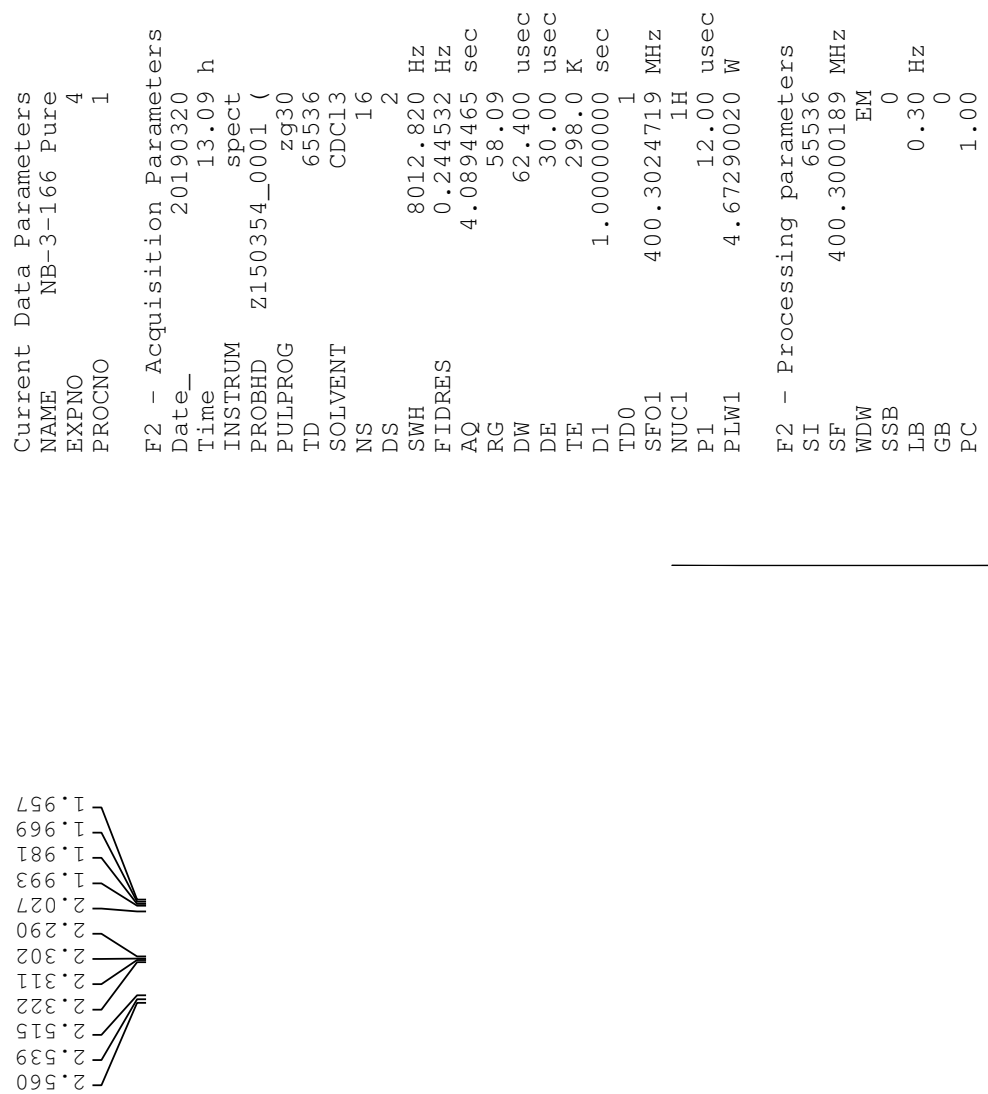

\}$=\frac{E}{2}$

틍
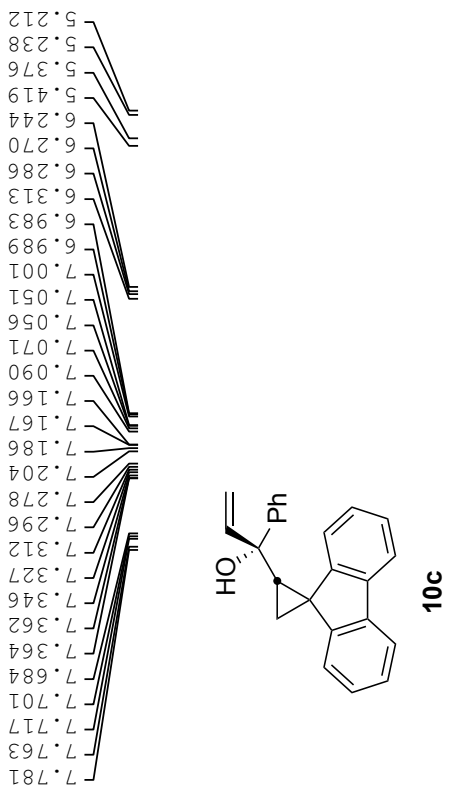

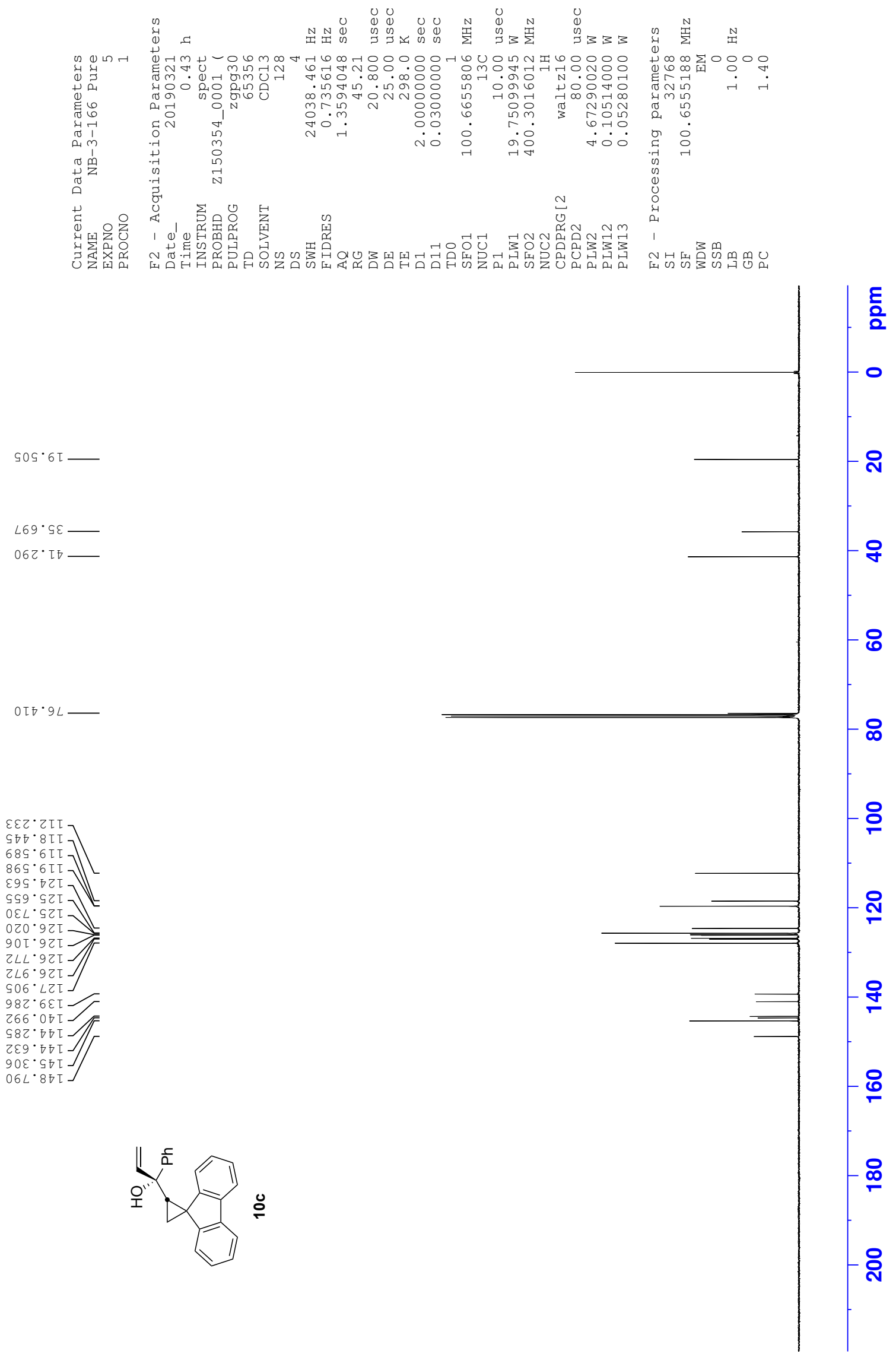

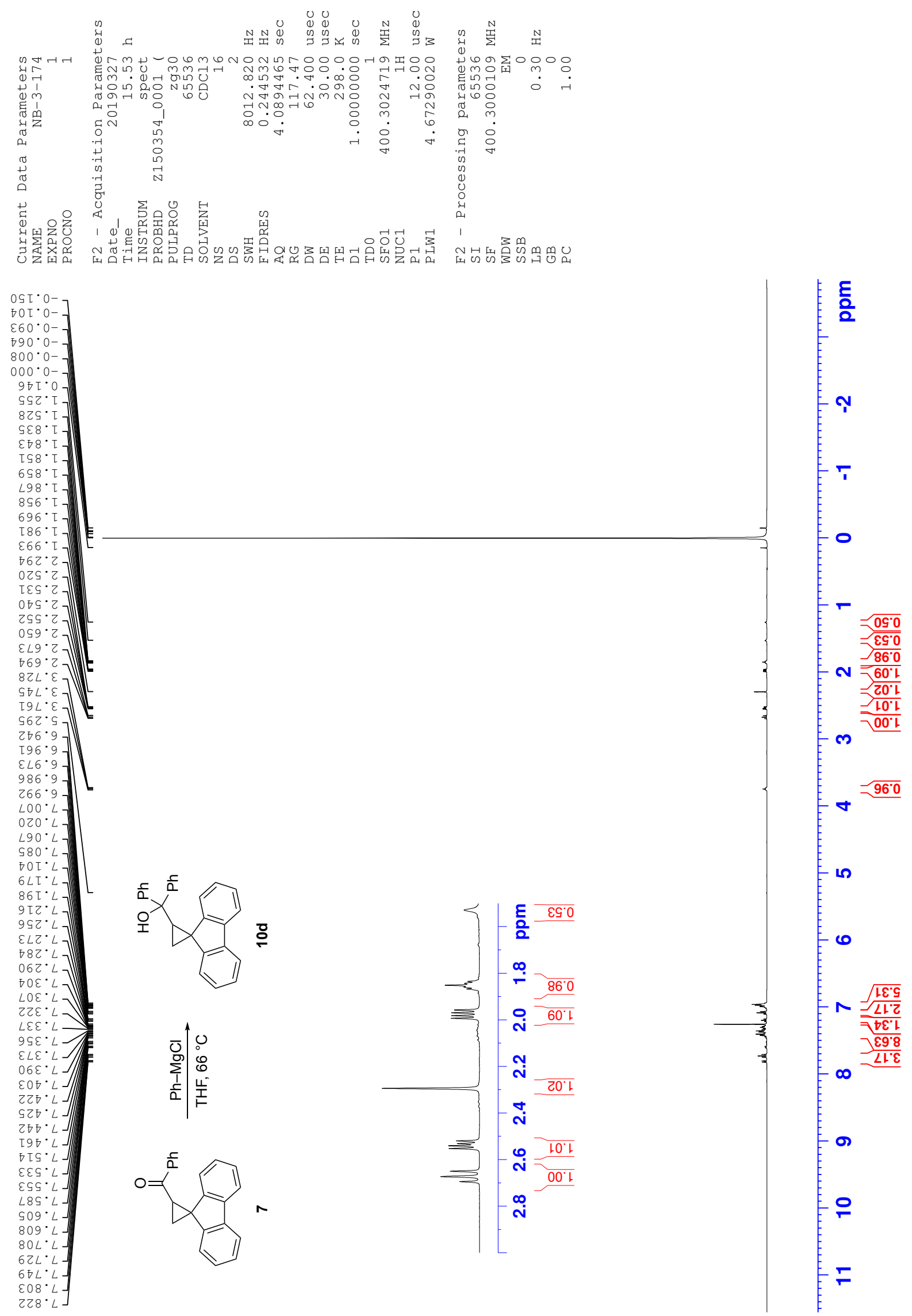

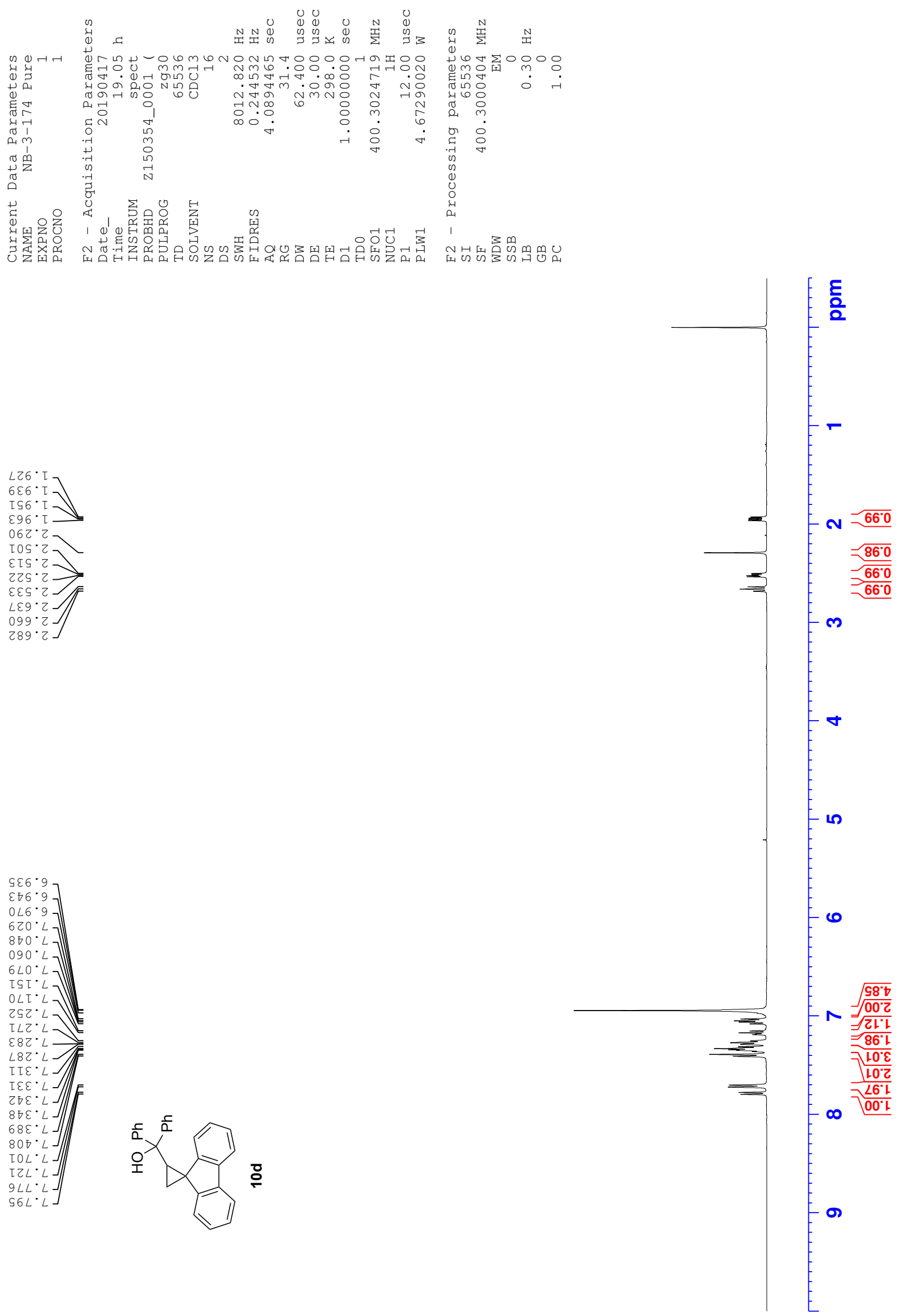

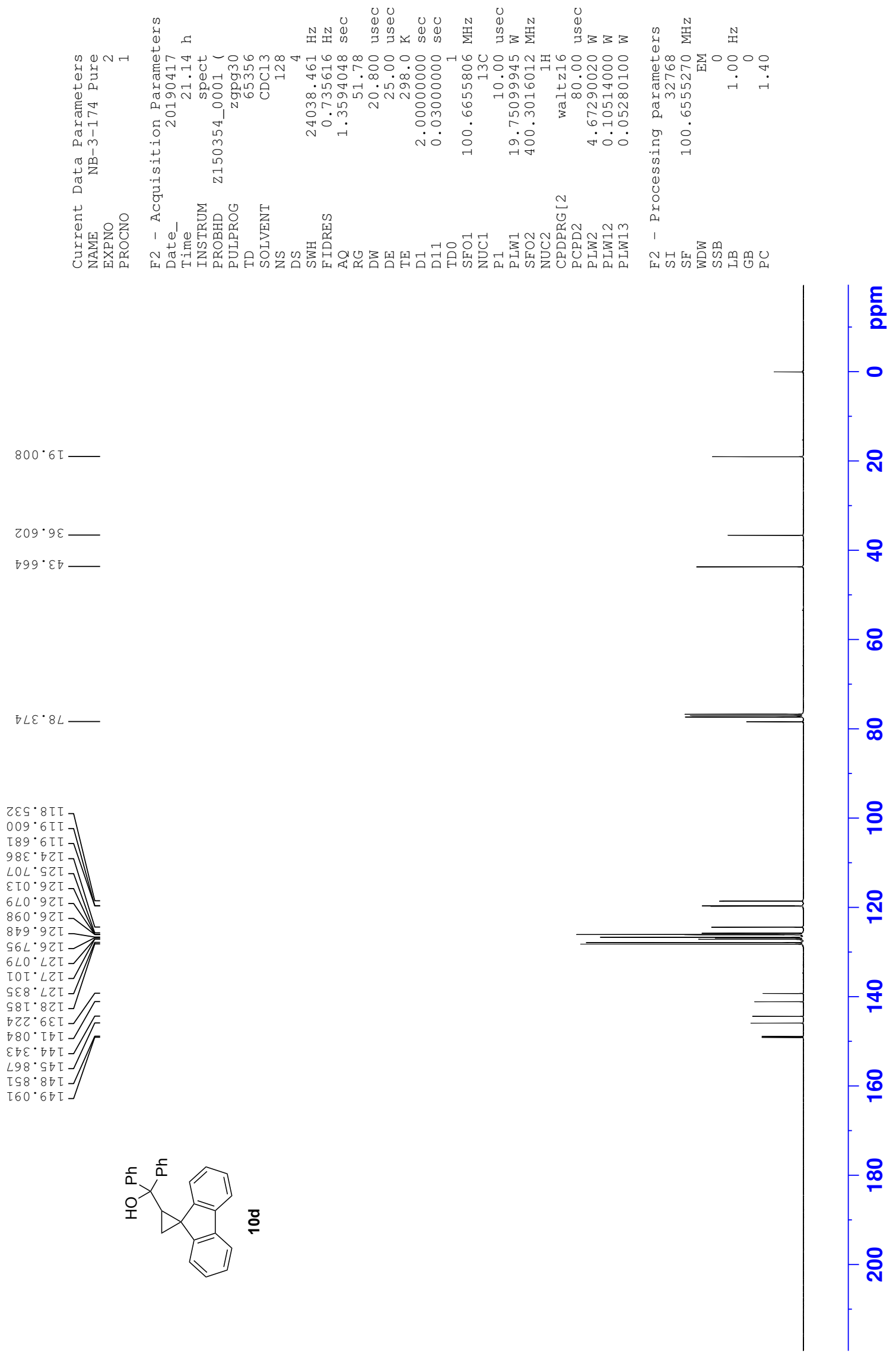

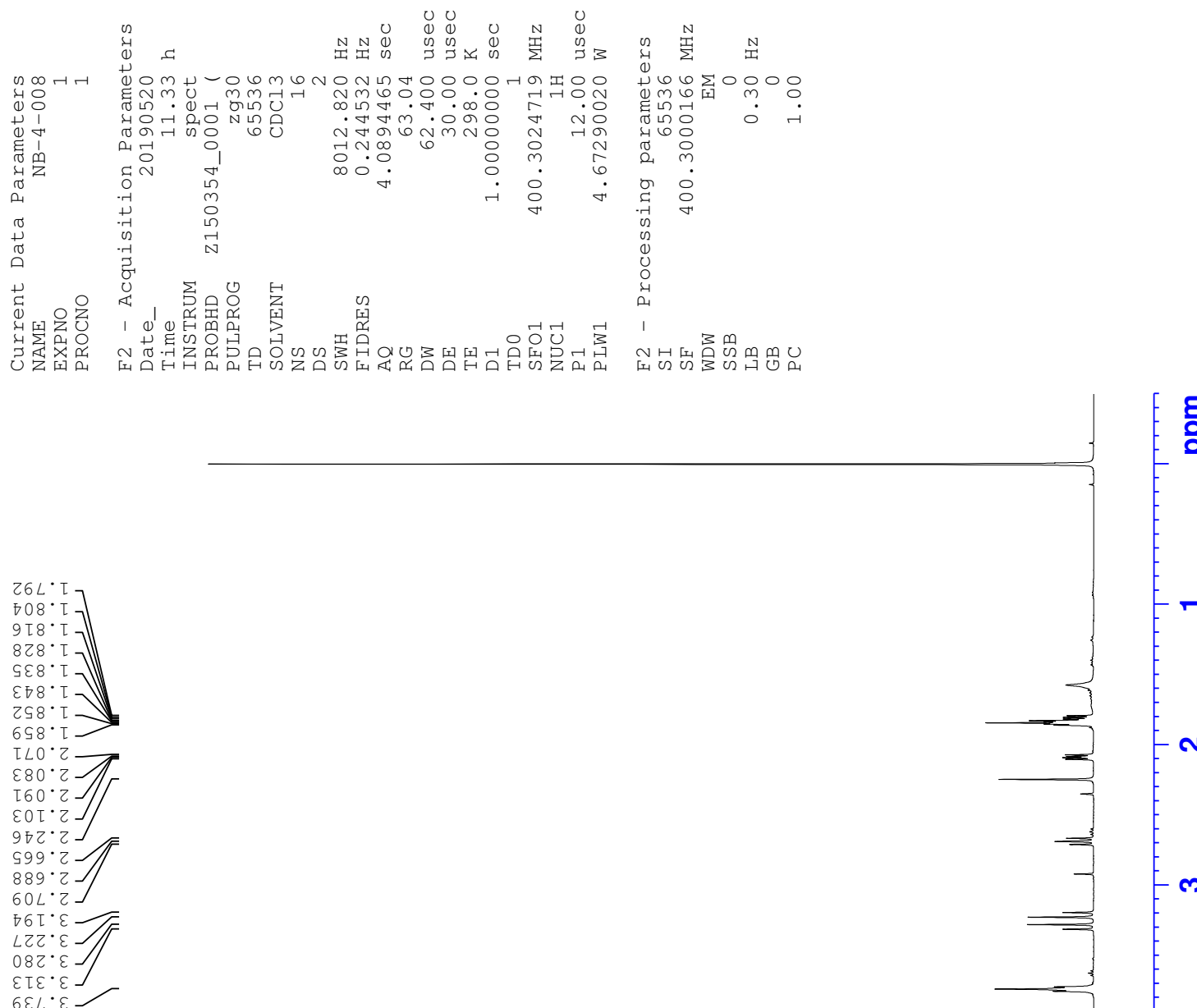

틍

$889^{\circ} \circ$
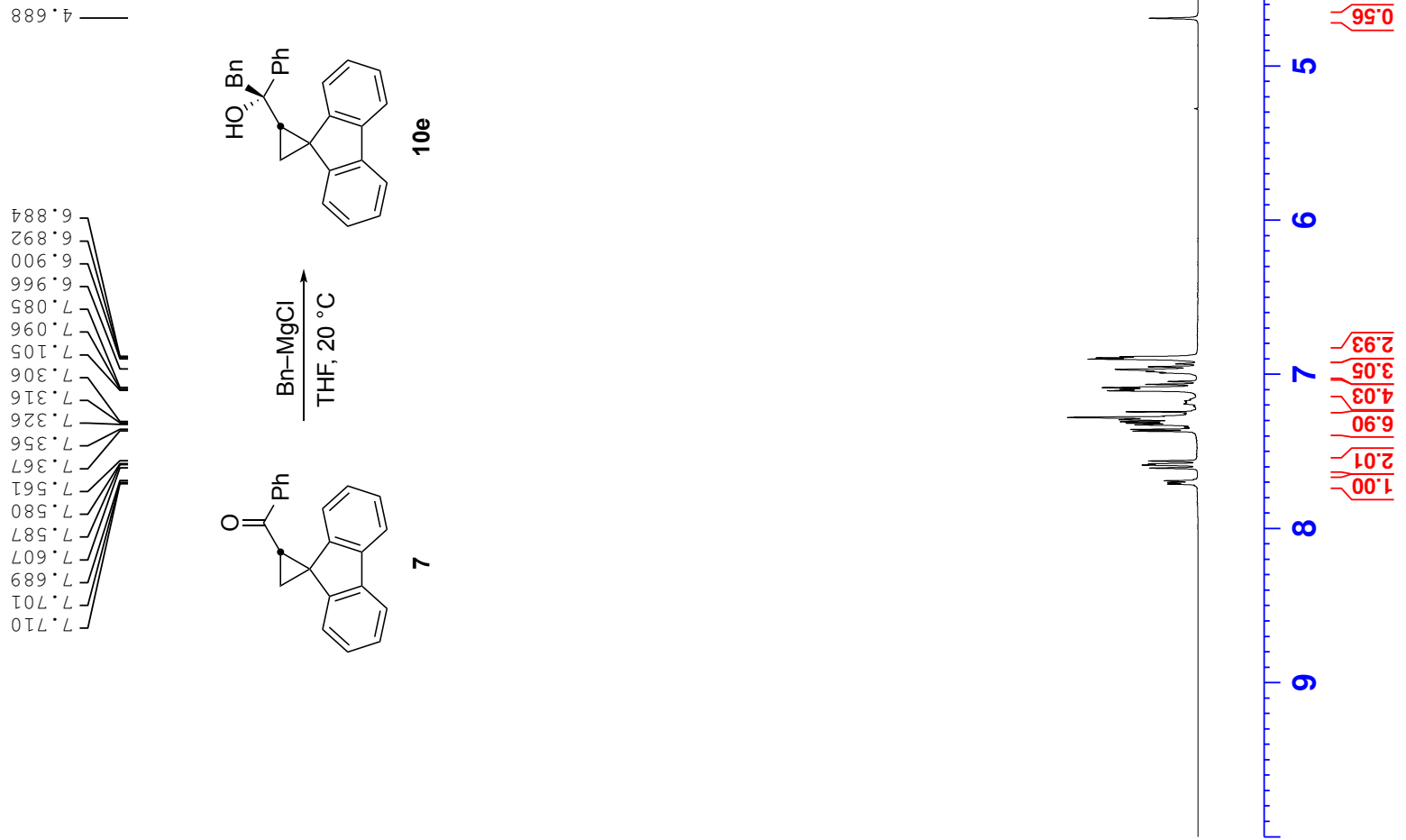

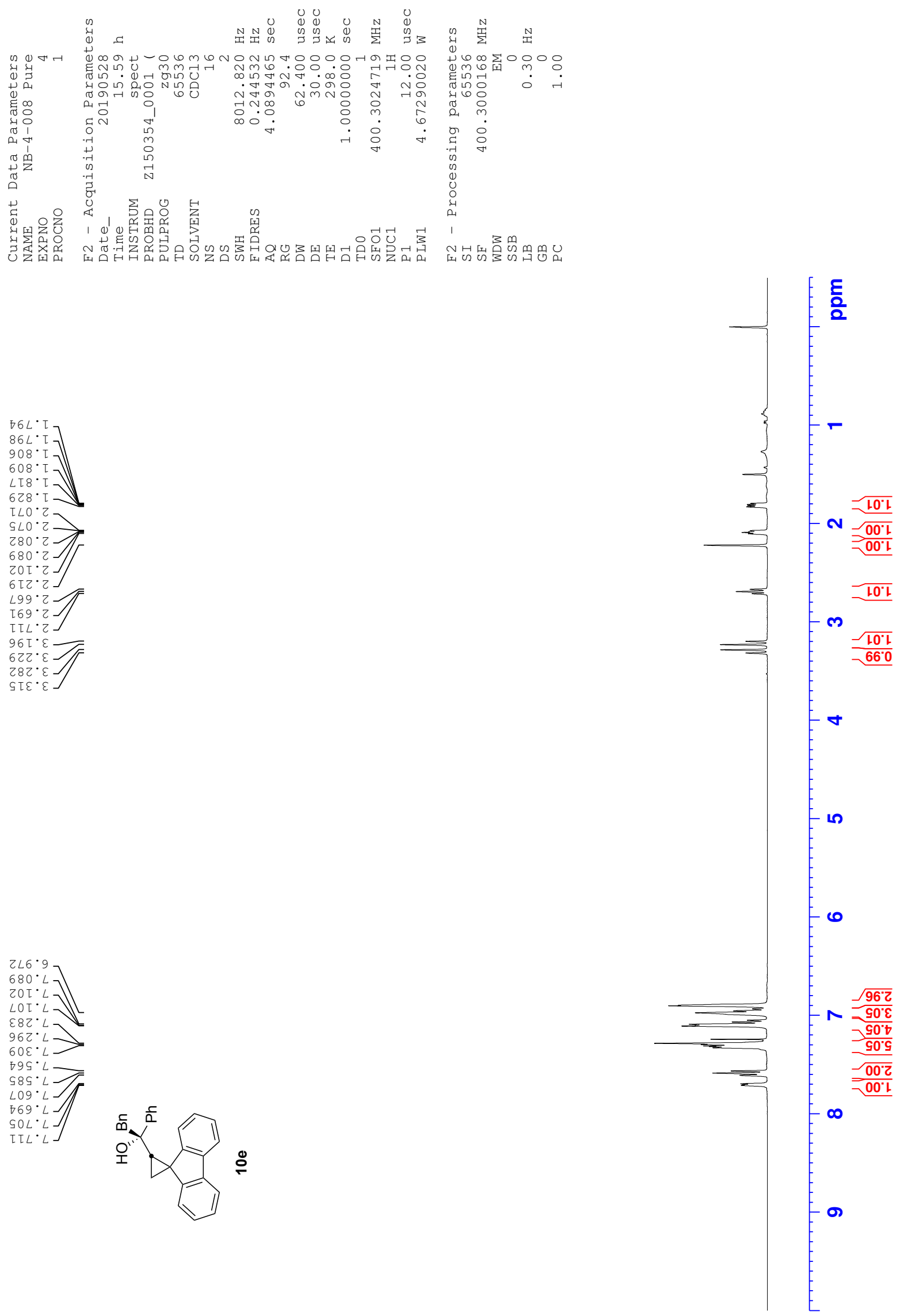

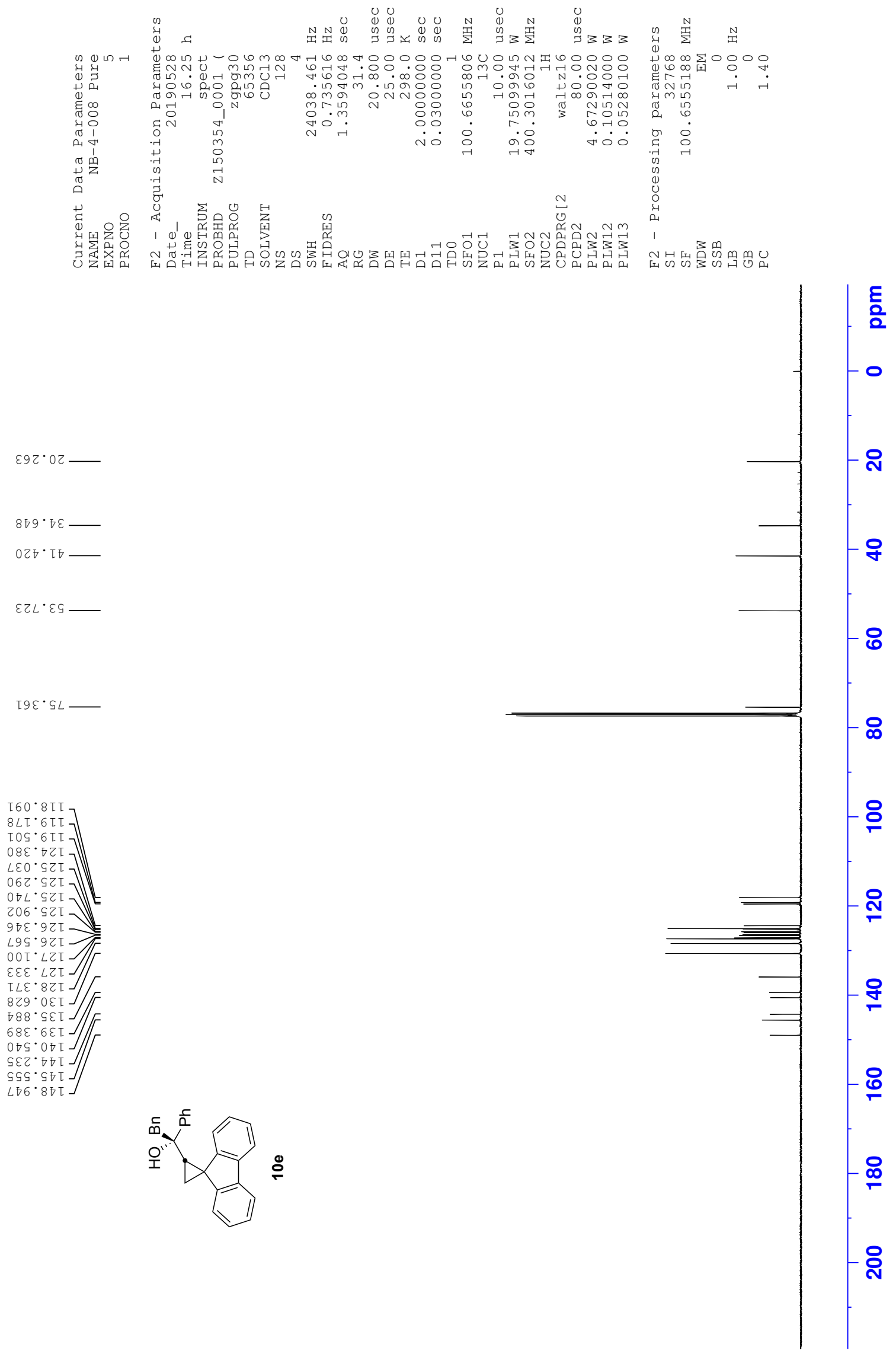

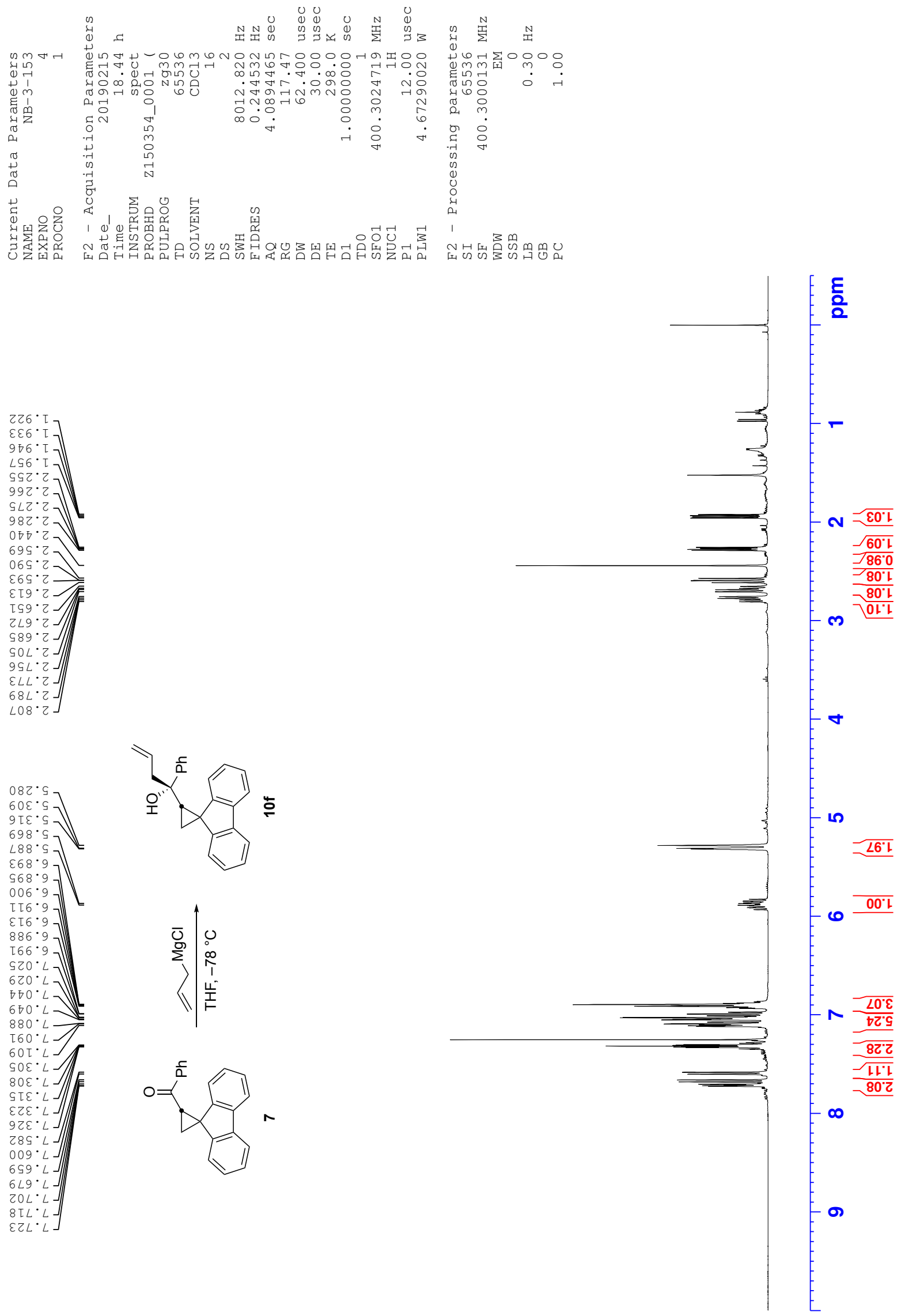

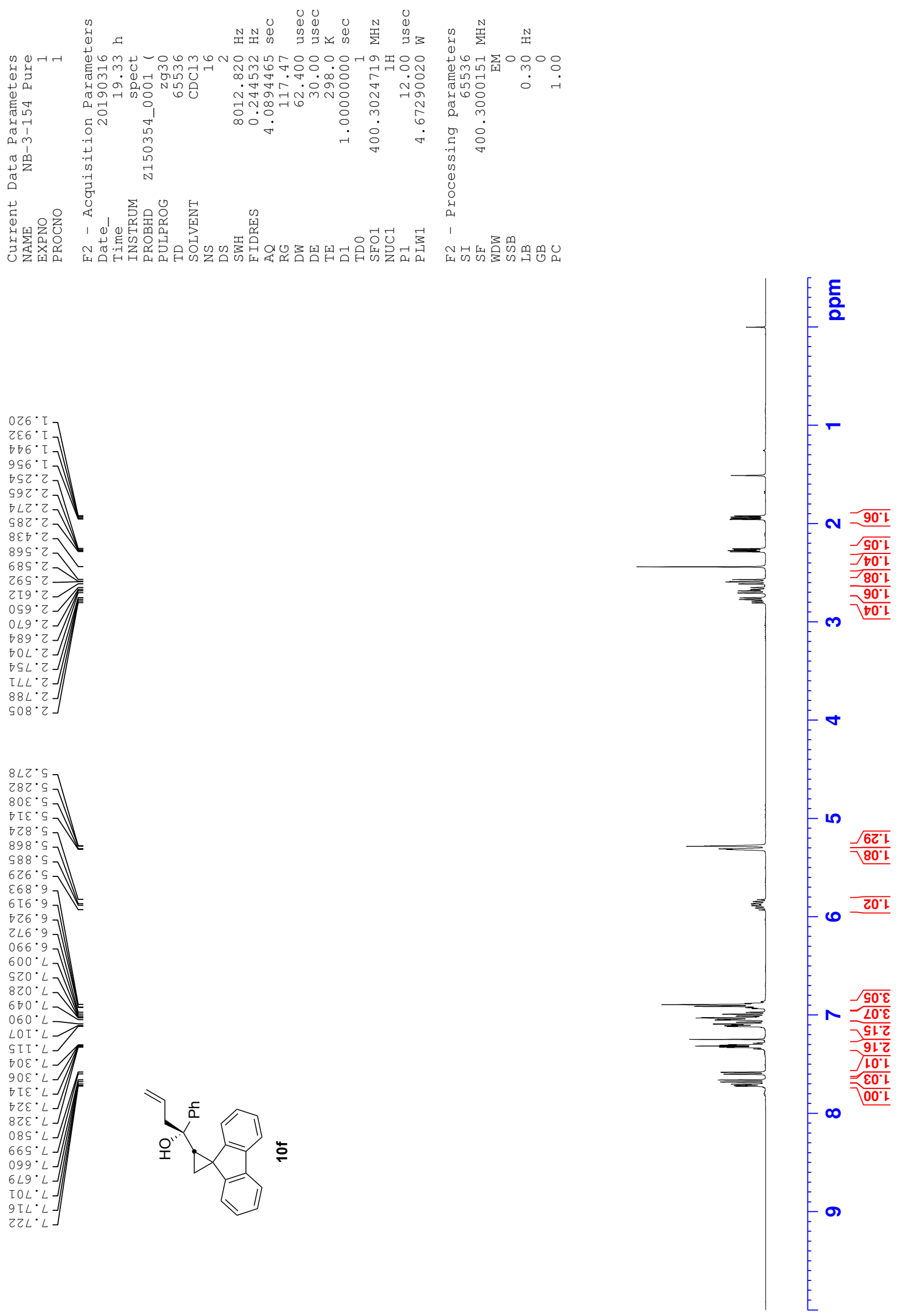

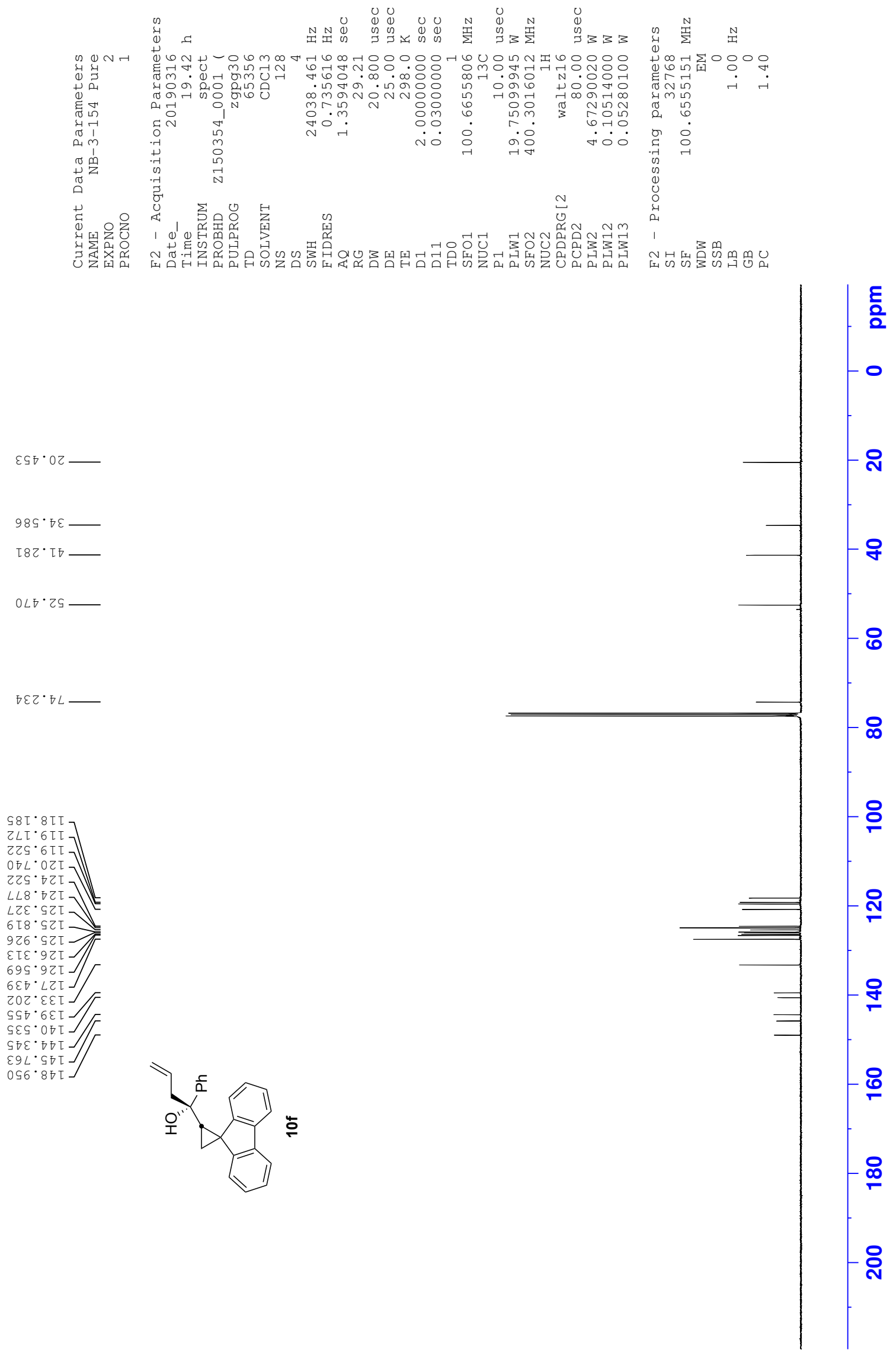

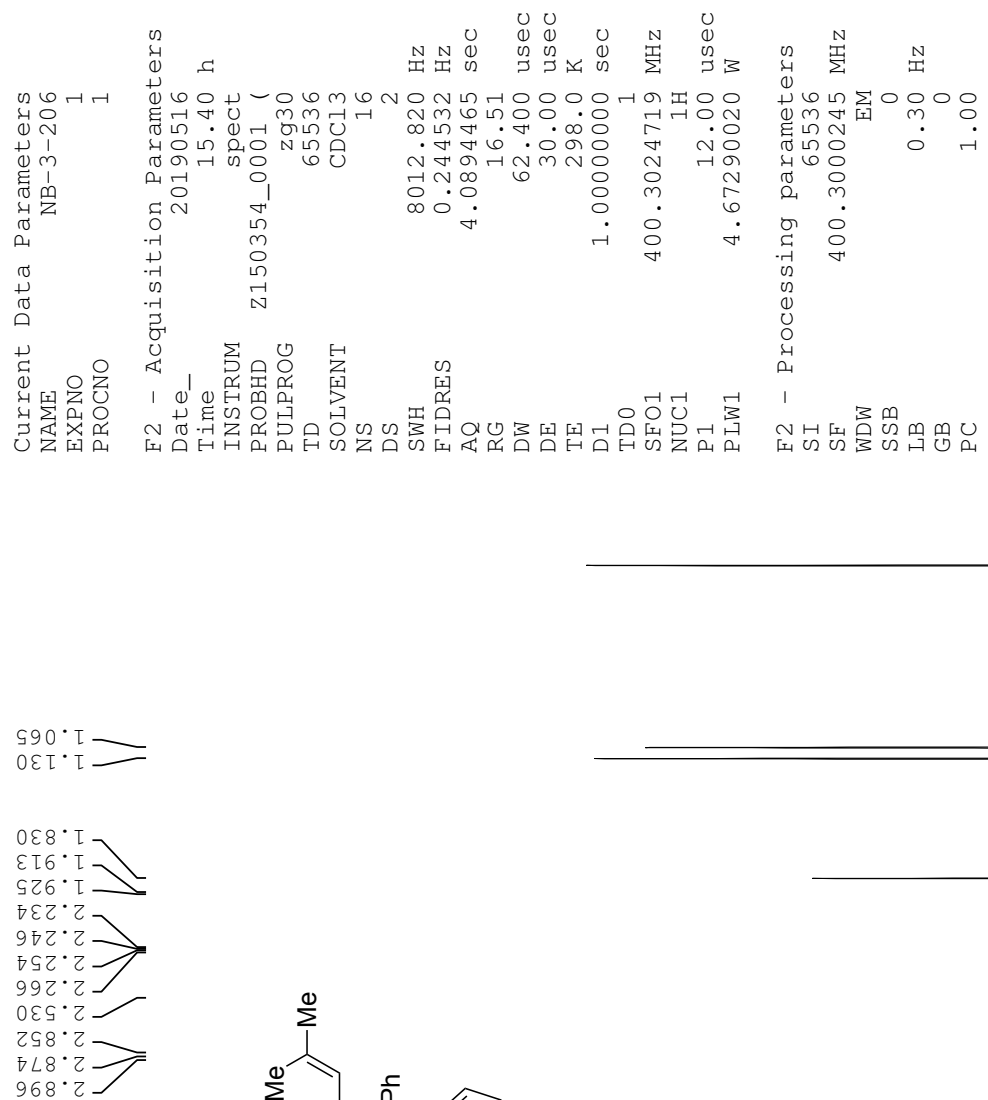

$\varpi \varepsilon L \cdot \varepsilon$
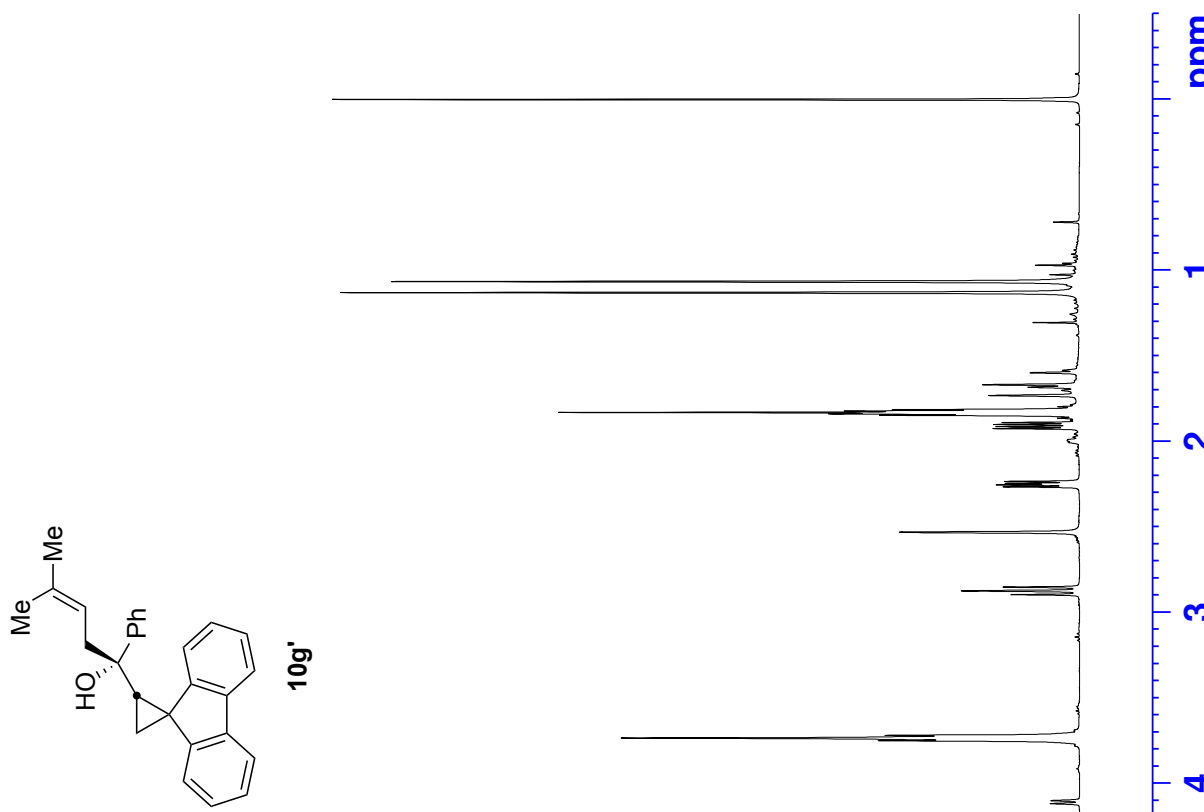

$\frac{\varepsilon}{}$
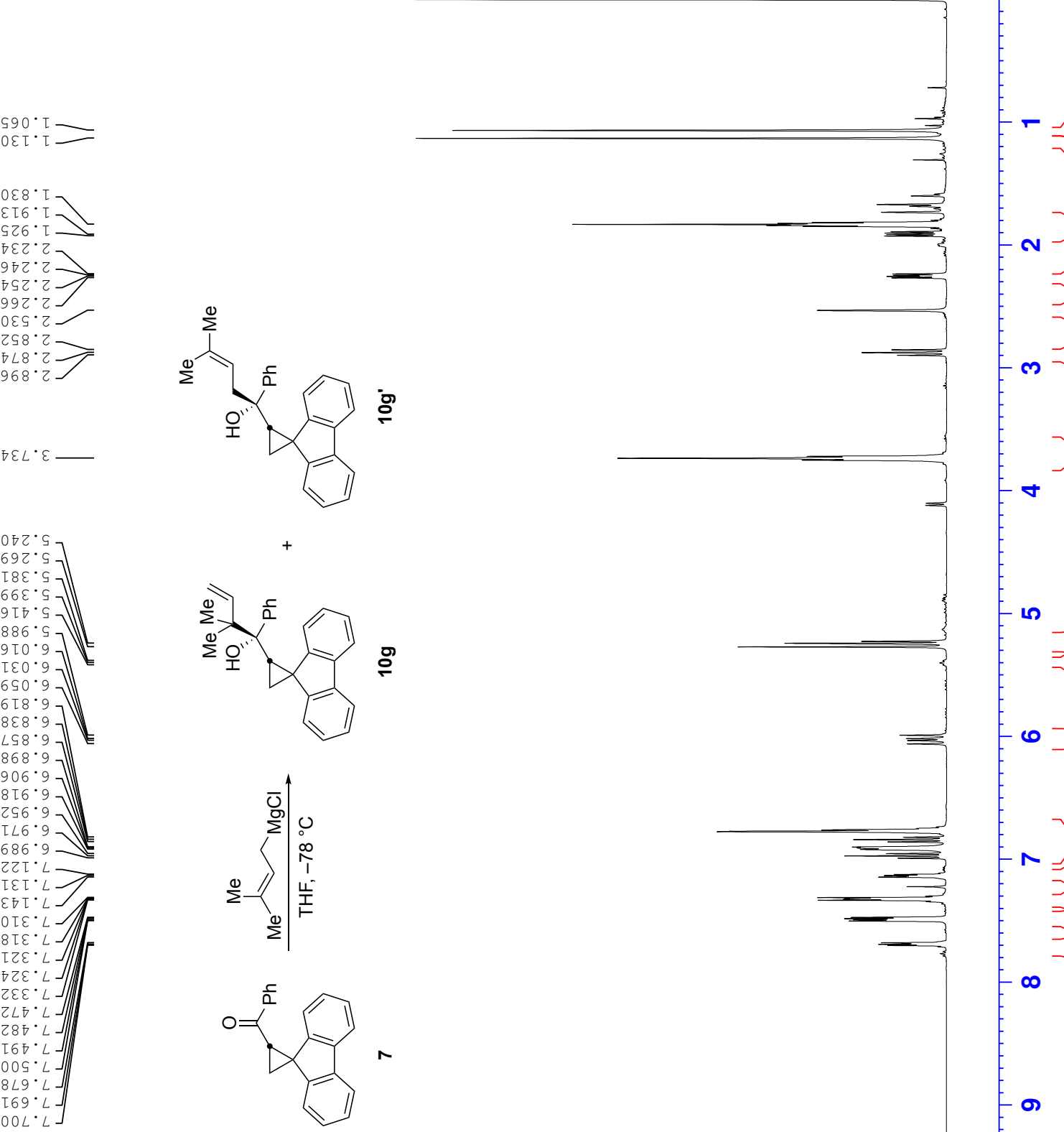

- 6

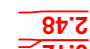

$2+0$

$- 6 \longdiv { 0 0 ^ { \circ } }$

$-\sqrt{20^{\circ} \mathrm{L}}$

$\sqrt{90^{\circ} \mathrm{L}}$

$\frac{\sqrt{9 L^{\prime} \mathrm{z}}}{\overline{\varepsilon 0^{\circ} \mathrm{z}}}$

II.

$-\infty$

$a$ 

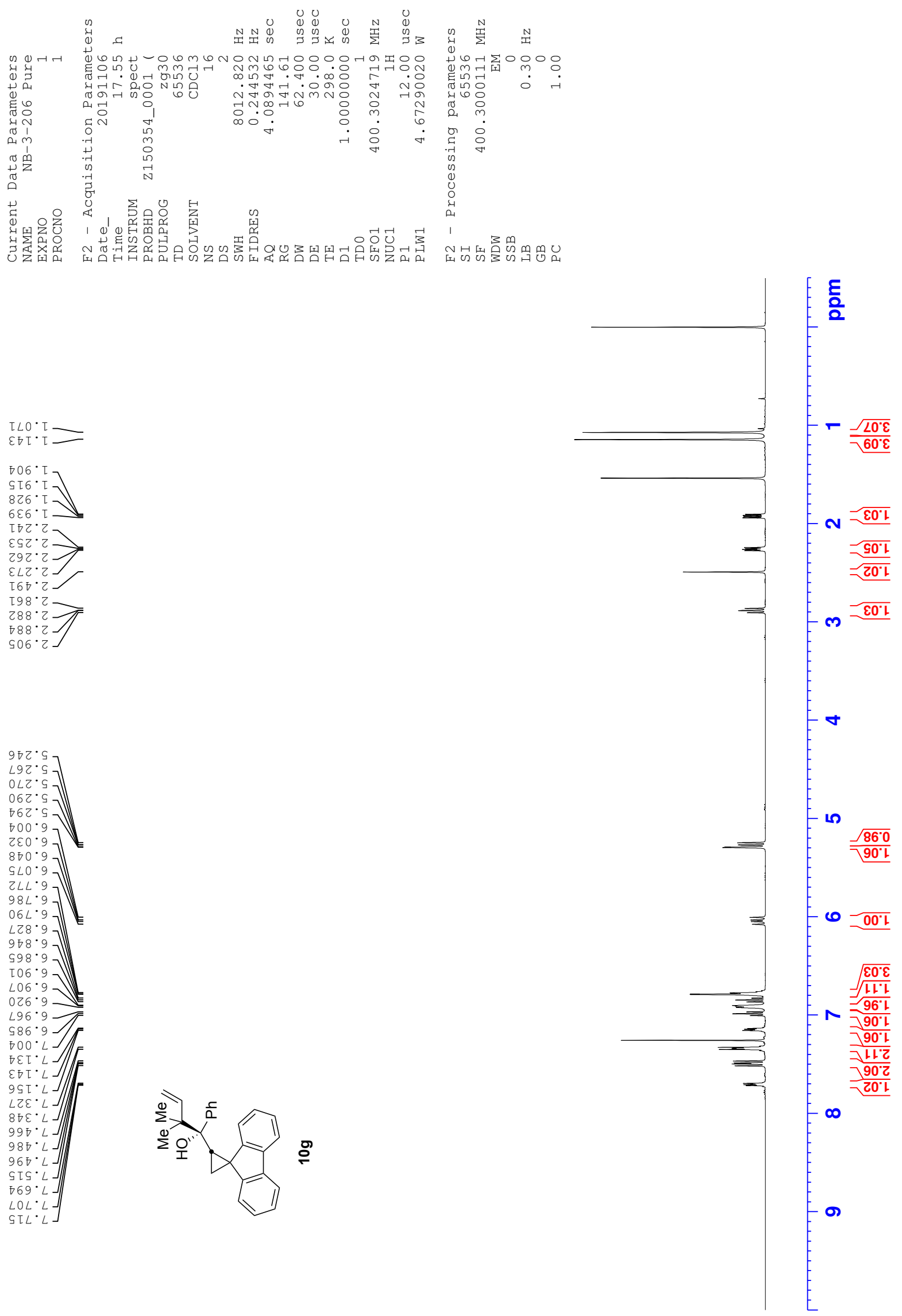

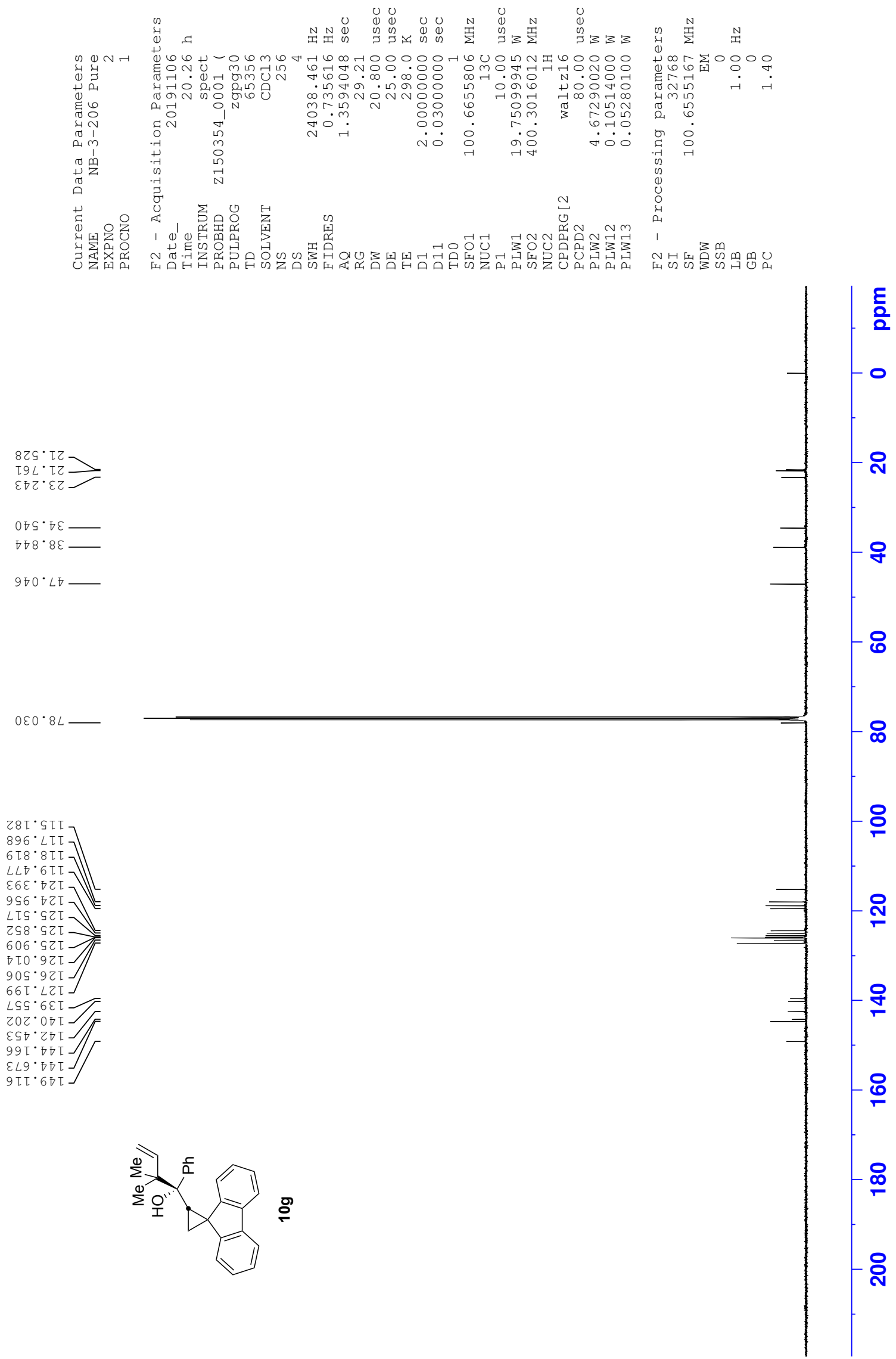


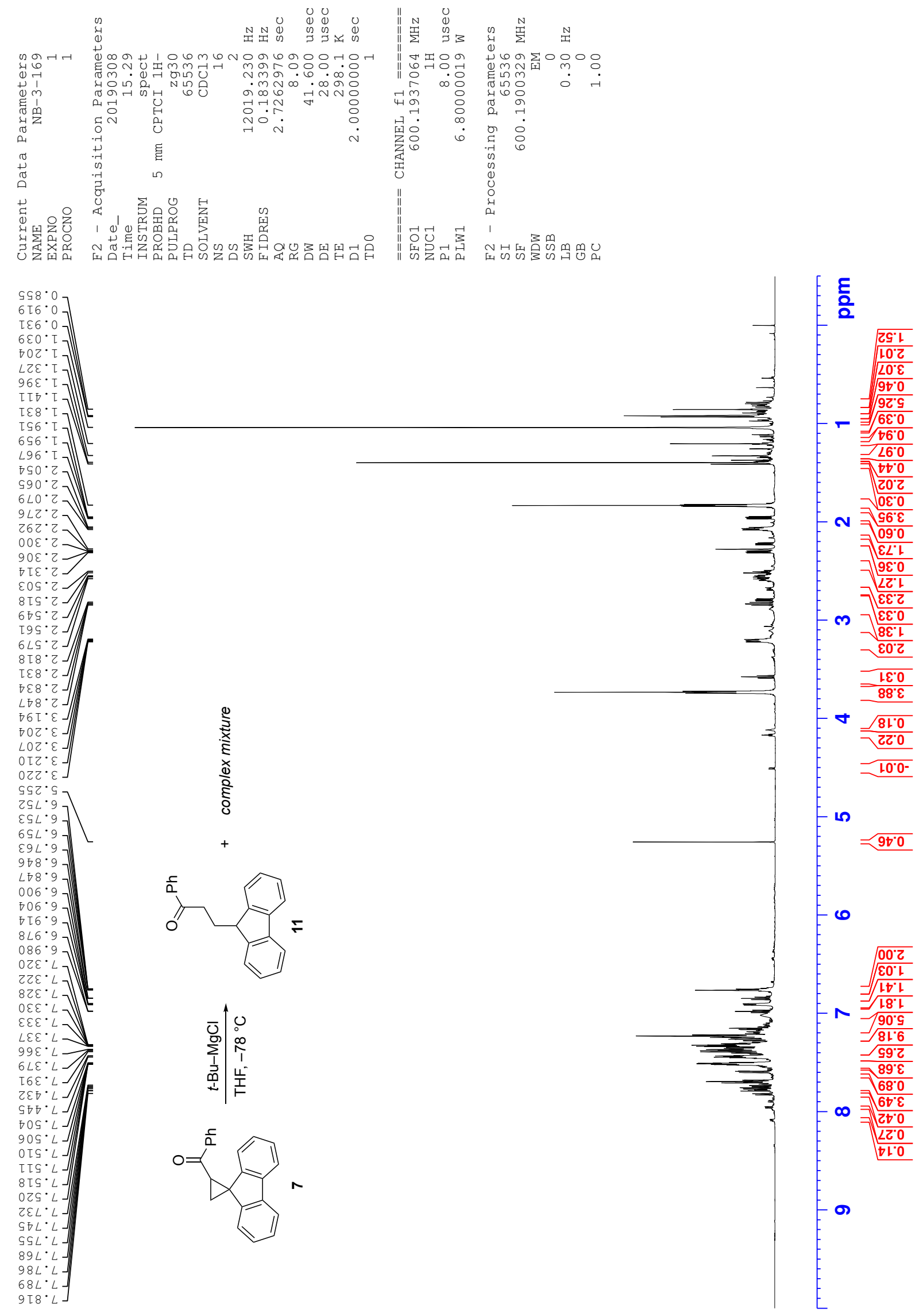



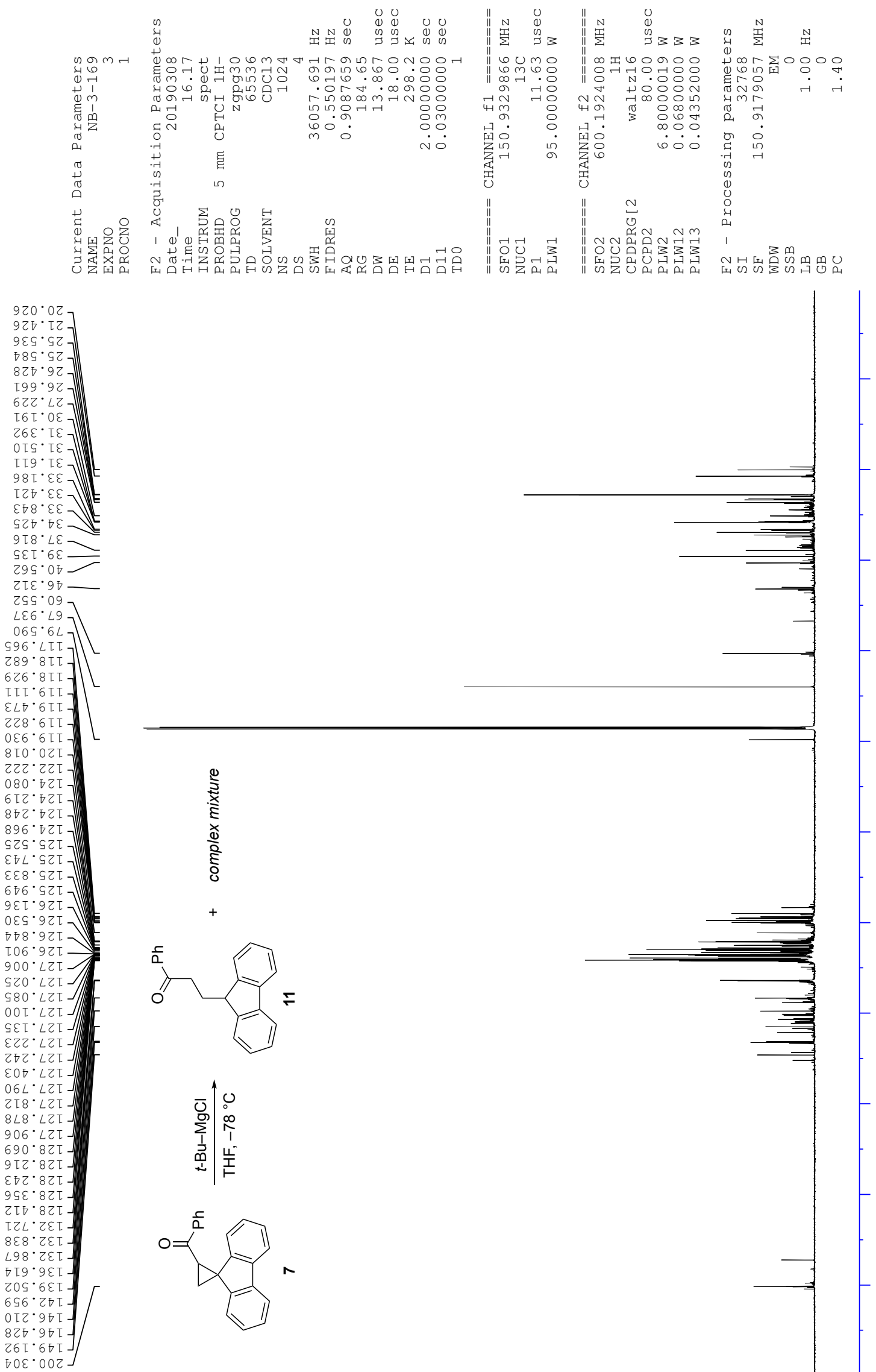

틍

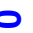

กิ
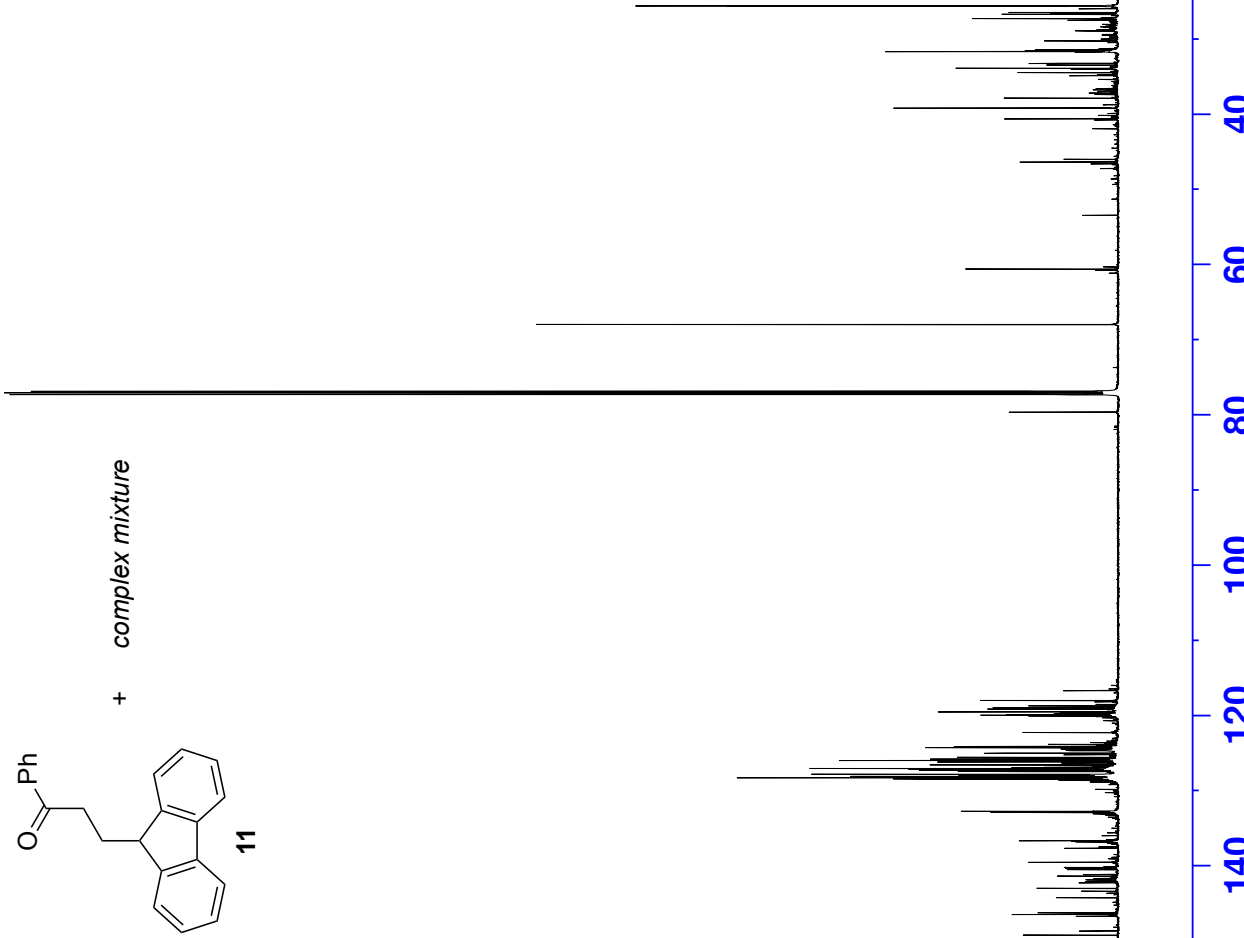

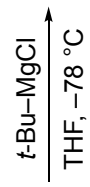

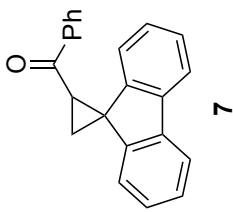

음

ิำ

和

$\stackrel{\circ}{\circ}$

$\stackrel{\circ}{\check{0}}$ 

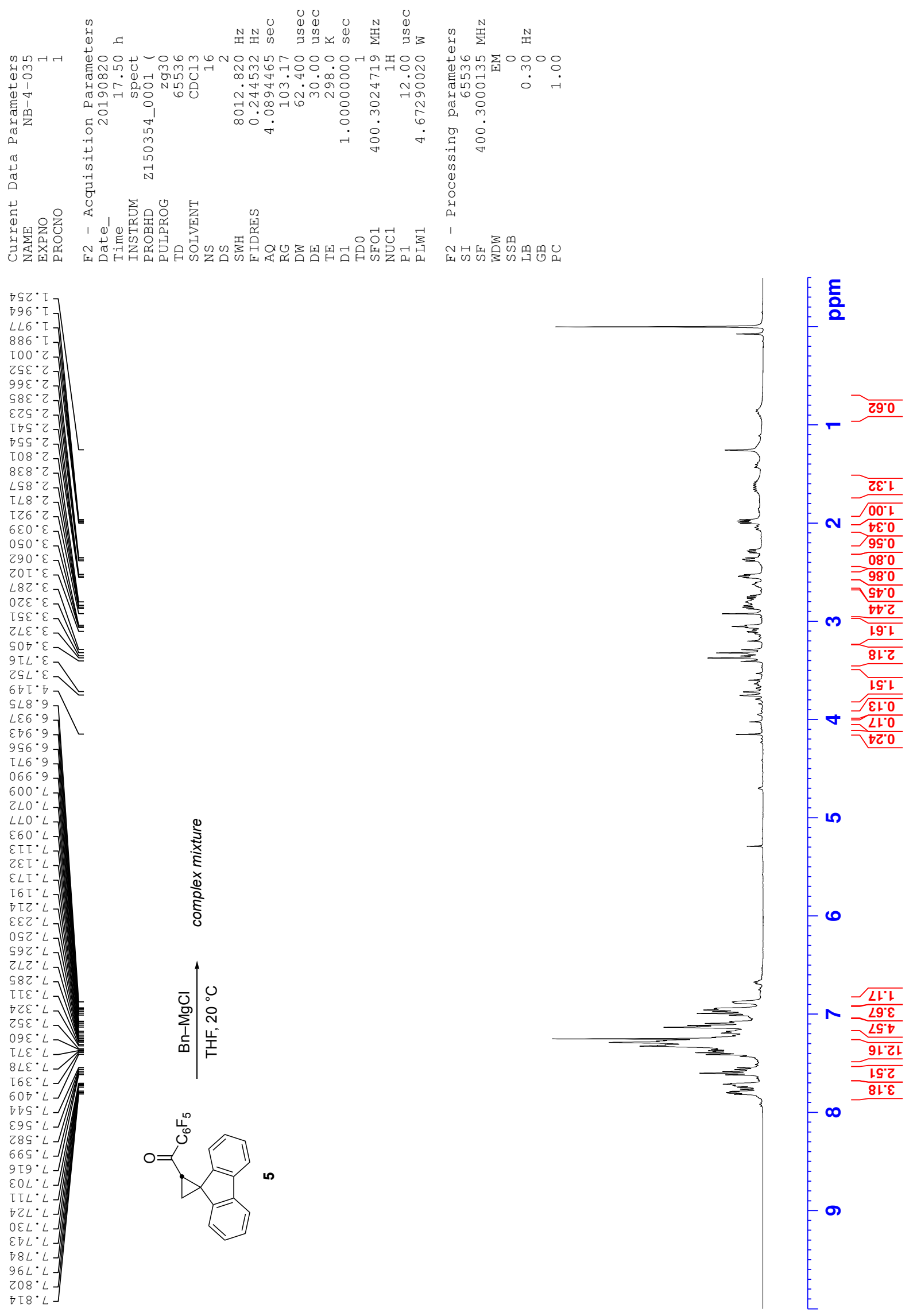

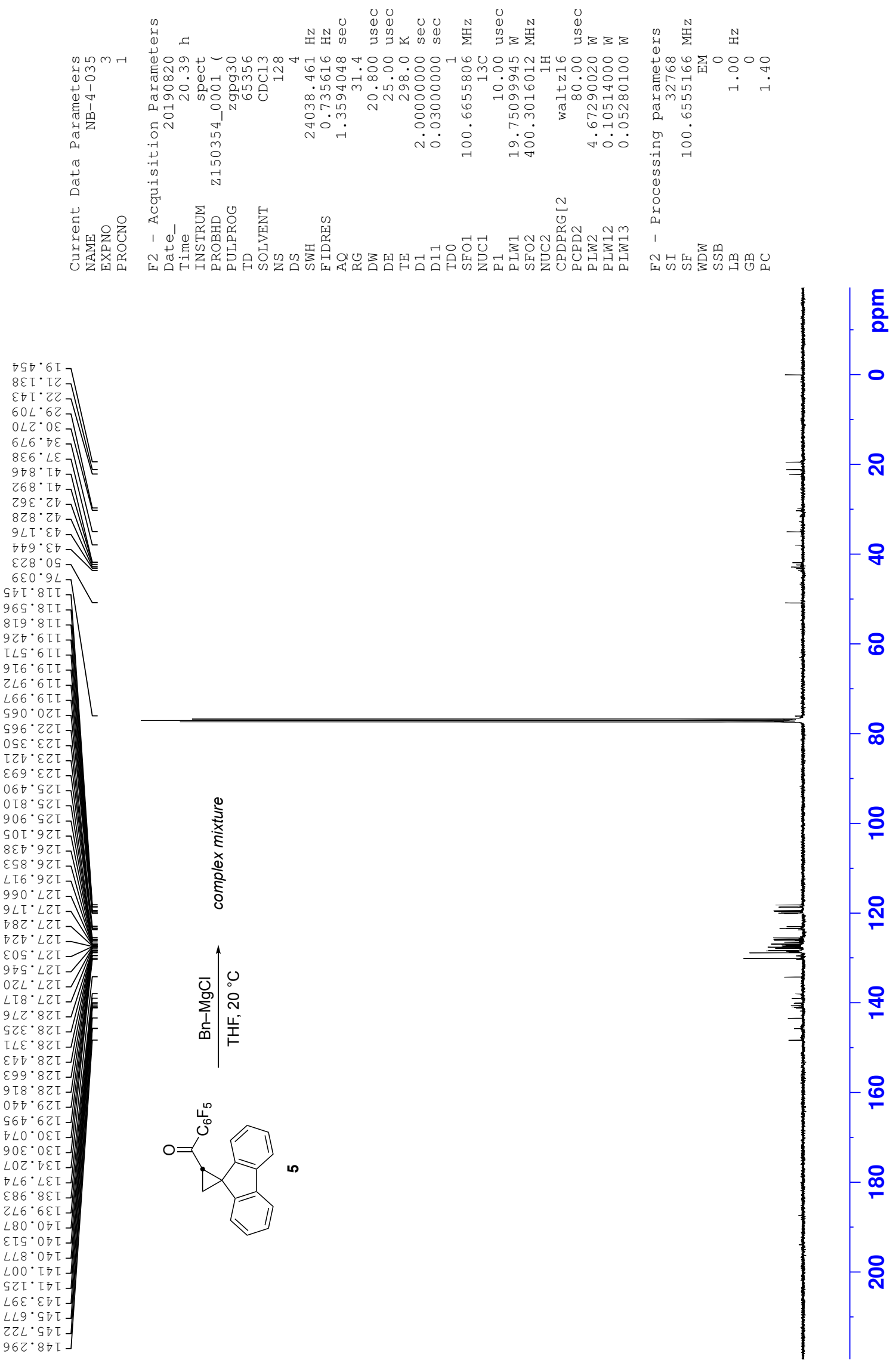

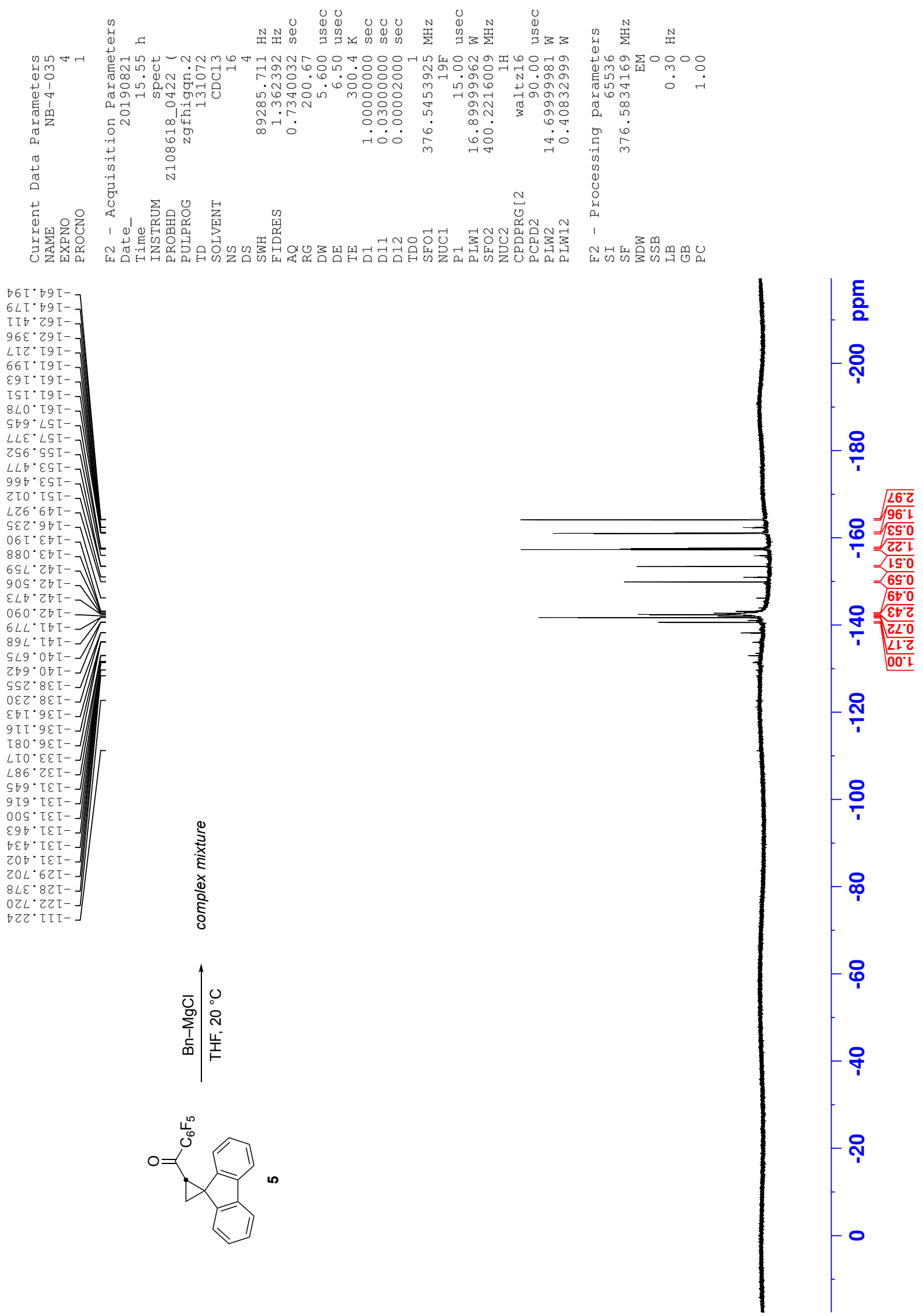

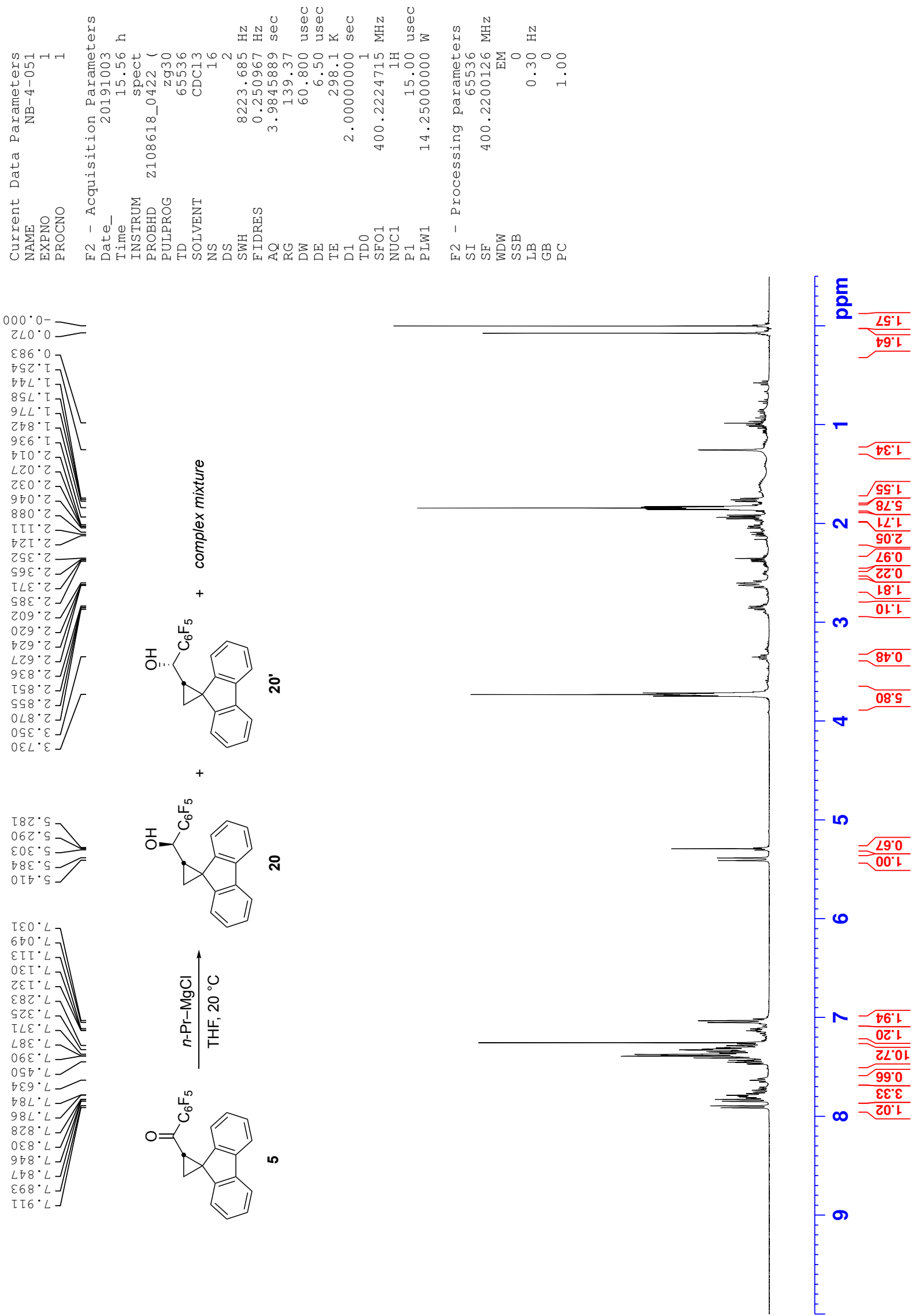

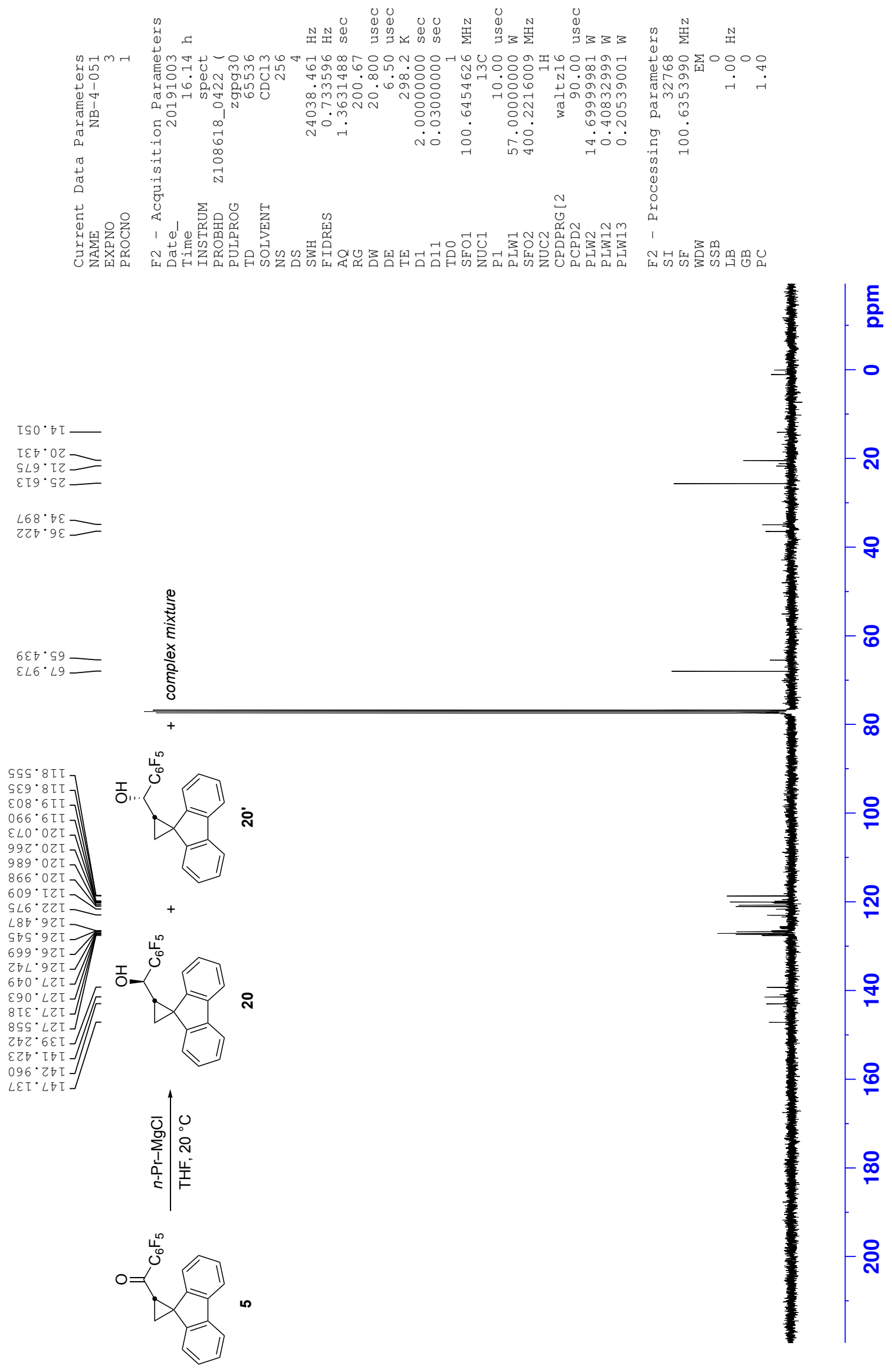

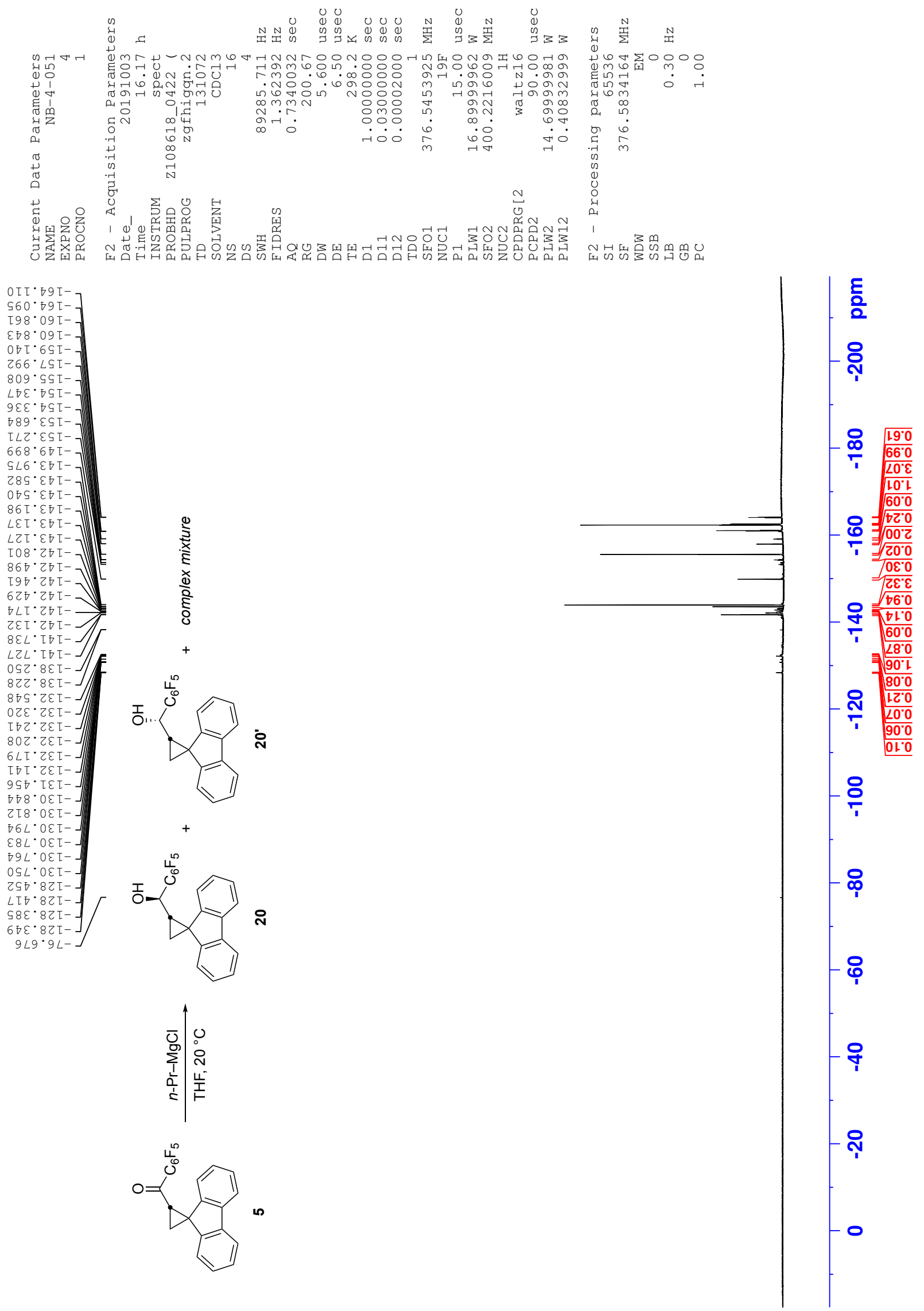

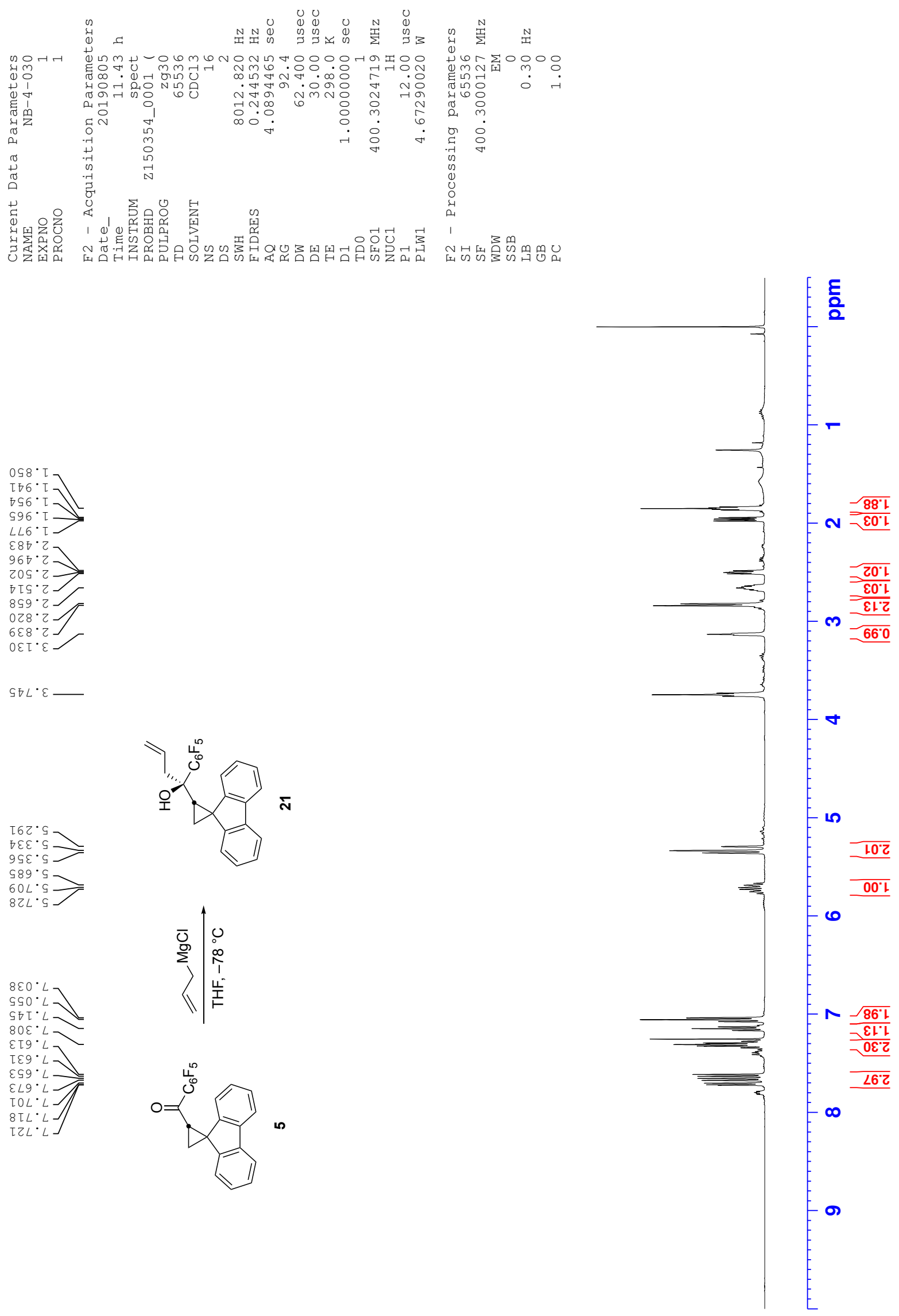

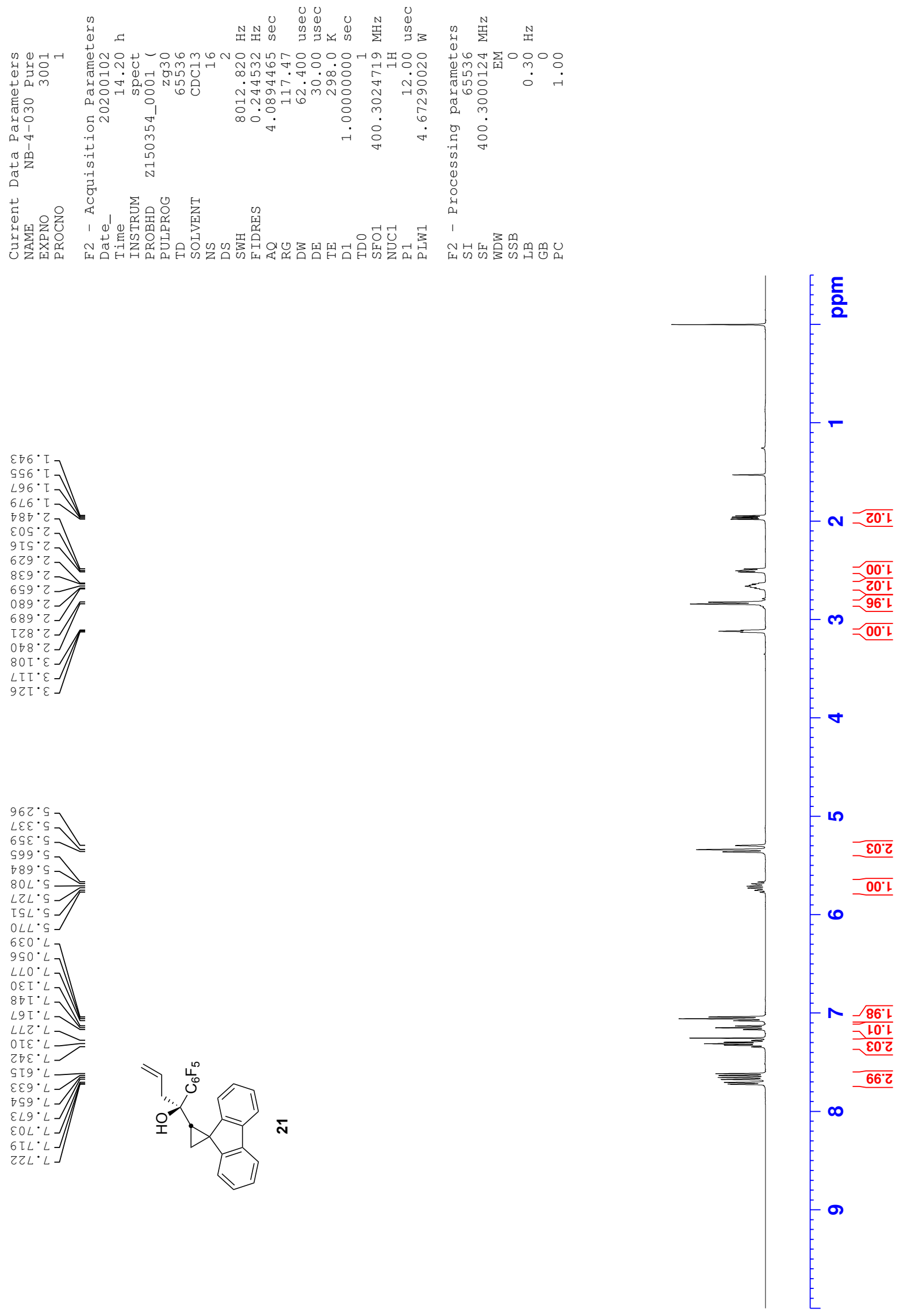

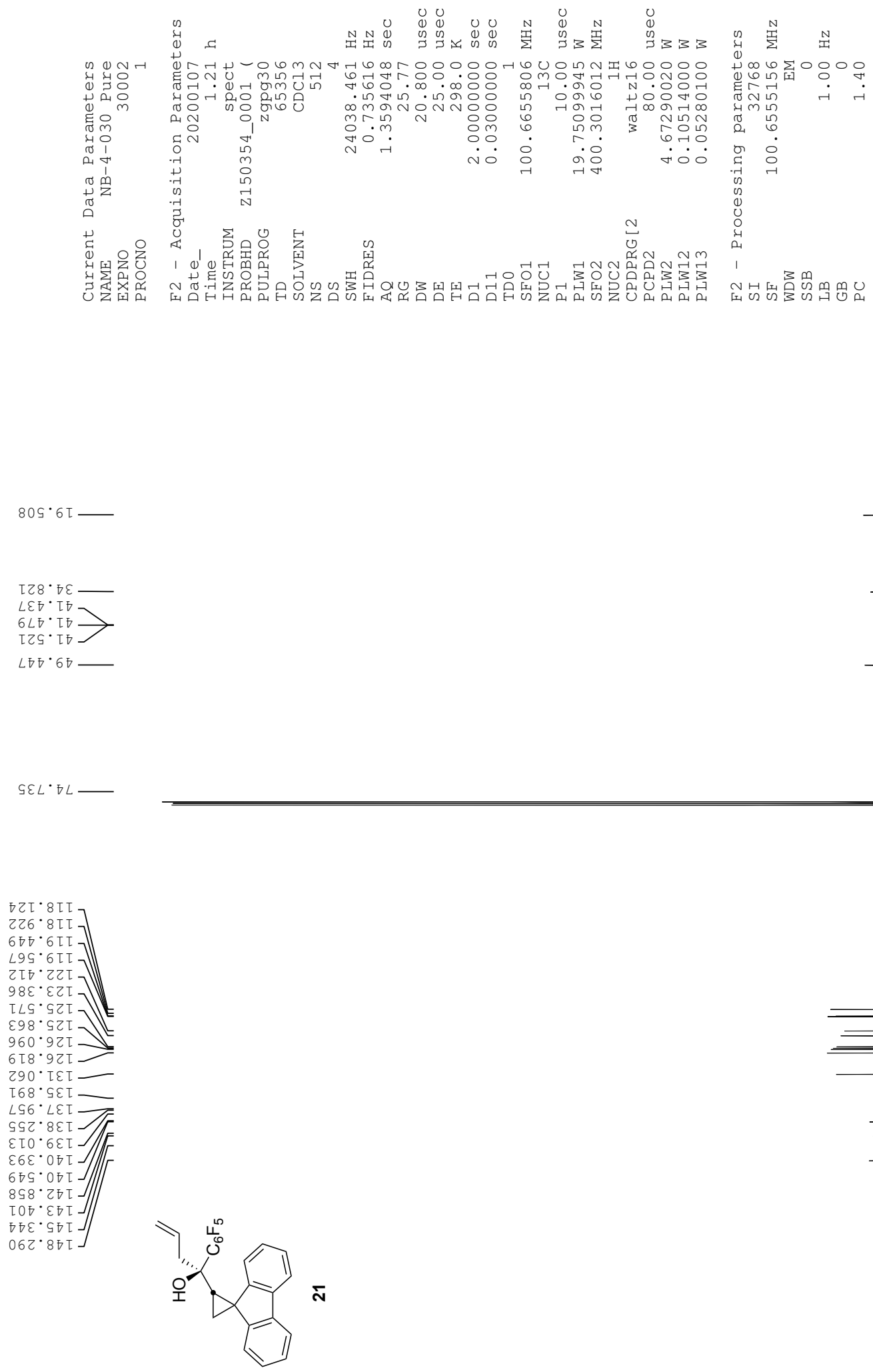

틀 

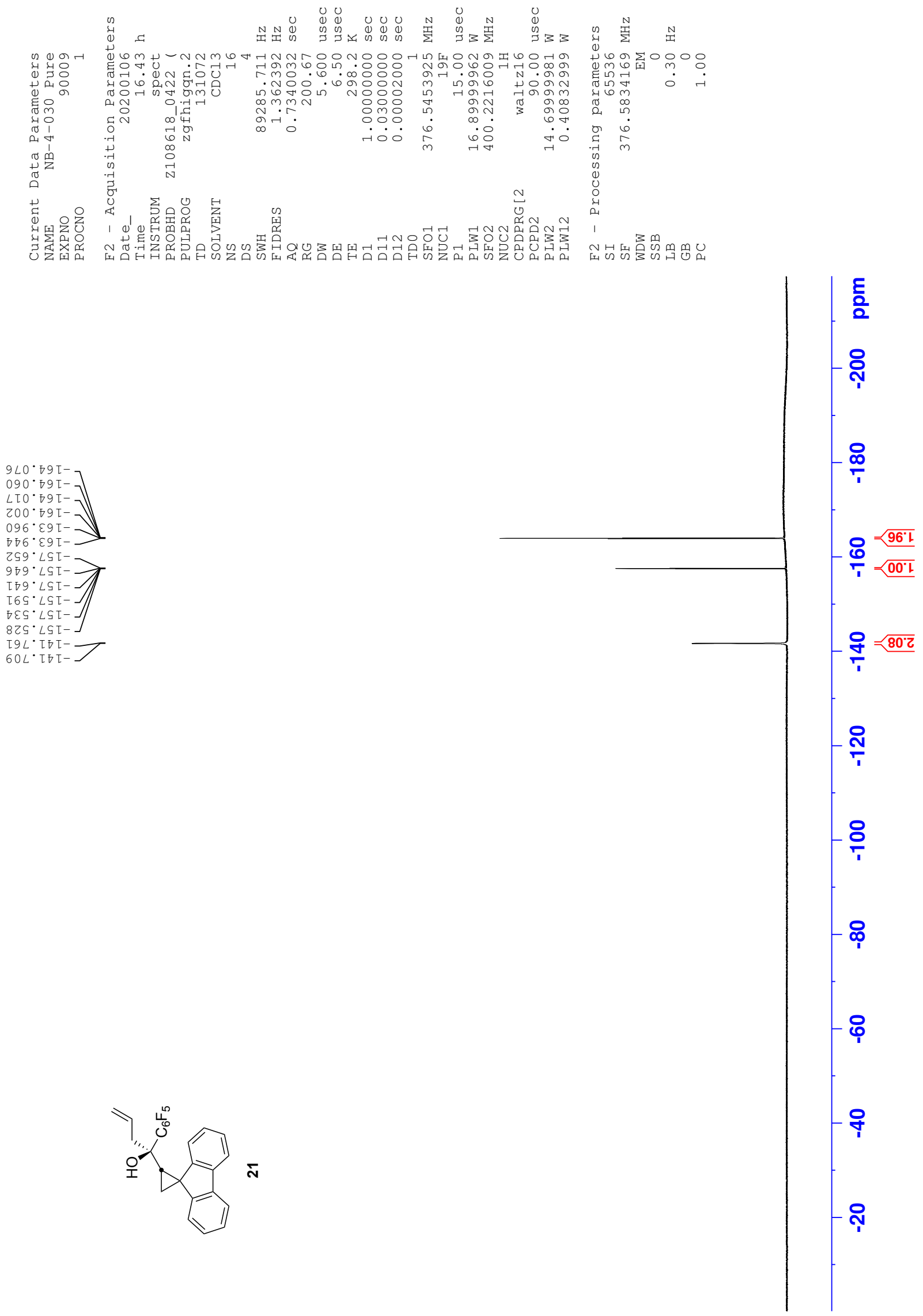

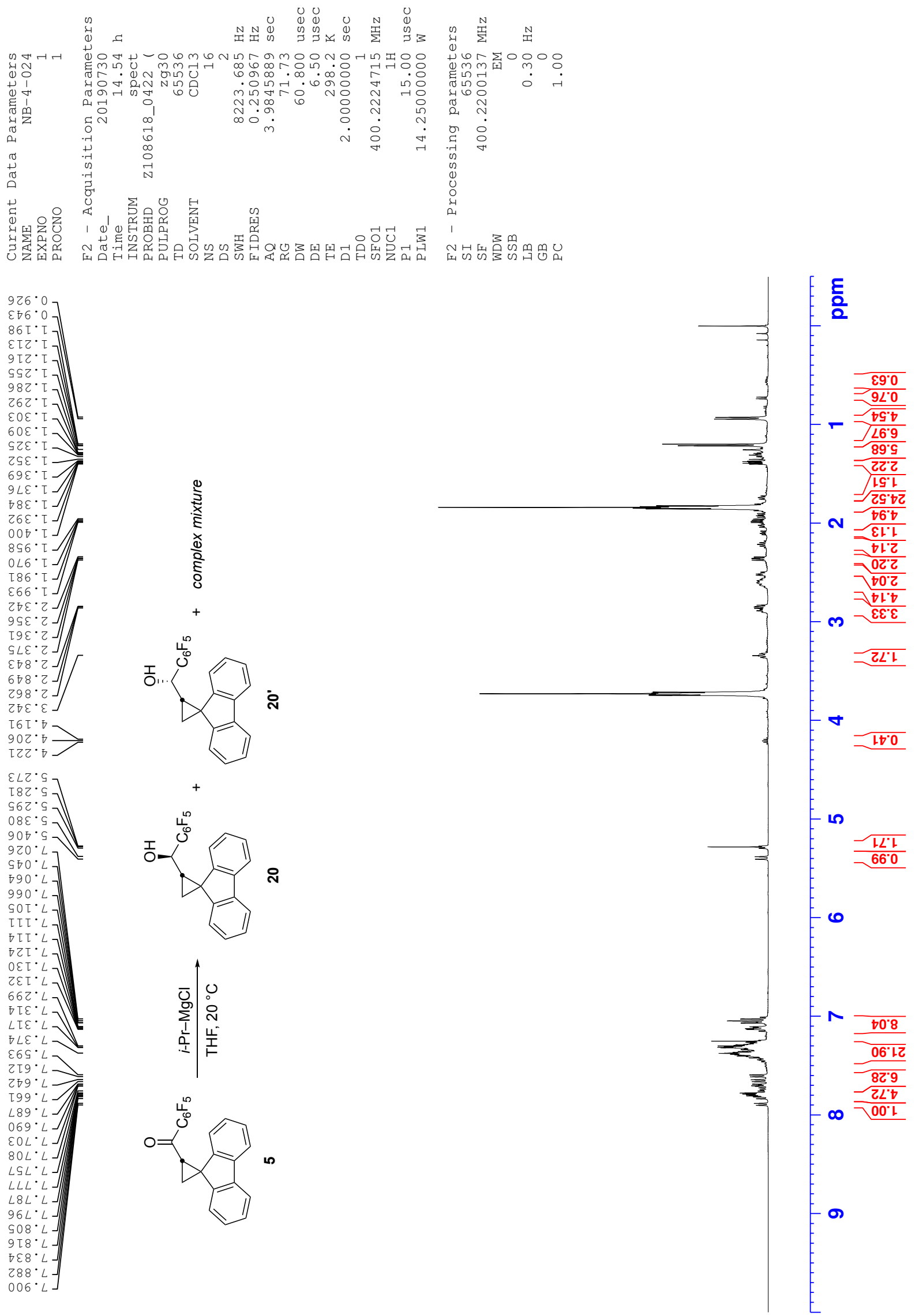

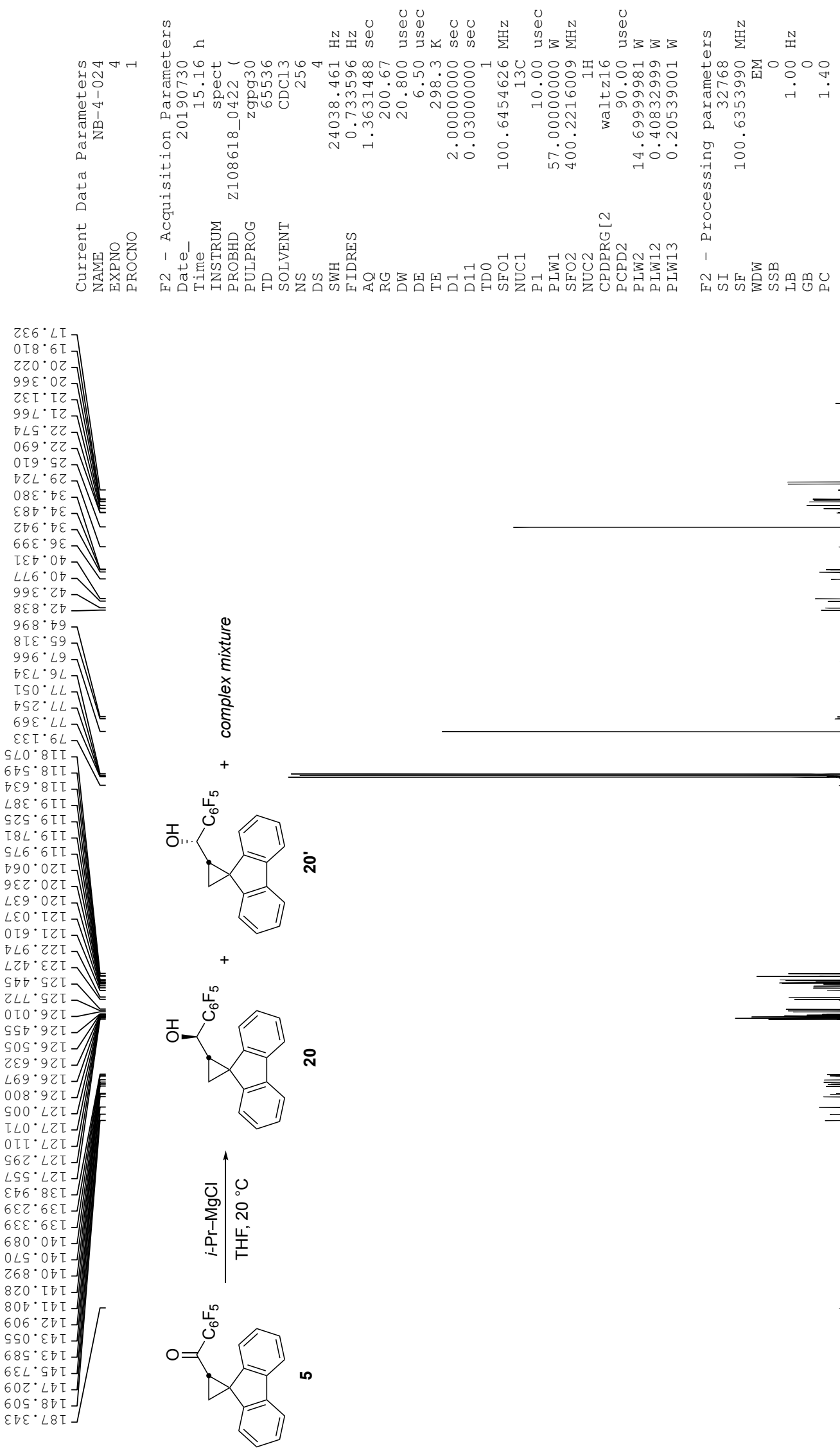

틍 

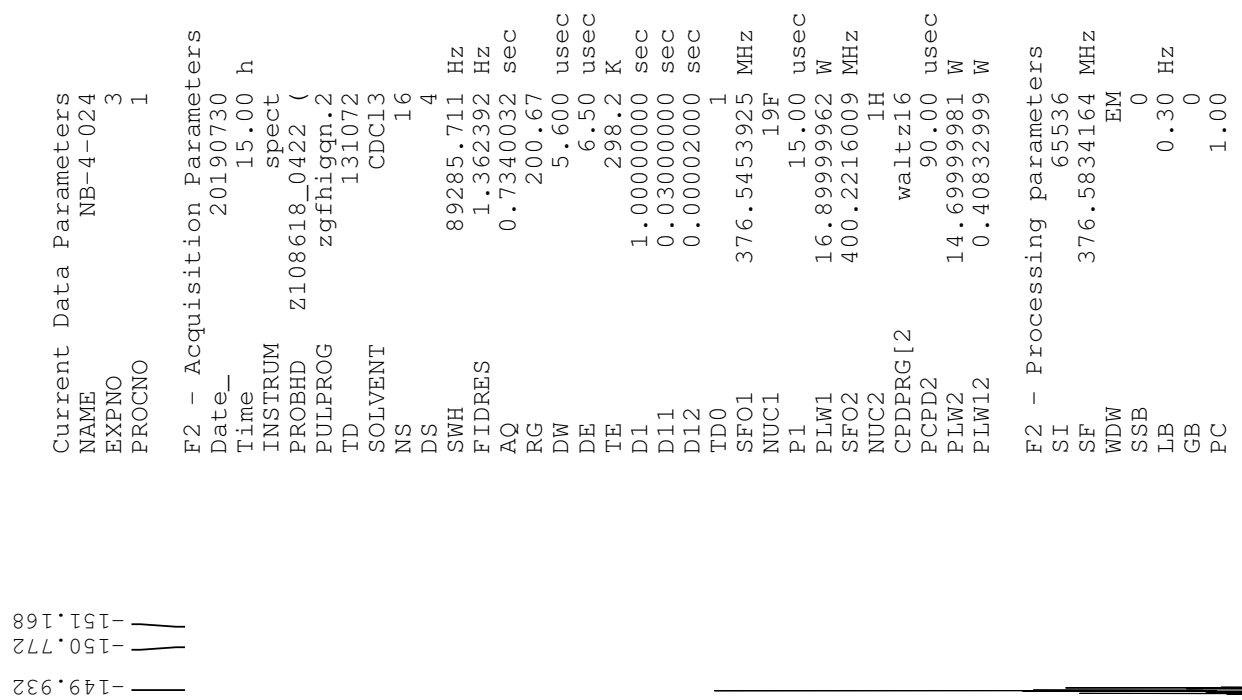

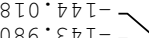

$086^{\circ} \varepsilon t[->$

$6 \tau \mathrm{I} \cdot \varepsilon \nabla \tau-\longrightarrow$

ZLI. Z⿱一𫝀-

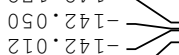

LLL $\operatorname{lot-}$

$9 \nabla z \cdot 8 \varepsilon \tau-\longrightarrow$
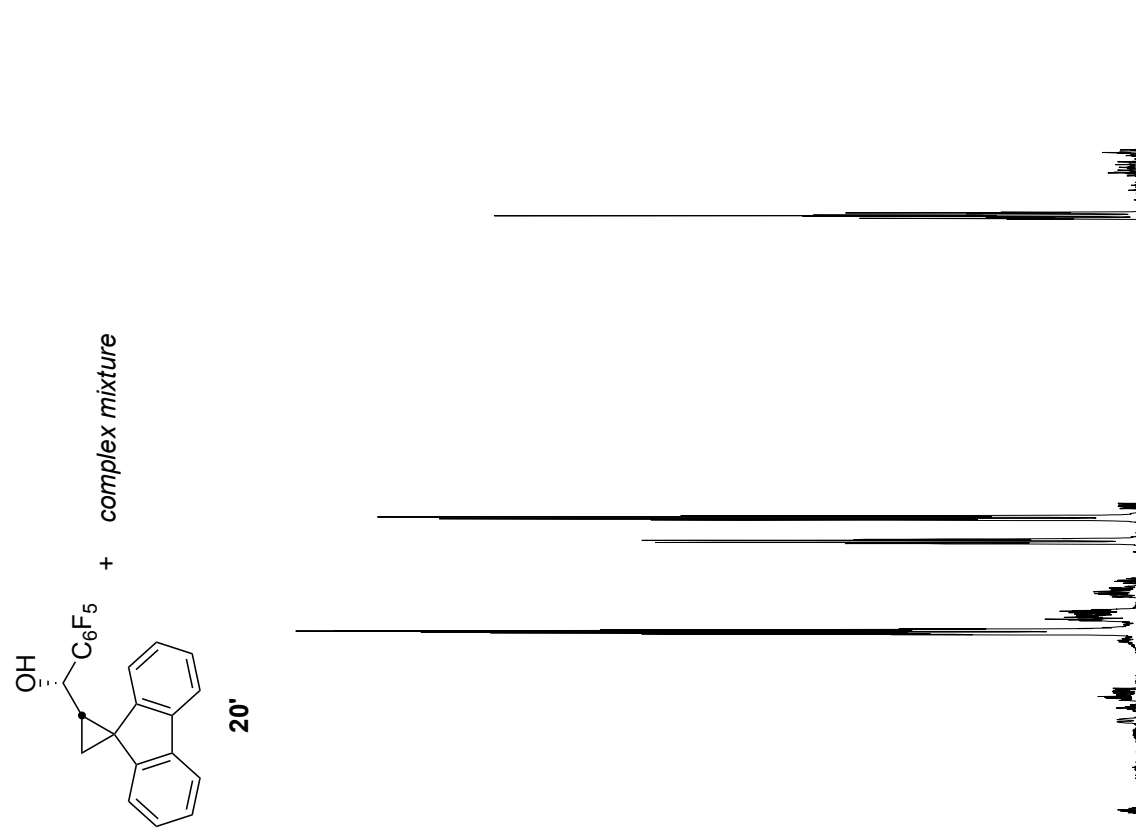

$\frac{\varepsilon}{}$

윧 $\overline{08^{\circ} 1}$

${ }_{0 \varepsilon \varepsilon \cdot \varepsilon \varepsilon \tau T-}^{20 \sigma^{\circ} \cdot \varepsilon \varepsilon \tau-}$

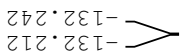

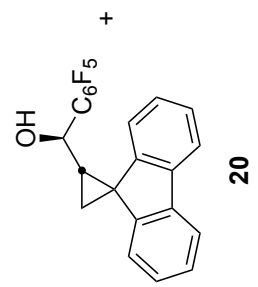

$\stackrel{\text { ๓n }}{\frac{m}{1}}$

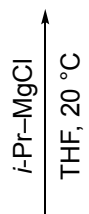

$\stackrel{2}{\frac{1}{2}}$

$\sqrt{t t^{\circ} \varepsilon}$

पहैं

IS.0

$\checkmark S^{\prime \prime}$

욷

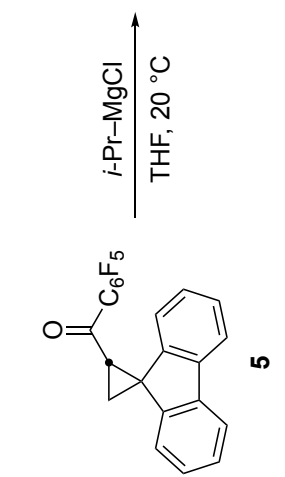

$\frac{0}{1}$

ำ

ํำ 

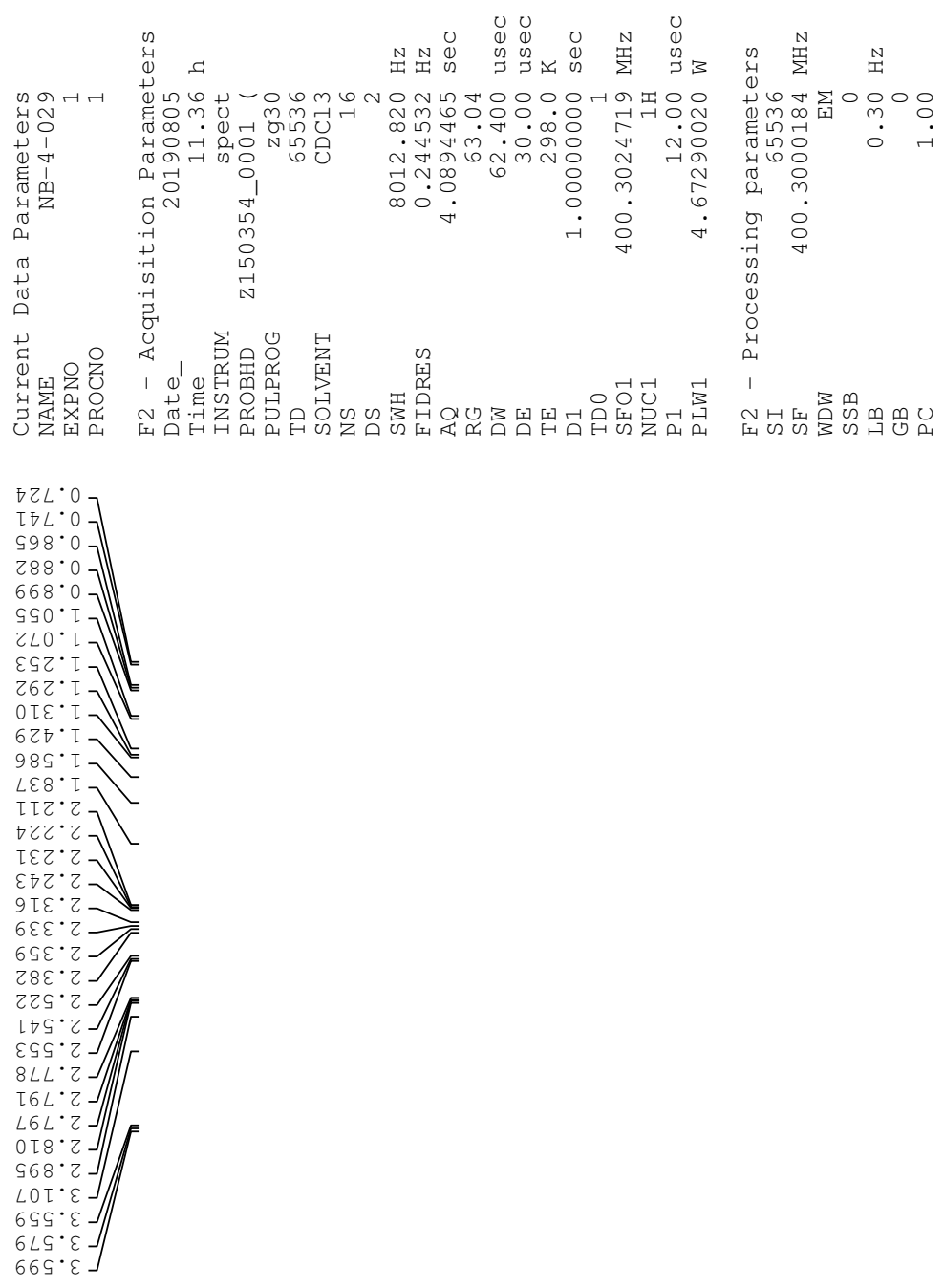

$\begin{array}{lll}810.9 & \\ \varepsilon \neq 0.9 & \end{array}$
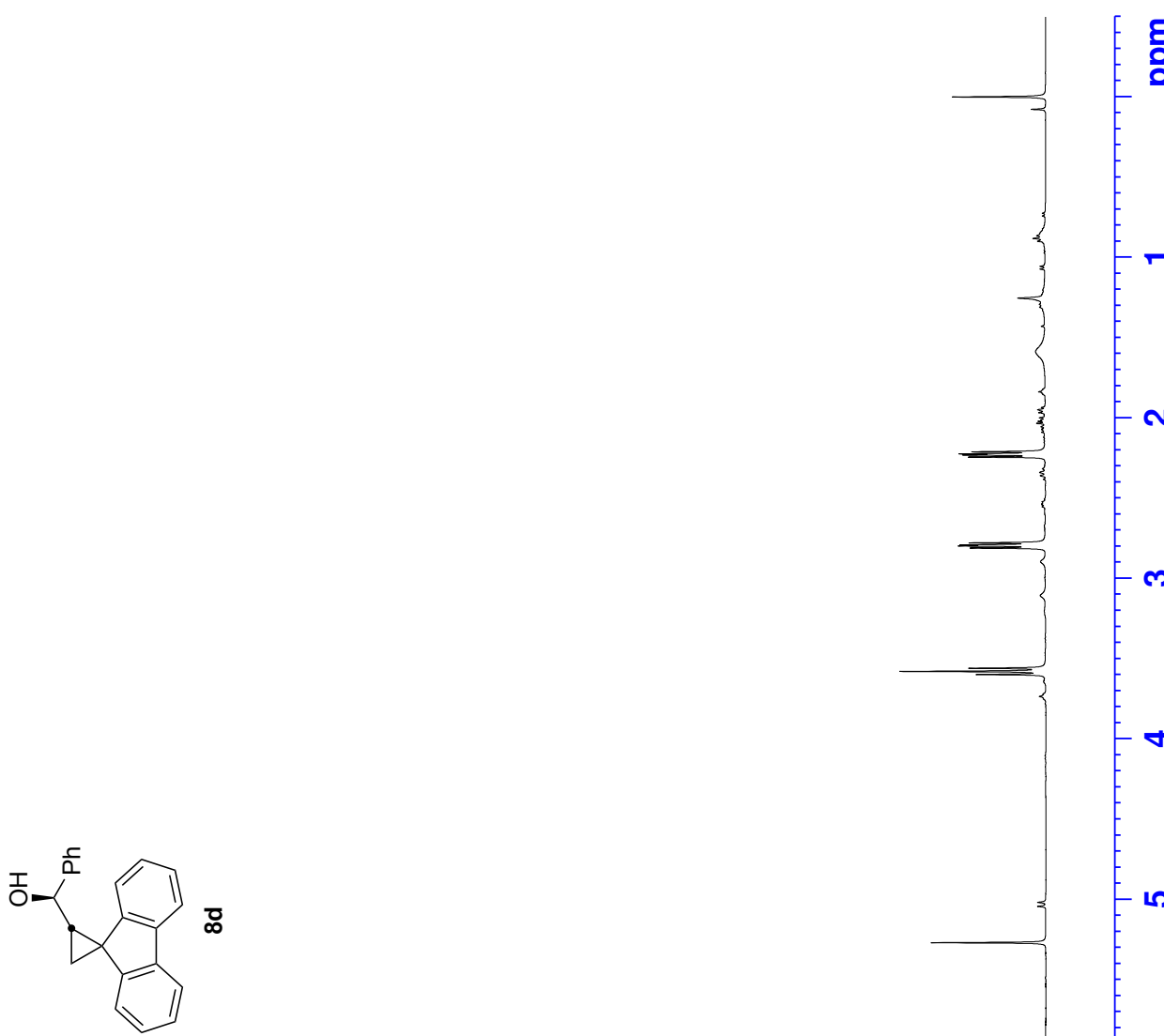

틍

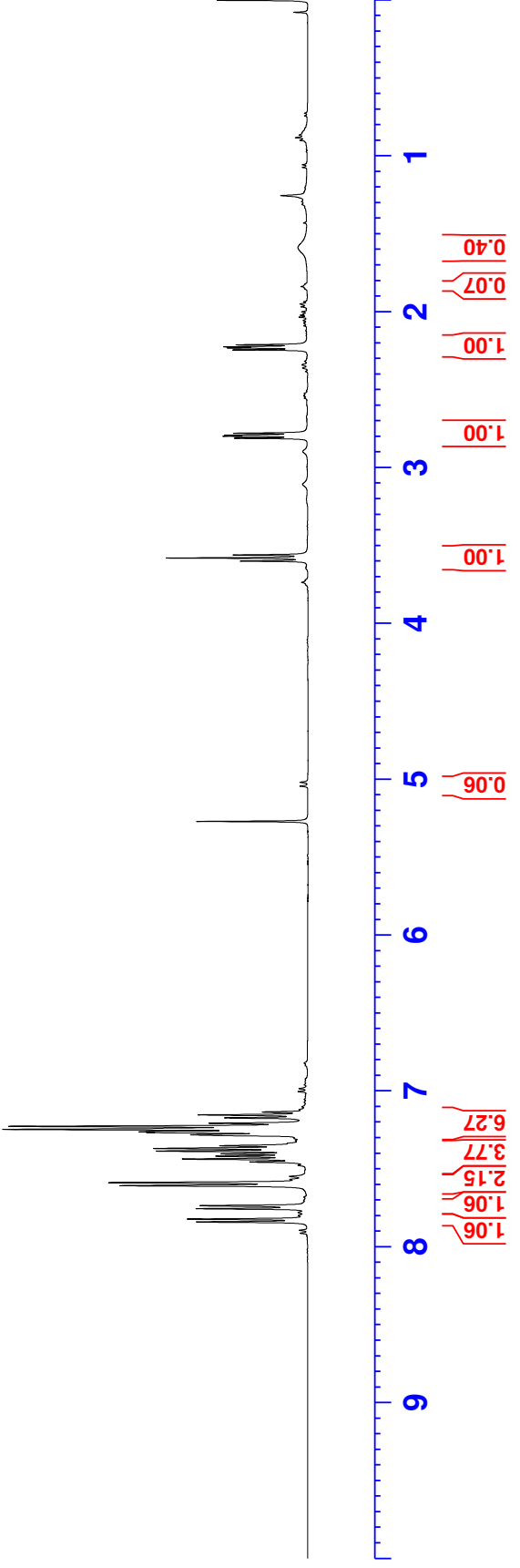



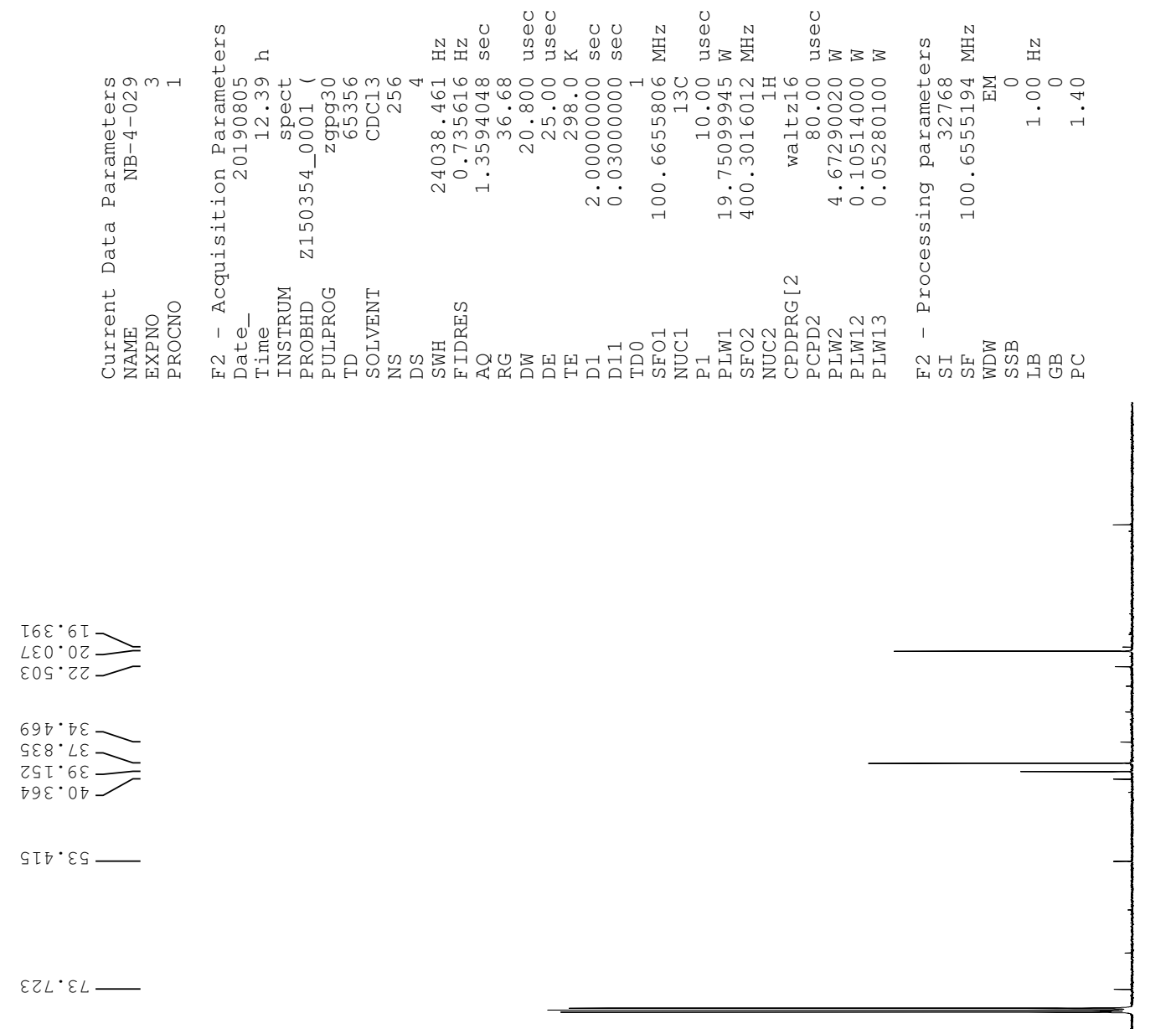

틍
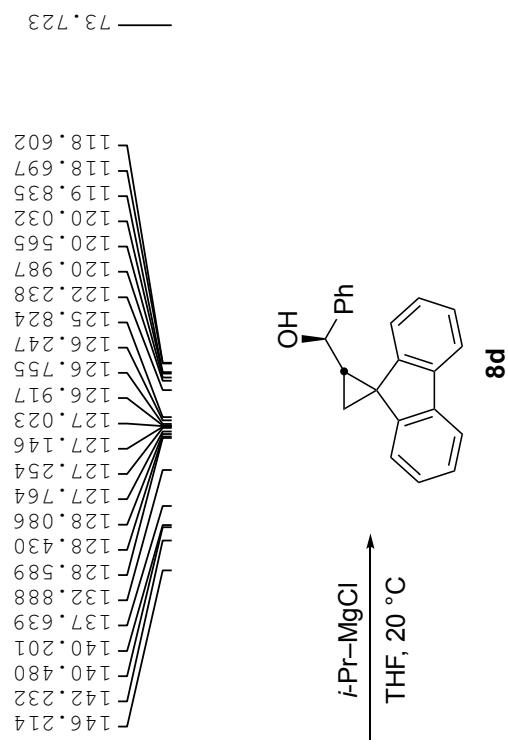

ㅇ

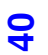

$-8$

$\infty$

움

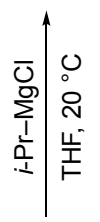

운

$\stackrel{8}{\circ}$

โ8 $\sigma^{\circ} \cdot 66 \tau$<smiles>CC(=O)C1CC12c1ccccc1-c1ccccc12</smiles> 

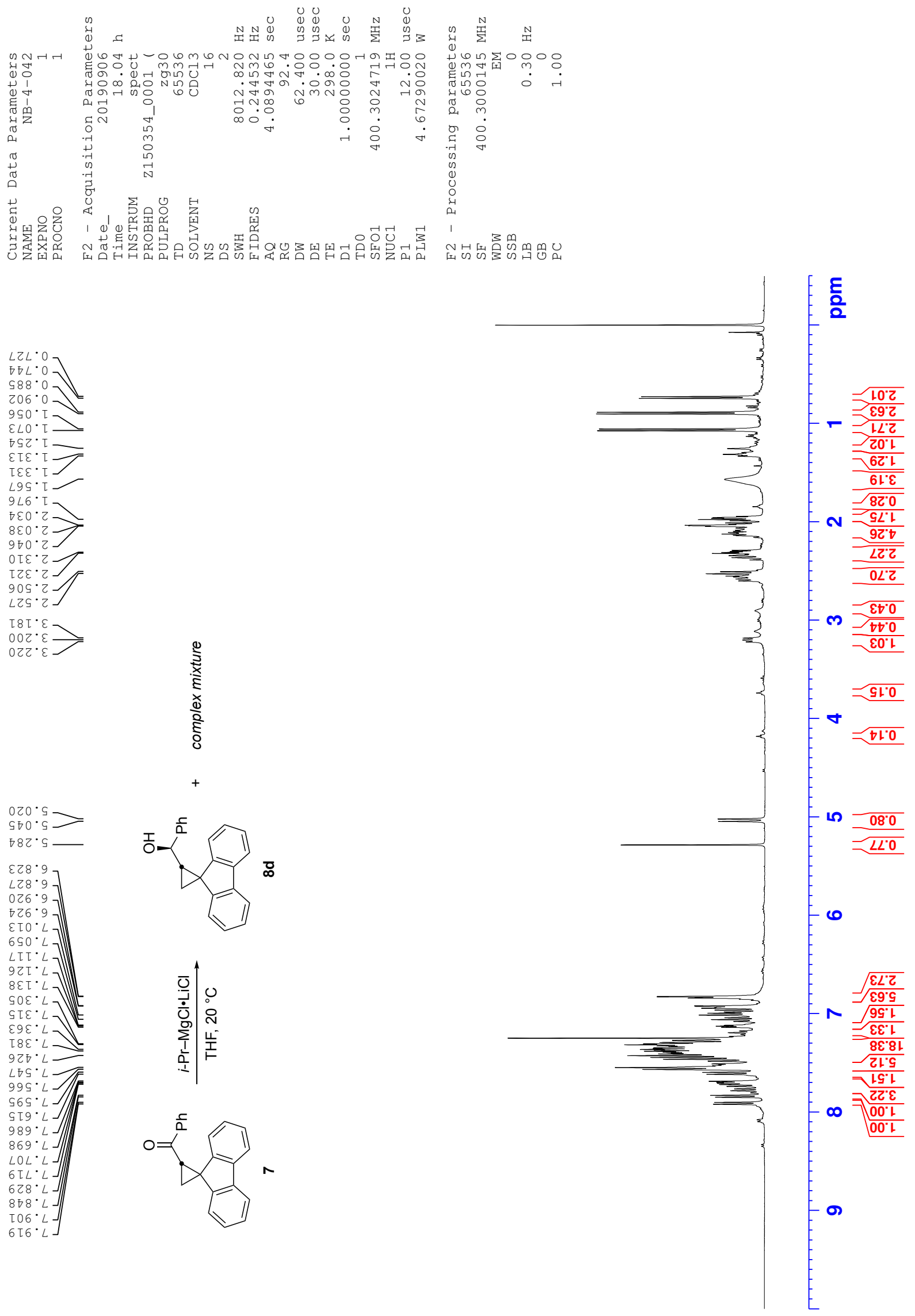

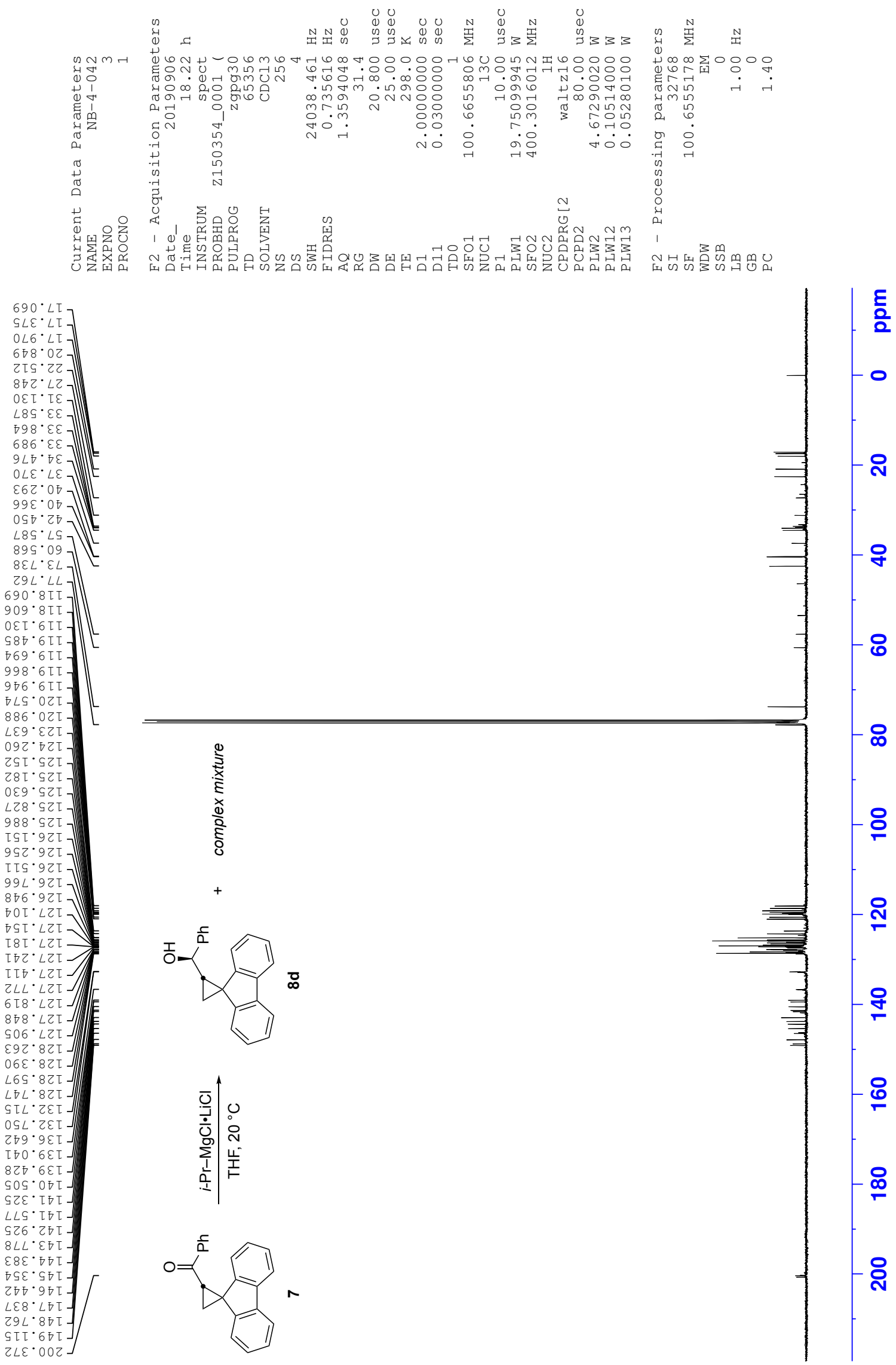

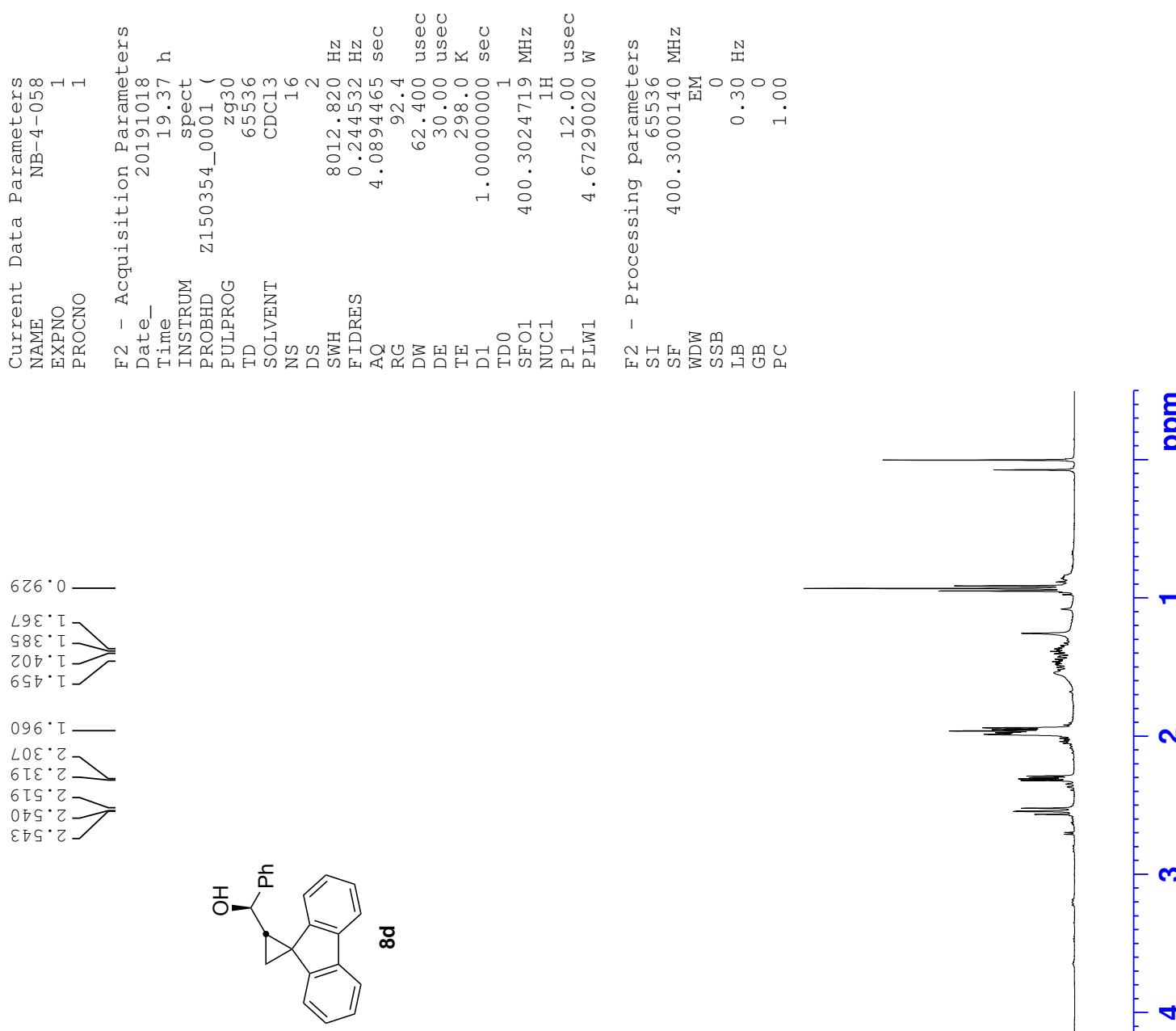

$\frac{\varepsilon}{}$
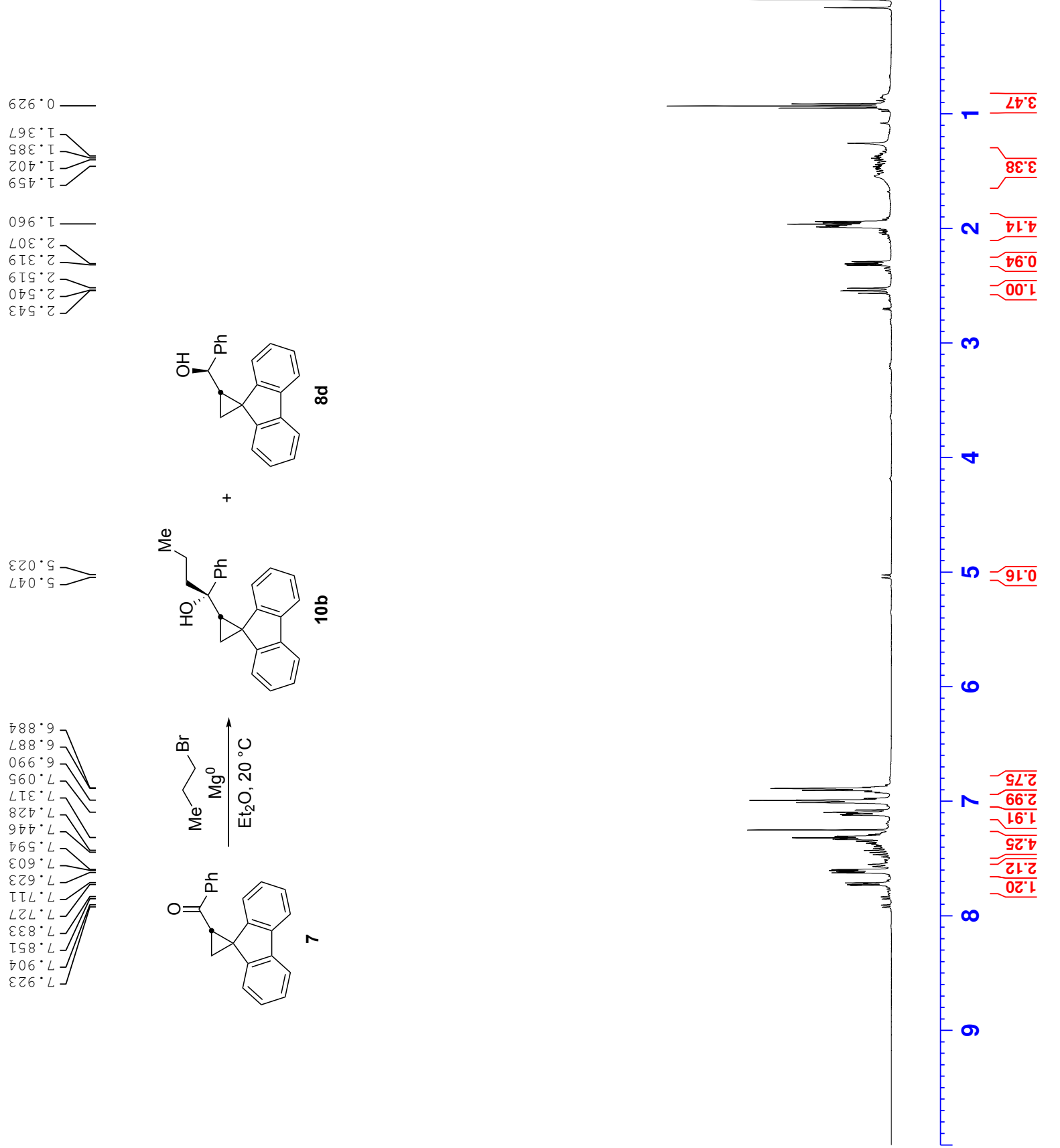

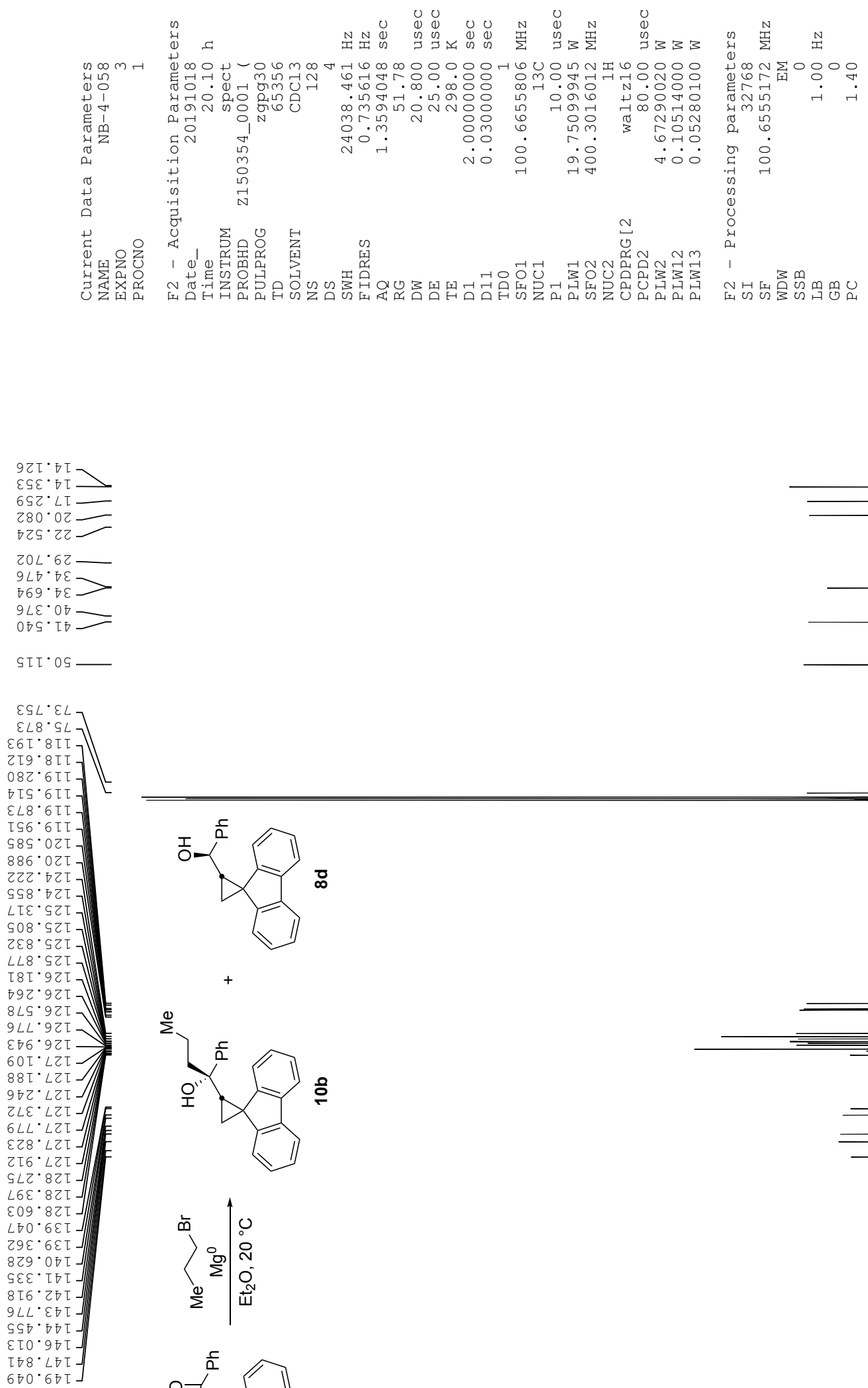

틍

$\stackrel{\infty}{\Sigma}$

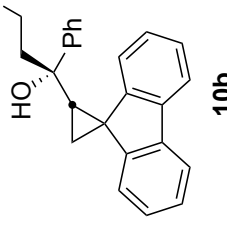

ํำ

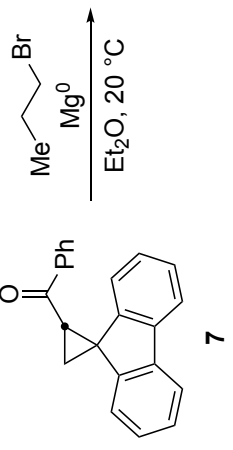

웅

$\stackrel{\infty}{\stackrel{0}{0}}$ 

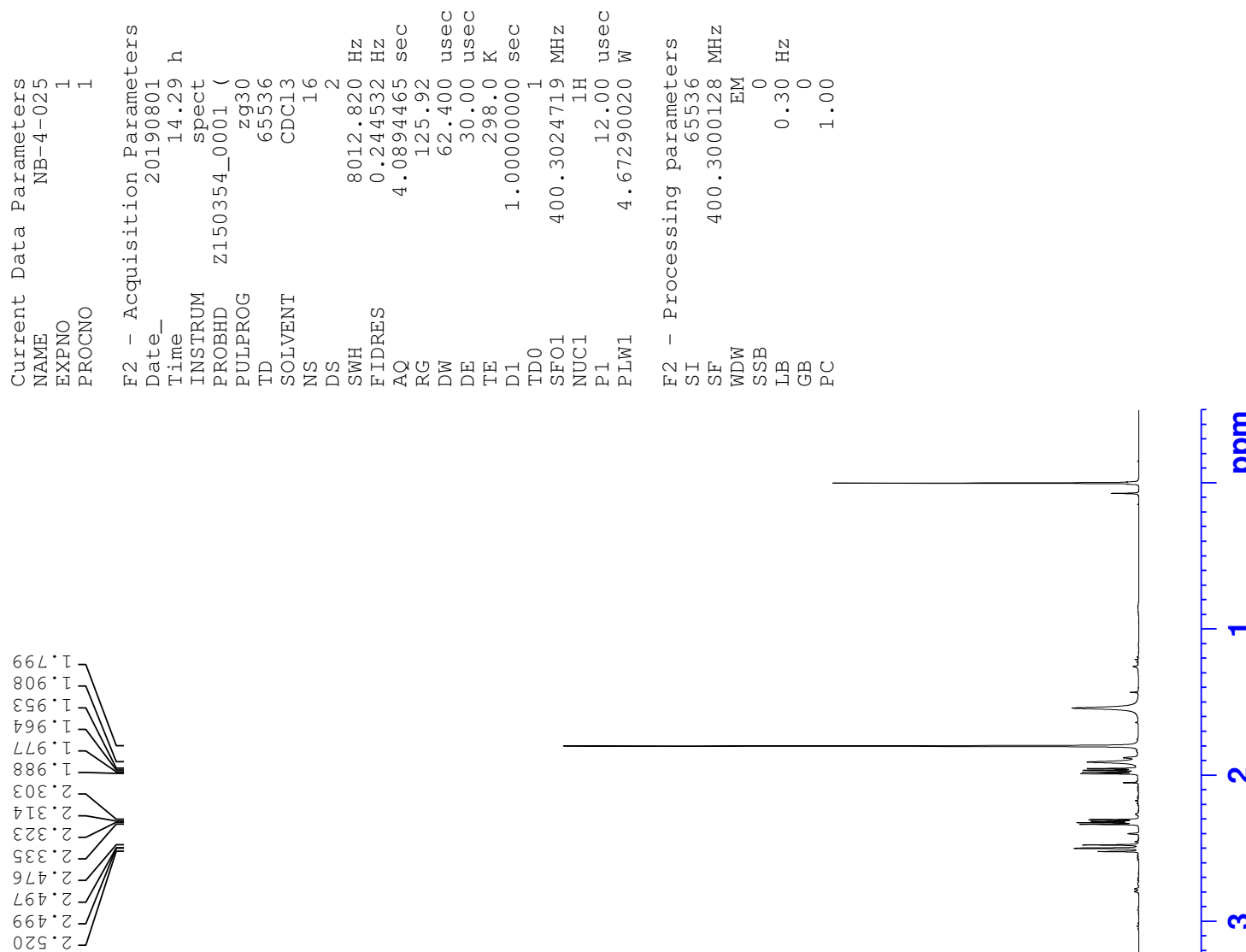

틍

$596 \cdot 9$

$590^{\circ}[$ ]

$690^{\circ} L$

$\left.\begin{array}{l}L \varepsilon[\cdot L \\ 6 \varepsilon[\cdot L]\end{array}\right]$

SSI. $L]$

$\angle S T^{\circ} L \backslash$

$9 L \tau \cdot L \perp$

9 IE.

$6 \tau \varepsilon \cdot L J$

ITg. $L$

โEg. $L$ -

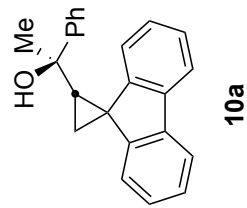

$\stackrel{\square}{\circ}$

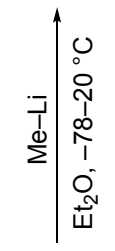

$289^{\circ} \mathrm{L} J$

$\operatorname{LDL}: L]$

$8 S L^{\circ} L J$

$[9 L \cdot L J$

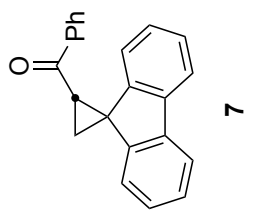

- 10

$-6$

$\sim \sqrt{\sqrt{10^{\circ} \mathrm{Z}}}$

2दम

86

$000 \%$

$-\infty$

86 '2

V $\triangle 0^{\circ}$

$\sqrt{\frac{10.1}{00.1}}$

m

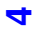



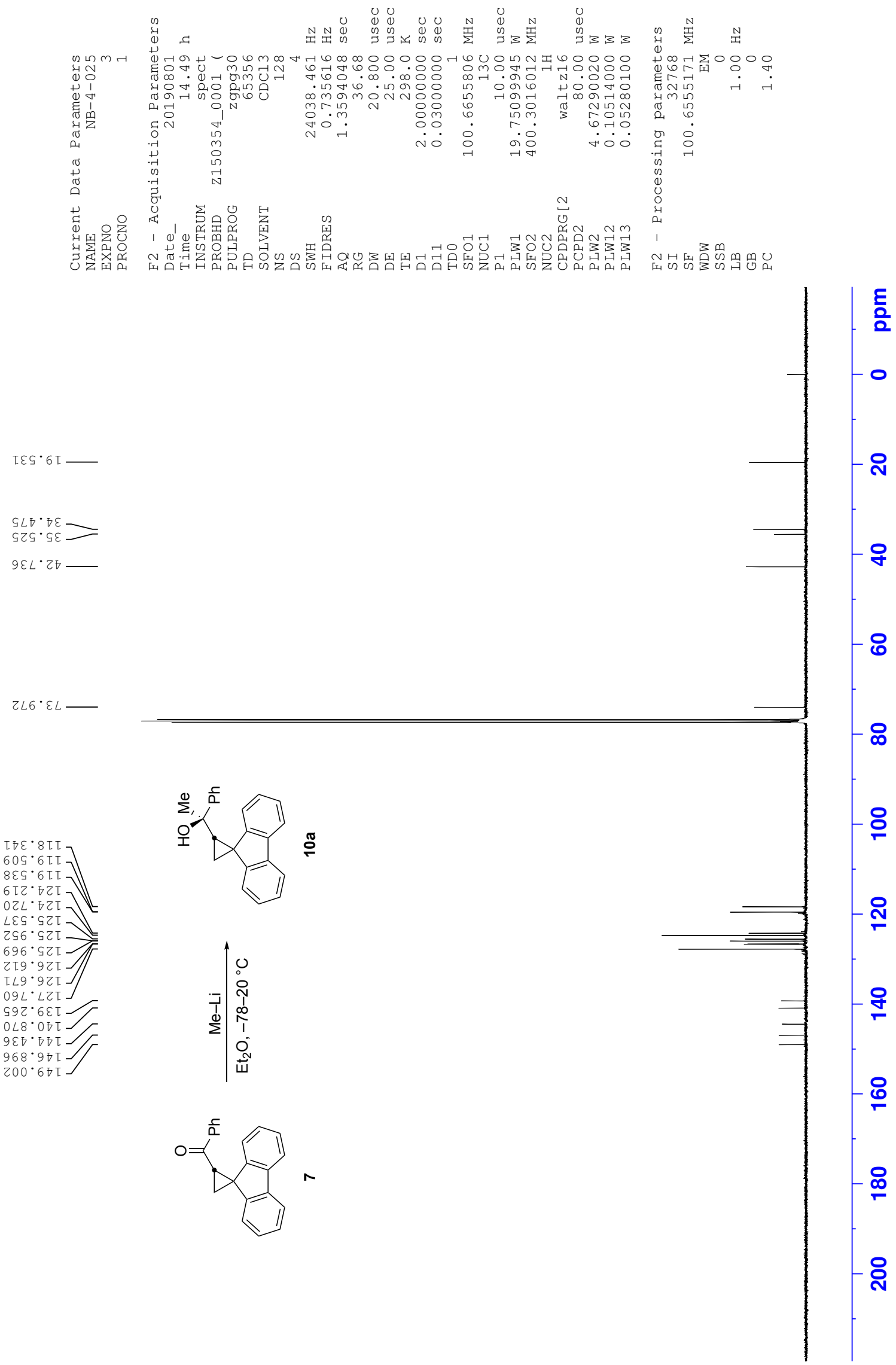

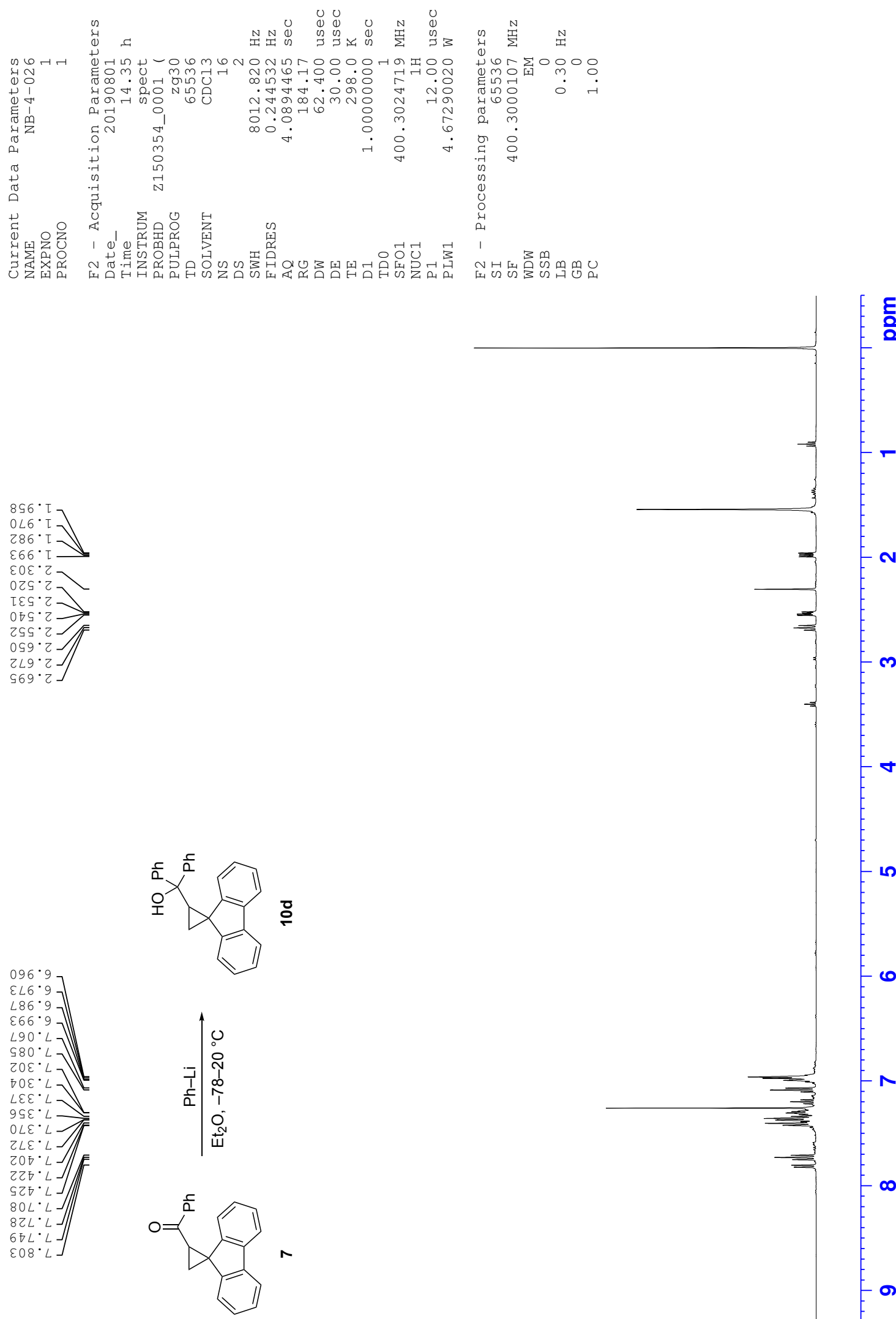

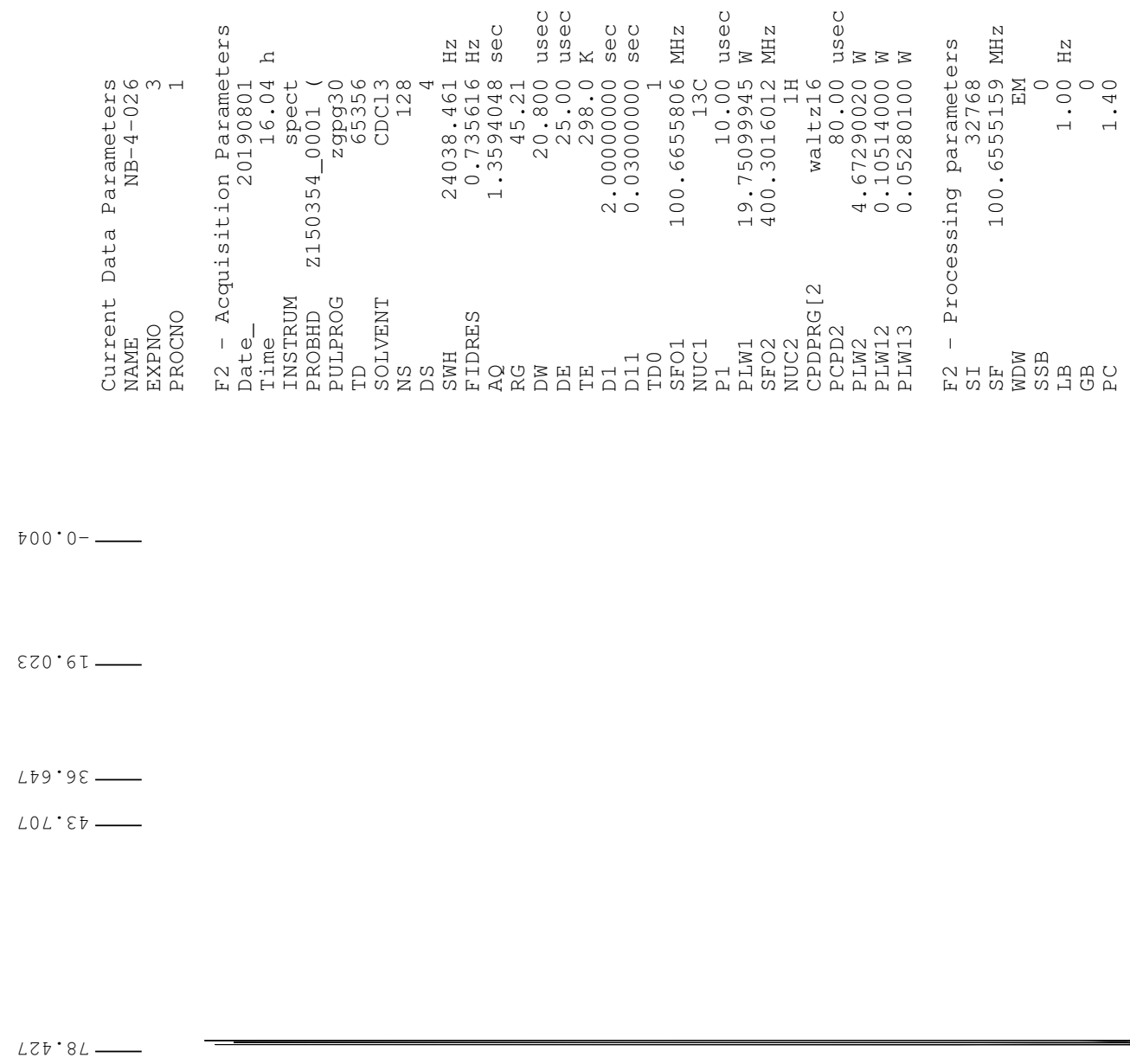

틍
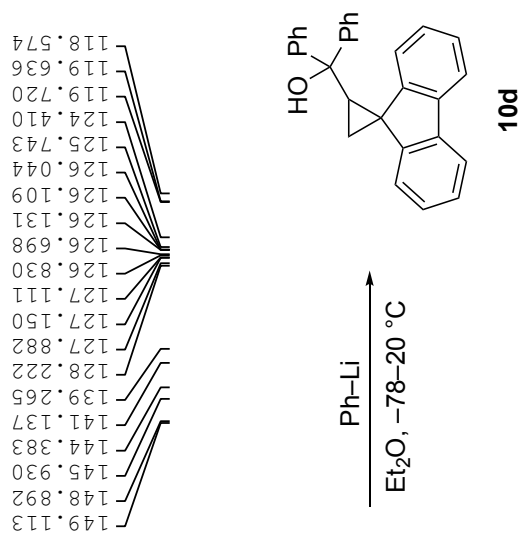

움

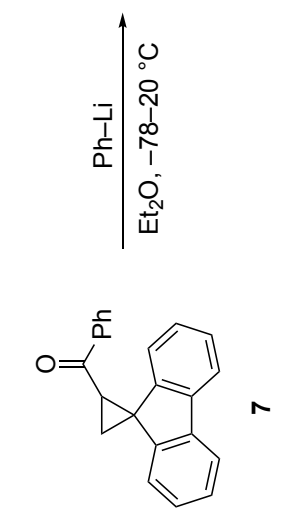

ํำ 

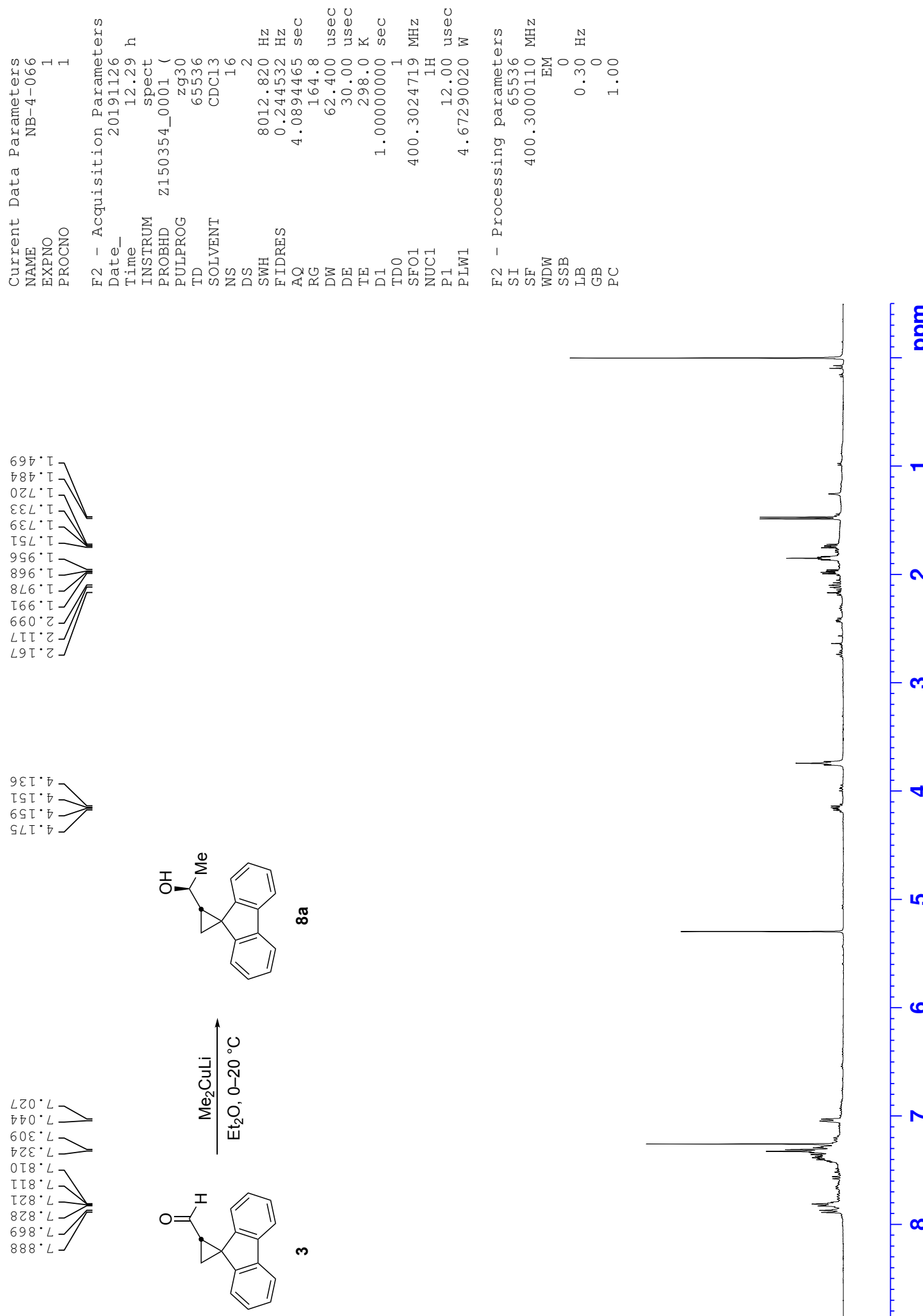

$\frac{\varepsilon}{}$

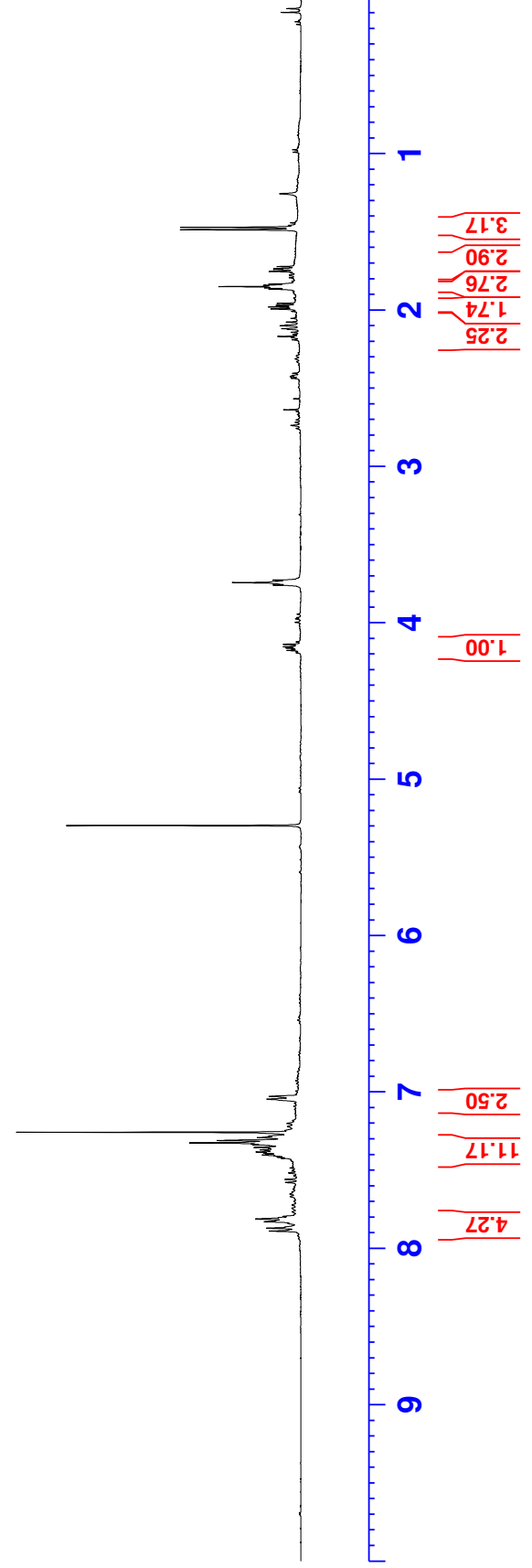



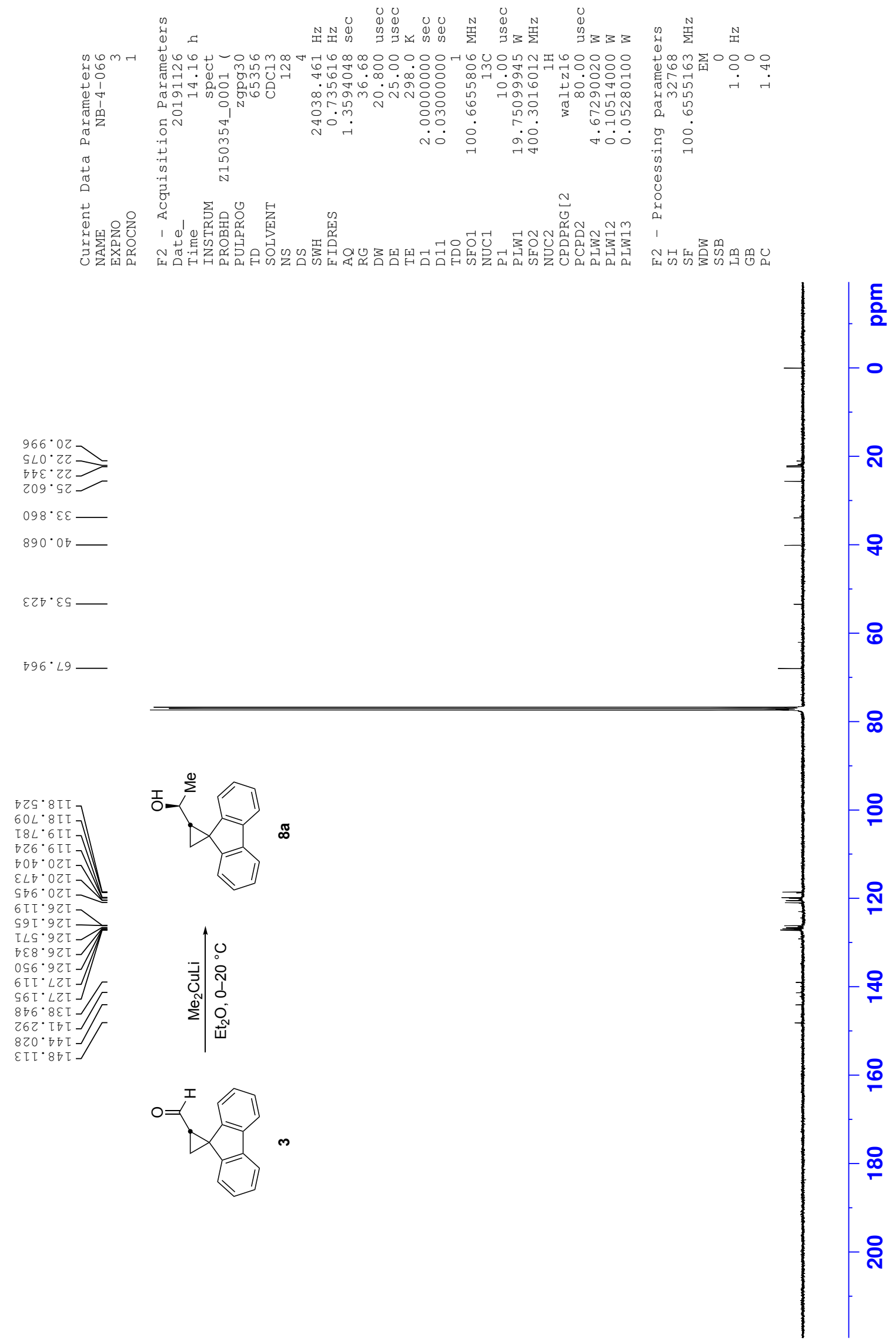

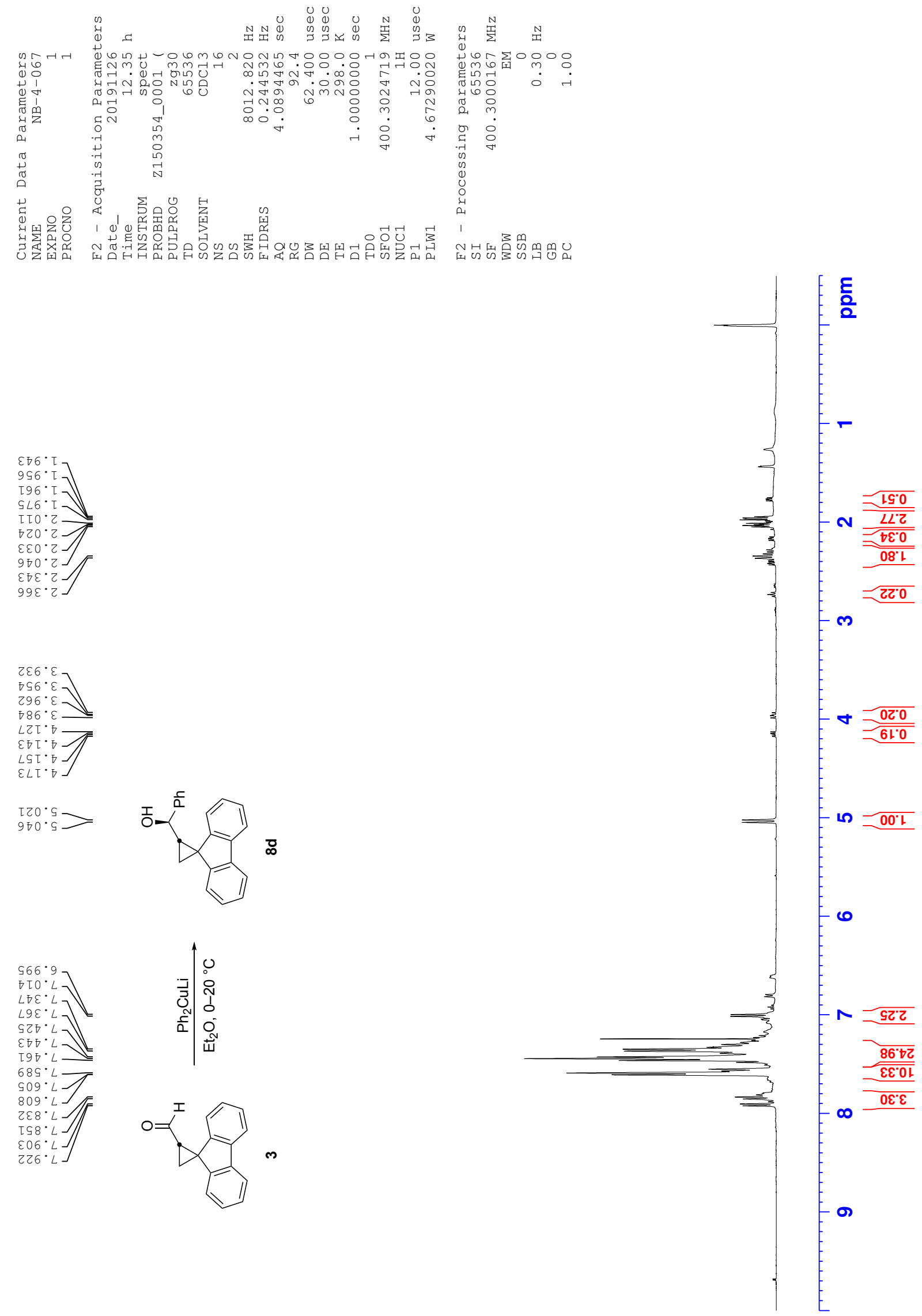

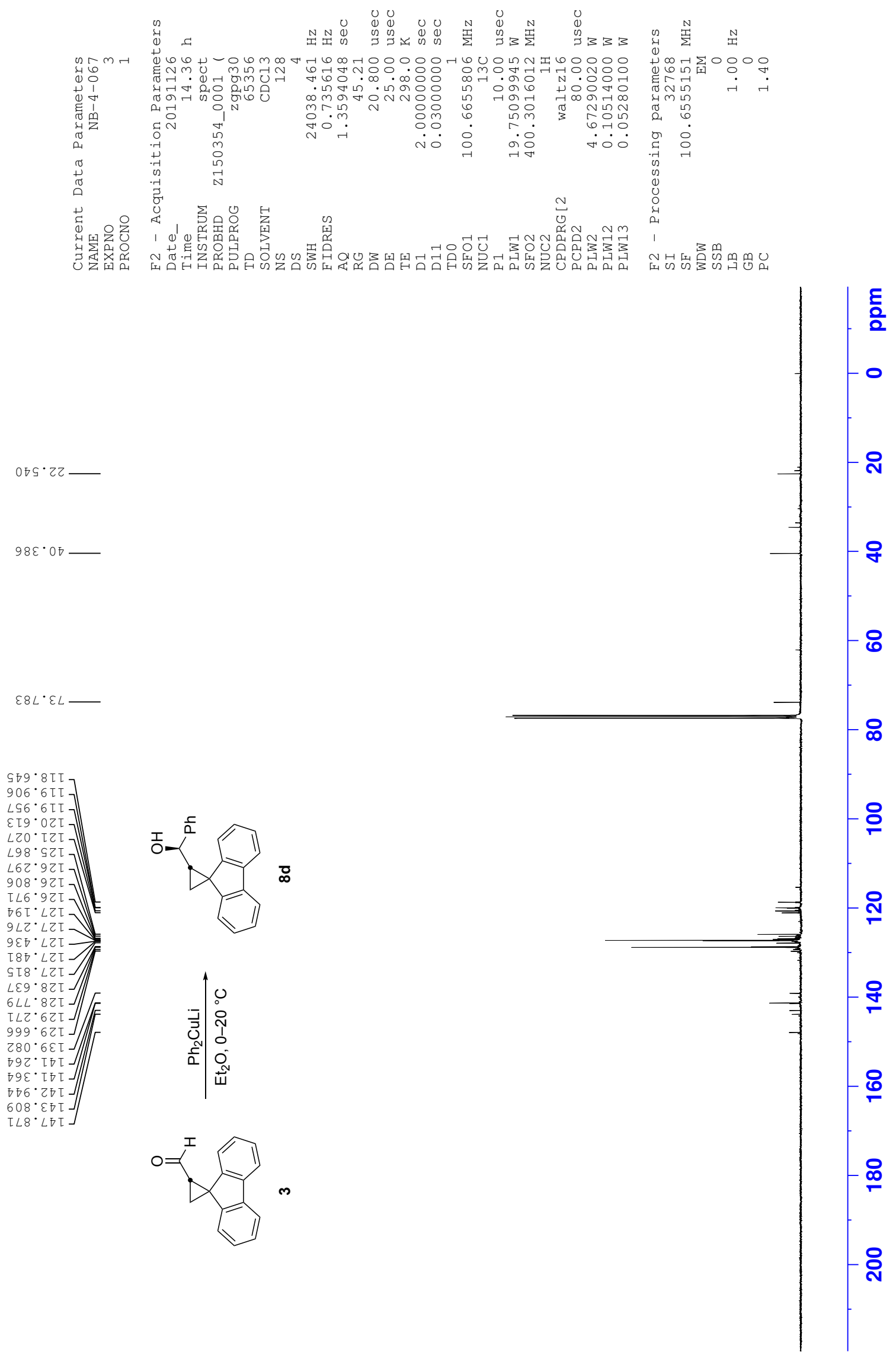

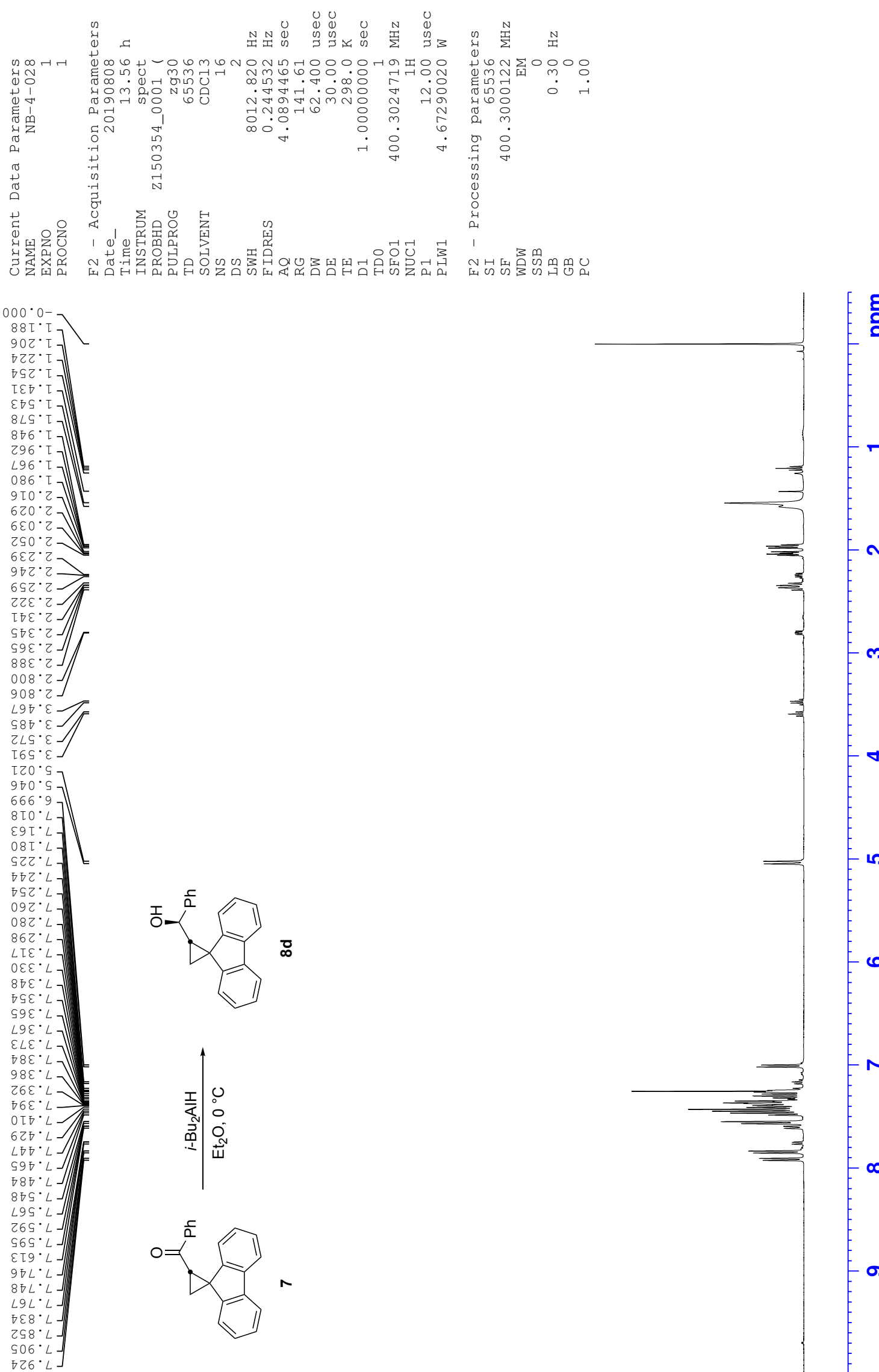

$\frac{\varepsilon}{}$

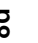<smiles>[CH2-]C(=O)C1CC12c1ccccc1-c1ccccc12</smiles> 

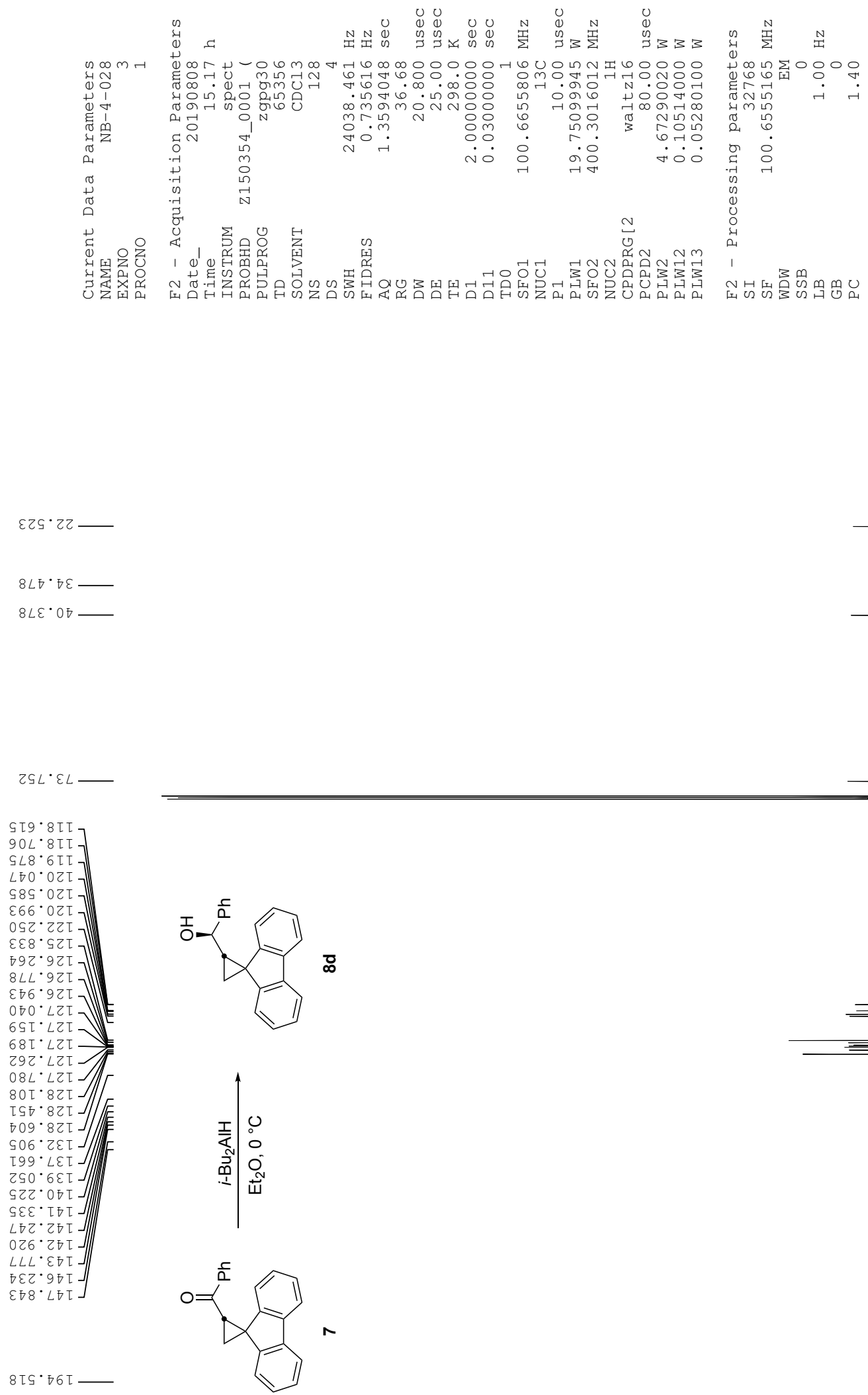

$-8$

q

8

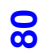

움

ิㅜ

g

$\stackrel{\circ}{\circ}$

$\stackrel{\infty}{\check{0}}$

우 

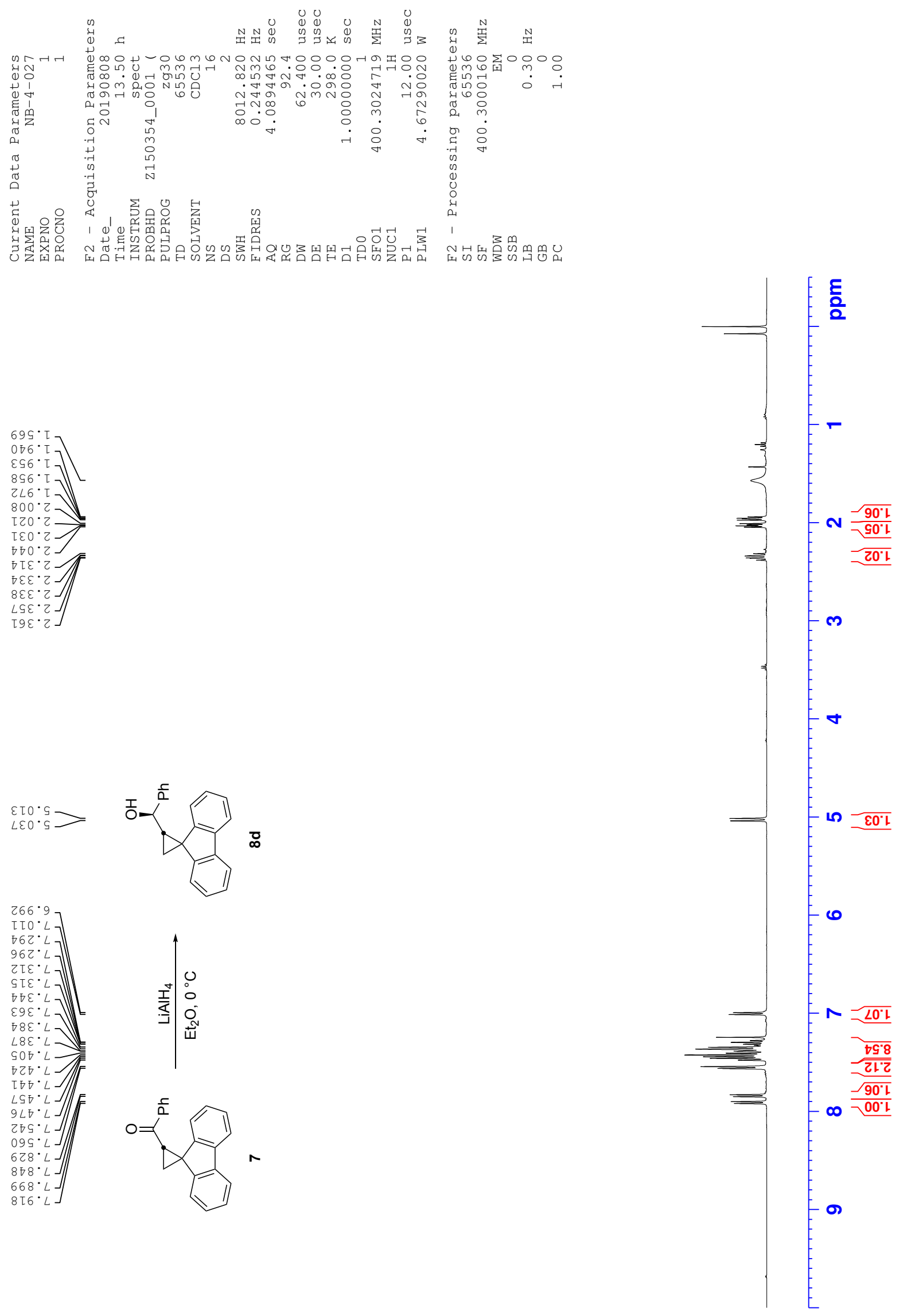

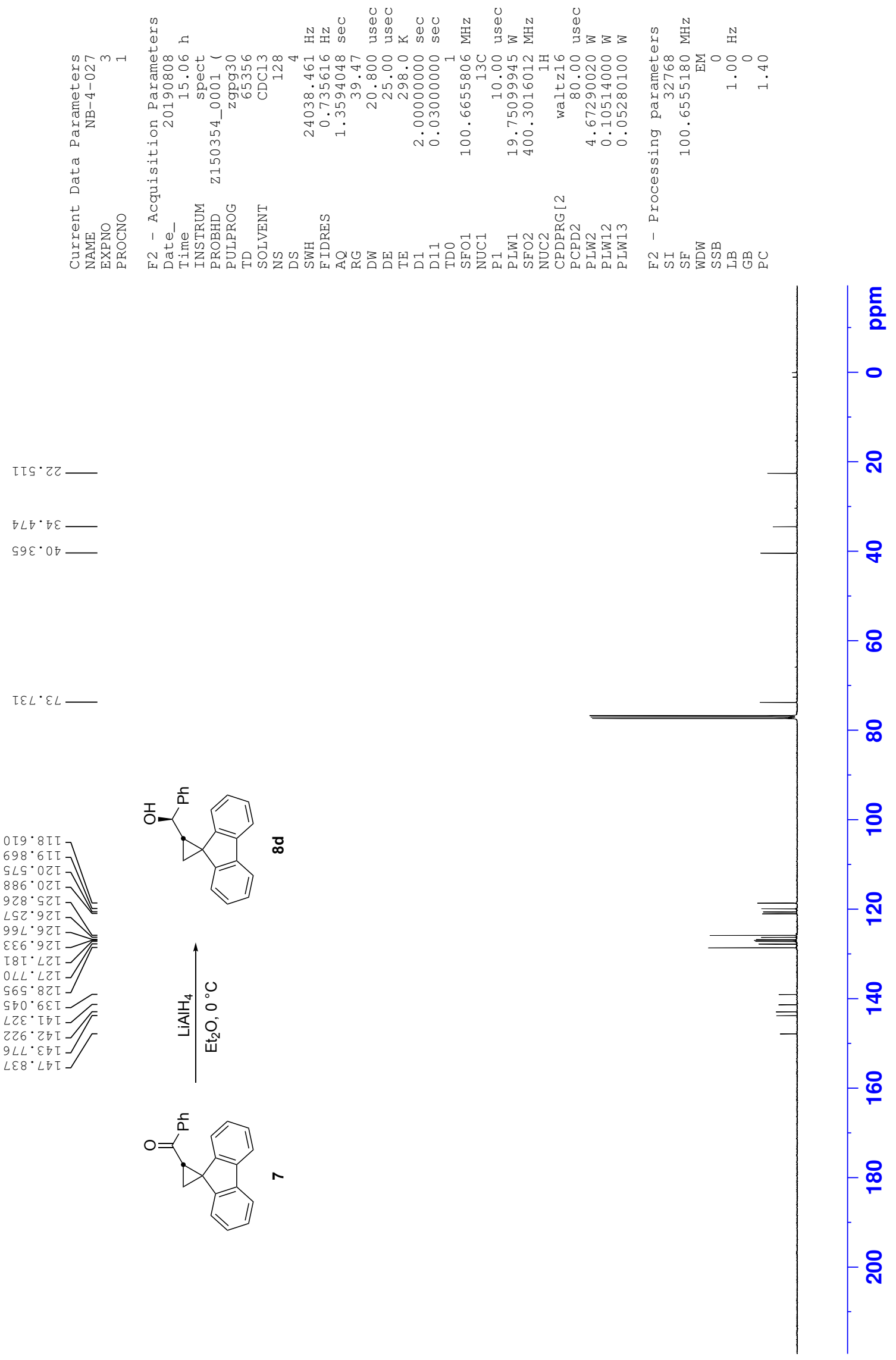

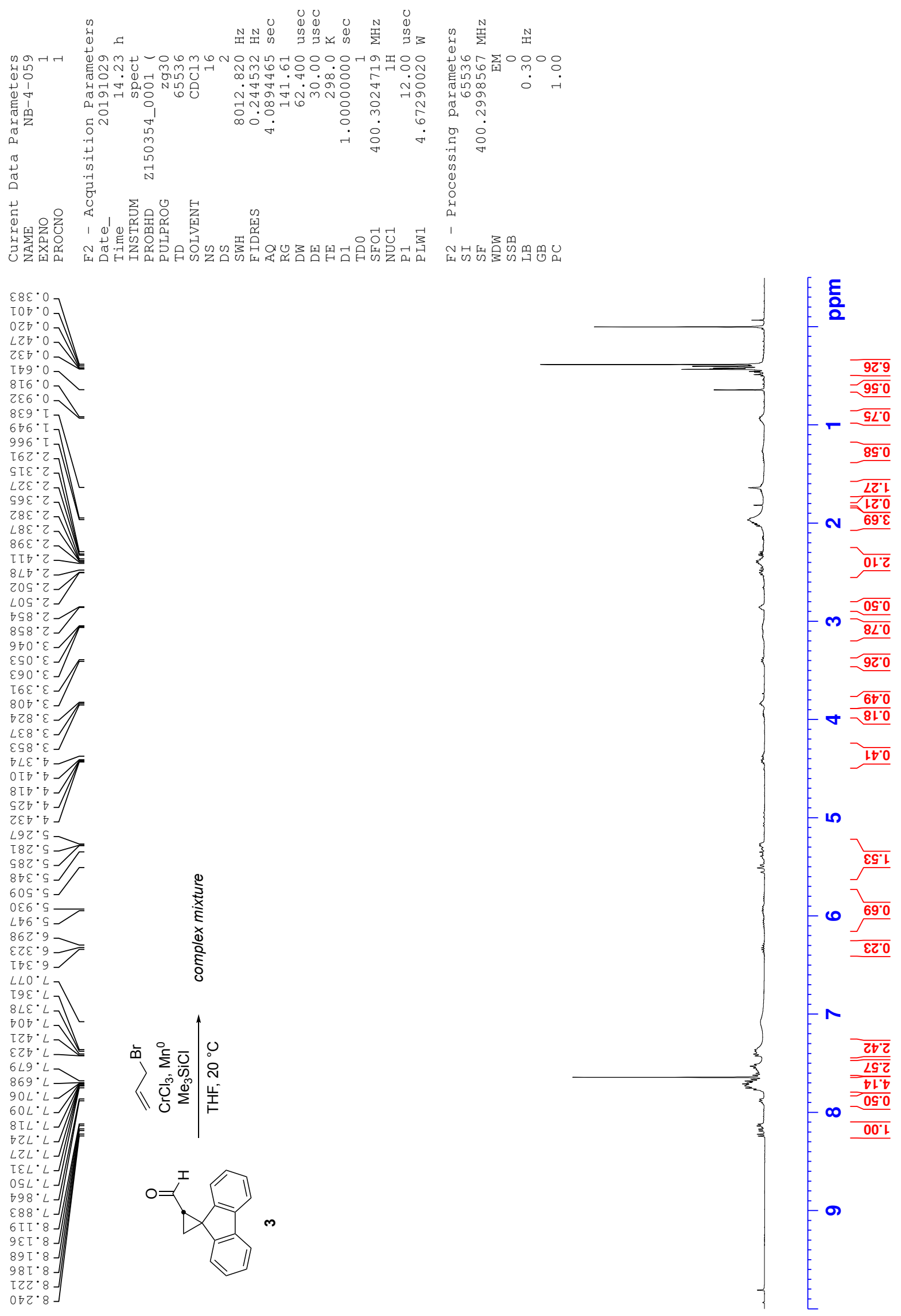

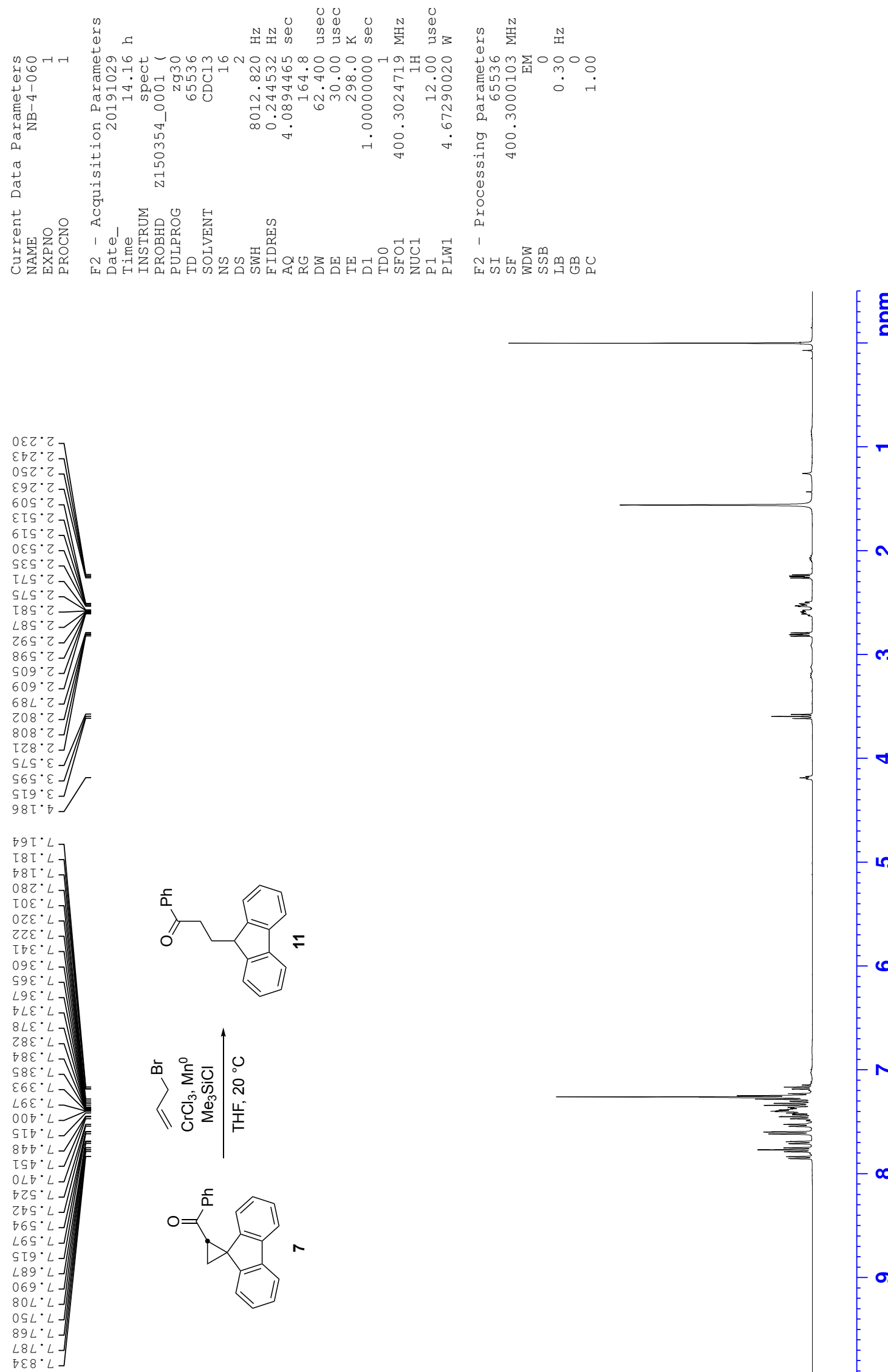

틍

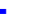

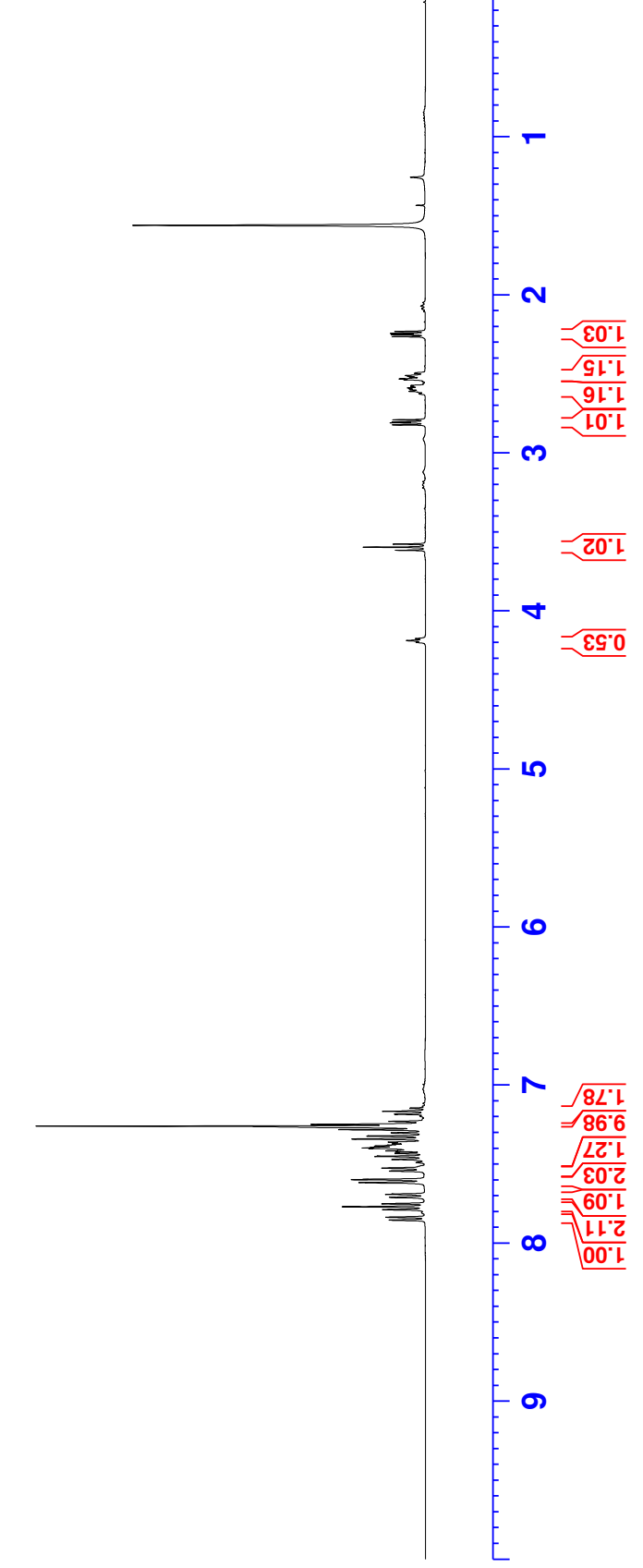



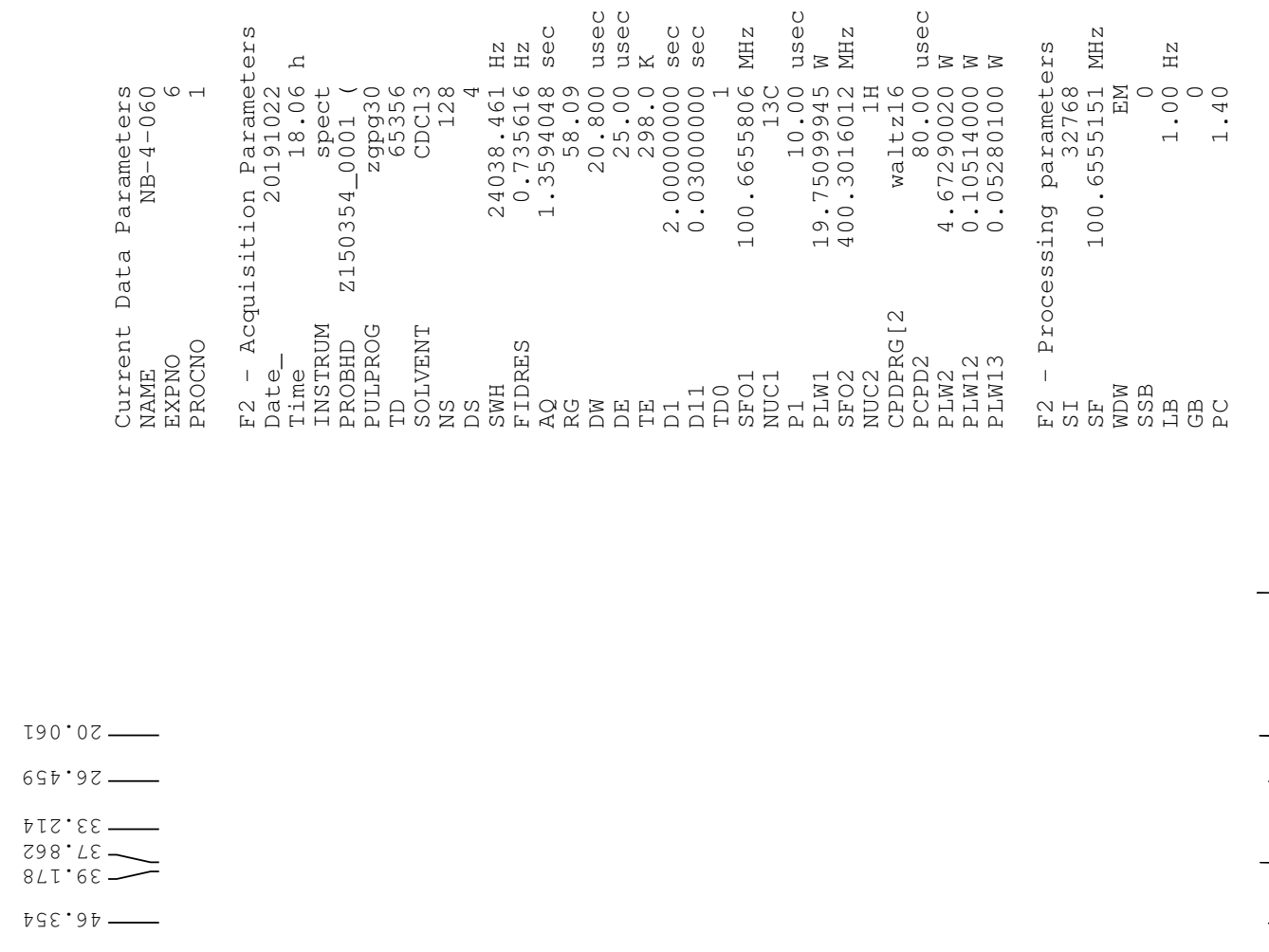

틍
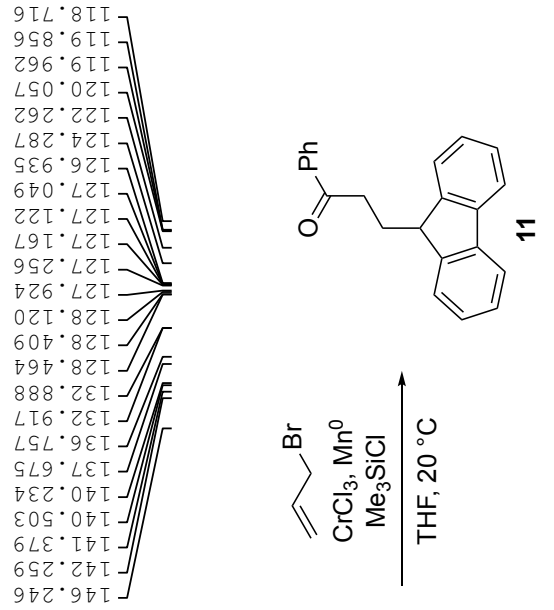

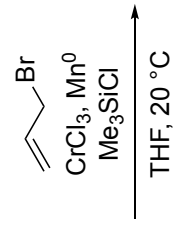

움

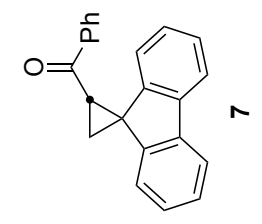



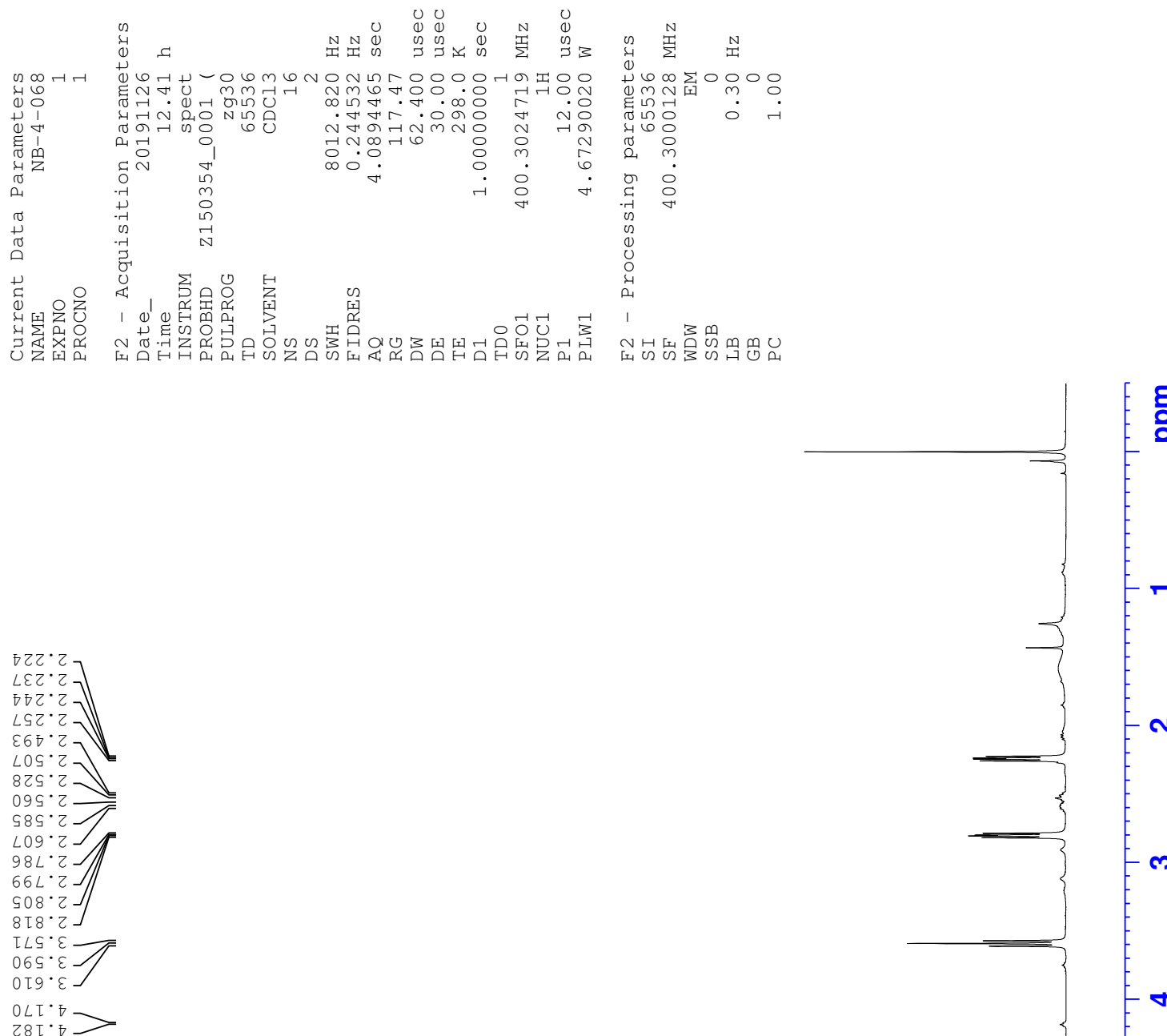

틍

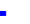
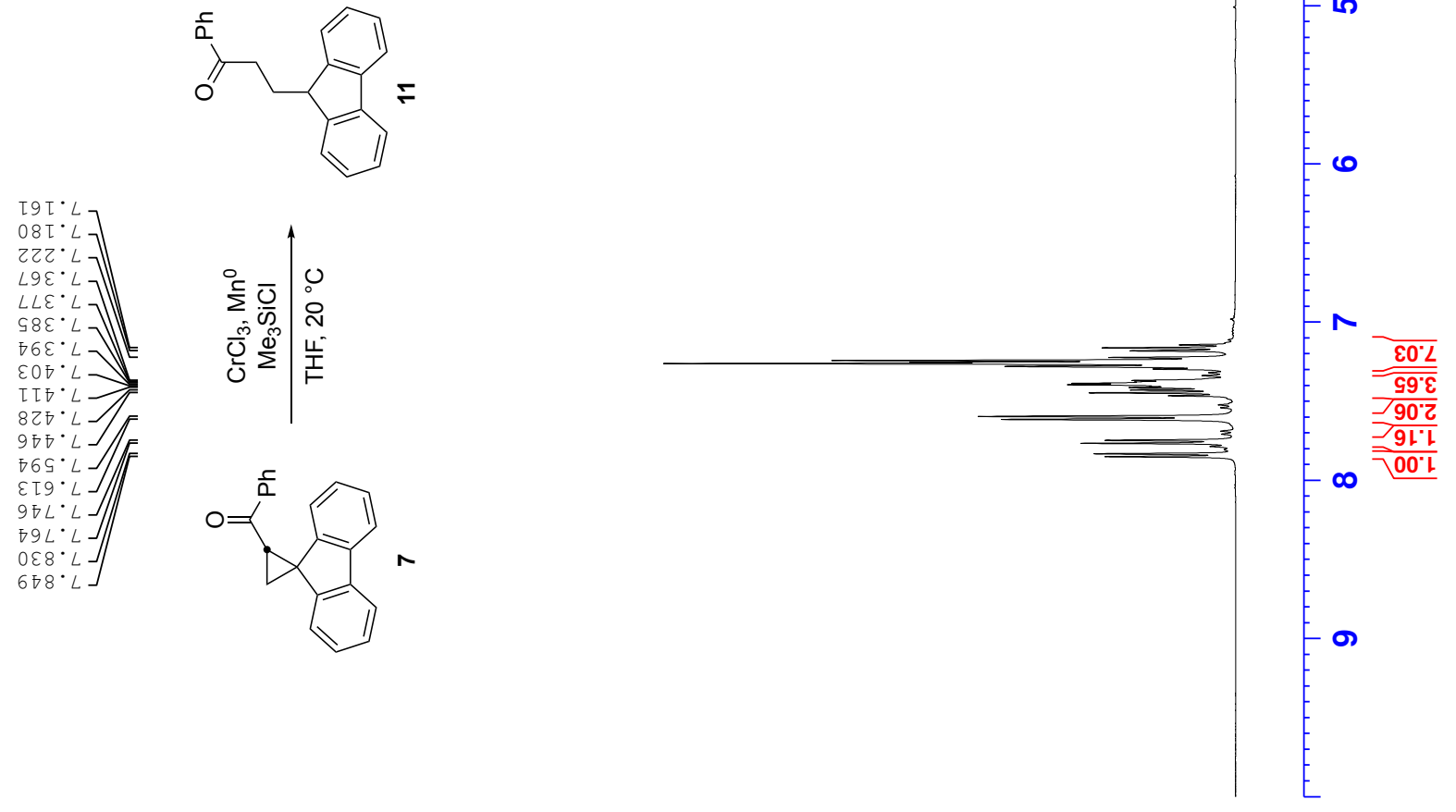

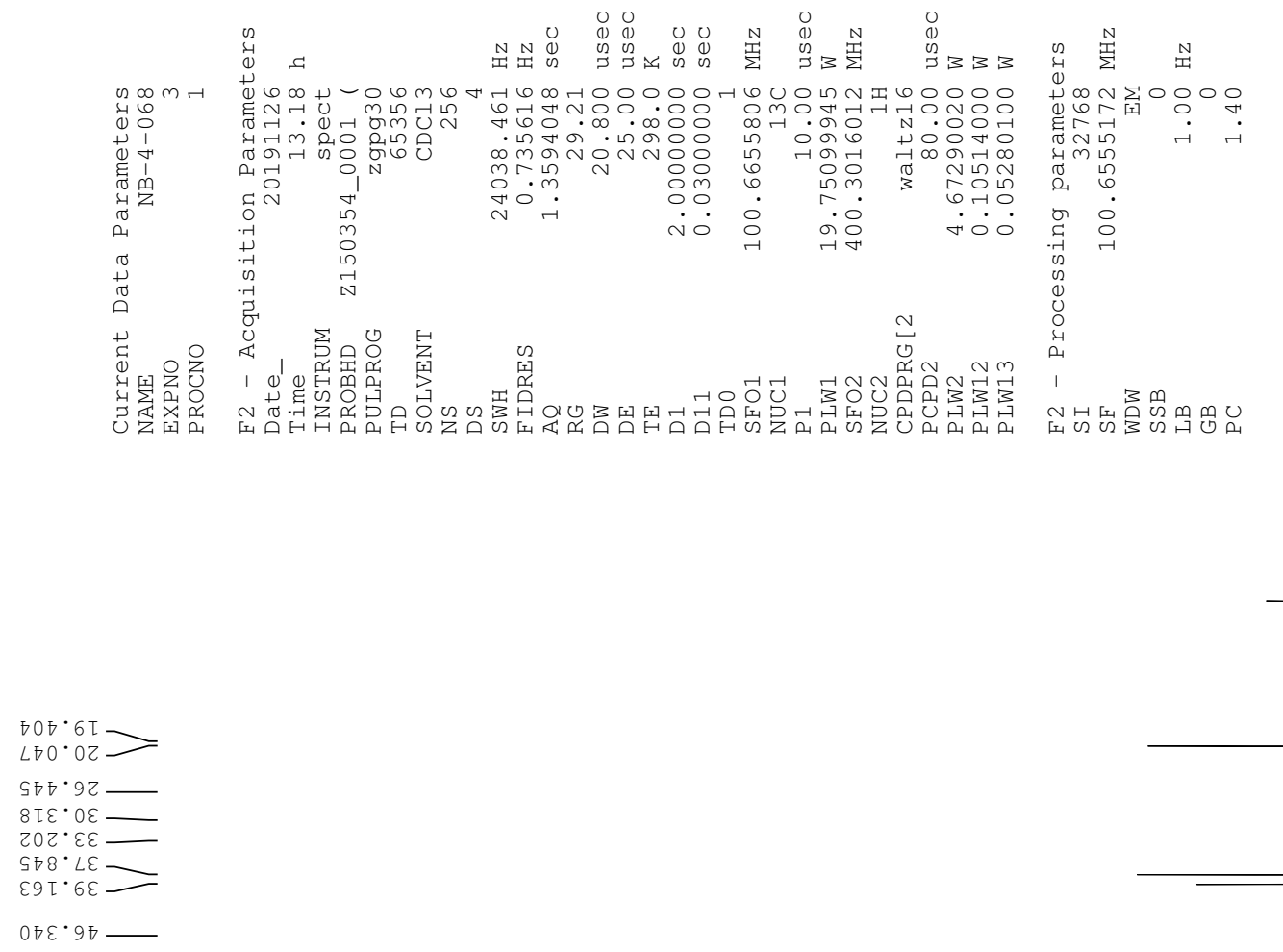

틍
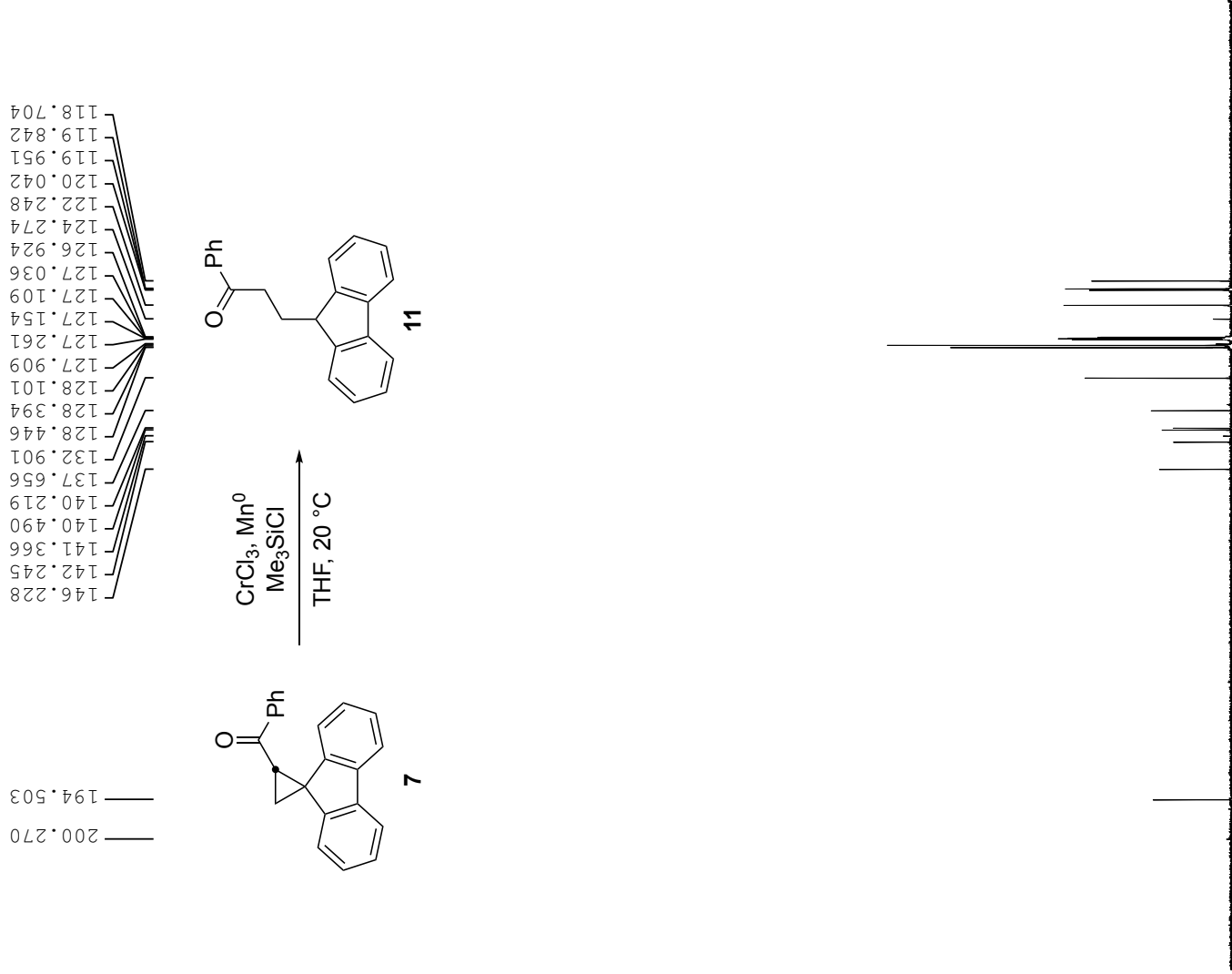

$\infty$

움

ำ

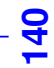

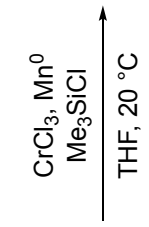

E05. $66 \mathrm{~T}$<smiles>CC(=O)C1CC12c1ccccc1-c1ccccc12</smiles> 

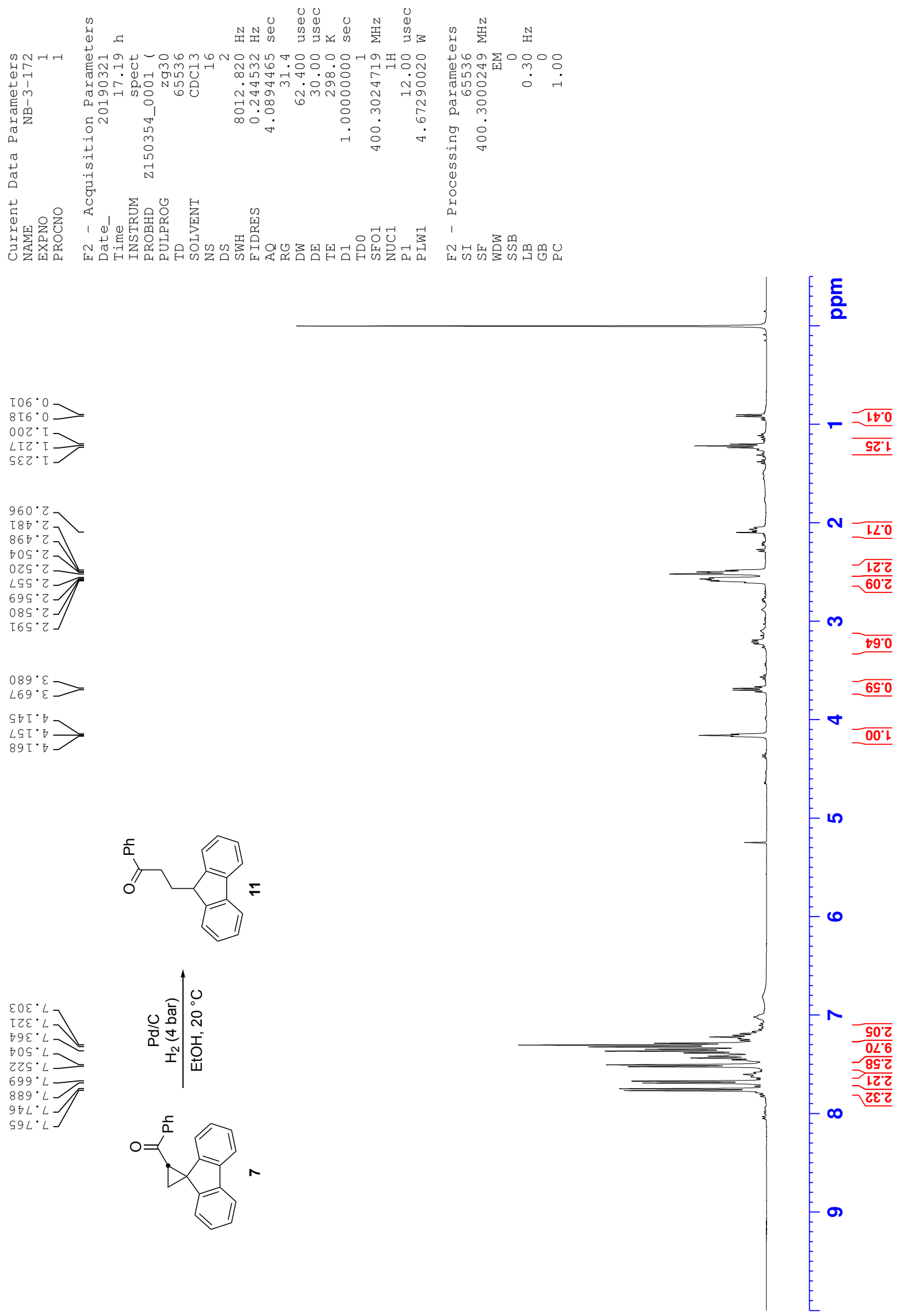

등

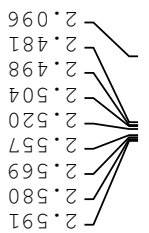

$089 \cdot \varepsilon>$

SET.

$\angle S T \cdot D>$

$89[\cdot 7$ 

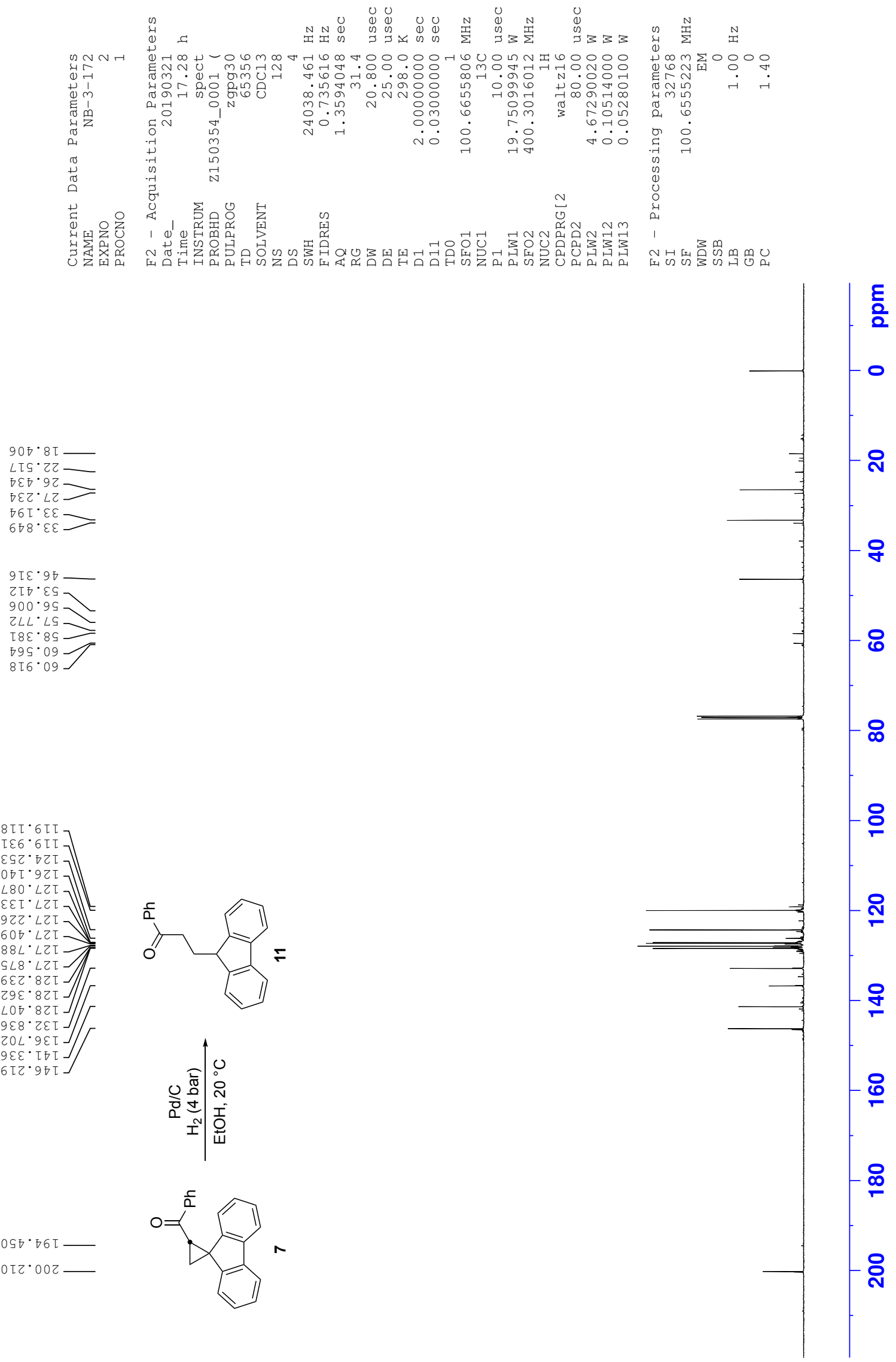